\title{
Laser Science \& Technology Program Annual Report - 2001
}

\author{
H.L. Chen
}

May 9, 2002

\section{Lawrence}

Livermore

National

Laboratory 


\section{DISCLAIMER}

This document was prepared as an account of work sponsored by an agency of the United States Government. Neither the United States Government nor the University of California nor any of their employees, makes any warranty, express or implied, or assumes any legal liability or responsibility for the accuracy, completeness, or usefulness of any information, apparatus, product, or process disclosed, or represents that its use would not infringe privately owned rights. Reference herein to any specific commercial product, process, or service by trade name, trademark, manufacturer, or otherwise, does not necessarily constitute or imply its endorsement, recommendation, or favoring by the United States Government or the University of California. The views and opinions of authors expressed herein do not necessarily state or reflect those of the United States Government or the University of California, and shall not be used for advertising or product endorsement purposes.

This work was performed under the auspices of the U.S. Department of Energy by the University of California, Lawrence Livermore National Laboratory under Contract No. W-7405-Eng-48.

This report has been reproduced directly from the best available copy.

Available electronically at http://www.doe.gov/bridge

Available for a processing fee to U.S. Department of Energy

and its contractors in paper from

U.S. Department of Energy

Office of Scientific and Technical Information

$$
\text { P.O. Box } 62
$$

Oak Ridge, TN 37831-0062

Telephone: (865) 576-8401

Facsimile: (865) 576-5728

E-mail: reports@adonis.osti.gov

Available for the sale to the public from

U.S. Department of Commerce

National Technical Information Service

5285 Port Royal Road

Springfield, VA 22161

Telephone: (800) 553-6847

Facsimile: (703) 605-6900

E-mail: orders@ntis.fedworld.gov

Online ordering: http://www.ntis.gov/ordering.htm

OR

Lawrence Livermore National Laboratory

Technical Information Department's Digital Library

http://www.llnl.gov/tid/Library.html 


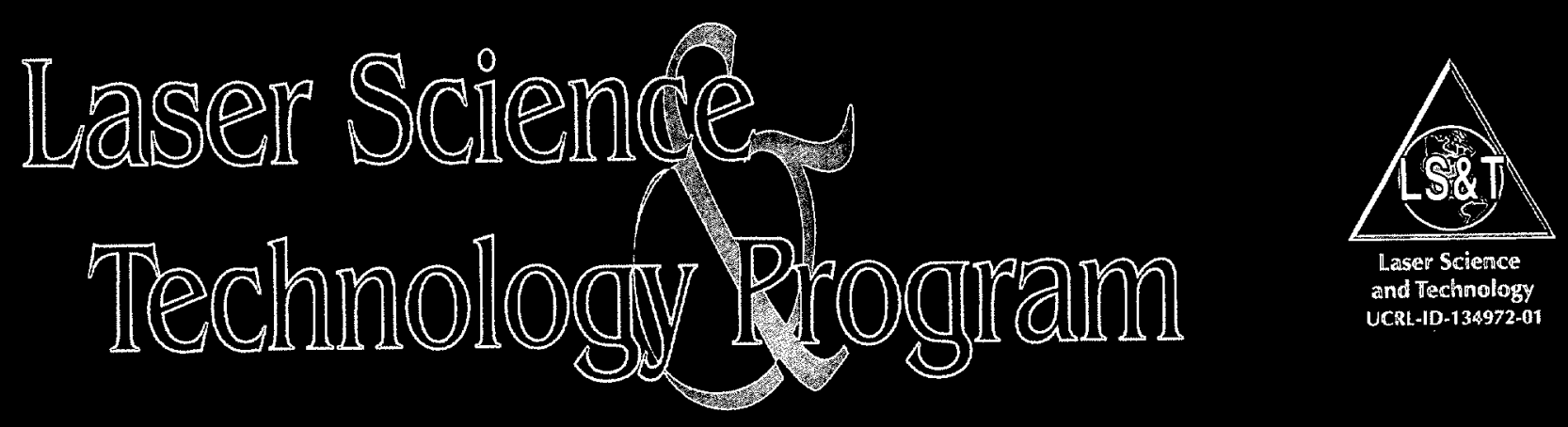

\begin{tabular}{l}
$\frac{2}{2}$ \\
$\frac{0}{0}$ \\
$\frac{8}{8}$ \\
\hline
\end{tabular}



Anmual Repont - 2001

NIIF Progirams Directorate

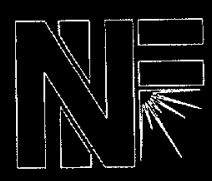




\section{FOREWORD}

The Laser Science and Technology (LS\&T) Program Annual Report 2001 provides documentation of the achievements of the LLNL LS\&T Program during the April 2001 to March 2002 period using three formats: (1) an Overview that is a narrative summary of important results for the year; (2) brief summaries of research and development activity highlights within the four Program elements: Advanced Lasers and Components (AL\&C), Laser Optics and Materials (LO\&M), Short Pulse Laser Applications and Technologies (SPLAT), and HighEnergy Laser System and Tests (HELST); and (3) a compilation of selected articles and technical reports published in reputable scientific or technology journals in this period. All three elements (Annual Overview, Activity Highlights, and Technical Reports) are also on the Web: http://laser./lnl.gov/lasers/pubs/icfq.html.

The underlying mission for the LS\&T Program is to develop advanced lasers, optics, and materials technologies and applications to solve problems and create new capabilities of importance to the Laboratory and the nation. This mission statement has been our guide for defining work appropriate for our Program. A major new focus of LS\&T beginning this past year has been the development of high peak power short-pulse capability for the National Ignition Facility (NIF). LS\&T is committed to this activity.

Questions and comments relating to the content of this report should be addressed to the Laser Science and Technology Program Office, Lawrence Livermore National Laboratory, P.O. Box 808, L-482, Livermore, CA 94551. 


\title{
LASER SCIENCE AND TEChNOLOGY Program UPdate 2001
}

\author{
L. A. Hackel AND H. L. Chen
}

May 2002

LAWRENCE LIVERMORE NATIONAL LABORATORY

University of California $\bullet$ Livermore, California $\bullet 94550$ 


\section{TABLE OF Contents}

1. Overview of the Laser Science and

Technology Program ................... I-1

Deploying NIF Hardware and Improving NIF Laser

Optics and Materials Performance

Construction and Testing of Z-beamlet Completed; Laser

Trigger System Delivered

KW- to MW-Class Solid-State Laser Technology for Inertial

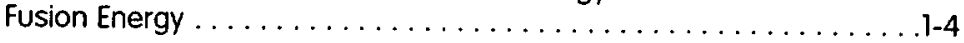

Solid-State Heat Capacity Laser for the Department of Defense . . . . . .1-6

Advanced Solid-State Lasers for Government and Industry . . . . . . . . . . 1-7

High Energy Ultrashort-Pulse Laser Technology . . . . . . . . . . . . . . . . . 1-9

Large-Scale Diffractive Optics and Adaptive Optics for Space,

NIF, and Petawatt Applications ........................1-10

Laser Guide Star Systems for Ground-Based Observatories . . . . . . 1-12

2. Advanced Lasers and Components .... . . . . . . . . 2-1

The Mercury Laser, a Diode-Pumped Solid-State Laser

Driver for Inertial Fusion, is Activated . . . . . . . . . . . . . . . . .

Development of High-Power-Microchannel-Cooled, Laser-Diode

Array is Underway $\ldots \ldots \ldots \ldots \ldots \ldots \ldots \ldots \ldots . . \ldots \ldots .2-2$

3. Laser Optics and Materials ... . . . . . . . . . . . 3-1

LS\&T Improves $\mathrm{CO}_{2}$ - Laser Treatment of NIF Final Optics ........ . .

Continuous Melting Process Produces High-Quality, Meter-Sized

Glass Slabs . . . . . ......................... .

Improving Antireflection Coatings for NIF KDP and DKDP Optics . . . .3-4

Shocked and Stressed, Metals Get Stronger . . . . . . . . . . . . . . 3-6

Lasershot Makes its Mark . . . . . . . . . . . . . . . . . . . . -9

4. Short Pulse Lasers, Applications and Technology . . 1

High-Bandwidth Technology for High-Power Short-Pulse Lasers. . . . . .

Meter-Scale Gratings Delivered to Rutherford for Petawatt

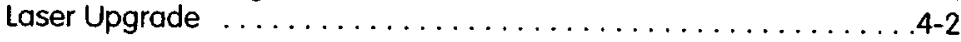

New Wet-Etching Process Figures Large-Aperture and

Ultrathin Optics .................................4-4

Segemented Fesnel Lens Developed for the Eyeglass

Space Telescope ...............................4-5

Further Developments in Ultrashort-Pulse Lasers . . . . . . . . . 4-7

5. Solid-State Heat-Capacity Laser and Tests . . . . . . . $5-1$

A 10-kW Solid-State Heat-Capacity Laser System Installed at HELSTF, White Sands Missile Range . ....................... .

$42-\mathrm{kW}$ Diode Array Delivered to the HELSTF Program . . . . . . . . . . 5-2

6. Special Project: Laser Material Processing . . . . . . 6-1

Laser Peening Increases the Corrosion Resistance of Metal ... . . . . . 6-1

Joint Research Develops Laser Peenforming—A New Precision

Metal-Forming Technology 


\section{Laser Science and Technology Program}

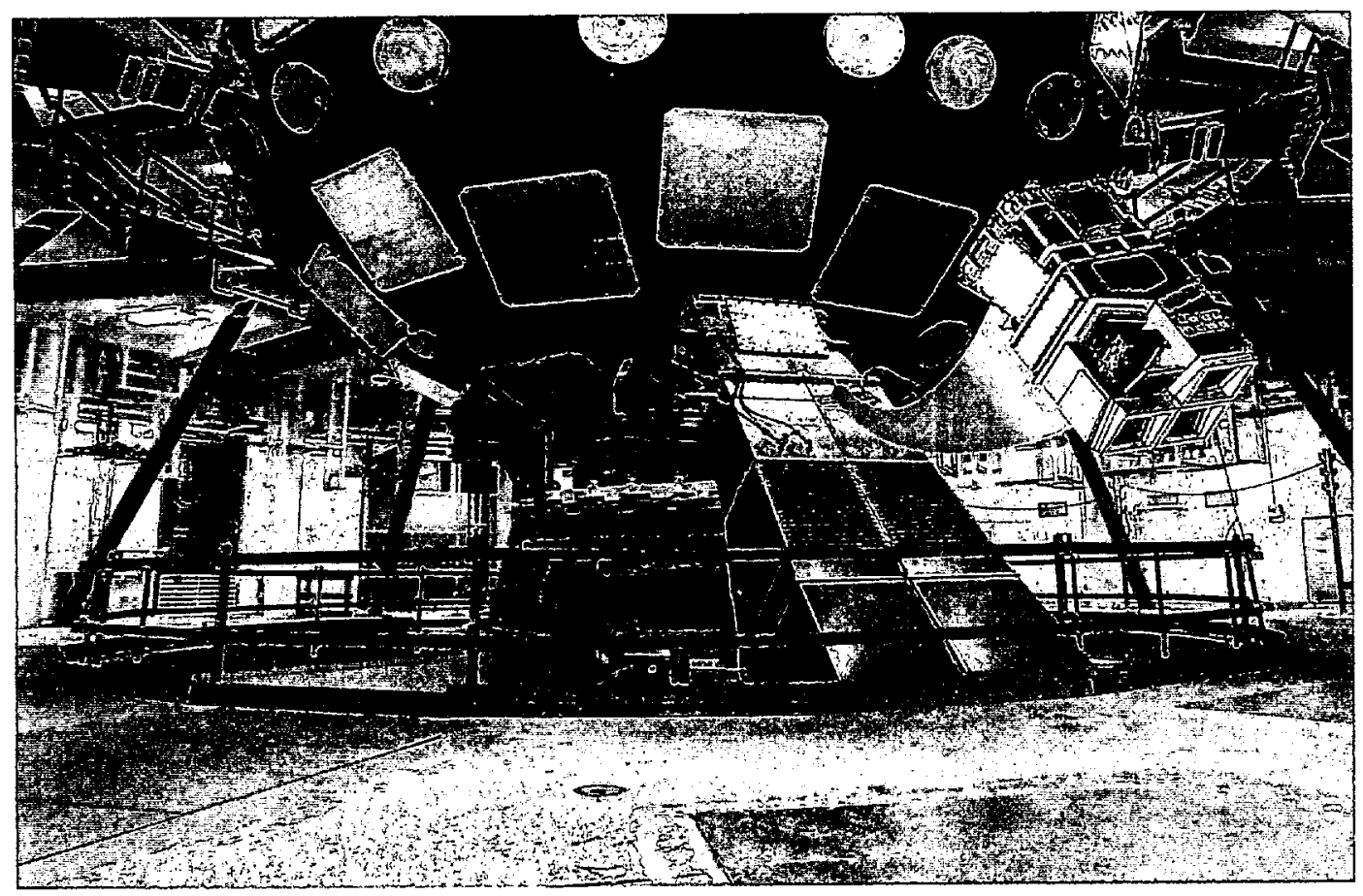

Final Optics Assembly simulated in target bay.

\section{Overview}

$\mathrm{T}$ he Laser Science and Technology (LS\&T) Program's mission is to develop advanced solid-state lasers, optics, materials technologies, and applications to solve problems and create new capabilities of importance to the Nation and the Laboratory. A top, near-term priority is to provide technical support and core competencies to the National Ignition Facility (NIF) to ensure commissioning success.

The primary objectives of LS\&T activities in fiscal year (FY) 2001 have been threefold(a) to support deployment of hardware and to enhance lasers and optics performance for NIF, (b) to develop advanced solid-state laser systems and optical components for the Department of Energy (DOE) and the Department of Defense (DoD), and (c) to invent, develop, and deliver improved concepts and hardware for other government agencies and U.S. industry. Special efforts have also been devoted to building and maintaining our capabilities in three technology areas: highpower solid-state lasers, high-power optical materials, and applications of advanced lasers. LS\&T activities during FY2001 focused on eight major areas:

1. NIF Project-LS\&T led major advances in technology and optical component development to improve the lifetime of $3 \omega \mathrm{UV}$ optics and enhance NIF's operations and performance capabilities.

2. Stockpile Stewardship Program (SSP)-LS\&T completed two advanced solid-state laser systems for SSP at the Sandia National Laboratories (SNL) in Albuquerque, New Mexico. Both lasers are currently operated by SNL to support experiments on SNL's Z-machine. 
3. High-energy-density physics and inertial fusion energy-LS\&T continued development of kW- to MW-class, diode-pumped, solid-state laser (DPSSL) drivers.

4. Department of Defense (DoD)_LS\&T developed and demonstrated a $13-\mathrm{kW}$ average-power heat-capacity laser as part of a longer term effort to develop a 100-kWclass solid-state heat-capacity laser (SSHCL) for missile defense.

5. Nuclear energy applications-LS\&T continued to develop laser-shock peening technology to improve the service lifetime of metal canisters designed for final disposal of highlevel radioactive waste and retired weapon components at Yucca Mountain, Nevada. Under cooperative research and development agreements (CRADA) with U.S. industry, we are also developing $\mathrm{kW}$-class solid-state lasers for shock peening and hole drilling of metals.

6. Ultrashort-pulse laser and extreme-field science for applications in SSP as well as national defense-To enhance the performance of kJ-class, ps Nd-glass laser amplifiers and high-average-power ultrashort laser systems, LS\&T developed a new, broadband, front-end laser technology utilizing optical-parametric chirped-pulse amplification (OPCPA).

7. Diffractive optics and precision optical figuring for space telescopes and petawatt lasers-LS\&T worked toward large-scale, lightweight diffractive optics for the next generation of space-based optical assets (such as the proposed Eyeglass space telescope), activating production facility for NIF beam sampling gratings, and demonstrating wet-etch optical figuring for producing custom continuous phase plate for NIF and other programs.

8. Laser guide star and adaptive optic systemsLS\&T installed and successfully activated advanced laser guide star and adaptive optic systems at both Lick and Keck Observatories.

\section{Deploying NIF Hardware and Improving NIF Laser Optics and Materials Performance}

LS\&T work on NIF has focused mainly on the deployment of the NIF Final Optics Assembly (FOA) and the development of $3 \omega$ optics processing and treatment technolo- gies to enhance the performance of optics in the FOA. The FOA converts the laser light from infrared to UV ( $3 \omega$ at $350 \mathrm{~nm}$ ), conditions the beam, and delivers it to the fusion target chamber. It consists of several large-aperture optical components including continuous phase plates for beam smoothing, fused silica vacuum windows, potassium dihydrogen phosphate (KDP) crystals for frequency conversion to $3 \omega$, a wedged focus lens and diffractive gratings for color separation, beam sampling gratings, and debris shields. To extend optics lifetimes in the FOA, we systematically studied the damage initiation mechanisms and measured the rate of damage-growth on optical components. Fused silica is an inherently good optical material for the NIF application, with $>99.999 \%$ of the surface being free of damage. Surface damage is initiated by surface cracks and impurities from the polishing process. The damage typically initiates in small size $<30 \mu \mathrm{m}$ and grows quickly with number of shots at fluences $>5 \mathrm{~J} / \mathrm{cm}^{2}$ for $\mathrm{ns}$ pulses at $3 \omega$ wavelength, creating excessive scatter and beam modulation. Surface damage can also be caused by beam modulation associated with bulk inclusions in some types of fused silica.

We developed a novel three-step approach to minimize damage-initiators and reduce damage-growth on $3 \omega$ optics in NIF. (1) Improve optical finishing process at the manufacturer to provide substrates with fewer defects. We qualified two vendors to manufacture inclusion-free blocks using an axial deposition process similar to that used for preforming of optical fiber without refractory material. We developed a series of surface treatment processes to reduce polish defects. By a combination of acid etching, $3 \omega$ laser conditioning, and magnetorheological finishing (MRF), we were able to achieve $\sim 50$ defects per fused silica optic at $12 \mathrm{~J} / \mathrm{cm}^{2}$ (the goal for $3 \omega$ optics), which is a $100 \times$ improvement in initiator reduction. (2) Preinitiate and mitigate surface damage on optics to eliminate growing craters during postprocessing. We refined a $\mathrm{CO}_{2}$ laser annealing process to spot-treat the residual surface damage and halt damage growth on fused silica optics. We also developed a spot machining process to stop surface damage growth on KDP crystals. (3) Perform routine inspection, retrieval, and refurbishment (after remitigation) of optics to stop damage growth in optics during NIF operations. 
Highest quality conventional polish

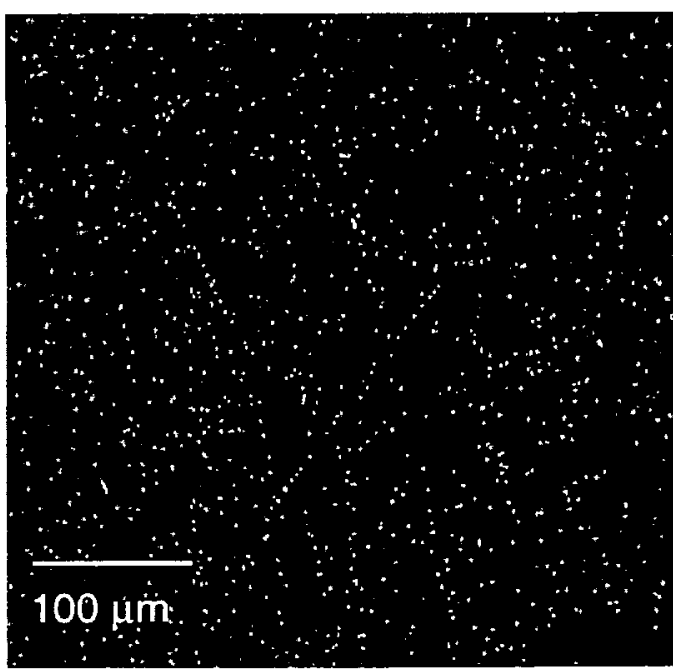

Advanced acid etching, laser conditioning, and MRF polishing

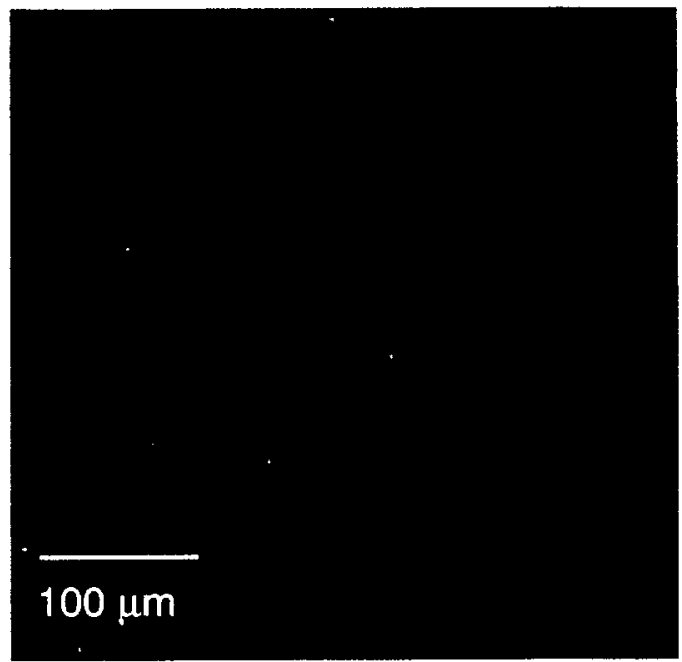

By a combination of acid etching, laser conditioning, and magnetorheological finishing, we produced optics with little or no defects. Dark-field microscopy of as-polished surfaces shows a near-absence of the subsurface damage normally associated with conventional finishing.

To manufacture KDP crystals capable of efficient conversion from $1 \omega$ to $3 \omega$, we developed orthogonal polarization interferometer to map frequency conversion efficiency over the entire KDP crystal area. A machine was built and installed at the NIF crystal manufacturer. To allow easier retrieval of optics for recycling and refurbishment, we completed the redesign of the FOA hardware. Statistical models of laser damage and growth were also developed to estimate lifetime of $3 \omega$ optics and optimize the refurbishment schedule for NIF. With these processing and recycling methods, we believe that the effective lifetime of $3 \omega$ optics will exceed 1000 shots, which meets NIF goals.

Advanced optical component fabrication technologies were also developed to fabricate large-aperture NIF optics including beam-sampling gratings (BSG) and phase plates. A wetchemical process machine and a patterningand-illumination station were assembled to fabricate full-size BSGs with 5- to 30-nm groove depth for damage testing in NIF. We have completed building and testing of production hardware for fabricating NIF BSGs used to perform critical power balance of the 192 NIF beamlines. We are currently beginning pilot production of full-size BSGs to be used on NIF early light. We have also developed controlled wetetching processes for figuring of NIF continuous contour phase plates.

\section{Construction and Testing of Z-Beamlet Completed; Laser Trigger System Delivered}

Under the auspices of DOE's Inertial Confinement Fusion (ICF) Program, LS\&T, in partnership with SNL, completed construction of a laser backlighter system (the Z-Beamlet) for $x$-ray radiography at SNL's $Z$ facility in Albuquerque, New Mexico. The $Z$ facility uses electrical pulsed power to drive a $z$-pinch implosion, which generates $x$-rays to drive ICF and high-energy-density physics experiments. We built the terawatt-class Nd:glass solid-state laser utilizing large-aperture optical components retrieved from former Beamlet and Nova lasers. To meet Z-Beamlet specifications $(2 \mathrm{~kJ}$ of $0.53-\mu \mathrm{m}$ laser energy in a picket fence of $<2 \mathrm{~ns}$ duration with a focus spot of $<50 \mu \mathrm{m}$ in diameter), we made a number of modifications to LLNL's Beamlet and utilized optical designs recently developed for NIF. These include: (1) Construction of a new fiber-optic Seed Pulse Generator (SPG) for the Master Oscillator Room (MOR) using NIF's diodepumped ytterbium-silica fiber-laser technology. (2) Increasing the regenerative amplifier ring length with improved stability. (3) Upgrading the plasma electrode Pockels cell (PEPC) to reduce electrode sputtering and improve switching uniformity. (4) Purging amplifiers 
with nitrogen gas to improve cleanliness of amplifier operation. (5) Re-engineering the entire Z-Beamlet computer control to a LabVIEW system using Windows NT, which enabled the laser and electrical pulses to fire simultaneously with synchronization of less than 3 ns. Additionally, LS\&T built beam sensors and alignment systems as well as designed a new final focus system (an analogy of NIF's FOA) to couple the Z-Beamlet laser output into the $Z$ target chamber. Z-Beamlet was activated in 2001. Its output was used to generate a powerful $x$-ray source for penetrating the $z$-pinch target. The first images of the z-pinch implosion target using the laser backlighter were produced in June 2001. The Z-Beamlet is currently used by SNL to support SSP experiments.

For DOE's ICF program, LS\&T also designed and built an all solid-state $\mathrm{Nd}$ laser that produces near diffraction-limited highenergy pulses at $263 \mathrm{~nm}$ for initiating breakdown in the $Z$ accelerators high-voltage switches. The solid-state laser we built offers several advantages relative to the $248-\mathrm{nm} \mathrm{KrF}$ excimer laser currently used at Sandia. Chief among these are superior beam quality and shorter pulse rise time (several hundred ps)properties expected to reduce switch timing jitter and improve synchronization of the $Z$ accelerator with its diagnostic x-ray backlighter pulse.

The laser trigger system generates pulses at

The Beamlet laser was modified and reassembled at Sandia for use as a backlighter beam on the $Z$ accelerator.
$1.053 \mathrm{~nm}$ using an injection-seeded Nd:YLF regenerative amplifier and a four-pass $1-\mathrm{cm}$ $\mathrm{Nd}$ :glass rod amplifier. A phase conjugator is used to maintain wavefront quality ( 2 to 3 times diffraction limit). The 10-ns output from

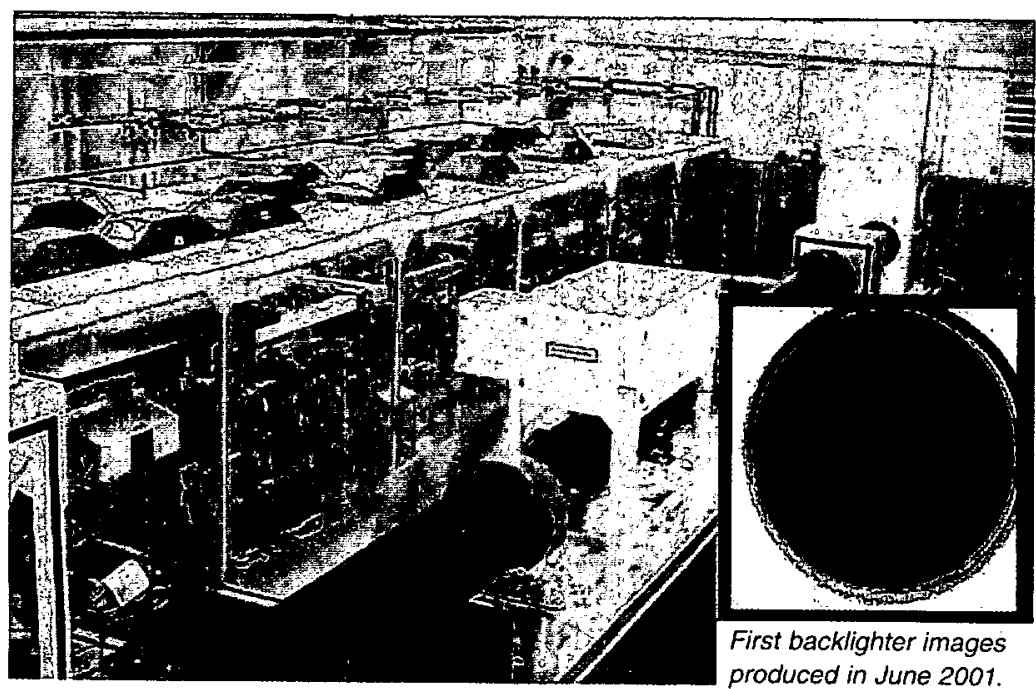

the four-pass amplifier feeds a single-pass $1-\mathrm{cm}$ rod amplifier and a pair of BBO crystals for frequency conversion to the fourth harmonic. We were able to generate an output of $850 \mathrm{~mJ}$ at $263 \mathrm{~nm}$ with $3.6-\mathrm{J}$ incident radiation at the $1.053 \mathrm{~nm}$. A maximum harmonic conversion efficiency of $25 \%$ was achieved, limited by two-photon absorption at $263 \mathrm{~nm}$. The laser trigger system was delivered to Sandia in September 2001 and is currently operated by SNL as a diagnostic tool in the z-pinch experiments.

\section{kW- to MW-Class Solid-State Laser Technology for Inertial Fusion Energy}

Under the support of the DOE Defense Programs, LS\&T continued to build a kW-class diode-pumped solid-state laser (Mercury) as the first in a series of next-generation lasers for inertial fusion energy (IFE). The Mercury laser system design is based on an IFE scalable architecture with goals achieving $10 \%$ electrical efficiency and $10-\mathrm{Hz}$ operation for $100 \mathrm{~J}$ per 5-ns pulses. Three component technologies were developed for this high-power, solid-state laser fusion driver: (1) large-scale, highperformance diode lasers; (2) high-speed gas cooling of the gain media; and (3) large $\mathrm{Yb}^{3+}: \mathrm{Sr}_{5}\left(\mathrm{PO}_{4}\right)_{3} \mathrm{~F}$ (Yb:S-FAP) crystal amplifiers. Our primary goals in FY2001 were to build and characterize the laser system with $320 \mathrm{~kW}$ of peak diode power (4 backplanes) and seven neodymium-doped glass slabs mounted in the gas-cooled amplifier. The $\mathrm{Nd}$ :glass slabs serve as surrogate material in the amplifier assembly until the Yb:S-FAP crystalline slabs are completed. The glass slabs are mounted in an aerodynamic aluminum vane structure to allow turbulent helium gas to flow across the faces. The concept of face cooling is advantageous over other technologies in that the thermal wavefront distortions are minimized, low order, and easily correctable. Measurements of the diode light delivery efficiency, uniformity, and polarization were made with $160 \mathrm{~kW}$ of diode power ( 2 backplanes). The remaining two backplanes were fabricated and are currently being assembled into the laser system for integrated tests. Propagation experiments of the seed beam through the amplifier were conducted along with the activation of the diagnostics packages. Major laser components 




The Mercury laser laboratory during alignment tests with major components labeled.

such as YLF oscillator, multipass filter, pump delivery system, and one gas-cooled amplifier were fully assembled and characterized. In the final configuration, the Mercury laser will require two amplifiers in order to produce $100 \mathrm{~J}$ of $1.047-\mu \mathrm{m}$ light.

The growth and fabrication of the Yb:S-FAP slabs constitute another major element of our program objectives. Our goal was to produce at least two half-sized defect-free crystals, which could be bonded into one large $4 \times 6 \mathrm{~cm}^{2}$ crystal. Growth efforts at LLNL and at Northrop Grumman Poly-Scientific, Blacksburg, Va., our industrial collaborator, resulted in the production of eight half-sized crystals that have been bonded together at ONYX Optics Inc., Dublin, Ca., to form four full-sized slabs. The slabs are in the final finishing and coating stages. Yb:SFAP crystals were chosen as the preferred gain media to neodymium-doped glass because they offer higher energy storage, reducing laser diode cost, and a practical gain cross section. In addition, the high thermal conductivity of $\mathrm{Yb}:$ S-FAP permits operation at $>10 \mathrm{~Hz}$ repetition rates. A total of 14 slabs will eventually be required for the two amplifiers. The diode bars needed to pump or excite the Yb:S-FAP crystals have been developed at Coherent Inc., Santa Clara, $\mathrm{Ca}$., and are now commercially available. The packaging technology, required to cool the diode bars was developed at LLNL (called V-BASIS), meets the stringent requirements pertaining to peak power, bandwidth, wavelength chirp, and reliability. Our goal was to fabricate one-half or 144 of the diode packages or "tiles" needed for Mercury and mount them on a copper-cooling block. Over 150 tiles were fabricated and tested. Through a close relationship with Directed Energy Inc., Fort Collins, Colo., off-the-shelf pulsers were upgraded to accommodate Mercury operating conditions and are now available commercially.

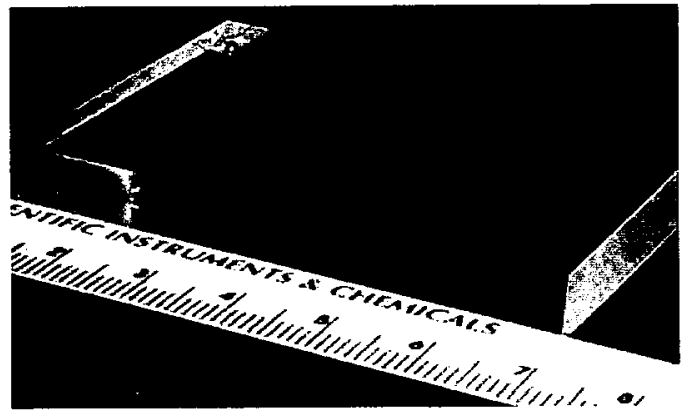

A full-sized Yb:S-FAP slab (amplifier medium) that has been bonded.

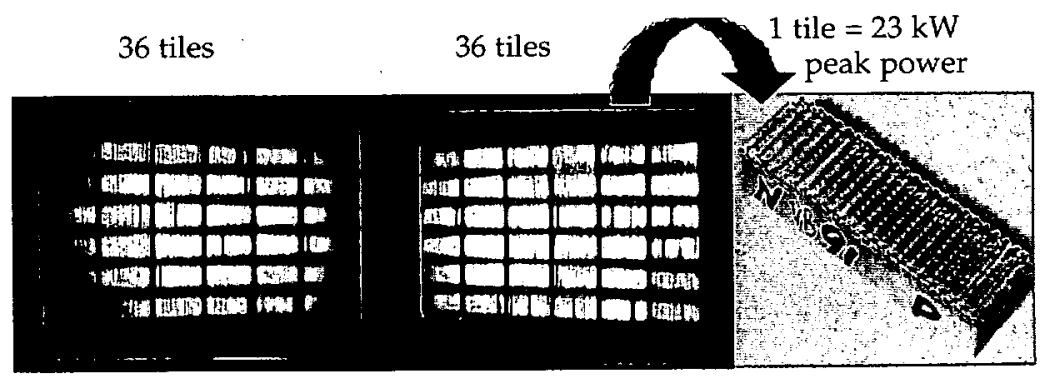

A picture of diode tiles (pump laser) firing producing up to $160 \mathrm{~kW}$ of peak power at $900 \mathrm{~nm}$. 
The performance of the $160-\mathrm{kW}$ diode package firing at $10 \mathrm{~Hz}$ has been characterized.

Activation of the remaining two backplanes is imminent and will allow us to pump the surrogate glass slab from both sides.

\section{Solid-State Heat Capacity Laser for the Department of Defense}

Under the support of the Army's Space and Missile Defense Command and in collaboration with industrial partners (Raytheon, Litton, and others), LS\&T is developing a high-averagepower (100-kW class), diode-pumped solidstate, heat-capacity laser technology for application in tactical short-range air defense missions. The ultimate vision is an electrically powered, diode-pumped, solid-state weapon that can be deployed on an electric vehicle. To establish a solid technical basis for the heatcapacity laser operation and risk reduction, we built and tested two flashlamp-pumped Nd:glass laser prototypes: 3-disk and 9-disk modules. In 2001, we completed the construction and testing of the 9-disk module. This 9disk module is designed to have $10-\mathrm{kW}$ average output power and deliver laser pulses with beam quality $<3 \times$ diffraction limit and energy of 500-J/pulse at $20 \mathrm{~Hz}$ for 10 -s bursts. We delivered this laser to the Army's High Energy Laser Systems Test Facility (HELSTF) at the White Sands Missile Range, New Mexico, to support material interaction testing. In August, we successfully demonstrated heat capacity operation for full $10 \mathrm{~s}$ with an average power of $13 \mathrm{~kW}(639 \mathrm{~J} /$ pulse at $20 \mathrm{~Hz})$.

Currently, the laser is operating with a stable

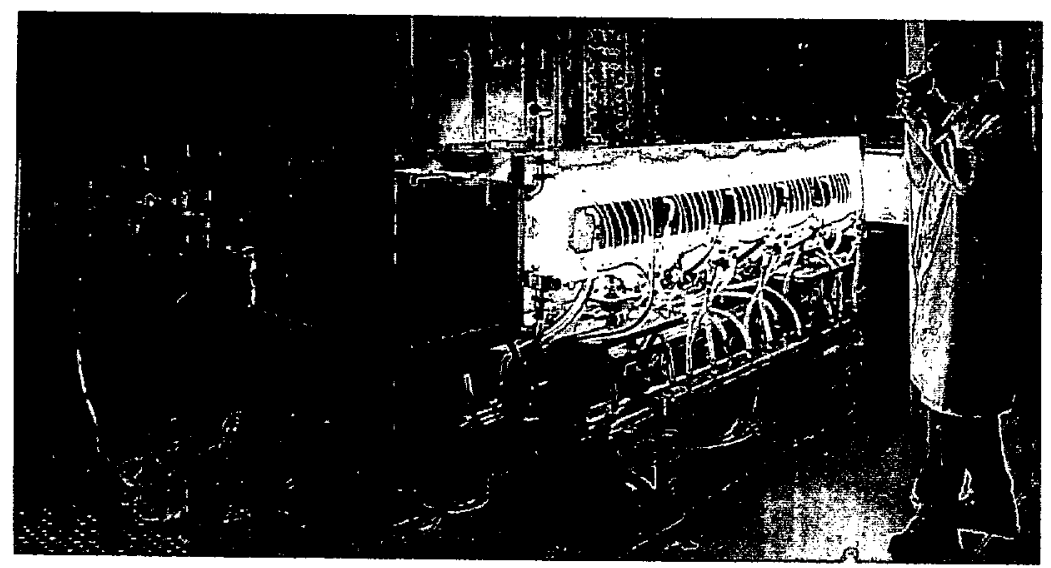

The 10-kW laser system installed at HELSTF to support material interaction testing.

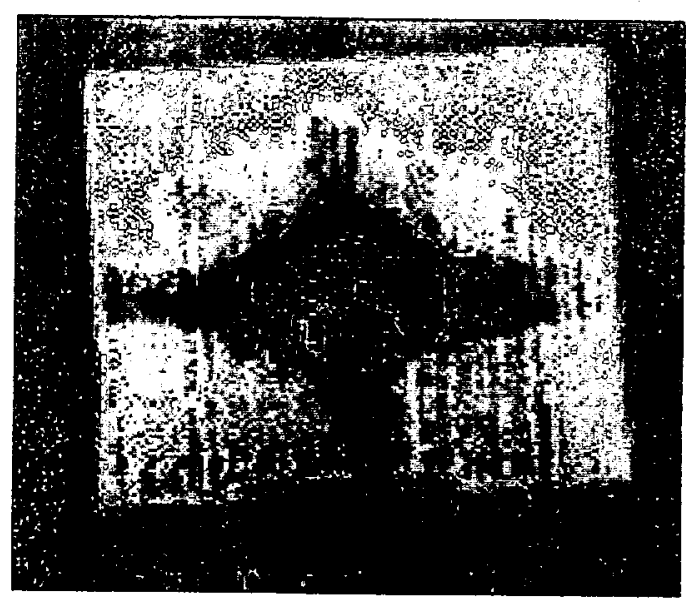

A 1-in.-thick aluminum target after a 2-sec laser burst produced a hole through it.

resonator, which is ideally suited to the target interaction experiments. Using a stable resonator with an output coupling of $29 \%$, we extracted up to $1000 \mathrm{~J} /$ pulse from the amplifier. A beam quality of approximately $3.5 \times$ the diffraction limit was demonstrated with an unstable resonator with no deformable mirror correction. Components for improving beam quality, such as deformable mirrors (DM), DM control electronics, and laser diagnostic sensor packages, have been assembled and tested. With the new DM and an unstable resonator, we expect to achieve the desired goal of $2 \times$ the diffraction limit over the entire 10-s run time.

Using the 9-disk prototype heat-capacity laser described above, we performed a series of target interaction experiments. During these experiments, the heat-capacity laser was operated at $3 \mathrm{~Hz}$ with energy of $650 \mathrm{~J}$ per pulse. The laser beam footprint is roughly square with a $6-\times 6.5-\mathrm{mm}$ spot on the target. The irradiation pulse has a temporal envelope of 300 to $400 \mu \mathrm{s}$ and consists of several relaxationoscillation spikes with peak intensities near $1 \mathrm{MW} / \mathrm{cm}^{2}$. We successfully removed about $0.9 \mathrm{~cm}^{3}$ of aluminum (11 mm deep) after irradiation with 38 laser pulses. To guide future target interaction experiments, we continued to develop numerical models to simulate the material removal process using hydrodynamics codes to model the vaporization and material ejection process. During a ribbon-cutting ceremony at HELSTF, we fired the 9-disk laser at a 1-in.-thick aluminum target and successfully penetrated the target in $2 \mathrm{~s}$.

The development of a $100-\mathrm{kW}$-class diodepumped neodymium-doped gadolinium 


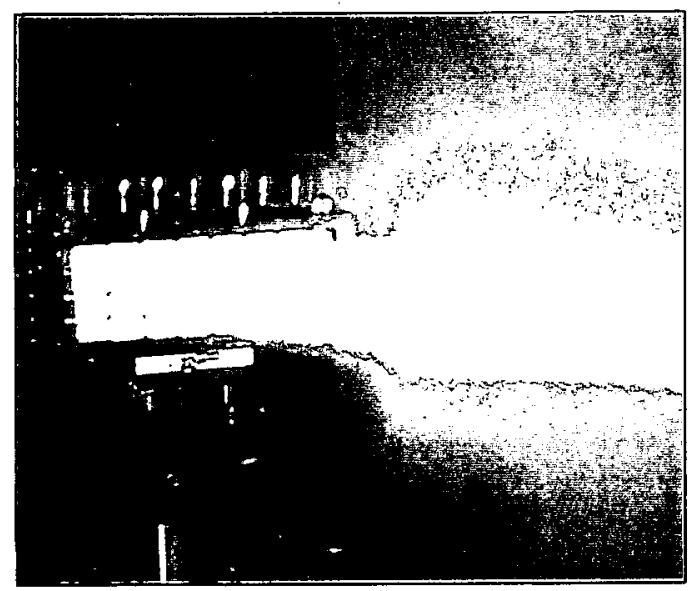

A $100-\mathrm{kW}$-class diode array module in operation. It was developed for pumping of high-average-power solid-state lasers.

gallium garnet (Nd:GGG) crystalline laser is proceeding rapidly. The diode-pumped Nd:GGG heat-capacity laser will have better thermal characteristics and operating efficiency enabling us to scale the output power to $100 \mathrm{~kW}(500 \mathrm{~J} /$ pulse at $200 \mathrm{~Hz})$. We have made significant progress toward the growth of high-quality Nd:GGG boules. Litton Airtron Synoptics, Charlotte, N.C., is now routinely growing $15-\mathrm{cm}$-diameter Nd:GGG boules with high optical quality. We have also completed the fabrication and testing of a $42-\mathrm{kW}$ laser diode array for pumping of Nd:GGG slabs. This high-power diode module is constructed from 280 edge-emitting laser-diodes mounted on a backplane packaged by 28 closely packed silicon microchannel monolithic tiles (SiMMs) arranged in $4 \times 7$ configuration. The brightness of the array is approximately $1 \mathrm{~kW} / \mathrm{cm}^{2}$ at a $10 \%$ duty factor. In order to utilize fully the light emitted from laser-diode bars, we designed microlenses to collect radiation along the fast axis and were able to reduce the beam divergence angle from $30^{\circ}$ to $<1^{\circ}$. The SiMMs package represents a significant breakthrough in the high-power diode-array packaging technology enabling us to scale the output of 2-D diode arrays to $100 \mathrm{~kW}$ or larger with extremely high brightness. Using diode bars procured from Coherent Semiconductor Group, Santa Clara, Ca., we successfully achieved $1.5 \mathrm{~kW}$ of output power from a single tile (10-bar SiMMs package) with electrical efficiency approaching $45 \%$. Using a diode array as pumping source, we completed emission cross-section and thermal deposition measurement on $\mathrm{Nd}: G G G$. We will demonstrate a small-scale Nd:GGG heatcapacity laser at $200 \mathrm{~Hz}$ in 2002 and replicate several features that would be found in a fieldable Army vehicle.

\section{Advance Solid-State Lasers for Government and Industry}

\section{Laser Peening Increases Fatigue Lifetime and the Corrosion Resistance of Metals}

LS\&T has an ongoing CRADA with Metal Improvement Company, Inc., Paramus, N.J., to develop and deploy a high-throughput laser peening technology to improve fatigue resistance of metals. Initial applications will significantly improve the service lifetime of military and commercial jet engines.

In a joint research effort with the Yucca Mountain Project (YMP), we are evaluating laser peening as a technique to improve the corrosion resistance and service lifetime of metal canisters designed for final disposal of high-level radioactive waste, dismantled reactors, and retired weapon components. Nuclear waste around the country will be stored underground at Yucca Mountain in welded canisters of Alloy 22. These canisters are required to last 10,000 years without leakage. However, the process of welding the end caps on these canisters can cause tensile stress that allows defects to grow into cracks and accelerate corrosion. Previous LS\&T work has demonstrated that laser peening can transform tensile stress into compressive stress deep into the material and prevent the growth of such cracks. In recent tests, we have found that laser peening not only stops crack propagation in welds but also retards the overall corrosion rate of metal. By optimizing the process parameters such as laser pulse duration (10 to $30 \mathrm{~ns}$ ), fluence intensity (50 to $300 \mathrm{~J} / \mathrm{cm}^{2}$ ) and number of treatment pulses, we were able to induce compressive stress deep into metals such as titanium, aluminum, steel, Alloy 22, etc. Compressive residual stress extending to depth of several millimeters has been achieved with laser pulses at various fluence intensities. We conducted a series of stress corrosion cracking (SCC) and surface corrosion experiments on 304 and 316 stainless-steel weld in a boiling $\mathrm{MgCl}_{2}$ solution. Our test results indicated that laser peening increases the free corrosion potential of metal, reduces the passive current density, and 


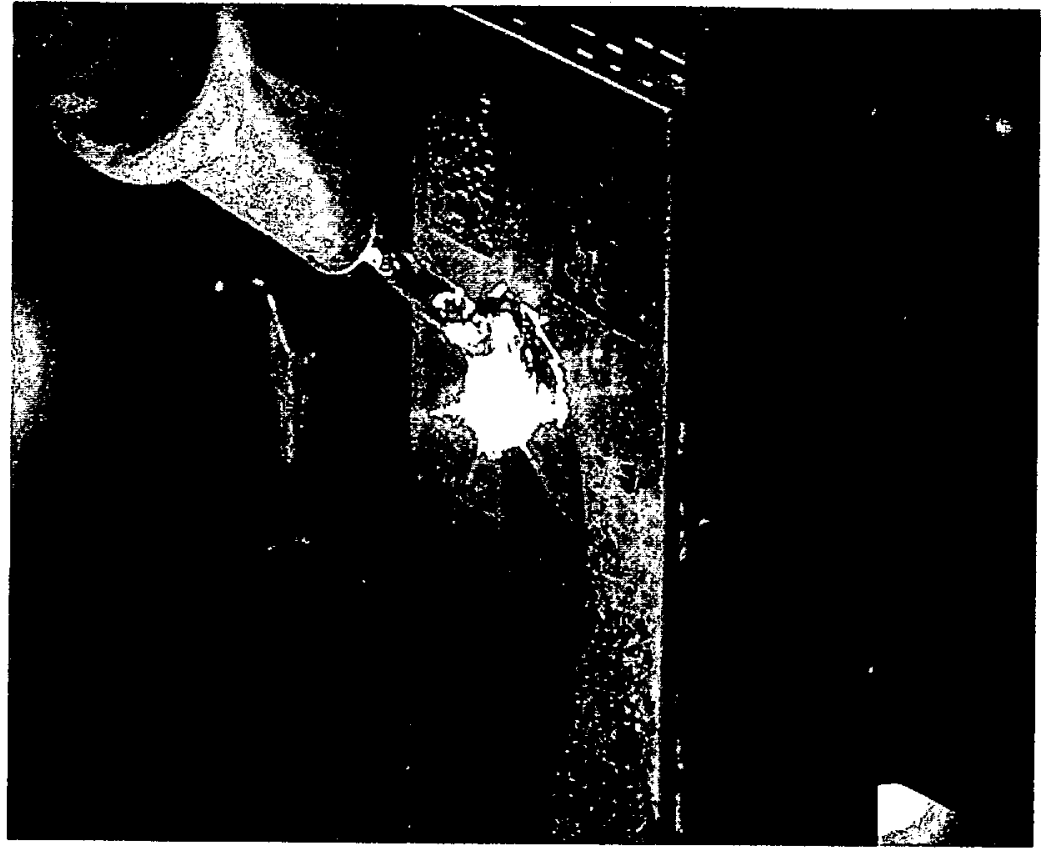

The Lasershotsm Marking System won an R\&D 100 Award in 2001. The technology imprints an identification mark that is permanent, machine readable, high resolution without weakening the surface of the marked part. In August 2001, NASA put three LLNL samples aboard the STS-105 space shuttle and subsequently bolted them on the space station facing the slipstream solar wind. The peenmarked parts will be examined after one year.

retards SCC on metals. We assembled a hightemperature reactor to perform SCC experiment on large-size (ft-scale) test samples. Laser peening was performed on a selected area of the welds. Under highly corrosive environment $\left(\mathrm{MgCl}_{2}\right.$ solution at $\left.160^{\circ} \mathrm{C}\right)$, the unpeened weld area showed cracks and corrosion within 5 days, while the laser-peened weld area showed no signs of cracking or corrosion even after 10 days of exposure. Cracks originating from the unpeened area appear to propagate, bypass, and cease at the peened zone. We are working closely with industry to commercialize this technology. Besides application in the YMP nuclear waste disposal for the government, there are potential applications of this technology for the U.S. industry in improving reactor safety and reliability and in extending the operational life of reactor components (internals, tubes, bolts, and pins) so that boilingwater reactors and pressurized water reactors will have a higher service life and lower operating cost.

We are also developing a laser peenmarking system to create permanent, high-resolution identification marks on safety-critical metal parts, without weakening the part. LS\&T and
Metal Improvement Company, Inc., are working with the National Aeronautics and Space Administration to determine the efficiency of laser peenmarking to identify and track millions of parts used in the space program. In August 2001, three laser peenmarked parts were put aboard the STS- 105 shuttle and subsequently bolted onto the space station to face the slipstream solar wind. After one year, they will be retrieved and examined to evaluate how well they held up in the hostile space environment.

\section{Short-Pulse, High-Average-Power Solid-State Laser for Micromachining}

Under the support of DOE's Stockpile Stewardship and Management Program, LS\&T continues to develop high-averagepower, short-pulse solid-state lasers and corresponding applications for use as precision cutting and drilling machines in the stockpile life extension process. The lasers developed for precision machining employ numerous advanced laser technologies of the LS\&T Program. These technologies include fiber mode-locked oscillators, solid-state regenerative and power chirped-pulse amplifiers using Ti:sapphire or direct diode-pumped Yb:YAG as gain media, optical parametric chirped-pulse amplifiers (OPCPA), and high-power multilayer dielectric gratings. These laser systems have an average output in the range of 5 to $100 \mathrm{~W}$ and pulse duration of 10 to $2000 \mathrm{fs}$. During the past year, LS\&T reactivated the short-pulse laser systems currently installed in Oak Ridge Y-12 Plant and LLNL's High Explosives Applications Facility (HEAF) to machine weapon parts, explosives, and energetic materials. Short-pulse laser processing enables machining of these components with high precision and negligible collateral damage. We were able to cut high explosives with no evidence of detonation or deflagration. For ICF and the Defense and Nuclear Technologies Directorate (DNT), we have also made good progress in the drilling of sub- $\mu \mathrm{m}$ holes through the Be capsules and sculpting of high-quality features on fusion targets. We were able to generate surface profiles with $<\mu \mathrm{m}$-scale roughness on polyimide, copper, and alumina targets. The short-pulse lasermachining tool significantly improves the precision and repeatability of the micromachining process. Under a work-for-others contract, 
LS\&T continues to develop a 100 -W-class picosecond, $\mathrm{KHz}$, solid-state laser system for precision drilling of shaped holes in metal.

\section{High-Energy Ultrashort-Pulse Laser Technology}

With support from the Laboratory Directed Research and Development (LDRD) Program, LS\&T has recently developed a new front-end technology utilizing optical parametric chirpedpulse amplification (OPCPA) to replace the regenerative amplifier used in a variety of chirped-pulse amplifiers, including the Nova petawatt, Falcon, and other short-pulse systems. The wavelength and pulse energy level obtained from this OPCPA is ideal for seeding $\mathrm{kJ}$-class Nd:glass amplifiers, such as NIF and other high-energy ultrashort-pulse laser systems. Previously. high-energy lasers employing chirped-pulse amplification (CPA) were built on a hybrid (Ti:sapphire-Nd:glass) laser technology. One difficulty with the hybrid laser system is its low pulse contrast and generation of prepulses by the regenerative amplifier. Single-pass OPCPA eliminates prepulses originating from regenerative amplifiers and reduces the amplified spontaneous emission (ASE) by approximately one order of magnitude compared to Ti:sapphire. It offers additional advantages when compared to regenerative chirped-pulse amplification: low thermal aberration, greater wavelength flexibility, higher gain, high beam quality, and lower B-integral as a result of short beam path through the gain medium. In 2001, we built a compact OPCPA using a commercial frequency-doubled Q-switched Nd:YAG laser as pumping source. Three $\beta$-barium borate (BBO) crystals were used as the gain media. Under the type I angular phase-matching condition, two crystals were configured as preamplifiers and one was used as the power amplifier. We have successfully demonstrated high gain $\left(\sim 10^{7}\right)$, high conversion efficiency $(>20 \%)$, good amplified pulse beam quality $\left(M^{2}<2\right)$, and high bandwidth fidelity in OPCPA (30 fs). We anticipate widespread use of OPCPA as frontend technology for glass-based petawatt laser systems and high-average-power systems. In the future, it may be possible to exploit the full bandwidth capability of OPCPA to produce $\mathrm{kJ}$-class pulses with duration of several tens of femtoseconds. This would potentially allow focused intensities of $10^{24} \mathrm{~W} / \mathrm{cm}^{2}$ or higher for experiments in the extreme-field science.

To study ultrafast response of materials for the Stockpile Stewardship Program, we continue to develop an yltrafast $x$-ray source based on Thomson scattering of fs laser pulses (Falcon laser) from 100-MeV relativistic-electron bunches. During FY2001, work on the integration of the multiterawatt ultrashort-pulse Falcon laser system and LLNL's linear accelerator (linac) began. We tested several techniques for timing synchronization between electron bunch and laser pulse to $\sim 2 \mathrm{ps}$ accuracy. The linac was reconfigured for installation of the $5-\mathrm{MeV}$ photoinjector. Considerable advances have also been made to the Falcon and photoinjector laser systems. Thomson scattering with the photoinjector was successfully

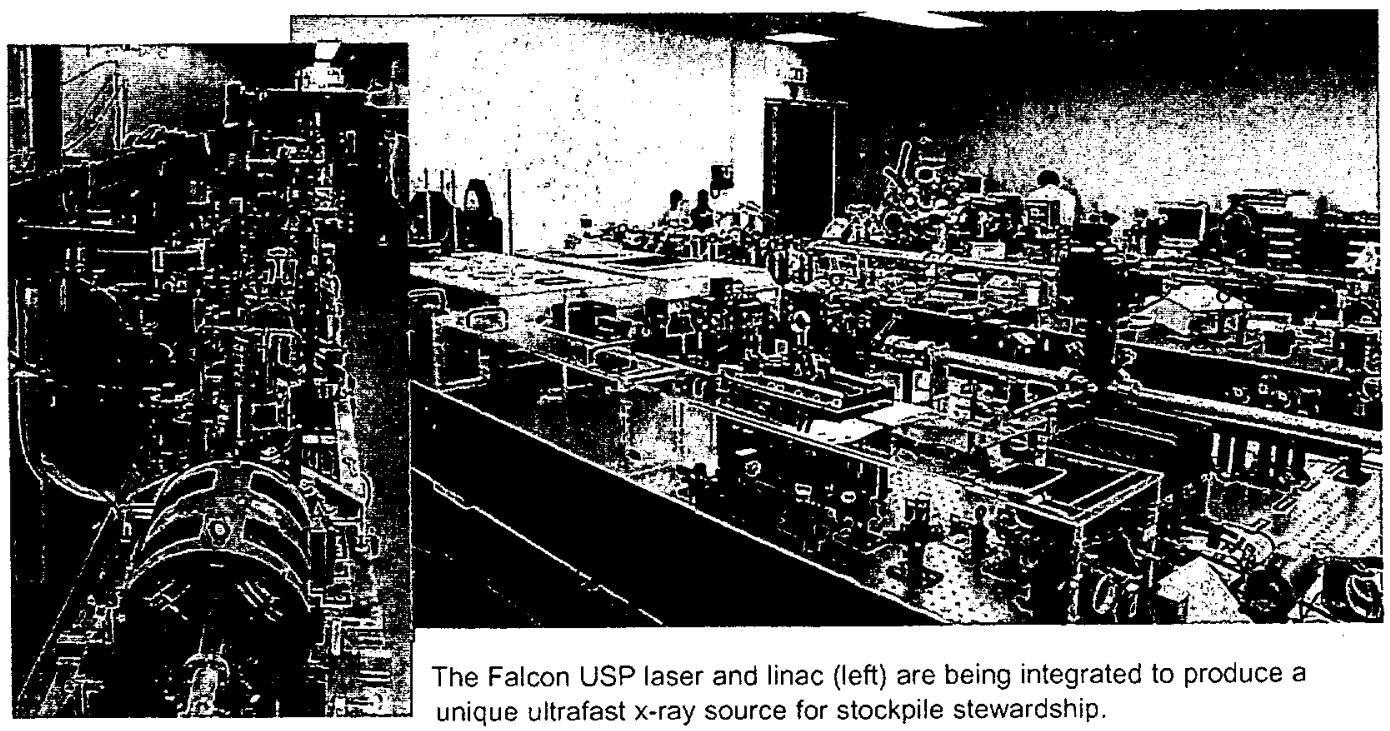


Thomson scattering with photoinjector was demonstrated.

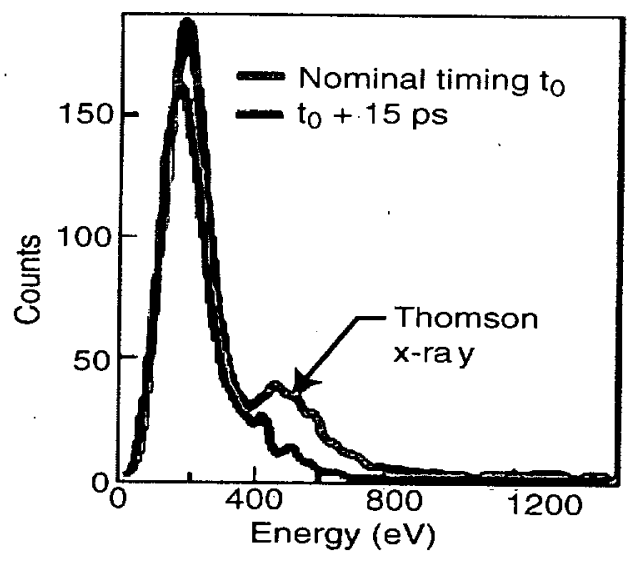

demonstrated; the photoinjector generated $600-\mathrm{eV} \mathrm{x}$-ray photons by scattering of the $5-\mathrm{MeV}$ electron beam with the 400 -fs laser pulses from Falcon. A new final power amplifier is being installed that will increase Falcon laser output by four times. We have developed a new front-end for Falcon based upon a hybrid system using both OPCPA and a Ti:sapphire amplifier. The hybrid design enables us to increase the pump efficiency and energy of the compressed pulses and simplify the overall architecture by eliminating the regenerative amplifier. We plan to complete the integration activities in 2002. When completed, this ultrafast $x$-ray source will be used to probe the dynamics of shock propagation and phase transitions in high- $Z$ metals that are relevant to Stockpile Stewardship.

\section{Large-Scale Diffractive Optics and Adaptive Optics for Space, NIF, and Petawatt Applications}

LS\&T continues to design and produce large-aperture high-efficiency diffractive optics for NIF and petawatt laser facilities around the world. These advanced optical components include meter-scale gold petawatt gratings, multilayer dielectric reflection gratings for pulse compression, fused silica transmission gratings, Fresnel lenses, and ultrathin optics $(<1 \mathrm{~mm})$. We are also advancing the adaptive optics and solid-state laser technologies for ground-based astronomy in the next decade.

\section{Meter-Scale Diffractive Optics for Petawatt Lasers}

During FY2001, under work-for-others contracts, we completed the fabrication of several 94-cm-diameter gold diffraction gratings for Rutherford Appleton Laboratory (RAL) in the United Kingdom and the University of Osaka, Japan. RAL has recently upgraded their 100-TW laser system using LLNL's large-aperture diffraction grating to generate subpicosecond pulses for high-intensity laser/matter interaction experiments. LLNL's large-aperture diffraction grating enables RAL to compress 500-J, 500picosecond pulses to achieve petawatt $\left(10^{15} \mathrm{~W}\right)$ of power and $10^{21} \mathrm{~W} / \mathrm{cm}^{2}$ at target. In 2001 , we successfully modified the exposure system (used originally for making LLNL's Petawatt laser gratings) to increase fringe stability during patterning of the grating. These gratings (with 1480 to 1800 line-pairs $/ \mathrm{mm}$ ) are currently optimized for high-efficiency and flat wavefront at the nominal $1053-\mathrm{nm}$ use wavelength. The damage threshold is greater than 400 $\mathrm{mJ} / \mathrm{cm}^{2}$ for a 10 -ps pulse. The diffraction efficiency of the gold-overcoated petawatt compressor gratings is $>93 \%$ in beam footprint, at

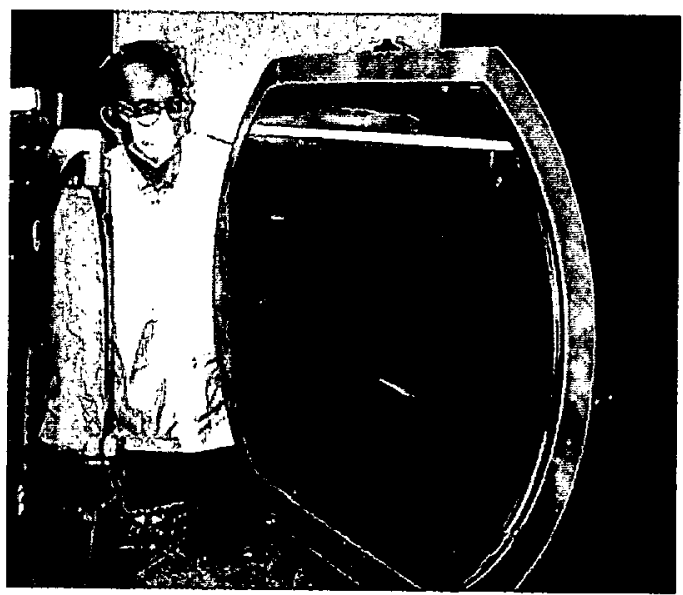

A meter-scale grating undergoing a wavefront test. LLNL supplies petawatt pulse compressor gratings to a variety of facilities both U.S. and foreign.
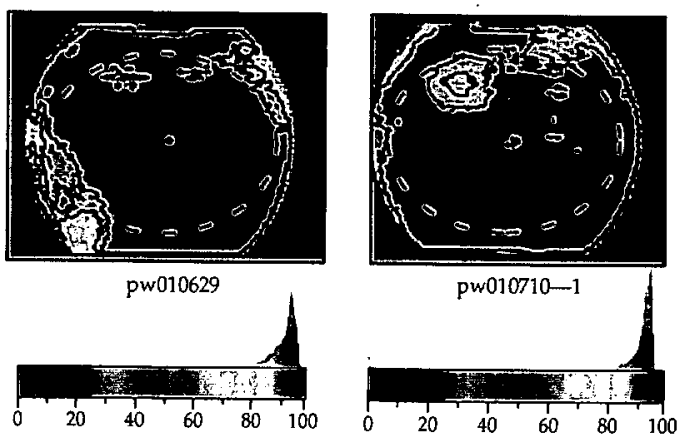

Full-aperture diffraction efficiency of two compressor gratings. Dashed lines show footprint of the compressed beam. 
use-wavelength and $54^{\circ}$ incidence angle, which represents the best uniformity we have been able to achieve to date at this scale. LS\&T is also developing large-aperture multilayer dielectric transmission and reflection gratings using cold-cathode ion-beam etching techniques for producing high-average-power ultrashort-pulse laser for laser machining and NIF applications. Using the dielectric reflective gratings, we were able to significantly improve the efficiency of pulse compression in highpower chirped-pulse amplifiers.

\section{New Wet-Etching Process for Figuring Large-Aperture and Ultrathin Optics}

With support from the Laboratory Directed Research and Development (LDRD) Program, LS\&T has recently developed a low-cost wetetching tool for precision optical figuring and finishing of large-aperture and ultrathin optical components. This wet-etch figuring (WEF) method uses applicator geometry and surface tension gradients (the Marangoni effect) to con-

fine the footprint of the flowing etchant (hydrofluoric acid) on the surface. In contrast to the conventional methods using abrasive slurries or ion milling, no mechanical or thermal stresses or residues are applied to the optic by this process. We have also developed a realtime interferometer to measure the thickness of the optical material while surfacing and figuring. This enables us to control the placement and dwell time of the wetted zone. We built both circular and linear etching toolheads for two- and one-dimensional (2- and 1-D) figuring. Several phase-correction and beam-shaping optics were fabricated using the 2-D WEF tool. An 80-mm-aperture corrective optics was recently fabricated for the Atomic Weapons Establishment (AWE), U.K., to correct wavefront aberrations in high-power lasers. The optic surface contour specified by AWE requires a maximum excursion of $12 \mu \mathrm{m}$ over this aperture. We were able to figure the surface profile within $100 \mathrm{~nm}$ of the target goal.
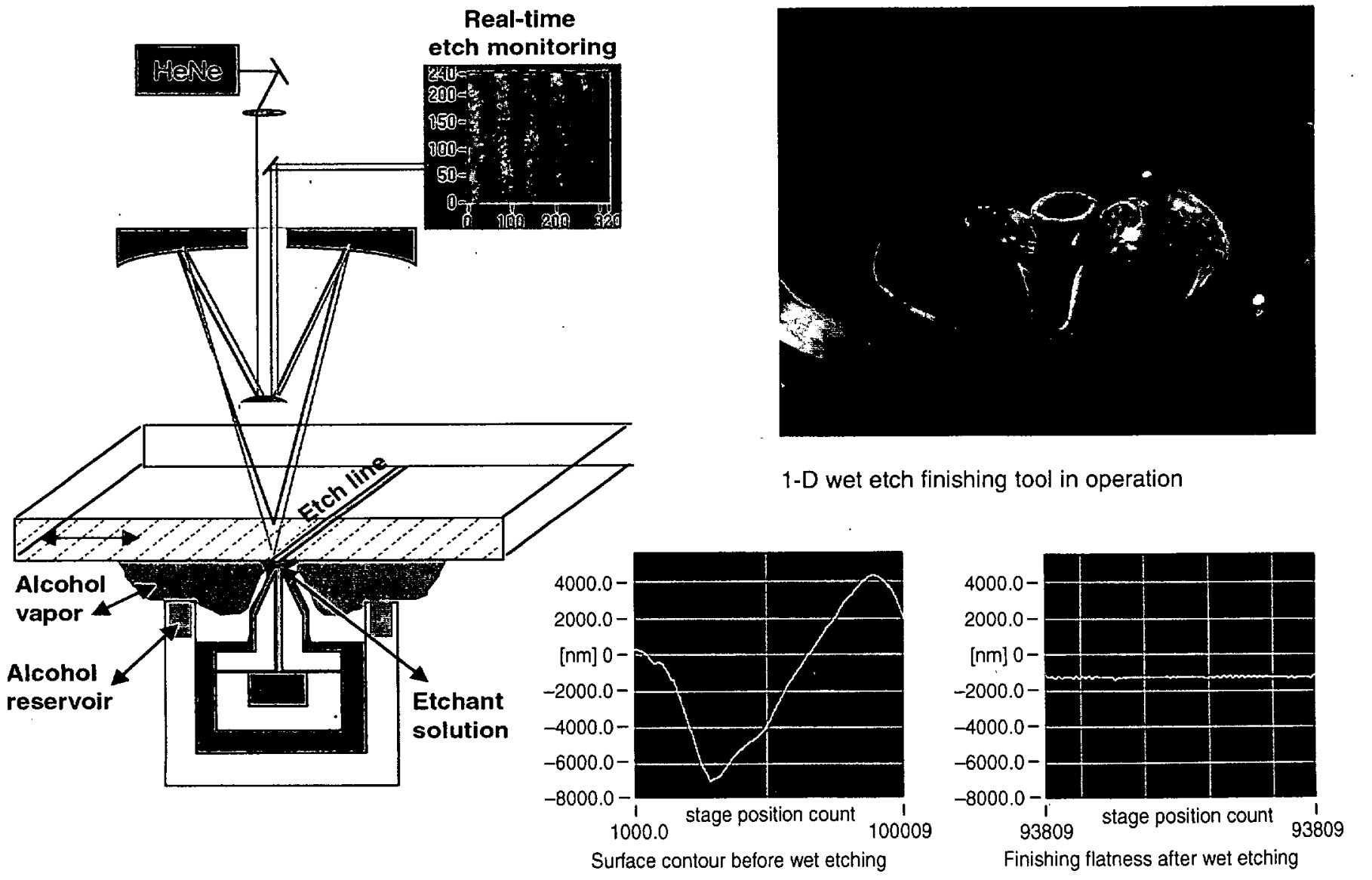

LS\&T developed 1-D and 2-D wet etch finishing tools with real-time interferometric feedback control. 
WEF is an ideal technology for the fabrication of large-aperture phase-modifying optics and optically flat optics. It is particularly useful for precision figuring of very thin $(\ll 1 \mathrm{~mm})$, lightweight optics for use in space and astronomy and high-power laser systems in which nonlinear effects due to bulk transmissive optics need to be minimized. LS\&T continues to improve the precision of the WEF machine and to use it for figuring of continuous phase plate for NIF.

\section{Lightweight Diffractive Optics for New Space Telescope: Eyeglass}

Working with the Physics and Advanced Technology (PAT) Directorate, LS\&T continues the development of a very largeaperture (25- to 50-m) diffractive space-based telescope (Eyeglass). Diffractive telescopes using thin-membrane Fresnel lenses offer several advantages over telescopes using mirrors. Thin-membrane lenses are lightweight $\left(\sim 0.1 \mathrm{~kg} / \mathrm{m}^{2}\right.$ areal density), packageable, and space deployable. During the past years, we built and tested a color-corrected diffractive telescope using a 50-cm-diameter, silica $\mathbf{f} / 100$ Fresnel lens. A near-term goal is to demonstrate that the technology can be scaled up to much larger size and engineered for deployment in space. In this context, we are developing technologies to fabricate a 5-m-diameter Fresnel lens. We completed the detailed design of this lens and the testing of a thin, foldable prototype in 2001. The Fresnel lens

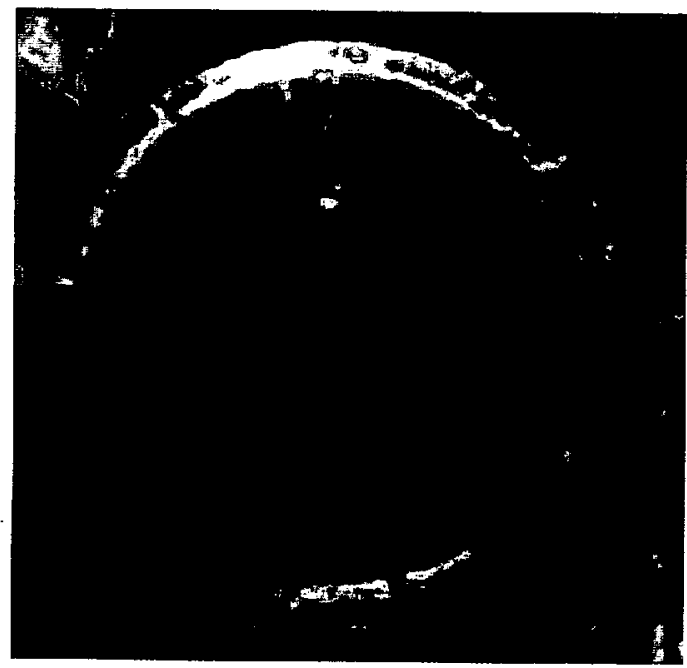

75-cm prototype lens in operation is composed of 81 panels approximately $1 \mathrm{~m}$ wide and $0.7 \mathrm{~mm}$ thick, patterned lithographically and joined together using metallic tabs.

As a first step in technology development, we patterned and assembled a six-segment, foldable Fresnel lens on 75-cm-diameter, 1-mm-thick fused silica plates and examined its focus quality when illuminated by a monochromatic, collimated beam. We were able to demonstrate a nearly diffraction-limited focal-spot shape using the foldable Fresnel lens. We plan to build the 5-m segmented Fresnel lens with metrologically verified optical precision in 2002 .

\section{Laser Guide Star Systems for Ground-Based Observatories}

Under the support of LDRD and University Relations Programs (UCDRD and the National Science Foundation Center for Adaptive Optics), LS\&T continues to work with PAT to develop fieldable sodium-layer laser guide star (LGS) systems for use on large astronomical telescopes in the Lick and Keck Observatories. Using an LGS at $589 \mathrm{~nm}$, artificial stars can be created in the direction of observation by laser-induced fluorescence in the mesospheric sodium layer, $90 \mathrm{~km}$ above the earth's surface. Using LGS beacons enables the collection of diffraction-limited images over $60 \%$ of the sky. During the past year, we facilitated, installed, and activated robust and reliable LGS and adaptive optic systems at Lick and

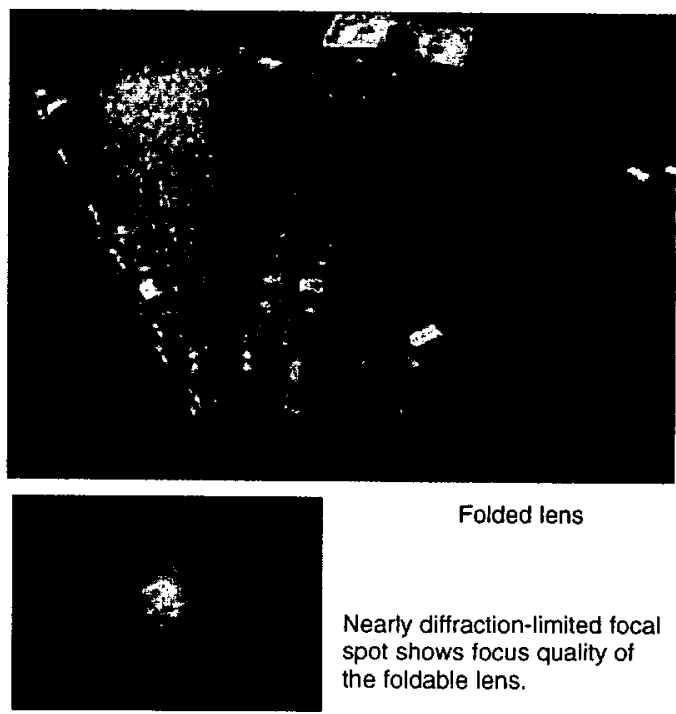

Eyeglass space telescope has demonstrated thin, foldable, six-panel diffractive lens prototype. 
Keck Observatories. These LGSs use frequency-doubled Nd:YAG lasers to pump a dye oscillator, preamplifier, and amplifier, and routinely produce up to $20 \mathrm{~W}$ at $26 \mathrm{kHz}$ with $1.5 \%$ energy stability and a Strehl ratio of 0.647 . The LGS system at Lick is currently being used for routine science observing.
The Keck system has recently completed performance and atmospheric propagation tests. Now we are developing, in collaboration with the European Southern Observatory, an all solid-state fiber guide star laser for next-generation telescopes.

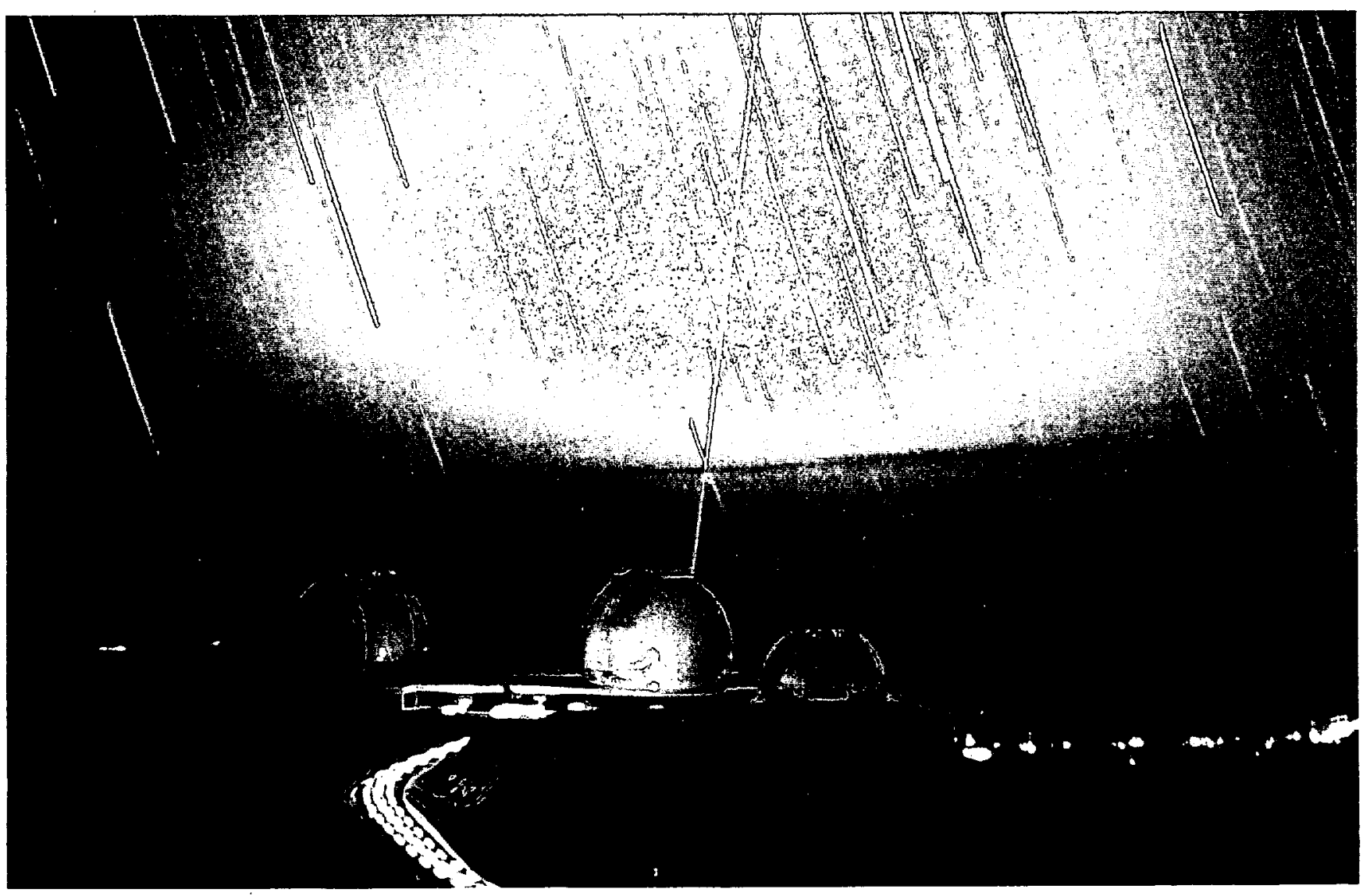

Photo of the Keck "virtual" guide star, showing LLNL-built orange laser beam emerging from the dome of the Keck $\|$ Telescope atop 14,000-ft. Mauna Kea volcano in Hawaii. (Photo by John McDonald, Canada-France-Hawaii Telescope Corp.) 


\section{Advanced Lasers ANd Components Research ACTIVIty Highlights}

\section{The Mercury Laser, a Diode- Pumped Solid-State Laser Driver for Inertial Fusion, is Activated}

The Mercury laser system design is based on a scalable architecture for iner-tial fusion with goals of achieving $10 \%$ electrical efficiencies and $10 \mathrm{Hertz}$ operation for $100 \mathrm{~J} / 5$ nsec pulses. Three component technologies had to be de-veloped for high power solid-state laser fusion drivers: large-scale high performance diode lasers, high speed gas cooling of the gain media, and $\mathrm{Yb} 3+: \mathrm{Sr} 5(\mathrm{PO} 4) 3 \mathrm{~F}$ (Yb:S-FAP) crystal amplifiers.

Currently, the hardware for one com-plete amplifier has been delivered and is being assembled and tested. All optics have arrived and been characterized. Three vacuum telescopes and the injec-tion spatial filter (Fig.1) are being vacuum leak tested. The pump delivery vacuum enclosures as well as the silver coated lens duct and homogenizer have been

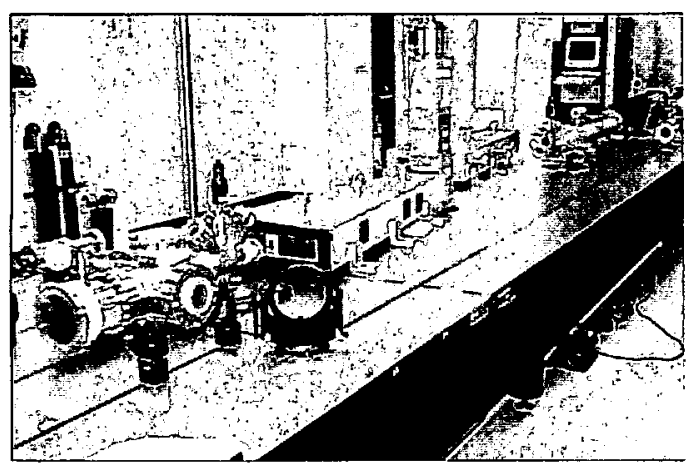

Figure 1. Mercury Laser Lab showing injection multipass filter, pump delivery hardware, and gas-cooled amplifier head. assembled. Two water-cooled copper back planes have been fabricated and tested. 72 diode laser tiles (each con-sisting of 23 bars operating at $>115 \mathrm{~W}$ peak/bar) capable of generating a total of over $160 \mathrm{~kW}$ of $900 \mathrm{~nm}$ light have been completed, characterized, and are under-going integrated testing with the backplane.

The Mercury Laser system has been designed to avert damage and to offer a scalable architecture. To achieve this, the telescope lenses are located near the relay image planes in the amplifiers to minimize beam modulation. Extensive ghost, amplified spontaneous emission (ASE), and pencil beam analysis were performed, validating the current archi-tecture and setting constraints on optical quality, surface reflectivity, and wedge angles, as well as theextinction required of a Pockel's cell in the reverser. The high average power Pockel's cell is comprised of an identical pair of $\mathrm{KD}^{*} \mathrm{P}$ crystals with their axes oriented such that the thermal birefringence cancels (Fig.2).

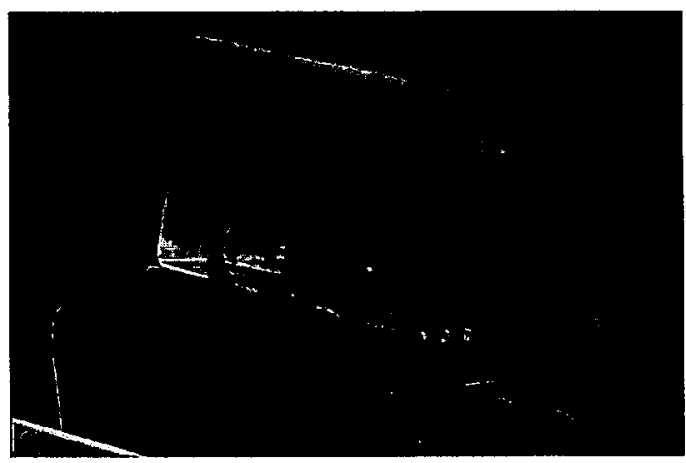

Figure 2. A half aperture $(1.5 \times 2.5 \mathrm{~cm})$ high averagepower Pockel s cell. 
Recent breakthroughs have been made in the growth of the ytterbium doped strontium fluorapatite (S-FAP) amplifier crystals. Crystals with large areas free of defects allow $1 / 2$ scale slabs to be diffusion bonded together to achieve full-scale amplifier slabs at Litton Airtron (Figure 3) and LLNL (Figure 4).

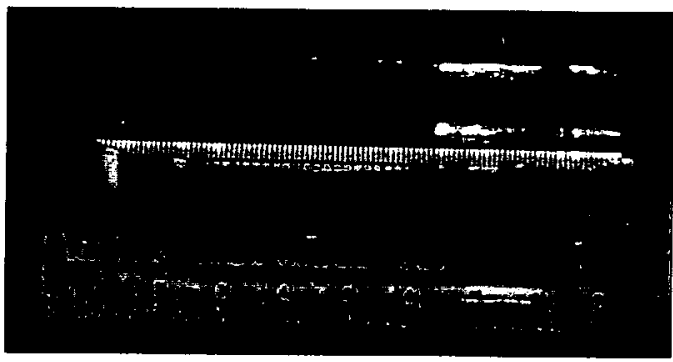

Figure 3. Litton Airtron boule is currently being fabricated into sub-slabs necessary for a full size diffusion bonded slab $(4 \times 6 \times 0.75 \mathrm{~cm})$

These Czochralski grown crystals have been challenged by defects including: cracking, cloudiness, bubble core defects, grain boundaries, anomalous ab-sorption, and crystal inclusions; these defects are essentially under control, although further fine-tuning is needed to assure reproducibility.

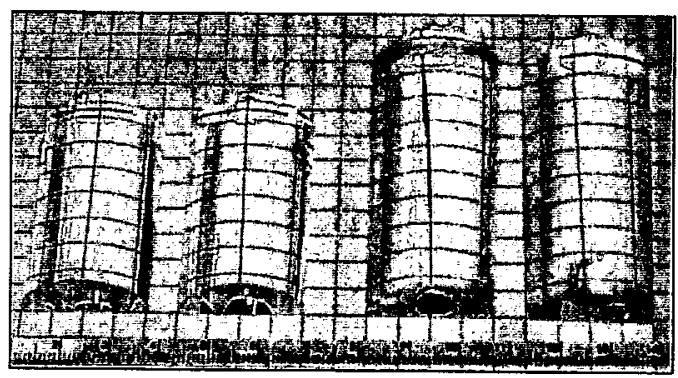

Figure 4.. Polished LLNL boules showing clarity. Boules are currently being fabricated into Mercury slabs.

High speed gas cooling allows operation at $10 \mathrm{~Hz}$ while maintaining wavefront quality for a $5 \times$ diffraction limited beam. A diagram of the gas-cooling system is shown in fig. 4 , along with interferometry results using surrogate $\mathrm{Nd}$ :glass slabs showing $<1 / 16$ wavefront distortion induced by the gas flow. Near term experiments include characterization of the diode backplanes and integrated beam propagation ex-periments in April. Goals for FY01 include demonstration of the half Mercury system including architecture, diodes, gas cooling, and material enough for seven full aperture S-FAP amplifiers.

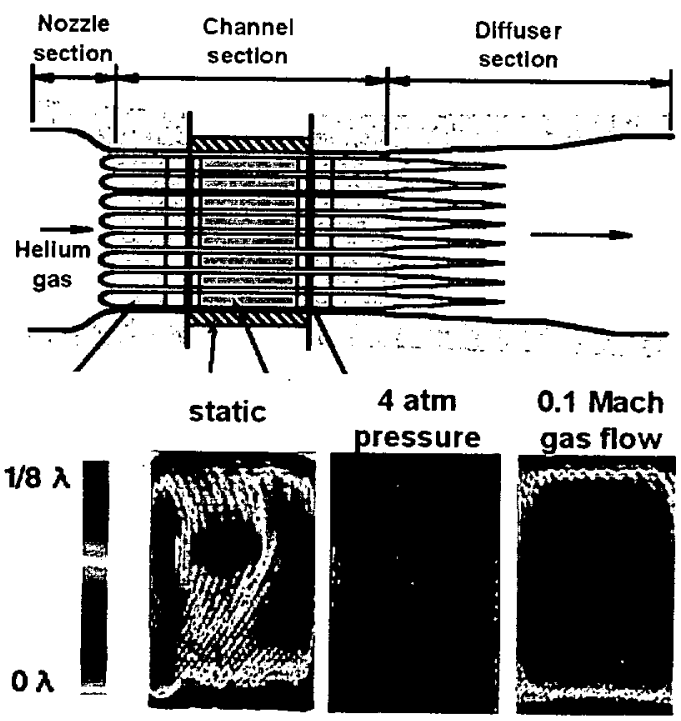

Figure 5. Wavefront distortions through the gas cooled amplifier head showing wavefront distortion for static, differential pressurized and differ-ential flow conditions.

\section{Development of High-Power- Microchannel-Cooled, Laser-Diode Arrays is UnderWay}

Under the support of the U.S. Army's Space and Missile Defense Command and in collaboration with industrial partners (Raytheon, Litton Airtron, and others), we are developing high-average-power (100-kW-class), diodepumped solid-state, heat-capacity laser technology for applications in tactical short-range air defense missions. To establish a solid technical basis for the 100-kW laser, we are building a testbed utilizing a neodymium-doped gadolinium gallium garnet (Nd:GGG) slab as laser media (with an active region of $5 \times 10 \mathrm{~cm} 2$, a third of the ultimate $100-\mathrm{kW}$ system) and four arrays of microchannel-cooled laser diodes as pump source.

The figures below show a schematic drawing of a silicon monolithic microchannelcooled (SiMM) laser-diode array and a diode array package. These SiMM packages are similar to those developed for the Mercury laser project (see LS\&T Program Update, September 2000 ), but contain only 10 diode bars instead of 23. Each array package contains 28 SiMM "tiles" arranged in a $4 \times 7$ configuration. 

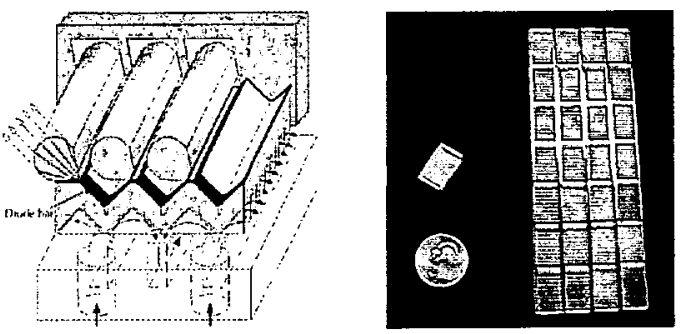

Schematic of a microchannel-cooled laser-diode array and a picture of a $4 \times 7$ diode array and individual tile.

One of the distinguishing features of the SiMM package is its ability to rapidly conduct heat away from the active region of the diode. This special feature is of particular importance to the Army's High-Energy Laser Systems Test Facility (HELSTF) applications because the laser is required to turn on rapidly and stay on at full power during the multisecond target engagement period. Long waiting period for thermal equilibrium is not an option for this application. Furthermore, if the temperature rise is too large, the laser wavelength might shift out of the absorption band (diode emission shift $\sim 0.3 \mathrm{~nm} /{ }^{\circ} \mathrm{C}$ ) and result in laser power loss.

To better understand the thermal behavior of the SiMM package, we performed a series of heat-conduction calculations. For comparison, we also analyzed the performance of a commercial system with a copper-based heatsink. The results of these modeling calculations are shown in the following figures.
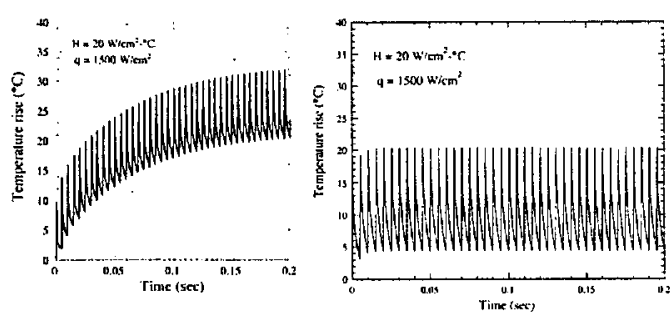

Plots display the temporal behavior of diode temperature (in the active region) calculated for a thermal input of $1.5 \mathrm{~kW} / \mathrm{cm}^{2}, 500-\mathrm{ms}$ pulse duration at $200 \mathrm{~Hz}$.

As shown in the charts, the SiMM package appears to have lower thermal impedance and shorter thermal equilibration time. The smaller thermal mass of the monolithic cooler allows the diodes to reach equilibrium much more quickly than the more massive copper heatsink. As a result, one would expect very little change in the time-integrated spectrum for the SiMM package over the duration of the excitation ( 10 seconds). However, the smaller thermal mass of the monolithic cooler could also cause greater temperature oscillation during each individual pulse and hence result in a larger intrapulse wavelength shift.

To verify this model, we measured the intrapulse as well as the long-term (time-integrated) spectrum of the diode light collected from a 10-bar SiMM package operated at $150 \mathrm{~W} /$ bar. We see in chart (a) that the rapid temperature rise during the 500-ms excitation pulse results in an intrapulse wavelength shift of about 4 $\mathrm{nm}$, which is much narrower than the absorption bandwidth $(10 \mathrm{~nm})$ of Nd:GGG material.

We also measured the long-term wavelength stability of the diode array operated at a $200-\mathrm{Hz}$ repetition rate and $10 \%$ duty cycle. As can be seen in chart (b), the wavelength varies very little $(<0.5 \mathrm{~nm})$ during the course of the burst, indicating very little long-term temperature increase, in good agreement with the model.

(a)

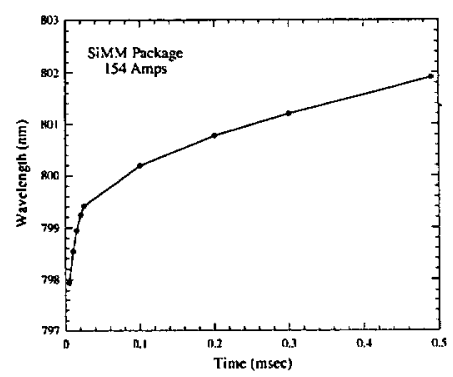

(b)

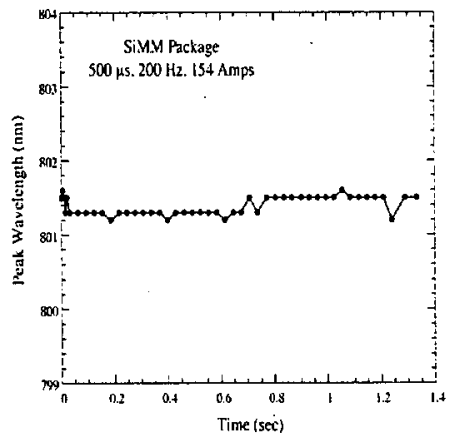


In recent tests, steady operation of a SiMM laser-diode array at $1.5 \mathrm{~kW} / \mathrm{cm} 2$ was successfully demonstrated with the 10-bar package (150 W/bar); see photo below. Output power degradation was found to be less than $3.4 \%$ after $5 \times 107$ pulses at $10 \%$ duty factor. We plan to test the Nd:GGG-slab testbed in the near future to optimize pump efficiency. We will also optimize the design of the SiMM package to further reduce costs of production.

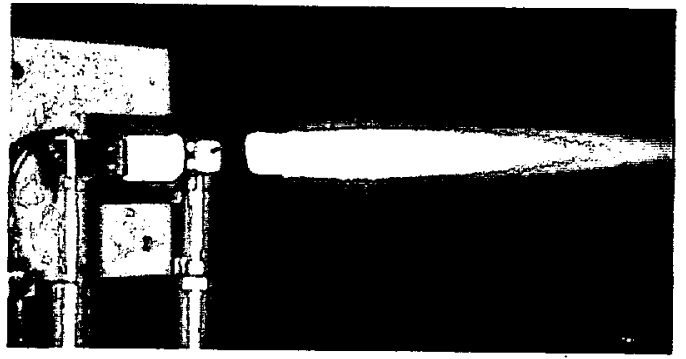

The 10-bar SIMM package in opereation. 


\title{
Scalable antiguided ribbon laser
}

\author{
Raymond J. Beach, Michael D. Feit, Ralph H. Page, LeAnn D. Brasure, Russell Wilcox, and \\ Stephen A. Payne \\ Lawrence Livermore National Laboratory, P.O. Box 808, Livermore, California 94551
}

\begin{abstract}
A new scalable fiber laser approach to phase locking of multiple gain cores in an antiguided structure is described and modeled. In essence, the waveguide comprises a periodic sequence of gain-loaded and no-gain segments that has a uniform refractive index (referred to as the ribbon) encapsulated within a reduced-index cladding region. Our calculations reveal that the constant-index profile within the ribbon structure provides optimal mode discrimination; the refractive index must be constant within \pm 0.001 to ensure single-mode operation for a five-core design. One-dimensional and two-dimensional calculations are pursued to support the design criteria. Slight periodic variation in the refractive index of the ribbon laser leads to the emergence of a photonic bandgap, in analogy to so-called holey fibers. Our constant-index design, together with the periodic gain profile, may be dessrihad.as a nhot.nnic metal.
\end{abstract} i.

\section{INTRODUCTION}

We describe a new, robustly scalable technique for phase locking multiple gain cores in a fiber structure based on antiguiding or radiative coupling. ${ }^{1}$ Our focus is on a ribbonlike geometry in which the waveguide region contains multiple gain cores that alternate with nongain regions in a periodic array. An outer, lower-index cladding surrounds the entire ribbon structure. The distinguishing feature of our design is its constant-refractive-index profile across the waveguide region, as opposed to alternating higher- and lower-index regions. Our modeling predicts that the constant-index design will provide modes that meet our two critical design requirements: strongly favored oscillation in a single transverse mode and good intensity uniformity across the waveguide structure. Interestingly, when the index profile is allowed to have a small index variation the model predicts formation of bandgaps in the allowed wave-vector values. Essentially, the ribbon fiber has a structure similar to that of photonic crystal fibers. ${ }^{2}$

Because the ribbon structure described in this paper contains a waveguiding region embedded in a lower-index outer cladding region, it has many features in common with single-core double-clad fiber lasers. The development of these double-clad fiber lasers has brought fiber lasers to the forefront of possible approaches to the design of high-beam-quality, high-average-power continuouswave laser sources. However, there are drawbacks to the implementation of a fiber-based system that the design described here should address. Individual fiber cores are believed to be limited to a capacity of roughly $100 \mathrm{~W}$ of average power ${ }^{3-6}$ owing to the output facet damage limit; therefore it is anticipated that high-average-power fiber systems will require phase combining of many individual apertures. The antiguiding design will allow the radiation output of the multiple cores to stay coherently phased together even as the number of cores increases. Conceptually, one could scale the device to higher powers simply by increasing the number of antiguided cores within the ribbon. Our modeling predicts that this design will scale to 100 embedded gain cores with reasonable tolerance limits on material construction and ribbon fabrication processes. A second drawback to achieving high-average-power fiber lasers is that the need to deliver the pump light into the end of the fiber requires that diodes have radiance-conditioned outputs. Our design benefits from the planar ribbon structure, which allows the fiber conceivably to be pumped from the side by diodes without radiance conditioning. This pumping concept is not further described in this paper, however. Additionally, the use of the ribbon structure is advantageous from a thermal management perspective and should allow all gain cores to be held at the same temperature.

The most commonly used approach to phase locking multiple apertures or gain cores is to couple the cores evanescently to their nearest neighbors. This technique, originally used with multistripe diode lasers, ${ }^{7}$ has been described in numerous papers. ${ }^{8-10}$ However, with evanescent phase locking the supermode or eigenmode tends to be localized on gain cores, and only nearest neighbors communicate with one another. When many cores are coupled evanescently, it is anticipated that a general degradation of the phase fidelity for cores will occur whose intensity will increase as the distance between cores increases. Additionally, there are techniques for diffractive coupling such as the Talbot plane methods ${ }^{11,12}$ and the more recently used 1-to- $N$-way phase-locking techniques. ${ }^{13,14}$ The ribbon structure described in this paper uses a nonevanescent approach to coherently phase together multiple gain-loaded cores. In this approach the gain elements are radiatively coupled in a leaky waveguide array, analogously to the most successful scheme for phasing laser diode elements. ${ }^{1,15,16}$ Because the eigenmode is delocalized across all gain cores in this case, all gain cores communicate with all other gain cores. The antiguided cores are arranged in a row in a long aspect ratio rectangle, or ribbon, with a slightly higher index than that of the outer pump-cladding medium. An

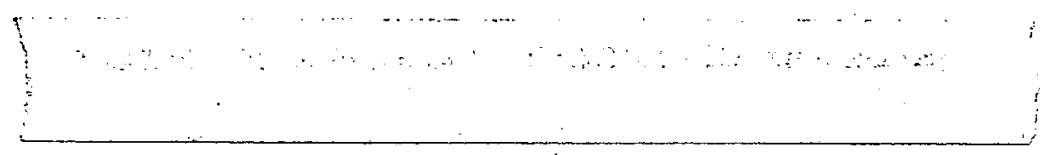


example structure with five cores is shown schematically in Fig. 1. The strong phase locking that is inherent in this approach should allow all the gain cores in the ribbon to communicate with one another and therefore scale to higher powers with higher phase fidelity across the aperture than can similar evanescently coupled structures.

Because of the ribbonlike structure, a one-dimensional transverse treatment provides a reasonable model for the properties of these devices. In this paper we describe a simple, but elegant, technique in which electric field propagators are used to generate the eigenmode spectrum of the structure. A full two-dimensional transverse analysis follows, with which modal gain discrimination is accurately assessed. The modeling shows some striking results in the eigenmode spectrum. Maximum mode discrimination occurs with $\Delta n=0$ (constant index) in the ribbon part of the structure, giving the best potential design for a high-average-power, single-mode laser. However, when the index is allowed to vary slightly between the gain and the nongain regions the spectrum shows formation of forbidden regions of wave-vector values. This is similar to the effect seen in holey fibers, i.e., photonic crystal fibers constructed with physical holes in the glass. ${ }^{17}$ The holes serve to provide a periodic modulation in the refractive index, something our design does by using materials with different refractive indices. One can adjust the width of the bandgap by varying the index difference between the gain and the nongain regions, providing an ability to tune the structure to best suit an application that requires bandgaps.

To explore key aspects of our proposed ribbon fiber we start by investigating a five-core device by onedimensional and then by two-dimensional analysis. As a specific example, the structure shown in Fig. 1 could be fabricated from pure fused silica along with Ge and F dopants to control the refractive index and $\mathrm{Yb}$ or $\mathrm{Nd}$ doping to provide the gain-loaded regions. In the structure shown in Fig. 1 the round doped-core sections serve as the gain regions for the optical wave that is confined to the $\sim 9-\mu \mathrm{m}$ high by $\sim 60-\mu \mathrm{m}$ wide rectangular waveguide region. The pump radiation is confined to the larger $\sim 350-\mu \mathrm{m}$ by $\sim 350-\mu \mathrm{m}$ square region, which we assume here is pure silica, and so has a lower refractive index than the waveguide region.

As we have already mentioned, there are two critical design considerations for the structures, which we con- sider here: First, the structure must strongly favor oscillation in a single transverse mode; second, the strongly favored mode must exhibit highly uniform intensity across the entire array of gain cores. We require singlemode operation such that a static phase corrector placed in the near field of the ribbon laser's output can optimize the phase across the aperture to achieve an output beam with a high Strehl ratio. The second requirement, that the strongly favored mode exhibit good uniformity across the entire array of gain cores, is necessary to ensure that the ribbon structure's gain saturates in a uniform manner such as not to increase the propensity of the device to operate in multiple transverse modes.

\section{THEORY OF THE ONE-DIMENSIONAL RIBBON FIBER WITH GAIN AND REFRACTIVE INDEX VARIATION}

To characterize our results we begin by analyzing the simpler problem of the one-dimensional structure that is related to the two-dimensional structure of Fig. 1. We take a lineout of the index profile along the center line of the Fig. 1 structure and in Fig. 2 plot a refractive-index profile to serve as an illustrative case. To further simplify our analysis we assume that the electric field polarization corresponds to that of a TE wave. We begin with the wave equation for the electric field in one of the constantindex strips along the waveguide:

$$
\left[\nabla^{2}-\left(\frac{n}{c}\right)^{2} \partial_{t}^{2}\right] \varepsilon(\mathbf{r}, t)=0 .
$$

Restricting the electric field to be TE polarized (the electric field is parallel to the interfaces between the index segments that make up the ribbon), we write the electric field in terms of its frequency $\omega$ and its longitudinal $k$-vector $\beta$ as

$$
\varepsilon(\mathbf{r}, t)=\hat{u}_{y} E(x) \exp [i(\omega t-\beta z)],
$$

where $\hat{u}_{y}$ is a unit vector in the $y$ direction. Substituting $\mathrm{Eq}$. (2) into Eq. (1) gives the equation that must be satisfied by $E(x)$ :

$$
\frac{\mathrm{d}^{2} E(x)}{\mathrm{d} x^{2}}=-\left[\left(\frac{n \omega}{c}\right)^{2}-\beta^{2}\right] E(x),
$$

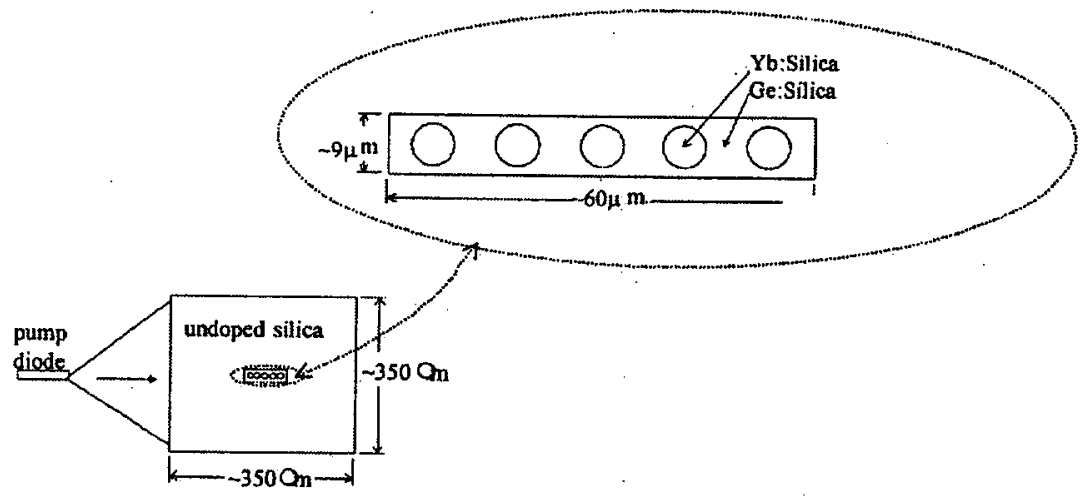

Fig. 1. Cross-sectional view of a five-core ribbon fiber. 


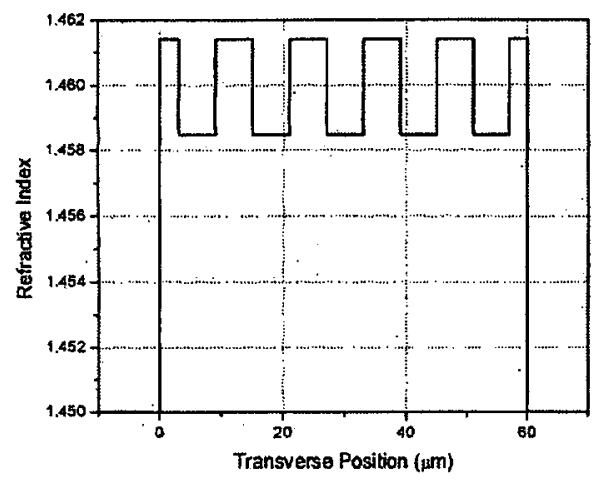

(a)

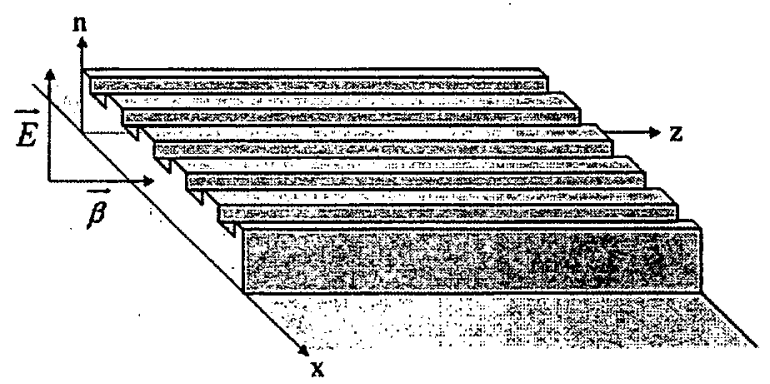

(b)

Fig. 2. (a) Refractive-index profile as a function of transverse position in the one-dimensional approximation to the ribbon waveguide structure shown in Fig. 1. The gain regions coincide with the lower index segments in the waveguide region. (b) Coordinate system used in our analysis for the TE-polarized wave (TE with respect to the refractive index boundaries) as shown. The $E$-vector is assumed to point in the $y$ direction. Assuming that fused silica is the base optical material, the pump cladding index is 1.45 , the gain regions have index 1.4585 , and the no-gain regions within the waveguide have index 1.4614. The central segments within the waveguide are each $6 \mu \mathrm{m}$ wide, and the end segments within the waveguide are $3 \mu \mathrm{m}$ wide.

which is the one-dimensional Helmholtz equation, ${ }^{18}$ or an eigenvalue equation for the Laplacian. Because of the polarization direction of the electric field (TE wave), the boundary condition to be satisfied at the interfaces between neighboring strips that have different refractive indices in Fig. 2 is one of continuity; i.e., the electric field amplitudes are the same on both sides of the boundary. In addition to the continuity of the electric field amplitude at the interfaces in the ribbon structure, second-order differential equation (3) for the electric field amplitude imposes a continuity condition on $\mathrm{d} E / \mathrm{d} x$ at the interfaces. If $\mathrm{d} E / \mathrm{d} x$ were not continuous at the interfaces, $\mathrm{d}^{2} E / \mathrm{d} x^{2}$ would blow up at those locations, leading to infinitely large values of the electric field. Finally, the boundary condition on the electric field amplitude outside the rectangular waveguiding structure of Fig. 2 is that it approach zero at large distances from the waveguide.

Summarizing, the boundary conditions to be satisfied by the electric field are

$$
\begin{gathered}
E\left(x_{i}^{-}\right)=E\left(x_{i}{ }^{+}\right), \\
\frac{\mathrm{d} E}{\mathrm{~d} x \mid x=x_{i}^{-}}=\frac{\mathrm{d} E}{\mathrm{~d} x \mid x=x_{i}{ }^{+}}
\end{gathered}
$$

$$
E(x) \rightarrow 0, \quad x \rightarrow \pm \infty,
$$

where $x_{i}{ }^{-}$and $x_{i}{ }^{+}$refer to the limiting values of $x$ at the $i$ th interface when it is approached from the negative and the positive sides, respectively. Because we are specifically interested in ensuring that we develop designs that will preferentially support only a single transverse mode, we must find all electric field eigenmode solutions to Eq. (3) that satisfy boundary conditions (4)-(6). We shall define these eigenmode solutions in terms of their longitudinal $k$-vector values, $\beta$.

Several methods of solution, for example, the transfer, or T-matrix, method, ${ }^{19,20}$ are available for generating solutions to the one-dimensional problem outlined above. Our method of solution, as outlined here, is to define arbitrarily an electric field amplitude of unit intensity at $x=0$ for the structure in Fig. 2 and then assume a $k$-vector value. To determine whether the assumed $k$-vector value corresponds to an actual eigenmode of the structure, we then propagate the electric field across the structure from $x=0$ to well beyond the waveguiding portion of the structure (past $60 \mu \mathrm{m}$ in Fig. 2). Applying boundary condition (6) then demands that, if the assumed $k$-vector value corresponds to an actual eigenmode, the electric field amplitude approach 0 as $x$ increases without bound.

The propagation of the electric field across the structure can be carried out numerically by use of Eq. (3) to incrementally step $E$ and $\mathrm{d} E / \mathrm{d} x$ across the structure, given initial values for both quantities at $x=0$. However, a quicker method and the one that we use here takes advantage of analytic propagators to propagate the field across an entire constant-index segment of the structure in a single step. The advantage of the analytic propagator method, which we outline below, is that it is extremely efficient and applicable to very large structures (hundreds of cores) that would bog down a calculation made by use of the more straightforward numerical incremental step calculation.

To begin the calculation, we arbitrarily choose a target $k$-vector value for $\beta$ in Eq. (3) and define both $E$ and $\mathrm{d} E / \mathrm{d} x$ at $x=0^{-}$in Fig. 2. Inasmuch as we require that $E(x)$ approach 0 as $x$ approaches $-\infty$, we must choose $\beta>n_{\text {clad }} \omega / c$, where $n_{\text {clad }}$ is the refractive-index value in the cladding region, $x<0$ or $x>60 \mu \mathrm{m}$, in Fig. 2. As we have already mentioned, we can arbitrarily set the value of $E\left(x=0^{-}\right)=1$; however, the value of $\mathrm{d} E /\left.\mathrm{d} x\right|_{x=0^{-}}$ is not arbitrary. The functional form of $E(x)$ for $x<0$ is

$$
E(x)=\exp \left[\beta^{2}-\left(\frac{n_{1} \omega}{c}\right)^{2}\right]^{1 / 2} x
$$

which decays exponentially to 0 as $x \rightarrow-\infty$. With $E(x)$ given by Eq. (7), the value of $d E /\left.d x\right|_{x=0}$ - is given by

$$
\left.\frac{\mathrm{d} E}{\mathrm{~d} x}\right|_{x=0^{-}}=\left[\beta^{2}-\left(\frac{n_{1} \omega}{c}\right)^{2}\right]^{1 / 2} .
$$

We denote by $n_{i}$ the refractive index of the $i$ th constantindex segment of the waveguide structure described in Fig. 2. Starting with the electric field amplitude and its first derivative at the left-hand side of one of the constant- 
index segments, the electric field amplitude and its derivative at the right-hand side of the constant-index segment are given by

$$
\begin{aligned}
E_{\mathrm{rhs}} & =E_{\mathrm{lhs}} \cos \left(\alpha_{i} l_{i}\right)+\frac{\frac{\mathrm{d} E}{\left.\mathrm{~d} x\right|_{\mathrm{lhs}}}}{\alpha_{i}} \sin \left(\alpha_{i} l_{i}\right), \\
\frac{\mathrm{d} E}{\left.\mathrm{~d} x\right|_{\mathrm{rhs}}} & =-E_{\mathrm{lhs}} \alpha_{i} \sin \left(\alpha_{i} l_{i}\right)+\frac{\mathrm{d} E}{\left.\mathrm{~d} x\right|_{\mathrm{lhs}}} \cos \left(\alpha_{i} l_{i}\right), \\
\beta & \leqslant \frac{n_{i} \omega}{c},
\end{aligned}
$$

where

$$
\alpha_{i}=\left[\left(\frac{n_{i} \omega}{c}\right)^{2}-\beta^{2}\right]^{1 / 2}
$$

or

$$
\begin{aligned}
E_{\mathrm{rbs}} & =E_{\mathrm{lhs}} \cosh \left(\hat{\alpha}_{i} l_{i}\right)+\frac{\frac{\mathrm{d} E}{\left.\mathrm{~d} x\right|_{\mathrm{lhs}}}}{\hat{\alpha}} \sinh \left(\hat{\alpha}_{i} l_{i}\right), \\
\frac{\mathrm{d} E}{\left.\mathrm{~d} x\right|_{\mathrm{rbs}}} & =E_{\mathrm{lhs}} \hat{\alpha}_{i} \sinh \left(\hat{\alpha}_{i} l_{i}\right)+\frac{\mathrm{d} E}{\left.\mathrm{~d} x\right|_{\mathrm{lhs}}} \cosh \left(\hat{\alpha}_{i} l_{i}\right), \\
\beta & >\frac{n_{i} \omega}{\mathrm{c}},
\end{aligned}
$$

where

$$
\hat{\alpha}_{i}=\left[\beta^{2}-\left(\frac{n_{i} \omega}{c}\right)^{2}\right]^{1 / 2},
$$

where $l_{i}$ is the width of the $i$ th constant-index segment in the ribbon fiber structure. The character of the electric field solution in any given index segment is strongly dependent on the relationship between the value of $\beta$ and $n_{i} \omega / c$. When $\beta<n_{i} \omega / c$, the solution is delocalized and the field propagates across the waveguide segment with an oscillatory behavior but does not decay in amplitude. When $\beta>n_{i} \omega / c$, the solutions are localized and the fields have an exponential dependence on the transverse coordinate in the waveguide segments, which is the situation that one normally thinks of as evanescent coupling. In our analysis we are interested primarily in the delocalized solutions $\left(\beta<n_{i} \omega / c\right)$ in which every gain region is coupled to every other gain region.

Using the transverse propagator method outlined above, we can propagate trial values of the wave vector $\beta$ in Eq. (2) across the waveguide structure. The requirement that the trial $\beta$ value correspond to an actual electric field eigenmode of the structure is that, as $x \rightarrow \infty$ on the right-hand side of the waveguide, the electric field amplitude go to zero. In general, a trial value for $\beta$ will generate an electric field amplitude that diverges either toward $+\infty$ or toward $-\infty$ as $x \rightarrow \infty$. This suggests a general method with which to search for electric field eigenmode $\beta$ values. If two nearby $\beta$ values can be found that generate fields that diverge in opposite directions as $x \rightarrow \infty$, then, by continuity, there must be an intermediate $\beta$ value such that the field that it generates goes asymptotically to 0 as $x \rightarrow \infty$, i.e., a $\beta$ value corresponding to an actual electric field eigenmode. An example of this method is illustrated by the field calculations displayed in Fig. 3. The $\beta$ values used for the calculations in Fig. 3 are as follows:

$$
\begin{aligned}
& \beta=8.7167195 \times(0.99999), \text { field diverges to }-\infty, \\
& \beta=8.7167195, \text { field converges to } 0, \\
& \beta=8.7167195 \times(1.00001), \text { field diverges to }+\infty .
\end{aligned}
$$

This technique is generally applicable to arbitrary waveguide structures and can quickly yield the entire spectrum of a given structure's allowed eigenvalues and eigenmodes.

The gain experienced by various laser modes is proportional to the overlap, $\Gamma$, of the mode's intensity envelope with the gain-loaded portion of the fiber:

$$
\Gamma=\frac{\int|E(x)|^{2} g(x) \mathrm{d} x}{\int|E(x)|^{2} \mathrm{~d} x},
$$

where $g(x)$ is a function with value unity in those portions of the fiber that are gain loaded and is proportional to 0 where there is no gain loading. This is a straightforward calculation once the eigenmode fields are known. Additionally, the effective index values $n_{\text {eff }}$ associated with the various eigenmodes of the structure can be calculated from

$$
\beta=\frac{n_{\mathrm{eff}} \omega}{c} .
$$

From Eq. (12) it can be seen that $c / n_{\text {eff }}$ is just the phase velocity associated with the eigenmode as it propagates in the ribbon structure. Using Eqs. (11) and (12), we can write the electric field that propagates down a gainloaded ribbon fiber structure as

$$
\varepsilon(\mathbf{r}, t)=E(x) \exp [i(\omega t-\beta z)] \exp \left(\xi \Gamma_{z} / 2\right),
$$

where $\xi$ is the gain per unit length in the gain-loaded portion of the ribbon fiber structure. Rewriting Eq. (13) and

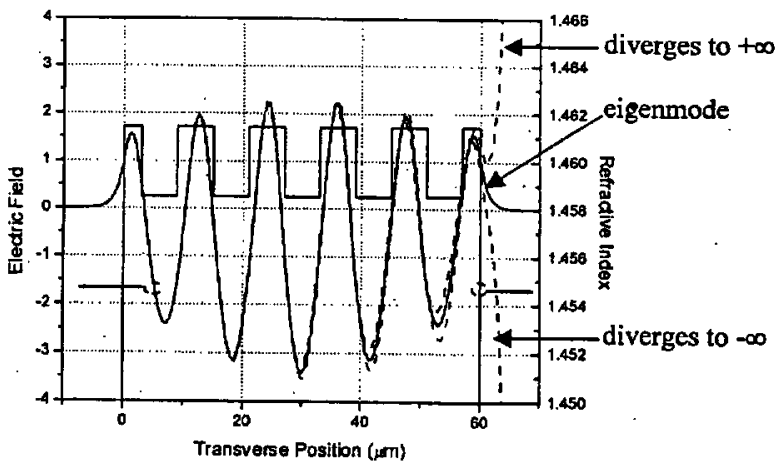

Fig. 3. Electric field calculations for three values of $\beta$ for the one-dimensional ribbon fiber structure described by the refractive-index profile in Fig. 2. The three profiles represent the fields calculated for the $\beta$ values described in the text. Also shown is the refractive-index profile (dashed curve). 
introducing the effective index defined in Eq. (12), but extending it to complex values, gives

$$
\begin{aligned}
\varepsilon(\mathbf{r}, t) & =E(x) \exp \left\{i\left[\omega t-\left(\beta+i \frac{1}{2} \xi \Gamma\right) z\right]\right\} \\
& =E(x) \exp \left[i\left(\omega t-\frac{n_{\mathrm{efr}} \omega}{c} z\right)\right] .
\end{aligned}
$$

With this expression, one immediately sees the connection between $\Gamma$ and the imaginary part of the complex effective refractive index:

$$
\operatorname{Im}\left(n_{\mathrm{eff}}\right)=\frac{c \xi \Gamma}{2 \omega} .
$$

\section{DESIGN CRITERIA FOR A SINGLE- INTENSITY LOBE IN EACH GAIN CORE}

Here we deduce the design rules for the one-dimensional ribbon structure that supports a mode that has a singleintensity lobe in each of its gain cores and constant peak intensity from gain core to gain core. Additionally, we re- concerns the design of the end segments of the ribbon structure to ensure that the intensity lobes are centered on the gain segments. Because the boundary conditions for the outer segments (the segments at the extreme ends of the waveguide structure) are different from those of the interior segments owing to the difference in the index steps there, separate consideration must be given to the widths of the segments beyond the constraint given by Eq. (16). For definiteness, we assume that the outer segments are gain loaded in our structure. With this constraint, we can then ensure that an intensity lobe will be centered on the first gain-loaded segment at the boundary by forcing the field to be null in the center of the first nogain segment, which is next to the boundary gain-loaded segment. To generate an equation to represent this constraint we use boundary condition (8) and the field propagators given in Eq. (9) to propagate the field first from the left-hand boundary of the waveguide region through the first gain-loaded segment and then to the midpoint of the first no-gain segment, where we force the field to null. After some algebraic manipulations, the resultant equation that represents this constraint is

$$
\begin{aligned}
& \tan \left\{l_{\text {edge }}\left[\left(\frac{n_{\mathrm{g}} \omega}{c}\right)^{2}-\beta^{2}\right]^{1 / 2}\right\}
\end{aligned}
$$

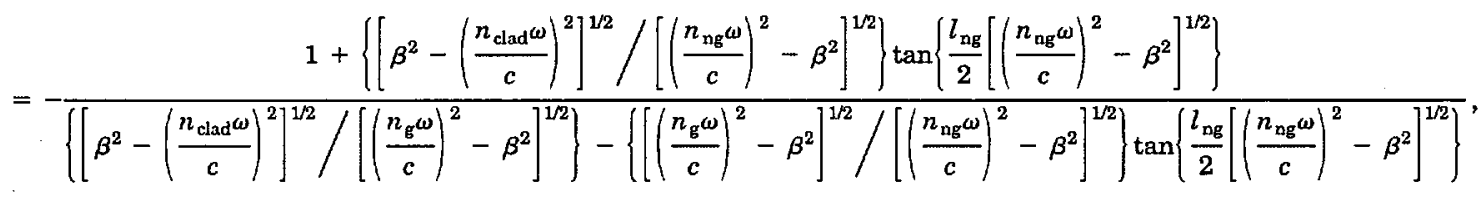

quire that this mode have the highest gain overlap of all the modes supported by the structure to ensure that it is the lowest threshold of all the modes supported and thereby lases preferentially over the other modes. Equation (3) hints at how to proceed. If we can arrange for the electric field to be null at the center of the undoped nogain segments that separate the doped gain segments within the waveguide region, then the lobes of the intensity envelope will center themselves on the gain regions and ensure a high overlap mode. To proceed, we fix a wave-vector value for this preferred mode and then construct the ribbon structure by varying the widths of the gain and the no-gain regions until the conditions described above pertain. Calling the refractive index values of the gain and the no-gain regions $n_{\mathrm{g}}$ and $n_{\mathrm{ng}}$, respectively, we adjust the widths of the gain and the nogain regions such that the following equation is satisfied:

$$
l_{\mathrm{g}}\left[\left(\frac{n_{\mathrm{g}} \omega}{c}\right)^{2}-\beta^{2}\right]^{1 / 2}+l_{\mathrm{ng}}\left[\left(\frac{n_{\mathrm{ng}} \omega}{c}\right)^{2}-\beta^{2}\right]^{1 / 2}=\pi,
$$

where $l_{\mathrm{g}}$ and $l_{\mathrm{ng}}$ are the widths of the gain and the nogain segments, respectively. Equation (16) ensures that one complete lobe will be associated with each gain segment that makes up the ribbon structure. Having ensured that only one lobe of the intensity envelope will be associated with each gain segment in the ribbon structure, we turn our attention to the remaining issue, which where $n_{\text {clad }}$ is the index of the pump cladding surrounding the waveguide region and $l_{\text {edge }}$ is the width of the gainloaded edge region. To illustrate in detail the procedure described above we investigate the ribbon structure defined in Table 1 below with five gain-loaded cores. In this structure the pump-cladding index region has refractive index 1.45, the gain-loaded portion of the waveguide has refractive index 1.4585 , and the no-gain regions of the waveguide have refractive index 1.4614. We arbitrarily take the vacuum wavelength of the waveguide radiation

Table 1. Details of Design of a One-Dimensional Ribbon Fiber Structure with a Step Index between Gain and No-Gain Segments

\begin{tabular}{ccc}
\hline Width $(\mu \mathrm{m})$ & Refractive Index & Gain Loaded? \\
\hline 10.00 & 1.45 & No \\
11.23 & 1.4585 & Yes \\
4.00 & 1.4614 & No \\
4.00 & 1.4585 & Yes \\
4.00 & 1.4614 & No \\
4.00 & 1.4585 & Yes \\
4.00 & 1.4614 & No \\
4.00 & 1.4585 & Yes \\
4.00 & 1.4614 & No \\
11.23 & 1.4585 & Yes \\
10.00 & 1.45 & No \\
\hline
\end{tabular}




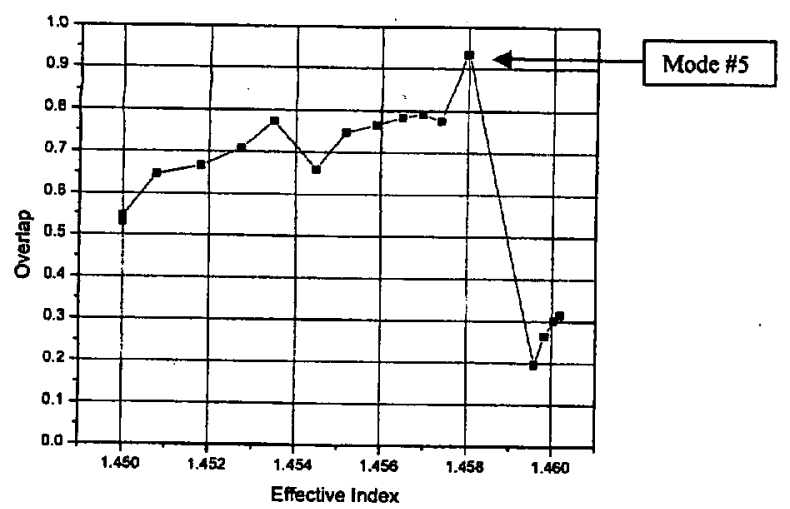

Fig. 4. Eigenmode overlap with the gain region plotted versus effective index. Mode $\# 5$ (counting from the right) was designed to have a single intensity lobe for each of the gain-loaded segments in the waveguide region.

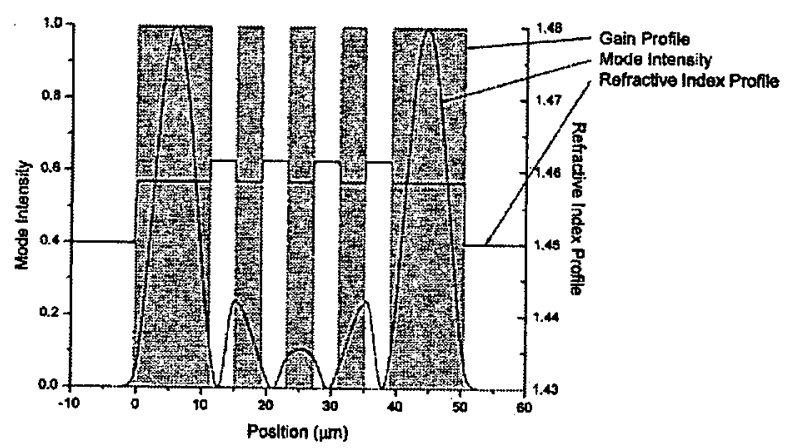

Fig. 5. Intensity envelope of the eigenmode for which the ribbon structure was designed (Mode \#5 in Fig. 4). The widths of the individual index segments are adjusted such that each gain region (shaded) sees a single intensity lobe. Superimposed is the refractive-index profile of the structure. The gain is located in the lower index regions within the waveguide structure.

to be $1.05 \mu \mathrm{m}$ and the widths of the interior gain and nogain segments to be $4 \mu \mathrm{m}$. With these choices, the mode that has one lobe associated with each gain region has a wave-vector value of $8.725 / \mu \mathrm{m}$ and the gain-loaded segments at the ends of the waveguide region have widths of $11.23 \mu \mathrm{m}$, as determined from Eq. (17), making the total width of the waveguide region $50.45 \mu \mathrm{m}$. Figure 4 gives a summary of this structure's eigenmode spectrum in a plot of mode overlap with gain regions versus effective index value. Figure 5 shows a plot of local mode intensity across the waveguide structure for the mode for which the waveguide structure was designed. The ribbon structure with the mode spectrum plotted in Fig. 4 was specifically designed to ensure that there would be a mode with wave vector $8.725 / \mu \mathrm{m}$ (the mode plotted in Fig. 5) and that this mode would have a single-intensity lobe in each of the gain-loaded segments of the waveguide. Having a singleintensity lobe in each of the gain-loaded regions of the structure ensures that this mode will have the highest gain overlap of all the modes supported by the structure.

Although the mode shown in Fig. 5 has the highest gain of all the modes supported by the structure, it does not have constant lobe intensity in the gain regions across the ribbon structure. The two outlying gain regions see almost 10 times the peak intensity of the central gain re- gion. Therefore under the conditions of strong gain saturation that would be required for efficient laser performance the outlying regions will be more strongly extracted than the central regions, leaving an unbalanced gain profile across the structure. This condition in turn may encourage additional modes to come in that will preferentially extract the central gain regions. Specific design of the ribbon structure to ensure that the peak intensity is unchanged from core to core is addressed in Section 4.

\section{ONE-DIMENSIONAL STRUCTURES WITH GAIN VARIATIONS AND CONSTANT REFRACTIVE INDEX}

As an alternative to the periodically modulated index structures just considered, we now evaluate a waveguide structure that has a uniform refractive index across its aperture, and we modulate the gain profile only periodically. To keep a connection with the previously analyzed case displayed in Fig. 4 we keep the outer cladding's index value at 1.45 , the waveguide region at a constant index of 1.4585, and the overall width of the waveguide at 50.45 $\mu \mathrm{m}$. Applying the same design procedure as has just been explained for the case in which the gain segments have a slightly lower refractive index than the no-gain segments, we obtain the five-core ribbon structure, as summarized in Table 2.

For the structure detailed in Table 2 the mode that has one lobe associated with each gain region has a wavevector value of $8.723 / \mu \mathrm{m}$. Figure 6 shows a summary of this structure's eigenmode spectrum in a plot of mode overlap with gain regions versus effective index value. Comparing Figs. 6 and 4, one can see that one of the advantages of the constant-refractive-index waveguide region is that the mode discrimination between the desired mode and all other modes supported by the structure is much better than for the variable-index waveguide region (Fig. 4). Figure 7 is a plot of local mode intensity across the waveguide structure for the mode for which the waveguide structure was designed (Mode \#5 in Fig. 6). Examining the mode structure in Fig. 7, we can see that the peak lobe intensity is constant across the waveguide structure, as we have already stated is one of the goals of

Table 2. Detailed Design of a One-Dimensional Ribbon Fiber Structure with a Constant-Index Waveguide Region

\begin{tabular}{ccc}
\hline Width $(\mu \mathrm{m})$ & Refractive Index & Gain Loaded? \\
\hline 10.00 & 1.45 & No \\
6.81 & 1.4585 & Yes \\
5.26 & 1.4585 & No \\
5.26 & 1.4585 & Yes \\
5.26 & 1.4585 & No \\
5.26 & 1.4585 & Yes \\
5.26 & 1.4585 & No \\
5.26 & 1.4585 & Yes \\
5.26 & 1.4585 & No \\
6.81 & 1.4585 & Yes \\
10.00 & 1.45 & No \\
\hline
\end{tabular}




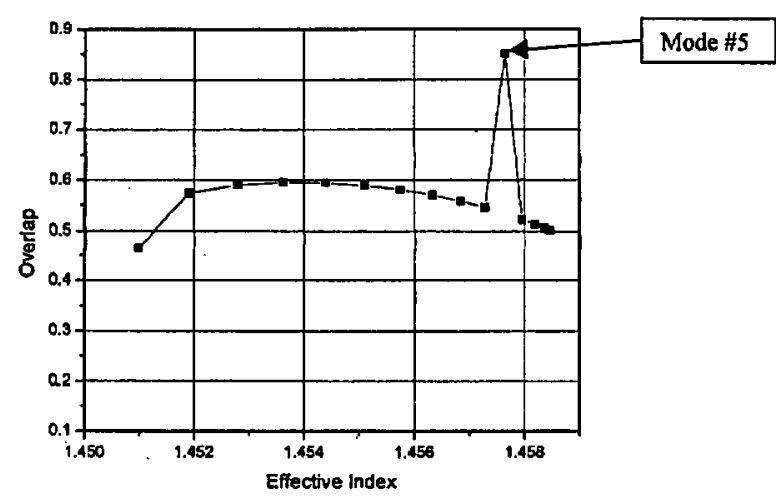

Fig. 6. Eigenmode overlap with the gain region plotted versus effective index. Mode \#5 (counting from the right) was designed to have a single intensity lobe for each of the gain-loaded segments in the waveguide region.

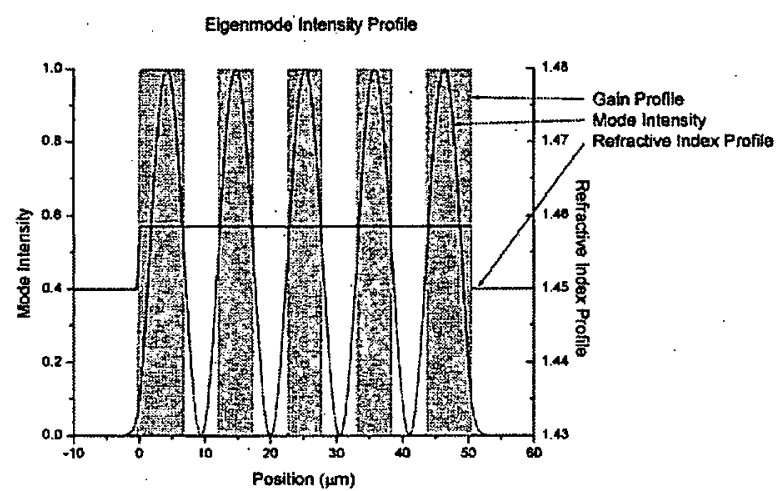

Fig. 7. Intensity envelope of the eigenmode for which the ribbon structure was designed (Mode \#5 in Fig. 6). The widths of the individual index segments are adjusted such that each gain region (shaded) sees a single intensity lobe. Superimposed is the refractive-index profile of the structure, which is constant across the waveguide region.

a robust design. This, combined with the enhanced mode discrimination afforded by the constant-index waveguide region compared with other structures that have variable indices in the waveguide region, makes the constantindex structure ideal for single-mode, power scalable devices. The realization of these advantages as a result of having a constant-index waveguide region is the central result of this study. It should be noted that the gain discrimination depicted in Fig. 6, which clearly favors the single mode plotted in Fig. 7 (Mode \#5), is calculated for the specific case in which the gain in the interior regions has a $50 \%$ fill factor; i.e., the interior gain and no-gain regions have the same width. By increasing the fill factor of the gain regions beyond the $50 \%$ fill design point considered above, we can increase the $\Gamma$ factor of the preferred mode beyond its value of 0.85 in Fig. 6. Doing so, however, will come at the expense of mode discrimination, as the $\Gamma$ factors of all other modes will also increase.

\section{ONE-DIMENSIONAL STRUCTURE WITH GAIN AND INDEX VARIATION}

Figures 8 and 9 reveal the effects of slightly increasing and decreasing, respectively, the refractive index of the gain-loaded segments by $\Delta n=0.001$. Comparing these calculations "of the overlap factor versus the effective index with the calculation of Fig. 6 makes it apparent that a bandgap emerges when $\Delta n \neq 0$. As is illustrated below, the refractive index acts as a potential, when an analogy is drawn between the paraxial wave equation of optics and the Schrödinger equation of quantum mechanics (see Section 6 below). Therefore we are able to say that each segment in the ribbon may be viewed as an atom.

For the case of Fig. 8, for which $\Delta n$ (gain - no-gain) $=+0.001$, it is as if a potential well had been introduced in the gain-loaded regions. This favors the overlap factors of modes that have five or fewer lobes (i.e., the first five points, counted from the right in Fig. 8). The sixth point (six lobes) fills the no-gain regions much more effectively, introducing a discontinuity in the plot of $\Gamma$ versus $n_{\text {eff }}$. Once there are many lobes in the mode, the overlap averages to approximately one half, as would be expected. The point of highest overlap may be regarded as the top of the valence band (maximal overlap with the gain-loaded segments); the point characterized by the lowest overlap factor (i.e., maximal overlap with the no-gain regions) may be regarded as the bottom of the conduction band. We thereby discern the photonic crystal nature of the ribbon laser.,21,22 The calculations in Fig. 9 are related but may be contrasted because the potential well (i.e., slightly higher index) is located in the no-gain segments. Therefore the field becomes concentrated in the no-gain regions and evidences a low overlap factor for fewer than four lobes. The discontinuity here may also be interpreted as a photonic bandgap.

We may now refer to Figs. 6 and 7, where Mode \#5 exhibits a greatly enhanced overlap factor than any of the other modes. The reader is reminded that this case corresponds to that of a constant refractive index across the ribbon. When we consider the solid-state analogies again, it is apparent that the constant-index, or constantpotential, model is closest to that of a nearly free electron in a metal. Of course, photonic metal would not have a bandgap, but the special mode (Mode \#5) would correspond to the boundary of the Brillouin zone. This analogy of a photonic metal holds most closely for the situation in which the electron is nearly free (i.e., does not sense the potential from individual atoms in the lattice).

\section{THEORY OF THE TWO-DIMENSIONAL RIBBON FIBER}

The one-dimensional theoretical model outlined above gives insight into the occurrence of the desired antiguided leaky modes in structures of the type considered here. To understand the effects on performance of possible fabrication constraints, we have considered a number of designs with either rectangular or circular cores that either touch the boundary or are immersed in the inner cladding region. For this reason we employed a full scalar-wave propagation method to simulate the performance of target designs as detailed below. Various implementations correspond to the same one-dimensional transverse structure (index distribution as seen on center line) analyzed in Section 5. 


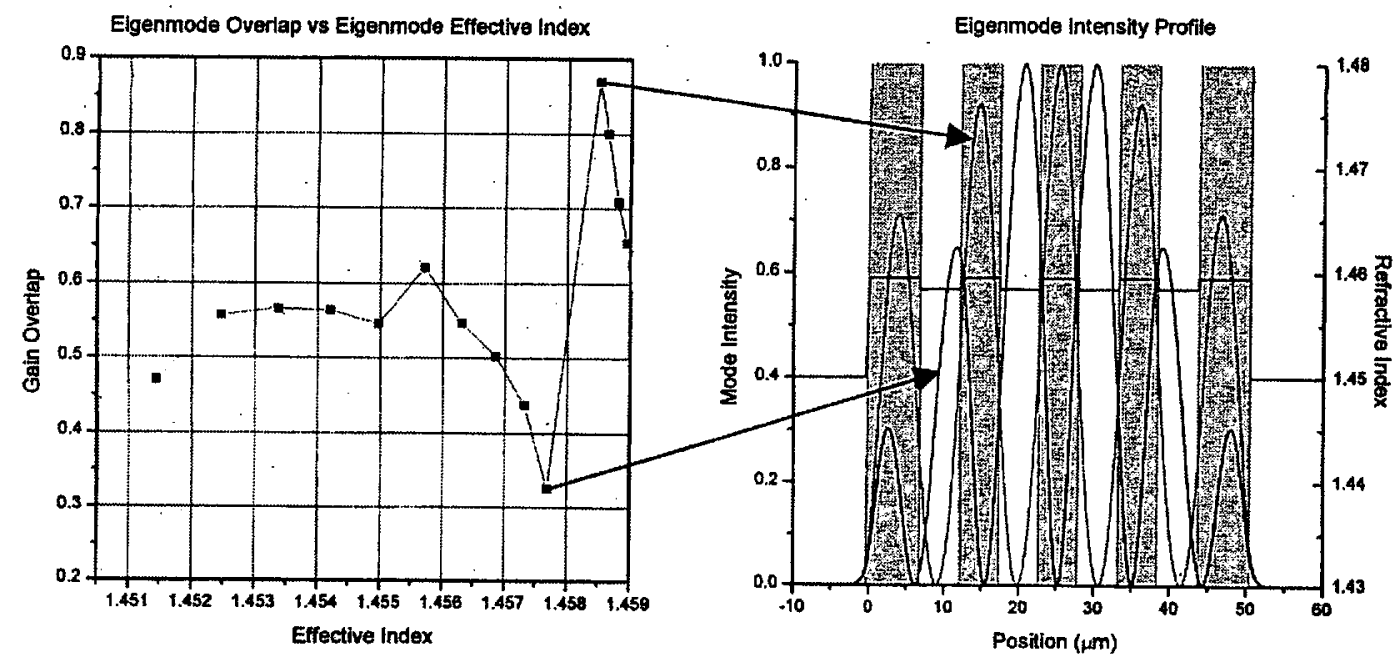

Fig. 8. Same structure as in Fig. 7 but with the gain-loaded segments' refractive index increased by 0.001 above that of the no-gain segments. Gain-loaded regions are shaded.

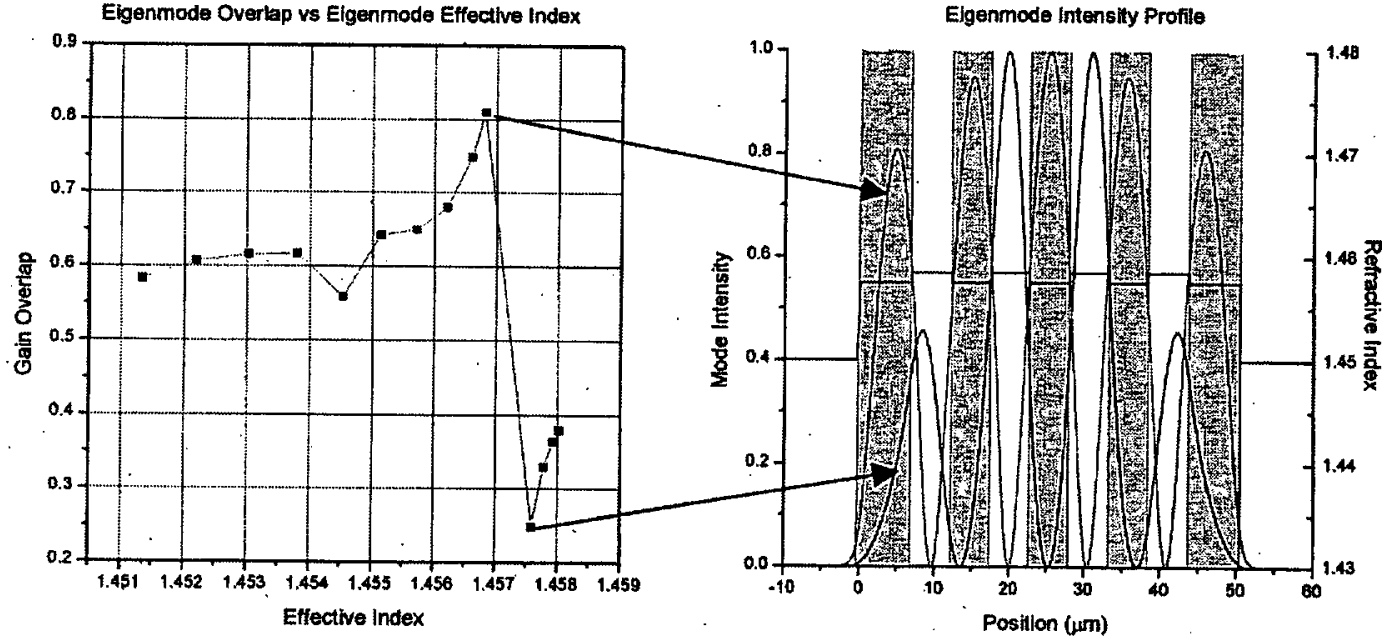

Fig. 9. Same structure as in Fig. 7 but with the gain-loaded segments' refractive index decreased by 0.001 below that of the no-gain segments. Gain-loaded regions are shaded.

Amplitude $\varepsilon$ of a propagating electric field in normal fiber and integrated optical waveguides with small index contrast, i.e., in which refractive-index differences are small compared with the index, is well described by the scalar Helmholtz wave equation ${ }^{18}$

$$
\nabla^{2} \varepsilon(x, y, z)+\left[\frac{n(x, y) \omega}{c}\right]^{2} \varepsilon(x, y, z)=0 .
$$

Here $n(x, y)$ is the refractive index, which is assumed to depend on the transverse spatial coordinates $x$ and $y$. Because the index is independent of $z$ (position along the ribbon fiber), such a structure supports modes whose shape is independent of $z$. Such a mode, $E_{i}(x, y)$, propagates with a characteristic propagation constant $\beta_{i}$, which can be found from the eigenvalue equation

$$
\nabla_{\perp}^{2} E_{i}(x, y)=-\left\{\left[\frac{n(x, y) \omega}{c}\right]^{2}-\beta_{i}^{2}\right\} E_{i}(x, y),
$$

where $\nabla_{\perp}^{2}=\partial^{2} / \partial x^{2}+\partial^{2} / \partial y^{2}$ is the transverse Laplacian operator. Equation (19) is equivalent to Eq. (3) but incorporates both tranverse dimensions. Because Eq. (18) is linear, the general solution is a linear combination of such modes with arbitrary amplitudes, each changing its phase with propagation distance according to its own characteristic modal propagation constant.

Numerical solution of Eq. (18) can be difficult. However, a convenient simplification is to make the slowly varying envelope approximation. We assume that amplitude $u$ varies mainly as $\exp \left(i K_{c} z\right)$, where $K_{c}$ is a reference wave number (see below); i.e., we let

$$
\varepsilon(x, y, z)=E(x, y, z) \exp \left(i K_{c} z\right)
$$

where $E$ is only a weak function of $z$. This means that $E$ varies little over an optical wavelength. In this case, substituting Eq. (20) into Eq. (18) and discarding the term $\partial^{2} E / \partial z^{2}$ because it is small compared with $K_{c} \partial E / \partial z$, we are left with the so-called paraxial wave equation: 
Beach et al.

$$
i \frac{\partial E}{\partial z}=-\frac{1}{2 K_{c}} \nabla_{\perp}{ }^{2} E+\frac{K_{c}}{2}\left[1-\frac{n^{2}(x, y)}{n_{c}{ }^{2}}\right] E=H E,
$$

where $n_{c}$ is the index that corresponds to $K_{c}$, i.e., $K_{c}$ $=n_{c} \omega / c$. Note that this equation has exactly the form of the Schrödinger equation of quantum mechanics. Propagation distance $z$ plays the same role in optical propagation as time does in the Schrödinger equation, and the potential energy is given by the second term on the right-hand side in Eq. (21), which involves the refractive index. There is thus a one-to-one analogy between optical waveguides and two-dimensional $(x, y)$ quantum mechanics. Equation (21) defines an optical Hamiltonian operator $H$ as in quantum mechanics. The eigenvalues and eigenfunctions of this operator are the fundamental quantities that describe the nature of the optical waveguide. It can be shown that the modal eigenfunctions of Eqs. (19) and (21) are exactly the same. ${ }^{23}$ However, the eigenvalues are different. The modes that compose field $E$ of Eq. (21) individually satisfy the eigenvalue equation

$$
H E_{i}=\beta_{i}{ }^{\prime} E_{i},
$$

with the relationship between $\beta_{i}$ and $\beta_{i}{ }^{\prime}$ given by

$$
\beta_{i}=\left(K_{c}{ }^{2}-2 K_{c} \beta_{i}{ }^{\prime}\right)^{1 / 2} \approx K_{c}-\beta_{i}{ }^{\prime} .
$$

That is, $\beta_{i}{ }^{\prime}$ represents the small change of modal wave number $\beta_{i}$ from reference wave number $K_{c}$. Because of the analogy with the Schrödinger equation, we see immediately that if we choose the cladding index to define $K_{c}$, then guided modes will have negative values of $\beta_{i}{ }^{\prime}$ (corresponding to bound states in quantum mechanics that have negative energy) and radiation modes will have positive values of $\beta^{\prime}$.

We solve Eq. (22) numerically, using the fast-Fouriertransform based beam propagation method. ${ }^{24,25}$ An advantage of this approach is that, for a lossless-gainless medium, it exactly conserves electromagnetic energy. This is important to enable reliable calculations to be carried out for a medium with small gain or loss. The solution found for Eq. (21) is of the form $E(x, y, z)$, where $E$ is, in general, a linear combination of modes excited at the start of the calculation by assumption of an initial field $E(x, y, 0)$. One can find the modal propagation constants by forming a correlation function $P(z)$ :

$$
P(z)=\iint E^{*}(x, y, 0) E(x, y, z) \mathrm{d} x \mathrm{~d} y .
$$

Because $E$ is a linear combination of orthonormal modes, the correlation will necessarily be of the form

$$
P(z)=\sum\left|A_{n}\right|^{2} \exp \left(i \beta_{n}^{\prime} z\right)
$$

where $A_{n}$ is the amplitude of mode $n$ and $\left|A_{n}\right|^{2}$ is proportional to the power in mode $n$. Fourier transforming $P(z)$ with respect to $z$ yields

$$
P(\beta)=\sum\left|A_{n}\right|^{2} \delta\left(\beta-\beta_{n}{ }^{\prime}\right) .
$$

That is, the spectrum of the correlation function consists of distinct lines centered at the modal propagation constants. Once the modal propagation constants are

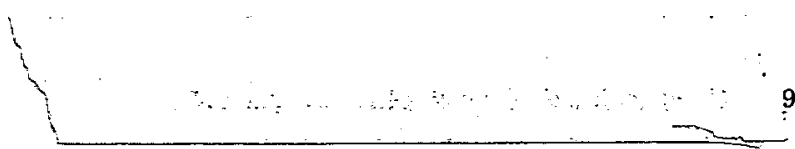

known, we can retrieve the nonnormalized mode shapes by projecting them from the propagated field, i.e.,

$$
E_{n}(x, y)=\iint E(x, y, z) \exp \left(i \beta_{n}^{\prime} z\right) \mathrm{d} z .
$$

This technique has been used effectively in modeling optical fibers, rib waveguides, $x$ and $y$ couplers, and optical resonators. ${ }^{26}$

This formalism remains the same for a nonpassive device, i.e., a device with distributed gain or loss. In that case the delta function line shapes in the spectrum of Eq. (26) are broadened by an amount proportional to the imaginary part of the modal wave number. A numerical technique that measures this width then gives a direct value for the modal gain.

\section{TWO-DIMENSIONAL STRUCTURE WITH GAIN VARIATION AND CONSTANT REFRACTIVE INDEX}

We used a numerical code that embodies the above formalism in our simulations of the two-dimensional transverse ribbon structure. Typically, the inserted field is propagated on a $256 \times 64$ grid and the propagated field is evaluated as a function of transverse coordinates $x$ and $y$

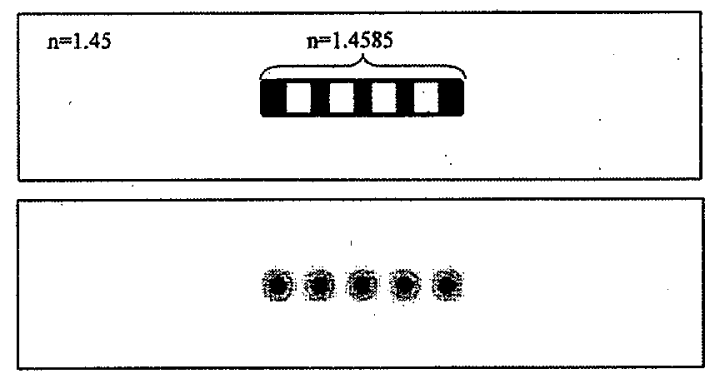

Fig. 10. Cross sectional view of the ribbon structure with two transverse dimensions that is analyzed in the text. The dark regions in the upper picture indicate the gain-loaded portions of the waveguide. The refractive index is constant throughout the waveguide region and equals 1.4585 . The waveguide region is 6 $\mu \mathrm{m}$ high, the end pieces are $4.5 \mu \mathrm{m}$ wide, and the central segments are $4 \mu \mathrm{m}$ wide.

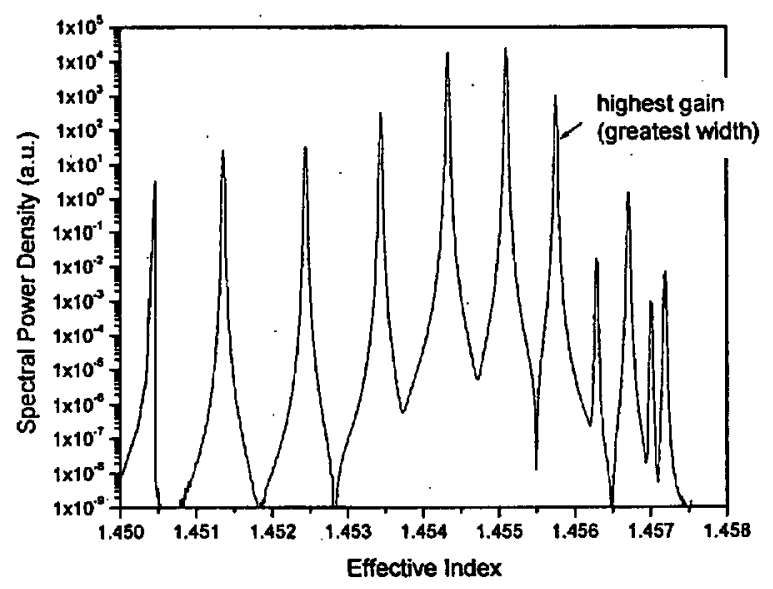

Fig. 11. Spectral power of modes excited by a Gaussian beam inserted into the structure described in Fig. 10. 
10
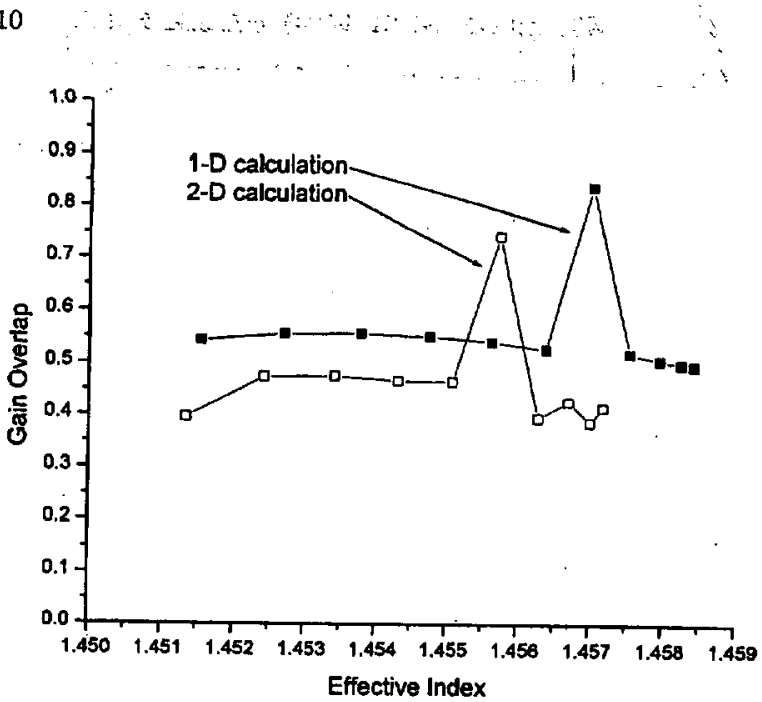

Fig. 12. Comparison of gain overlap and effective mode index for one-dimensional (1-D) and two-dimensional (2-D) calculations.

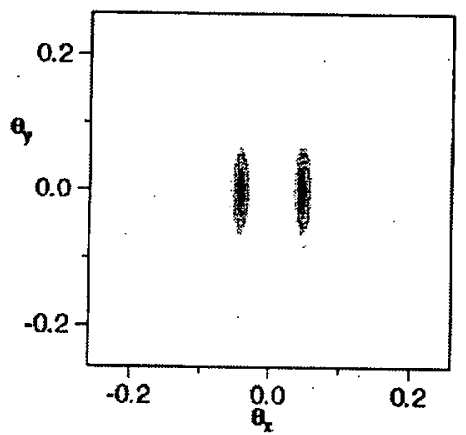

(a)

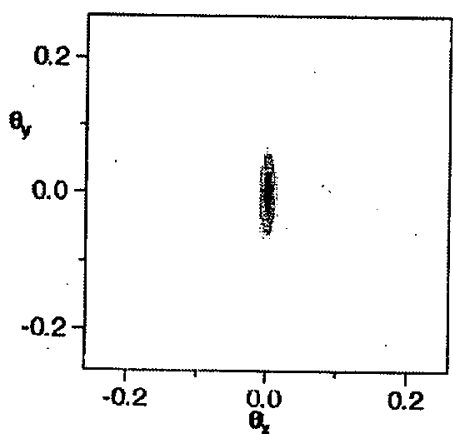

(b)

Fig. 13. (a) Uncorrected far field of the ribbon structure of Fig. 10. Far-field lines are separated by 132 mrad. (b) Far field after correction with a simple phase plate. Approximately two thirds of the total energy is contained in the central peak.

at 32,800 longitudinal $z$ values. The complex modal propagation constants and modal field patterns can then be calculated. A cross section of a sample ribbon laser structure is shown in Fig. 10. Both refractive index and small-signal gain are spatially distributed. Simulations are started with an initial field. Part of this field projects onto waveguide modes and is propagated. The rest is radiated away from the structure. To prevent this radiated energy from reflecting from the numerical boundaries of
Beach et al.

the simulation, we placed an absorbing layer around the outer boundary. Propagating the initial field and calculating the correlation function defined in Eq. (25) and its Fourier transform over the propagation distance lead to the spectrum shown in Fig. 11. Excited modes appear as distinct spectral lines for which the spectral width is proportional to the modal gain. Figure 12 plots the gain overlap versus the effective mode index for the structure shown in Fig. 10 for both the one- and two-dimensional formalisms. The one-dimensional calculation corresponds to the one-dimensional structure that we generated by taking a lineout along the $x$ axis of the twodimensional structure depicted in Fig. 10 . In both the one- and two-dimensional analyses, the fifth mode is the one with the highest gain. As one would expect, the twodimensional calculation gives both smaller gain overlaps and smaller effective index values because of the extra degree of freedom in the transverse direction.

As can be seen, e.g., from Fig. 7, the optimal antiguided modal field is nearly a pure sinusoid, which implies that the far field will consist principally of two lines with an angular spread of $\pm \lambda / n p$ in the horizontal direction. Here $\lambda / n$ is the wavelength in the medium and $p$ is the period. Similarly, the angular spread in the vertical direction is determined by the structure height. These expectations are met in our numerical simulations. Figure 13(a) shows the simulated far-field pattern for the ribbon structure of Fig. 10. Because the modal field is coherent, collimation can be achieved by use of a phase plate. Additionally, because the modal structure is so simple, the most convenient approach to collimation is to adjust the phase of periods of the structure by either 0 or $\pi$, successively, to effectively yield the absolute value of the modal field. This elimination of zero crossings greatly improves the far-field pattern, as shown in Fig. 13(b), where the simulation reveals that two thirds of the modal power has been concentrated in a central peak. The horizontal width of this peak depends on the number of cells in the structure and will improve as $1 / N$ for the ideal structure.

\section{ROBUSTNESS TO VARIATIONS IN REFRACTIVE INDEX AND DIMENSIONS}

Our ultimate goal in this study is to develop an understanding of robust fiber ribbon designs that will guarantee single-transverse-mode operation in the presence of strong gain saturation. Therefore it is useful to develop a criterion for how tightly design tolerances, both cell refractive indices and cell dimensions, must be held for the various cells that constitute a given structure. We investigate this issue in two regimes. First we look at the effect of systematic variations in cell refractive indices and cell dimensions. This type of systematic error represents what we shall likely see in structures fabricated from conventional fiber and by use of preform pulling technology. Cell dimensions will tend to vary together, shrinking below or expanding above the design point as a result of the manner in which the ribbons are pulled. Also, because we anticipate using the same starting material for all the gain and all the no-gain portions of the various cells that make up the waveguide region of the ribbon, we expect in- 


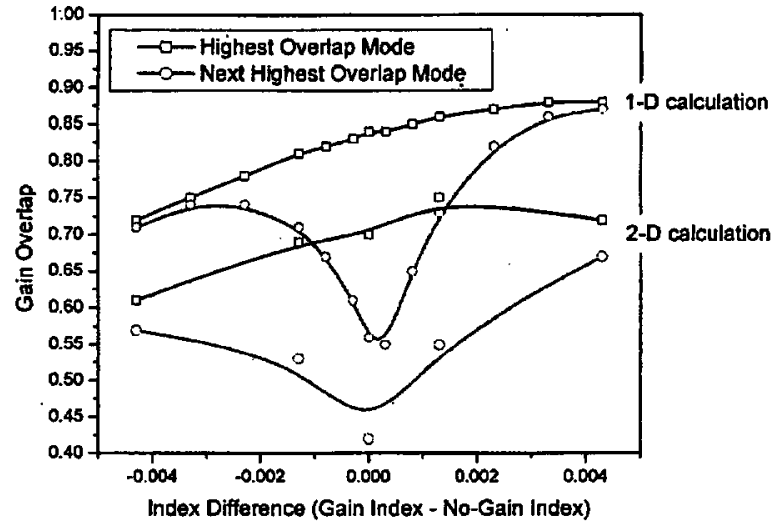

Fig. 14. Overlap versus index difference of the two highest overlap modes for one-dimensional (1-D) and two-dimensional (2-D) cases with systematic index variation between the gain and nogain regions.

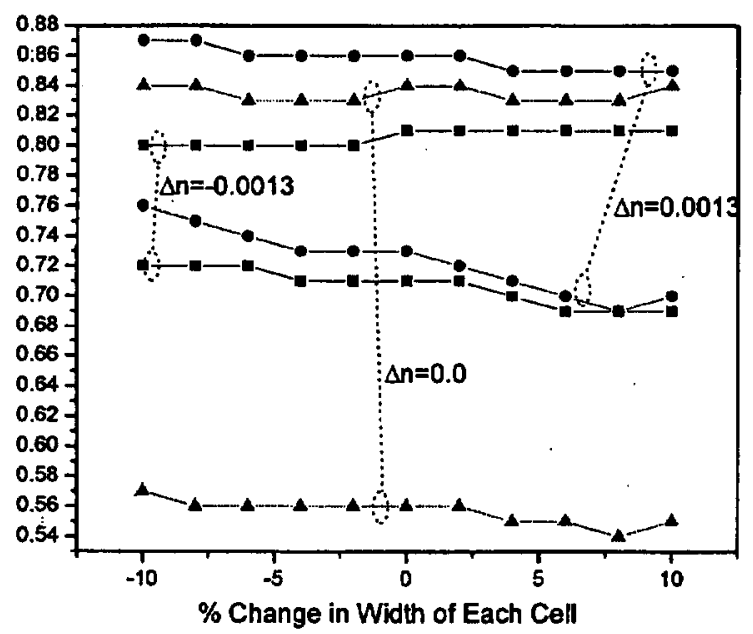

Fig. 15. Gain overlap versus index difference of the two highest overlap modes for three values of cell $\Delta n$. All data points were calculated with the one-dimensional model.

dex errors from the desired design point to occur uniformly throughout the structure. In addition to investigating these systematic errors, we go on to investigate random errors in both cell refractive indices and cell dimensions for very large, 100-core structures. Such errors characterize uncontrolled aspects of the ribbon structure fabrication and could be an issue in the developing technology that we anticipate using.

Here we determine what the tolerance in the refractive indices of the gain and the no-gain regions must be to ensure that the structure will support only a single transverse mode. Because the one-dimensional code can be quickly executed, it is better suited to running the large number of cases required for this parameter study. The two-dimensional code is then used on a smaller scale to confirm the results of the one-dimensional code. Using the same-constant index five-core structure as described in Section 7, we made a series of runs to determine sensitivity to systematic variations in the refractive indices of the gain and the no-gain cells. In this series of runs we kept the geometry constant, allowing only the index difference to vary from run to run. We used an index of 1.45 for the cladding, while the gain-doped regions were held fixed at an index of 1.4585 and the nongain regions were allowed to vary from 1.4628 to 1.4542 . The data were compiled by the index difference value of gain index - no-gain index. This range of allowed indices for the no-gain regions represents both guidedlike (gain index > non-gain index) and anti-guidedlike (gain index $<$ non-gain index) variations. For each index difference, the largest and the second largest gain overlap modes were recorded. We ran a total of 11 cases, using the one-dimensional model and reran 5 of the 11 cases using the two-dimensional model. Figure 14 is a plot of overlap versus index difference and presents both the one- and two-dimensional results. Although the gain overlap increases as the index difference increases for the highest gain-overlap mode, Fig. 14 shows that the point of maximum discrimination occurs with an index difference of zero. In addition, the qualitative similarity between the one- and two-dimensional calculations is evident in Fig. 14; both show maximum gain discrimination for an index difference of zero. Remember that one of the critical design requirements is that the structure oscillate in a single transverse mode, making good mode discrimination a strong figure of merit in the design. As the index difference increases (in both the positive and the negative directions), the overlap values of the two strongest modes get closer, increasing the chance that the structure will hop from one mode to the other during operation.

We next explored the structure's sensitivity to geometry (cell width) variations. The dimensions of all cells within the structure were varied by the same amount, mimicking the type of systematic error that might be expected if one were fabricating the structure by using a fiber preform pulling technique. For this parameter study we varied each cell width by a specified amount $( \pm 2 \%$, $\pm 4 \%$, $\pm 6 \%$, $\pm 8 \%$, and $\pm 10 \%$ ) from its original value. This parameter study was repeated for systematic index variations of $-0.0013,0.0$, and 0.0013 . All runs were made with the one-dimensional model. As can be seen from Fig. 15, in which the results of this study are plotted, varying the cell widths has little effect on either the gain overlap value or the mode discrimination out to the $\pm 10 \%$ systematic variations investigated here. Based on these results, we believe that an acceptable index difference is \pm 0.001 , with a target difference of 0.0 . Within this index difference range, we should be able to handle cell width fabrication errors of $\pm 10 \%$ and possibly more.

Important for power scaling is the question of required index tolerance as a function of the number of cores. To determine this value we repeated the foregoing five-core structure analysis in which the refractive index of the gain and no-gain cells was systematically varied for a 20core and a 100-core ribbon fiber structure. Figure 16 depicts the results of this study. The data in Fig. 16 were generated by use of the one-dimensional code with subcell dimensions identical to those used for Fig. 14. It is evident from Fig. 16 that, as the number of cells increases, the requirements on the systematic variation of the refractive index become more stringent. To better quantify this scaling law, we determined the FWHM spread in index for which the gain discrimination between the two 
highest overlap modes is just one half of its peak value at $\Delta n=0$. These FWHM values appear in Fig. 16. In Fig. 17 we have plotted these FWHM index spreads relative to the inverse number of gain cores $\left(1 / n_{\text {cores }}\right)$ for the three cases studied here. As can be seen from the data plot in Fig. 17, the $\Delta n_{\text {FWHM }}$ requirement varies almost linearly with $1 / n_{\text {cores }}$, which is what we intuitively expect. The straight line in Fig. 17 represents a linear least-squares fit to the data points that is constrained to pass through the origin, $\Delta n_{\text {FWHM }}=0.0127 / n_{\text {cores }}$.

In addition to the systematic errors just considered, random variations in cell index and cell dimension may be an issue, particularly in fiber structures that consist of a large number of cells. To investigate the effect of random index variations we modeled a 100-core ribbon fiber with nominally constant index throughout the waveguiding re-

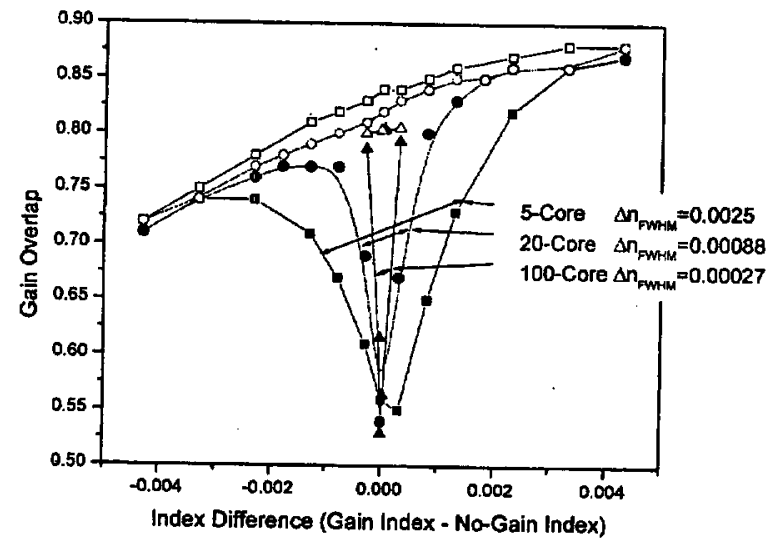

Fig. 16. Overlap versus index difference of the two highest overlap modes for one-dimensional structures that have a systematic index variation between the gain and the no-gain regions. Three structures are investigated here that consist of, 20 , and 100 cores. For each structure investigated, the gain overlap of the highest overlap and the next-highest overlap mode are plotted against the systematic index variation.

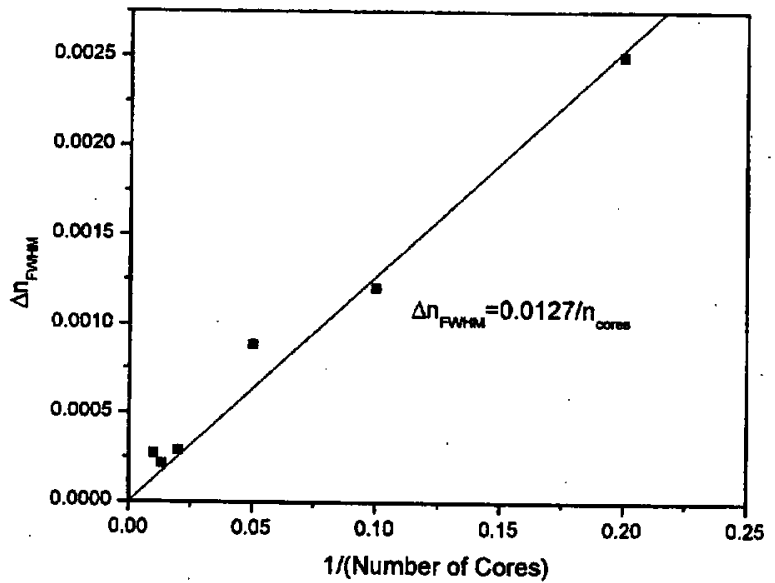

Fig. 17. Gain discrimination requirements versus number of mode cores taken from the data in Fig. 16. Additionally, data points are included for 10,50 , and 75-gain-core structures. The FWHM spread in index plotted here is defined by the two points at which the gain discrimination between the two highesto verlap modes is just halved from its peak value at $\Delta n=0$. Straight line, linear least-squares fit to the data points that is constrained to pass through the origin.

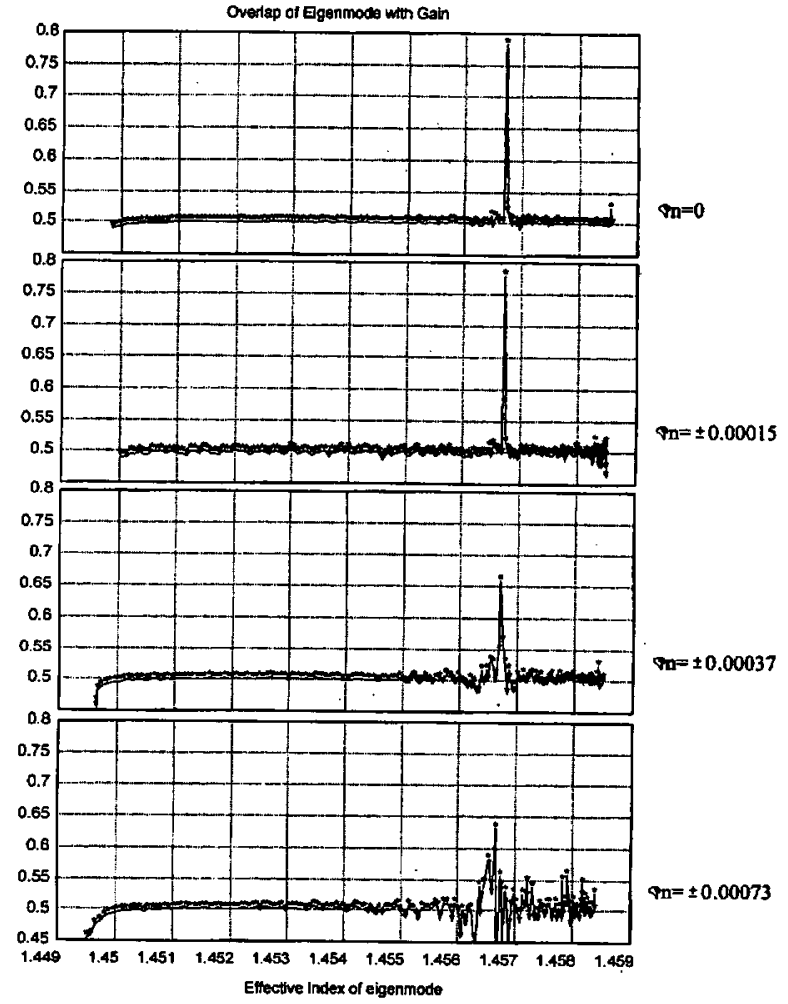

Fig. 18. Effects ofran dom variations in cellre fractive index illustrated with a 100-core structure in which gain overlaps of the structure's eigenmodes are plotted against the various modes' effective refractivein dices. In each caseth ein dividual cells that constitute the waveguide have had their refractive indices randomly varied with a uniform spread about the design point. The magnitudes of the uniform random distributions were taken to be $\Delta n=0, \Delta n= \pm 0.00015, \Delta n= \pm 0.00037$, and $\Delta n= \pm 0.00073$, as indicated.

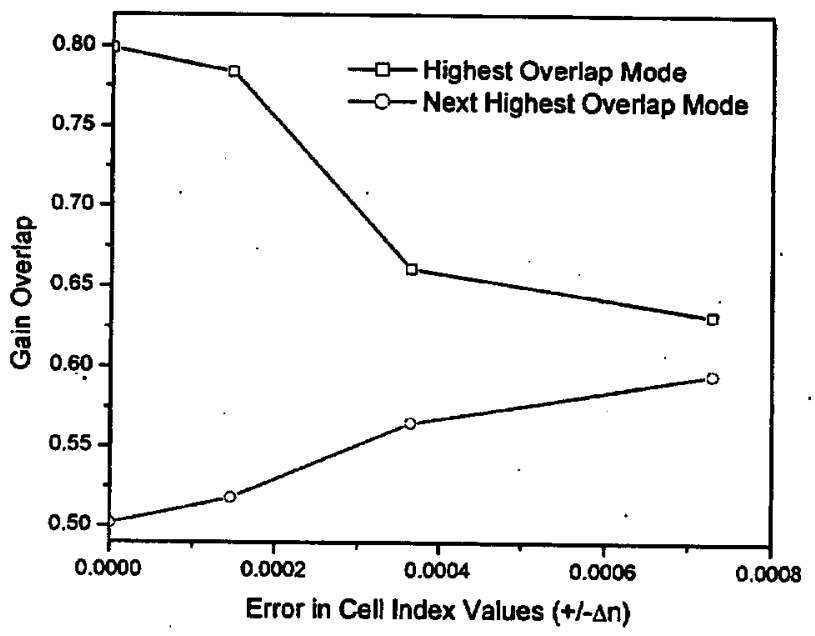

Fig. 19. Gain overlap for a 100 -core ribbon fiber. The overlap of the two highest gain overlap modes from Fig. 18 is plotted as a function of the random error introduced in the individual cell refractive indices. Random errors in refractive indicesar e uniformly distributed with the indicated amplitudes.

gion. The one-dimensional waveguide design investigated here consists of a structure similar that in Fig. 10 but with cell dimensions of $3.65 \mu \mathrm{m}$ for the central cells 
and $4.36 \mu \mathrm{m}$ for the end cells. The effect of random variations in cell refractive index is illustrated in Fig. 18, where we have plotted the structure's eigenmode gain overlap against the various mode effective refractive indices. In each case the individual cells that compose the waveguide have had their refractive indices randomly varied with a uniform spread about the design point. The magnitudes of the uniform random distributions were taken to be $\Delta n=0, \Delta n= \pm 0.00015, \Delta n$ $= \pm 0.00037$, and $\Delta n= \pm 0.00073$, as indicated. In Fig. 19 the overlaps of the two highest-gain overlap modes for this structure are plotted as a function of the random error introduced in the individual cell refractive indices. Examining this plot, one can see that the gain discrimination between the two highest overlap modes begins to degrade substantially when random index variations are approximately $\Delta n= \pm 0.00037$, in approximate accord with the FWHMs deduced in Figs. 16 and 17 . We have also modeled the effect of random variations in the cell dimensions for the 100-core structure studied here and found that in general the structure is highly robust to these types of variation. Based on our modeling, random dimensional variations at the $\pm 10 \%$ level do not signifcantly degrade mode overlap and mode overlap discrimination and so are probably not issues in device fabrication.

\section{CONCLUSION}

In this paper we have described a new, robustly scalable technique for phase locking of multiple gain cores in a fiber structure based on antiguiding or radiative coupling. Our focus has been on a ribbonlike geometry in which the waveguide region contains multiple gain cores alternating with nongain regions in a periodic array. An outer, lower-index cladding surrounds the entire ribbon structure. The distinguishing feature of our design is a constant refractive-index profile across the waveguide region as opposed to alternating higher- and lower-index regions. Our modeling predicts that the constant-index design will provide modes that meet our two critical design requirements: strongly favored oscillation in a single transverse mode and good intensity uniformity across the waveguide structure. The constant-index case is also more robust in terms of design tolerances that must be held with both the refractive-index values across the waveguide region as well as physical dimensional tolerances.

\section{ACKNOWLEDGMENTS}

This research was supported by the U.S. Air Force U.S. Air Force Research Laboratory (AFRL), Albuquerque, N. Mex., under contract L-8958.1, and by the U.S. Department of Energy under contract W-7405-ENG-48. We are grateful to Craig Denman of the AFRL for his interest and support and to Nathan Brilliant of the AFRL for his technical suggestions. We also thank William Krupke for many useful suggestions and comments during the course of this research.

\section{REFERENCES}

1. D. Botez and D. R. Scifres, Diode Laser Arrays (Cambridge U. Press, Cambridge, 1994), Chap. 1.

2. W. J. Wadsworth, J. C. Knight, W. H. Reeves, P. St. J. Russell, and J. Arriaga, " $\mathrm{Yb}^{3+}$-doped photonic crystal fibre laser," Electron. Lett. 36, 1452-1454 (2000).

3. V. Dominic, S. MacCormack, R. Waarts, S. Sanders, S. Bricknese, R. Dohle, E. Wolak, P. S. Yeh, and E. Zucker, "110 W fibre laser," in Conference on Lasers and ElectroOptics (CLEO/US) (Optical Society of America, Washington, D.C., 1999), paper CPD11-1.

4. V. Dominic, S. MacCormack, R. Waarts, S. Sanders, S. Bricknese, R. Dohle, E. Wolak, P. S. Yeh, and E. Zucker, "110 W fibre laser," Electron. Lett. 35, 1158-1160 (1999).

5. M. Muendal, B. Engstrom, D. Kea, B. Lalierte, R. Minns, R. Robinson, B. Rockney, Y. Zhang, R. Collins, P. Gavrolovic, and A. Rowley, "35-watt $\mathrm{cw}$ singlemode ytterbium fiber laser at 1.1 micron," in Conference on Lasers and ElectroOptics, Vol. 11 of 1997 OSA Technical Digest Series (Optical Society of America, Washington, D.C., 1997), paper CPD30-1.

6. H. Zeller, U. Willamowski, A. Tunnermann, H. Welling, S. Unger, V. Reichel, H.-R. Muller, J. Kirchof, and P. Albers, "High-power $\mathrm{cw}$ neodymium-doped fiber laser operating at $9.2 \mathrm{~W}$ with high beam quality," Opt. Lett. 20, 678-560 (1995).

7. D. Meyhuys, K. Mitsunaga, L. Eng, W. K. Marshall, and A. Yariv, "Supermode control in diffraction-coupled semiconductor laser arrays," Appl. Phys. Lett. 63, 1165-1167 (1988).

8. P. Glas, M. Naumann, A. Schirrmacher, and Th. Pertsch, "A $\mathrm{cw}$ diode-pumped single-silica fiber comprising 40 cores used as active elements for a high power fiber laser at 1050 $\mathrm{nm},{ }^{,}$in Conference on Lasers and Electro-Optics (CLEO/ US), Vol. 6 of 1998 OSA Technical Digest Series (Optical Society of America, Washington, D.C., 1998), paper CtuK 5.

9. M. Wrage, P. Glas, M. Leitner, T. Sandrock, N. N. Elkin, A. P. Napartovich, and A. G. Sukharev, "Experimental and numerical determination of coupling constant in multicore fiber," Opt. Commun. 175, 97-102 (2000).

10. P. L. Cheo, A. Liu, and G. G. King, "A high-brightness laser beam from a phase-locked multicore $\mathrm{Yb}$-doped fiber laser array," IEEE Photon. Technol. Lett. 13, 439-441 (2001).

11. M. Wrage, P. Glas, D. Fischer, M. Leitner, D. V. Vysotsky, and A. P. Napartovich, "Phase locking in a multicore fiber laser by means of a Talbot resonator," Opt. Lett. 25, 1436$1438(2000)$.

12. D. Mehuys, W. Streifer, R. G. Waarts, and D. F. Welch, "Modal analysis of linear Talbot-cavity semiconductor lasers," Opt. Lett. 16, 823-825 (1991).

13. J. Banerji, A. R. Davies, and R. M. Jenkins, "Comparison of Talbot and 1-to- $N$-way phase-locked array resonators," Appl. Opt. 36, 1604-1609 (1997).

14. E. K. Gorton and R. M. Jenkins, "Theory of $1-N$-way phaselocked resonators," Appl. Opt. 40, 916-920 (2001).

15. D. Botez and A. P. Napartovich, "Phase-locked arrays of antiguides: analytical theory," IEEE J. Quantum Electron. 30, 975-980 (1994).

16. D. Botez, A. P. Napartovich, and C. A. Zmudzinski, "Phaselocked arrays of antiguides: analytic theory II," IEEE J. Quantum Electron. 31, 244-253 (1995).

17. T. M. Monro, D. J. Richardson, N. G. R. Broderick, and P. J. Bennet, "Holey optical fibers: an efficient modal model," J. Lightwave 'Technol. 17, 1093-1102 (1999).

18. D. Marcuse, Theory of Dielectric and Optical Waveguides, 2nd ed. (Academic, New York, 1991).

19. A. P. Napartovich and D. Botez, "Analytic theory of the structure of collective modes in antiguided semiconductors," Quantum Electron. 26, 670-675 (1996).

20. A. P. Napartovich and D. Botez, "Analytic theory of phaselocked arrays of antiguided diode lasers," in Physics and Simulation of Optoelectronic Devices, W. W. Chow and M. Osinski, eds., Proc. SPIE 2994, 600-610 (1997). 
21. E. Yablonovitch, "Inhibited spontaneous emission solidstate physics and electronics," Phys. Rev. Lett. 58, 20592062 (1987).

22. J. Broeng, T. Sondergaard, S. E. Barkou, P. M. Barbeito, and A. Bjarklev, "Waveguidance by the photonic bandgap effect in optical fibres," J. Opt. A 1, 477-482 (1999).

23. M. D. Feit and J. A. Fleck, Jr., "A spectral approach to optical resonator theory," Appl. Opt. 20, 2843-2851 (1981).
24. M. D. Feit and J. A. Fleck, Jr., "Mode properties of optical fibers with lossy components by the propagating beam theory," Appl. Opt. 20, 848-856 (1981).

25. M. D. Feit and J. A. Fleck, Jr., "Computation of mode eigenfunctions in graded index optical fibers by the propagating beam method," Appl. Opt. 19, 2240-2246 (1980).

26. M. D. Feit and J. A. Fleck, "Analysis of rib waveguide and couplers by the propagating beam method,"J. Opt. Soc. Am. A 7, 73-79 (1990). 


\title{
Narrow-line ytterbium fiber master-oscillator power amplifier
}

\author{
Nathan A. Brilliant \\ U.S. Air Force Research Laboratory, Directed Energy Directorate, 3550 Aberdeen SE, Kirtland Air Force Base, \\ New Mexico 87117
}

Raymond J. Beach, Alexander D. Drobshoff, and Stephen A. Payne

Lawrence Livermore National Laboratory, Livermore, California 94551

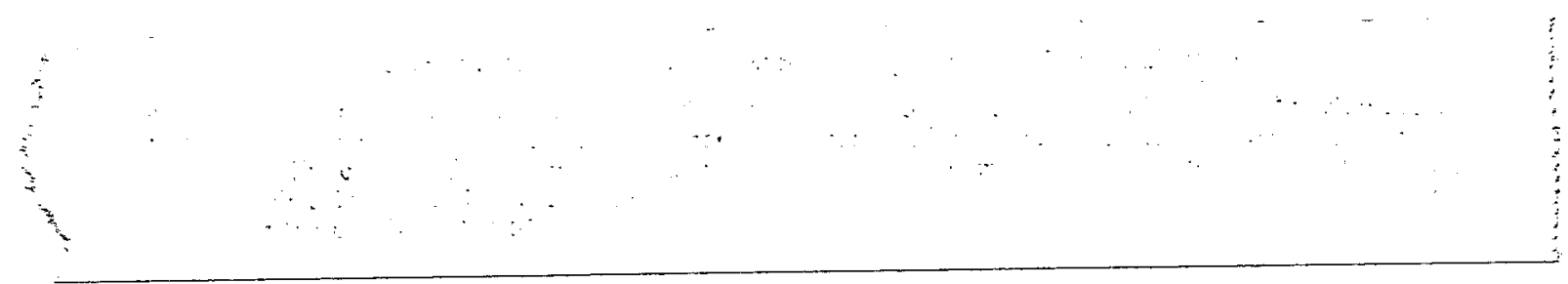

\section{INTRODUCTION}

A great deal of experimental and theoretical research has been done in the past 20 years on active fiber devices. ${ }^{1-3}$ Much of this research has been directed toward telecommunications applications at relatively low power and with erbium as the active ion. More recently, dual-clad fiber lasers and amplifiers have received much attention as potential sources for high-power applications. However, it is unlikely that a single diffraction-limited source will emit more than a few hundred watts. Therefore coherent combination of many sources may be a path to higher powers. For coherent beam combination, there is a trade between the linewidth of the sources and the precision required in matching the optical path lengths. This is easily understood in terms of fringe visibility in a Michelson interferometer. For a narrow-line light source, the fringe visibility is high even for a large mismatch in the interferometer arms. For white light, the fringes are visible only for a small path mismatch. Since coherent combination becomes far easier with narrow-band sources, single-frequency lasers and master-oscillator power amplifiers become attractive.

While great strides have been made in power-scaling fiber lasers, the research is not universally applicable to coherent combination. Dominic et al. reported a fiber laser with greater than $100-\mathrm{W}$ cw output. ${ }^{4}$ Free-running fiber lasers operate with very broad linewidths, $1 \mathrm{~nm}$, which make coherent combination nearly impossible. Other groups have reported amplifiers with high gain over much of the ytterbium emission spectrum. ${ }^{5}$ However, they do not mention the seed linewidth. Lasers and amplifiers with narrow linewidths have been limited to much lower powers.

We developed a 2-W narrow-linewidth source consisting of a fiber distributed-feedback (DFB) oscillator (Ionas, Denmark), a preamplifier, and an amplifier. Our system is based on Page's ${ }^{6}$ design for a Yb:silica DFB master os- cillator and dual-clad amplifier. The preamplifier is a short section of doped single-mode fiber pumped with a 200-mW fiber-coupled diode. The main amplifier is a dual-clad fiber end pumped by a fiber-coupled diode with up to $12 \mathrm{~W}$ of available power. We built this source as a seed for much higher power amplifiers to investigate stimulated Brillouin scattering, which will be the limiting factor for high-power single-frequency fiber amplifiers.

This amplifier offers an excellent opportunity to test our model against experiment since we have cross sections, lifetime, and dopant concentration measured from the preform from which the fiber was drawn. We compare amplifier performance, saturation, and spectra.

We are also pursuing an accurate and comprehensive model for high-power fiber sources to use as a design tool for future systems. While there are many models for fiber amplifiers in the literature, we have based our model primarily on the research by Marcerou et al., ${ }^{7}$ Giles et $a l .,{ }^{8,9}$ and Oron and Hardy. ${ }^{10,11}$ It is a rate-equations model with spectrally resolved amplified spontaneous emission. Our modeling effort is unique in that it uses results of a simplified model as input to the more complete model. This is necessary for model convergence for an unsaturated amplifier. The simplified model makes the assumption that the inversion is constant along the length of the fiber and is referred to as the mean excited fraction model ${ }^{12}$ (MEFM). The differential equations and methods of solving them are reported in many papers; we include them for completeness in Appendix A. The mean excited fraction model follows in the next section.

\section{MEAN EXCITED FRACTION MODEL}

The algorithms for solving the differential equations describing the fiber amplifier often require an accurate initial guess. This is especially true when ASE is strong and contributes to amplifier saturation. The mean ex- 
cited fraction model is used to find an estimate for the signal power, transmitted pump, and ASE spectrum. The MEFM assumes that the population inversion is uniform along the length of the fiber. One balances the number of atoms excited by the pump and the number de-excited by stimulated and spontaneous emission. While this can be far from accurate in many cases, this assumption does allow for a quick solution that can be used as an initial guess for the more complex model. Because the singlepass gain can be high, small reflections at the fiber end can provide enough feedback for oscillation. To adequately model such situations, we must account for multiple passes of the fiber caused by finite reflectivities. A similar model has been presented by Nielson et al.,${ }^{13}$ however, they do not account for multiple passes of the fiber or for spectrally resolved ASE.

This model looks for a zero of the change in population inversion as a function of the population inversion. $\Delta n_{2}$ is a monotonically decreasing function $n_{2} \cdot n_{2}$ is the fractional of ions in the excited state. For zero inversion, $\Delta n_{2}>0$. The pump sees absorption, but there is no gain or spontaneous emission. For $n_{2}=1$, there are no ions in the ground state. Only emission takes place: $\Delta n_{2}$ $<0$. The correct steady-state inversion is the zero crossing for this function.

\section{A. Differential Equations}

The equations for pump, signal, ASE, and inversion depend on a variety of parameters such as cross sections, dopant concentration, and fiber geometry. In normalized variables, the differential equation describing the evolution of the power is

$$
\pm \frac{\mathrm{d} p_{i}^{ \pm}(z)}{\mathrm{d} z}=\left[n_{2}(z) k_{2 i}-k_{3 i}\right] p_{i}^{ \pm}(z)+n_{2} k_{4 i} .
$$

A complete development of the differential equations is provided in Appendix A. The constants are defined in Table 1. Buried within the normalized variables are the familiar parameters; the definition of the symbols as well as the values used for the modeled data are in Table 2. Two important parameters are the mode overlap, $\Gamma$, and the capture fraction, $\Omega$. The mode overlap is a measure of how well the guided mode overlaps the core dopant. ${ }^{8,9}$ The mode overlap affects the stimulated emission and the

Table 1. Normalized Model Parameters ${ }^{a}$

\begin{tabular}{ll}
\hline Variable & \multicolumn{1}{c}{ Value } \\
\hline$z$ & $z / L$ \\
$p_{i}^{+}$ & $\frac{P_{i}^{+} \tau_{s}}{h \nu_{i} N_{0} A_{\text {core }} L}$ \\
$k_{1}$ & $\tau_{s} / r_{2}$ \\
$k_{2 i}$ & $\left(\sigma_{i}^{e}+\sigma_{i}^{a}\right) N_{0} \Gamma_{i} L$ \\
$k_{3 i}$ & $\sigma_{i}^{a} N_{0} \Gamma_{i} L+\alpha_{i} L$ \\
$k_{4 i \text { field }}$ & $4 n^{2} \Delta \lambda \sigma_{i}^{e} \Gamma_{i} L\left(\lambda^{2} A_{\text {core }}\right)^{-1}$ \\
$k_{4 i \text { gea }}$ & $2 \pi n \Delta \lambda \sigma_{i}^{e} \Gamma_{i} L \lambda^{-4} \mathrm{NA}^{2}$ \\
$k_{4 i \text { arb }}$ & $16 \pi n^{3} \Delta \lambda \sigma_{i}^{e} \Gamma_{i} L \lambda^{-4} \Omega$ \\
$k_{5 i}$ & $\sigma_{i}^{a} N_{0} \Gamma_{i} L$ \\
\hline
\end{tabular}

a There are three entries for the spontaneous-emission coefficient, depending on how it is calculated.
Table 2. Model Parameters and Input Values

\begin{tabular}{llcl}
\hline Symbol & \multicolumn{1}{c}{ Parameter } & Source $^{\alpha}$ & \multicolumn{1}{c}{ Value } \\
\hline$\tau_{s}$ & Upper-state lifetime & $\mathrm{m}$ & $940 \mu \mathrm{s}$ \\
$N_{0}$ & Yb Number density & $\mathrm{m}$ & $9 \times 10^{25} \mathrm{~m}^{-3}$ \\
$a$ & Core radius & $\mathrm{s}$ & $4 \mu \mathrm{m}$ \\
$\mathrm{NA}$ & Core numerical aperture & $\mathrm{s}$ & 0.1 \\
$n_{\text {core }}$ & Core index & $\mathrm{s}$ & 1.453 \\
$\lambda_{p}$ & Pump wavelength & $\mathrm{m}$ & $975 \mathrm{~nm}$ \\
$\alpha_{p}$ & Pump scattering & $\mathrm{n}$ & $0.004 \mathrm{~m}^{-1}$ \\
$\eta_{p}$ & Pump coupling & $\mathrm{f}$ & 0.53 \\
$\lambda_{s}$ & Signal wavelength & $\mathrm{m}$ & $1100 \mathrm{~nm}^{-1}$ \\
$\alpha_{s}$ & Signal scattering & $\mathrm{n}$ & $0.001 \mathrm{~m}^{-1}$ \\
$\eta_{s}$ & Signal coupling & $\mathrm{f}$ & 0.5 \\
$L$ & Fiber length & $\mathrm{m}$ & $9.4 \mathrm{~m}$ \\
$A_{\text {clad }}$ & Cladding area & $\mathrm{s}$ & $2.25 \times 10^{-8} \mathrm{~m}$ \\
$R_{1}$ & Reflectivity at side 1 & $\mathrm{f}$ & 0 \\
$R_{2}$ & Reflectivity at side 2 & $\mathrm{f}$ & 0 \\
$\Omega$ & Capture fraction & $\mathrm{c}$ & 0.001 \\
$\Gamma$ & Overlap & $\mathrm{f}$ & $0.35,0.58$ \\
\hline
\end{tabular}

${ }^{a} \mathrm{~m}$ indicates that the value was measured. $\mathrm{s}$ indicates manufacturer specification. $c$ indicates calculated from manufacturer specification. $n$ indicates a nominal value for fiber of this type. $f$ indicates that the value was allowed to float to achieve the best fit with experiment.

absorption. The capture fraction determines how much of the spontaneous emission enters the guided mode. There is spontaneous emission in all directions; only a small fraction is within the critical angle for a singlemode core. The length is normalized to the length of the fiber and runs from 0 to 1 . In this form, $k_{2 i}$ represents gain, $k_{3 i}$ represents absorption and scattering loss, and $k_{4 i}$ represents spontaneous emission. The steady-state population inversion is

$$
n_{2}(z)=\frac{\sum_{i} k_{5 i}\left[p_{i}^{+}(z)+p_{i}^{-}(z)\right]}{k_{1}+\sum_{i} k_{2 i}\left[p_{i}^{+}(z)+p_{i}^{-}(z)\right]}
$$

where $k_{5 i}$ is the normalized excitation rate, $k_{2 i}$ accounts for stimulated emission, and $k_{1}$ accounts for spontaneous emission and nonradiative de-excitation.

\section{B. Pump}

One can determine the change in population inversion by following the spatially localized group of photons as they bounce back and forth between the fiber ends. The notation in this subsection is the convention that the upper index represents the pass number, and the lower index represents the wave of interest. For example, $p_{i 1}^{(3)}$ is the photon number after the third pass for the $i$ th ASE bin whose first pass traveled from $z=1$ to $z=0$. The same convention applies to the other parameters such as the change in photon number $(\Delta p)$, change in scattered photons $(\Delta s)$, and change in population inversion $\left(\Delta n_{2}\right)$. Let $p_{p 1}^{\text {in }}$ be the number of pump photons incident upon the fiber at $z=1$. The lower index $p 1$ indicates that this is the pump field starting at $z=1$. The number coupled into the pump cladding is $p_{p 1}^{(0)}=\eta_{p} p_{p 1}^{\text {in }}$. The number of photons remaining after a single transit of the fiber is 


$$
p_{p 1}^{(1)}=p_{p 1}^{(0)} \exp \left(g_{p}\right),
$$

where $g_{p}=n_{2} k_{2 p}-k_{3 p}$, and the upper index is the pass number. $G_{p}=\exp \left(g_{p}\right)$ is the single-pass integrated gain. Equation (3) results from Eq. (1) under the assumption that $n_{2}$ is not a function of position and $k_{4 p}$ is zero. The net change in the photon number is

$$
\Delta p_{p 1}^{(1)}=p_{p 1}^{(0)}\left(G_{p}-1\right) .
$$

Since the pump sees absorption, $n_{2} k_{2 i}-k_{3 i} \leqslant 0$ and $\Delta p_{p} \leqslant 0$. In these normalized variables, the change in photon number corresponds exactly to a change in the relative population inversion, given by

$$
\Delta n_{2 p 1}^{(1)}=-\Delta p_{p 1}^{(1)}
$$

The indices on $n_{2}$ follow the same convention as the indices for the photon numbers. The lower index, $p 1$, and upper index, 1 , indicate that this is the change in fractional inversion associated with $\Delta p_{p 1}^{(1)}$. Some of the pump photons are lost to scattering and not to the absorption process. The number of pump photons lost to scattering in a single pass is given by

$$
\Delta s_{p 1}^{(1)}=\int_{0}^{1} \alpha_{p} p_{p 1}^{(1)}(z) \mathrm{d} z=s_{p} \Delta p_{p 1}^{(1)},
$$

where $s_{p}=\alpha_{p} / g_{p}$. Since these photons do not contribute to excitation, the total change in population inversion caused by pump absorption on the first pass is

$$
\Delta n_{2 p 1}^{(1)}=-\left(\Delta p_{p 1}^{(1)}-\Delta s_{p 1}^{(1)}\right)=-\Delta p_{p 1}^{(1)}\left(1-s_{p}\right) .
$$

For each subsequent pass of the fiber, there is a gain (or loss) of $G_{p}$ and a multiplicative factor $R_{0}$ or $R_{1}$. For an arbitrary odd pass of the fiber, the number of photons left at the end of the pass is

$$
p_{p 1}^{(2 m+1)}=p_{p 1}^{(0)}\left(R_{0} R_{1}\right)^{m} G_{p}^{2 m+1} .
$$

$m$ runs from 0 to $\infty$. The number of photons lost to absorption is given by.

$$
\Delta p_{p 1}^{(2 m+1)}=p_{p 1}^{(0)}\left(G_{p}-1\right)\left(R_{0} R_{1}\right)^{m} G_{p}^{2 m} .
$$

Similarly for an even pass, the number of photons left at the end of the pass is given by

$$
p_{p 1}^{(2 m+2)}=p_{p 1}^{(0)} R_{0}\left(R_{0} R_{1}\right)^{m} G_{p}^{2 m+2} .
$$

Every even pass sees an odd number of reflections: an additional interaction with $R_{0}$. The change in photon number for an arbitrary even pass is

$$
\Delta p_{p 1}^{(2 m+2)}=p_{p 1}^{(0)} R_{0}\left(G_{p}-1\right)\left(R_{0} R_{1}\right)^{m} G_{p}^{2 m+1} .
$$

The number of scattered photons in any pass is simply $s_{p} \Delta p_{p 1}$, where $\Delta p_{p 1}$ is the number of absorbed pump photons for that pass. The total number of ions excited into the upper state, including an infinite number of reflections, is

$$
\begin{aligned}
\Delta n_{2 p 1}= & -p_{p 1}^{(0)}\left(1-s_{p}\right)\left(G_{p}-1\right)(1 \\
& \left.+R_{0} G_{p}\right) \sum_{m=0}^{\infty}\left(R_{0} R_{1} G_{p}^{2}\right)^{m} .
\end{aligned}
$$

Equation (12) comes from Eqs. (9) and (11). The factor $\left(1-s_{i}\right)$ accounts for the reduction in excitation caused by the scattered photons. The lack of a superscript on $\Delta n_{2 p 1}$ indicates that this is the total contribution to excitation for all passes. If the quantity $\left(R_{0} R_{1} G_{p}^{2}\right)<1$, then the sum converges. Equation (12) becomes

$$
\Delta n_{2 p 1}=-p_{p 1}^{(0)}\left(1-s_{p}\right)\left(G_{p}-1\right) \frac{1+R_{0} G_{p}}{1-R_{0} R_{1} G_{p}^{2}} .
$$

For the pump, it is clear that the infinite sum converges. Both reflectivities are strictly less than one; the gain is also less than one since the pump sees absorption. AIthough not obvious, the sum also converges for the signal and ASE. The exponential term is the gain. Even if there is enough gain for the signal or ASE to oscillate, the gain always clamps just under the loss. The denominator in Eq. (13) gives the familiar laser threshold condition. Since $G_{p}<1$, the change in photon number is positive. For a pump injected at the other end of the fiber the result is the same with 1's and 0 's exchanged.

\section{Signal}

The signal evolves in an manner analogous to the pump. The only difference is for the scattered light. For the signal, and ASE, the scattered photons resulted from deexcitation and must be counted. For the signal injected at $z=0$, the total change in population is

$$
\Delta n_{2 s 0}=-p_{p 0}^{(0)}\left(G_{s}-1\right)\left(1+s_{s}\right) \frac{1+R_{1} G_{s}}{1-R_{0} R_{1} G_{2}^{2}} .
$$

Equation (14) differs from Eq. (13) only in the sign of the $s$. Since $G_{s}>1$, the change in photon number is negative.

\section{Amplified Spontaneous Emission}

The evolution of the ASE is somewhat different. On the first pass of the fiber, the ASE is generated by the constant term in Eq. (1), which is exactly soluble. On subsequent passes, the power evolves only through stimulated emission and absorption. The ASE spectrum is resolved by dividing it into bins of finite width and accounting for the photons in each bin separately. For the first pass for the ASE generated in the direction $z=0$ toward $z=1$, the photon number as a function of $z$ is

$$
p_{i 1}(z)=\left(p_{i 1}{ }^{(0)}+a_{i}\right) \exp \left(g_{i} z\right)-a_{i},
$$

where $a_{i}=n_{2} k_{4 i} / g_{i}$. Since there are no photons injected at the fiber end, $p_{i 1}{ }^{(0)}=0$, giving

$$
p_{i 1}(z)=a_{i}\left[\exp \left(g_{i} z\right)-1\right] \text {. }
$$

The change in photon number after a full pass is

$$
\Delta p_{i 1}^{(1)}=a_{i}\left(G_{i}-1\right) .
$$

Since there are no photons injected, the change in photon number at the end of the first pass is the same as the total number of photons at the end of the pass. The light scattered out of this bin in the first pass is

$$
\Delta s_{i 1}^{(1)}=\int_{0}^{1} \alpha_{i} p_{i 1}(z) \mathrm{d} z=\alpha_{i} a_{i}\left(\frac{G_{i}-1}{g_{i}}-1\right) .
$$

with the definition of $s_{i}$, Eq. (18) becomes 


$$
\Delta s_{i 1}^{(1)}=a_{i} s_{i}\left(G_{i}-1\right)-\alpha_{i} \alpha_{i} .
$$

The total contribution to de-excitation for the first pass is

$$
\Delta n_{2 i 1}^{(1)}=-a_{i}\left(1+s_{i}\right)\left(G_{i}-1\right)+\alpha_{i} a_{i} .
$$

For all passes after the first of the fiber, we need only account for the stimulated-emission gain seen by the power in this bin. We have already accounted for the spontaneous process. Just as for the pump, each pass picks up a multiplicative factor of $G_{i}$ and a multiplicative factor $R_{0}$ or $R_{1}$. For all odd passes except the first, the photon number after the pass is given by

$$
p_{i 1}^{(2 m+3)}=a_{i}\left(G_{i}-1\right)\left(R_{0} R_{1}\right)^{m+1} G_{i}^{2 m+2} .
$$

The change in photon number is

$$
\Delta p_{i 1}^{(2 m+3)}=a_{i}\left(G_{i}-1\right)^{2}\left(R_{0} R_{1}\right)^{m+1} G_{i}^{2 m+1} .
$$

The number of scattered photons for all odd passes except the first follows the same rule as for scattered signal photons: $\Delta s_{i 1}^{(2 m+3)}=s_{i} \Delta p_{i 1}^{(2 m+3)}$. The change in the population inversion for an odd pass becomes

$$
\Delta n_{2 i 1}^{(2 m+3)}=-\left(1+s_{i}\right) \Delta p_{i 1}^{(2 m+3)} .
$$

The total contribution for all odd passes except the first is found by

$$
\Delta n_{2 i 1}^{\text {odd }}=-\left(1+s_{i}\right) a_{i} R_{0} R_{1} G_{i}\left(G_{i}-1\right)^{2} \sum_{m=0}^{\infty}\left(R_{0} R_{1}\right)^{m} G_{i}^{2 m} .
$$

Since the infinite sum converges, Eq. (24) becomes

$$
\Delta n_{2 i 1}^{\text {odd }}=-a_{i}\left(1+s_{i}\right)\left(G_{i}-1\right) \frac{R_{0} R_{1} G_{i}\left(G_{i}-1\right)}{1-R_{0} R_{1} G_{i}^{2}}
$$

For all even passes,

$$
\begin{aligned}
p_{i 1}^{(2 m+2)} & =a_{i} R_{0}\left(G_{i}-1\right)\left(R_{0} R_{1}\right)^{m} G_{i}^{2 m+1}, \\
\Delta p_{i 1}^{(2 m+2)} & =a_{i} R_{0}\left(G_{i}-1\right)^{2}\left(R_{0} R_{1}\right)^{m} G_{i}^{2 m} .
\end{aligned}
$$

The total contribution to de-excitation for all even passes is similar to Eq. (25),

$$
\Delta n_{2 i}^{\text {even }}=-a_{i}\left(1+s_{i}\right)\left(G_{i}-1\right) \frac{R_{0}\left(G_{i}-1\right)}{1-R_{0} R_{1} G_{i}^{2}}
$$

Accounting for all reflections and scattered light, the total change in the population inversion is the sum of the contributions in Eqs. (20), (25), and (28):

$$
\begin{aligned}
\Delta n_{2 i 1}= & \alpha_{i} a_{i}-a_{i}\left(1+s_{i}\right)\left(G_{i}-1\right) \\
& \times\left[1+\frac{R_{0}\left(G_{i}-1\right)\left(1+R_{1} G_{i}\right)}{1-R_{0} R_{1} G_{i}^{2}}\right] .
\end{aligned}
$$

Again, the lower index on $n_{2}$ in Eq. (29) indicates that this is the de-excitation caused by the $i$ th ASE bin whose first pass traveled from $z=1$ to $z=0$. For ASE starting in the opposite direction, we switch the lower indices. If the reflectivities are the same, then $\Delta n_{2 i 0}=\Delta n_{2 i 1}$.

Since only a small fraction of the spontaneous emission is captured, the model must explicitly account for all of the photons radiated outside of the guided mode. The total inversion change due to all spontaneous emission is related to the constant $k_{1}$ (the normalized decay rate). The uncaptured photon number is just the total decay rate minus the captured photon for which we have already accounted:

$$
\delta n_{2 u}=-k_{1}+\sum_{i} 2 n_{2} k_{4 i}
$$

The sum is over all ASE bins, pump, and signal; the factor of 2 accounts for beam propagation in both directions.

The total change in the population inversion becomes

$$
\Delta n_{2}=-k_{1}+\sum_{i}\left[\left(\Delta n_{2 i 0}+\Delta n_{2 i 1}\right)+2 n_{2} k_{4 i}\right]
$$

\section{E. Output Powers}

Consider the pump light exiting the fiber at $z=1$. This is a sum of the odd passes of the pump injected at $z=0$ plus the sum of the even passes of the pump injected at $z=1$. Based on Eq. (8), the contribution for the odd passes is

$$
\eta_{p} p_{p 0}^{\text {in }} G_{p} \sum_{m=0}^{\infty}\left(R_{0} R_{1}\right)^{m} G_{p}^{2 m}
$$

where $p_{p 0}^{\text {in }}$ is the power injected at $z=0$. An infinite sum of the even passes, Eq. (10), gives the other contribution:

$$
\eta_{p} p_{p 1}^{\text {in }} R_{0} G_{p}^{2} \sum_{m=0}^{\infty}\left(R_{0} R_{1}\right)^{m} G_{p}^{2 m}
$$

The total power output at $z=1$, including the transmission of the fiber end, $T_{1}$, is

$$
p_{p 1}^{\text {out }}=T_{1} \eta_{p} G_{p} \frac{p_{p 0}^{\text {in }}+p_{p 1}^{\text {in }} R_{0} G_{p}}{1-R_{0} R_{1} G_{p}^{2}}
$$

A similar equation holds true for the power coming out the other end. The signal can be found in the same fashion.

The ASE power exiting the fiber is somewhat different since it starts from noise and not an externally injected power. The power exiting at $z=1$ for bin $i$ is

$$
p_{i 1}^{\text {out }}=T_{1} a_{i}\left(G_{i}-1\right) \frac{1+R_{0} G_{p}}{1-R_{0} R_{1} G_{i}^{2}} .
$$

For ASE bins at short wavelengths, the population inversion may not be great enough to provide net gain, $G_{i}-1 \leqslant 0$, and Eq. (35) is less than zero. For all ASE bins with no net gain, the MEFM returns zero output power. This is also used to limit the number of ASE channels for the integrated solution. This can lead to some difficulty for asymmetric population inversion. For a highly asymmetric inversion, there may be no net gain for a photon starting at the fiber end. However, a photon starting in the middle could see significant gain. Including these wavelengths in the full solution presents a problem because they do not allow the model to converge. Equation (35) illustrates the primary failing of this simplified model. If the reflectivities are equal, the powers emitted from the fiber ends are also equal. For a long fiber pumped from one end, the measured spectra are not 


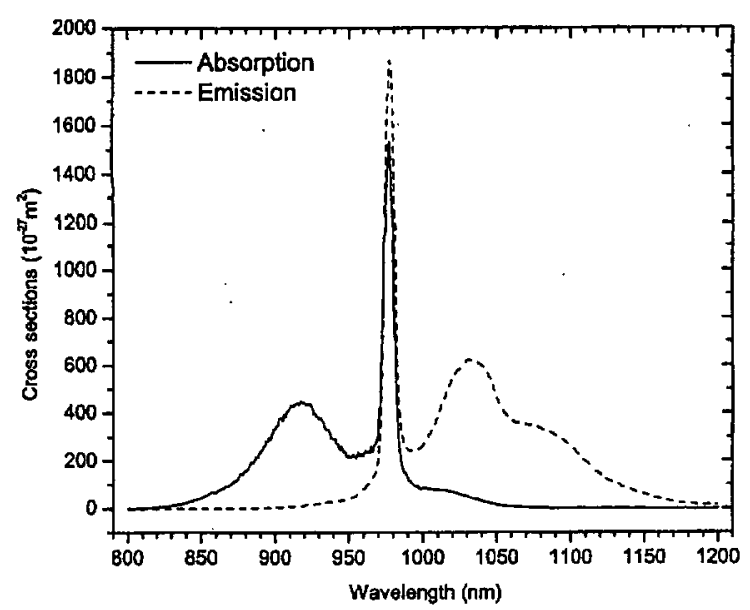

Fig. 1. Cross sections measured by Patel. ${ }^{14}$ Absorption cross section (solid curve) and emission cross section (dashed curve).

the same. This is caused by asymmetry in the population inversion that is ignored in the MEFM.

\section{F. Model Input}

The numerical model requires a significant amount of input data. In some cases, the values were measured. In other cases, the values were specified by the fiber manufacturer. Some parameter values were not available and were set at nominal values measured or specified for similar fibers. Some parameters were set to find the best fit with the experimental data. The most critical parameters are the emission and absorption cross sections, which depend strongly on the composition of the fiber core. The cross sections and the upper-state lifetime were measured by Falgun Patel from a piece of preform from which the fiber was drawn. ${ }^{14}$ The cross sections are shown in Fig. 1. There are a number of other critical parameters that are difficult to measure: the overlap, the capture fraction, pump coupling, signal coupling, and reflectivities. The pump coupling and signal coupling can be obtained by measuring transmission for several lengths of fiber and extrapolating to zero. Since we did not have sufficient fiber, we could not make these measurements. Therefore we left the pump and signal coupling as parameters that we could alter to match the modeled and experimental data. The fiber end faces were angle polished to $\sim 8^{\circ}$. The feedback into the fiber from an angled end can be very low, nearly $10^{-5}$. For the modeled data presented here, we assumed no reflection. For the other critical parameters, we adjusted them to find the best agreement with experiment. The input parameters and their values are given in Table 2. Table 2 also indicates the source for the parameter values: measured, manufacturer specification, nominal, or adjusted to fit experiment. The parameters marked nominal were taken either from manufacturer specifications for a similar fiber or from the literature.

\section{AMPLIFIER}

The master-oscillator power amplifier consists of three parts: master oscillator, preamplifier, and amplifier. The master oscillator and preamplifier were fusion spliced together. We use bulk optics to couple both the signal and the pump into the amplifier. The configuration is shown in Fig. 2. The master oscillator is a fiber DFB laser pumped with a fiber-coupled single-mode diode at $975 \mathrm{~nm}$. The specifications state that the laser linewidth is of the order of $10 \mathrm{kHz}$; no time scale was given for this measurement. The maximum output of the laser is $\sim 5 \mathrm{~mW}$. Based on experience with previous amplifiers, we were concerned that this power would be insufficient to saturate the higher-power amplifier and eliminate selfpulsing. To ensure saturation of the final amplifier, we added a preamplifier consisting of a $2-\mathrm{m}$ section of $\mathrm{Yb}$ doped single-mode ( $3-\mu \mathrm{m}$ core-diameter) fiber, a wavelength division multiplexer, and a 975-nm fiber-coupled diode. We also included a fiber pig-tailed isolator (KAIFA) designed for $1053 \mathrm{~nm}$ to prevent feedback from the preamp into the master oscillator. The maximum output of the preamplifier is $>40 \mathrm{~mW}$. We did not seed the amplifier with the DFB alone. The final amplifier is a $10-\mathrm{m}$ section of dual-clad Yb-doped fiber pumped with a high-brightness fiber-coupled diode. The pump cladding of the dual-clad fiber is octagonal with a diameter of $\sim 165$ $\mu \mathrm{m}$ and numerical aperture (NA) of $\sim 0.4$. The core is single mode with a diameter of $4 \mu \mathrm{m}$ and NA of 0.1 . The pump diode emits nearly $12 \mathrm{~W}$ of $975-\mathrm{nm}$ light from a $250-\mu \mathrm{m} \quad 0.22-\mathrm{NA}$ fiber. The pump light is collimated with an 18-mm focal-length aspheric lens and refocused into the amplifier with a $12-\mathrm{mm}$ aspheric lens. The magnification of the two lenses is such that the pump fiber exactly fills the pump cladding in the absence of any misalignment or aberration. As a result, the coupling efficiency is less than optimal. The preamplifier was isolated from the final amplifier by a free space isolator designed for $1064 \mathrm{~nm}$. Although this isolator was not ideal, it had a transmission of $\sim 50 \%$ and an isolation of $\sim 13 \mathrm{~dB}$. The signal was separated from the pump with a dichroic beam splitter. The fiber ends were angle polished in order to reduce feedback from end reflections. We also removed the last few millimeters of polymer outer cladding to prevent damage by uncoupled pump light. We observed self-pulsation early in the experiment. In some cases, the pulsation did not hinder the experiment. However, on other occasions, the pulsation damaged the end of the fiber into which we coupled the pump. A damaged fiber end required removal of a few millimeters of fiber and repolishing. By eliminating reflections from optical ele-

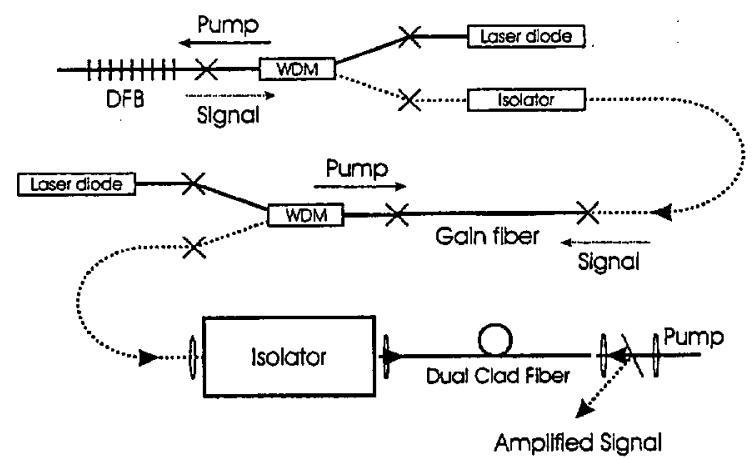

Fig. 2. Master oscillator, preamplifier, and amplifier. WDM indicates a wavelength-division multiplexer.' DFB indicates the distributed feedback laser. X's are fusion splices. 
ments after the amplifier output, we eliminated the pulsing. The self-pulsing is strongly influenced by feedback into the amplifier.

\section{RESULTS}

Figures 3-5 show experimental amplifier data compared with two sets of modeled data. We did not have an instrument capable of measuring the linewidth of the signal. We did not make a measurement of the phase or amplitude noise caused by the amplifier. Figure 3 shows the amplifier performance as a function of pump power. The output powers shown are total power: amplified signal and forward propagating ASE. The circles are the experimental data for the saturated amplifier with an injected seed of $26.6 \mathrm{~mW}$. We see a slope efficiency of $\sim 38 \%$. This is not exceptionally good unless one believes the numerical simulations and accepts that the pump coupling is only $53 \%$. In that case the optical to optical efficiency is $\sim 70 \%$. Although pump coupling of $53 \%$ seems low, we did not have the ideal optics for pump coupling. The brightness of the pump delivery fiber was very close to the minimum brightness required by the pump cladding in the amplifier. As a result, the image of the pump delivery fiber almost completely filled the pump cladding of the amplifier. Any aberration or misalignment would have significantly reduced the coupling. The triangles are data for the unseeded amplifier acting as a superfluorescent source. The slope efficiency of the unseeded amplifier is equal to the slope efficiency of the seeded amplifier for pump powers beyond $4 \mathrm{~W}$. The upper lines are the modeled data for the saturated amplifier; the lower lines are the modeled data for the unseeded amplifier. Figure 4 shows saturation data. The dots represent experimental data for three different pump powers: squares, $1.14 \mathrm{~W}$; circles, $3.37 \mathrm{~W}$; triangles, $6.92 \mathrm{~W}$. The curves closest to each set of dots are modeled data for the same pump powers. Figure 5 is the ASE spectrum for light traveling opposite the pump for an unseeded amplifier. The data were taken with an injected pump power of $2.56 \mathrm{~W}$. The experimental and modeled data are normalized to their peak values for easier comparison. In all figures, the dotted curves represent data modeled with $\Gamma=0.35$. The dashed curves represent data modeled with $\Gamma=0.58$

The first set of modeled data $(\Gamma=0.35)$ give the best fit for the saturated amplifier with predicted powers within $10 \%$. However, the model with these input parameters does not accurately predict either the amplifier performance for small-seed or the ASE spectrum for the unseeded amplifier. The $\Gamma$ used is also low compared with what we would calculate for a fiber with NA $=0.1$ and $a=4 \mu \mathrm{m}$. $\Gamma=0.35$ corresponds to $\mathrm{NA}=0.06$, which is low but not unreasonably so considering the variability in the core size and numerical aperture. Although the NA of the fiber was nominally 0.1 , it was not actually measured. We have other fiber with the same nominal NA but with measured NA as small as 0.076. A small overlap could also be caused by a nonuniform dopant density across the core. In particular, a small dip in the center where the intensity is highest could cause a smaller overlap. Assuming that $\Gamma=0.35$ is correct, the

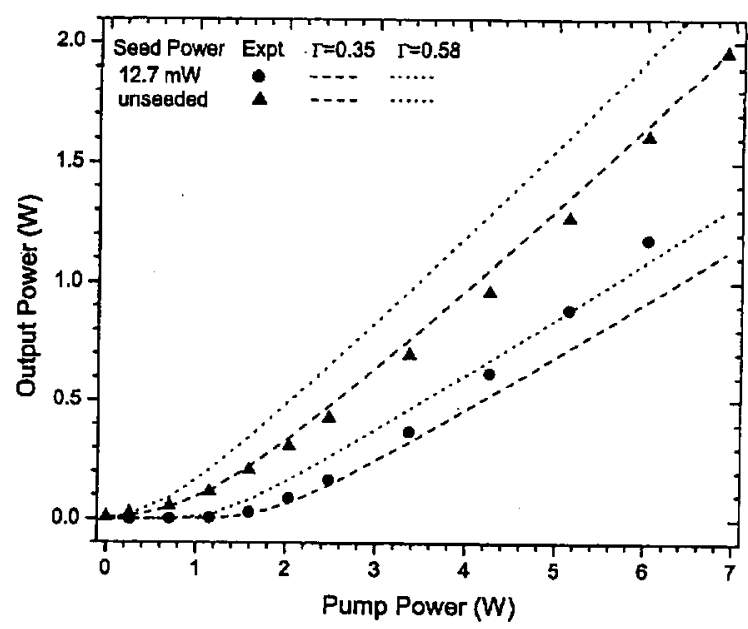

Fig. 3. Experimental and modeled performance as a function of incident pump power. Experimental data for $12.7-\mathrm{mW}$ seed (triangles) and no seed (circles). Modeled data for $\Gamma=0.35$ (dashed curve) and $\Gamma=0.58$ (dotted curve).

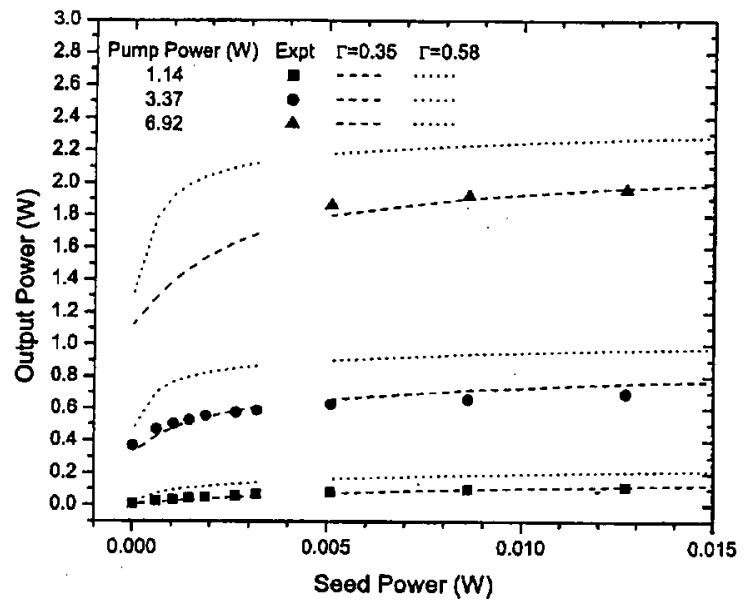

Fig. 4. Experimental and modeled saturation as a function of incident seed power. Experimental data for pump powers of $1.14 \mathrm{~W}$ (squares), $3.37 \mathrm{~W}$ (circles), and $6.92 \mathrm{~W}$ (triangles). Modeled data for $\Gamma=0.35$ (dashed curve) and $\Gamma=0.58$ (dotted curve).

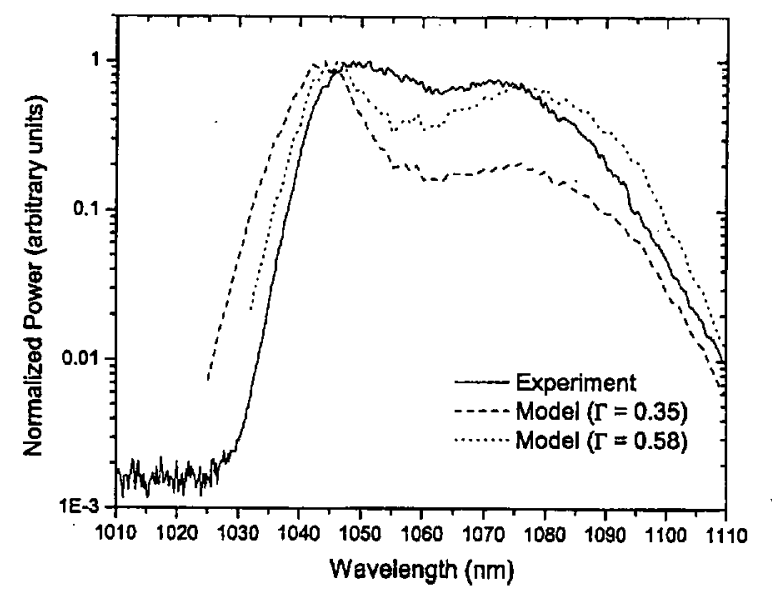

Fig. 5. Experimental and modeled amplifier ASE spectra. Experimental data (solid curve) and modeled data for $\Gamma=0.35$ (dashed curve) and $\Gamma=0.58$ (dotted curve). 
poor fit for the spectrum could be caused by a number of things. The absorption cross section near $1070 \mathrm{~nm}$ is $\sim 5 \times 10^{-27} \mathrm{~m}^{2}$. The precision in the measurement is $\pm 1 \times 10^{-27} \mathrm{~m}^{2}$. For the section of the fiber with low pump intensity, the relative inversion can be as low as $n_{2}=0.02$. The reabsorption can be significant, and a difference of $20 \%$ in the absorption cross section would change the shape of the spectrum dramatically.

The second set of modeled data $(\Gamma=0.58)$ has better correspondence with the ASE spectrum and unseeded amplifier performance. The overlap used in the second set is also lower than the calculated value and would require $\mathrm{NA}=0.075$. The price for better ASE prediction is a poorer fit with the saturated amplifier data. The potential cause of the discrepancy in the extraction is inhomogeneous broadening of the emission and absorption cross sections. We treat the ytterbium as homogeneously broadened. Significant inhomogeneous broadening caused by variations in the local electric field would make some excited atoms unavailable for extraction by a narrow-line seed but still available to the much broader ASE. We tried to account for this by using a different overlap factor for the signal and ASE. The results were not encouraging since the model then predicted a much higher ratio of ASE power to amplified signal power. In addition, we see no spectral hole burning in this or other experiments.

The correspondence between the measured and modeled data is quite good with powers accurate to within 10\%. The primary difficulties limiting the fidelity of the model are the large number of input parameters and the uncertainty in their values. In particular, the overlap should be measured and not calculated because of uncertainties in the doping and the index profile in the core. The assumption that the overlap is the same for absorption and emission may be invalid. This assumption is related to an assumption that the radial dependence of the excited- and ground-state distributions are the same. Under heavy saturation, this may be far from correct because the strong field at the center of the core burns a hole in the inversion. If the inversion dips in the center where the intensity is high, the overlap is much smaller. The overlap may have a strong dependence on the power.

\section{SUMMARY}

We have presented a simple model for pump, signal, and ASE. We have also presented a comparison between model prediction and measured data for a 2 -W fiber narrow-linewidth master-oscillator power amplifier. We saw good agreement, with some caveats. The input pump coupling was lower than expected but not unreasonable given the closely matched brightness of the pump and the dual-clad fiber. The model required a smaller mode overlap than calculated from the fiber geometry. However, the overlap is not unreasonably small considering the variability in manufacturing fibers. Unfortunately, a reduced overlap led to a predicted spectrum that did not agree well with experiment. In any case, this source will be an ideal seed to investigate stimulated Brillouin scattering and mitigation techniques in high-power amplifiers.

\section{APPENDIX A: NUMERICAL MODEL AND DIFFERENTIAL EQUATIONS}

An ytterbium-doped fiber amplifier in the steady state can be described by many differential equations in the optical intensity. For a complete description, one must account for the pump, signal, amplified spontaneous emission (ASE), and the population inversion. Inclusion of pump and signal only will give inaccurate results. Since fiber amplifiers can have very high single-pass gains, ASE can make a significant contribution to the extracted power and saturation of the inversion. An expanded model including Rayleigh backscattering and stimulated Brillouin scattering can be found in Ref. 15 .

There are many publications about modeling in fiber amplifiers and lasers. J. F. Marcerou et al. ${ }^{7}$ present the most complete model addressing signal, ASE, and the population inversion. This model has no approximations concerning the spatial mode of the guided waves, the spatial distribution of ions, or the saturation of the amplifier by signal or ASE. The vast majority of other models make approximations. The most common approximation is to remove the transverse dependence by introducing an overlap factor that quantifies how well the guided mode overlays the dopant in the core. ${ }^{8-11}$ The other common approximations involve the treatment of ASE. Many models assume that the ASE is small compared with the amplified signal and has no effect on the population inversion. Other models treat the ASE as a single parameter and do not resolve the spectrum. There are also many papers that present analytical solutions for predicting signal and ASE. These, however, have even more approximations that limit their application. Several papers present models similar to the model of amplifiers in this paper. $^{10,11,16,19}$

All of the differential equations are strongly coupled to each other. All of these beams are coupled to each other through the population inversion. The forward and backward beams at a particular wavelength are coupled by the reflections at the end of the fiber. With counterpropagating and copropagating beams, there are boundary conditions to enforce at both ends of the fiber. For a problem with boundary conditions at one end, a simple numerical integration solves the problem. With boundary conditions at both ends, the numerical solution becomes vastly more complex. However, there are standard methods for solving such equations. The algorithm estimates the powers of the counterpropagating beams at the front of the fiber. The code then integrates the coupled differential equations to the fiber end and compares the powers to the boundary conditions. This is known as the shooting method. ${ }^{17}$ Based on the comparison to the boundary conditions, the code refines the estimate. This continues until some convergence criteria are met. The algorithm to refine the estimate and decide on convergence is the globally convergent Newton-Raphson algorithm. $^{18}$

\section{A. Absorption and Stimulated Emission}

Consider a wave traveling in the positive $z^{\prime}$ direction along the fiber. The intensity of the wave is a function of $z^{\prime}, r$, and $\phi$, and it evolves according to 


$$
\begin{aligned}
\frac{\mathrm{d} I_{i}^{+}\left(r, \phi, z^{\prime}\right)}{\mathrm{d} z^{\prime}} & \\
= & -\alpha_{i} I_{i}^{+}\left(r, \phi, z^{\prime}\right)+\left[N_{2}\left(r, \phi, z^{\prime}\right)\left(\sigma_{i}^{e}+\sigma_{i}^{a}\right)\right. \\
& \left.-N_{0}\left(r, \phi, z^{\prime}\right) \sigma_{i}^{a}\right] I_{i}^{+}\left(r, \phi, z^{\prime}\right) .
\end{aligned}
$$

$N_{2}$ is the volume number density of ytterbium ions in the excited state; $N_{0}$ is the total volume number density $\sigma_{i}^{e}$ and $\sigma_{i}^{a}$ are the emission and absorption cross sections. $\alpha_{i}$ represents an additional loss due to scattering. The upper index on $I_{i}^{+}$indicates the direction of propagation; the lower index identifies the wave (signal, pump, or ASE bin). Assume that $I_{i}^{+}$is a guided mode of the fiber and that the fiber geometry does not change as a function of $z^{\prime}$. In this case, $I_{i}^{+}$is separable into a function of $z^{\prime}$ and a function of $r$ and $\phi ; I_{i}^{+}\left(r, \phi, z^{\prime}\right)=P_{i}^{+}\left(z^{\prime}\right) \Phi_{i}(r, \phi)$. $P_{i}^{+}\left(z^{\prime}\right)$ is the power. $\Phi_{i}(r, \phi)$ is the normalized transverse dependence and integrates to one. Since the transverse spatial dependence of each mode is determined by the fiber geometry and index profile, it is the same for forward- and backward-propagating beams. There is, however, a wavelength dependence. Consider the case where the number densities are separable into longitudinal and transverse functions as well: $N_{0}\left(r, \phi, z^{\prime}\right)$ $=N_{0}^{\prime}\left(z^{\prime}\right) \Psi(r, \phi)$. Further assume that the transverse dependence for the upper-state number density is the same as the transverse dependence of the total number density: $N_{2}\left(r, \phi, z^{\prime}\right)=N_{2}^{\prime}\left(z^{\prime}\right) \Psi(r, \phi)$. This assumption is valid only if the saturation at the center of the core is not significantly greater than at the edge. ${ }^{9} N_{2}^{\prime}$ and $N_{0}^{\prime}$ are linear number densities. If $N_{0}$ is a constant inside the core, zero outside, and uniform along the length of the fiber, then $N_{0}^{\prime}(z)=N_{0} A_{\text {core }}$. Equation (A1) can be integrated to find a differential equation in power:

$$
\begin{aligned}
\frac{\mathrm{d} P_{i}^{+}}{\mathrm{d} z^{\prime}}= & -\alpha_{i} P_{i}^{+}+\left[\left(\sigma_{i}^{e}+\sigma_{i}^{a}\right) N_{2}^{\prime}\right. \\
& \left.-\sigma_{i}^{a} N_{0}^{\prime}\right] P_{i}^{+} \int_{0}^{\infty} \int_{0}^{2 \pi} \Phi_{i} \Psi r \mathrm{~d} r \mathrm{~d} \phi .
\end{aligned}
$$

For clarity, the explicit dependence on $z^{\prime}, r$, and $\phi$ has been dropped. Since the integral over the transverse variables appears quite often, we define a new parameter $\Gamma_{i}$ by

$$
\Gamma_{i}=A_{\text {core }} \int_{0}^{\infty} \int_{0}^{2 \pi} \Phi_{i} \Psi r \mathrm{~d} r \mathrm{~d} \phi
$$

$\Gamma_{i}$ is a unitless parameter that quantifies how well the guided mode overlaps the rare-earth ions. At this point, it is also convenient to define a relative population inversion,

$$
n_{2}\left(z^{\prime}\right)=\frac{N_{2}^{\prime}\left(z^{\prime}\right)}{N_{0}^{\prime}\left(z^{\prime}\right)}
$$

By substituting Eq. (A3) and Eq. (A4) into Eq. (A2), the equation for the evolution of the power through stimulated emission and absorption becomes

$$
\frac{\mathrm{d} P_{i}^{+}}{\mathrm{d} z^{\prime}}=\left[\left(\sigma_{i}^{e}+\sigma_{i}^{a}\right) n_{2}-\sigma_{i}^{a}\right] N_{0} \Gamma_{i} P_{i}^{+}-\alpha_{i} P_{i}^{+} .
$$

\section{B. Overlap}

If the core-index profile and the dopant density are known, the overlap for the modes confined to the core can be calculated. Ideally, the dopant is uniform throughout the core with no ions outside the core. The guided mode can be calculated from a step-index profile. Approximating the guided mode by a Gaussian, the overlap is given by $\Gamma=1-\exp \left(-2 a^{2} / \omega^{2}\right)$, where $\omega$ is the mode radius for the field. For a fiber with core radius of $4 \mu \mathrm{m}$ and numerical aperture of $0.1, \Gamma=0.78$. This calculation of $\Gamma$ was made with a mode field radius determined by the Marcuse formula. ${ }^{20}$ Since the shape of the guided mode is wavelength dependent, the overlap is also wavelength dependent. For a wavelength of $1 \mu \mathrm{m}, \Gamma=0.82$. It is unclear that these approximations (step index and uniform dopant) are valid. During the drawing process, the rare earth may diffuse from the core, and the index profile may soften. It is also well known that the core dopants often have a dip in the center due to the preform fabrication process. Therefore the computer model can calculate the overlap or accept a user-specified value.

The situation for the pump in a dual-clad fiber is different. For a highly multimode pump cladding, the pump intensity is approximately constant within the pump cladding and zero outside. Under this assumption, the overlap for the pump is the ratio of core area to cladding area.

\section{Spontaneous Emission}

The inclusion of spontaneous emission requires an additional term in the differential equation. The amount of spontaneous emission captured into the guided mode can be calculated in two ways. There is an approach using geometric optics that defines a capture fraction by the solid angle of rays that see total internal reflection at the core-cladding boundary. Another approach defines the capture fraction by looking at the overlap between the dipole radiation far field and mode field. The contribution of spontaneous emission to the intensity is

$$
m \frac{g\left(\nu_{i}\right) \Delta \nu}{\tau_{2}} h \nu_{i} N_{2}\left(r, \phi, z^{\prime}\right) \Omega_{i}^{+}(r, \phi) .
$$

$\Omega_{i}$ is the capture fraction and is unitless. $\tau_{2}$ is the lifetime of the upper state, $g\left(\nu_{i}\right)$ is the normalized line shape, $\Delta \nu$ is the bandwidth, and $m$ is the number of modes. ASE is included by adding Eq. (A6) to the righthand side of Eq. (A1).

In practice, spontaneous emission into the pump and signal is neglected. Emission into the pump beam is quickly reabsorbed. For narrow-line sources, the spontaneous emission into the signal is negligible.

\section{Geometric Approach}

The solid angle for a fiber of numerical aperture NA and core index $n$ is approximately $\pi \mathrm{NA}^{2} / n^{2}$ for small NA. The capture fraction, $\Omega_{i}$, is independent of the location within the core and is given by

$$
\Omega_{i}=\frac{\mathrm{NA}^{2}}{4 n^{2}} .
$$


For a single-mode core, $n=1.45$ and N.A. $=0.1$, the capture faction is 0.0012 . The power radiated into this solid angle per unit length is

$$
m h \nu_{i} \frac{g\left(\nu_{i}\right) \Delta \nu}{\tau_{2}} N_{0} n_{2} A_{\text {core }} \frac{\mathrm{NA}^{2}}{4 n^{2}} .
$$

The factor of $A_{\text {core }}$ comes from the integral over the transverse spatial variables. This equation can be simplified by use of the relationship between the lifetime and emission cross section,

$$
2 \pi m \frac{\Delta \lambda h c^{2} n}{\lambda^{5}} \sigma_{i}^{e} n_{2} N_{0} A_{\text {core }} N^{2} .
$$

\section{Field Approach}

The capture fraction can also be defined by looking at the overlap integral between the far field for dipole radiation and the guided-mode field. The capture fraction according to Marcerou et $a l .^{7}$ is given by

$$
\Omega_{i}(r, \phi)=\frac{\pi a^{2}}{V^{2}} \frac{\mathrm{NA}^{2}}{n^{2}} \Phi_{i}(r, \phi) .
$$

$a$ is the core radius. Integrating to find the power change,

$$
m \frac{g\left(\nu_{i}\right) \Delta \nu}{\tau_{2}} \frac{\pi a^{2}}{V^{2}} \frac{\mathrm{NA}^{2}}{n^{2}} N_{2}^{\prime} \int_{0}^{\infty} \int_{0}^{2 \pi} \Phi_{i} \psi r \mathrm{~d} r \mathrm{~d} \phi .
$$

Using the definition of the overlap and assuming that the total ion density does not change with $z^{\prime}$,

$$
m h \nu_{i} \frac{g\left(\nu_{i}\right) \Delta \nu}{\tau_{2}} N_{0} n_{2} \frac{\pi a^{2}}{V^{2}} \frac{\mathrm{NA}^{2}}{n^{2}} \Gamma_{i}^{+} .
$$

The total capture fraction integrated over the core is

$$
\Omega_{i}=\frac{1}{V^{2}} \frac{\mathrm{NA}^{2}}{n^{2}} \Gamma_{i}
$$

This equation can be simplified with the relationships between the lifetime, cross section, core radius, numerical aperture, and $V$ number,

$$
\frac{2 m \Delta \lambda h c^{2} n}{\lambda^{3}} \sigma_{i}^{e} N_{0} \Gamma_{i} n_{2}
$$

The field approach gives a result very similar to the geometric approach. If we compare Eqs. (A7) and (A13), Eq. (A13) is smaller by the factor $4 \Gamma V^{-2}$. For reasonable numbers ( $\Gamma=0.8$ and $V=2$ ), the capture fraction calculated with Eq. (A7) is 0.00119. With Eq. (A13), the capture fraction is $\sim 0.00095$. For a single-mode core, there are two orthogonal polarization modes: $m=2$.

The computer model allows the user to choose between either method for calculating the capture fraction. In principle, the capture fraction can be calculated just as the overlap. However, for the same reasons, this may not be accurate. Therefore the code accepts an arbitrary capture fraction, as well. Experimentally, there is significant ASE over a broad range of wavelengths. Since the cross sections change dramatically over this wavelength region, it must be divided into sufficiently small bins to determine an accurate spectrum. However, the number of differential equations to solve has an inverse relationship to the bin size. The computer code provides flexibility in the bin size, but most of the simulated data uses a 1-nm width. This allows for good resolution in the spectrum and for a quick solution. In addition, the crosssection data were taken at 1-nm intervals.

\section{Population Inversion}

The individual transitions between ytterbium energy levels cannot be resolved at room temperature, and the reported cross sections are measured between the lower and upper manifolds. The cross sections are different for absorption and emission, which allows a two-level approximation for the energy levels. The absorption cross section for the pump is larger than the emission cross section. The difference in the cross section and the rather low inversion allow for net absorption. The emission cross section for the signal is much greater than the absorption cross section. Even when there are fewer atoms in the upper manifold than in the lower manifold, the signal experiences gain. In steady state, the normalized inversion is

$$
n_{2}=\frac{\sum_{i} \sigma_{i}^{a} \Gamma_{i}\left(P_{i}^{+}+P_{i}^{-}\right)\left(A_{\text {core }} h \nu_{i}\right)^{-1}}{\frac{1}{\tau_{2}}+\sum_{i}\left(\sigma_{i}^{e}+\sigma_{i}^{a}\right) \Gamma_{i}\left(P_{i}^{+}+P_{i}^{-}\right)\left(A_{\text {core }} h \nu_{i}\right)^{-1}} .
$$

\section{E. Normalized Variables}

These differential equations are unwieldy with all of the constants. The definition of some normalized variables makes the equations less cumbersome and the numerical solution faster by requiring fewer operations. We define a normalized distance and time by $z=z^{\prime} L^{-1}$ and $\tau=t \tau_{s}^{-1}$, where $\tau_{s}$ is the time for a single transit of the fiber $\left(\tau_{s}=n L c^{-1}\right)$. We define a normalized photon number as

$$
p_{i}^{+}=\frac{P_{i}^{+} \tau_{s}}{h \nu_{i} N_{0} A_{\text {core }} L} .
$$

This is the photon number divided by the total number of ions in the core. With these normalized variables, the complete description of the evolution of the power [Eqs. (A5) and (A12)] is

$$
\pm \frac{\mathrm{d} p_{i}^{ \pm}}{\mathrm{d} z}=\left(n_{2} k_{2 i}-k_{3 i}\right) p_{i}^{ \pm}+n_{2} k_{4 i}
$$

The constants are defined in Table 1. Equation (A17) is the sum of contributions for each process. The $k_{2 i}$ term represents gain due to stimulated emission, and the $k_{3 i}$ term is absorption loss. The $k_{4 i}$ term is the spontaneous emission. The steady-state population inversion is

$$
n_{2}(z)=\frac{\sum_{i} k_{5 i}\left(p_{i}^{+}+p_{i}^{-}\right)}{k_{1}+\sum_{i} k_{2 i}\left(p_{i}^{+}+p_{i}^{-}\right)}
$$

where $k_{5 i}$ is the normalized pumping rate. 


\section{F. Boundary Conditions}

The boundary conditions are set by the end-face reflectivities and the power injected into each end. In principle, this model can have signal and pump injected at either $z=0$ or $z=1$. For the modeling in this paper, signal and pump are counterpropagating. The pump is injected at $z=1$; the signal is injected at $z=0$. In the most general case, the reflectivities at each end, $R_{0}$ and $R_{1}$, can be different for pump, signal, and each ASE bin. One must be careful in treating the reflections from the fiber end faces because the amount of light reflected can be different from the light that is recoupled into the guided mode. Consider the case of the fiber end polished at $8^{\circ}$. The reflectivity of the air-glass interface is $\sim 4 \%$. For the core mode, the acceptance angle is small, $\sim 6^{\circ}$. Of the reflected light, only a small fraction recouples to the guided mode. However, for the cladding, the acceptance angle is very high, and most of the light couples back into the cladding. For this model, reflectivity refers to the fraction of light that re-enters the core mode or the cladding mode.

\section{G. Model Implementation}

For a set of differential equations with boundary conditions at two points, there are several options for finding a solution. ${ }^{17}$ The shooting method starts at $z=0$ and propagates all of the equations in the positive $z$ direction. For the forward-propagating beams, one imposes the boundary conditions at $z=0$. For the counterpropagating beams, one estimates the values at $z=0$. After integrating to $z=1$, the boundary conditions for the counterpropagating beams are subtracted from the integrated values. If the guess is correct, the subtraction will be zero for each beam. The comparison is then used to reestimate the values at $z=0$ and to reintegrate. The first estimate is provided by the mean excited fraction model. The algorithm ends after the comparison shows that all of the counterpropagating beams are sufficiently close to their boundary conditions. We generally use a Bulirsch-Stoer ${ }^{17}$ algorithm for the actual integration. The computer code implements a globally convergent Newton-Raphson algorithm to refine the estimates for each shot. ${ }^{18}$ At the heart of the globally convergent Newton-Raphson is the Newton-Raphson algorithm, which is locally convergent. Consider the estimates at $z=0$ as an $N$-dimensional vector, $x$. The numerical integration and subtraction of the boundary values is a vector-valued function $F(x)$. The Newton-Raphson algorithm searches for another vector, $p$, such that $F(x+p)=0$. To first order,

$$
F(x+p)=F(x)+p \mathbf{M}(x)=0 .
$$

$\mathbf{M}(x)$ is the Jacobian matrix evaluated at $x$. Finding $p$ requires solving Eq. (A19). This, however, requires inverting the Jacobian matrix, which must not be singular. This requirement on the Jacobian in effect requires that we deal only in ASE bins with net gain along the full length of the fiber. For an ASE bin with no net gain, the power as a function of $z$ decreases until it reaches 0 at some point $z<1$. The power at $z=1$ for that bin is 0 and is independent of the initial guess for all of the counterpropagating beams. This means that there is a 0 row in the Jacobian, and it is singular. Because Eq. (A19) is only a local approximation to the problem, there is no guarantee that the $p$ calculated actually forces $F(x+p)$ $=0$. The globally convergent variant of the NewtonRaphson method searches along the direction of $p$ (or a vector near $p$ ) until $F(x+p)$ has decreased sufficiently. It then forms a new local approximation for another iteration. This continues until the convergence criteria are met. The model in this paper applies both relative and absolute convergence criteria.

\section{ACKNOWLEDGMENTS}

This research was performed at Lawrence Livermore National Laboratories and was funded by the U.S. Air Force Research Laboratory's Directed Energy Directorate. We would like to acknowledge the advice of Ralph Page and Don Browning.

\section{REFERENCES}

1. M. Digonnet, "Closed-form expressions for the gain in three- and four-level laser fibers," IEEE J. Quantum Electron. 26, 1788-1796 (1990).

2. D. C. Hanna, R. M. Percival, I. R. Perry, R. G. Smart, P. J. Suni, and A. C. Tropper, "An ytterbium-doped monomode fiber laser: broadly tunable operation from $1.010 \mu \mathrm{m}$ to $1.162 \mu \mathrm{m}$ and three-level operation at $974 \mathrm{~nm}$, ${ }^{\mathrm{J}}$. Mod. Opt. 37, 517-525 (1990).

3. R. Paschotta, J. Nilsson, A. C. Tropper, and D. C. Hanna, "Ytterbium-doped fiber amplifiers," IEEE J. Quantum Electron. 33, 1049-1056 (1997).

4. V. Dominic, S. MacCormack, R. Waarts, S. Sanders, S. Bicknese, R. Dohle, E. Wolak, P. S. Yeh, and E. Zucker, " 110 W fibre laser," Electron. Lett. 35, 1158-1160 (1999).

5. J. M. Sousa, J. Nilsson, C. C. Renaud, J. A. Alvarez-Chavez, A. B. Grudinin, and J. D. Minelly, "Broadband diodepumped ytterbium-doped fiber amplifier with $34-\mathrm{dBm}$ output power," IEEE Photonics Technol. Lett. 11, 39-41 (1999).

6. R. H. Page, R. J. Beach, C. A. Ebbers, R. B. Wilcox, S. A. Payne, W. F. Krupke, C. C. Mitchell, A. D. Drobshoff, and D. F. Browning, "High-resolution, near-diffraction-limited, tunable solid-state visible light source using sum frequency generation," Conference on Lasers and Electro-Optics, Vol. 39 of OSA Trends in Optics and Photonics (Optical Society of America, Washington, D.C., 2000), paper CMD3.

7. J. F. Marcerou, H. A. Fevrier, J. Ramos, J. C. Auge, and P Bousselet, "General theoretical approach describing the complete behavior of the erbium-doped fiber amplifier," $F i$ ber Laser Sources and Amplifiers II, Proc. SPIE 1373, 168$186(1990)$.

8. C. R. Giles and D. di Giovanni, "Spectral dependence of gain and noise in erbium-doped fiber amplifiers," IEEE Photonics Technol. Lett. 2, 797-800 (1990).

9. C. R. Giles and E. Dersurvire, "Modeling erbium-doped fiber amplifiers," J. Lightwave Technol. 9, 271-283 (1991).

10. A. Hardy and R. Oron, "Signal amplification in strongly pumped fiber amplifiers," IEEE J. Quantum Electron. 33, 307-313 (1997).

11. R. Oron and A. A. Hardy, "Rayleigh backscattering and amplified spontaneous emission in high-power ytterbiumdoped fiber amplifiers," J. Opt. Soc. Am. B 16, 695-701 (1999).

12. R. Beach, Lawrence Livermore National Laboratories, P.O. Box 808, L-482, Livermore, Calif. 94551 (personal communication, 2000).

13. A. O. Neilson, J. H. Povlsen, A. Bjarklev, O. Lumholt, T. P.

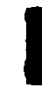

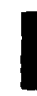

]

】

】

!

】

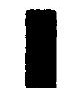

】

I

】

】

】

】

】 
Rasmussen, and K. Rottwitt, "Fast method for accurate prediction of fibre laser oscillation wavelength," Electron. Lett. 27, 1644-1645 (1991).

14. F. Patel, "Solid-state rare-earth doped media for applications in waveguide lasers," Ph.D. dissertation (University of California, Davis, Calif., 2000).

15. N. A. Brilliant, "Ytterbium-doped dual-clad fiber amplifiers," Ph.D. dissertation (University of New Mexico, Albuquerque, N.M., 2001).

16. G. C. Valley, "Modeling cladding-pumped $\mathrm{Er} / \mathrm{Yb}$ fiber amplifiers," Opt. Fiber Technol. Mater. Devices Syst. 7, 21-44 (2001).
17. W. H. Press, S. A. Teukolsky, W. T. Vetterling, and B. P. Flannery, Numerical Recipes in C (Cambridge University, Cambridgè, England 1997).

18. J. E. Dennis Jr. and R. B. Schnabel, Numerical Methods for Unconstrained Optimization and Nonlinear Equations (Society for Industrial and Applied Mathematies, Philadelphia, Pa., 1996).

19. E. Desurvire and J. R. Simpson, "Amplification of spontaneous emission in erbium-doped single-mode fibers," J. Lightwave Technol. 7, 835-845 (1989).

20. C. Vasallo, Optical Waveguide Concepts (Elsevier, New York, 1991). 


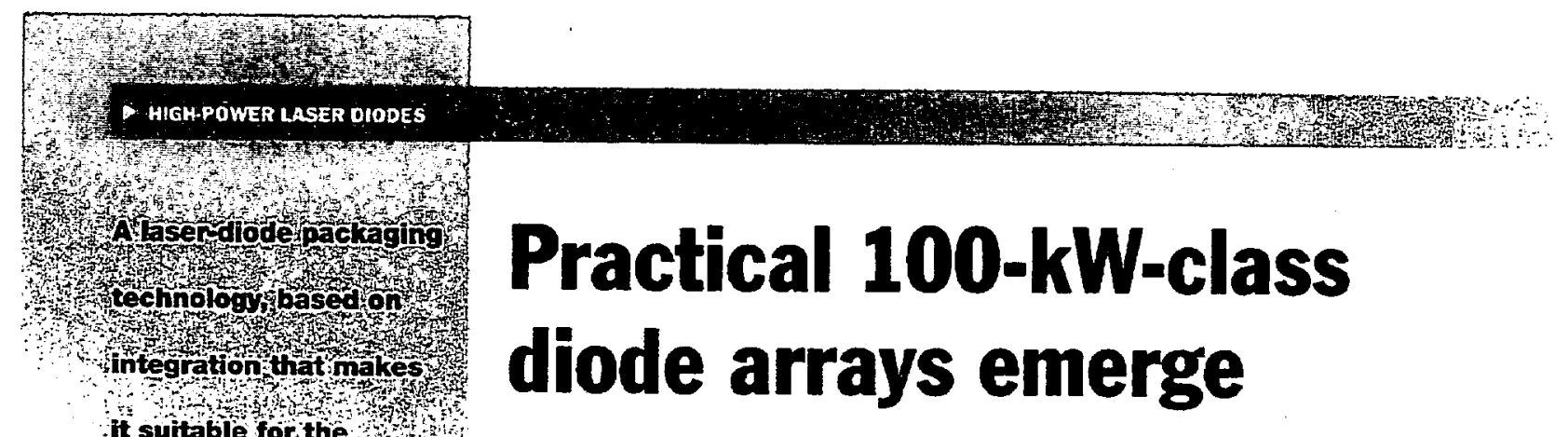

It suitable for the

construction of large

laser-diode arrays,

Raymond d. Beach and Barry L, Freitas

boasts 10- to 100-kW

output power

capabilities.

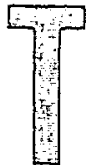

0 imagine $100-\mathrm{kW}$ diode arrays becoming a common reality, two key elements of the technology need to fall into place: high-performance diode bars, and heat sinks that offer excellent thermal management and precision diode-bar mounting. Lawrence Livermorc National Laboratory (LLNL; Livermore, CA) has developed a silicon-based packaging technology to solve the challenge of packaging diode bars.

\section{Silicon monolithic microchannels}

Photolithography and etching techniques are applied to produce thousands of miniscule 30- $\mu \mathrm{m}$-wide channels in silicon substrates. Water flowing through these microchannels aggressively cools the laser diode bars, which are mounted
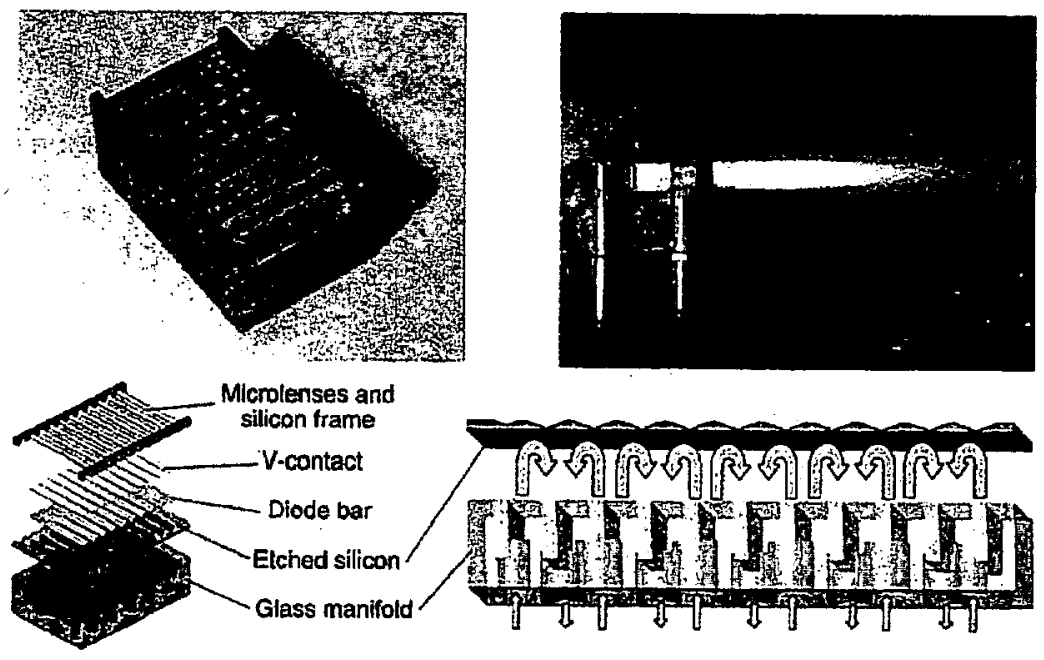

The sillcon monolithic microchannels (SiMMS) package holds 10 individual laser-diode bars in a single monolithically cooled silicon tile. Shown are an individual SiMMMs package (top left), a SMMMs package in operation (top righth, and SiMMs package components with the water flow path through the microchannels and glass manifold structure (bottom), .

on the silicon at a location less than $200 \mu \mathrm{m}$ from the channels (see figure this page). By combining 10 diode bars onto a single heat sink, a 10-bar package (referenced as a tile) serves as the unit cell from which large two-dimensional diode arrays can be built up through tiling.

Individual tiles that make up these large arrays are known as silicon monolithic microchannels (SIMM's; see Fig. 11. ${ }^{1}$ Considerations that drove the SiMMs package design included ease of fabrication and the ability to construct large laser-diode axrays with 10- to 100-kW output power capabilities. Of paramount importance in the design was the requirement that the same aggressive heat-removal capability that characterized the original rack-and-stack silicon microchannel-cooled package be preserved in the SiMMs (see "Original microchannel-cooled package is still

RAYMOND J. BEACH is the group leader for diade-pumped solid-state lasers, and BARRY L. FRETAS is the group leader for laser diode packaging at the US Department of Energy's Lawrence Livemore National Laboratory, P.O. Box 808, Livermore, CA 94551 ; e-mall: beach2Qinlinl.gov. in use," p. 105). ${ }^{2}$ This challenge was met in the same manner as the original rack-and-stack package-by incorporating microchannels into the silicon directly below the location of the attached laser-diode bars. As in the original rackand-stack silicon microchannel cooler, the SiMMs design maintains a very tight thermal circuit with only $177 \mu \mathrm{m}$ of silicon separating the heat-generating laser-diode bars and the microchannel fins that define the cooling channels.

\section{Integrating mierolensing attachment technology}

Because nearly all applications at Livermore Laboratory now require microlensed arrays, the development of an easy and efficient microlensing attachment technology was an important consideration during the design phase of the SiMMs package. Easy and efficient microlens attachment translated into the requirement that an entire SiMMs package be microlensed in a single step. This eliminates the production steps necessary to individually attach a microlens to each diode bar as we do in our rack-and-stack package. From previous experience with the rack-and-stack package, we knew that 


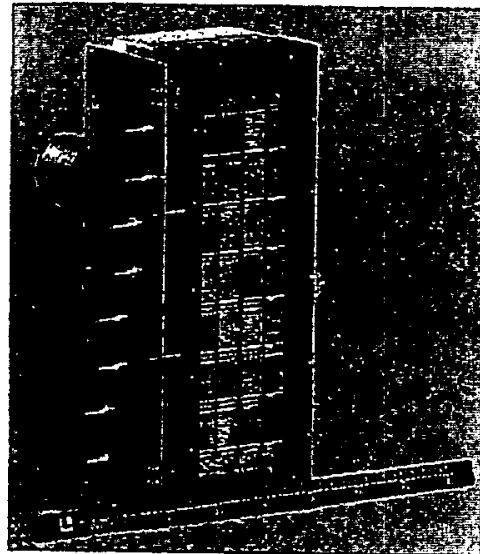

FIGURE 1 . This diode array of 28 closely packed SiMMs is capable of genterating in excess of $40 \mathrm{~kW}$ of optical power. Brightress of the array is high due to the compact tiling of the heat sinks and the precision placement of 10 -element microlens amays on each tile.

sion placement of the laser-diode bars on the SiMMs package is accomplished by using V-groove teclunology on the front surface of the package. V-grooves are generated with the same etching technology used to fabricate the microchannels on the backside of the silicon. In particular, starting with $<110>$ oriented silicon wafers, an etch mask is defined such that the <111> crystallographic planes form etch stops that terminate the etching process and generate the characteristic $V$-groove contour over the entire surface (see Fig. 2). These V-grooves serve as pads to which the laser-diode bars are registered and attached. V-grooves are defined lithographically, so this technique allows us to locate the output facet of the bars with micrometer precision relative to one another over the entire SiMMs package as required for one-step microlensing.

An unusual feature of the SiMMs package is the $55^{\circ}$ angle from the normal at which light is enitted. The emission direction of the diodes at $55^{\circ}$ to the normal of the SiMMs front face is due to the orientation of the V-grooves that serve as pads for the laser-diode bars. With the diode bars located, the microlenses can be held in precise frames fabricated in the form of silicon runners in a ladder-like structure consisting of 10 lenses. The entire 10-lens assembly is then attached to the SiMMs package in a single step. This microlens array serves to collimate the fast axis radiation of the laser-diode bars from its original $30^{\circ}$ divergence angle into a collimated beam with a divergence angle of less than $1^{\circ}$.

To maintain the aggressive cooling capability of the original rack-and-stack package, microchannels are etched into the backside of the silicon just below the laser-diode bars at their V-groove defined locations. These microchannels etch up into the silicon following the same V-groove contour that characterizes the front of the package--bringing cooling water within $177 \mu \mathrm{m}$ of the heat-generating diode bars. Microchannels in the SiMMs package are $30 \mu \mathrm{m}$ wide and located on $60-\mu \mathrm{m}$ centers. The channels are sourced and drained through a

\section{Otginal microchannel cooled pactiog \\ and} Driven primanly by government applications requiring state of the-ant as LLNL has maintained LLNL has maintained a vibrant program focised on the development of high average-power laser diode arrays sncethe mat 1980 . The onginal rack and-

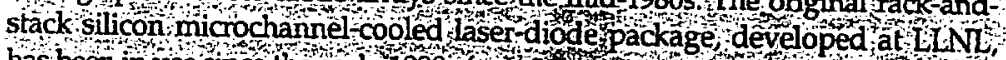

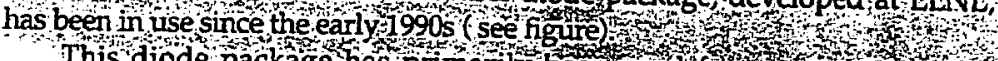

This diode package has primariy ben ws ad for onstructing kilowattlass arrays consisting of 10 to 50 packats ity that stackng bings to building custom arrays has been instumental

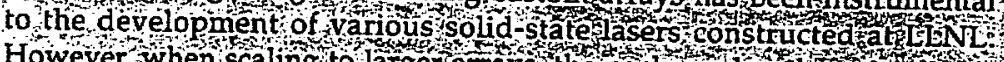
However, when scaling to larger arrays the package does have its disad vantages since eachpackag has to be hydraulically sealed to the pack age next to it the multiple water sals required inarays largerthansey

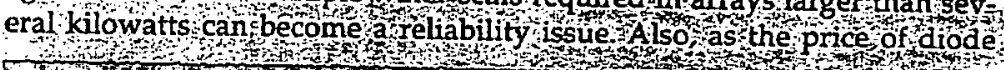

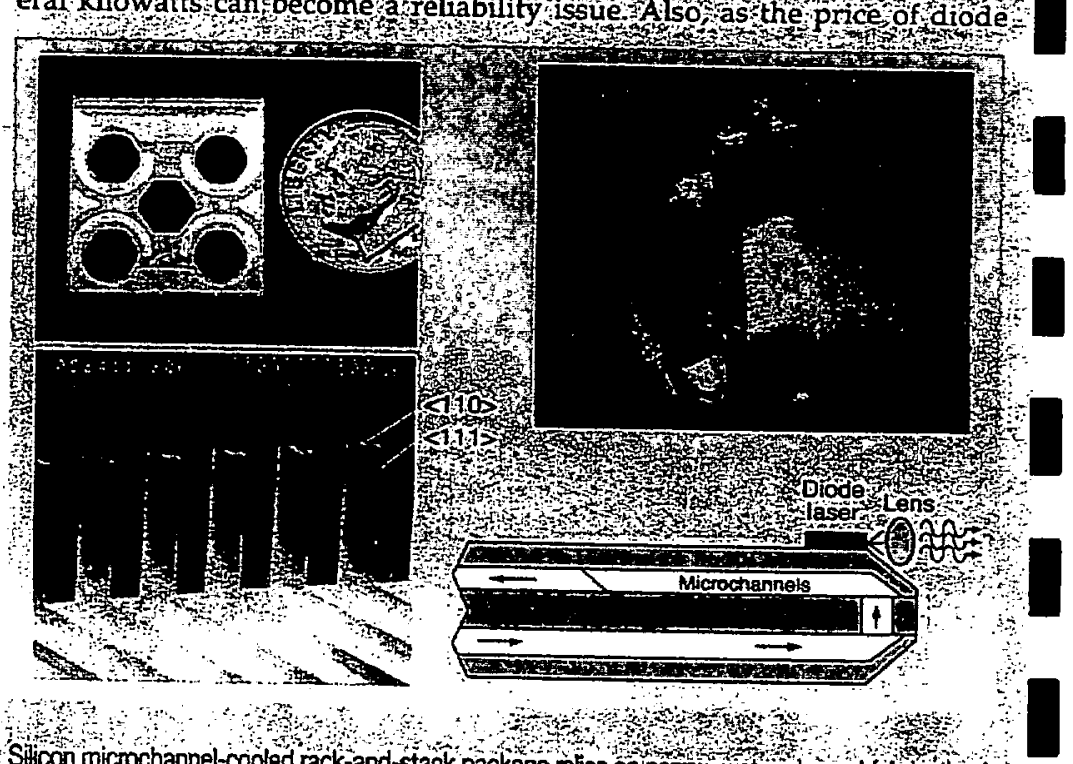

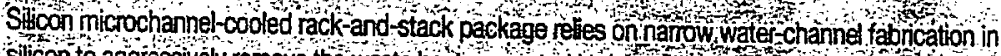
silicon to aggressively remove the waste heat genered by laserolode bas top view of the microchannel-cooled package (top leff) two-dimensional array illustrates the stacking of individua packages to form a larger multibar aray fop nght) microchannels (appoximately 25 um wide and $150 \mathrm{\mu m}$ deep) in silicon fabricated by anisotropic etching, (bottom left, and a side view showing the laminated silicon-glass-silicon structure, with arows depicting wator flowi dinection (bottom right)

bars continues to drop and therr reliability continues to increase, is advantageous to mount multiple bars on a single mocochannel-cooler package to dilute the impact of the cost overmany bars rather than just one or two. With these considerations in mind, a second-generation microchannel-cooled package (the SiMMs) has been developed that is capable of holding 101 -cm bars:

backing glass block that has a manifold structure fabricated into it using glassmilling technology. The backing glass block is attached to the silicon front face using the same anodic bonding technology that is used to join the silicon-glasssilicon laminates in the original rackand-stack package.

\section{Package perfomance}

Typical SiMMs package performance generated from a single tile at a $10 \%$ duty factor produced a peak optical output power of $1.5 \mathrm{~kW}$ (see Fig. 3) This performance was obtained using length and $90 \%$ fill. The measurec laser-diode bars with a 1-mm cavity 


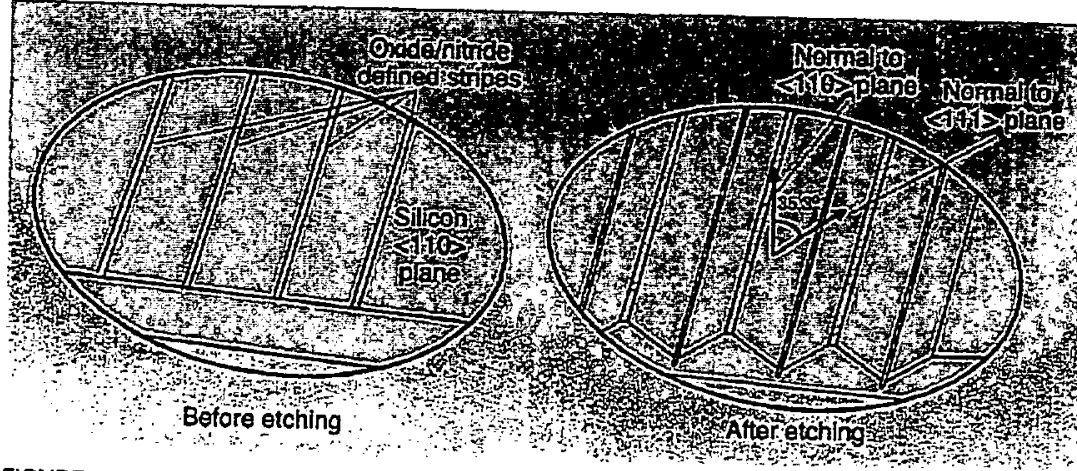

FIGURE 2. V-groove fabrication technology starts with <110>-oriented sillcon wafers having an oxide/nitride-defined etch mask (leff). Using a $65^{\circ} \mathrm{C}$ potassium hyoroxide solution, the $\langle 110\rangle$ planes are etched away at a rate of $40 \mu \mathrm{m} / \mathrm{hr}$. The $\langle 111\rangle$ planes etch approximately 600 times slower than $\langle 110\rangle$ planes, and efiectively serve as etch stops defining the characteristic V-groove (right).

thermal resistance of the SiMMs package is $0.32^{\circ} \mathrm{C} / \mathrm{W}_{\text {the }}$ referenced to a single 1-cm diode bar and the cold inlet water temperature. For example, a diode bar generating $100 \mathrm{~W}_{\text {th }}$ of waste heat would experience a $32^{\circ} \mathrm{C}$ temperature rise at its junction above the temperature of the inlet coolant water.
The backing glass blocks of the by-side on a backplane structure containing cooling-water source drains. The package is specifically designed for close packing to give high effective fill factors.

Low cost and aggressive thermal performance requirements drove the develSiMMs package are designed to sit side- opment of the silicon microchannel approach. ${ }^{4}$ To understand in more detail how the channels function, it is useful to use a very simplified picture of the heat flow in the structure and break down the temperature rise between the heatgenerating laser-diode $p-n$ junction and the cold inlet coolant water into three different components.

The first component corresponds to the temperature rise associated with the heat flowing through the solid silicon material from the laser-diode bar down into the regions between the water flow channels.

The next component corresponds to the temperature rise associated with heat flowing across the stagnant boundary layer of water and into the moving fluid. This stagnant boundary layer exists because of the "no slip" boundary condition at the channel wall-to-wall interface.

The last component corresponds to the temperature rise associated with

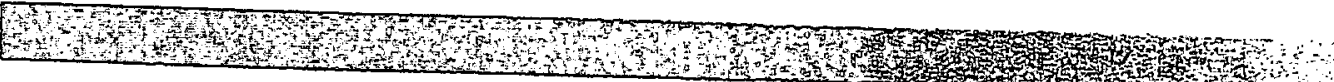

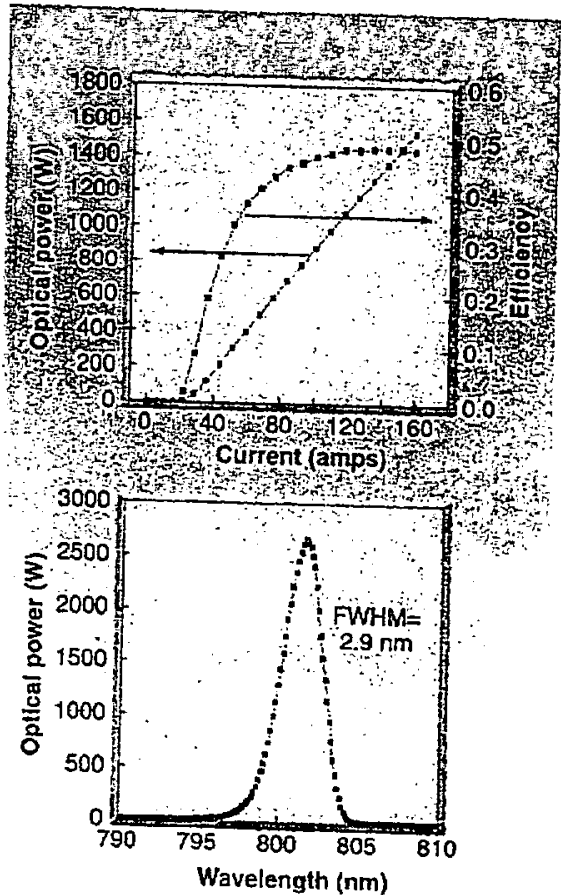

FIGURE 3. An optical output of $1.5 \mathrm{~kW}$ was achieved from a single 10-bar SiMMs package with wall piug efficiencies approaching $50 \%$; the spectral output from a SiMMs package at a $1.5-\mathrm{kW}$ peak output power and a 500-is pulse length is shown. For both data plots, the inlet coolant water was at $22^{\circ} \mathrm{C}$ and the flow rate was $1.8 \mathrm{~L}$ min. at $35 \mathrm{psi}$

the heat absorbed by the flowing water as it goes from the cold inlet side of the channel to the hot outlet side of the channel. It turns out that the temperature rise associated with the heat flowing across the stagnant water boundary layer at the solid-to-water interface is generally the largest temperature rise in flowing water heat-sink structures, such as our microchannel cooler. To understand this, consider that the thermal conductivity of water at $0.0061^{\circ} \mathrm{C} / \mathrm{W}_{1 \mathrm{~h}}-\mathrm{cm}$ is 250 times less than that of silicon at room temperature. Thus, in com- pact flowing water heat-sink structures the biggest leverage in controlling the overall temperature rise is generally associated with minimizing the thickness of the stagnant boundary layer. Boundary layer thickness scales with the channel width for the laminar flow conditions used in our coolers, so the solid material used to construct the cooler should be one that permits easy fabrication of narrow channels. This result explains the apparently paradoxical choice of silicon for the sulid material in our heat sink over other more obvious materials with higher thermal conductivities, such as copper.

\section{REFERENCES}

1. J. A. Skidmore et al.. Appl. Phys. Lett. 77. 10 (2000).

2. R. J. Beach el al., IEEE J. Quant. Elect, 28. 966 (1992).

3. D. Tuckerman et al., IEfE Elect. Device Letr. (EDL-2), 126 (1981).

4. R. J. Beach ot al., Proc. SPIE Photonics West, 2383 (1995). 


\title{
Activation of the Mercury Laser System: A Diode-Pumped Solid-State Laser Driver for Inertial Fusion
}

\author{
A.J. Bayramian \\ C.A. Ebbers \\ S.A. Payne \\ L.K. Smith
}

\author{
R.J. Beach, \\ B.L. Freitas \\ K.I. Schaffers
}

J.B. Tassano

\author{
C. Bibeau
}

V.K. Kanz

K.M. Skulina

\begin{abstract}
Initial measurements are reported for the Mercury laser system, a scalable driver for rep-rated inertial fusion energy. The performance goals include $10 \%$ electrical efficiency at $10 \mathrm{~Hz}$ and $100 \mathrm{~J}$ with a 2-10 ns pulse length. We report on the first $\mathrm{Yb}: \mathrm{S}$-FAP crystals grown to sufficient size for fabricating full size $(4 \mathrm{x} 6 \mathrm{~cm}$ ) amplifier slabs. The first of four $160 \mathrm{~kW}$ (peak power) diode arrays and pump delivery systems were completed and tested with the following results: $5.5 \%$ power droop over a $0.75 \mathrm{~ms}$ pulse, $3.95 \mathrm{~nm}$ spectral linewidth, far field divergence of $14.0 \mathrm{mrad}$ and $149.5 \mathrm{mrad}$ in the microlensed and unmicrolensed directions respectively, and $83 \%$ optical-to-optical transfer efficiency through the pump delivery system.
\end{abstract}

\section{INTRODUCTION}

The Mercury laser design is based on creating a scalable architecture for inertial fusion energy applications (Orth and Payne[1]). This architecture builds on our knowledge of flashlamp-pumped glass laser systems such as Nova, Omega, and the National Ignition Facility, namely multi-pass amplification, energy storage and extraction, pumping, linear and nonlinear wavefront distortions, frequency-conversion, and beam smoothing. This diodepumped solid state laser approach adds several imposing challenges including: repetition rate, efficiency, reliability, and cost. It is noteworthy that Matsui et al. [2] are developing a Nd:glass diode-pumped zigzag driver $(10 \mathrm{~J}$ at $10 \mathrm{~Hz}$ ) for IFE, and have recently demonstrated $8.5 \mathrm{~J}$ at $0.5 \mathrm{~Hz}$ and $1.9 \mathrm{~J}$ at $2.5 \mathrm{~Hz}$. To achieve the Mercury goals of $10 \mathrm{~Hz}$ operation at $10 \%$ electrical efficiency, three major technologies were developed: reliable high-peak-power diode arrays, high optical quality $\mathrm{Yb} 3+: \mathrm{Sr} 5(\mathrm{PO} 4) 3 \mathrm{~F}$ (Yb:S-FAP) crystalline slabs, and efficient helium gas cooling of the gain media. Integration of these technologies into a modular, scalable architecture will add reliability to the operation of Mercury system, and great impact to the modest output energies at this scale. These technologies will allow us to step beyond the single shot devices of the past three decades to yield efficient inertial fusion drivers for the power plants of the future.

\section{ARCHITECTURE}

The optical layout of the amplifiers, pump delivery, optics, and relay telescopes was arranged to reduce the probability of damage, and yet create a compact laser system. Since the packaging of the diode bars causes the diode light to exit at an angle of $55^{\circ}$, the diode arrays were tilted to accommodate for this angle and split into two elements to allow for the passage of the $1047 \mathrm{~nm}$ extraction beam through the center as shown in Fig. 1. A unique challenge to the gas-cooled design of Mercury was in the coupling of the diode pump light to the amplifier medium. We chose a pumping scheme in which the diode pump and extraction light are collinear. This design minimizes the number of optics in the beam line relative to earlier designs thereby lowering the overall Bintegral of the laser extraction, while maximizing the pump transport efficiency. The diode array light is guided to the amplifier through multiple reflections within a hollow lens duct and homogenizer. In designing these optics, the diode tiles are first arranged to produce a uniform angular distribution at the input of the lens duct. Next, the duct length is optimized for maximum transfer efficiency and the output aperture size is nearly matched to the beam size. A slightly larger aperture $(3.2 \times 5.2 \mathrm{~cm})$ is needed to allow for the passage of the angularly multiplexed extraction beams. Finally, the homogenizer length is optimized to create a homogeneous near field profile at the exit. A ray tracing code was used to find the optimal configuration of the tiles with the result being an arrangement of six tiles wide by six tiles high in each backplane. Measurements of this system resulted in a , transport efficiency of $77 \%$ in a $3 \times 5 \mathrm{~cm}$ area at the first amplifier slab. Using a 4-pass double amplifier system, a $40 \mathrm{~mJ}$ narrowband input pulse will be amplified to $100 \mathrm{~J}$ with an extraction efficiency of $63 \%$, and a pump optical to optical efficiency of $22 \%$. This is possible because of the low saturation fluence of Yb:S-FAP (3.2 $\mathrm{J} / \mathrm{cm} 2$ ), which allows efficient extraction at approximately $7 \mathrm{~J} / \mathrm{cm} 2$, a fluence level that is well below the damage threshold. 


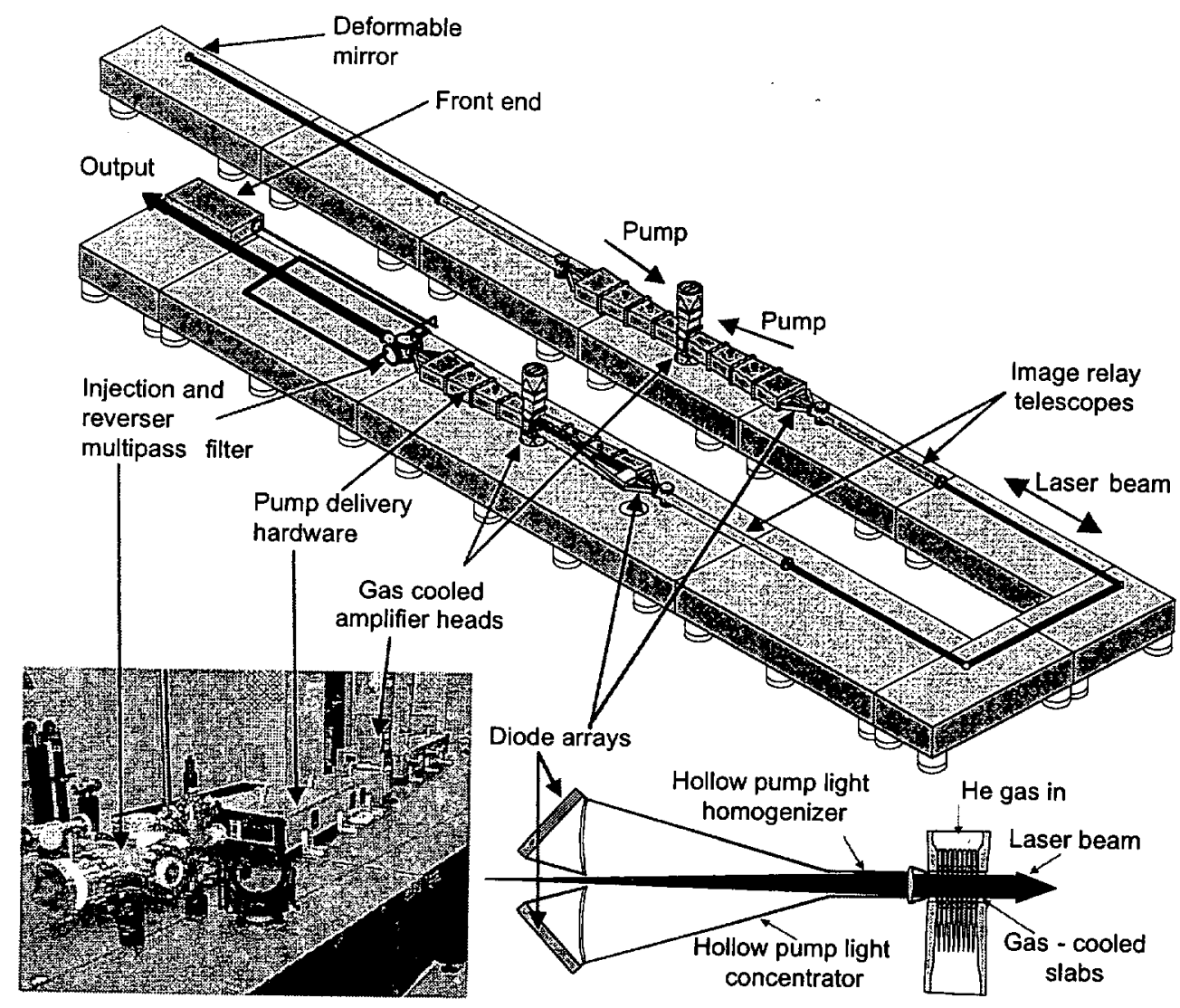

Fig. 1. The architecture design for the Mercury laser employs eight $80 \mathrm{~kW}$ diode arrays arranged in a backplane design. In addition, one-to-one telescopes are used to relay image the extraction beam from one amplifier to another. One lens in each telescope is located near the amplifier to reduce modulation, thereby helping to avoid optical damage.

In order to extract all the stored energy, a four-pass configuration is employed by angularly multiplexing the beams, which eliminates the need for a large-aperture high average power Pockels cell at the output where energies are high. An image relayed system is used since optics which are located out of the image plane encounter greater intensity modulation on the beam and are thus at higher risk for optical damage. One lens of each one-to-one telescope is located very close to the amplifier to help reduce modulation and thus prevent optical damage. After the final pass, where the average fluence exceeds $7 \mathrm{~J} / \mathrm{cm} 2$, the out of relay output lens provides a $2 \mathrm{X}$ magnification to reduce the peak fluence to acceptable levels.

Extensive ghost and amplified spontaneous emission analysis was performed, validating the current architecture and setting constraints on optical quality, surface reflectivity, wedges, and the extinction required of a Pockels cell in the reverser (an image relay beam path which turns the beam and re-injects it for the 3 and 4th pass through the system). Though the Pockels cell sees relatively low energy pulses $(<0.5 \mathrm{~J} / \mathrm{cm} 2)$, the average power loading $(100 \mathrm{~W})$ requires an advanced design since thermal birefringence compromises the extinction ratio of the cell. A design was explored in which two nearly identical $\mathrm{KD} * \mathrm{P}$ crystals are oriented with a $90^{\circ}$ rotator between them such that the thermally induced birefringence is nearly cancelled. The average power Pockels cell shown in Fig. 2 has an aperture of $1.5 \times 2.5 \mathrm{~cm}$, is capable of extinction of $200: 1$ at an incident power of $100 \mathrm{~W}$, and will be used to prevent ghost beams from reaching high levels of amplification.

The ytterbium doped S-FAP slabs have a $1^{\circ}$ wedge between faces, and have $5^{\circ}$ canted edges to suppress: parasitics, etalon effects, stimulated Brillouin, and stimulated Raman scattering. Preliminary laser propagation modeling of the full system, using the MIRO code developed by scientists at CEA in France (adapted from Prop 92 at LLNL), indicates that greater than $90 \%$ of the beam is contained in a $5 \mathrm{x}$ diffraction limited spot with a Bintegral of 1.7 for a $2 \mathrm{~ns}$ pulse. We are currently updating our thermal model to include the pump deposition profiles for the new wedged slab design and will incorporate these dynamic distortions in our MIRO propagation 
model (earlier models gave 3.6 waves total thermal distortion for the 4 pass system). A deformable mirror will be used in the final system to help correct for low order distortions.

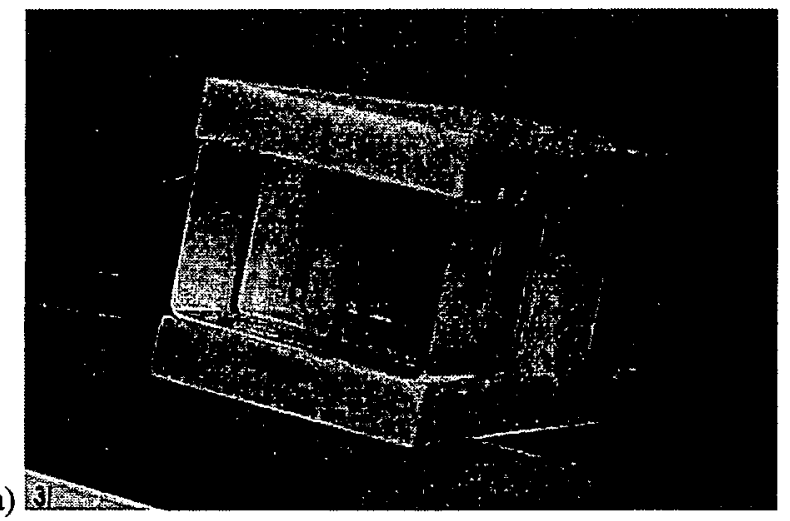

b)

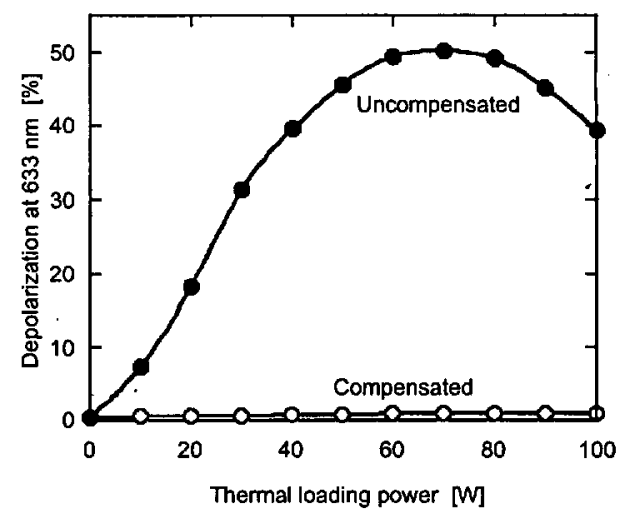

Fig. 2 a) Picture of the thermal birefringence compensated average power Pockels cell, b) A plot showing the advantage of birefringence compensation by demonstrating extremely low depolarization loss up to $100 \mathrm{~W}$ average power.

\section{CRYSTAL GROWTH}

Recent breakthroughs have been made in the growth of the ytterbium doped strontium fluorapatite (S-FAP) crystals. Current growths have produced four $3.4 \mathrm{~cm}$ diameter boules free of major defects. These boules have been fabricated into _ size amplifier slabs (Fig. 3a and 3b), which will then be diffusion bonded together by Onyx Optics to achieve full-scale $4 \times 6 \mathrm{~cm}$ amplifier slabs[3]. These Czochralski grown crystals were initially plagued by a number of defects including: cracking, cloudiness, bubble core defects, grain boundaries or slip dislocations, anomalous absorption, and crystal inclusions. The remaining defects include a small annulus of inclusions on the outer perimeter of the boule, and a small number of bubble core defects concentrated to the center of the boule. Our current method of fabricating _ slabs allows us to work around the defects while continuing to explore ways to eliminate them. The first full completed amplifier slab of $\mathrm{Yb}: \mathrm{S}-\mathrm{FAP}$ is anticipated by October, 2001.

a)

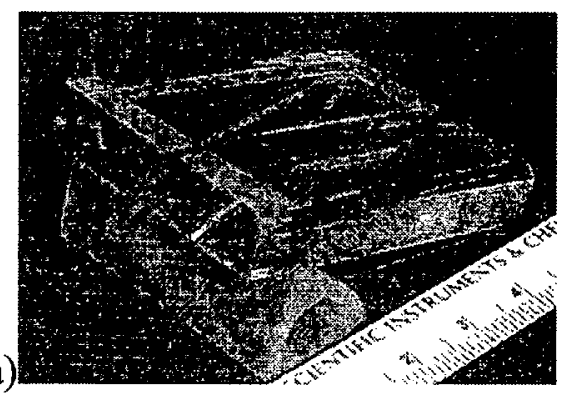

b)

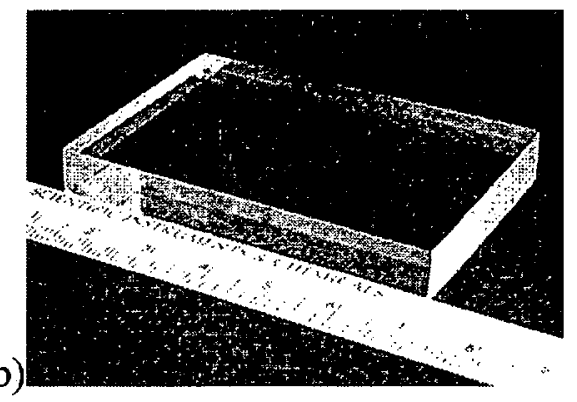

Fig. 3 a) Sub-slab components from a Livermore boule to be fabricated into full size amplifier slabs, b) A bonded full size slab from a Litton-Airtron boule currently being finished.

\section{DIODE LASERS}

A 23-bar monolithic diode laser package was developed for the Mercury Laser, referred to as Bars And Springs In Slots (V-BASIS)[4]. In this scheme, diodes are soldered to a V-groove etched silicon wafer, which is then bonded to a Molybdenum block for structural support and heat sinking. Next microlens arrays are precision mounted onto the assembly to increase brightness. The final unit or "tile" is then mounted to a water-cooled copper backplane. Electrical feed-throughs and coolant channels are located inside the backplane to increase the diode tile packing density and the overall brightness of the array. High peak irradiance is desirable which leads to compact diode spacing. However, the diode spacing must be kept to some minimum spacing to accommodate the $1 \mathrm{~mm}$ cavity length of the bars. The package is consistent with our low duty factor (1\%) and the need for low cost packaging ( 6624 diode bars in the system). The V-BASIS design, which balances both of these requirements 
and allows for compact mounting onto the copper backplane cooler. Each backplane delivers up to $80 \mathrm{~kW}$ of peak power in a $750 \mathrm{~ms}$ pulse with a divergence of $1^{\circ}$ in the microlensed axis, $9^{\circ}$ in the unmicrolensed axis, and a $4 \mathrm{~nm}$ bandwidth. Recent experiments based on Coherent bars demonstrate that these diodes can operate with an electrical efficiency of at least $45 \%$ per bar. Experimental life tests of a 23 bar V-BASIS tiles at $10 \mathrm{~Hz}$ and 115 $\mathrm{W}$ yield a lifetime of greater than 108 shots.

\section{ACTIVATION}

Initial activation included investigation of several key components in the system. Amplifier slabs are mounted into aerodynamic vanes[5] that accelerate the gas to produce turbulent flow across the amplifier slabs for maximum cooling, and then decelerate the gas with minimum vibration. The pressure balance across the eight gas channels in an amplifier head was found to be an average of 0.775 psi pressure drop with maximum variations of +0.056 and $-0.052 \mathrm{psi}$, which is adequate to preclude the formation of wake disturbances as the flows merge at the trailing edge of the vane. These results were confirmed experimentally by measuring the differential wavefront distortion of a gas-cooled amplifier head loaded with surrogate $\mathrm{Nd}$ :glass slabs and found to be less than 1/16, which for a two amplifier system corresponds to half wave distortion due to effects of gas flow.

Currently two backplanes have been activated $(160 \mathrm{~kW}$ total) and we hope to complete fabrication of two more backplanes by mid-October. Results for the initial backplane characterization are shown in Figs. 4 . The measured droop (6\%) in the temporal pulse, the linewidth $(4.2 \mathrm{~nm})$ of the output spectrum, and far field divergence (13 $\mathrm{x}$ $146 \mathrm{mrad})$ of the full backplane are well within the design point requirements. Measurements of the transfer efficiency and pump homogeneity after the pump delivery system (diode lenses, lens duct, homogenizer, and laser transport lens) at the input to the first slab are depicted in Fig. 5. The transfer efficiency was experimentally measured to be $83 \%$, which is similar to the ray-trace prediction of $86 \%$. The pump uniformity at the amplifier locations matches well with ray trace predictions as can be seen in the overlay of the lineouts in Fig. $5 \mathrm{~b}$.

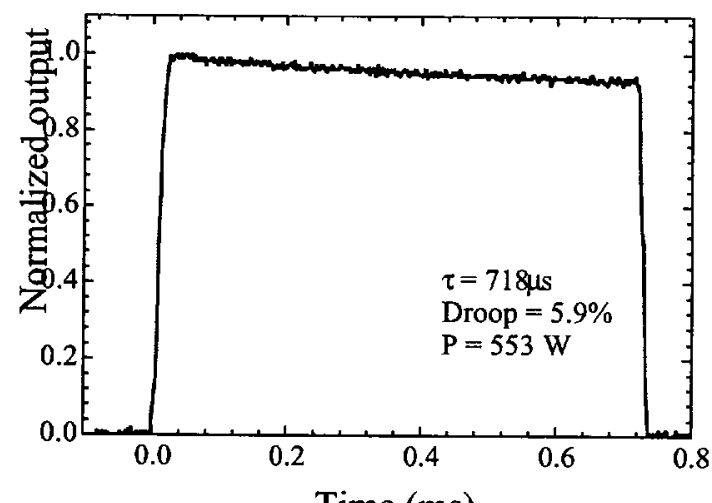

a)

c)

\section{FWHM}

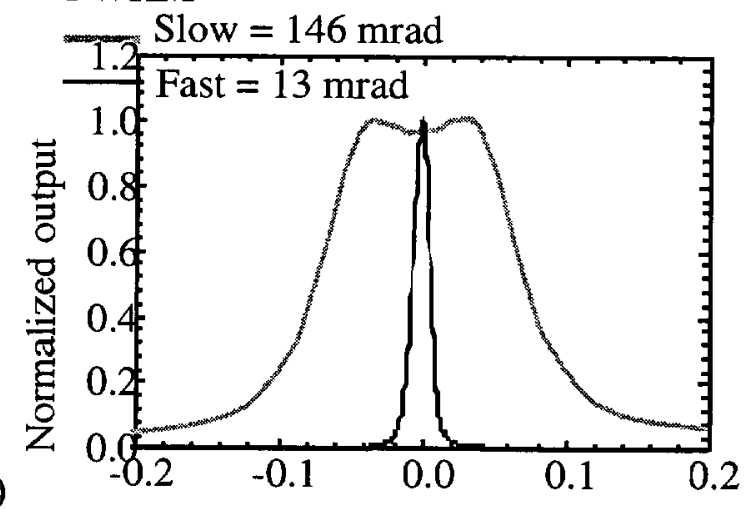

$$
\text { - * Yb:S-FAP absorption }
$$

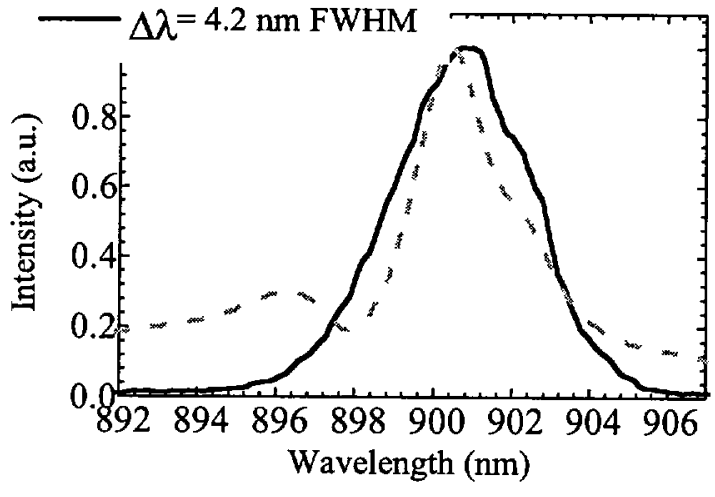

b)

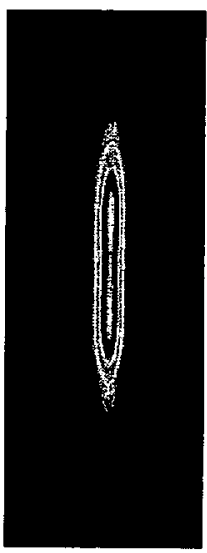

Fig. 4 a) The power droop of temporal pulsewidth for each backplane is within specifications $(<10 \%)$ for the current backplane cooling design. b) The linewidth of the diode backplanes overlaps well with Yb:S-FAP pump linewidth of $4.6 \mathrm{~nm} . \mathrm{c})$ The divergence matches predictions and ensures a longer Rayleigh range after the homogenizer. 
Our front end laser, based on a Continuum master oscillator power amplifier system, produces over $500 \mathrm{~mJ}$ in a $20 \mathrm{~ns}$ FWHM pulsewidth. The spatially gaussian profile will be apodized to produce a spatially flat-topped supergaussian profile. This front end laser will be used in preliminary gain experiments employing surrogate $\mathrm{Nd}$ :glass slabs to measure gain uniformity and wavefront distortion. These experiments will be used to validate beam propagation codes as well as the optical layout of the architecture and allow for fine-tuning the system before the amplifiers are assembled with Yb:S-FAP.
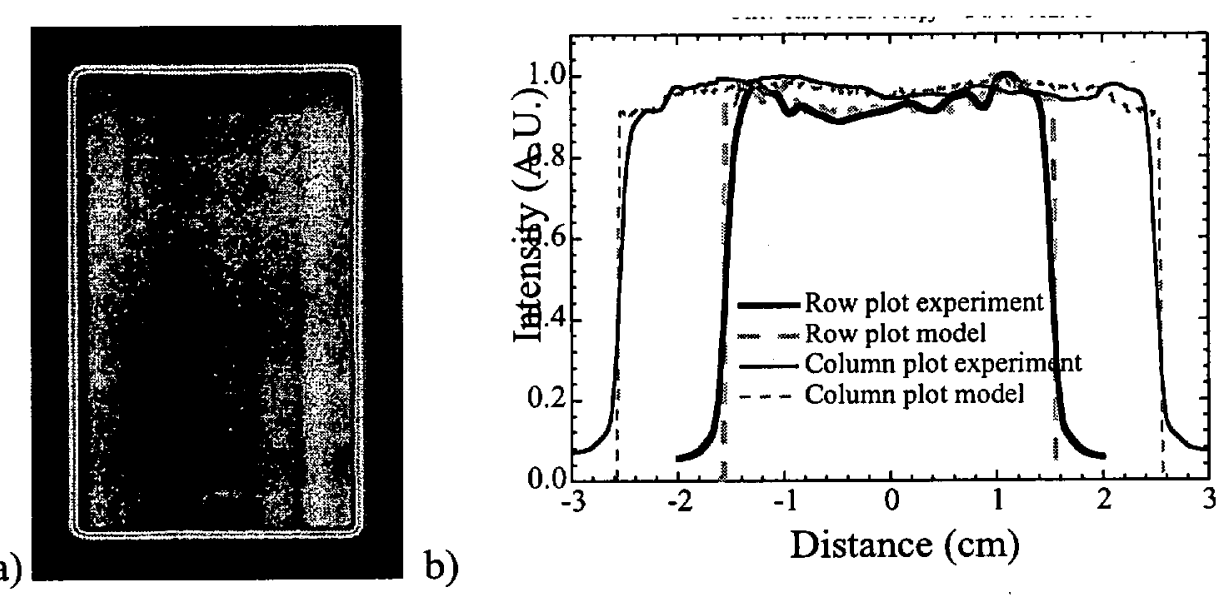

Fig. 5 a) A $4 \times 6 \mathrm{~cm}$ intensity plot of the output of the pump delivery system shows a nearly homogeneous output. b) Lineouts of the plot show the intensity variation is less than $\pm 3 \%$ in the vertical direction and approximately $\pm 8 \%$ in the horizontal direction.

\section{SUMMARY}

Initial measurements were reported for the Mercury laser system, a scalable driver for inertial fusion energy. The first $\mathrm{Yb}$ :S-FAP crystals of sufficient size to fabricate full aperture amplifiers have been grown with four crystals in various stages of fabrication. The first full diode backplane and pump delivery system was completed and tested with the following average results: $6 \%$ power droop, $4.2 \mathrm{~nm}$ spectral linewidth, far field divergence of 13 mrad and $146 \mathrm{mrad}$ microlensed and unmicrolensed axis respectively, and $83 \%$ transfer efficiency through the pump delivery system.

We would like to thank Mark Emanuel and Jay Skidmore for their prior work developing the laser diodes. This article is dedicated to the memory of Howard Powell for his unwavering support of the Mercury Laser and his personal commitment to inertial fusion energy. This work was performed under the auspices of the U.S. Department of Energy by the University of California Lawrence Livermore National Laboratory under contract No. W-7405-Eng-48.

\section{REFERENCES}

1. C. D. Orth, S. A. Payne, and W. F. Krupke, Nuc. Fus. 36, 75-116, (1996)

2. H. Matsui; T. Eguchi; T. Kanabe; M. Yamanaka; M. Nakatsuka; Y. Izawa; and S. Nakai, Review of Laser Engineering, 28, 176-81, (2000) (also T.Kawashima et al. in IFSA 2001).

3. A.J. Bayramian, C. Bibeau, K.I. Schaffers, J.K. Lawson, C.D. Marshall, and S.A. Payne, in OSA Trends in Optics and Photonics Series, M.M. Fejer, H. Injeyan, U. Keller, ed(s)., Vol. 26 of OSA Proceedings Series (Optical Society of America, Washington, D.C., 1999), 635-641.

4. J.A. Skidmore, B.L. Freitas, J. Crawford, J. Satariano, and others, Appl. Phys. Lett., 77, 10-12, (2000).

5. C.D. Marshall, L.K. Smith, S. Sutton, M.A. Emanuel, K.I. Schaffers, S. Mills, S.A. Payne, and W.F. Krupke, in OSA Trends in Optics and Photonics Series, S.A. Payne, C. Pollock, ed(s).,Vol. 1 of OSA Proceedings Series (Optical Society of America, Washington, D.C., 1996), 208-212.S 


\title{
High Average Power Yb:YAG Laser
}

\author{
Luis E. Zapata \\ Captain Steven M. Massey* \\ Raymond J. Beach \\ Stephen A. Payne
}

*United States Air Force

\begin{abstract}
We are working on a composite thin-disk laser design that can be scaled as a source of high brightness laser power for tactical engagement and other high average power applications. The key component is a diffusion-bonded composite comprising a thin gain-medium and thicker cladding that is strikingly robust and resolves prior difficulties with high average power pumping/cooling and the rejection of amplified spontaneous emission (ASE). In contrast to high power rods or slabs, the one-dimensional nature of the cooling geometry and the edge-pump geometry scale gracefully to very high average power. The crucial design ideas have been verified experimentally ${ }^{l}$. Progress this last year included: extraction with high beam quality using a telescopic resonator, a heterogeneous thin film coating prescription that meets the unusual requirements demanded by this laser architecture, thermal management with our first generation cooler. Progress was also made in design of a second-generation laser.
\end{abstract}

\section{INTRODUCTION}

With recent funding provided by the Joint Technology Office, our thin-disk laser project has gained momentum. We refer to this project as the "HiBriTE" laser an acronym for High Brightness Tactical Engagement. A new set of experiments has started and we report here a snapshot in time for the results of these ongoing tests while covering our planned tests. The recent experimental results give us confidence in our scalability and high brightness arguments. To affirm the viability of the concept, the first step is to demonstrate $300 \mathrm{~W}$ of sustained output. Beam quality issues at high average power are next in our agenda. Shown in Fig. 1 are the diode arrays, hollow lens ducts, and output coupler, in addition to a blow-up of the 15\% Yb:YAG / YAG composite laser disk situated on the cooler (soldered with indium). The inset picture in figure 1 shows finished, diffusion-bonded Yb:YAG/YAG composite laser gain media we are using in our tests. This is the equipment we are testing. It has already produced 260 watts in low duty factor quasi$\mathrm{cw}$ operation $(\sim 10 \%, 5 \mathrm{~ms}$ pulses $)$ and 50 watts in true $\mathrm{cw}$ operation. With the hardware upgrades enabled by the new funding, we will demonstrate our average power goal of 300 watts and tractable high brightness by using a telescopic resonator ${ }^{2}$. We have already collected data with a telescopic resonator at low duty factor and have generated low order gaussian modes of predictable high beam quality. We are nearing similar tests at high average power.

\section{THE THIN DISK ADVANTAGE}

One of the crucial advantages of the thin-disk design is that the thermal gradients are aligned with the beam propagation direction, so that they do not impart significant wavefront distortion onto the light field. To first order, the thin-disk laser geometry mitigates the effects of $\mathrm{dn} / \mathrm{dT}$ and stress-optic effects. A second order effect remains, relating to the pump uniformity. Deformations previously were the main source of wavefront error in a thin-disk however, because "stiffness" is proportional to the cube of the thickness. A major advantage of our approach over conventional thin-disk lasers is therefore the diffusion bonded undoped cap serving as a "stiffness" member for the HiBriTE laser-disk, keeping the deformations to a minimum. We will present interferometry data of a thermally loaded thin-disk-composite laser element, which we plan to benchmark with our thermo-mechanical/optics calculations. Another advantage provided by the diffusion bonded cap is that it allows for side pumping with laser diodes. Last but not least, the index of refraction matched cap layer also provides a larger volume which dilutes spontaneous emission greatly diminishing the adverse impact of amplified spontaneous emission (ASE). There is no downside to the undoped cap which simply rises to a constant temperature and has no impact on the heat handling advantage of the thin disk. 


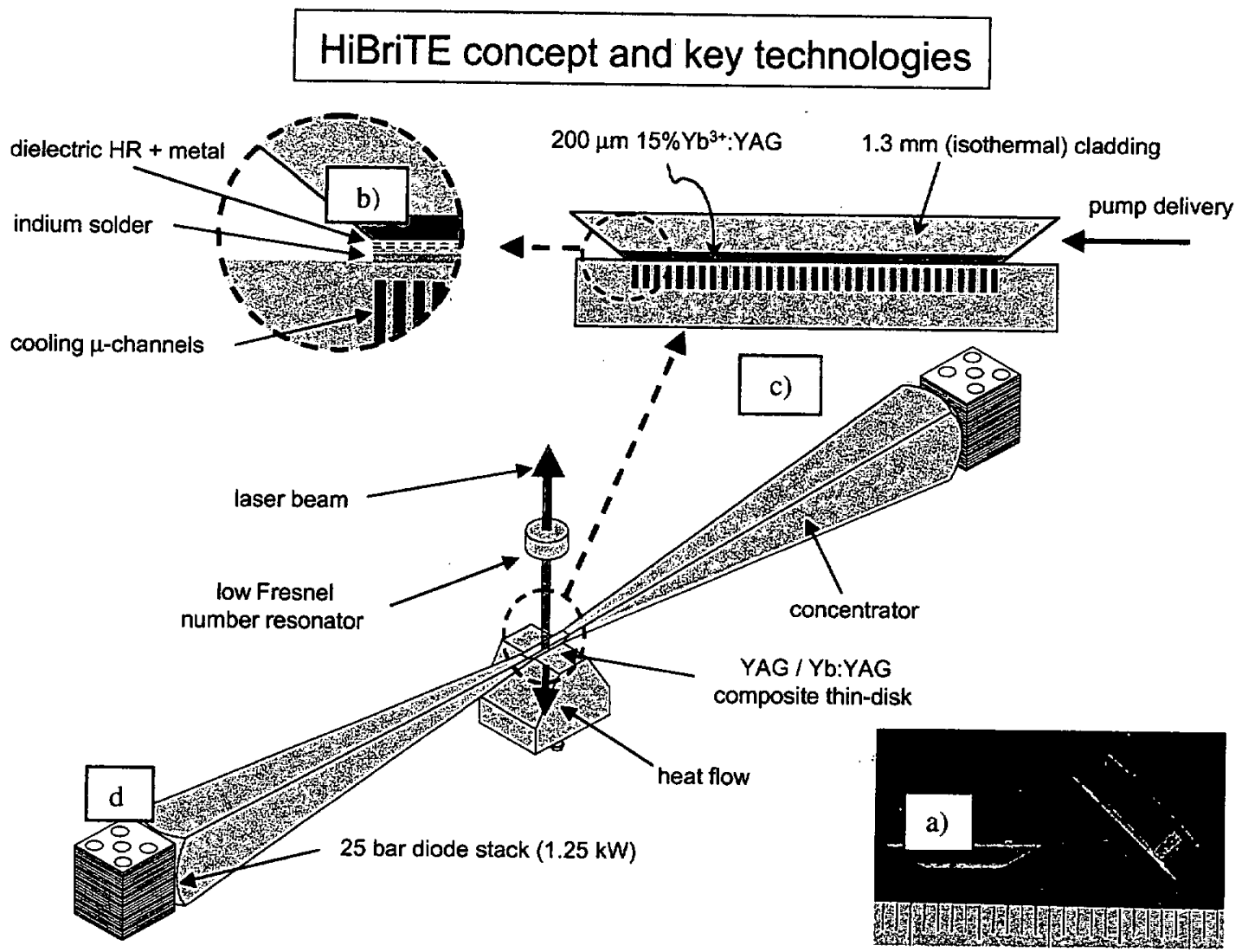

Figure 1.- The HiBriTE thin-disk-laser concept. The enabling technologies are depicted in this figure. (a) The Yb:YAG/YAG diffusion-bonded composite gain medium is cut and polished with specified shape; (b) a complex thin film coating we developed acts as the laser back-mirror and for pump containment, (c) Indium soldering conducts heat intensity $\geq 350 \mathrm{~W} / \mathrm{cm}^{2}$ to the c) high efficiency micro-channel cooler. (d) High brightness pump diodes and lens-duct pump delivery.

\section{INITIAL EXPERIMENTS -THEMAL MANAGEMENT}

The thin film coating was a subject of intense activity over the past year. Demands placed on the thin film by the unusual architecture are: 1) reflect at the laser wavelength $(1030 \mathrm{~nm})$ with high efficiency, 2) reflect diode pump light at $940 \mathrm{~nm}$ over a broad phase space, 3) be compatible with indium solder for low thermal impedance and 4) conduct a heat flux of $350 \mathrm{~W} / \mathrm{cm}^{2}$. Development took place in house. YAG coupons were coated with a dielectric stack followed by several metal films and finally an indium film prior to soldering to a gold coated CuW surface (similar to a cooler's surface). Two major problems were encountered: Adhesion of the metal film to the dielectric stack and eutectic mixing of indium with candidate metallic film during soldering. An additional metal film barrier was added to prevent indium migration during soldering. Adhesion of the films was taken care off by judicious choice of the metal films and surface activation via plasma sputtering of the dielectric surface prior to depositing the first metal layer. We now routinely coat and solder gain samples to coolers. We have tested one slab at a measured 590 $\mathrm{W} / \mathrm{cm}^{2}$ continuous heat flux with no signs of damage to the coatings.
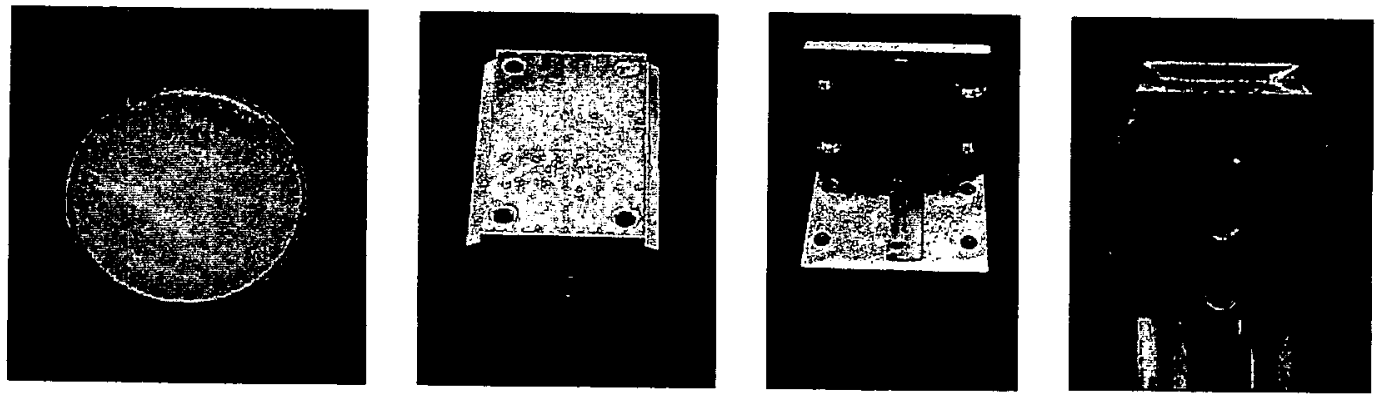

Figure 2. From left to right: first successful YAG coupon, ready cooler, precision solder fixture, fully coated slab and cooler tested at $590 \mathrm{~W} / \mathrm{cm}^{2}$ with no signs of damage. 


\section{INITIAL EXPERIMENTS -BEAM QUALITY}

The characteristic one-dimensional thermal gradient of a thin-disk laser can be exploited if we make the transverse dimension of the laser aperture the principal means of scaling the average power output. High radiance is required for tactical engagement missions and therefore a resonator capable of extracting with beam quality is required. Unstable resonators are not a good choice due to intrinsically low gain through the thin dimension of the gain medium. Since a high gain to loss ratio is required for efficient laser extraction, the resonator transverse mode must have low loss. The resonator Fresnel number $N_{f}=a^{2} /(L \cdot \lambda)$ (where $a$ is the aperture radius and $L$ the cavity length), determines the highest order Gaussian mode that can oscillate without significant diffractive loss $\left(\mathrm{N}_{\max }=\pi \cdot \mathrm{N}_{\mathrm{f}}\right.$ for a confocal resonator). A cavity with low Fresnel number is desired however; it is not practical to use the length of the resonator for mode selection. An intra-cavity telescope was first proposed by Steffen et al. and later analyzed and experimented upon by

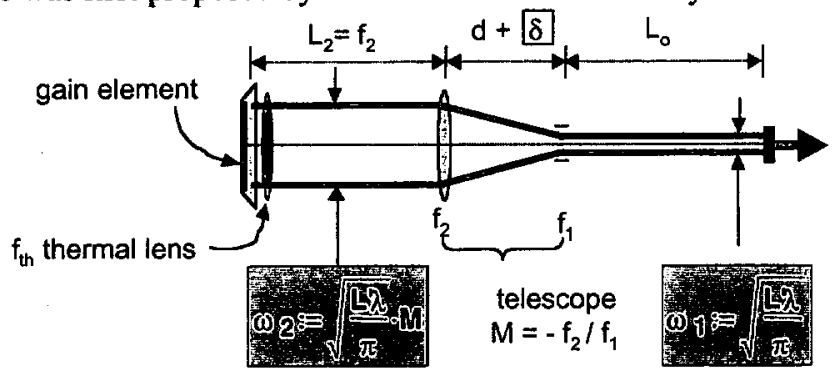

Figure 3-The telescopic resonator. The simple solutions of the special case shown above are both useful and illustrative. The thermal lens is considered attached to the back-mirror. The parameter $\delta$ is adjusted to compensate for thermal lensing as well as to bring it into stability.

Hanna ${ }^{3}$. A dynamically stable resonator with an effective length $M^{2} \cdot L_{0}$ can be realized (where $L_{0}$ is the length at the small beam end of the cavity and $M$ is the telescope magnification). Figure 3 shows the key features of the telescopic resonator. The telescopic resonator is an attractive choice for the HiBriTE laser for several reasons. One is the ability to easily adjust for thermal lensing under varied pumping conditions.

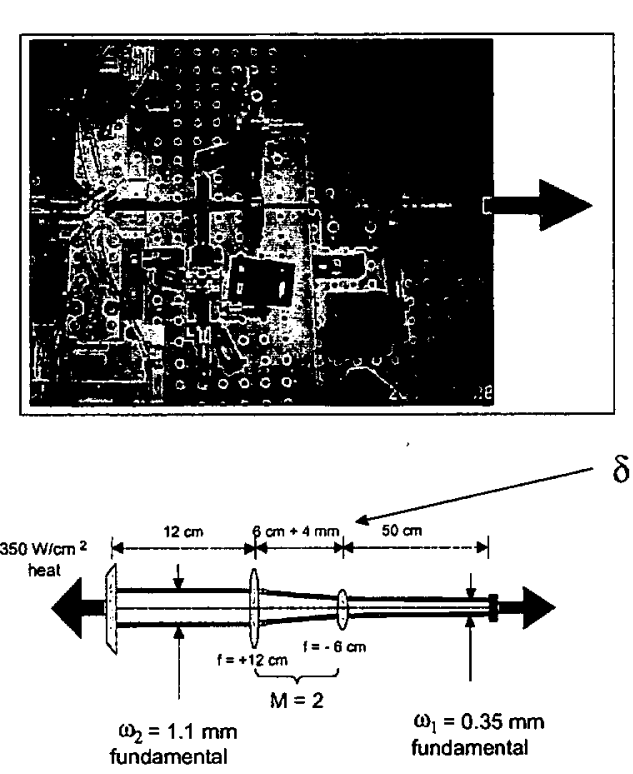

$$
\text { Pure modes Mixed modes }
$$
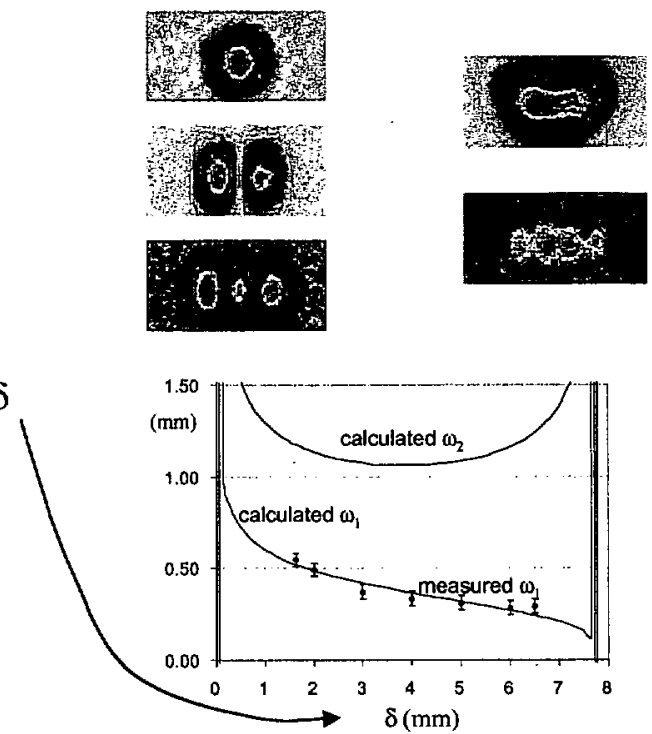

Figure 4-Clockwise from the top-left comer: top view picture of the HiBriTE laser and intracavity (Galilean) telescope; a sample of output modes ranging from TEM ${ }_{0,0}$ to TEM $M_{4,1}$ obtained adjusting the output coupler tilt for a given $L_{o}$ and $\delta$. The mixed modes were more prevalent away from $g_{1} \cdot g_{2}=$; the measured (vertical) beam parameter $\left(\omega_{2}\right)$ at the output coupler (the only one accessible) was compared to the theory; The desired operating stability point is found for operation. 
Another, as Hanna showed in his appendix, is that once adjusted, the diffraction losses produced are those of an equivalent confocal resonator, which offers the greatest degree of mode selectivity and insensitivity to thermal fluctuations. Figure 4 summarizes our initial experiments calibrating our laboratory telescopic resonator and verifying its mode selectivity. Experiments at average power will verify the dynamically stable operation of this resonator in the near future.

\section{PROGRESS WITH A SECOND GENERATION DESIGN}

The scaling to truly high average powers requires optical isolation "grooves" between gain "islands". The optically-passive volume adjacent to the gain-sheet drastically reduces the solid angle factor available for the internal fluorescence to experience gain however, there is a maximum laser aperture size that is limited by ASE due to unconfined photons traveling transversely within the thin gain medium. This aperture can be increased by strategically cut "grooves" into the thin material but not penetrating the "cap-disk". We feel encouraged by the data and believe that our thin-disk laser designs will enable a new class of high beam quality, high average power lasers. The future holds promise. We have calculated that a single ASE limited aperture based on YbAG should enable up to $8 \mathrm{~kW}$ of laser power while $>100 \mathrm{~kW}$ can be envisaged based on near contiguous placement of several such apertures. A reasonable next step in the near term based on standard $15 \% \mathrm{Yb}: Y A G$ was also investigated computationally. Figure 5 shows an ASE limited design with a hexagonal cross section pumped from 6 sides. The absorbed power distribution computed (ray-trace) peaks in the center of the aperture. The design can be optimized for efficient extraction when coupled with the suitable low order mode of a telescopic resonator.

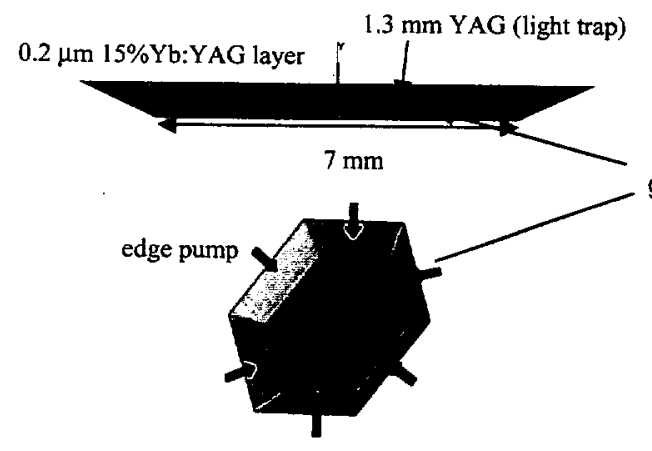

Six sides will be used to pump the thin disk
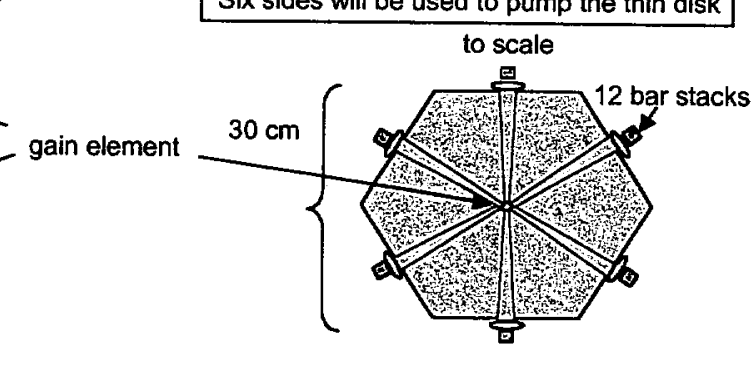

Absorbed power distribution
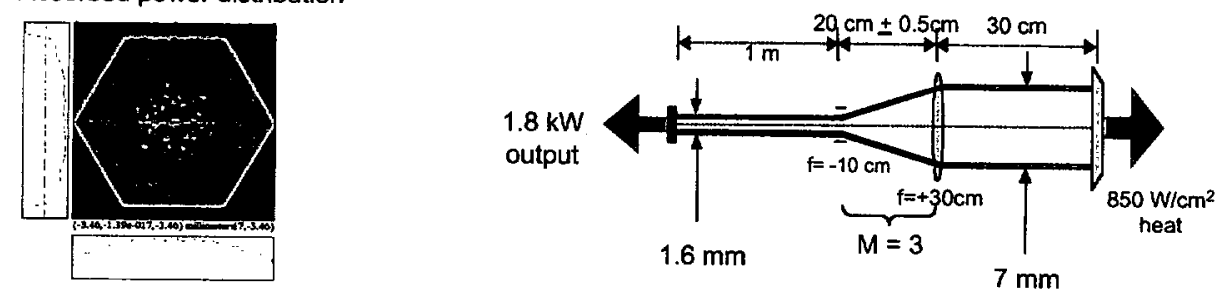

Figure 5-A reasonable next step using available crystals would be the ASE limited design shown here.

\footnotetext{
'Luis E. Zapata, Raymond J. Beach and Stehen A. Payne, Lawrence Livermore National Laboratory ,"Composite Thin-Disk Laser Scaleable to $100 \mathrm{~kW}$ Average Power Output and beyond”, SSLDTR-2000 Technical Digest

${ }^{2}$ D. C. Hanna, C. G. Sawyers, M. A. Yuratich, University of Southhampton, UK, "Telescopic resonators for largevolume TEMoo -mode operation", Optical and Quantum Electronics 13 (1981) 493-507
}

${ }^{3}$ J. Steffen, J. P. Lortscher and G. Herziger, ibid QE-8 (1972) 239-45 


\title{
Progress in the growth of $\mathrm{Yb}: \mathrm{S}-\mathrm{FAP}$ laser crystals
}

\author{
K.I. Schaffers*,1 J.B. Tassano, P.A. Waide, S.A. Payne, R.C. Morris \\ Lawrence Livermore National Laboratory, P.O. Box 808, L-482, Livermore, CA 94551, USA
}

\begin{abstract}
Growth of $\mathrm{Yb}: \mathrm{S}-\mathrm{FAP}\left[\mathrm{Yb}^{3+}: \mathrm{Sr}_{5}\left(\mathrm{PO}_{4}\right)_{3} \mathrm{~F}\right]$ crystals is being studied for $1.047 \mu \mathrm{m}$ laser operation. These crystals are not yet routinely available and the growth of high optical quality, low loss crystals poses a challenge due to a number of crystal growth issues, including, cloudiness, bubble core defects, anomalous absorption, low-angle grain boundaries, and cracking. At this time, a growth process has been formulated to simultaneously eliminate or greatly diminish each of the defects to yield high quality material. Laser slabs of dimension $4.0 \times 6.0 \times 0.75 \mathrm{~cm}$ are being fabricated from subscale pieces using the diffusion bonding technique.i
\end{abstract}

Keywords: A2. Czochralski method; B1. Inorganic compounds; B1. Oxides; B3. Solid state lasers

\section{Introduction}

Host materials doped with the $\mathrm{Yb}^{3+}$ ion have sparked a great deal of interest in the last several years for their use as solid-state lasers that can be pumped by InGaAs-based diode lasers [1,2]. These materials have longer storage lifetimes than $\mathrm{Nd}^{3+}$. doped hosts and can have favorable cross-sections [3]. For example, the material and laser performance have been examined for $\mathrm{Yb}$ : YAG [4] and the $\mathrm{Yb}$ : fluoroapatite (FAP) family of crystals [5$7]$ including, $\mathrm{Ca}_{5}\left(\mathrm{PO}_{4}\right)_{3} \mathrm{~F}$ (C-FAP), $\mathrm{Sr}_{5}\left(\mathrm{PO}_{4}\right)_{3} \mathrm{~F}$ (S-FAP), $\quad \mathrm{Ca}_{5-x} \mathrm{Sr}_{x}\left(\mathrm{PO}_{4}\right)_{3} \mathrm{~F}, \quad \mathrm{Sr}_{5-x} \mathrm{Ba}_{x}\left(\mathrm{PO}_{4}\right)_{3} \mathrm{~F}$, and $\mathrm{Sr}_{5}\left(\mathrm{VO}_{4}\right)_{3} \mathrm{~F}$. Although $\mathrm{Yb}: \mathrm{YAG}$ is much

${ }^{*}$ Corresponding author. + 1-925-422-5084; fax; + 1-925-4236195.

E-mail address: schaffers1@linl.gov (K.I. Schaffers).

'Work done under the auspices of the US Department of Energy by the University of California Lawrence Livermore National Laboratory under Contract W-7405-ENG-48. better suited for high power/high thermal load operations because of its significantly better thermal properties, $\mathrm{Yb}$ :fluoroapatites are well suited for diode pumping in moderate thermal load applications owing to the relaxed diode brightness requirement and the $3 \times$ greater gain cross-section. The laser performance of $\mathrm{Yb}: \mathrm{S}$ FAP has been investigated for use in a diode pumped gas-cooled-slab laser geometry [8]. It was shown to produce $50 \mathrm{~W}$ of optical power at $1047 \mathrm{~nm}$ with a laser output efficiency of $51 \%$ with a laser-diode array pump source operating at $900 \mathrm{~nm}$. Currently there is interest in this crystal for a $100 \mathrm{~J}, 1 \mathrm{~kW}, 1 \mathrm{~ns}$ gas-cooled slab laser system (Mercury Laser). [9]. Yb:S-FAP crystals are not yet routinely available and the growth of high optical quality, low loss crystals poses a challenge due to a number of crystal growth issues, including cloudiness, bubble core defects, anomalous absorption, cracking, and low-angle grain boundaries. A significant effort has been made to 
understand the defect chemistry of $\mathrm{Yb}: \mathrm{S}-\mathrm{FAP}$ and a growth process has been developed to yield high optical quality crystals.

\section{Experimental}

Crystals of $\mathrm{Yb}: \mathrm{S}-\mathrm{FAP}$ are grown by using the Czochralski method from a $\mathrm{SrF}_{2}$-rich melt. Growth is done in a 4 in. diameter crucible having a height of $4 \mathrm{in}$. at $1810^{\circ} \mathrm{C}$ in an inert flowing nitrogen atmosphere with rotation rates from $6-$ $15 \mathrm{rpm}$ and a pull rate of $0.5 \mathrm{~mm} / \mathrm{h}$. As described later in this article, excess $\mathrm{SrF}_{2}$ in the melt has been chosen because it produces clear crystals with no cloudiness. The melt is prepared by decomposing $\mathrm{SrHPO}_{4}, \mathrm{SrCO}_{3}$, and $\mathrm{Yb}_{2} \mathrm{O}_{3}$ starting materials to yield a $\mathrm{Yb}$-doped $\left.\mathrm{Sr}_{3} \mathrm{PO}_{4}\right)_{2}$ melt to which $\mathrm{SrF}_{2}$ is added to yield the appropriate composition. The pre-decomposition reaction prevents $\mathrm{SrF}_{2}$ from oxidizing to $\mathrm{SrO}$ thereby removing fluorine from the melt. $\mathrm{Yb}_{2} \mathrm{O}_{3}$ has been typically used as the dopant to aid in the charge compensation process where a $\mathrm{Yb}^{3+}-\mathrm{O}^{2-}$ ion pair exchanges for a $\mathrm{Sr}^{2+}-$ $\mathrm{F}^{-}$pair [10]. An excess of $\mathrm{Yb}_{2} \mathrm{O}_{3}$ is added to the starting composition to accommodate the approximate $12 \%$ distribution coefficient from melt to crystal. Current boule dimensions are $2.8 \mathrm{~cm}$ diameter by $7 \mathrm{~cm}$ length grown along the $c$-axis. Efforts are centered around growing crystals of sufficient size to produce pieces for diffusion bonding to form a $4.0 \mathrm{~cm} \times 6.0 \mathrm{~cm}$ ( $c$-axis) $\times 0.75 \mathrm{~cm}$ slab for the Mercury Laser being developed at Lawrence Livermore National Laboratory.

\section{Defect chemistry and growth strategies}

A number of defects in $\mathrm{Yb}: \mathrm{S}-\mathrm{FAP}$ crystals have been overcome to produce high quality material for laser slabs. Five prominent defects encountered are cloudiness in as-grown boules, bubble core defects, low-angle grain boundaries, anomalous absorption, and cracking. An understanding of each of these defects has been achieved in an effort to engineer methods to eliminate or sufficiently reduce their magnitude to lessen the threat to laser performance.
A $\mathrm{SrF}_{2}$-rich melt composition has proven effective in eliminating the cloudiness in boules of $\mathrm{Yb}: \mathrm{S}-\mathrm{FAP}$ grown from a near-stoichiometric melt. The cloudiness is believed to be a result of second-phase particles that precipitate out in the crystal lattice. The identity of the second phase has not yet been verified, but it is likely of phosphatebased origin resulting from a deficiency of $\mathrm{SrF}_{2}$ at the growth interface with the evaporation from the melt surface. As a result, an excess of $\mathrm{SrF}_{2}$ beyond the stoichiometric composition is added to yield $33 \mathrm{~mol} \%$ total fluoride in the melt. A $33 \mathrm{~mol} \%$ $\mathrm{SrF}_{2}$ composition in the $\mathrm{Sr}_{3}\left(\mathrm{PO}_{4}\right)_{2}: \mathrm{SrF}_{2}$ phase system is equivalent to a congruently melting compound with the stoichiometry $\mathrm{Sr}_{7}\left(\mathrm{PO}_{4}\right)_{4} \mathrm{~F}_{2}$. However, Inductively-Coupled Plasma/Mass Spectrometry has identified the composition of the crystals to be $\mathrm{Sr}_{5}\left(\mathrm{PO}_{4}\right)_{3} \mathrm{~F}$. These phase relationships have been extrapolated from the $\mathrm{Ca}_{3}\left(\mathrm{PO}_{4}\right)_{2}: \mathrm{CaF}_{2}$ phase diagram [11] since the phase diagram for the strontium analog does not yet exist. It has been found that the amount of excess $\mathrm{SrF}_{2}$ that is added is significant where $<33 \mathrm{~mol} \%$ yields cloudy crystals and large excesses lead to the formation of new, long "stringtype" defects that propagate perpendicular to the $c$-axis at the core. However, compositions near $33 \mathrm{~mol} \%$ are optimal for eliminating the supersaturation of the second phase and clear crystals are produced.

A growth orientation along the $c$-axis for $\mathrm{Yb}: \mathrm{S}-$ FAP crystals is critical for eliminating the anomalous absorption that occurs as a broad band from approximately $925-1000 \mathrm{~nm}$ with a peak at $975 \mathrm{~nm}$. Crystals grown along both the $a$-axis and $45^{\circ}$ to the $c$-axis intermittently display the unwanted absorption whereas there is no evidence for this absorption in the $c$-axis boules that have been grown to date. The absorption is attributed to the $\mathrm{Yb}^{3+}$ ion on a second site in the crystal lattice, based on excitation and emission spectra. The spectrum most closely resembles that of $\mathrm{Yb}^{3+}$ in a phosphate environment such as is seen in $\mathrm{LuPO}_{4}$ [3], where there are no characteristics of the desired spectrum arising from the vibration interactions of the $\mathrm{Yb}^{3+}-\mathrm{O}^{2-}$ charge compensation pair [10]. A possible explanation for the elimination of the anomalous absorption in $c$-axis crystals 


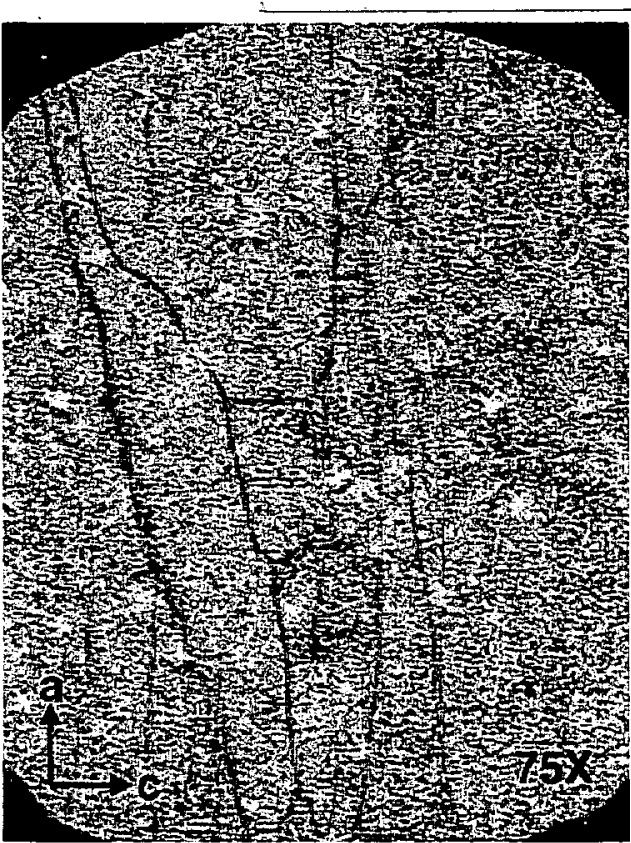

Fig. 1. Five defect structures are present in Yb:S-FAP crystals including: (a) bubble core; (b) anomalous absorption; (c) cloudiness; (d) cracking and (e) low-angle grain boundaries.

relates to the presence of $\mathrm{F}^{-}$ion "conduction" channels that parallel the $c$-axis in the apatite lattice. The preferred orientation may allow for easier charge compensation substitution of the $\mathrm{Yb}^{3+}-\mathrm{O}^{2-}$ pair for the $\mathrm{Sr}^{2+}-\mathrm{F}^{-}$pair giving rise to the appropriate absorption spectrum thereby reducing the probability of $\mathrm{Yb}$ achieving charge compensation by another mechanism.

By growing "seed extensions" and choosing a small cone angle to maintain a stable growth interface, low-angle grain boundaries have been eliminated. The grain boundaries appear as slight shifts in the refractive index or waves running through the crystal in sheets oriented perpendicular to the $c$-axis (see Fig. 1). Ref. [12] provides an extensive explanation of the crystal lattice effects that lead to the formation of these grain boundaries and therefore, these effects will not be reiterated here. However, it is understood that the dislocations are formed by propagation from the seed to the crystal and strain-induced deformations from an unstable growth interface [13]. The growth of a seed extension makes it possible to grow beyond any grain boundaries propagating from the seed or formed during the seeding process. In addition, a gradual increase in diameter in the cone section helps to maintain stable growth conditions reducing hoop stress preventing new grain boundaries from forming. Further, $c$-axis growth makes it easier to grow beyond grain boundaries because they propagate perpendicular to the growth direction.

Crystals of $\mathrm{Yb}: \mathrm{S}-\mathrm{FAP}$ are grown in a higher thermal gradient furnace and at small $2-3 \mathrm{~cm}$ diameter to stabilize the growth interface and control the formation of bubble core defects. The bubble core is attributed to constitutional supercooling where a supercooled liquid is formed from concentration gradients of rejected melt components pushed along in front of the advancing interface. In the $\mathrm{Yb}: \mathrm{S}$-FAP system, $\mathrm{SrF}_{2}$ and $\mathrm{Yb}_{2} \mathrm{O}_{3}$ are in excess to alleviate cloudiness and account for the distribution coefficient of dopant, respectively, and therefore, may be of higher concentration near the interface after being turned away from the growing crystal. Instabilities deviating from steady-state conditions, resulting from this phenomena, can then cause small protuberances that can come in contact with the supercooled liquid and grow very fast away from the interface into the melt. Eventually these protuberances grow together and close off, trapping low melting temperature liquid, which contracts and leaves a void upon cooling (see Fig. 2). SEM/EDX analysis has pinpointed the approximate composition associated with the bubbles to be $\mathrm{Yb}_{2} \mathrm{O}_{3}$ and $\mathrm{Sr}_{3}\left(\mathrm{PO}_{4}\right)_{2}$. To prevent the growth of any protuberances formed at the interface, we have used higher thermal gradients, typically axial gradients of $\sim 35^{\circ} \mathrm{C} / \mathrm{cm}$ near the melt. In addition, it has been observed that the introduction of the bubble core consistently begins at diameters of approximately $\geqslant 3.0 \mathrm{~cm}$. Recent growths of smaller, $2.2 \mathrm{~cm}$ diameter boules, in the higher gradient have completely eliminated the bubble core defects.

Cracking is managed by not separating the crystals from the melt during the cooling process and by significant reduction of defects. The main source of cracking was related to high thermal gradients in the crystals, especially at larger $(5 \mathrm{~cm})$ 


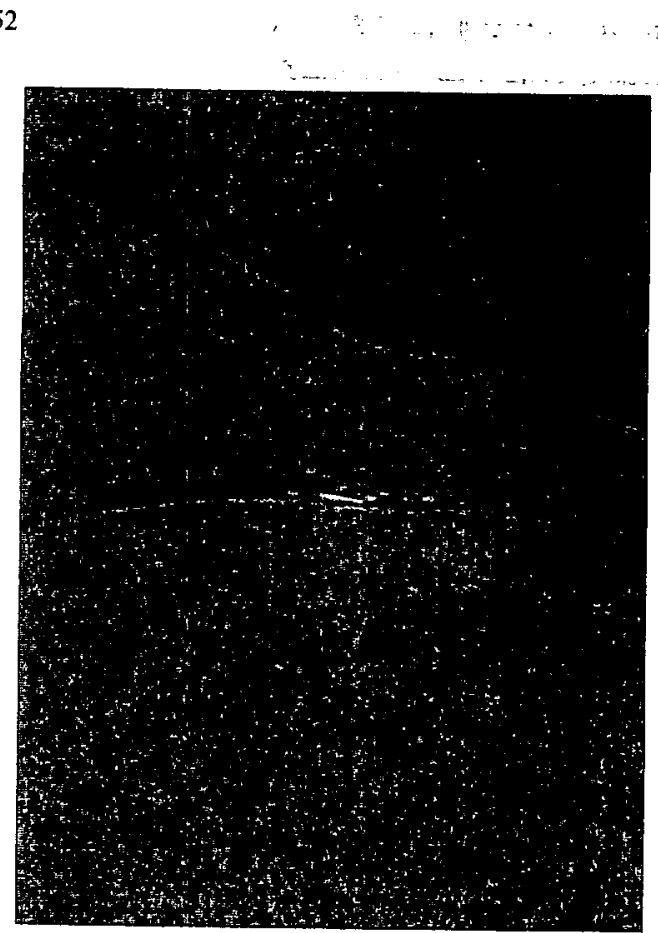

Fig. 2. Photograph of the bubble core defects in $\mathrm{Yb}: \mathrm{S}-\mathrm{FAP}$ crystals at a magnification of $78 \times$.

diameters, as well as numerous bubble core defects. Cooling the crystals still attached to the melt reduces the thermal stress along the length of the boule by using the large thermal load of the melt to conduct heat into the crystal. As a result, cracking is no longer an issue in the growth of small diameter $\mathrm{Yb}$ : S-FAP crystals.

\section{Conclusions}

A growth process has been developed to eliminate each of the five defects encountered in $\mathrm{Yb}: \mathrm{S}-\mathrm{FAP}$ crystals! Excess $\mathrm{SrF}_{2}$ in the initial melt and $c$-axis growth have been successful in preventing cloudiness and the anomalous absorption. Also, seed extensions, higher thermal gradients, and small diameter crystals have proven effective in handling the formation of grain boundaries and bubble core defects. Finally, with the significant reduction in defects together with cooling the crystals while attached to the melt, cracking is no longer an issue. Boules grown using this method

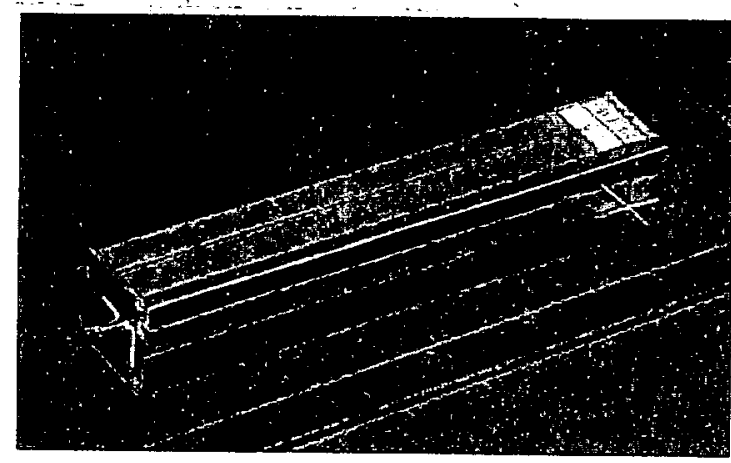

Fig. 3. Photograph of a high quality, polished section of an $\mathrm{Yb}: \mathrm{S}-\mathrm{FAP}$ crystal.

yield small crystals with high optical quality, as shown in Fig. 3. The diffusion bonding technology will be used to stitch together sub-slabs to form a full size $(4 \times 6 \mathrm{~cm})$ slab. $^{2}$ This process has been proven successful in test samples where there is no visible evidence of the bond or by using interferometry. Currently, high quality sub-slabs are being produced for laser testing.

\section{Acknowledgements}

The authors wish to acknowledge Scientific Materials, Inc., for their collaborative work on this project and for performing the SEM/EDX measurements that identified the bubble core defects. We greatly appreciate the help of Camille Bibeau and Andrew Bayramian for their advice and for performing all of the spectroscopy and interferometry throughout the development of $\mathrm{Yb}: \mathrm{S}-\mathrm{FAP}$ crystals. In addition, we are indebted to Peter Thelin, Ron Vallene, and James Embree, for their expertise in cutting and polishing crystals for inspection and optical studies.

\section{References}

[1] A.R. Reinberg, L.A. Riseberg, R.M. Brown, R.W. Wacker, W.C. Holton, Appl. Phys. Lett. 19 (1971) 11.

\footnotetext{
${ }^{2}$ All diffusion bonding is done at Onyx Optics, Inc. in Dublin, CA.
} 
[2] T.Y. Fan, Opt. Lett. 16 (1991) 1089.

[3] L.D. DeLoach, S.A. Payne, L.L. Chase, L.K. Smith, W.L. Kway, W.F. Krupke, IEEE J. Quantum Electron. 29 (1993) 1179.

[4] T.Y. Fan, S. Klunk, G. Henien, Opt. Lett. 18 (1993) 423.

[5] S.A. Payne, L.K. Smith, L.D. DeLoach, W.L. Kway, J.B. Tassano, W.F. Krupke, IEEE J. Quantum Electron. 30 (1) (1994) 170.

[6] C.D. Marshall, L.K. Smith, R.J. Beach, M.A. Emanuel, K.I. Schaffers, J. Skidmore, S.A. Payne, B.H.T. Chai, IEEE J. Quantum Electron. 32 (4) (1996) 650.

[7] K.I. Schaffers, A.J. Bayramian, C.D. Marshall, J.B. Tassano, S.A. Payne, in: C.R. Pollock W.R. Bosenberg (Eds.), OSA Trends in Optics and Photonics on Advanced Solid State Lasers, January 1996 Conference, p. 420.

[8] C.D. Marshall, L.K. Smith, S. Sutton, M.A. Emanuel, K.I. Schaffers, S. Mills, S.A. Payne, W.F. Krupke, B.HT. Chai, in: S.A. Payne, C.R. Pollock (Eds.), OSA Trends in Optics Photonics on Advanced Solid State Lasers, January 1996 Conference p. 208.

[9] C.D. Marshall, R.J. Beach, C. Bibeau, C.A. Ebbers, M.A. Emanuel, E.C. Honea, W.F. Krupke, S.A. Payne, H.T. Powell, K.I. Schaffers, J.A. Skidmore, S.B. Sutton, Advanced Solid State Lasers Conference Technical Digest, 1998, p. 76.

[10] L.D. DeLoach, S.A. Payne, W.L. Kway, J.B. Tassano, S.N. Dixit, W.F. Krupke, J. Luminescence 62 (1994) 85.

[11] J. Berak, I. Tomczak-Hudyna, Rocz. Chem. 12 (1972) 2157.

[12] G.M. McManus, R.H. Hopkins, W.J: Takei, J. Appl. Phys. 40 (1) (1969) 180.

[13] Private communication with Robert Morris, consultant, 1998. 


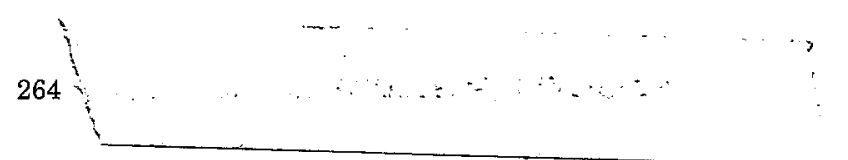

\title{
Optical properties of $\mathrm{Dy}^{3+}$ - and $\mathrm{Nd}^{3+}$-doped $\mathrm{KPb}_{2} \mathrm{Cl}_{5}$
}

\author{
M. C. Nostrand, R. H. Page, and S. A. Payne \\ Lawrence Livermore National Laboratory, University of California, L-482, Livermore, California 94550
}

\author{
L. I. Isaenko and A. P. Yelisseyev
}

Design and Technological Institute for Monocrystals, Siberian Branch, Russian Academy of Sciences, 43 Russkaya, Novosibirsk 630058, Russia

\begin{abstract}
$F$
Optical properties including radiative quantum efficiencies, cross-relaxation coefficients, refractive index, energy-gap law parameters, and maximum phonon energy are presented for a new low-phonon-frequency, nonhygroscopic host crystal potassium lead chloride $\left(\mathrm{KPb}_{2} \mathrm{Cl}_{5}\right)$ doped with $\mathrm{Dy}^{3+}$ and $\mathrm{Nd}^{3+}$. Assuming that the total decay rate $(W)$ from each level is composed of radiative $\left(A^{\mathrm{rad}}\right)$, multiphonon $\left(W^{\mathrm{MP}}\right)$, and concentrationdependent cross-relaxation ( $\left.W^{c}\right)$ rates $\left(W=A^{\text {rad }}+W^{\mathrm{MP}}+W^{c}\right.$ ), we determined radiative quantum efficiencies $\left(\eta^{\mathrm{rad}}=A^{\mathrm{rad} / W}\right)$ from emission data for five samples of various $\mathrm{Dy}^{3+}$ concentrations $\left(N_{0}\right)$. These results were compared with values calculated from a Judd-Ofelt analysis of the absorption spectrum. This technique required identification of cross-relaxation pathways. A cross-relaxation coefficient $k=1.83 \times 10^{-37} \mathrm{~cm}^{6} \mathrm{~s}^{-1}$ (where $W^{c}=k N_{0}^{2}$ ) was measured for the $\mathrm{Dy}^{3+}{ }^{6} \mathrm{H}_{9 / 2}+{ }^{6} F_{11 / 2}$ level. The measured multiphonon decay rates yielded energy-gap law ( $\left.W^{\mathrm{MP}}[\Delta E] \approx B \exp [-\beta \Delta E]\right)$ parameters $B=3.72 \times 10^{9} \mathrm{~s}^{-1}$ and $\beta=1.16$ $\times 10^{-2} \mathrm{~cm}$, indicating that laser action should be possible to near $9 \mu \mathrm{m}\left(\Delta E=1100 \mathrm{~cm}^{-1}\right)$ in this laser host at ronm temperature.

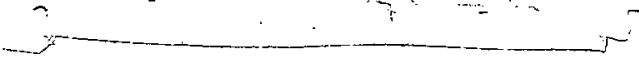

\section{INTRODUCTION}

Solid-state host materials with low maximum phonon energies and high-luminescence quantum yields have been subjects of a great deal of research for applications in $1.3-\mu \mathrm{m}$ telecommunications amplifiers and longwavelength $(4-10-\mu \mathrm{m})$ lasers. ${ }^{1-5}$ We recently demonstrated 4.3- $\mu \mathrm{m}$ laser action in $\mathrm{CaGa}_{2} \mathrm{~S}_{4}: \mathrm{Dy}^{3+}, 6$ and 4.8and $4.9-\mu \mathrm{m}$ emission was recently observed in $\mathrm{Tb}^{3+}$ - and $\mathrm{Ho}^{3+}$-doped gallium lanthanum sulfide (GLS) glass, respectively. ${ }^{7,8}$ High quantum efficiency of these devices requires low-phonon-energy hosts for prevention of luminescence quenching. A rule of thumb indicates that, for acceptable quantum efficiency for emission from a given energy level, the energy gap to the next lowest level must span at least four or five (maximal energy) phonons. Sulfide and chloride hosts have received the most attention because oxide and fluoride hosts typically have maximum phonon energies in excess of $500 \mathrm{~cm}^{-1}$ (rendering them inefficient above $4 \mu \mathrm{m}$ ). Currently, optical parametric oscillator (OPO) systems are typically used to cover wavelengths beyond $4 \mu \mathrm{m}$. They incorporate both nonlinear media and pump sources, leading to some complexity and sensitivity in the optical system. These challenges could be mitigated if direct solid-state mid-IR lasers were found. The longest wavelength of which we are aware in a room-temperature, solid-state host medium is $7.2 \mu \mathrm{m}$ in $\mathrm{Pr}^{3+}$-doped $\mathrm{LaCl}_{3},{ }^{9}$ a low-phonon-energy chloride host that is notoriously moisture sensitive. Identifying a lowphonon-energy crystal that incorporates rare-earth ions and resists attack by moisture has been difficult. We have identified $\mathrm{KPb}_{2} \mathrm{Cl}_{5}$ as a promising new host for mid-IR applications. ${ }^{10-14}$ Previous studies were concerned with $\mathrm{KPb}_{2} \mathrm{Cl}_{5}$ as the luminescence mechanism of divalent lead emission centers that are important for visualization of UV radiation ${ }^{15}$ and as a low-phonon host for trivalent erbium. ${ }^{16}$ This host is not hygroscopic, incorporates rare-earth ions, and has a very low maximum phonon frequency $\left(203 \mathrm{~cm}^{-1}\right)$. Direct determination of its quantum efficiencies, decay rates, and optical properties is important in the evaluation of this host as a candidate for applications that require mid-IR operation. In this paper we study five samples of potassium lead chloride doped with trivalent dysprosium, $\mathrm{KPb}_{2} \mathrm{Cl}_{5}: \mathrm{Dy}^{3+}$, to assess some of its capabilities and limits. Inasmuch as the study of this low-phonon-energy host is in its early stages, it is important in establishing that $\mathrm{KPb}_{2} \mathrm{Cl}_{5}$ is a credible laser host to note that laser action in $\mathrm{KPb}_{2} \mathrm{Cl}_{5}: \mathrm{Dy}^{3+}$ at $2.4 \mu \mathrm{m}$ (Ref. 17) and in $\mathrm{KPb}_{2} \mathrm{Cl}_{5}: \mathrm{Nd}^{3+}$ at $1.06 \mu \mathrm{m}$ (Ref. 18) was reported previously.

This paper consists of five sections. In Section 1 we describe the crystal growth and physical properties of $\mathrm{KPb}_{2} \mathrm{Cl}_{5}$. Section 2 includes the spectroscopic data, Judd-Ofelt analysis, and concentration-dependent energy-transfer rates of $\mathrm{KPb}_{2} \mathrm{Cl}_{5}: \mathrm{Dy}^{3+}$. The quantum efficiency model for $\mathrm{KPb}_{2} \mathrm{Cl}_{5}: \mathrm{Dy}^{3+}$ is developed in Section 3 . Spectroscopic and quantum efficiency data for $\mathrm{KPb}_{2} \mathrm{Cl}_{5}: \mathrm{Nd}^{3+}$ are given in Section 4. We use the results of Sections 3 and 4 in Section 5 to determine the energygap dependence of the nonradiative decay to be able to predict the maximum room-temperature laser wavelength achievable in $\mathrm{KPb}_{2} \mathrm{Cl}_{5}$. 
Table 1. $\mathrm{Dy}^{3+}$ Concentrations of the Five Samples Used in Our Study ${ }^{\alpha}$

\begin{tabular}{ccc}
\hline & \multicolumn{2}{c}{$\mathrm{Dy}^{3+}$ Concentration, $N_{0}$} \\
\cline { 2 - 3 } Sample Number & $\times 10^{20} \mathrm{~cm}^{-3}$ & mol. \% \\
\hline 1 & 0.35 & 0.76 \\
2 & 0.40 & 0.87 \\
3 & 0.43 & 0.93 \\
4 & 0.54 & 1.17 \\
5 & 0.73 & 1.59 \\
\hline
\end{tabular}

a The concentration of sample 1 was measured directly, and those of samples 2-5 were determined by comparative absorption.

\section{CRYSTAL GROWTH AND PHYSICAL PROPERTIES}

$\mathrm{KPb}_{2} \mathrm{Cl}_{5}$ is biaxial and belongs to the monoclinic crystal class (space group $P 2_{1} / c-C_{2 h}^{5}$ ) with lattice parameters $a$ $=0.8831 \mathrm{~nm}, b=0.7886 \mathrm{~nm}, c=1.2430 \mathrm{~nm}, \alpha=\gamma$ $=90^{\circ}$, and $\beta=90^{\circ} 8^{\prime} .{ }^{15}$ The $\beta$ value near $90^{\circ}$ indicates that the crystal is nearly orthorhombic.

Single crystals of $\mathrm{KPb}_{2} \mathrm{Cl}_{5}$ (melting point, $434^{\circ} \mathrm{C}$ ) typically $2.5 \mathrm{~cm}$ long and $8 \mathrm{~mm}$ in diameter were grown at the Design and Technological Institute for Monocrystals in Novosibirsk, Russia, by the Bridgman technique in a twozone furnace with a vertical gradient of $20 \% \mathrm{~cm}$. The $\mathrm{Dy}^{3+}$ was doped onto the $\mathrm{Pb}^{2+}$ site, with $\mathrm{K}^{+}$vacancies assumed to be responsible for charge compensation. We found that in $\mathrm{KPb}_{2} \mathrm{Cl}_{5}$ there are four nonequivalent sites of $\mathrm{Pb}^{2+}{ }^{15,19}$ The $\mathrm{Dy}^{3+}$ content did not exceed $3 \mathrm{~mol}$. \% in the melt, and the segregation coefficient was determined to be $\sim 1$, based on measurements of bulk $\mathrm{Dy}^{3+}$ content with the use of an inductively coupled plasma mass spectrometer.

The refractive index was measured with a Metricon prism coupler along two orthogonal axes of an oriented crystal with two orthogonal polarizations of a $\mathrm{He}-\mathrm{Ne}$ laser. By rotating the crystal about each axis, we could determine maximum and minimum indices for each polarization. By this method, we found that $n_{z}=2.019$ \pm 0.001 and $n_{x}=1.982 \pm 0.001$, giving $\Delta n=0.037$ \pm 0.003 (it appears that $n_{x} \approx n_{y}$ ).

Five $\mathrm{KPb}_{2} \mathrm{Cl}_{5}$ samples of various $\mathrm{Dy}^{3+}$ concentrations $(0.76-1.6 \mathrm{~mol}$. \%; see Table 1) were used in this study. The samples were grown individually over the course of a few years; in each attempt we strove for better optical quality. Consequently the crystals varied in optical quality; samples 1-3 were generally of good optical quality; sample 4 was fair; and sample 5 was poor. The poor optical quality was generally attributed to the presence of $\mathrm{OH}$ impurities.

\section{SPECTROSCOPY OF $\mathrm{KPb}_{2} \mathrm{Cl}_{5}: \mathrm{Dy}^{3+}$}

\section{A. Infrared Cutoff}

The multiphonon infrared cutoff (shown in Fig. 1) of $\sim 20$ $\mu \mathrm{m}$ was measured with a Perkin-Elmer 983 infrared spectrophotometer. The absorption feature near $7.2 \mu \mathrm{m}$ was been identified as $\mathrm{NH}_{4}{ }^{+}$, which had been introduced in a purification step of the crystal-growth process. The maximum transmission of $\sim 80 \%$ is consistent with Fresnel and scattering losses in the 5-mm length of sample. In particular, because the losses in this plot are due mainly to Fresnel reflections (which are index dependent), an index of refraction can be estimated from the transmission data of Fig. 1. For example, with no internal scattering losses the index is related to the transmission by $n=\left(1+\sqrt{1-T^{2}}\right) / T$. Figure 1 indicates that $T \approx 0.8$ from 4 to $10 \mu \mathrm{m}$, so $n \approx 2$ in this region. This result agrees with the data measured at the $\mathrm{He}-\mathrm{Ne}$ wavelength of $0.6 \mu \mathrm{m}$, so $n$ remains relatively constant from 0.6 to $10 \mu \mathrm{m}$.

\section{B. Energy-Level Estimation}

Absorption spectra at 15 and $300 \mathrm{~K}$ are shown in Fig. 2. The cold spectrum shows line narrowing that is due to the thermal depopulation of the $(2 J+1)$ Stark levels that constitute the ground state. Energy levels were assessed based on the peaks of the $15-\mathrm{K}$ spectrum and are shown in Fig. 3. These data are used below in Judd-Ofelt analysis and evaluation of multiphonon decay rates. The Russell-Saunders assignments and letter designations of the energy levels are taken after Dieke. ${ }^{20}$ Notice the large relative intensity of the $1.3 \mu \mathrm{m} \mathrm{Z} \rightarrow W$ peak, indicating the hypersensitive nature of this transition.

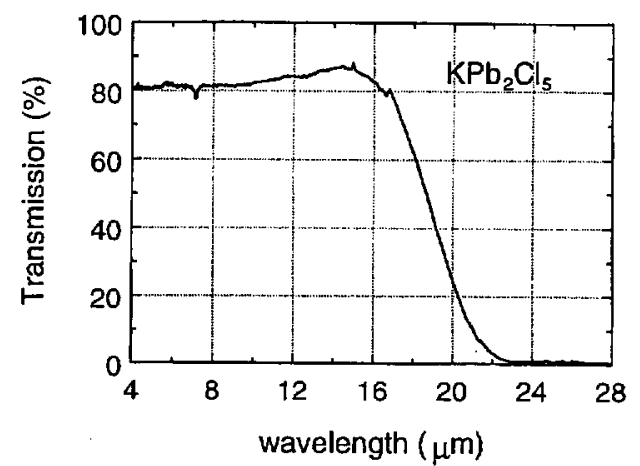

Fig. 1. Mid-IR transmission spectrum through $5 \mathrm{~mm}$ of $\mathrm{KPb}_{2} \mathrm{Cl}_{5}$. Long-wave cutoff owing to multiphonon absorption. A transmission of $\approx 80 \%$ indicates an average index $n \approx 2.0$ in the $4-10-\mu \mathrm{m}$ region.

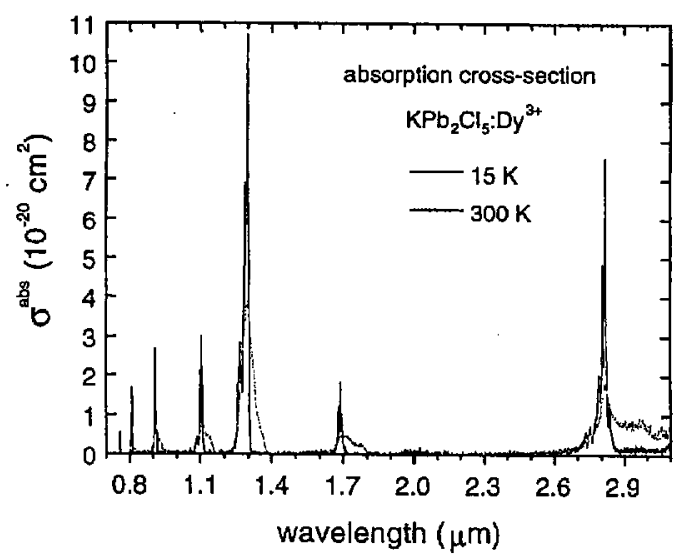

Fig. 2. Absorption cross section of $\mathrm{KPb}_{2} \mathrm{Cl}_{5}: \mathrm{Dy}^{3+}$ at $15 \mathrm{~K}$ and at room temperature $(300 \mathrm{~K})$, showing line broadening with temperature. Energy-level values were determined based on peakto-peak values of the 15-K spectrum and appear in Fig. 3 . 


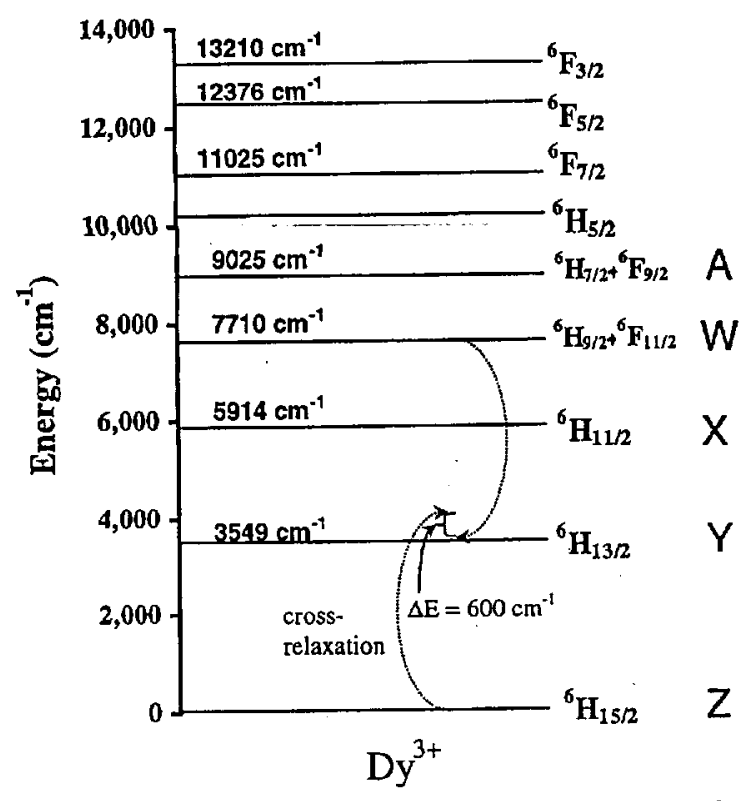

Fig. 3. Energy-level diagram of trivalent Dy. The letter designations of the levels are taken after Dieke. ${ }^{20}$ The proposed phonon-assisted energy-transfer pathway is indicated. Note that this pathway actually involves two neighboring ions: a donor ion in the $W$ level and an acceptor ion in the $Z$ level interacting to promote both ions into the $Y$ level.

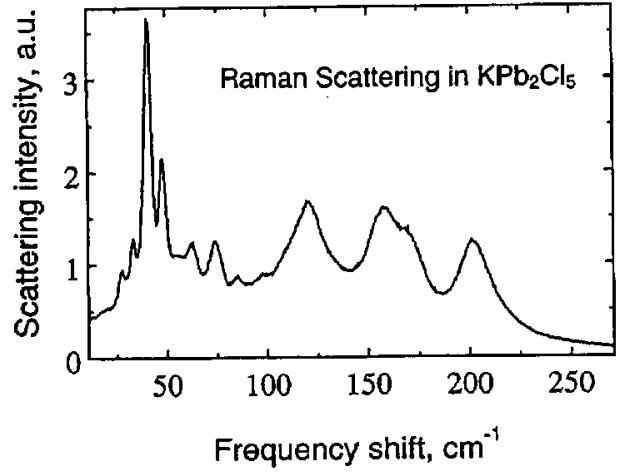

Fig. 4. Raman phonon spectrum of $\mathrm{KPb}_{2} \mathrm{Cl}_{5}$. The highest feature occurs near $203 \mathrm{~cm}^{-1}$, which we assign as $\nu_{\max }$.

\section{Absorption Cross Section and Doping Density}

Polarized and unpolarized absorption data were taken with a Perkin-Elmer Lambda 9 spectrophotometer for five $\mathrm{KPb}_{2} \mathrm{Cl}_{5}: \mathrm{Dy}^{3+}$ samples. The orthogonal polarization directions were identified at extinction positions between crossed polarizers. As the unpolarized spectra were essentially indistinguishable from the polarized spectra, the unpolarized spectra were used in this study. We determined the absorption cross section $\sigma^{\text {abs }}(\lambda)$ from the measured absorption coefficient $\alpha(\lambda)$ for one sample (1) by measuring the total $\mathrm{Dy}^{3+}$ concentration $N_{0}$ with the inductively coupled mass-spectrometer technique and taking the ratio $\sigma^{\text {abs }}(\lambda)=\alpha(\lambda) / N_{0}$. The $\mathrm{Dy}^{3+}$ concentration for the four other samples (2-5) was then established by a least-squares fit of the absorption coefficient curves of samples 2-5 to the absolute cross-section curve of sample 1. Table 1 lists the results of this analysis. The estimated error in the data is $\pm 10 \%$.

\section{Maximum Phonon Energy}

The maximum phonon energy was determined from a Raman scattering spectrum in $\mathrm{KPb}_{2} \mathrm{Cl}_{5}$ powder by use of a Ramanor U-1000 spectrometer $\left(1-\mathrm{cm}^{-1}\right.$ spectral resolution) with 514-nm argon-laser excitation. The results, shown in Fig. 4, indicate a maximum phonon energy of $h \nu_{\max }=203 \mathrm{~cm}^{-1}$, measured at the peak of the highestenergy feature. (These data appeared in a previous publication, ${ }^{13,14}$ but there the spectrum for $\mathrm{KPb}_{2} \mathrm{Cl}_{5}$ was labeled a spectrum for $\mathrm{BaCl}_{2}$. This figure rectifies that error with a new spectrum for $\mathrm{KPb}_{2} \mathrm{Cl}_{5}$.) According to factor group analysis for $\mathrm{KPb}_{2} \mathrm{Cl}_{5}$ related to the $P 2_{1} / c-C_{2 h}^{5}$ group with $Z=4$ and the $C_{2}$ axis along $b$ in the unit cell, 96 dispersion branches are expected, as $24 A_{g}+24 B_{g}+24 A_{u}+24 B_{u}$. The superposition of these branches produces the $\sim 15$ bands resolved in the figure. Because the monoclinic $\mathrm{KPb}_{2} \mathrm{Cl}_{5}$ structure contains no molecular groups with specific internal vibrations, the observed spectrum can be described in terms of $\mathrm{K}^{+}, \mathrm{Cl}^{-}$, and $\mathrm{Pb}^{2+}$ activity. The features below $75 \mathrm{~cm}^{-1}$ are due to $\mathrm{Pb}^{2+}$ translational modes; the features from 75 to $100 \mathrm{~cm}^{-1}$ are probably due to $\mathrm{K}^{+}$translational modes. The bands above $100 \mathrm{~cm}^{-1}$, including the band at 203 $\mathrm{cm}^{-1}$, which is believed to be related to vibrations of $\mathrm{Cl}-\mathrm{K}$ bonds, are associated with vibrations that involve $\mathrm{Cl}^{-}$.

\section{E. Judd-Ofelt Analysis}

A widely used method to predict radiative lifetimes, quantum efficiencies, and branching ratios was developed by Judd $^{21}$ and Ofelt. ${ }^{22}$ According to the Judd-Ofelt model, the line strength of induced electric dipole (ED) transitions between states $\left|f^{n}[S L] J\right\rangle$ and $\left|f^{n}\left[S^{\prime} L^{\prime}\right] J^{\prime}\right\rangle$ is

$$
S^{\mathrm{ED}}=\sum_{t=2,4,6} \Omega_{t}\left|\left\langle f^{n}[S L] J\left\|U^{(t)}\right\| f^{n}\left[S^{\prime} L^{\prime}\right] J^{\prime}\right\rangle\right|^{2},
$$

where $\left|\left\langle f^{n}[S L] J\left\|U^{(t)}\right\| f^{n}\left[S^{\prime} L^{\prime}\right] J^{\prime}\right\rangle\right|^{2}$ are the squared reduced-matrix elements that are commonly found in the literature ${ }^{23}$ and $\Omega_{2}, \Omega_{4}$, and $\Omega_{6}$ are the host-dependent Judd-Ofelt intensity parameters. These parameters can be determined by a best-fit calculation, because the line strengths can also be determined from the integrated absorption cross section

$$
\int \sigma(\lambda) \frac{d \lambda}{\lambda}=\frac{4 \pi^{2}}{3} \frac{e^{2}}{\hbar c} \frac{1}{2 J+1}\left[\frac{\left(n^{2}+2\right)^{2}}{9 n} S^{\mathrm{ED}}+n S^{\mathrm{MD}}\right] .
$$

We use the method discussed by Krupke ${ }^{24}$ to perform the best fit. This method requires the contributions from magnetic dipole (MD) transitions to be subtracted from the integrated absorption cross section. The line strength of induced magnetic dipole transitions is given by

$$
S^{\mathrm{MD}}=(\hbar / 2 m c)^{2}\left|\left\langle f^{n}[S L] J\|\mathbf{L}+2 \mathbf{S}\| f^{n}\left[S^{\prime} L^{\prime}\right] J^{\prime}\right\rangle\right|^{2},
$$

where the bracketed quantities indicate the matrix elements that must be computed in the intermediate coupling scheme. These matrix elements, however, are most easily computed in the $L S$ coupling scheme,${ }^{25}$ so coupling coefficients $C(S, L)$ are needed where 


$$
\left|4 f^{n}[S L] J\right\rangle=\sum_{S, L} C(S, L)\left|f^{n} S L J\right\rangle .
$$

The $C(S, L)$ for $\mathrm{Dy}^{3+}$ were taken from the eigenfunctions of the energy matrix found in Ref. 26. Once the line strengths are determined, the spontaneous emission rates and radiative branching ratios can be calculated:

$$
A_{J^{\prime}}=\frac{32 \pi^{3}}{3 \lambda^{3}} \frac{e^{2}}{\hbar c} \frac{c}{2 J+1}\left[\frac{n\left(n^{2}+2\right)^{2}}{9} S^{\mathrm{ED}}+n^{3} S^{\mathrm{MD}}\right]
$$

$$
\begin{aligned}
& A_{\mathrm{J}}=\sum_{\mathrm{J}^{\prime}} A_{\mathrm{JJ}^{\prime}}=1 / \tau_{\mathrm{J}}{ }^{\mathrm{rad}}, \\
& \beta_{J^{\prime}{ }^{\prime}}{ }^{\mathrm{rad}}=A_{\mathrm{J}^{\prime}} / A_{\mathrm{J}} \text {, }
\end{aligned}
$$

where $\tau_{\rfloor}^{\text {rad }}$ is the total radiative lifetime of level $\mathrm{J}$. The radiative quantum efficiency $\eta_{\lrcorner}^{\text {rad }}$ is given by

$$
\eta_{\mathrm{J}}^{\mathrm{rad}}=\tau_{\mathrm{J}}{ }^{\mathrm{meas}} / \tau_{\mathrm{J}}{ }^{\mathrm{rad}},
$$

where $\tau_{\lrcorner}$meas is the total measured fluorescence lifetime of level J. The results of this Judd-Ofelt analysis are shown in Table 2. The $W \leftarrow Z(1.3-\mu \mathrm{m})$ transition has sometimes been omitted from the analysis in the literature because of its hypersensitive nature. ${ }^{27,28}$ Because the rms error of the fit was lower with the 1.3- $\mu \mathrm{m}$ transition excluded $(0.16 \%$ versus $2.44 \%)$, we also omitted it from this analysis. The inclusion or exclusion of the 1.3- $\mu \mathrm{m}$ transition can significantly affect the Judd-Ofelt analysis (particularly the value of $\Omega_{2}$ in this case), which can compromise the reliability of the results obtained. This is the main reason why in Section 3 we explore a different method of arriving at such results. Previously reported $\mathrm{KPb}_{2} \mathrm{Cl}_{5}: \mathrm{Dy}^{3+}$ Judd-Ofelt data included the $1.3-\mu \mathrm{m}$ feature, ${ }^{13,14}$ used an inaccurate value of $n=1.7$ for the average refractive index, and included magnetic dipole data only for the ${ }^{6} H_{15 / 2} \rightarrow{ }^{6} H_{13 / 2}$ transition. ${ }^{14}$

\section{F. Decay Rates and Concentration-Dependent Energy} Transfer

To gain a more comprehensive picture of the fluorescence dynamics of the $\mathrm{KPb}_{2} \mathrm{Cl}_{5}: \mathrm{Dy}^{3+}$ system, we investigated concentration-dependent phenomena by studying the fluorescence decay rates of samples 1-5. The total decay rate $W_{\mathcal{L}}$ of a level $\mathrm{J}(\mathrm{J}=\mathrm{A}, \mathrm{W}, \mathrm{X}, \mathrm{Y})$ was assumed to obey the relation

$$
W_{\mathrm{J}}=A_{\mathrm{J}}^{\mathrm{rad}}+W_{\mathrm{J}}^{\mathrm{MP}}+W_{\mathrm{J}}{ }^{\mathrm{c}} \equiv W_{\mathrm{J}}{ }^{0}+W_{\mathrm{J}}{ }^{c},
$$

where $W_{\mathrm{J}}{ }^{0}$ is the radiative and multiphonon component of the decay (which is not concentration dependent) and $W_{J}^{c}$ is the concentration-dependent component of the decay from the level designated $\mathrm{J}$.

Decay intensities for the $W$ level, shown in Fig. 5, display an approximately single-exponential transient and indicate the total decay rates increase with concentration. We propose that the origin of this concentration dependence is the phonon-assisted cross-relaxation process depicted in Fig. 3, whereby an ion in the ground level interacts with a second ion in the $W$ level such that both ions end up in the $Y$ level. This process must be phonon assisted because the energy of the final state is $600 \mathrm{~cm}^{-1}$ below the initial state (exothermic). Lifetimes were determined by a least-squares exponential fit according to $I(t)=I(0) \exp \left[-\left(W_{\mathrm{J}}^{0}+W_{\mathrm{J}}^{c}\right) t\right]$, where $I(t)$ is the decay intensity at time $t$. Such an exponential decay is predicted when excitation migration among donors precedes a dipole-dipole energy transfer between a donor (D) and an acceptor (A). For low-moderate concentration levels, the decay rate for such a transfer is generally given by

$$
W_{\mathrm{J}}^{c}=k_{\jmath}{ }^{c} N_{\mathrm{D}} N_{\mathrm{A}}=k_{\mathrm{J}}{ }^{c} N_{0}^{2},
$$

where $k_{\mathrm{J}} c$ is the cross-relaxation coefficient and in this case the donors and acceptors are the same species: $N_{\mathrm{D}}$

\begin{tabular}{|c|c|c|c|c|c|c|c|}
\hline Transition & $\lambda(\mu \mathrm{m})$ & $S^{E D}\left(\times 10^{-20} \mathrm{~cm}^{2}\right)$ & $S^{\mathrm{MD}}\left(\times 10^{-20} \mathrm{~cm}^{2}\right)$ & $A^{\mathrm{ED}}\left(\mathrm{s}^{-1}\right)$ & $A^{\mathrm{MD}}\left(\mathrm{s}^{-1}\right)$ & $\beta^{\mathrm{rad}}$ & $\tau_{\text {rad }}(\mathrm{ms})$ \\
\hline \multicolumn{8}{|l|}{${ }^{6} H_{13 / 2}(\mathrm{Y})$} \\
\hline $\begin{aligned} & \rightarrow{ }^{6} H_{15 / 2} \\
{ }^{6} H_{11 / 2} & (\mathrm{X})\end{aligned}$ & 2.82 & 3.55 & 1.02 & 65.7 & 18.9 & 1.000 & 11.8 \\
\hline$\rightarrow{ }^{6} H_{13 / 2}$ & 4.23 & 1.88 & 1.49 & 12.0 & 9.5 & 0.091 & 4.24 \\
\hline $\begin{array}{c}\rightarrow{ }^{6} H_{15 / 2} \\
{ }^{6} H_{9 / 2}+{ }^{6} F_{11 / 2}(\mathrm{~W})\end{array}$ & 1.69 & \multicolumn{2}{|c|}{${ }^{6} H_{9 / 2}+{ }^{6} F_{11 / 2}(\mathrm{~W})$} & 214.3 & & 0.909 & - \\
\hline$\rightarrow{ }^{6} H_{11 / 2}$ & 5.57 & 2.27 & 0.76 & 6.84 & 2.5 & 0.010 & 1.09 \\
\hline$\rightarrow{ }^{6} H_{13 / 2}$ & 2.40 & 2.73 & 0.00 & 99.2 & - & 0.108 & - \\
\hline \multicolumn{8}{|l|}{${ }^{6} H_{7 / 2}+{ }^{6} F_{9 / 2}$ (A) } \\
\hline$\rightarrow{ }^{6} H_{9 / 2}+{ }^{6} F_{11 / 2}$ & 7.60 & 1.64 & 0.97 & 2.5 & 1.5 & 0.004 & 1.06 \\
\hline$\rightarrow{ }^{6} H_{11 / 2}$ & 3.21 & 1.48 & 0.01 & 28.1 & 0.1 & 0.030 & - \\
\hline$\rightarrow{ }^{6} H_{13 / 2}$ & 1.83 & 2.87 & 0.00 & 281.7 & - & 0.297 & - \\
\hline$\rightarrow{ }^{6} H_{15 / 2}$ & 1.11 & 1.47 & 0.00 & 633.0 & - & 0.669 & - \\
\hline
\end{tabular}
$=N_{\mathrm{A}}=N_{0}$. The cross-relaxation coefficient depends on the critical radius $R_{\mathrm{DX}}$ for nonradiative dipole-dipole energy transfer developed by Förster and Dexter, which can be estimated from the overlap of the emission and absorption cross sections ${ }^{29}$ :

Table 2. Results of the Judd-Ofelt Analysis for Sample $1^{\alpha}$ a The W (and A) level data were calculated with the assumption that the constituent levels are in thermal equilibrium. $\Omega_{2}=5.41 \times 10^{-20} \mathrm{~cm}{ }^{2}, \Omega_{4}$
$=0.99 \times 10^{-20} \mathrm{~cm}^{2}, \Omega_{6}=2.96 \times 10^{-20} \mathrm{~cm}^{2}$. 


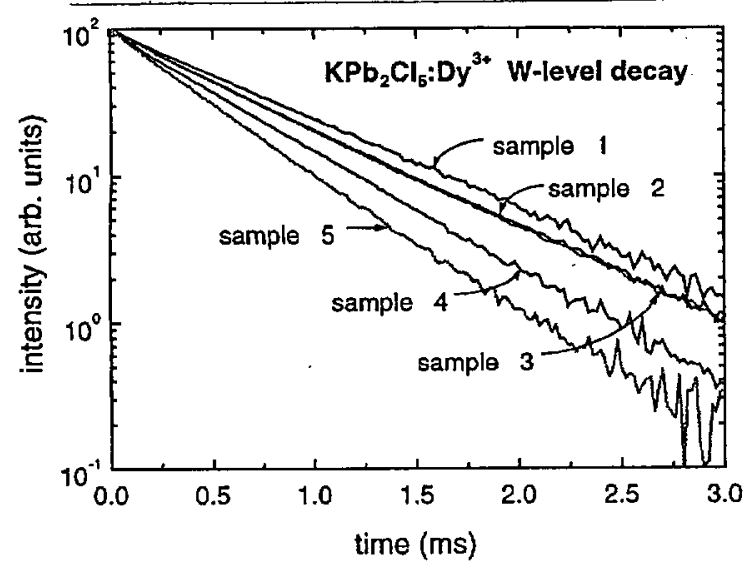

Fig. 5. Temporal decay of the $W$ level on $1.3-\mu \mathrm{m}$ excitation. The decay rate increases as the sample concentration increases, which is indicative of concentration-dependent cross relaxation. Notice that the decay is largely singly exponential for each sample.

$$
R_{\mathrm{D} X}^{6}=\frac{3 c}{8 \pi^{4} n^{2} A_{\mathrm{J}}^{\mathrm{rad}}} \int \sigma_{D}^{\mathrm{em}}(\lambda) \sigma_{X}^{\mathrm{abs}}(\lambda) \mathrm{d} \lambda,
$$

where $n$ is the refractive index and $X=D$ or $X=A$ for donor-donor (D-D) or donor-acceptor (D-A) transfer.

When the overlap of the D-D interaction is much stronger than the overlap of the D-A interaction (e.g., when $R_{\mathrm{DD}} \gg{ }_{\mathrm{DA}}$ ), Burshtein's hopping model ${ }^{30}$ is assumed to be applicable. In this case the cross-relaxation coefficient is given by

$$
k_{\mathrm{J}}^{c}=\pi(2 \pi / 3)^{5 / 2} R_{\mathrm{DA}}{ }^{3} R_{\mathrm{DD}}{ }^{3} A_{\mathrm{J}}{ }^{\mathrm{rad}} .
$$

This situation typically arises for rare-earth ions for which the Stokes shifts are relatively small, giving rise to relatively large D-D overlaps and thus to a large value of $R_{\mathrm{DD}}$.

To verify that $R_{\mathrm{DD}} \gg{ }_{\mathrm{DA}}$ for $\mathrm{KPb}_{2} \mathrm{Cl}_{5}: \mathrm{Dy}^{3+}$, we show in Fig. 6 the D-D and D-A cross sections that are relevant to the cross-relaxation process. Emission cross sections were determined from the relation

$$
\sigma_{J^{\prime} J^{\prime}}{ }^{\mathrm{em}}(\lambda)=\frac{\lambda^{5} \beta_{\mathrm{JJ}^{\prime}}{ }^{\mathrm{rad}} A_{\mathrm{J}^{\mathrm{rad}}}^{\mathrm{rad}}}{8 \pi c n^{2}} \frac{I(\lambda)}{\int_{\mathrm{J} \rightarrow \mathrm{J}^{\prime}} I(\lambda) \lambda \mathrm{d} \lambda},
$$

where $I(\lambda)$ is the blackbody-calibrated emission spectrum (arbitrarily scaled units of watts per nanometer) and where the radiative decay rate $A_{\downarrow}{ }^{\mathrm{rad}}$ and the radiative branching ratio $\beta_{\mathrm{JJ}}{ }^{\mathrm{rad}}$ were estimated from the JuddOfelt analysis of Subsection 2.E.

The assistance of phonons in the D-A energy-transfer model can be incorporated by use of the exponential relation developed by Auzel $^{31}$ to include the multiphonon sidebands of the emission and absorption cross sections. The Stokes sideband cross section is given $b^{32}$

$$
\sigma^{\text {Stokes }}=\sigma^{\text {elec }} \exp \left(-\alpha_{s} \Delta \dot{E}\right),
$$

where $\Delta E$ is the energy mismatch between the electronic spectra [found in Eq. (13)] and the Stokes vibronic spectra. The parameter $\alpha_{s}$ depends only on the host and is given $\mathrm{by}^{31}$

$$
\alpha_{s}=\left(h \nu_{\max }\right)^{-1}\left(\ln \left\{\left(\bar{N} / S_{0}\right)\left[1-\exp \left(-h \nu_{\max } / k T\right)\right]\right\}-1\right)
$$

where $h \nu_{\max }$ is the maximum phonon energy of the host, $\bar{N}$ is the number of phonons required for assisting the nonresonant energy transfer, $S_{0}$ is an electron-phonon coupling constant, and $k T \approx 208 \mathrm{~cm}^{-1}$ at room temperature. Auzel reports that $S_{0}$ does not vary greatly from host to host, with a typical value of $S_{0}=0.04$. Using $\bar{N}=3$ and $h \nu_{\max }=203 \mathrm{~cm}^{-1}$, we obtain an estimate of $\alpha_{s}=0.014 \mathrm{~cm}$. These exponential tails are shown appended to the electronic spectra in Fig. 6(b). Using Eqs. (11)-(14), we estimate that $R_{\mathrm{DD}}=19.4 \AA$ and $R_{\mathrm{DA}}$ $=8.37 \AA$, so $k_{\mathrm{W}^{c}}{ }^{c}=0.79 \times 10^{-37} \mathrm{~cm}^{6} \mathrm{~s}^{-1}$.

The cross-relaxation coefficient $k_{W}{ }^{c}$ can also be determined experimentally. The total decay rates for the $W$ level are plotted in Fig. 7 against the square of the $\mathrm{Dy}^{3+}$ concentration, along with the fit according to Eqs. (9) and (10):

$$
W_{W}=W_{W}^{0}+k_{W}{ }^{c} N_{0}^{2} .
$$

Thus the slope of the straight-line fit yields the crossrelaxation coefficient $k_{W}{ }^{c}$, whereas the intercept yields the intrinsic decay rate $W_{W}{ }^{0}$. Values of $k_{W}{ }^{c}=(1.83$ $\pm 0.20) \times 10^{-37} \mathrm{~cm}^{6} \mathrm{~s}^{-1}$ and $W_{W}^{0}=1197 \pm 59 \mathrm{~s}^{-1}$ are obtained. These results are in reasonable agreement with the hopping model, establishing the credibility of the cross-relaxation pathway. Based on a similar analysis, we have concluded that no appreciable concentrationdependent processes occur from the $A$ or the $X$ level.

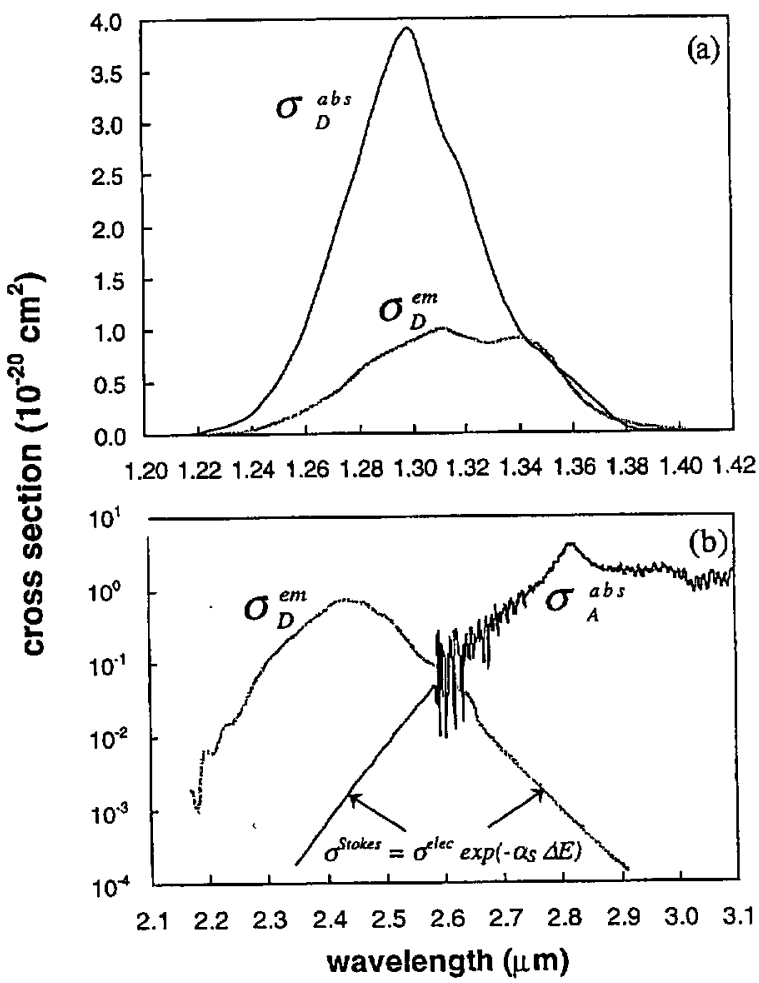

Fig. 6. Spectral overlap of (a) two donor ions and (b) a donor and an acceptor ion. The relatively large overlap of the donor ion absorption and emission suggests that hopping is likely to occur. Bottom (note the log scale), way in which multiphonon Stokes sideband contributions to the cross sections can increase the spectral overlap. 


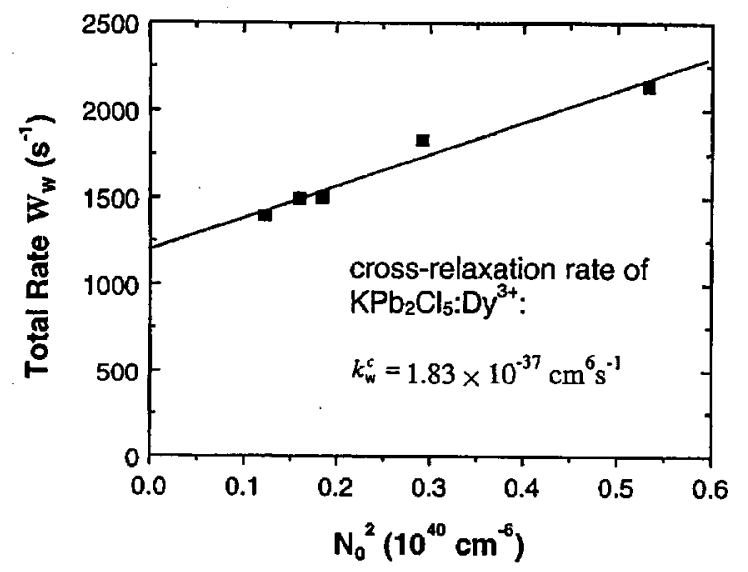

Fig. 7. Total decay rate for the $W$ level $\left(W_{W}\right)$ plotted against the square of the $\mathrm{Dy}^{3+}$ concentration $\left(N_{0}^{2}\right)$. Also shown is the fit $W_{W}=W^{0}+k_{W}{ }^{c} N_{0}^{2}$, where $k_{W}{ }^{c}$ is the cross-relaxation coeffcient. The slope implies that $k_{W}{ }^{c}=1.83 \times 10^{-37} \mathrm{~cm}^{6} \mathrm{~s}^{-1}$. $W_{\mathrm{W}}=2 W^{0}$ when $N_{0}=0.8 \times 10^{20} \mathrm{~cm}^{-3}$.

\section{QUANTUM EFFICIENCY MODEL FOR $\mathrm{KPb}_{2} \mathrm{Cl}_{5}: \mathrm{Dy}^{3+}$}

A. Method

With ground-state absorption at 2.9, 1.7, 1.3, 1.1, and 0.9 $\mu \mathrm{m}$, the low-lying energy levels in $\mathrm{KPb}_{2} \mathrm{Cl}_{5}: \mathrm{Dy}^{3+}$ are accessible by direct laser excitation. By exciting each level individually and examining the relative emission from each lower-lying level, we can determine the radiative quantum efficiencies of these levels. In practice this has been done by measurement of the ratios of emission rate intensities, as we shall see. The concept is pedagogically illustrated in Fig. 8 for a hypothetical three-level system. Figure 8(a) shows the energy levels, indicating the pump level (level 3) and the possible output wavelengths $\lambda_{3}$ and $\lambda_{2}$. For this example we assume that $\eta_{2}{ }^{\mathrm{rad}}=1$ and that $\beta_{32}{ }^{\mathrm{rad}}=0$, so only nonradiative decay connects level 3 to level 2. Figures $8(\mathrm{~b}), 8(\mathrm{c})$, and $8(\mathrm{~d})$ show the emission spectra (photon emission rate $\phi$ versus wavelength $\lambda$ ) in cases when $\eta_{3}{ }^{\text {rad }}=1,0.5,0$, respectively. For this example it is apparent that $\eta_{3}{ }^{\mathrm{rad}}=\phi_{3} /\left(\phi_{2}+\phi_{3}\right)$, where $\phi_{\mathrm{J}}$ is the photon emission rate for level $\mathrm{J}$ represented by the emission peak at $\lambda_{\mathrm{J}}$.

In the following more general model we assume that decay from each level is possibly influenced by radiative, multiphonon, and concentration-dependent transfer processes only and that multiphonon decay occurs only to the next lowest energy level. This method is an extension of that used by Quimby et al. ${ }^{33}$ We shall use the results of this analysis to calculate accurate emission cross sections and multiphonon decay rates that affect the potential use of $\mathrm{KPb}_{2} \mathrm{Cl}_{5}: \mathrm{Dy}^{3+}$ as a long-wavelength laser medium.

The model is based on solutions to steady-state rate equations. It has been assumed that the pump intensities involved $\left(I_{\text {pump }} \sim 10 \mathrm{~W} / \mathrm{cm}^{2}\right)$ do not significantly deplete $\left(I_{\text {sat }} \sim 10 \mathrm{~kW} / \mathrm{cm}^{2}\right)$ the ground-state population. With the inclusion of the cross-relaxation pathway identified in Subsection 2.F, and with ${ }^{P} N_{\mathrm{J}}$ denoting the population density of the $J$ level $(J=A, W, X, Y)$ under direct excitation of the $P$ level $(P=A, W, X)$, the steady-state rate equations of levels $W, X$, and $Y$ take the form

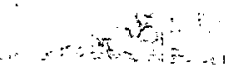

$$
\begin{aligned}
& \frac{\mathrm{d}\left({ }^{\mathrm{w}} N_{\mathrm{w}}\right)}{\mathrm{d} t}=0={ }^{\mathrm{w}} R-{ }^{\mathrm{w}_{N}}{ }_{\mathrm{w}} / \tau_{\mathrm{W}}, \\
& \frac{\mathrm{d}\left({ }^{w} N_{\mathrm{X}}\right)}{\mathrm{d} t}=0=b_{\mathrm{WX}}{ }^{{ }^{w}} N_{\mathrm{W}} / \tau_{\mathrm{W}}-{ }^{{ }} N_{\mathrm{X}} / \tau_{\mathrm{X}}, \\
& \frac{\mathrm{d}\left({ }^{W_{N}} N_{Y}\right)}{\mathrm{d} t}=0=\left(b_{W Y}+\eta_{W^{c}} \beta_{W Y}\right)^{W_{W}} N_{W} / \tau_{W} \\
& +b_{X Y}{ }^{w_{X}} N_{X} / \tau_{X}-w_{N_{Y}} / \tau_{Y},
\end{aligned}
$$

when the $W$ level is directly pumped and

$$
b_{\mathrm{JJ}}=\eta_{\mathrm{J}}^{\mathrm{rad}} \beta_{\mathrm{JJ}^{\prime}}+\eta_{\mathrm{J}}^{\mathrm{MP}} \beta_{\mathrm{JJ}}{ }^{\mathrm{MP}}+\eta_{\mathrm{J}}^{c} \beta_{\mathrm{J} J^{\prime}}{ }^{\mathrm{c}}
$$

$\left(\eta_{J}{ }^{\mathrm{rad}}+\eta_{J}^{\mathrm{MP}}+\eta_{\mathrm{J}}{ }^{c}=1\right)$ represents the total branching ratio through downward relaxation from level $\mathrm{J}$ to level $\mathrm{J}^{\prime}$ in all processes (radiative, multiphonon, and cross relaxation), ${ }^{W}$ is the rate at which level $W$ is pumped, and $\tau_{J}$ is the total fluorescence lifetime of level $\mathrm{J}$. The second term in parentheses in Eq. (19) represents the (upward) energy transfer from the ground state to the $Y$ level in the cross-relaxation pathway (see Fig. 3). The quantum efficiencies and branching ratios are all defined similarly: $\eta_{\mathrm{J}}{ }^{a}=W_{\mathrm{J}}{ }^{a} / W_{\mathrm{J}} ; \quad \beta_{\mathrm{J}^{\prime}}{ }^{a}=W_{\mathrm{J}^{\prime}}{ }^{a} / W_{\mathrm{J}}{ }^{a} ;$ and $a=$ radiative (rad), multiphonon (MP), and cross relaxation (c). The cross-relaxation fraction $\eta_{W}{ }^{c}$ (the fraction of the $W$ level population that decays by cross relaxation) is determined from the measured values of $W_{W}$ and $W_{W}{ }^{0}$ by use of Eq. (9):

$$
\eta_{W}^{c} \equiv W_{W}^{c} / W_{W}=1-W_{W} / W_{W}
$$

The following assumptions were used in this analysis: (a)

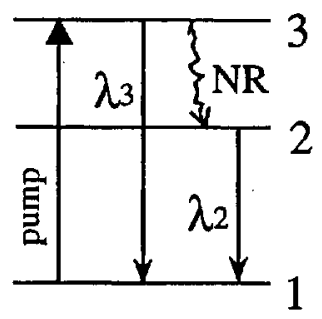

(c)

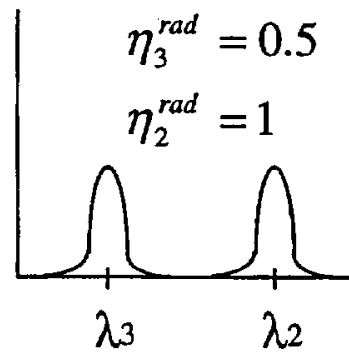

(b)

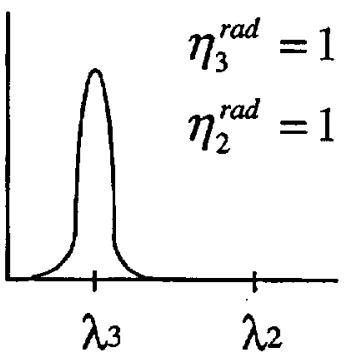

(d)
Fig. 8. Pedagogical illustration of the concept of the radiative quantum efficiency model. (a) Level 3 is pumped directly, and we assume that $\eta_{2}{ }^{\mathrm{rad}}=1$ and $\beta_{32}{ }^{\mathrm{rad}}=0$. The wavy arrow indicates nonradiative decay (NR). Hypothetical emission rates $\phi$ (in arbitrary photons per second) are shown as a function of wavelength $\lambda$ for (b) $\eta_{3}{ }^{\text {rad }}=1$, (c) $\eta_{3}{ }^{\text {rad }}=0.5$, and (d) $\eta_{3}{ }^{\text {rad }}=0$. 
- $\beta_{\mathrm{JJ}}{ }^{\mathrm{MP}}=1$ if $\mathrm{J}^{\prime}$ is the next level below $\mathrm{J} ; \beta_{\mathrm{JJ}}{ }^{\mathrm{MP}}$ $=0$ otherwise (multiphonon emission occurs only to the next lowest energy level).

- $\beta_{W r}{ }^{c}=1$ (concentration-dependent energy transfer from the $W$ level occurs only to the $Y$ level).

- $\eta_{Y}{ }^{M P}=0$ [the $Y-Z$ gap is sufficiently large $(>15$ phonons) to inhibit all multiphonon decay from the $Y$ level].

Solving the rate equations for the individual populations ${ }^{W_{N}} N_{\mathrm{J}}$ in the steady state, and writing the radiative emis-
In a similar manner we can write the rate equations for the levels $X$ and $Y$ when level $X$ is directly pumped and arrive at the relation

$$
\mathrm{x}_{\phi_{Y} X} \equiv \frac{{ }^{\mathrm{x}} \phi_{\mathrm{Y}}}{\mathrm{x}_{\phi_{\mathrm{X}}}}=\frac{b_{\mathrm{XY}} \eta_{\mathrm{Y}}^{\mathrm{rad}}}{\eta_{\mathrm{X}}^{\mathrm{rad}}} .
$$

Equations (27), (28), and (29) [in conjunction with Eq. (20)] represent three equations for the three unknowns $\eta_{\mathrm{W}}{ }^{\mathrm{rad}}, \eta_{\mathrm{X}}^{\mathrm{rad}}$, and $\eta_{\mathrm{Y}}{ }^{\mathrm{rad}}$, permitting a unique determination of these parameters. The solutions are

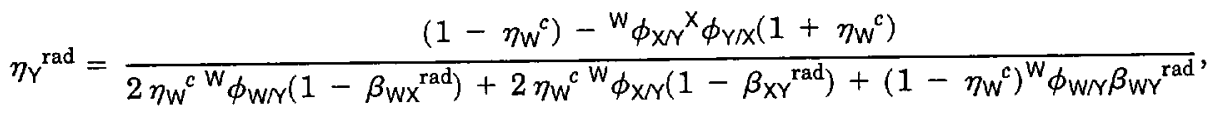

sion rate (photons per second) from level $J$ as $\left[{ }^{W_{N}} N_{J} \tau_{J}\right] \eta_{J}^{\mathrm{rad}}$, we have

$$
\begin{aligned}
& {\left[{ }^{W_{N}} N_{\mathrm{W}} / \tau_{\mathrm{W}}\right] \eta_{\mathrm{W}}{ }^{\mathrm{rad}}={ }^{\mathrm{W}} R \eta_{\mathrm{W}}{ }^{\mathrm{rad}},} \\
& {\left[{ }^{\mathrm{W}} N_{\mathrm{X}} / \tau_{\mathrm{X}}\right] \eta_{\mathrm{X}}{ }^{\mathrm{rad}}={ }^{\mathrm{W}} R b_{\mathrm{WX}} \eta_{\mathrm{X}}{ }^{\mathrm{rad}}} \\
& {\left[{ }^{W_{N}} N_{Y} / \tau_{Y}\right] \eta_{Y}{ }^{\mathrm{rad}}={ }^{W_{R}} R\left(b_{\mathrm{WY}}+\eta_{\mathrm{W}}{ }^{\mathrm{c}} \beta_{\mathrm{WY}}{ }^{c}+b_{\mathrm{WX}} b_{\mathrm{XY}}\right) \eta_{\mathrm{Y}}{ }^{\mathrm{rad}} \text {. }}
\end{aligned}
$$

The radiative emission rate can also be determined from the blackbody-calibrated emission spectrum ${ }^{\mathrm{P}} I(\lambda)$ (units of arbitrarily scaled watts per nanometer), that results from direct excitation of the $P$ level $(P=A, W, X)$ because

$$
\left[{ }^{\mathrm{P}} N_{\mathrm{J}} / \tau_{\mathrm{J}}\right] \eta_{\mathrm{J}}{ }^{\mathrm{rad}} \propto \sum_{\mathrm{J}^{\prime}} \int_{\mathrm{J} \rightarrow \mathrm{J}^{\prime}} \frac{{ }^{\mathrm{P}} I(\lambda)}{h c / \lambda} \mathrm{d} \lambda \equiv{ }^{\mathrm{P}} \phi_{\mathrm{J}},
$$

where the integral is carried out over all bands $\mathrm{J} \rightarrow \mathrm{J}^{\prime}$, where $J$ ' lies below $J$. Thus ${ }^{P} \phi_{\lrcorner}$represents the radiative emission rate from level $\mathrm{J}$ after level $\mathrm{P}$ is pumped and can be determined experimentally (to within a proportionality constant) from the emission spectrum ${ }^{P} I(\lambda)$. The radiative branching ratios can also be determined experimentally from ${ }^{P} I(\lambda)$. From Eq. (25), for a single transition $\mathrm{J} \rightarrow \mathrm{J}^{\prime}$ with radiative branching ratio $\beta_{\mathrm{JJ}^{\prime}}{ }^{\mathrm{rad}}$, we have

$$
\beta_{J J^{\prime}}{ }^{\mathrm{rad}}=\frac{\int{ }_{J \rightarrow J^{\prime}}{ }^{P} I(\lambda) \lambda d \lambda}{\sum J_{J^{\prime}} \int_{J \rightarrow J^{\prime}}{ }^{P} I(\lambda) \lambda d \lambda} .
$$

Notice that the form of Eq. (26) makes the value of the branching ratio invariant to the pumping level $P$.

We eliminate arbitrary constants that arise from collection efficiency and absolute intensity calibrations of the system by taking ratios of Eqs. (22)-(24) and employing relation (25). Labeling the ratio of the radiative emission rate from the $\mathrm{J}$ th level to the radiative emission rate from the Kth level as ${ }^{\mathrm{P}} \phi_{\mathrm{J} / \mathrm{K}}$ (i.e., ${ }^{\mathrm{P}} \phi_{\mathrm{J} / \mathrm{K}} \equiv{ }^{\mathrm{P}} \phi_{\mathrm{J}} /{ }^{\mathrm{P}} \phi_{\mathrm{K}}$ ), we can use expressions (22)-(25) to write

$$
\begin{aligned}
& w_{\phi_{X / W}} \equiv \frac{w_{\phi_{X}}}{w_{\phi_{W}}}=\frac{b_{\mathrm{WX}} \eta_{\mathrm{X}}^{\mathrm{rad}}}{\eta_{\mathrm{X}}^{\mathrm{rad}}}, \\
& w_{\phi_{\mathrm{Y} / \mathrm{W}}} \equiv \frac{{ }^{w_{\phi_{Y}}}}{w_{\phi_{\mathrm{W}}}}=\frac{\left(b_{\mathrm{WY}}+\eta_{\mathrm{W}}{ }^{c} \beta_{\mathrm{WY}}{ }^{c}+b_{\mathrm{WX}} b_{\mathrm{XY}}\right) \eta_{\mathrm{Y}}^{\mathrm{rad}}}{\eta_{\mathrm{W}}^{\mathrm{rad}}} .
\end{aligned}
$$

$$
\begin{aligned}
& \eta_{X}^{\mathrm{rad}}=\frac{\eta_{Y}^{\mathrm{rad}}}{\mathrm{x}_{\phi_{Y X}}+\eta_{Y}^{\mathrm{rad}}\left(1-\beta_{X Y}{ }^{\mathrm{rad}}\right)}, \\
& \eta_{W}{ }^{\mathrm{rad}}=\frac{\eta_{\mathrm{X}}^{\mathrm{rad}}\left(1-\eta_{\mathrm{W}}{ }^{c}\right)}{{ }_{\mathrm{W}_{\mathrm{XW}}}+\eta_{\mathrm{X}}^{\mathrm{rad}}\left(1-\beta_{\mathrm{WX}}{ }^{\mathrm{rad}}\right)} .
\end{aligned}
$$

The quantum efficiency for the $A$ level can also be determined from a similar analysis. Including the $A$ level in the rate-equation analysis, where it now becomes the level directly pumped, and proceeding as before, we arrive at the expression

$$
\eta_{\mathrm{A}}^{\mathrm{rad}}=\frac{\eta_{W}^{\mathrm{rad}}}{{ }_{\mathrm{A}}^{\mathrm{r}}{ }_{\mathrm{W} / \mathrm{A}}+\eta_{\mathrm{W}}{ }^{\mathrm{rad}}\left(1-\beta_{\mathrm{AW}}{ }^{\mathrm{rad}}\right)}
$$

Finally, it is worthwhile to note that, in the absence of energy transfer (as for low $\mathrm{Dy}^{3+}$ concentration), Eq. (30) takes on the much simpler form

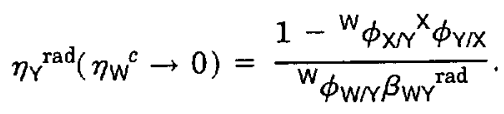

\section{B. Experimental Setup and Data}

Emission spectra were taken with a computer-controlled 1-m scanning monochromator. The samples were directly excited by a cw source, and a reference chopper was placed at the entrance slit of the monochromator to modulate the emission signal at $77 \mathrm{~Hz}$. The $W$ level was pumped with a $1.319-\mu \mathrm{m}, 100-\mathrm{mW} \mathrm{Nd}: Y A G$ laser (manufactured by Amoco) with an absorption coefficient $\alpha$ $\approx 0.9 \mathrm{~cm}^{-1}$; the A level was pumped with $500 \mathrm{~mW}$ of 1.064- $\mu \mathrm{m}$ light from a Nd:YAG laser (manufactured by Lightwave Electronics) whose absorption coefficient was $\alpha \approx 0.01 \mathrm{~cm}^{-1}$. Dichroic filters, an InSb detector $(77 \mathrm{~K})$, and a stimulated Raman scattering lock-in amplifier were used to isolate, detect, and process the signals. For spectra with emission wavelengths below 3:5 $\mu \mathrm{m}$, a $2.0-\mu \mathrm{m}$ blazed 300-grating $/ \mathrm{mm}$ grating was used; a $4.0-\mu \mathrm{m}$ blazed, 150-grating/mm grating was used for spectra that included wavelengths greater than $3.5 \mu \mathrm{m}$. Individual scans were obtained for wavelengths in the range 1.2-2.6 $\mu \mathrm{m}$ (by A-level pumping), 1.7-3.5 $\mu \mathrm{m}$, and 2.6-5.5 $\mu \mathrm{m}$ (both by $W$-level pumping). We used the $1.2-2.6-\mu \mathrm{m}$ run with A-level pumping to obtain the line shape of the 

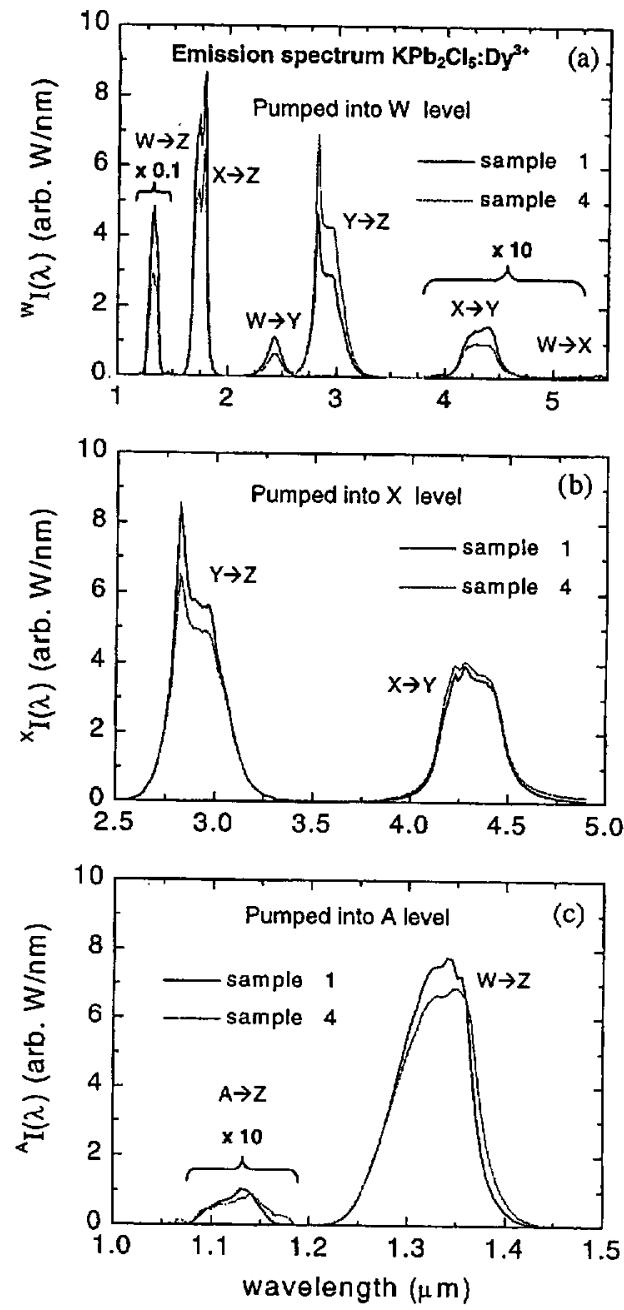

Fig. 9. Emission data used to determine branching ratios $\beta_{\mathrm{J}}^{\text {rad }}$ and fluorescence ratios ${ }^{P} \phi_{J / K}$ for samples 1 and 4 . The vertical scales have been adjusted to conserve the total emission rate for each sample. (a) Emission spectrum ${ }^{W} I(\lambda)$ obtained by direct excitation of the $W$ level. Note the scale change near $1.3 \mu \mathrm{m}$ and beyond $4 \mu \mathrm{m}$. The transfer of population from the $W$ level to the $Y$ level in the higher doped sample (4) is shown by the relative peak heights of the $W \rightarrow Z$ and $Y \rightarrow Z$ transitions. (b) Emission spectrum obtained by direct excitation of the $X$ level. The $1.7-\mu \mathrm{m}(X \rightarrow Z)$ feature is not shown. Note that the $2.9-\mu \mathrm{m}(Y$ $\rightarrow$ Z) feature is dramatically reduced compared with that of the $W$-level-pumped spectrum in (a). (c) Emission spectrum obtained by direct excitation of the $A$ level. Only the $A \rightarrow Z$ and $W \rightarrow Z$ transition are shown.

1.3- $\mu \mathrm{m}$ emission because this datum was too difficult to obtain in the presence of the $1.3-\mu \mathrm{m}$ (X-level) pump signal. These individual curves were then overlapped and scaled by a least-squares fit of the amplitudes of the appropriate overlapping regions to form the continuous curve shown in Fig. 9(a). Emission data were limited to $\lambda<5.5 \mu \mathrm{m}$ by the cutoff of the InSb detector. Level X was pumped with an $\sim 1-W \mathrm{cw}$ InGaAsP-InP diode source at $1.7 \mu \mathrm{m}$ developed at the Lawrence Livermore National Laboratory. ${ }^{34}$ The data shown in Fig. 9(b) were collected in a single run with the $4.0-\mu \mathrm{m}$ blazed grating and InSb detector. The branching ratio of the $4.3-\mu \mathrm{m}$ emission was taken from the W-level-pumped data. A-level- pumped data are shown in Fig. 9(c). However, because only the emission at $1.1 \mu \mathrm{m}(\mathrm{A} \rightarrow \mathrm{Z})$ was discernible the emissions at 1.8 and $3.1 \mu \mathrm{m}(A \rightarrow Y$ and $A \rightarrow X)$ could not be resolved because of the presence of the much stronger emissions at 1.7 and $2.9 \mu \mathrm{m}(X \rightarrow Z$ and $Y \rightarrow Z)$ ], these data were generated by pumping of the ${ }^{6} F_{7 / 2}$ level at 0.9 $\mu \mathrm{m}$ with AlGaAs diodes and collected by the InSb detector with a 600 -grating $/ \mathrm{mm}$ grating blazed at $1 \mu \mathrm{m}$. Accordingly, we used the branching ratios from the Judd-Ofelt data in Table 2 to estimate ${ }^{A} \phi_{W / A}$ in Eq. (33).

The raw intensity data were spectrally calibrated with a blackbody source (Electro-Optical Industries Model WS142) operating at $1013 \mathrm{~K}$, where the peak emission wavelength is $2.9 \mu \mathrm{m}$. In this way, the raw emission intensity could be converted into units of arbitrary watts per nanometer. We obtained measured fluorescence lifetimes for the $W, X$, and $Y$ levels by pumping the $W$ level with the $100-\mathrm{mW}$ Amoco laser at $1.3 \mu \mathrm{m}$, chopped to produce $250-\mu$ s pulses at $7 \mathrm{~Hz}$, and fitting the observed 2.4-, 1.7-, and $2.9-\mu \mathrm{m}$ emission, respectively, to an exponential decay. We used bandpass filters and the InSb detector at $77 \mathrm{~K}$ to record the data. We obtained measured lifetimes for the A level by pumping with the 900-nm AlGaAs diodes in pulsed operation ( $35 \mu \mathrm{s}, 160 \mathrm{~Hz}$ ) and fitting the $1.1-\mu \mathrm{m}(\mathrm{A} \rightarrow \mathrm{Z})$ decay. We used a $1-\mu \mathrm{m}$ blazed grating (600 gratings $/ \mathrm{mm}$ ) monochromator and a dry-ice-cooled photomultiplier tube to record the data.

The measured branching ratios $\beta_{\mathrm{J}}{ }^{\mathrm{rad}}$ from Eq. (26) are given in Table 3 , and the fluorescence ratios ${ }^{\mathrm{P}} \phi_{\mathrm{J} / \mathrm{J}}$, from Eqs. (27)-(29) are given in Table 4. The resultant quantum efficiencies and radiative lifetimes are shown in Table 5. For the $Y$ level, it was assumed (as noted above) that $\eta_{Y}{ }^{\mathrm{MP}}=0$ and that the $\eta_{Y}{ }^{c}$ values from Table 4 are due to oxygen impurities in the host, which is evident in

Table 3. Measured Radiative Branching Ratios According To Eq. $(26)^{a}$

\begin{tabular}{cccccc}
\hline Sample Number & $\beta_{W X}{ }^{\mathrm{rad}}$ & $\beta_{W Y^{\mathrm{rad}}}$ & $\beta_{\mathrm{WZ}}{ }^{\mathrm{rad}}$ & $\beta_{X Y}{ }^{\mathrm{rad}}$ & $\beta_{\mathrm{XZ}}{ }^{\mathrm{rad}}$ \\
\hline 1 & 0.008 & 0.076 & 0.916 & 0.118 & 0.882 \\
2 & 0.009 & 0.080 & 0.911 & 0.154 & 0.846 \\
3 & 0.010 & 0.083 & 0.907 & 0.132 & 0.868 \\
4 & 0.018 & 0.073 & 0.909 & 0.118 & 0.882 \\
5 & 0.020 & 0.088 & 0.892 & 0.116 & 0.884 \\
Average & 0.013 & 0.080 & 0.907 & 0.128 & 0.872 \\
\hline
\end{tabular}

${ }^{a}$ In principle, these are sample independent.

Table 4. Experimental Values for the Fluorescence Ratios As Defined in Eqs. (27)-(29) ${ }^{a}$

\begin{tabular}{cccccr}
\hline Sample Number & ${ }^{w_{\phi_{W N}}}$ & ${ }^{w_{\phi_{X Y}}}$ & ${ }^{w_{\phi_{X W}}}$ & ${ }^{\mathrm{x}} \phi_{Y / X}$ & ${ }^{{ }^{A} \phi_{W / A}}$ \\
\hline 1 & 1.971 & 0.673 & 0.341 & 0.115 & 115.4 \\
2 & 1.250 & 0.459 & 0.367 & 0.145 & 86.1 \\
3 & 1.180 & 0.450 & 0.381 & 0.090 & 98.5 \\
4 & 0.859 & 0.348 & 0.405 & 0.091 & 105.3 \\
5 & 0.954 & 0.594 & 0.623 & 0.059 & 83.9 \\
\hline
\end{tabular}

a The superscripts refer to the pumping level; the subscripts refer to the originating levels of the emissions. 


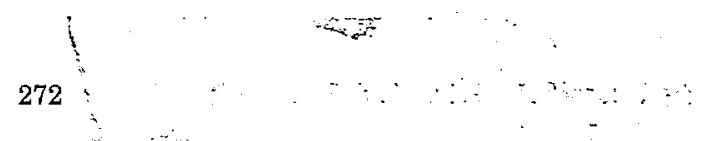

Table 5. Radiative, Multiphonon, and Cross-Relaxation Quantum Efficiencies as Determined by the Analysis of Section $4^{a}$

\begin{tabular}{|c|c|c|c|c|c|c|c|c|}
\hline Level & Sample Number & & $\eta^{\mathrm{rad}}$ & & $\eta^{\mathrm{MP}}$ & $\eta^{c}$ & $\begin{array}{l}\tau_{\text {meas }}^{\text {mo }} \\
(\mathrm{ms})\end{array}$ & $\begin{array}{c}\tau^{\mathrm{rad}}=\underset{(\mathrm{ms})}{\tau^{\mathrm{meas}} / \eta^{\mathrm{rad}}} \\
\end{array}$ \\
\hline \multirow[t]{6}{*}{ A } & 1 & 0.006 & \pm & 0.002 & $0.994 \pm 0.002$ & $0.000^{c}$ & 0.0052 & 0.94 \\
\hline & 2 & 0.007 & \pm & 0.002 & $0.993 \pm 0.002$ & $0.000^{c}$ & 0.0051 & 0.74 \\
\hline & 3 & 0.006 & \pm & 0.002 & $0.994 \pm 0.002$ & $0.000^{c}$ & 0.0049 & 0.82 \\
\hline & 4 & 0.004 & \pm & 0.002 & $0.996 \pm 0.002$ & $0.000^{c}$ & 0.0056 & 1.26 \\
\hline & $\cdot 5$ & 0.004 & \pm & 0.002 & $0.996 \pm 0.002$ & $0.000^{c}$ & 0.0048 & 1.17 \\
\hline & Average & 0.005 & \pm & 0.001 & $0.995 \pm 0.001$ & 0.000 & 0.0052 & 0.99 \\
\hline \multirow[t]{6}{*}{ W } & 1 & 0.642 & \pm & 0.022 & $0.216 \pm 0.014$ & $0.142 \pm 0.007^{b}$ & 0.717 & 1.12 \\
\hline & 2 & 0.593 & \pm & 0.020 & $0.210 \pm 0.012$ & $0.197 \pm 0.010^{b}$ & 0.671 & 1.13 \\
\hline & 3 & 0.590 & \pm & 0.019 & $0.208 \pm 0.011$ & $0.202 \pm 0.010^{b}$ & 0.667 & 1.13 \\
\hline & 4 & 0.471 & \pm & 0.020 & $0.184 \pm 0.011$ & $0.346 \pm 0.017^{b}$ & 0.547 & 1.16 \\
\hline & 5 & 0.344 & \pm & 0.021 & $0.216 \pm 0.012$ & $0.441 \pm 0.022^{b}$ & 0.467 & 1.36 \\
\hline & Average & $\ldots d$ & & & $0.207 \pm 0.005$ & $\ldots d$ & $\ldots d$ & 1.18 \\
\hline \multirow[t]{6}{*}{$x$} & 1 & 0.991 & \pm & 0.049 & $0.009 \pm 0.049$ & $0.000^{c}$ & 5.964 & 6.02 \\
\hline & 2 & 1.010 & \pm & 0.047 & $0.000 \pm 0.047$ & $0.000^{c}$ & 6.394 & 6.39 \\
\hline & 3 & 1.049 & \pm & 0.048 & $0.000 \pm 0.048$ & $0.000^{c}$ & 6.493 & 6.49 \\
\hline & 4 & 0.994 & \pm & 0.059 & $0.006 \pm 0.059$ & $0.000^{c}$ & 6.939 & 6.98 \\
\hline & 5 & 0.962 & \pm & 0.100 & $0.038 \pm 0.100$ & $0.000^{c}$ & 6.565 & 6.83 \\
\hline & Average & 1.001 & \pm & 0.027 & $0.011 \pm 0.027$ & 0.000 & 6.471 & 6.54 \\
\hline \multirow[t]{6}{*}{$Y$} & 1 & 0.904 & \pm & 0.073 & $0.000^{c}$ & $0.096 \pm 0.073^{e}$ & 14.62 & 16.2 \\
\hline & 2 & 1.003 & \pm & 0.082 & $0.000^{c}$ & $0.000 \pm 0.082^{e}$ & 15.46 & 15.5 \\
\hline & 3 & 1.061 & \pm & 0.084 & $0.000^{c}$ & $0.000 \pm 0.084^{e}$ & 15.31 & 15.3 \\
\hline & 4 & 0.731 & \pm & 0.071 & $0.000^{c}$ & $0.269 \pm 0.071^{e}$ & 16.16 & 22.1 \\
\hline & 5 & 0.381 & \pm & 0.047 & $0.000^{c}$ & $0.619 \pm 0.047^{e}$ & 11.40 & 29.9 \\
\hline & Average $f$ & 0.989 & \pm & 0.046 & 0.000 & $0.032 \pm 0.046$ & 15.13 & 15.7 \\
\hline
\end{tabular}

${ }^{a}$ Radiative lifetimes were determined as the ratio of the fluorescence lifetime ( $\tau^{\text {meas }}$ ) to the radiative quantum efficiency ( $\eta^{\text {rad }}$ ).

${ }^{b}$ Determined from measured lifetime data with Eq. (21).

${ }^{c}$ Assumed value.

${ }^{d}$ Concentration dependent.

${ }^{e}$ Assumed to be due to oxygen impurities in the host.

$f$ Samples 4 and 5 have been omitted from the average.

the sample 1 absorption spectrum of Fig. 2 (300 K) near 3 $\mu \mathrm{m}$. The total branching ratios $b_{\mathrm{JJ}}$ as defined in Eq. (20) are shown in Table 6.

\section{Discussion}

The justification for the use of macroscopic rate equations in our model is not obvious. Although there is a random variation in the distance between the two components in each microscopic donor-acceptor pair, the donor decay is observed to be exponential, as occurs in the case of fast

Table 6. Total Branching Ratios As Defined in Eq. (20)

\begin{tabular}{cccccc}
\hline Sample & $b_{W X}$ & $b_{W Y}$ & $b_{W Z}$ & $b_{X Y}$ & $b_{X Z}$ \\
\hline 1 & 0.221 & 0.191 & 0.588 & 0.126 & 0.874 \\
2 & 0.216 & 0.244 & 0.540 & 0.154 & 0.846 \\
3 & 0.214 & 0.251 & 0.535 & 0.132 & 0.868 \\
4 & 0.192 & 0.380 & 0.428 & 0.124 & 0.876 \\
5 & 0.223 & 0.471 & 0.306 & 0.150 & 0.850 \\
Average & 0.213 & $\ldots a$ & $\ldots a$ & 0.137 & 0.863 \\
\hline
\end{tabular}

${ }^{a}$ Concentration dependent. donor migration (i.e., in the hopping model). The rationalization lies in the notion that the donor decay can then be described by a collective macroscopic rate, which can be modeled with rate equations. The self-consistent nature of our data seems to support this justification.

Further self-consistency can be seen in the data in Table 5 and 6 . We expect (and observe) that, as the sample concentration increases, cross-relaxation between the $W$ and $Y$ levels will tend to increase the crossrelaxation fraction of the $W$ level $\left(\eta_{W}{ }^{c}\right)$ at the expense of the radiative fraction ( $\eta_{W}{ }^{\mathrm{rad}}$ ), leaving the (concentrationindependent) multiphonon fraction $\left(\eta_{W}{ }^{\mathrm{MP}}\right)$ unchanged. Thus the multiphonon-dominated $b_{W x}$ remains constant while the cross-relaxation-dominated $b_{W Y}$ increases and $b_{W Z}$ decreases with increasing concentration. As $\eta_{\mathrm{X}}{ }^{\mathrm{rad}}$, $b_{X Y}$, and $b_{X Z}$ are unaffected by cross relaxation, they too remain independent of concentration.

Another self-consistent result is the value of $\eta_{\mathrm{X}}{ }^{\mathrm{rad}}$ (from Table 5). As level $X$ lies $\sim 2350 \mathrm{~cm}^{-1}$ (more than 11 phonons) above the $Y$ level, we expect the multiphonon contribution to the $X$-level decay to be minimal, making the radiative quantum efficiency near unity. Figure 10 shows the values of the radiative quantum efficiencies $\eta_{\mathrm{W}}{ }^{\mathrm{rad}}, \eta_{\mathrm{X}}^{\mathrm{rad}}$, and $\eta_{\mathrm{Y}}{ }^{\mathrm{rad}}$ for sample 1 plotted as a function 
of $\eta_{\mathrm{W}}{ }^{c}$ according to Eqs. (30)-(32). In this figure the curves reflect the radiative branching ratios $\beta_{\mathrm{JJ}}{ }^{\mathrm{rad}}$ and fluorescence ratios ${ }^{\mathrm{P}} \phi_{\mathrm{J} / \mathrm{K}}$ that were actually measured for sample 1 ; only $\eta_{W}{ }^{c}$ (the fraction of the W-level population taking the cross-relaxation pathway) was allowed to vary independently. We see that $\eta_{\mathrm{X}}{ }^{\mathrm{rad}}$ is near unity at precisely the value of $\eta_{W}$ determined by means of Eq. (21) from lifetime measurements, as indicated by the dashed vertical line in the figure. The assumption of a crossrelaxation path with a decay rate of the form $W^{c}$ $=k N_{0}^{2}$ is critical in obtaining this result, because $\eta_{W}{ }^{c}$ $=1-W_{W}{ }^{0} / W_{W}$ and $W_{W}{ }^{0}$ is obtained from the straightline fit to the plot of $W_{W}$ versus $N_{0}^{2}$ (e.g., Fig. 7). Notice from Fig. 10 that values of $\eta_{W}{ }^{c}$ less than 0.13 and greater than 0.85 are unphysical because the radiative quantum efficiencies there are outside the physical boundary 0 $\leqslant \eta^{\mathrm{rad}} \leqslant 1$.

The radiative lifetimes, determined from Eq. (8) as the ratio of the measured lifetime to the radiative quantum efficiency, can also be used as a consistency check because $\tau^{\text {meas }}$ and $\eta^{\text {rad }}$ are independently determined. We expect $\tau^{\mathrm{rad}}$ values to be intrinsic to $\mathrm{KPb}_{2} \mathrm{Cl}_{5}$ and thus independent of concentration. The relatively constant values for the $A, W, X$, and $Y$ levels in Table 5 generally point to selfconsistency. Samples 4 and 5 , however, seem to be inconsistent with samples $1-3$ for the $Y$ level. The lower

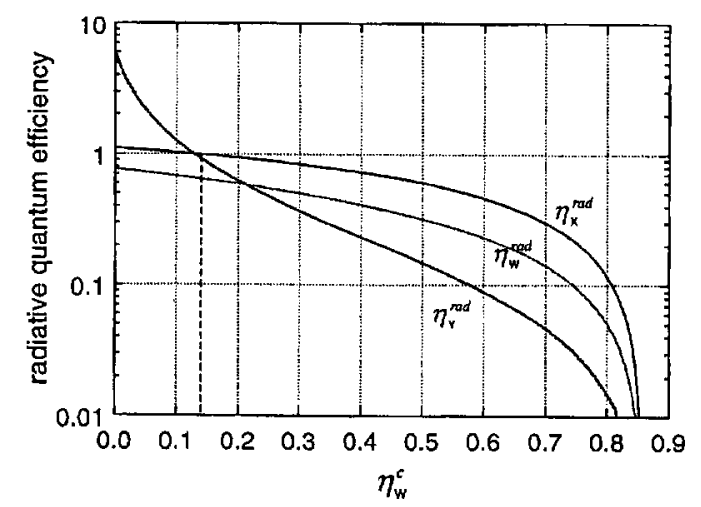

Fig. 10. $W, X$, and $Y$ level radiative quantum efficiencies plotted as a function of the W-level cross-relaxation fraction for sample 1 according to Eqs. (30), (31), and (32), respectively. The dashed vertical line indicates the value of $\eta_{W}{ }^{c}$ determined from temporal measurements according to Eq. (21). The $\eta>1$ region is inaccessible. values of $\eta_{Y}{ }^{\text {rad }}$ (and the relatively large values of $w_{\phi_{W}}$ and ${ }^{w} \phi_{W / Y}$ in . Table 4) are attributed to $\mathrm{OH}$ quenching of the $Y$ level near $3 \mu \mathrm{m}$ in samples 4 and 5. A corresponding decrease in the measured $Y$-level lifetimes for these samples was expected but not observed (although a small decrease was observed). This inconsistency has not yet been resolved.

The small value $(<1 \%)$ for the A-level radiative quantum efficiency is surprising because the energy gap to the $W$ level of $\sim 1300 \mathrm{~cm}^{-1}$ corresponds to more than six phonons. Indeed, there may be processes other than nonradiative multiphonon decay at work, as evidenced by the fact that Dieke did not observe fluorescence from the A level in $\mathrm{LaCl}_{3}{ }^{20}$ In this case, the $\mathrm{NH}_{4}{ }^{+}$vibration [absorption feature near $\left.7.2 \mu \mathrm{m}\left(\sim 1390 \mathrm{~cm}^{-1}\right)\right]$ is probably responsible for reducing the radiative decay rate from the A level.

The results of this analysis that can be directly compared with the Judd-Ofelt analysis of Subsection 2.E are shown in Table 7. We calculated the $W={ }^{6} H_{9 / 2}$ $+{ }^{6} F_{11 / 2}$ (and $A={ }^{6} H_{7 / 2}+{ }^{6} F_{9 / 2}$ )-level data by assuming that the constituent levels were in thermal equilibrium. The Judd-Ofelt predictions of radiative branching ratios were generally good (within 10\%), but the radiative lifetimes have been somewhat underestimated. The branching ratios and radiative lifetimes measured here can be used to produce more accurate emission cross-section data from Eq. (13). In fact, through Eqs. (13) and (26) and the analysis of Section 3 , these cross sections require only the measured refractive index, fluorescence lifetimes, and calibrated emission spectra of the selectively pumped energy levels. The measured peak cross sections are included in Table 7 and in general are smaller than those predicted by the Judd-Ofelt data.

\section{SPECTROSCOPY AND ANALYSIS FOR $\mathrm{KPb}_{2} \mathrm{Cl}_{5}: \mathrm{Nd}^{3+}$}

$\mathrm{KPb}_{2} \mathrm{Cl}_{5}: \mathrm{Nd}^{3+}$, whose absorption spectrum is shown in Fig. 11, was included in this study because the ${ }^{4} F_{5 / 2}$ and ${ }^{4} F_{3 / 2}$ levels of $\mathrm{Nd}^{3+}$ (see Fig. 11, inset) provide a convenient energy gap (1030 $\mathrm{cm}^{-1}$ peak to peak) for the multiphonon plot in Section 6 . The analysis (similar to that in Section 3) for these levels is quite simple, as we may assume that the radiative quantum efficiency for the ${ }^{4} F_{3 / 2}$ level is $\approx 1$ and that the radiative branching ratio

Table 7. Measured Data from Tables 3 and 5 Compared with Judd-Ofelt Data from Table ${ }^{a}$

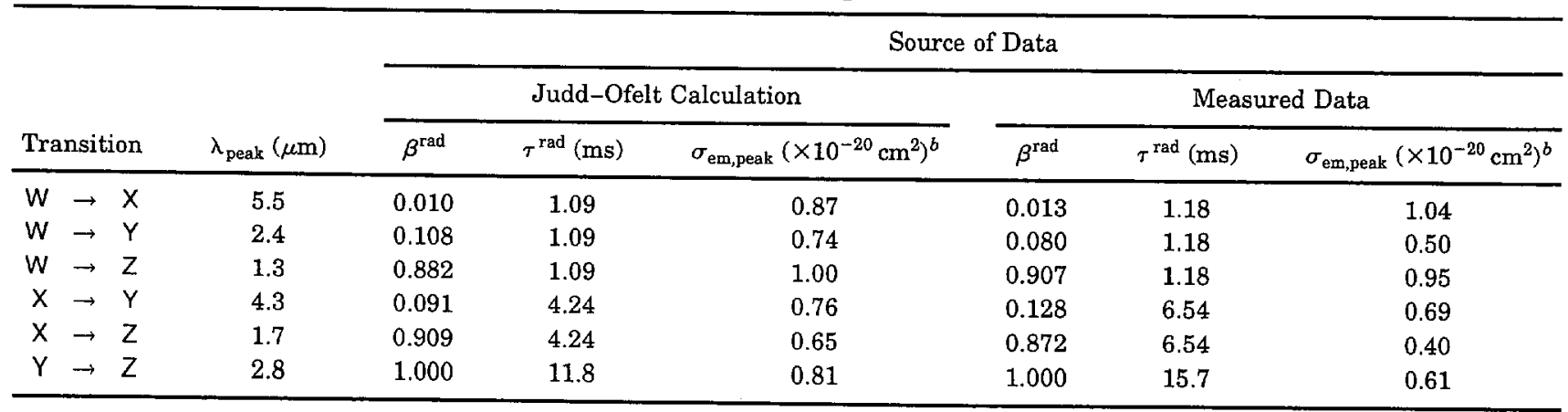

\footnotetext{
average values were used for the measured data whenever applicable.
}

${ }^{b}$ Calculated with Eq. (13). 


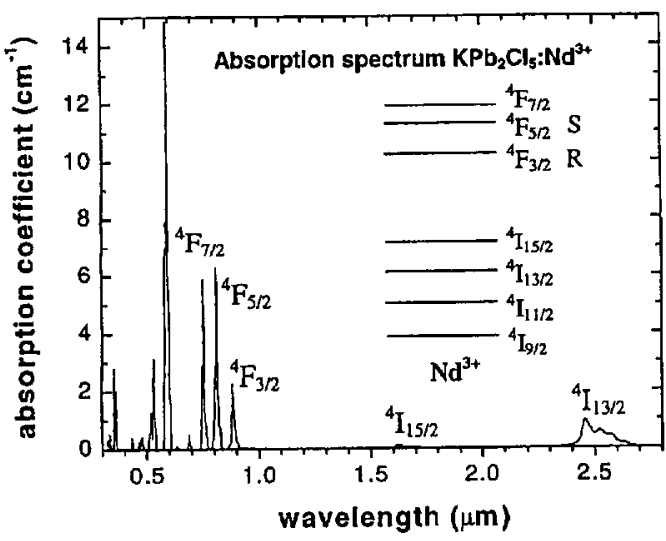

Fig. 11. $\mathrm{Nd}^{3+}$ absorption spectrum and energy levels. Note the letter designations of the ${ }^{4} F_{5 / 2}$ and ${ }^{4} F_{3 / 2}$ levels. The ${ }^{4} F_{3 / 2} \mathrm{R}$ level is assumed to have unit quantum efficiency. The ${ }^{4} F_{5 / 2} \mathrm{~S}$ level was pumped for quantum efficiency experiments.

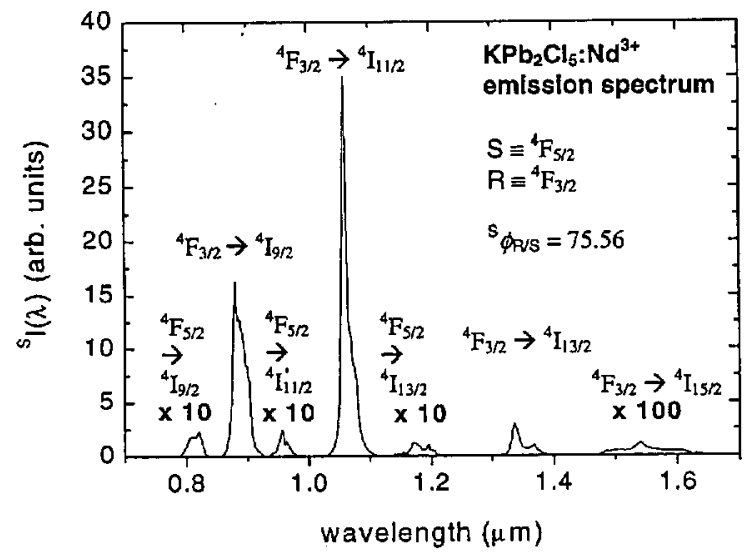

Fig. 12. Emission spectrum obtained by direct excitation of the $S$ level of $\mathrm{KPb}_{2} \mathrm{Cl}_{5}: \mathrm{Nd}^{3+}$. Emission from both the ${ }^{4} F_{3 / 2} \mathrm{R}$ and the ${ }^{4} F_{5 / 2} \mathrm{~S}$ (energy gap, $\approx 1000 \mathrm{~cm}^{-1}$ ) levels is observed. The data imply a fluorescence ratio ${ }^{\mathrm{s}} \phi_{\text {RUS }}$ of 75.56 .

between the levels is $\approx 0$, so multiphonon decay is the only pathway that connects the two levels. Labeling the $\mathrm{Nd}^{3+}{ }^{4} F_{5 / 2}$ level $S$ and the ${ }^{4} F_{3 / 2}$ level R, we find the result analogous to Eqs. (31) and (33):

$$
\eta_{\mathrm{S}}^{\mathrm{rad}}=\frac{1}{\mathrm{~s}_{\phi_{\mathrm{A} / \mathrm{S}}}+1} .
$$

The S-pumped emission data for $\mathrm{Nd}^{3+}$ are shown in Fig. 12 , in which emission peaks that originate from the $\mathrm{S}$ level at $0.81,0.95$, and $1.18 \mu \mathrm{m}$ are visible. A cw Ti:sapphire laser tuned to $0.81 \mu \mathrm{m}$ was used to pump the sample, and a liquid-nitrogen-cooled InSb detector (along with appropriate bandpass filters) was used to record the emission from 0.83 to $1.8 \mu \mathrm{m}$ at the exit port of a $1-\mathrm{m}$, $1-\mu \mathrm{m}$ blazed grating monochromator. The emission near $810 \mathrm{~nm}$ was obtained on a second run spanning 0.78-1.1 $\mu \mathrm{m}$ by use of a Ti:sapphire laser tuned to $0.77 \mu \mathrm{m}$ (pumping the ${ }^{4} F_{7 / 2}$ level) and a dry-ice cooled photomultiplier tube. We then combined the two runs by matching the emission peaks in the overlapping region. The value of

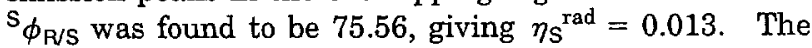
fluorescence lifetime for the $S$ level was measured to be $3.4 \mu \mathrm{s}$; that of the R level was measured to be $340 \mu \mathrm{s}$.

\section{NONRADIATIVE DECAY RATE FOR $\mathrm{KPb}_{2} \mathrm{Cl}_{5}$}

\section{A. Energy-Gap Law}

The analysis of Section 5 permits calculation of the nonradiative (multiphonon) decay rates, so we may plot the multiphonon emission rate versus energy gap and deduce the phenomenological parameters described by the energy-gap law. The energy-gap law states the empirically derived phenomenological dependence of the nonradiative multiphonon decay rate $W_{J}^{M P}$ on the energy gap $\Delta E$ between a level $\mathrm{J}$ and the next lowest level:

$$
W_{\rfloor}^{M P}=B \exp (-\beta \Delta E)\left[1-\exp \left(-h \nu_{\max } / k T\right)\right]^{-p},
$$

where $p=\Delta E / h \nu_{\max }$ is the number of phonons (each characterized by $h \nu_{\max }$ ) required for bridging the energy gap and $k T \approx 208 \mathrm{~cm}^{-1}$ at room temperature. $B$ and $\beta$ are the two fitted parameters that characterize the decay. These phenomenological constants are dependent on the host but are largely independent of the rare-earth ion dopant.

\section{B. Fitted Parameters}

Using the $\mathrm{Dy}^{3+}$ quantum efficiency and measured lifetime data for sample 1 from Table 4 and the $\mathrm{Nd}^{3+}$ data from Section 5 , we can determine the multiphonon decay rates $W^{\mathrm{MP}}$ as $W^{\mathrm{MP}}=\eta^{\mathrm{MP}} / \tau^{\text {meas }}$. Combining this result with the energy gaps calculated (peak to peak) from the energy levels shown in Fig. 3, we obtain the plot shown in Fig. 13. The fitted parameters for $\mathrm{KPb}_{2} \mathrm{Cl}_{5}$ are $B=3.721$ $\times 10^{9} \mathrm{~s}^{-1}$ and $\beta=1.156 \times 10^{-2} \mathrm{~cm}$. The data point at $\Delta E=1315 \mathrm{~cm}^{-1}$ that arises from the A-level data was not included in the fit. As discussed in Subsection 4.C, we believe that the relatively weak emission from this level involves processes other than just nonradiative multiphonon decay. It is notable that Dieke also observed fluorescence from the ${ }^{4} F_{5 / 2} \mathrm{~S}$ level in $\mathrm{LaCl}_{3}: \mathrm{Nd}^{3+}(\Delta E$ $\approx 1000 \mathrm{~cm}^{-1}$ ). For comparison, plots of $W^{\mathrm{MP}}$ versus the energy gap for other hosts ${ }^{23}$ are included in Fig. 13. $\mathrm{KPb}_{2} \mathrm{Cl}_{5}$ is comparable with $\mathrm{LaCl}_{3}$ in its multiphonon decay characteristics.

From the multiphonon plot we can conclude that to achieve at least $1 \%$ radiative quantum efficiency for a

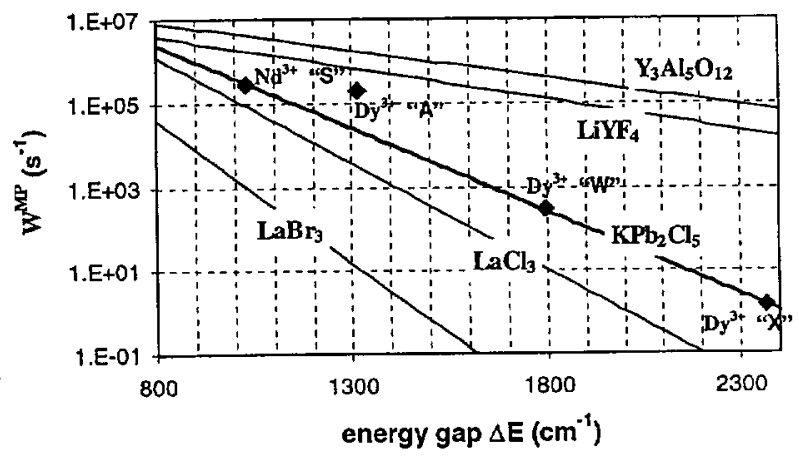

Fig. 13. Multiphonon relaxation rate versus energy gap in $\mathrm{KPb}_{2} \mathrm{Cl}_{5}$ (filled diamonds). The source of each data point is indicated. The curves for YAG, $\mathrm{YLF}, \mathrm{LaCl}_{3}$, and $\mathrm{LaBr}_{3}$ are shown for comparison. These data are taken from Ref. 23. 
typical radiative rate of $10^{3} \mathrm{~s}^{-1}$ requires a multiphonon rate of at most $10^{5} \mathrm{~s}^{-1}$, which occurs near $\Delta E$ $=1150 \mathrm{~cm}^{-1}$ in $\mathrm{KPb}_{2} \mathrm{Cl}_{5}$ and corresponds to a wavelength of $\sim 9 \mu \mathrm{m}$. This conclusion is consistent with the measured radiative quantum efficiency of $1.3 \%$ for the $1030-\mathrm{cm}^{-1}$ gap in $\mathrm{KPb}_{2} \mathrm{Cl}_{5}: \mathrm{Nd}^{3+}$. We are currently working to identify a rare-earth ion transition that is capable of demonstrating this long-wavelength laser potential. The difficulty lies in the fact that, in addition to the proper energy gap, this transition must also possess a suitable pumping scheme and emission cross section.

\section{CONCLUSIONS}

We have studied the optical properties of rare-earthdoped $\mathrm{KPb}_{2} \mathrm{Cl}_{5}$, a novel low-phonon-energy chloride host that is not moisture sensitive. Concentration-dependent cross-relaxation processes were identified and characterized for the ${ }^{6} \mathrm{H}_{9 / 2}+{ }^{6} F_{11 / 2} \mathrm{~W}$ level with a cross-relaxation coefficient of $k_{\mathrm{W}}{ }^{c}=(1.83 \pm 0.20) \times 10^{-37} \mathrm{~cm}^{6} \mathrm{~s}^{-1}$. Using only fluorescence lifetimes, refractive index, and calibrated emission intensities, we determined radiative quantum efficiencies and indicated that laser action in this host should be possible to $\sim 9 \mu \mathrm{m}$.

\section{ACKNOWLEDGMENTS}

Our gratitude is extended to Peter Thelin, who polished the laser crystal, to Theresa Duewer, who measured its $\mathrm{Dy}^{3+}$ content, and to Jay Skidmore, who provided the laser diodes. We thank Denise Krol and Howard Powell for their contributions and support. This research, supported by the Defense Advanced Research Projects Agency and the U.S. Department of Energy's Office of Basic Energy Sciences, was performed under the auspices of the U.S. Department of Energy, Lawrence Livermore National Laboratory, contract W-7405-ENG-48.

M. C. Nostrand's e-mail address is nostrand1@llnl.gov.

\section{REFERENCES}

1. T. Schaafsma, L. B. Shaw, B. Cole, J. S. Sanghera, and I. D. Aggarwal, "Modeling of $\mathrm{Dy}^{3+}$-doped GeAsSe glass 1.3- $\mu \mathrm{m}$ optical fiber amplifiers," IEEE Photon. Technol. Lett. 10, 1548-1550 (1998).

2. S. Tanabe, T. Hanada, M. Watanabe, T. Hayashi, and N. Soga, "Optical properties of dysprosium-doped low-phononenergy glasses for a potential 1.3- $\mu \mathrm{m}$ optical amplifier," J. Am. Ceram. Soc. 78, 2917-2922 (1995).

3. K. Wei, D. P. Machewirth, J. Wenzel, E. Snitzer, and G. H. Sigel, Jr., "Spectroscopy Of $\mathrm{Dy}^{3+}$ in Ge-Ga-S glass and its suitability for 1.3- $\mu \mathrm{m}$ fiber-optic amplifier applications," Opt. Lett. 19, 904-906 (1994).

4. D. W. Hewak, B. N. Samson, J. A. M. Neto, R. I. Laming, and D. N. Payne, "Emission at $1.3-\mu \mathrm{m}$ from dysprosiumdoped GaLaS glass," Electron. Lett. 30, 968-970 (1994).

5. Y. Guimond, J. L. Adam, A. M. Jurdyc, J. Mugnier, B. Jacquier, and X. H. Zhang, "Dy ${ }^{3+}$-doped stabilized GeGaS glasses for $1.3 \mu \mathrm{m}$ optical fiber amplfiers," Opt. Mater. 12, 467-471 (1999).

6. M. C. Nostrand, R. H. Page, S. A. Payne, W. F. Krupke, and P. G. Schunemann, "Room-temperature laser action at 4.3$4.4 \mu \mathrm{m}$ in $\mathrm{CaGa}_{2} \mathrm{~S}_{4}: \mathrm{Dy}^{3+}$," Opt. Lett. 24, 1215-1217 (1999).

7. T: Schweizer, B. N. Samson, J. R. Hector, W. S. Brocklesby, D. W. Hewak, and D. N. Payne, "Infrared emission and ion-ion interactions in thulium- and terbium-doped gallium lanthanum sulfide glass," J. Opt. Soc. Am. B 16, 308316 (1999).

8. T. Schweizer, B. N. Samson, J. R. Hector, W. S. Brocklesby, D. W. Hewak, and D. N. Payne, "Infrared emission from holmium doped gallium lanthanum sulphide glass," Infrared Phys. Technol. 40, 329-335 (1999).

9. S. R. Bowman, L. B. Shaw, B. J. Feldman, and J. Ganem, "A 7- $\mu \mathrm{m}$ praseodymium-based solid-state laser," IEEE J. Quantum Electron. 32, 646-649 (1996).

10. R. H. Page, K. I. Schaffers, S. A. Payne, and W. F. Krupke, "Dy-doped chlorides as gain media for $1.3 \mu \mathrm{m}$ telecommunications amplifiers," J. Lightwave Technol. 15, 786-793 (1997).

11. A. M. Tkachuk, S. E. Ivanova, L. I. Isaenko, A. P. Eliseev, W. F. Krupke, S. A. Payne, R. W. Solarz, M. C. Nostrand, and R. H. Page, "Dy" ${ }^{3+}$-doped crystals of double chlorides and double fluorides as the active media of IR solid-state lasers and telecommunication amplifiers," J. Opt. Technol. 66, 460-462 (1999).

12. A. Tkachuk, S. Ivanova, L. Isaenko, A. Yelisseyev, S. A. Payne, R. W. Solarz, M. C. Nostrand, and R. H. Page, "Comparative spectroscopic study of the $\mathrm{Dy}^{3+}$ doped double chloride and double fluoride crystals for telecommunication amplifiers and IR lasers," Acta Phys. Pol. A 95, 381-394 (1999).

13. L. I. Isaenko, A. Yelisseyev, V. A. Nadolinny, V. I. Pashkov, M. C. Nostrand, S. A. Payne, R. H. Page, and R. W. Solarz, "Spectroscopic investigation of rare-earth-doped chloride single crystals for telecommunications amplifiers," in Solid State Lasers VII, R. Scheps, ed., Proc. SPIE 3265, 242-249 (1998).

14. M. C. Nostrand, R. H. Page, S. A. Payne, W. F. Krupke, P. G. Schunemann, and L. I. Isaenko, "Spectroscopic data for infrared transitions in $\mathrm{CaGa}_{2} \mathrm{~S}_{4}: \mathrm{Dy}^{3+}$ and $\mathrm{KPb}_{2} \mathrm{Cl}_{5}: \mathrm{Dy}^{3+}$," in Advanced Solid-State Lasers, W. R. Bosenberg and M. M. Fejer, eds., Vol. 19 of OSA Topics in Optics and Photonics Series (Optical Society of America, Washington, D.C., 1998), pp. 524-528.

15. K. Nitsch, M. Dusek, M. Nikl, K. Polak, and M. Rodova, "Ternary alkali lead chlorides-crystal-growth, crystalstructure, absorption and emission properties," Prog. Cryst. Growth Charact. 30, 1-22 (1995).

16. S. R. Bowman, S. K. Searles, J. Ganem, and P. Schmidt, "Further investigations of potential $4 \mu \mathrm{m}$ laser materials," in Advanced Solid-State Lasers, M. M. Fejer, H. Injeyan and U. Keller, eds., Vol, 26 of OSA Topics in Optics and Photonics Series (Optical Society of America, Washington, D.C., 1999), pp. 487-490.

17. M. C. Nostrand, R. H. Page, S. A. Payne, W. F. Krupke, P. G. Schunemann, and L. I. Isaenko, "Room temperature $\mathrm{CaGa}_{2} \mathrm{~S}_{4}: \mathrm{Dy}^{3+}$ laser action at 2.43 and $4.31 \mu \mathrm{m}$ and $\mathrm{KPb}_{2} \mathrm{Cl}_{5}: \mathrm{Dy}^{3+}$ laser action at $2.43 \mu \mathrm{m}$," in Advanced Solid. State Lasers, M. M. Fejer, H. Injeyan, and U. Keller, eds., Vol. 26 of OSA Topics in Optics and Photonics Series (Optical Society of America, Washington, D.C., 1999), pp. 441449.

18. M. C. Nostrand, R. H. Page, S. A. Payne, W. F. Krupke, P. G. Schunemann, and L. I. Isaenko, "Laser demonstrations of rare-earth ions in low-phonon chloride and sulfide crystals," in Advanced Solid-State Lasers, H. Injeyan, U. Keller, and C. Marshall, eds., Vol. 34 of OSA Topics in Optics and Photonics Series (Optical Society of America, Washington, D.C., 2000), pp. $459-463$.

19. M. Nikl, K. Nitsch, I. Velicka, J. Hybler, K. Polak, and T. Fabian, "Photoluminescence of $\mathrm{KPb}_{2} \mathrm{Cl}_{5}$," Phys. Status Solidi B 168, K37-K42 (1991).

20. G. Dieke, Spectra and Energy Levels of Rare-Earth Ions in Crystals (Interscience, New York, 1968).

21. B. R. Judd, "Optical absorption intensities of rare-earth ions," Phys. Rev. 127, 750-761 (1962).

22. G. S. Ofelt, "Intensities of crystal spectra of rare-earth ions," J. Chem. Phys. 37, 511-520 (1962).

23. A. A. Kaminskii, Crystalline Lasers: Physical Processes and Operating Schemes (CRC Press, New York, 1996). 
24. W. F. Krupke, "Radiative transition probabilities within the $4 f^{3}$ ground configuration of Nd: YAG," IEEE J. Quantum Electron. QE-7, 153-159 (1971).

25. B. G. Wybourne, Spectroscopic Properties of Rare Earths (Interscience, New York, 1965)

26. B. G. Wybourne, "Structure of $f^{n}$-configurations. II. $f^{5}$ and $f^{9}$ configurations," J. Chem. Phys. 36, 2301-2310 (1962).

27. C. K. Jorgensen and B. R. Judd, "Hypersensitive pseudoquadrupole transitions in lanthanides," Mol. Phys. 8, 281-290 (1964).

28. R. S. Quimby, "Active phenomena in doped halide glasses," in Fluoride Glass Fiber Optics, I. D. Aggarwal and G. Lu, eds. (Academic, San Diego, Calif., 1991), p. 356.

29. J. A. Caird, A. J. Ramponi, and P. R. Staver, "Quantum efficiency and excited-state relaxation dynamics in neodymium-doped phosphate laser glasses," J. Opt. Soc. Am. B 8, 1391-1403 (1991)

30. A. I. Burshtein, "Hopping mechanism of energy transfer," Sov. Phys. JETP 35, 882-885 (1972).
31. F. Auzel, "Multiphonon-assisted anti-Stokes and Stokes fluorescence of triply ionized rare-earth ions," Phys. Rev. B 13, 2809-2817 (1976).

32. A. Braud, S. Girard, J. L. Doualan, and R. Moncorge, "Spectroscopy and fluorescence dynamics of $\left(\mathrm{Tm}^{3+}, \mathrm{Tb}^{3+}\right)$ and $\left(\mathrm{Tm}^{3+}, \mathrm{Eu}^{3+}\right)$ doped $\mathrm{LiYF}_{4}$ single crystals for 1.5- $\mu \mathrm{m}$ laser operation," IEEE J. Quantum Electron. 34, 2246-2255 (1998).

33. R. S. Quimby, K. T. Gahagan, B. G. Aitken, and M. A. Newhouse, "Self-calibrating quantum efficiency measurement technique and application to $\mathrm{Pr}^{3+}$-doped sulfide glass," Opt. Lett. 20, 2021-2023 (1995).

34. J. A. Skidmore, B. L. Freitas, C. E. Reinhardt, E. J. Utterback, R. H. Page, and M. A. Emanuel, "High-power operation of InGaAsP-InP laser diode array at $1.73 \mu \mathrm{m}$," IEEE Photon. Technol. Lett. 9, 1334-1336 (1997); D. T. Schaafsma, L. B. Shaw, B. Cole, J. S. Sanghera, and I. D. Aggarwal, "Modeling of $\mathrm{Dy}^{3+}$-doped GeAsSe glass $1.3-\mu \mathrm{m}$ optical fiber amplifiers," IEEE Photon. Technol. Lett. 10, 15481550 (1998). 


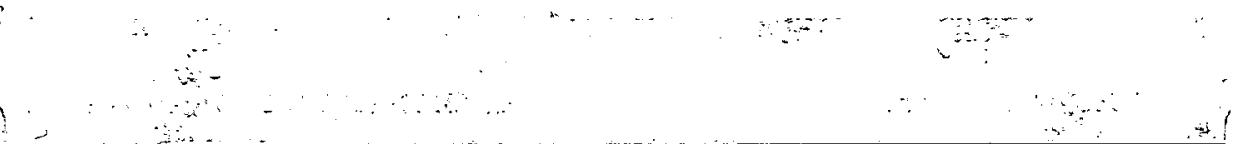

\title{
Plasma turbulence imaging using high-power laser Thomson scattering
}

\author{
S. J. Zweben ${ }^{\text {a) }}$ \\ Princeton Plasma Physics Laboratory, Princeton, New Jersey 08543 \\ J. Caird \\ Lawrence Livermore National Laboratory, Livermore, California 94550
}

W. Davis, D. W. Johnson, and B. P. Le Blanc

Princeton Plasma Physics Laboratory, Princeton, New Jersey 08543

(Presented on 21 June 2000)

The two-dimensional (2D) structure of plasma density turbulence in a magnetically confined plasma can potentially be measured using a Thomson scattering system made from components of the Nova laser of Lawrence Livermore National Laboratory. For a plasma such as the National Spherical Torus Experiment at the Princeton Plasma Physics Laboratory, the laser would form an $\approx 10$-cm-wide plane sheet beam passing vertically through the chamber across the magnetic field. The scattered light would be imaged by a charge coupled device camera viewing along the direction of the magnetic field. The laser energy required to make $2 \mathrm{D}$ images of density turbulence is in the range 1-3 kJ, which can potentially be obtained from a set of frequency-doubled $\mathrm{Nd}$ :glass amplifiers with diameters in the range of $208-315 \mathrm{~mm}$. A laser pulse width of $\leqslant 100 \mathrm{~ns}$ would be short enough to capture the highest frequency components of the expected density fluctuations.

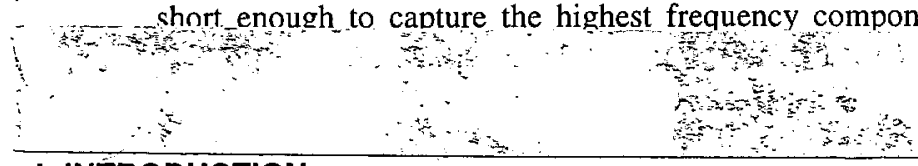

\section{INTRODUCTION}

Plasma turbulence is one of the most difficult phenomena to understand in plasma physics. Compared with neutral fluid turbulence, there is still relatively little experimental information about the space-time structure of small-scale plasma density turbulence, which is probably the dominant turbulence in many magnetic fusion plasmas (here "small scale" refers to a range of turbulence wave numbers across the magnetic field of $k_{\perp} a \ll 1$, where $a$ is the plasma size). Given the well-recognized importance of such turbulence for magnetic fusion plasma confinement, ${ }^{1}$ it is important to improve our diagnostic ability in this area.

This article discusses the feasibility of making a significant improvement in diagnosing plasma density turbulence by applying the high-power laser equipment developed by the inertial confinement fusion (ICF) program at Lawrence Livermore National Laboratory (LLNL) to measure plasma density turbulence in a suitable magnetic fusion device. We propose to use the well-understood technique of Thomson scattering to measure the two-dimensional (2D) structure of density turbulence in an experiment such as the National Spherical Torus Experiment (NSTX) at the Princeton Plasma Physics Laboratory (PPPL), which has a large magnetically confined plasma suitable for such a study. This initial assessment shows that the required laser would need $\approx 1-3 \mathrm{~kJ}$ of energy in a pulse of width $\leqslant 100 \mathrm{~ns}$ to make a single $2 \mathrm{D}$ image of the expected density turbulence in a NSTX discharge. This appears feasible using a laser built from components that could be obtained from the recently decommissioned Nova laser system at LLNL.

A schematic illustration of this concept is shown in Fig.

a)Electronic mail: szweben@pppl.gov
1. The laser would enter the plasma through a large window at the bottom of the machine in a beam with a radial width of $\approx 10 \mathrm{~cm}$. The laser beam would exit through a window at the top, and stop in a specially constructed beam dump remote from the machine. A very small fraction of the laser light $\left(\approx 10^{-9}\right)$ will be Thomson scattered by the free electrons in the beam path. The local intensity of the scattered light will be proportional to the local electron density. Therefore spatial fluctuations in the electron density in the radial versus

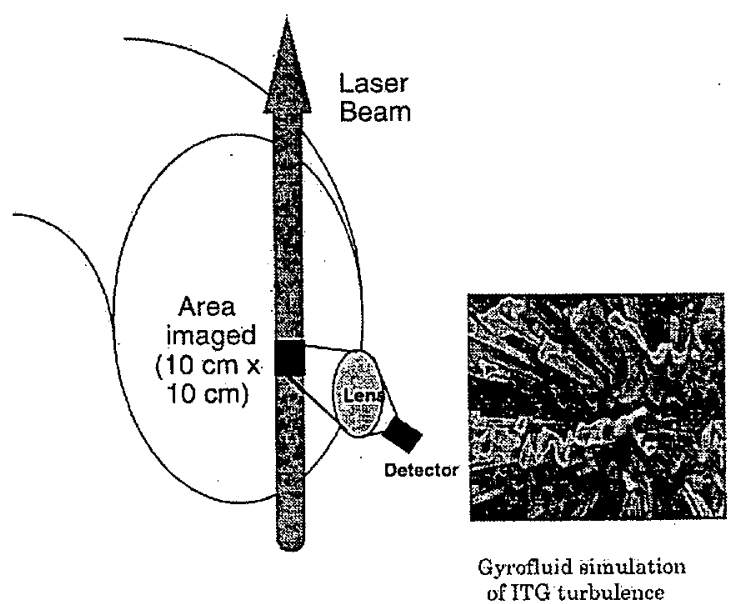

FIG. 1. Schematic illustration of a 2D Thomson scattering measurement of density turbulence in NSTX. The camera viewing along the magnetic field line would make a $2 \mathrm{D}$ radial vs poloidal image of the scattered light from electrons within the area imaged. At the right is a 3D rendering of a theoretical simulation of ITG density turbulence, which has short correlation lengths in the radial vs poloidal plane but a long correlation length along the magnetic field. The laser beam duration of $\leqslant 100 \mathrm{~ns}$ is shorter than the turbulence autocorrelation time, so the instantaneous structure of the turbulence should be visible. 
TABLE I. Assumed NSTX parameters for 2D Thomson scattering.

\begin{tabular}{ll}
\hline \hline Plasma major radius & $R_{o}=0.85 \mathrm{~m}$ \\
Plasma minor radius & $a=0.68 \mathrm{~m}$ \\
Radius of measurement & $R \approx R_{o}+a / 2 \approx 1.3 \mathrm{~m}$ \\
Density at this location & $n=3 \times 10^{13} \mathrm{~cm}^{-3}$ \\
Electron temperature at this radius & $1 \mathrm{keV}$ \\
Estimated turbulence size scale & $\lambda \approx 1-10 \mathrm{~cm}$ \\
Estimated turbulence magnitude & $\tilde{n} / n \approx 1 \%$ \\
Estimated viewing area & $10 \mathrm{~cm} \times 10 \mathrm{~cm}$ \\
Estimated turbulence time scale & $\leqslant 1 \mu \mathrm{s}$ \\
\hline
\end{tabular}

poloidal plane can be measured by making a $2 \mathrm{D}$ image of the scattered light as viewed approximately along the magnetic field line, thus integrating over the width of the laser beam in the direction parallel to the magnetic field (in which the fluctuations are constant). If the laser pulse width is shorter than the turbulence autocorrelation time (eddy turnover time), an image of the small-scale density turbulence will be produced.

Similar scattering techniques are used in fluid turbulence experiments, ${ }^{2}$ but a plasma scattering experiment is more difficult due to the $\geqslant 10^{6}$ times lower density of plasma with respect to neutral fluids. However, this measurement in a magnetic fusion plasma is important because the results could be directly compared with the recent three-dimensional (3D) gyrokinetic and gyrofluid simulations of toroidal plasma turbulence, such as that illustrated in Fig. $1 .^{3} \mathrm{~A}$ comparison between the measured $2 \mathrm{D}$ density turbulence structure and these models would be a significant test of the validity of these models, even if this structure could only be measured at one point in time per discharge.

\section{SIMULATION OF THE EXPECTED 2D IMAGES}

The main technical difficulty in this diagnostic arises from the very small cross section for incoherent Thomson scattering, $\quad \sigma_{\mathrm{TS}}=(8 \pi / 3) r_{e}{ }^{2} \approx 6.6 \times 10^{-25} \mathrm{~cm}^{2}$. Although small, this cross section is completely understood, making Thomson scattering measurements the most reliable method for plasma density and temperature measurements in magnetic fusion research. ${ }^{4}$ In this section we estimate the laser energy needed to produce 2D images of plasma density turbulence in NSTX and show a simulation of such an image.

The assumed plasma and turbulence parameters for NSTX are shown in Table I, as estimated from previous experimental studies on tokamak plasmas. ${ }^{1}$ The plasma density in the region of interest is typically $n=3 \times 10^{13} \mathrm{~cm}^{-3}$ with a local density fluctuation level of $\approx 1 \% \mathrm{rms}$ and a range of spatial scales from $\lambda_{\perp} \approx 1-10 \mathrm{~cm}$. The structure of these fluctuations is generally isotropic and turbulent across the magnetic field but very elongated along the magnetic field $\left(\lambda_{\text {parallel }} \gg 1 \mathrm{~m}\right)$, as illustrated in Fig. 1.

First we give a rough estimate of the laser energy required to resolve these density fluctuations using Thomson scattering. The fraction of scattered photons is $F=\sigma_{\mathrm{TS}} n L$, where $L$ is the vertical distance along the laser path length. For an incident laser beam with $P$ photons $/ \mathrm{cm}$ in the radial direction, the total number of photons per $\mathrm{cm}$ scattered into a detector subtending a solid angle $\Omega$ str. is thus $N_{\text {tot }}$ $\approx F P \Omega / 4 \pi$. For imaging a $1 \mathrm{~cm}$ vertical $\times 1 \mathrm{~cm}$ radial area

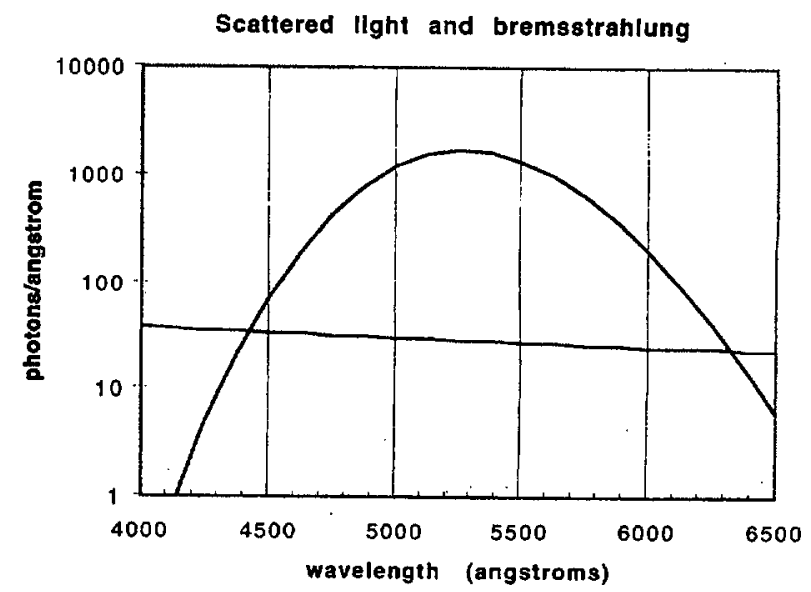

FIG. 2. Calculated spectrum of the laser light scattered at $90^{\circ}$ to the laser beam by a $\mathrm{I} \mathrm{cm} \times 1 \mathrm{~cm}$ area of a NSTX plasma with $n=3 \times 10^{13} \mathrm{~cm}^{-3}$ and $T_{e}=1 \mathrm{keV}$, compared with the plasma bremsstrahlung spectrum (straight line). The estimated signal/noise level for a density measurement within this area is $S / N \approx 430$ for a $1 \mathrm{~kJ}$ laser beam spread over a radius of $10 \mathrm{~cm}$ radially.

of the NSTX plasma, $F \approx 2 \times 10^{-11}, P \approx 3 \times 10^{18}$ photons $/ \mathrm{cm}$ for a $1 \mathrm{~J} / \mathrm{cm}$ green laser, and $\Omega / 4 \pi \approx 2 \times 10^{-3}$ (corresponding to $f 16$ ). Thus the total number of scattered photons from this area reaching the detector is $N_{\mathrm{tot}} \approx 10^{5} \mathrm{per} \mathrm{Joule} / \mathrm{cm}$ of laser energy. We now assume a total photon-tophotoelectron detection efficiency of $\approx 3 \%$, which includes both the losses in the optics and the detector photon counting efficiency. Thus the number of photoelectrons detected is $\approx 3 \times 10^{3}$ per Joule/cm of laser energy. For an assumed laser of $1 \mathrm{~kJ}$ energy over a beam width of $10 \mathrm{~cm}$, this implies 3 $\times 10^{5}$ photoelectrons will be detected from the plasma area of $1 \mathrm{~cm} \times 1 \mathrm{~cm}$. This corresponds to a statistical fluctuation level of $\leqslant 0.2 \%$, which should be small enough to measure the $\approx 1 \%$ density fluctuations.

A more detailed simulation of this Thomson scattering process has been done taking into account the electron temperature, the assumed scattering angle $\left(90^{\circ}\right.$ to the laser beam), and the detection solid angle and efficiency used above. A typical scattered spectrum is shown in Fig. 2, along with the expected visible bremsstrahlung background spectrum calculated for this plasma assuming a $Z_{\text {eff }}=3$. The result is that by integrating over the scattered spectrum, the expected signal/noise $(\mathrm{S} / \mathrm{N})$ level for a density measurement made within a $1 \mathrm{~cm} \times 1 \mathrm{~cm}$ area is $S / N \approx 430$ at a laser energy of $E=100 \mathrm{~J} / \mathrm{cm}$, which is approximately consistent with the rough estimate above. The ratio of signal-tobremsstrahlung signal is also $\geqslant 10$ over most of the linewidth, assuming a detector gating time of $100 \mathrm{~ns}$.

The conclusion from this analysis is that the expected density fluctuations in NSTX can be imaged using a laser with $\geqslant 1 \mathrm{~kJ} /$ pulse in a $2 \mathrm{D}$ configuration such as is illustrated in Fig. 1. This laser energy can be contrasted with the conventional NSTX Thomson scattering system, ${ }^{5}$ which uses a frequency doubled Nd:yttrium-aluminum-garnet laser with an energy $\approx 1.5 \mathrm{~J} / \mathrm{pulse}$ in a single line passing through the plasma. That system is expected to have a few- $\%$ statistical accuracy for measuring density in a $\mathrm{cm}$-sized region. Thus it 


\section{$100 \times 100$ Resolution}
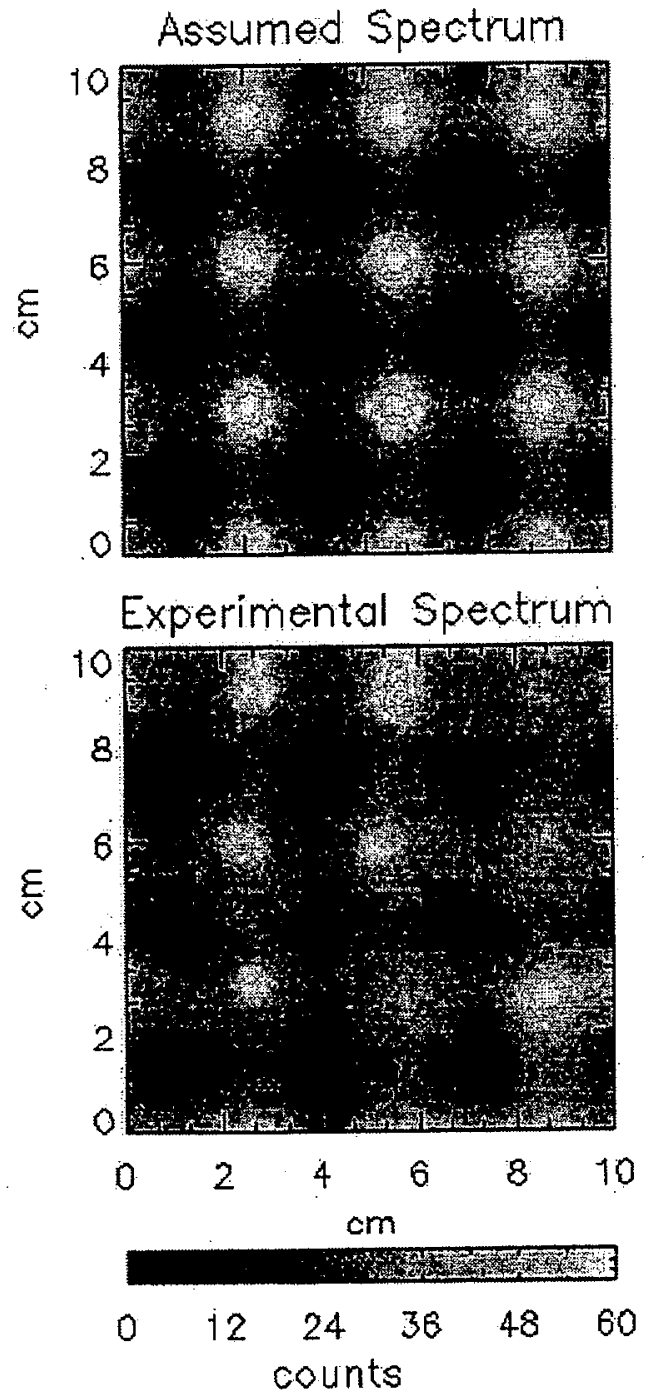

FIG. 3. Simulation of expected $2 \mathrm{D}$ image for an assumed $3 \mathrm{~cm}$ wavelength density fluctuation in NSTX. The top shows the assumed spatial structure of the $1 \%$ density fluctuations in a $10 \mathrm{~cm} \times 10 \mathrm{~cm}$ area. The bottom shows the expected image after taking into account the statistical fluctuations expected for a $2 \mathrm{~kJ}$ laser scattering system. The average plasma density has been subtracted from both these images.

is not surprising that a $\mathrm{kJ}$-class laser is needed to measure $\mathrm{cm}$-scale density fluctuations to $\approx 0.2 \%$ accuracy over a 10 $\mathrm{cm} \times 10 \mathrm{~cm}$ region.

The quality of the $2 \mathrm{D}$ imaging which can be expected from a kJ-class laser Thomson scattering system in NSTX is illustrated in Fig. 3. The assumed "theoretical" spatial density fluctuation spectrum (top) was a coherent mode with a 3 $\mathrm{cm}$ wavelength in both directions perpendicular to the magnetic field. The amplitude of this perturbation was assumed to be $1 \%$ of its mean value (the mean is subtracted out in both parts of this figure). The "experimental" spectrum (bottom) was obtained by (a) binning this theoretical image in a set of $100 \times 100$ pixels, (b) randomly changing the number of counts in each pixel by the expected statistical noise level (proportional to the square root of the amplitude in each pixel), and (c) smoothing the results over $10 \times 10$ pixels $(1 \mathrm{~cm} \times 1 \mathrm{~cm})$ to simulate the experimental image. The case shown in Fig. 3 corresponds to a signal level of 3 $\times 10^{5}$ counts $/ \mathrm{cm}^{2}$, i.e., to a total laser energy corresponding to $\approx 1 \mathrm{~kJ}$ in the NSTX case. The conclusion of this analysis is that such a laser should be able to make a reasonably clear image of the expected density turbulence in NSTX.

\section{HIGH POWER LASER SYSTEM}

The $\approx 1-3 \mathrm{~kJ}$ laser required for this diagnostic can be made using potentially available components from the $\mathrm{Nd}$ : glass Nova laser, which was operated at LLNL at a maximum energy level of $40 \mathrm{~kJ} /$ pulse $(351 \mathrm{~nm}){ }^{6}$ Laser components from LINL have already been used to design a $2 \mathrm{~kJ}$ laser diagnostic for use on the Z-facility at Sandia National Laboratory. ${ }^{7}$ This is about $10-100$ times more laser energy per pulse than has been used in the highest-power laser Thomson scattering systems previously used in tokamaks.

The requirements on the laser system for the present Thomson scattering diagnostic are relatively modest compared with the typical requirements for ICF experiments. The $\mathrm{Nd}$ :glass frequency would most likely be doubled to $526 \mathrm{~nm}$ to facilitate detection of the scattered signals using high spatial resolution charged coupled device (CCD) detectors (see Sec. IV). The pulse duration should be $<100$ ns pulse to reduce background light. The laser linewidth will easily be orders of magnitude smaller than the width of the scattered spectrum (Fig. 2). The beam needs a single pass propagation distance of $\approx 10 \mathrm{~m}$ to propagate through a device like NSTX, and a far-field spot size of $\approx 1 \mathrm{~cm}$ inside the plasma to achieve the desired spatial resolution. Note that the toroidal extent of the laser beam can be well over $10 \mathrm{~cm}$, since the camera is viewing turbulent filaments which are highly correlated in the toroidal direction. The laser profile versus radius would need to be measured accurately to calibrate the scattered signals versus radius.

It would be highly desirable but not necessary to make several pulses per shot with a time separation comparable to the turbulence autocorrelation time $(\approx 10 \mu \mathrm{s}$ ), in order to measure the dynamics of the turbulence. This could be achieved in principle by dividing the available laser energy into multiple pulses, since the Nova glass amplifiers have an energy storage time of several hundred microseconds. However, there is a trade-off between the number of such pulses per shot and the energy in each pulse, such that the highest spatial resolution would be obtained with a single laser pulse. A single 2D image obtained with a repetition rate of 1 high-power laser pulse per hour should be sufficient for the initial research goals of this diagnostic.

These requirements could be met by a laser constructed using Nova components similar to those outlined in Table II. The designs are based on laser amplifiers of aperture size 208-315 mm, which are potentially available for this purpose. The generic laser system design using Nova disk amplifiers, as illustrated in Fig. 4, can produce a 1200-3000 J pulse at $526 \mathrm{~nm}$, the second harmonic of the $\mathrm{Nd}$ :glass laser wavelength. This system design uses four laser passes through the glass amplifiers, and assumes frequency doubling with $75 \%$ conversion efficiency. It should be stressed 
TABLE II. Preliminary summary of potential main cavity laser amplitier configurations using Nova components (with angle-multiplexed four-pass arrangement).

\begin{tabular}{|c|c|c|}
\hline Amplifier size (mm) & $208^{a}$ & 315 \\
\hline Beam diameter (mm) & 180 & 273 \\
\hline Disks per amplifier unit & 3 & 2 \\
\hline Disk thickness (mm) & 32 & 43.2 \\
\hline Single pass gain per amplifier & 2.2 & 1.75 \\
\hline Small signal gain $\left(\mathrm{cm}^{-1}\right)$ & 0.068 & 0.054 \\
\hline Stored energy density $\left(\mathrm{J} / \mathrm{cm}^{3}\right)$ & 0.32 & 0.25 \\
\hline Stored energy per amplifier (J) & 1182 & 1924 \\
\hline Damage limit (J, est. at $20 \mathrm{~ns})$ & 1600 & 15000 \\
\hline Number of amplifiers in four-pass & 3 & 4 \\
\hline Four-pass small signal gain & 1804 & 2294 \\
\hline Total stored energy (J) & 3545 & 7695 \\
\hline Gain saturation energy (J) & 1527 & 3502 \\
\hline Available $1 \omega$ output energy $(\mathrm{J})$ & 1600 & 4000 \\
\hline $2 \omega$ output at $75 \%$ conversion (J) & 1200 & 3000 \\
\hline
\end{tabular}

${ }^{2}$ The $208 \mathrm{~mm}$ amplifiers are currently unavailable due to heavy demand, but may become available at a later date depending on project funding availability.

that a detailed conceptual design for this laser system would be required to make a final determination of the laser requirements, identify all components, and make accurate cost and schedule estimates before implementation could begin.

\section{POSSIBLE IMPLEMENTATION}

There are many technical issues to be resolved before this diagnostic could be implemented on a magnetic fusion energy (MFE) device like NSTX. Some of these are:

(a) the vacuum and optical integrity of the windows must be maintained, which requires a laser energy limit of about $5 \mathrm{~J} / \mathrm{cm}^{2}$ at the windows;

(b) the beam dump must be capable of absorbing the laser pulse without damage or scattering of light back into the plasma;

(c) the laser beam must be handled safely, and requires a large clean area near the machine;

(d) the detector and laser wavelength need to be optimized for the desired spatial resolution and $\mathrm{S} / \mathrm{N}$ ratio, e.g., a
Sub-system

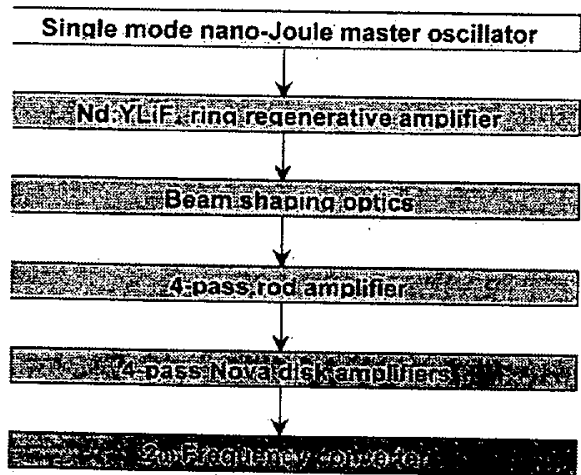

Output energy

$1-3 \mathrm{~nJ}$

$1-10 \mathrm{~mJ}$

$0.1-1 \mathrm{~ms}$

$0.3-3 \mathrm{~J}$

$1.6-4.0 \mathrm{~kJ}$

$1.2=3.0 \mathrm{kj}$
FIG. 4. Generic laser system design using Nova disk amplifiers to produce a $1200-3000 \mathrm{~J}$ pulse at $526 \mathrm{~nm}$, the second harmonic of the Nd:glass laser wavelength. Two options are considered, one with a final frequency-doubled laser energy of $\approx 1200 \mathrm{~J}$ and another with a laser energy of $\approx 3 \mathrm{~kJ}$. high resolution image might use a photocathode/CCD detector at a laser wavelength of $532 \mathrm{~nm}$, while a low resolution (e.g., $10 \times 10$ ) image could be made at a higher $\mathrm{S} / \mathrm{N}$ level using an array of avalanche photodiodes at $1064 \mathrm{~nm}$; and

(e) a realistic evaluation needs to be made of the cost and schedule, including the laser systems, interface with the machine, and detection system.

It should be noted that this diagnostic could in principle be used on any MFE device similar in size and accessibility to NSTX. However, the implementation of this diagnostic would obviously be considerably more costly and time consuming than for a conventional Thomson scattering system of much lower energy.

\section{SUMMARY}

This article described a diagnostic to measure the 2D structure of plasma density turbulence in a MFE fusion plasma using high-power laser Thomson scattering. It appears feasible to construct the required $\approx 1-3 \mathrm{~kJ}$ pulsed laser source with components developed for the Nova program at LLNL. This system could also make multiple images within the laser energy storage time of several hundred microseconds, but only at lower spatial resolution. Considerably more analysis is needed before a practical system could be implemented on a device such as NSTX.

\section{ACKNOWLEDGMENTS}

The authors thank the following people for helpful comments: R. Goldston, G. Hammett, R. Hawryluk, G. Kerbel, E. Mazzucato, T. Munsat, H. Park, S. Payne, T. Munsat, and A. J. Wootton. This work was performed under the auspices of the U. S. Department of Energy by Princeton Plasma Physics Laboratory under Contract No. DE-AC02-76CH03073 and by Lawrence Livermore National Laboratory under Contract No. W-7405-ENG-48.

${ }^{1}$ A. J. Wootton, B. A. Carreras, H. Matsumoto, K. McGuire, W. A. Peebles, C. P. Ritz, P. W. Terry, and S. J. Zweben, Phys. Fluids B 2, 2879 (1990).

${ }^{2}$ U. Frisch, Turbulence (Cambridge University Press, Cambridge, 1995).

${ }^{3}$ G. D. Kerbel, T. Pierce, J. L. Milovich, D. E. Shumaker, A. Verlo, R. E. Waltz, G. W. Hammett, M. A. Beer, and W. Dorland, Int. J. Supercomput. Appl. 10, No. 213 (1996).

${ }^{4}$ I. H. Hutchinson, Principles of Plasma Diagnostics (Cambridge University Press, Cambridge, 1987), Chap. 7.

${ }^{5}$ D. Johnson, N. Bretz, B. LeBlanc, R. Palladino, D. Long, and R. Parsells, Rev. Sci. Instrum. 70, 776 (1999).

${ }^{6}$ J. K. Lawson, D. R. Speck, C. Bibeau, S. C. Burkhart, M. A. Henesian, C. W. Laumann, T. L. Weiland, and R. B. Wilcox, Appl. Opt. 31, 5061 (1992); C. Bibeau, D. R. Speck, R. B. Ehrlich, C. W. Laumann, D. T. Kyrazis, M. A. Henesian, J. K. Lawson, M. D. Perry, P. J. Wegner, and T J. Weiland, ibid. 31, 5799 (1992); P. J. Wegner, M. A. Henesian, D. R Speck, C. Bibeau, R. B. Ehrlich, C. W. Laumann, J. K. Lawson, and T. L. Weiland, ibid. 31, 6414 (1992).

${ }^{7}$ R. Adams, A. Anderson, R. Aragon, J. Auerbach, B. Behrendt, D. Boomquist, J. Caird, E. Dawson, G. Edwards, and A. Erlandson et al., Report for UCRL-ID-134409, Lawrence Livermore National Laboratory, Livermore, CA, 31 May 1999.

${ }^{8}$ M. N. A. Beurskens, C. J. Barth, N. J. L. Cardozo, and H. J. van der Meiden, Plasma Phys. Controlled Fusion 41, 1321 (1999).

${ }^{9}$ M. Yu. Kantor and D. V. Kourprienko, Rev. Sci. Instrum. 70, 780 (1999). 


\title{
Damage Threats and Response of Final Optics for Laser-Fusion Power Plants
}

\author{
M. S. Tillack ${ }^{I}$ \\ S. A. Payne ${ }^{2}$ \\ N. M. Ghoniem ${ }^{3}$ \\ M. R. Zaghloul \\ J. F. Latkowski \\ ${ }^{1}$ UC San Diego, La Jolla, CA 92093-0417 \\ ${ }^{2}$ Lawrence Livermore National Laboratory, P.O. Box 5508, Livermore, CA 94551 \\ ${ }^{3}$ UCLA,Los Angeles, CA 90024-1597
}

The final optics for laser-IFE (inertial fusion energy) power plants will be exposed to a variety of damage threats, including high-energy neutrons and gamma rays, $x$-rays and ions from the target explosion, contamination by condensable gases and particulate, and the laser itself. Experiments and modeling have been performed in order to understand damage mechanisms and possible solutions for those threats that are judged to be the most serious concerns for a power plant.

\section{INTRODUCTION}

Survival of the final optic is one of the most critical issues for a laser-driven IFE power plant. Laserinduced damage is compounded by the presence of contaminants and various prompt radiations that emanate from repeated target explosions. Table I summarizes the various damage threats and also provides nominal goals for a power plant. These goals are driven by the desire for modest-sized optics (operating at $\sim 5 \mathrm{~J} / \mathrm{cm}^{2}$ fluence), lifetime on the order of years, target physics requirements imposed on beam characteristics, and acceptable cost-ofelectricity. Direct drive targets require illumination symmetry of the order of $1 \%$ and positioning accuracy of $10-100 \mathrm{~mm}$.
Table I. Damage threats and nominal goals

\begin{tabular}{|l|l|}
\hline Final Optic Threat & Requirement \\
\hline $\begin{array}{l}\text { Defects and swelling } \\
\text { (g-rays and neutrons) }\end{array}$ & $\begin{array}{l}\text { Absorption loss }<5 \% \\
\text { Wavefront distortion }<1 / 2 \\
\mathrm{~mm}\end{array}$ \\
\hline $\begin{array}{l}\text { Optical damage by laser } \\
\text { (LIDT) }\end{array}$ & $\begin{array}{l}>5 \mathrm{~J} / \mathrm{cm}^{2} \text { threshold (normal } \\
\text { to beam) for }>108 \text { shots }\end{array}$ \\
\hline Contamination & $\begin{array}{l}\text { Absorption loss }<5 \% \\
>5 \mathrm{~J} / \mathrm{cm}^{2} \text { damage threshold }\end{array}$ \\
\hline Ablation by x-rays & $<10-4$ monolayer per shot \\
\hline Sputtering by ions & $<10-4$ monolayer per shot \\
\hline
\end{tabular}

For transmissive optics, radiation-induced darkening is a particularly serious concern. Highly pure $\mathrm{SiO}_{2}$ and $\mathrm{CaF}_{2}$ have been irradiated and the increased absorption coefficients arise from known color centers. The possibility of reducing the damage through annealing at elevated temperature has been evaluated.

For reflective optics, the primary design option considered is grazing-incidence metal mirrors. For these, the most serious concern is reduction in laser damage threshold due to long-term exposure and contamination. Unstable surface deformations due to operation at grazing incidence angles are possible and have been evaluated. Both experimental results on LIDT and modeling of defects are presented.

\section{NEUTRON DAMAGE TO TRANSMISSIVE OPTICS}

After $\mathrm{n}^{0}$ irradiating fused silica samples for a IFE-equivalent dose of several months at several temperatures (performed at LANSCE, Los Alamos), the optical absorption spectrum plotted in Fig. 1 is obtained. For the case of irradiations at $105^{\circ} \mathrm{C}$ and $179^{\circ} \mathrm{C}$, the spectra nearly overlap (sample path length of $1 \mathrm{~cm}$ ). The peak at 630 $\mathrm{nm}$ is due to Non-Bridging Oxygen Hole Centers (NBOHC), while the rising edge at $350 \mathrm{~nm}$ arises from Oxygen Deficient Centers (ODC) [1]; the slow rise in the sample irradiated at $425{ }^{\circ} \mathrm{C}$ is due to scattering. The mechanism illustrated in Fig. 2 explains the experimental observations, including the fact that the ODCs and NBOHCs are created in roughly equal numbers, $\gamma$-rays form E' centers from the ODCs, and the defects can be thermally annealed away. The annealing is illustrated in Fig. 3 , where the defects in the sample originally $n^{\circ}-$ irradiated at $105^{\circ} \mathrm{C}$ are shown to return to its original state at $380^{\circ} \mathrm{C}$. In addition, a type of "radiation annealing" is observed to occur, which leads to a maximum absorption coefficient of $\sim 1 \mathrm{~cm}^{-1}$ at $350 \mathrm{~nm}$, irrespective of 
dose. This process operates because the collisional cascades overlap at high dose, and a maximum level of defects forms. The plausibility of employing thin $(<1 \mathrm{~mm})$ fused silica as the final optic is being explored.

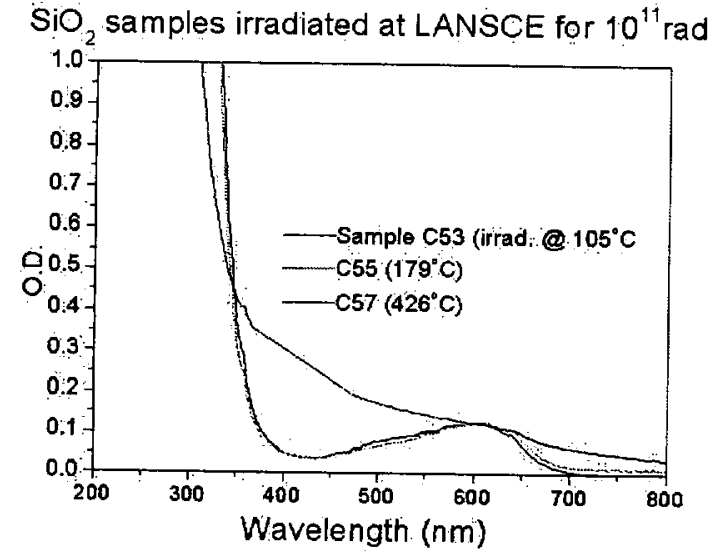

Fig. 1. Results for irradiating fused silica for $\sim 1011$ rads at the indicated temperature.

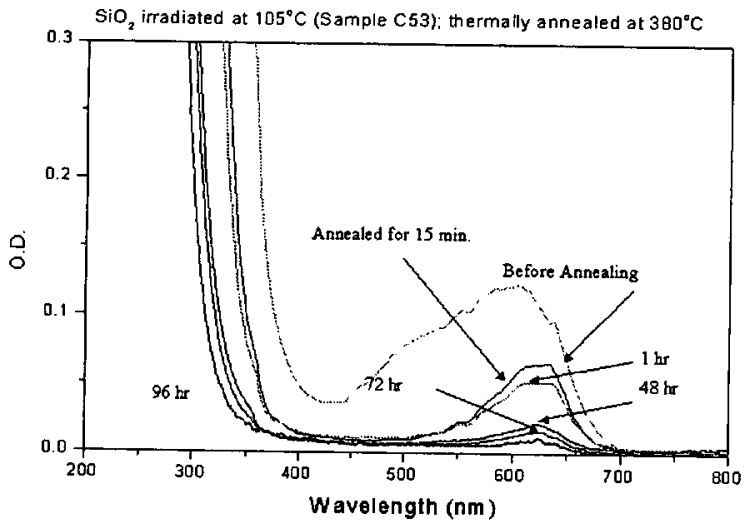

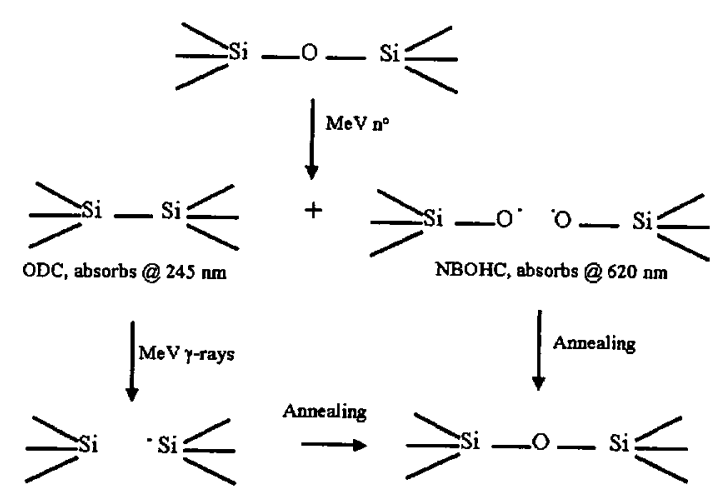

E' Center, absorbs @ $213 \mathbf{~ n m}$

Fig. 2. Mechanism by which neutron-irradiation of $\mathrm{SiO} 2$ leads to the formation of Oxygen Deficient Centers $(O D C)$ and Non-Bridging Oxygen Hole Centers (NBOHC).

Fig. 3. Thermal annealing of the $N B O H C s$ (near $630 \mathrm{~nm}$ ) and the $O D C s$ (edge observed at $300-350 \mathrm{~nm}$ ).

\section{LASER-INDUCED DAMAGE TO GRAZING INCIDENCE MIRRORS}

For $\mathrm{KrF}$ lasers, color centers near the operating wavelength of $248 \mathrm{~nm}$ may rule out transmissive final optics. Very high performance is possible using multi-layer dielectric mirrors; however, fears over the effect of neutroninduced damage have led to the adoption of metal mirrors as the primary candidate [2]. Unfortunately, metal mirrors suffer from relatively low reflectivity (and consequently low damage threshold), especially for UV wavelengths. Aluminum maintains relatively high reflectivity for UV light. By operating at a grazing angle of incidence with s-polarized light, reflectivities in excess of $99 \%$ are possible.

One of the unresolved issues for grazing incidence metal mirrors is whether or not they can operate for long periods of time at laser fluences higher than the normal incidence damage threshold (for aluminum this can be as low as $0.2 \mathrm{~J} / \mathrm{cm}^{2}$ ). In order to keep the optic size modest, a goal of $5 \mathrm{~J} / \mathrm{cm}^{2}$ normal to the beam was chosen as a reasonable goal. Although laser-induced damage to metal mirrors has been explored previously, uncertainties still exist in the fundamental mechanisms of LIDT for multiple-shot beams, the influence of erosion (sputtering, pitting and cratering) mechanisms, and possible changes in the mechanical properties by pulsed neutron irradiation. Modeling of unstable thermomechanical deformations of metal surfaces was performed in order to predict the long-term damage threshold. In addition, experiments were performed on diamond-turned $\mathrm{Al}$ surfaces at $85^{\circ}$ angle of incidence for up to 10,000 shots in order to provide experimental validation. 


\section{MODELING OF GRADING INCIDENCE METAL MIRROR DAMAGE}

Structural changes induced by a laser beam occur in the crystal lattice subsystem of the material, which can be considered as an elastic continuum. On the other hand, laser radiation excites only the electronic subsystem, creating a non-equilibrium plasma state in a shallow layer of the material. Relaxation of this sub-surface plasma leads to intense local heating and generation of a variety of lattice (point and extended) defects. These point and extended defects (e.g., dislocations) - viewed as rigid inclusions in the elastic continuum - will thus deform it.

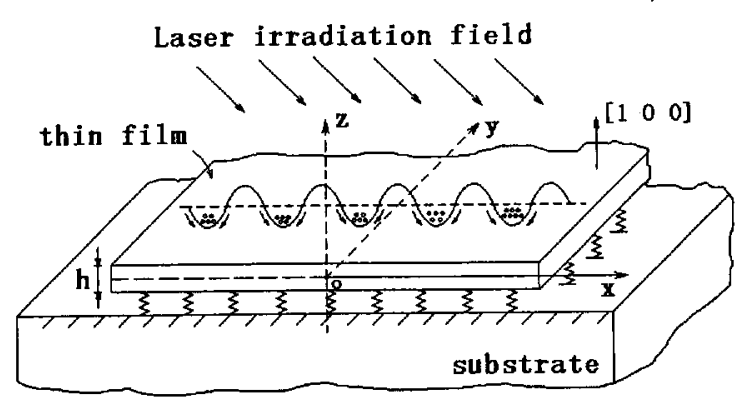

Fig. 4. Schematic of the model for laser-induced surface instabilities and the formation of "ripple" patterns.

Experimental evidence for the formation of point and extended defects in laser-irradiated materials were first reported in the early seventies by Metz and Smidt $[3,4]$. The deformation of the elastic continuum itself changes the transport characteristics of these defects in such a way as to minimize the total free energy of the system. An unstable feedback loop is set up, driven by laser irradiation, and controlling the interaction between the defect and deformation fields of the material, as illustrated in Fig. 4.

We developed a model which describes the dynamics of such systems in the case of uniform laser irradiation [5]. We also extended this model to the case of focused laser irradiation [6]. The model applies well to the description of the mechanical deformation behavior of thin coatings on substrates (as in coated laser optics), and is in very good agreement with experimental data. Through linear, nonlinear and numerical analyses, we determined how rose deformation patterns, with petal number increasing with laser intensity, naturally arise in this model, in agreement with experimental observations on focused laser irradiation, as can be seen in Fig. 5 .
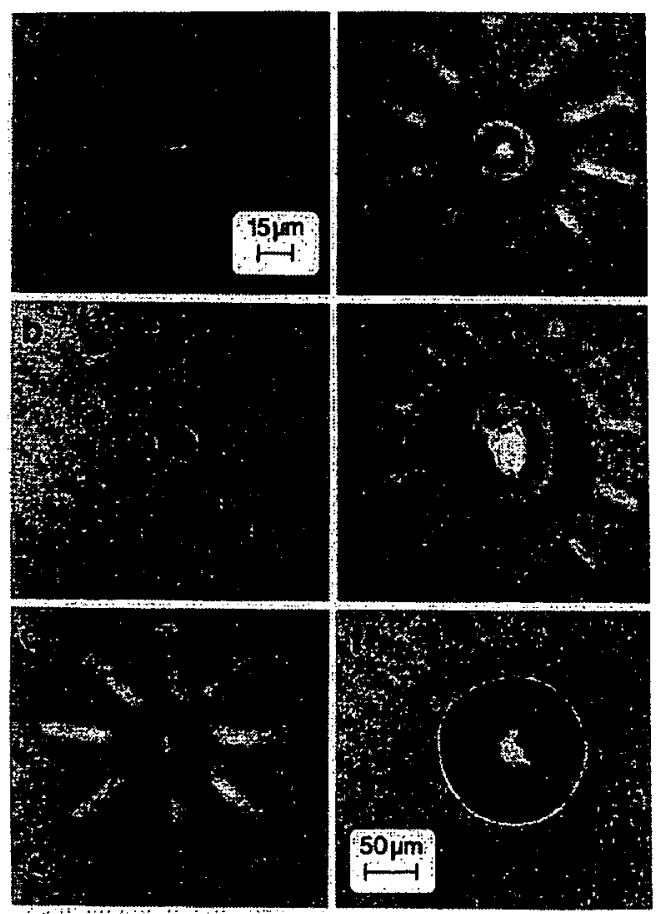

Fig. (5a): experimental observations of "Rose" deformation patterns in focused laser [7].

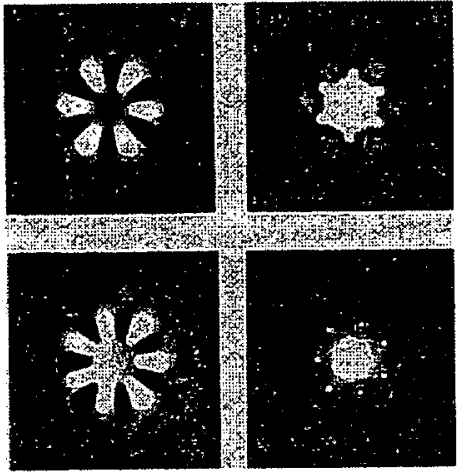

Fig. (5b): Computed "Rose" surface deformation [6].

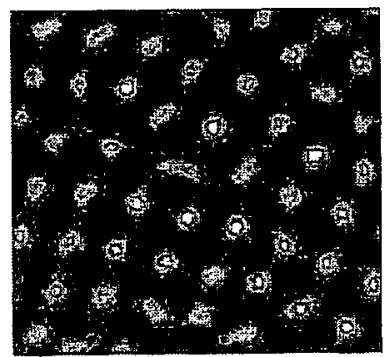

Fig. (5c): Results of numerical solutions for surface deformation patterns in laser-irradiated surfaces [5]. 


\section{EXPERIMENTAL DATA ON GRAZING INCIDENCE METAL MIRROR DAMAGE}

Experiments were performed using a frequency-doubled YAG laser with a beam size of $1.2 \mathrm{~cm}$ and maximum energy of $800 \mathrm{~mJ}$. Mirrors were fabricated by diamond turning $99 \%$ pure (Al-1 100) and $99.999 \%$ pure aluminum with a natural oxide coating $\sim 30 \mathrm{~nm}$ thick. An example undamaged surface profile is shown in Fig. 6 . The lathe marks are clearly evident, with a maximum surface height variation of $\sim 30 \mathrm{~nm}$.

Experiments were performed for single and multiple shots up to $10^{4}$, all at $85^{\circ}$ angle of incidence. The Al-1100 mirrors survived single shot exposure up to $18 \mathrm{~J} / \mathrm{cm}^{2}$ normal to the beam. Below $8 \mathrm{~J} / \mathrm{cm}^{2}$ no visible damage is observed up to the maximum number of shots tested. Between $8-18 \mathrm{~J} / \mathrm{cm}^{2}$, visible changes appear on the surface, indicating the onset of microscopic damage. After continued exposure at this fluence level, eventually the damage becomes sufficiently severe to cause enhanced absorption and eventually ablation (see Fig. 7). Fig. 8 summarizes damage measurements for Al-1100. For comparison, Fig. 9 shows the response of a pure Al surface which survives up to the melting threshold without exhibiting an intermediate damage regime. Pure $\mathrm{Al}$ appears to have a significantly increased damage threshold as compared with Al-1100. Experiments are ongoing.

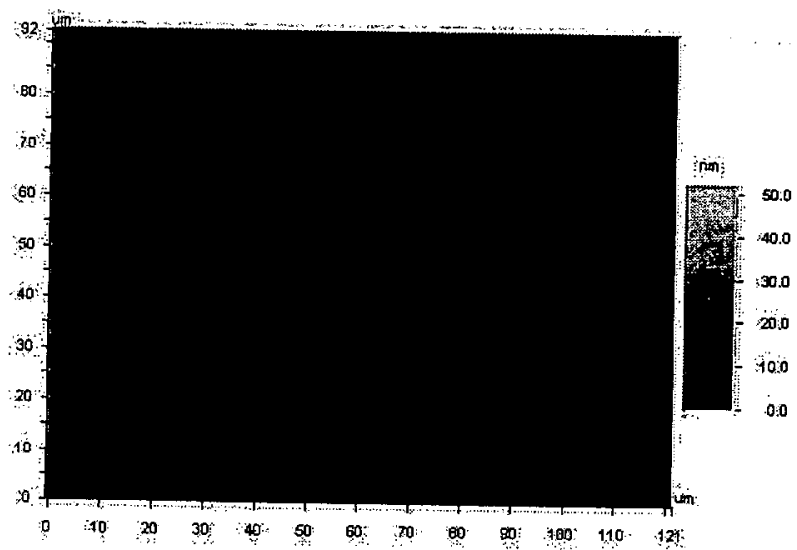

Figure 6. Surface profile of an undamaged Al-1100 mirror

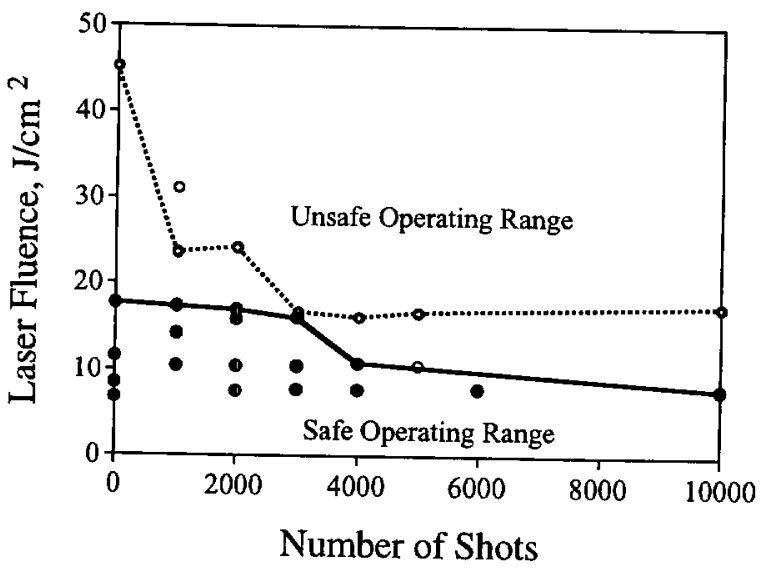

Figure 8. Damage regimes for Al-1100

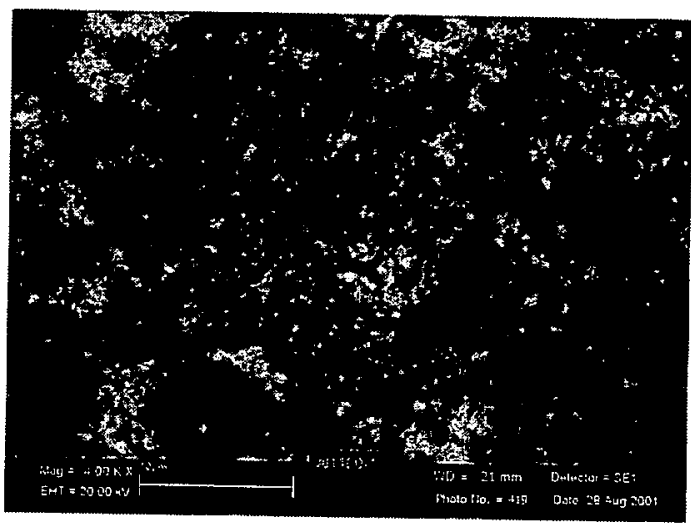

Figure 7. SEM photo of Al-1100 mirror exposed to $20 \mathrm{~J} / \mathrm{cm} 2$ for 104 shots

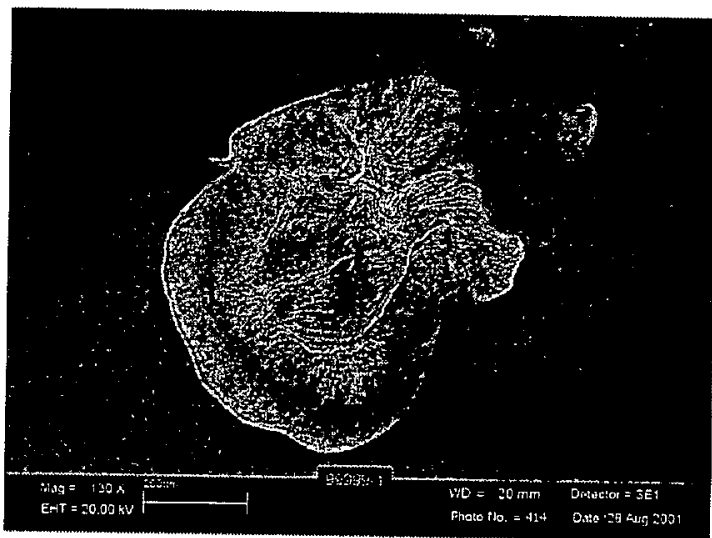

Figure 9. SEM photo of pure Al exposed to one shot at $180 \mathrm{~J} / \mathrm{cm} 2$

\section{REFERENCES}

[1] C. D. Marshall, J. A. Speth, and S. A. Payne, "Induced optical absorption in gamma, neutron and ultraviolet irradiated fused quartz and silica," Journal of Non-Crystalline Solids 212 (1997) 59-73.

[2] R. L. Bieri and M. W. Guinan, "Grazing Incidence Metal Mirrors as the Final Elements in a Laser Driver for Inertial Confinement Fusion," Fusion Tech. 19 (May 1991) 673-678. 
[3] S.A. Metz and F.A. Smidt, Jr., Appl. Phys. Lett., 19 (1971) 207.

[4] F.A. Smidt, Jr. and S.A. Metz, Proc. Conf. on Radiation Induced Voids in Metals, Albany, New York, June 9-11, 1971, p.613.

[5] D. Walgraef, N.M. Ghoniem, and J. Lauzeral, "Deformation Patterns in Thin Films Under Uniform Laser Irradiation," Phys. Rev. B, 56, No. 23 (1997) 15361.

[6] Lauzeral, D. Walgraef, and N.M. Ghoniem, "Rose Deformation Patterns in Thin films Irradiated By Focused Laser Beams," Phys. Rev. Lett. 79, No. 14 (1997) 2706.

[7] P.Mogyorosi, K.Piglmayer and D.Bauerle, Surface Science, 208 (1989) 232. 


\title{
Hydro*Star - A Direct Water-Cooled DD-Fueled IFE Fusion- Chamber Concept
}

\author{
Charles D. Orth
}

\begin{abstract}
We introduce a new IFE fusion-chamber concept called Hydro*Star that uses DT-ignited DD targets and a water blanket. The driver can be either a 13 to 16-MJ diode-pumped solid-state laser (DPSSL) with fast ignition, or a 4-MJ heavy-ion accelerator operating at a reprate 10 times faster than the fusion chamber to accumulate sufficient energy in storage rings to direct $40 \mathrm{MJ}$ at the target. The driver employs a prepulse system to burn an ionized path through the ambient fusion-chamber vapors, whose operating pressure is about $20 \mathrm{~atm}$. We assume that the targets, which have a yield of about $2800 \mathrm{MJ}$, can be indirectly driven with two-sided illumination. The blanket, which is 1 to $2-\mathrm{m}$ thick and placed immediately inside the structural wall, is operated just over $100 \mathrm{C}$ either in a liquid or frothed-liquid state, the latter being preferred to reduce stresses in the structural wall. The structural wall, at a radius of 4 to $5 \mathrm{~m}$, is composed of low-carbon steels to avoid the stress-corrosion cracking problems that have plagued certain light-water-reactor (LWR) systems. The functions of the blanket are (1) to shield the structural wall and exterior components from neutron and gamma-ray target emissions, and (2) to supply water for the direct generation of steam. Each fusion pulse vaporizes nearly one-half centimeter of the inside surface of the water blanket, thereby creating hot steam which is vented directly from the fusion chamber into ordinary steam turbines. Thus, Hydro*Star operates just like a simple steam engine, with a basic reprate of only $0.8 \mathrm{~Hz}$ per GWe of net output. Because the steam temperature is 900 to $1200 \mathrm{~K}$, the plant thermal efficiency is nearly $50 \%$. This efficiency is much better than the typical $35-40 \%$ now being achieved in commercial reactors, and much better than the efficiencies estimated for previous fusion-chamber concepts except CASCADE (55\%). Other advantages for the new concept include reduced plant radioactivity (reduced radionuclides inventory), longer component lifetimes, nearly self-cleaning operation, reduced risk for catastrophic accidents, and potentially lower cost of electricity. Although Hydro*Star has many advantages, we identify many serious design issues that require future investigation. These include the problems associated with (1) how to interface the evacuated driver beam lines to the high-pressure fusion chamber, (2) how to propagate the driver beams through the high-pressure steam, and (3) how to obtain the necessary tritium supplies without breeding tritium in the water blanket.
\end{abstract}

\section{CONCEPT SUMMARY}

We propose a new concept of a fusion chamber for inertial fusion energy (IFE) utilizing driver beams from either a diode-pumped solid-state laser (DPSSL) or a heavy-ion (HI) driver. The chamber is operated at high pressure, and features DT-ignited DD fuel targets, a (frothed) water blanket, and direct steam power conversion. Because of its water blanket, Hydro*Star operation avoids any significant vaporization, condensation, and neutron deterioration of structural wall materials. In brief, a 1-GWe Hydro*Star operating at $0.8 \mathrm{~Hz}$ has the following features (see Fig. 1 and Tables 1 and 2):

Driver - either a 13 to $16-\mathrm{MJ} 0.826-\mathrm{Hz}$ DPSSL operating with fast ignition (DD target gain $\mathrm{G}_{\mathrm{DD}}=\mathrm{G}_{\mathrm{DT}}$ * $(347 / 340)^{*}\left[\left(\rho \Delta \mathrm{r}+5.5 \mathrm{~g} / \mathrm{cm}^{2}\right) /\left(\rho \Delta \mathrm{r}+60 \mathrm{~g} / \mathrm{cm}^{2}\right)\right]$ with $\mathrm{DT}$ target gain $\mathrm{G}_{\mathrm{DT}}=400 \mathrm{E}_{\mathrm{dr}}^{0.4}$ for driver energy $\mathrm{E}_{\mathrm{dr} r}$ see Ref. 5), or a $4-\mathrm{MJ} \mathrm{HI}$ accelerator operating at $8.26-\mathrm{Hz}$ to accumulate sufficient energy in storage rings to direct $40 \mathrm{MJ}$ at a target at $0.826 \mathrm{~Hz}$. A prepulse system clears channels through the high-pressure fusionchamber vapors before each pulse on target. Each $\mathrm{HI}$ beam is focused outside the fusion chamber before passing through the $3-\mathrm{mm}$ hole in the $\sim 1.5-\mathrm{m}$-long baffled diaphragm system separating the highpressure fusion-chamber environment from the beam-tube vacuum. Each beam is transported to the target inside the ionized column created by the prepulse system. The selection of beam ion $(\mathrm{Z}>50, A>130)$ has not yet been made. For a DPSSL, the wavelength is assumed to be 0.35 to $0.50 \mu \mathrm{m}$.

Target - an advanced design using DT-ignited DD fuel ( $\mathrm{D}^{3} \mathrm{He}$ could be used if it becomes available). We assume that the targets are indirectly driven with two-sided illumination (i.e., each target consists of a 
capsule mounted inside a hohlraum). The targets are assumed to have a gain of $\sim 180$ (DPSSL) or $\sim 70$ (HI) thereby producing a yield of $\sim 2.8 \mathrm{GJ}$ per target for either driver type.

Containment Vessel \& Blanket - a 3-to-5-m-radius, nearly spherical, vented, low-carbon-stainless-steel vessel lined with a $1-\mathrm{m}$ to $2-\mathrm{m}$-thick blanket of water. The water is supported by a compartmental or wick substructure that prevents unrestricted gravitational flow. The preferred mode of blanket operation (although not necessarily essential) is with many small compressible vapor bubbles in the water, thus creating a frothed water blanket that reduces wall stresses. The containment vessel reprate and size can be adjusted to obtain the desired dynamical water vapor chemistry at $T \approx 900$ to $1200 \mathrm{~K}$ and the desired turbine pressure.

Power-Conversion System - ablation and vaporization of the water blanket and direct steam conversion. The vaporized water exits the fusion chamber through its vents and goes directly into metal-bladed turbines. The plant thermal efficiency $(70 \%$ of Carnot) is about $50 \%$.

Plant Energetics - For every 1 GWe of electrical output, the plant energy and power flow are those described in Table 2. We emphasize that the values shown in Table 2 are merely illustrative for one possible design, in which the chamber contains $\leq 2.9 \mathrm{GJ}$ every pulse, and the plant gross thermal power is $\leq 2.5 \mathrm{GW}$ with recycled power fractions of $17 \%$ or less. The optimum plant output power has not yet been established.

Table 1 Main Features of Hydro*Star

- Self-cleaning protected first wall

- 1 to 2 -m-thick frothed-liquid water blanket

- Simplified chamber dynamics with increased target repetition rate

- Direct steam-boiler operation without intermediate heat exchangers

- Manageable tritium consumption and handling

- Plant thermal efficiency of $\sim 50 \%$

- Either DPSSL or HI driver beams

- Lower risk, lower cost, and naturally safer

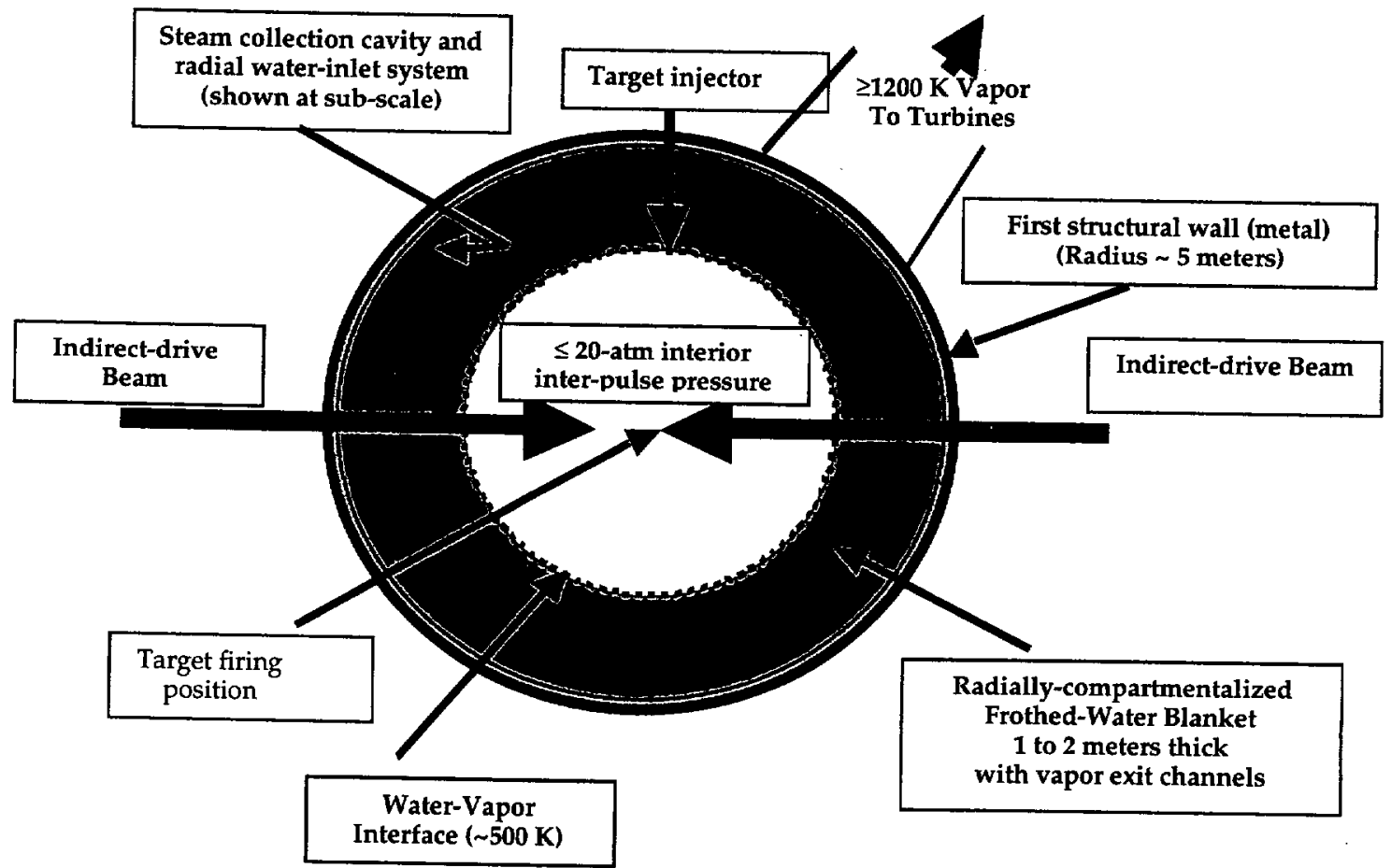

Figure 1 Conceptual Layout of Hydro*Star. 
This concept has many unresolved and difficult design issues, which we will address below. The concept does have many advantages, however, and Table 3 displays a partial list of these advantages.

Table 2 Energy and Power Flow in 1-GWe Hydro*Star at $0.826 \mathrm{~Hz}$

\begin{tabular}{|c|c|c|}
\hline Item & $\begin{array}{l}\text { DPSSL Driver } \\
\text { (calculated) }\end{array}$ & $\begin{array}{l}\text { HI Driver } \\
\text { (estimated) }\end{array}$ \\
\hline Driver reprate on target $(\mathrm{Hz})$ & 0.826 & 0.826 \\
\hline Driver internal reprate $(\mathrm{Hz})$ & 0.826 & 8.26 \\
\hline Assumed energy $E_{d r}$ on target (MJ) & 16.143 & 40.00 \\
\hline Driver input power (MW) & 203.4 & 165.1 \\
\hline Driver output power (MW) & 13.33 & 33.02 \\
\hline Driver efficiency & $6.55 \%$ & $20 \%$ \\
\hline Total number of driver beams on target & 1231. & (unknown) \\
\hline Driver total cost $(\mathrm{B} \$)$ & 9.425 & (unknown) \\
\hline Target fuel (DT-hot-spot ignited) & $\mathrm{DD}$ & $\mathrm{DD}$ \\
\hline Target gain G (DPSSL with FI) & 178.2 & $\begin{array}{c}69.00 \\
\text { (assumed) }\end{array}$ \\
\hline $\begin{array}{l}\text { Energy contained per pulse in chamber, } \\
\text { including driver energy (MJ) }\end{array}$ & 2,893 . & 2,800 \\
\hline Chamber neutron multiplier & 1.05 & 1.05 \\
\hline Gross thermal power (MW) & $2,508.0$ & $2,427.3$ \\
\hline Plant thermal efficiency & 0.50 & 0.50 \\
\hline Gross electric power (MW) & $1,254.0$ & $1,213.7$ \\
\hline $\begin{array}{l}\text { Fraction of gross electric power sent to auxiliary } \\
\text { equipment }\end{array}$ & $4 \%$ & $4 \%$ \\
\hline Recycled power fraction & $16.9 \%$ & $14.17 \%$ \\
\hline Recycled power (MW) & 203.4 & 165.1 \\
\hline Net output power (MW) & $1,000.0$ & $1,000.0$ \\
\hline Projected COE (cents $/ \mathrm{kWh}$ ) & 19.4 & (unknown) \\
\hline
\end{tabular}

Table 3 Advantages of Hydro*Star

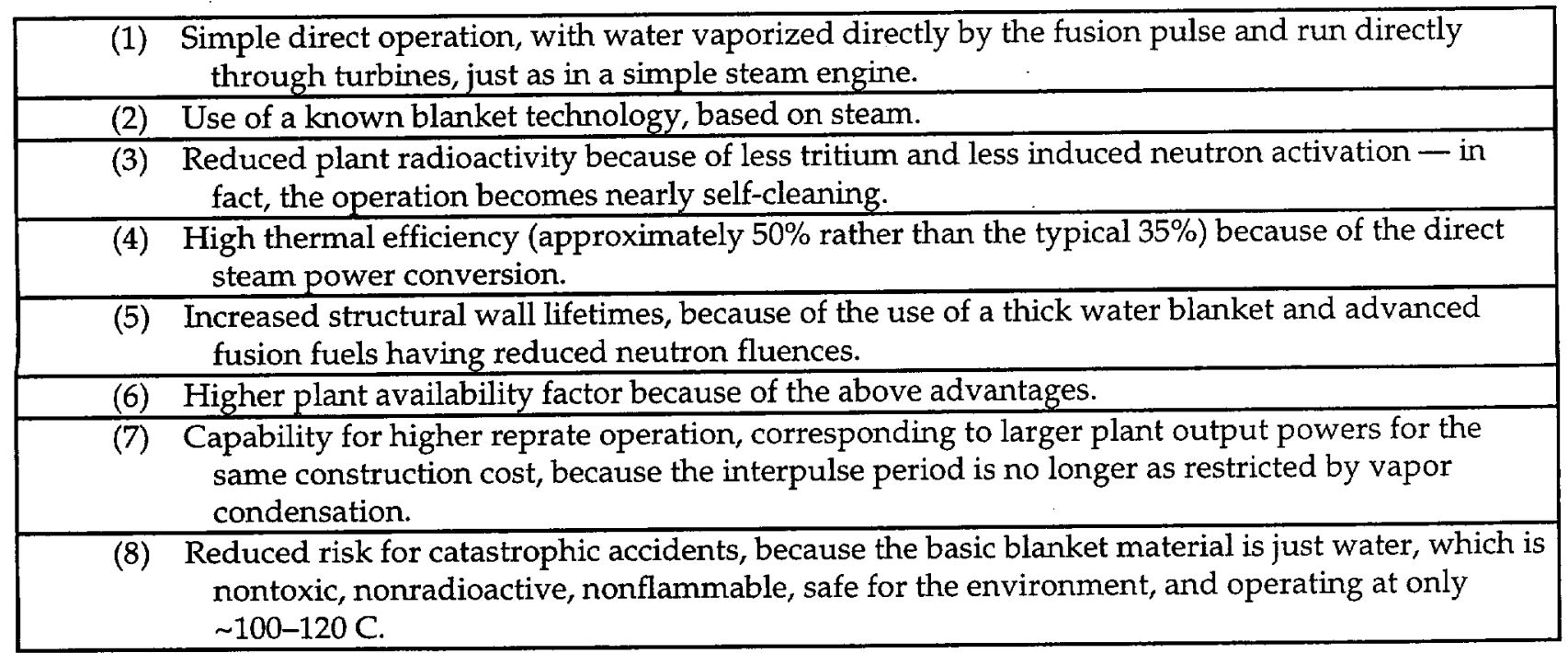




\section{MOTIVATION}

Previous fusion-chamber designs have generally avoided the use of DD targets because such targets are difficult to ignite and burn with a gain much less than that for DT targets. Use of DT targets, however, requires that the blanket for the containment system have capabilities to absorb neutron energy and breed tritium. The latter requirement severely constrains the available solid and liquid blanket materials, essentially to compounds of lithium. In liquid form, these compounds (1) can have safety hazards due to flammability and/or chemical reactivity, (2) generally require high pumping powers, (3) are usually arranged in complicated geometries, (4) typically introduce uncertainties in their isochoric breakup and condensation, and (5) usually dictate low thermal efficiencies because of their low vaporization temperatures. In solid form, the lithium compounds can be ceramics with which we have little experience. In addition, the abundance of tritium for DT targets causes increased radioactivity problems, even though the ICF concepts have reduced plant radioactivity and reduced safety risks when compared with other fusion or fission concepts. Furthermore, use of DT reduces component lifetimes because of the large fluence of $14-\mathrm{MeV}$ neutrons.

We therefore chose to abandon the reliance on DT fuel. This decision removes the necessity to breed tritium, thereby allowing a wide variety of blanket materials. The safest and cheapest material is probably water, which allows for direct conversion of the ablated and vaporized blanket material through turbines (if the residual tritium level proves to be manageable). We felt that the best configuration for a water blanket would be an annulus directly adjacent to the containment wall and supported in a compartmental or wick substructure (and not a system of isolated fluid jets)
because:

(1) Interior jet structures may not break up as much as one might expect under isochoric neutron heating, so they may not be an aid to condensation of vaporized blanket material to facilitate higher reprates, and definitely introduce reprate constraints corresponding to the time to re-establish the jet structure after any shot;

(2) Jet structures involve greater complexity (higher risk) and greater pumping-power losses, and can introduce a potentially serious reprate constraint due to splash;

(3) Solid interior annuli can be accelerated by ablation and/or vaporization pressures and cause serious impact problems for first walls; and

(4) Unconstrained (unwicked) liquid flows at the containment wall at large radii generally involve very large flow volumes and hence large pumping-power expense if recycled using normal pumping methods.

Previous fusion-chamber designs have avoided operation of a containment vessel at pressures above roughly 0.01 Torr to allow the driver beams to propagate without significant attenuation through the ambient vapors preceding any pulse. However, this requirement heavily constrains the containment concepts, so we chose to abandon this requirement as well. Such a decision shifts the design burden from the multitude of problems associated with the constrained containment concepts to two (serious!) problems associated with Hydro*Star, namely, (1) how to interface the evacuated driver beam lines to the high-pressure fusion chamber, and (2) how to propagate the driver beams through the high-pressure fusion-chamber gas.

Previous fusion-chamber designs have considered laser, HI, or light-ion drivers. Although Hydro*Star might accommodate any of these drivers, we shall consider only HI and DPSSL drivers.

In addition, Hydro*Star is similar to the General Electric Boiling-Water fission system in the sense that the primary heat-exchange fluid is transferred in the vapor state, not in the liquid or solid state. Consequently, Hydro*Star does not need intermediate heat-exchange systems that lower plant thermal efficiency. Another benefit from using the vaporized blanket material directly is that Hydro*Star is thereby free of reprate restrictions due to recombination chemistry and condensation physics associated with trying to obtain a fusion-chamber pressure below 0.01 Torr prior to the next driver pulse, as is required in most other designs. In addition, because the Hydro*Star fluid is water, which has a low vaporization temperature, high Carnot efficiencies can be realized without invoking the use of ceramics, which might prove to be costly and/or risky, especially for turbine blades.

\section{ISSUES}

There are many serious issues that must be addressed with this fusion-chamber concept. We discuss some below, and request the help of readers in discovering others and in overcoming difficulties with those listed here. 


\section{Target Gain \& Driver Cost}

The cost of the driver to ignite DD fuel targets is a serious issue because the driver energy for DD targets must be substantially larger than for DT targets for the same target gain. Although the only way to establish the optimum parameters for such things as the target gain and the driver efficiency is through a detailed cost analysis of all plant systems, it is possible to determine approximate relationships through a simplified analysis as follows. Independent of the fusion-chamber reprate (or the number of targets ignited simultaneously per driver pulse), the so-called fusion cycle gain is

$$
\eta(G+1) M \varepsilon_{t h}=\frac{1}{0.96 f}
$$

where $\eta$ is the driver efficiency, $G$ is the target gain ( $G+1$ includes the driver energy), $M$ is the blanket energy multiplication factor (typically 1.15 for past blankets, but not yet calculated for water, so we conservatively use 1.05), $\varepsilon_{\text {th }}$ is the power-conversion thermal efficiency, $f$ is the recirculated power fraction to run the driver, and the 0.96 assumes a $4 \%$ loss of gross electric power to operate auxiliary systems. For example, with $M \approx 1.05$ and using $f=0.10$, we get $\eta G \varepsilon_{\text {th }} \approx 10$. Normally $\varepsilon_{\text {th }}$ is about 0.35 , so $\eta G$ would be near 30 . [Note that $\eta G$ estimates do in fact proceed from Eq. (1) and not from an economic analysis because fusion-chamber ("reactor") construction costs are not linearly related to plant thermal power]. ${ }^{1}$ As shown below, however, the Hydro*Star concept allows $\varepsilon$ th $\approx 0.50$, so we desire $\eta G \approx 20$ (we probably need only half this, corresponding to an $f$ of $20 \%$ ). With a HI driver operated with storage rings and a prepulse system as described below, we assume $\eta \approx 0.20$ so the target gain must be 50 to 100 to satisfy simplified plant economics. We shall conservatively assume a gain of 70 including the HI driver energy itself (i.e., $\mathrm{G}+1=70$ ).

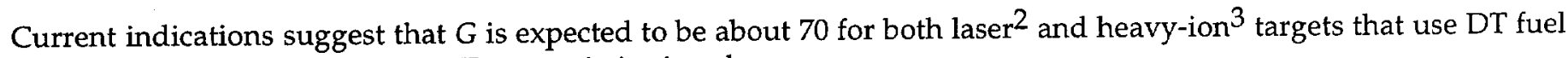
and a driver energy of 5 to $10 \mathrm{MJ}$. Target gain is given by

$$
G=\frac{M_{\text {fuel }} E_{\mathrm{TN}} \Phi}{E_{\mathrm{dr}}}
$$

where $M_{\text {fuel }}$ is the mass of fusion fuel, $E_{\mathrm{TN}}$ is the fusion energy released per unit mass ( $340 \mathrm{MJ} / \mathrm{mg}$ for DT; 347 $\mathrm{MJ} / \mathrm{mg}$ for DD, assuming that all of the produced tritium and ${ }^{3} \mathrm{He}$ burn; and $353 \mathrm{MJ} / \mathrm{mg}$ for $\mathrm{D}^{3} \mathrm{He}$ ), $\Phi$ is the fuel burn-up fraction,

$$
\Phi=\frac{\rho \Delta r}{\rho \Delta r+\Psi}
$$

$E_{\mathrm{dr}}$ is the energy of the driver, $\rho \Delta r$ is the compressed fuel column density, and $\Psi$ is a fuel-dependent and burntemperature-dependent constant. For DT fuel, $\Psi$ is $6 \mathrm{~g} / \mathrm{cm}^{2}$ for burn temperatures near $80 \mathrm{keV}$. For DD fuel, $\Psi$ is about $60 \mathrm{~g} / \mathrm{cm}^{2}$ for $80 \mathrm{keV}$, but 40 to $30 \mathrm{~g} / \mathrm{cm}^{2}$ for 200 to $300 \mathrm{keV}$, respectively (see Fig. 2). Therefore, if DD and DT targets have the same fuel mass, the same $\rho \Delta r$ (e.g., about $3 \mathrm{~g} / \mathrm{cm}^{2}$ ), and the same burn temperatures, then the gain for a given driver energy should be a factor of 7 lower for the DD target [because the DT target has $\Phi=3 /(3+6)$ while the DD target has $\Phi=3 /(3+60)]$.

We need not accept such low gains for DD targets, however, for several reasons. First, although a DT reaction produces $80 \%$ of its $340 \mathrm{GJ} / \mathrm{g}$ of yield in fast neutrons,

$$
\mathrm{D}+\mathrm{T} \rightarrow(3.5-\mathrm{MeV}) \alpha+(14.1-\mathrm{MeV}) \mathrm{n}
$$

a DD reaction produces only two-thirds of its $347 \mathrm{GJ} / \mathrm{g}$ of yield in fast particles 


$$
\begin{gathered}
6 \mathrm{D} \rightarrow(3.5-\mathrm{MeV}) \alpha+(3.6-\mathrm{MeV}) \alpha+(2.45-\mathrm{MeV}) \mathrm{n}+ \\
(14.1-\mathrm{MeV}) \mathrm{n}+(3.02-\mathrm{MeV}) \mathrm{p}+(14.7-\mathrm{MeV}) \mathrm{p}+1.83 \mathrm{MeV} \text { K.E. }
\end{gathered}
$$

assuming that the DD reactions are fully catalyzed (i.e., the tritium and ${ }^{3} \mathrm{He}$ that are produced burn completely). We can thus increase the size of the DD fuel mass, and the target would stop a significantly larger portion of the fusion energy, thereby affording a higher burn temperature and a lower $\Psi$ in Eq. (3) (see Fig. 2). This would make the gains for DT and DD targets for a given driver energy differ by less than a factor of 7 . In addition, there are advanced design concepts that allow DD target gains near 70, but for larger driver energies (i.e., larger ignition thresholds). Target gain for standard designs scales with driver energy according to the old relationship $4 G \approx E_{\mathrm{dr}}{ }^{1 / 2}$ or the more current relationship ${ }^{2} G \approx E_{\mathrm{dr}}$, where the exponents on $E_{\mathrm{dr}}$ in both relationships tend to decrease towards higher $E_{\mathrm{dr}}$; however, $G$ for advanced DD designs can have a completely different dependence. The correct dependence on $E_{\mathrm{dr}}$

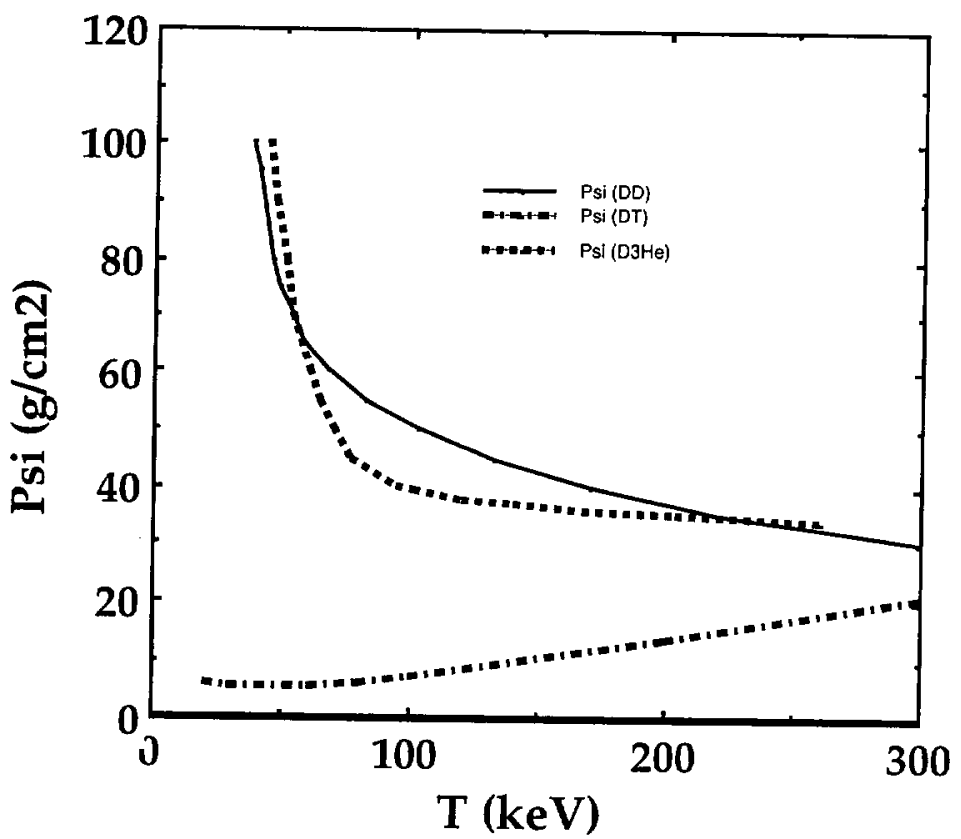

must be determined before we know how much to increase $E_{\mathrm{d} r}$ to offset the lower gain expected for DD targets. We estimate, however, that $E_{\mathrm{dr}}$ must be less than the product of the 5 to $10 \mathrm{MJ}$ estimated for $E_{\mathrm{dr}}$ for standard designs and the factor of 7 that would be dictated by $G \approx E_{\mathrm{dr}}$ to make up the maximum factor of 7 difference in gain. For the discussion here, we assume that $E_{\mathrm{dr}}$ must be $40 \mathrm{MJ}$ for the DD targets used with a HI driver.

Figure 2: Y in Eq. (3) as a function of fuel burn temperature.

For a DPSSL driver using fast ignition ${ }^{5}(\mathrm{FI})$, we have chosen an old DT gain curve via Ref. 5 as $\mathrm{G}_{\mathrm{DT}}=400 \mathrm{E}_{\mathrm{dr}}^{0.4}$ and scaled this by Eqs. (2) and (3) to get the DD gain curve $G_{D D}=G_{D T} *(347 / 340)^{*}\left[\left(\rho \Delta r+5.5 \mathrm{~g} / \mathrm{cm}^{2}\right) /\left(\rho \Delta \mathrm{r}+60 \mathrm{~g} / \mathrm{cm}^{2}\right)\right]$. FI is a speculative target-ignition scheme with many unresolved issues, especially the overall gain curve, so the gains used here are merely illustrative. FI employs a capsule compression (with most any driver) that is less sophisticated than the standard compression, which attempts to achieve a central hot spot in the fuel. The modest compression is followed by a $\sim 200-p s 10^{18} \mathrm{~W} / \mathrm{cm}^{2}$ laser pulse to "channel" through the ablated corona to any part of the compressed fuel, and then a $\sim 30-p s 10^{20} \mathrm{~W} / \mathrm{cm}^{2}$ laser pulse to ignite the fuel through the channel.

The construction cost of a $\mathrm{HI}$ driver scales typically as $E_{\mathrm{dr}} 0.4$, according to Bangerter. ${ }^{3}$ According to this scaling, a 40 MJ driver would cost 2.5 times more than a 4-MJ driver. Although it is possible that driver costs might be reduced to accommodate such an increase, it is more advantageous to be creative and try to keep the cost near the 4-MJ-driver level. One way to do this is to reprate the 4-MJ driver at 10 times the reprate of the fusion chamber, with the pulses being stored in suitable storage rings at the end of the driver chain, and then delivered simultaneously to one target. 
Assuming that the cost of the storage apparatus is much less than the cost of increasing the energy of the whole driver, the cost scales much less than $E_{\mathrm{dr}}{ }^{0.4}$, and Eq. (1) is unaffected. Further cost studies are necessary to see if such tricks are viable. We will assume here that they are.

The situation becomes even more tricky, if not impossible, for $\mathrm{D}^{3} \mathrm{He}$ fuel. Such fuel is even harder to light (the fusion and Bremsstrahlung rates are equal for temperatures of $4.5 \mathrm{keV}$ for DT, $16 \mathrm{keV}$ for DD, but $38 \mathrm{keV}$ for $\mathrm{D}^{3} \mathrm{He}$ ). In addition, the cost of ${ }^{3} \mathrm{He}$ is prohibitive (very limited quantities exist naturally on the earth). We therefore chose to use DD fuel. Nevertheless, $D^{3} \mathrm{He}$ has a similar $\mathrm{Q}$ value

$$
\mathrm{D}+\mathrm{He}^{3} \rightarrow(3.6-\mathrm{MeV}) \alpha+(14.7-\mathrm{MeV}) \mathrm{p}^{+},
$$

and a similar $E_{\mathrm{TN}}\left(353 \mathrm{MJ} / \mathrm{mg}\right.$ ); moreover, $\mathrm{D}^{3} \mathrm{He}$ has nearly $100 \%$ of the fusion release in charged particles (some DD fusion occurs) and has a slightly higher gain than DD at burn temperatures above about $50 \mathrm{keV}$ [see Fig. 2 and Eq. (3)]. Use of $\mathrm{D}^{3} \mathrm{He}$ thus does have advantages (e.g., requiring a thinner blanket), so $\mathrm{D}^{3} \mathrm{He}$ should be considered whenever it becomes more available. However, Tabak ${ }^{14}$ has shown that a $14-\mathrm{MeV}$ neutron from a DT hot spot, which can be used to light $D^{3} \mathrm{He}$, can convert ${ }^{3} \mathrm{He}$ to $\mathrm{T}$ which then reacts with a $\mathrm{D}$, thus making most of the yield from $\mathrm{D}^{3} \mathrm{He}$ targets arise from DT reactions. Careful calculations of yield products are therefore required.

The cost of targets must also be considered in detail. If advanced DD target designs are required to keep the driver cost acceptable, and we think that they are, there is a serious question whether the advanced target designs can be fabricated at economical cost. Further studies of the target fabrication facility are required.

\section{Wick Operation \& Splash}

What we designate here as the wick is either a compartmental substructuring of the blanket's water inlets, much like a honeycomb with each compartment having its own water inlet, or an actual wick substructure relying on capillary action. In either case, the purpose is to restrict any free flow of the blanket water under the pull of gravity, thereby avoiding high-volume flows and hence high pumping-power losses. Although design studies are needed to determine whether compartmental substructures are viable, we will assume that they are not, and consider just wicktype substructures.

There are serious questions relating to the wick composition, its mechanical stability, and its lifetime. The water blanket must be supplied with pressurized water throughout its spherical contact with the main containment wall to offset the outward pressure caused by (1) shock impact of the target fireball with the blanket, and perhaps also by (2) some $x$-ray ablation of the inner surface of the water. The fireball is a hot interior gas region produced because the ambient water vapor is opaque to all of the $x$ rays and most of the charged particles emitted from the target. Expansion of this hot region creates a spherical shock wave much like that from an explosion of HE in the atmosphere. The shock travels outward in the ambient fusion-chamber vapors and impacts the blanket creating an impulse that pressurizes the water. In addition, the isochoric heating from the faster charged particles and the neutrons will cause vibrational loads as the water continually releases its stress due to sudden temperature rises. These temperature rises may be only a few degrees $\mathrm{K}$ or less at radii of several meters, but would be larger for smaller fusion chambers. Under all of these conditions, it is not clear that a wick can be designed to support the water mass and not deteriorate due to fatigue and/or radiation exposure. Moreover, it is not clear how much (if any) of the inner surface of the wick would ever be exposed to $x$ rays or extremes temperatures, and thereby be eroded, reducing its lifetime.

In addition, it is desirable (but not necessarily essential) to have compressible voids in the blanket to reduce the shock pressures transmitted to the structural wall. Voids might be fabricated into the wick material, although this option might seriously constrain the wick design and/or materials available. Voids might alternatively be introduced either by operating the blanket in a two-phase vapor-liquid realm or by frothing the water upon its entry into the blanket. A future investigation should address whether frothing is viable and just how it might be accomplished. Frothing is not essential, however, even though it is advantageous, because shocks formed in the water will decay rapidly over the first several millimeters of transit toward the structural wall to a pressure level corresponding to the yield strength for uni-axial strain. Such pressures should be below the spallation threshold for the wall material, even when we 
consider the shock coupling from the water into the structural wall. However, disruption of the blanket due to shock reflections at the water-wall interface may be an issue, so we must consider such a possibility in future investigations.

The water throughput at $50 \%$ thermal efficiency is $0.56 \mathrm{~cm} / \mathrm{s}$ per GW of plant electrical power, if the inner water surface is at a radius of $3 \mathrm{~m}$ and we ignore energy used to dissociate or ionize the vapor (see below). The wick structure must allow for this throughput under continuous operation, but must also accommodate some startup procedure.

Whether there will be water splash to impede the delivery of driver beams for the next pulse is a question needing further study. We assume here that the presence of the wick structure will stabilize the water enough to avoid oscillations that might disrupt the spherical nature of the inner surface and lead to ablative forces or shock impacts that would cause splash. A design with a water annulus at the structural wall is certainly less prone to splash than a design with jets or interior annuli; the question is whether splash is avoided or reduced to a manageable level.

\section{Containment Vessel Vapor Dynamics and Chemistry}

For either driver, each pulse produces $\sim 2800 \mathrm{MJ}$ of fusion energy. Because roughly $1 / 3$ of the output is in high-energy neutrons according to Eq. (5), we assume that about two-thirds or $1870 \mathrm{MJ}$ is contained in the spherical volume interior to the water blanket, and that this energy acts to vaporize, dissociate, and ionize water every pulse. Because it takes about $3.7 \mathrm{MJ} / \mathrm{kg}$ to vaporize water at $100 \mathrm{C}$ and raise its temperature to 900 to $1200 \mathrm{~K}, 53 \mathrm{MJ} / \mathrm{kg}$ to dissociate water completely, and $220 \mathrm{MJ} / \mathrm{kg}$ to ionize water once, one might expect the sum or $277 \mathrm{MJ} / \mathrm{kg}$ to represent the net expenditure to remove water from the blanket. This is not true, however, because the ionization and dissociation states merely store the energy until it can be transferred to the first wall (the water). Thus, each fusion pulse releases enough energy to vaporize roughly $1870 / 3.7 \approx 500 \mathrm{~kg}$ of water (i.e., a 4.4-mm thickness at a radius of $3 \mathrm{~m}$ ). This mass of water in a 3-m-radius cavity produces a density about 3 times atmospheric density (i.e., about $4.4 \mathrm{~kg} / \mathrm{m}^{3}=4.4 \times$ $10^{-3} \mathrm{~g} / \mathrm{cm}^{3}$, or 1.5 to $4.5 \times 10^{20}$ particles $/ \mathrm{cm}^{3}$, the latter value applying if the vapor is $\left.\mathrm{H}, \mathrm{H}, \mathrm{O}\right)$. The column density of this vapor along a $3-\mathrm{m}$ radius is $1.3 \mathrm{~g} / \mathrm{cm}^{2}$, and the pressure is about 2040T Pascal (i.e., about $22 \mathrm{~atm}$ for $\mathrm{T}=$ $1100 \mathrm{~K})$. The mean free path of neutrons in water is about $11 \mathrm{~g} / \mathrm{cm}^{2}$ at $2 \mathrm{MeV}$ and $20 \mathrm{~g} / \mathrm{cm}^{2}$ at $14 \mathrm{MeV}$. Thus, the vapor will not stop the neutrons. The range of protons in water is $0.015 \mathrm{~g} / \mathrm{cm}^{2}$ at $3 \mathrm{MeV}$ and $0.22 \mathrm{~g} / \mathrm{cm}^{2}$ at $14 \mathrm{MeV}$. Thus, the vapor (and possibly even the target) will stop most of the protons. The vapor will certainly absorb the $x$ rays, but possible resonance re-emission of the $x$ rays and line transport through the vapor might make the vapor effectively transparent to the $x$ rays at microsecond time scales.

A fusion pulse in Hydro*Star is therefore quite different than in an evacuated fusion chamber, in several respects. First, only about $1 / 3$ of the energy is transported to the blanket in the form of neutrons, not the usual 60 to $80 \%$. This reduces neutron fluences and extends component lifetimes. Second, the near-target absorption of the $x$ rays, target debris, and proton emissions will produce a fireball that transports energy to the blanket primarily through a shock wave (although there may be some radiation too). This makes the Hydro*Star explosion similar to the explosion of TNT in the atmosphere. Although there is more experience with this kind of explosion, it definitely creates more difficulty in trying to contain the fusion processes, which are thereby more hydrodynamic in nature rather than radiative. Third, it is not clear just how much $x$-ray ablation of the surface of the water will occur. If resonance line transport is not significant, $x$-ray ablation will be significantly suppressed. Fourth, the ambient state of the fusion chamber is one at high pressure, not the usual 0.01 Torr or less. Nevertheless, the exact dynamics occurring inside Hydro*Star are currently not well known and must be determined through future investigation.

In any case, the end result of every pulse is to vaporize some water and to raise its temperature to one to tens of eV, thereby ionizing and dissociating the water. This hot vapor then cools by vaporizing more water until the rate of heat conduction through the water blanket can match the heat-transfer rate from the vapor. The vapor then cools by blanket heat conduction until reaching the boiling temperature of the water at the ambient pressure. During such cooling, which occurs on a time scale near $1 \mathrm{~ms}$, the energies stored in ionization and dissociation are released through recombination, thereby delaying the cooling. If cooling below the boiling temperature were allowed, condensation would occur.

The vapor that exits the containment vessel through the vents therefore has a composition that depends on its temperature, and therefore on its pressure, but also on the residence time at that temperature. Initially, when very 
hot, the vapor species include ionized elements and the dissociated species $\mathrm{H}_{1} \mathrm{H}_{2}, \mathrm{O}, \mathrm{O}_{2}, \mathrm{OH}$, along with $\mathrm{H}_{2} \mathrm{O}$. Water above $3300 \mathrm{~K}$ is fully dissociated, while $\mathrm{H}$ and $\mathrm{O}$ below $800 \mathrm{~K}$ will not combine without a catalyst or a flame source. ${ }^{6}$ Thus, dissociated hydrogen and oxygen between $800 \mathrm{~K}$ and $3300 \mathrm{~K}$ will burn depending on the time spent at these temperatures, with a typical burn delay time of perhaps $2 \mathrm{~ms}$. Thus, at any particular temperature, a kinetics computer code must be used to establish the constituents.

The operating temperature (and pressure) of the vapor exiting the fusion chamber through its vents must be chosen with several constraints in mind:

(1) The primary constituent of the vapor entering the turbines should be steam, not an explosive mixture of $\mathrm{H}_{2}$ and $\mathrm{O}_{2}$.

(2) The temperature should not exceed about $1200 \mathrm{~K}$, or ceramic turbine blades are required, and cost and risk would thereby increase. The current upper limit to avoid materials problems for the turbines is in fact about $900 \mathrm{~K}$, but some materials development can be expected.

(3) The steam temperature $T_{\text {hot }}$ and turbine pressure should both be as high as possible to increase plant thermal efficiency $\varepsilon_{t h}$, which is the product of the turbine efficiency $\varepsilon_{t b}$ and the Carnot efficiency:

$$
\varepsilon_{\mathrm{th}}=\varepsilon_{\mathrm{tb}} \frac{T_{\text {hot }}-T_{\text {cold }}}{T_{\text {hot }}}
$$

We assume that the thermal dump temperature $T_{\text {cold }}$ is near $300 \mathrm{~K}$, so the Carnot efficiency is near $70 \%$. For standard steam turbines, which currently operate at $2000 \mathrm{psig}$ (136 atm) with a maximum of 5000 psig (340 atm) ${ }^{7}$ the turbine efficiency is composed of a mechanical efficiency above $90 \%$ and an electrical-conversion efficiency near $75 \%$, thus making $\varepsilon_{\mathrm{tb}}$ approximately $70 \%$. Because Hydro*Star operates at only tens of atm, we must determine by how much $\varepsilon_{t b}$ is reduced below $70 \%$ by having the less efficient turbine operation at the lower pressures. For sure, plant operation (reprate and venting) must be arranged to maximize turbine pressure and not let the chamber pressure drop significantly between pulses (as it otherwise would tend to do).

(4) The steam temperature must not be so high that turbine maintenance decreases the plant availability factor. Current operating temperatures for standard steam turbines for high availabilities are 1000 to $1050 \mathrm{~F}(810$ to $840 \mathrm{~K})$, with a maximum of $1150 \mathrm{~F}(900 \mathrm{~K}) \cdot{ }^{7}$ What range will be appropriate in the future is unknown.

(5) The energy required for the prepulse system to prepare a path for the heavy ions can not be large compared with the $40 \mathrm{MJ}$ required by the target, or significant cost could be added to the driver systems. The prepulse energy increases for larger fusion-chamber pressures because larger pressures increase the ambient mass in the beam paths. The prepulse energy also increases for higher ambient temperatures because even higher channel temperatures are required to cause expansions to obtain the channel densities that will permit efficient beam propagation.

With these constraints, it appears as though the maximum steam temperature should be 900 to $1200 \mathrm{~K}$, with 1000 to $1100 \mathrm{~K}$ being preferred if we allow for some future materials development. It is for this reason that we have specified the internal chamber pressure to be about $20 \mathrm{~atm}$, in which case the chamber vapor is $0.44 \mathrm{~g} / \mathrm{cm}^{2}$ thick per meter of length. We have not yet run a kinetics code to understand the chemistry at these conditions, but it is extremely likely that such vapor, after transit to the turbines, will not contain hydrogen gas or oxygen gas unless these species have formed unmixed pockets. Issues include (1) the likelihood of fractionation into $\mathrm{H}_{2}$ and $\mathrm{O}_{2}$ at bends in the vent pipes and (2) the necessity for using catalysts in the steam pipes to ensure a strict $\mathrm{H}_{2} \mathrm{O}$ composition.

Another issue is whether the pressure pulses associated with the fusion explosions can be smoothed out enough as seen at the turbines. We don't know how much pressure variation the turbines can withstand.

\section{Pressure Barriers at Fusion chamber Ports}

Because beam pipes for a $\mathrm{HI}$ accelerator, a laser driver, and a target-injection accelerator normally operate at vacuum, while the fusion chamber in Hydro*Star operates at a pressure of tens of atmospheres, there are very serious interface 
difficulties at the ports where these beam pipes connect to the fusion chamber. Not only do the ports have to separate the high-pressure and vacuum environments, but they also must allow for a path through the water blanket, with all essential apparatus appropriately shielded with non-activating materials. The design of the ports therefore becomes very difficult.

Because the design of the target-injection accelerator is unknown, we must leave the formidable task of its port design to future studies. The simplest case may be for the injector to operate through the same ports used by the driver beams, but this is far from obvious if we consider the interface difficulties that would result.

For each driver beam port, it may be possible to use a solid barrier with a hole whose size $(3 \mathrm{~mm})$ is slightly larger than the focused spot size of the accelerator system $(2 \mathrm{~mm})$. Downstream of this barrier, a series of expansion subchambers between baffles immersed in the water blanket should rapidly reduce the steam from the fusion chamber to a moderate pressure (significantly below $1 \mathrm{~atm}$ ?) provided the subchambers can condense the steam rapidly enough to permit large Prandtl-Meyer expansion angles of the steam entering each subchamber. Studies must be conducted to optimize the design of such baffled subchambers, and ensure the desired thermal-transfer rates to the surrounding $100 \mathrm{C}$ water. Differential pumping (e.g., a series of Roots blowers, to avoid water getting in the pump oil) could then be considered immediately upstream of the beam-entrance barrier in an expansion chamber (not shown in Fig. 1) to form the isolation of the moderate pressure from the beam-tube vacuum.

The differential pumping capacity required can be estimated by assuming that the baffle system reduces the pressure only to $1 \mathrm{~atm}$ (760 Torr), and computing the conductance $C$ of a $1.5-\mathrm{m}$-long pipe with a $3-\mathrm{mm}$ diameter. The conductance is given by

$$
C=\frac{A^{2} P}{8 \pi \eta_{\nu} L}=5305 \frac{\left[A\left(m^{2}\right)\right]^{2} P(\text { Torr })}{\eta_{v}(P a \cdot s) L(m)} \quad \text { liters } / \text { second }
$$

where the pipe area is $A, P=760$ Torr, the pipe length is $L=1.5 \mathrm{~m}$, and the steam viscosity is $\eta_{v} \approx 1.4 \times 10^{-5} \mathrm{~Pa}-\mathrm{s}$. The conductance is then $10 \mathrm{l} / \mathrm{s}$, so at 760 Torr, the pumping capacity required is $7.6 \times 10^{3} \mathrm{Torr}-\mathrm{l} / \mathrm{s}$. State-of-the-art oil pumps have a capacity 10 to 100 times this value, and the Nova Roots blowers had a capacity 10 times this value. In addition, the baffle system is likely to reduce the pressure well below $1 \mathrm{~atm}$; moreover, the conductance of the system will be much less than $10 \mathrm{l} / \mathrm{s}$ if sonic choked flow is established. In short, the required pumping capacity seems to be well within capabilities, but more study is required to determine more accurate pumping parameters.

These techniques to couple vacuum lines with the chamber would require that the beam be focused before arriving at the outside of the fusion chamber, and be propagated for roughly $5 \mathrm{~m}$ of pathlength at its focused state inside the ionized channel formed inside the entrance port and into the fusion chamber. We do not know if it is possible to transport a HI beam in such a manner, but such transport is undoubtedly easier to accomplish for a laser beam. In addition, the prepulse system must somehow be interfaced to operate through this same hole, and all holes must be operated so that they do not ice shut. Moreover, we must worry about the effects of the shock that travels up the baffled system following each fusion pulse, and the rate of erosion of the first upstream 3-mm orifice. These issues have not been addressed for a laser beam as well, so we view these difficulties as some of the most severe in attempting to establish the viability of Hydro*Star.

\section{Driver Beam Propagation Through Ambient Vapor}

According to current understanding, ${ }^{3}$ heavy ions $(Z>50, A>130)$ can propagate through ionized channels if we consider the following constraints on the ambient fusion-chamber vapors:

(1) The ion number density in the ionized channel must be larger than about $3 \times 10^{16} \mathrm{~cm}^{-3}$ to suppress microinstabilities in a neutralized beam (two-stream, hose-type, et cetera), but ballistic propagation without significant charge stripping requires number densities less than $10^{11} \mathrm{~cm}^{-3}$.

(2) The column density of the channel must be small in comparison with the ion range (which is usually selected to be about $0.1 \mathrm{~g} / \mathrm{cm}^{2}$ ). That is, the column density of the channel must not be so large that collisional $\mathrm{dE} / \mathrm{dx}$ losses are comparable to the beam energy. 
(3) The column density (i.e., radiation length) of the channel must not be so high that beam emittence is seriously degraded by multiple Coulomb scattering of the ions in the channel.

(4) The channel densities must not be so large that the propagation of the HI beam causes significant ohmic losses in the channel from return currents flowing in response to the induced electric fields.

Even though any discussion of beam instabilities other than the limit imposed by (1) above is outside the scope of this report, more study of beam instabilities is required. Item (4) is also outside the scope of this report. The net result of all of the above constraints for a $\mathrm{HI}$ beam, as assessed in 1981,8 is to expect suitable transport over lengths of $<10 \mathrm{~m}$ for pressures less than $10^{-3}$ Torr or perhaps in a window from 0.1 to 1 Torr, all at $273 \mathrm{~K}$.

The assumption here is that a prepulse system, composed of an electron, (10- $\mu \mathrm{m}$ ?) laser, or HI beam, can blast its way through the ambient fusion-chamber vapors and create the desired ionized channel. The ability to use such a prepulse system depends on many issues, including the following:

(1) How to interface the prepulse system with the HI system prior to entering the HI beam ports on the fusion chamber.

(2) How to focus the prepulse system on the target.

(3) How much energy is lost in coupling the prepulse beam to the water vapor.

(4) How much energy is required to prepare a channel having a suitable density and ionization level.

(5) How much time is required to expand the ambient channel to obtain the required channel densities.

(6) How much energy is lost because of radiation from the hot channel.

(7) How much damage, if any, is done to the target by the prepulse beams.

The energy loss in using a prepulse can be estimated by calculating the amount of energy required to completely dissociate, nearly completely ionize, and heat up each prepulse channel so that expansion of the channel would produce an acceptable ion density, say, $5 \times 10^{16}$ ions $/ \mathrm{cm}^{3}$. If we assume that the channel diameter is $3 \mathrm{~mm}$ with length $5 \mathrm{~m}$, the channel mass is $4.7 \times 10^{-4} \mathrm{~kg}$. Dissociation requires $53 \mathrm{MJ} / \mathrm{kg}$ or $0.025 \mathrm{MJ}$ per channel, and complete ionization requires $6720 \mathrm{MJ} / \mathrm{kg}$ or $3.2 \mathrm{MJ}$ per channel (but ionization of all levels except the last 739-eV oxygen level requires only $40 \%$ of this or $1.3 \mathrm{MJ}$ per channel). If we assume the heated channel will expand adiabatically, we need to heat the vapor to a temperature that scales with the $2 / 3$ power of the number density (assuming a specific heat ratio $\gamma=$ $5 / 3)$. If $5 \times 10^{16}$ ions $/ \mathrm{cm}^{3}$ is acceptable, then we need to heat the vapor to roughly $1000\left(4.5 \times 10^{20} / 5 \times 10^{16}\right)^{2 / 3}=4.3 \times 10^{5}$ $\mathrm{K}=37 \mathrm{eV}$, which requires about $1.7 \mathrm{MJ}$ per channel. Thus, each channel can be prepared with a minimum energy of only $3 \mathrm{MJ}$. This analysis ignores any radiation losses, and assumes that enough time is available for the heated channel to expand. Of course, half of the prepulse beam energy is recoverable with a plant thermal efficiency of 0.50 .

Collisional $\mathrm{dE} / \mathrm{dx}$ losses of the heavy ions in the ionized channel are the most restrictive. Such losses are best discussed in terms of the range of the heavy ions, which is usually chosen to be near $0.1 \mathrm{~g} / \mathrm{cm}^{2}$. If this were the case, we would desire a channel length less than $0.01 \mathrm{~g} / \mathrm{cm}^{2}$. Because each $5-\mathrm{m}$-length channel is $2.2 \mathrm{~g} / \mathrm{cm}^{2}$, the prepulse would have to expand the channel cylindrically by at least a factor of $(220)^{2}$, thereby reducing the ion density from $4.5 \times 10^{20}$ to less than $9.3 \times 10^{15} \mathrm{~cm}^{-3}$. Because we assume that beam micro-instabilities may become bothersome below a few times $10^{16} \mathrm{~cm}^{-3}$, some tradeoff may be necessary between the beam total range and the channel ion density.

Degradation of beam emittence because of multiple Coulomb scatterings of the heavy ions off water-vapor nuclei depends on how the product of the beam size $(2 \mathrm{~mm})$ and the rms scattering angle compares to the beam emittence of typically $2 \mathrm{~mm} \mathrm{mrad} .^{3}$ The multiple Coulomb scattering angle in mrad is 9

$$
\Theta=\frac{14.1 \mathrm{MeV} / \mathrm{c}}{p \beta} Z_{\mathrm{in}} \sqrt{\frac{x}{36.1 \mathrm{~g} / \mathrm{cm}^{2}}}\left[1+\frac{1}{9} \log _{10}\left(\frac{x}{36.1 \mathrm{~g} / \mathrm{cm}^{2}}\right)\right]
$$

where $p$ is the beam momentum in $\mathrm{GeV} / \mathrm{c}, \beta$ is the ratio of beam speed to the speed of light, $Z_{\text {in }}$ is the charge of the beam, $x$ is the column density of the channel in $\mathrm{g} / \mathrm{cm}^{2}$, and $36.1 \mathrm{~g} / \mathrm{cm}^{2}$ is the radiation length for water. If we assume that $Z_{\text {in }}$ is about 70 and that $p \beta$ is about $20 \mathrm{GeV} / \mathrm{c}$, then $\theta$ is $1.0 \mathrm{mrad}$ when $x$ is $0.035 \mathrm{~g} / \mathrm{cm}^{2}$ and $0.1 \mathrm{mrad}$ when $x$ is $6.6 \times 10^{-4} \mathrm{~g} / \mathrm{cm}^{2}$. For a $5-\mathrm{m}$ length channel, these values correspond to vapor densities of $7.0 \times 10^{-5} \mathrm{~g} / \mathrm{cm}^{3}\left(7.0 \times 10^{18}\right.$ 
ions $\left./ \mathrm{cm}^{3}\right)$ and $1.3 \times 10^{-6} \mathrm{~g} / \mathrm{cm}^{3}\left(1.3 \times 10^{17}\right.$ ions $\left./ \mathrm{cm}^{3}\right)$. Therefore, because the scattering contribution adds to the beam emittence in quadrature, multiple Coulomb scattering will not significantly degrade the beam if the channel ion density is less than $10^{17}$ to $10^{18}$ ions $/ \mathrm{cm}^{3}$. Consequently, ionizational $\mathrm{dE} / \mathrm{dx}$ losses are more restrictive than scattering losses.

For the discussion here, we will assume that $\mathrm{HI}$ prepulsing is feasible, preferably using the HI beam system itself, and that the required channel density is $5 \times 10^{16}$ ions $/ \mathrm{cm}^{3}$. The parameters required for a laser pre-pulse system must be addressed in a future study, but we believe that pre-pulsing may be more easily accomplished for a laser beam than for a HI beam because higher residual chamber gas densities can be permitted for laser propagation.

\section{Target Injection Through Ambient Vapor}

Target injection into a chamber operating at about $20 \mathrm{~atm}$ has not been studied, and must therefore be addressed. Issues include (1) thermal control for cryogenic targets, (2) deflective instabilities in propagation through a possibly turbulent ambient vapor, (3) interface of the injector to the fusion chamber, (4) deceleration of the target in propagating through the fusion chamber, and (5) suitable alignment and tracking techniques.

An estimate of the problems to be encountered because of the deceleration of the target after injection can be obtained by computing the terminal velocity of a target injected vertically using gravity. The terminal velocity is 10

$$
v_{T}=\frac{2 r^{2} g\left(\rho_{t g}-\rho_{v p}\right)}{9 \eta_{v}}
$$

where $r$ is the radius of the target (let's assume it is $1 \mathrm{~cm}), g$ is the acceleration due to gravity $\left(980 \mathrm{~cm} / \mathrm{s}^{2}\right), \rho_{\mathrm{tg}}$ is the effective target density (let's assume $\left.0.1 \mathrm{~g} / \mathrm{cm}^{3}\right), \rho_{\mathrm{vp}}$ is the vapor density $\left(4.4 \times 10^{-3} \mathrm{~g} / \mathrm{cm}^{3}\right.$ ), and $\eta_{\mathrm{v}}$ is the viscosity of the vapor (about $1.4 \times 10^{-4} \mathrm{~g} / \mathrm{cm} \mathrm{s}$ ). With these assumptions, the terminal velocity is $1.6 \mathrm{~m} / \mathrm{ms}$. Thus, deceleration is expected to be an issue, but not a significant issue.

\section{Neutron Shielding for Driver Beam Ports and Vent Tubes}

There is no problem introducing a bend at the last $\mathrm{HI}$ focusing magnet so that upstream driver components are not in a direct line-of-sight to the target. Nevertheless, neutron scattering can result in leakage of neutrons along escape paths (vent tubes, beam ports). Therefore, port designs must attempt to minimize this leakage through adequate additional shielding, and must account for the possibility of reduced lifetimes of components exposed to the neutron fluence. Optimum design of such additional shielding is straight forward, but nontrivial. Similar comments apply for a laser driver.

\section{First Wall Stresses}

The possibility of high structural wall stresses is a serious problem for Hydro*Star. The fusion yield per target is 2.8 GJ, or $2 / 3$ ton TNT-equivalent. This is up to 3 times more than planned for the LLNL LMF chamber. The fact that the explosion occurs at a pressure of about $20 \mathrm{~atm}$ makes the stresses even higher, because the transfer of energy from the target to the blanket (and finally to the wall) is primarily through hydrodynamic shocks, not radiation. However, the shocks are attenuated considerably by propagation through the water vapor because complete dissociation of water requires $53 \mathrm{MJ} / \mathrm{kg}$, and complete first ionization requires $220 \mathrm{MJ} / \mathrm{kg}$, and up to $500 \mathrm{~kg}$ of ambient vapor is produced in the chamber prior to venting. Nevertheless, similar dissociation and ionization energies apply for air (e.g., 33.8 $\mathrm{MJ} / \mathrm{kg}$ to dissociate nitrogen, $100 \mathrm{MJ} / \mathrm{kg}$ to first-ionize nitrogen), and experience from explosions in air suggest that high stresses may be involved. In any case, we know of no calculations of the shocks transferred to a blanket at conditions anywhere near those applicable here, so calculations must be forthcoming.

If the blanket can be operated with vapor bubbles introduced in whatever manner possible, the structural wall stresses will probably be well within structural limits for chamber radii exceeding roughly $3 \mathrm{~m} .{ }^{11}$ The worst case possible is thus a water blanket without any bubbles. For such a system, the momentum impulse transferred to the structural wall is actually less than produced through shock impact at the inner surface of the water, because of the increase in area of the structural wall. The impulse on the structural wall increases as the inner radius of the water is 
decreased, and would become intolerable at some radius that is dependent on target yield. However, radii larger than about $3 \mathrm{~m}$ should be sufficient to reduce all stresses to a manageable level because shocks attenuate to the uni-axialstrain levels before traveling $1 \mathrm{~cm}$ in most liquid and solid materials. Although interior post-fusion pressures do rise briefly to very high values, no acceleration of the water annulus is possible, as there would be for interior annuli or jet structures.

Another serious problem is the potential for stress corrosion cracking at welds in the structural wall. Because of experience with G.E.'s boiling water (fission) reactor, it was discovered that chloride (and molybdenum, etc.) ions originally at low concentration (parts per billion levels) slowly increase in water that is being vaporized until the conductivity allows carbon to be extracted from the heat-affected zones near the welds in a structural wall composed of ordinary steels. The carbon then reacts with the chromium, and the chromium removal leads to corrosion. However, they discovered that use of low-carbon steels (series 300 steels with carbon less than $0.04 \mathrm{ppb}$ ) prevents the occurrence of this problem. Therefore, Hydro*Star must use such low-carbon steels for any surfaces in contact with the water being vaporized in the fusion chamber to avoid the stress-corrosion cracking problems. With use of such steels, stress-corrosion cracking should not be a problem.

\section{Blanket Purity \&Waste-Stream Cleanup}

Radiological management and waste-stream cleanup for the water blanket involves serious issues, not from the water itself, but from contaminants added to the water. The induced activity in the water itself arises primarily though ${ }^{14} \mathrm{C}$ production off ${ }^{17} \mathrm{O}$ via ${ }^{17} \mathrm{O}(\mathrm{n}, \alpha){ }^{14} \mathrm{C}$. We have not yet estimated the induced activity from this process, but we don't expect it to present a serious problem.

For a plant output power $P$, unburned tritium will get into the water from the fuel targets at a rate

$$
R_{\mathrm{T}}=\frac{P(1+f)}{\varepsilon_{\mathrm{th}} M E_{\mathrm{TN}} \Phi_{\mathrm{DD}}}\left(1-\Phi_{\mathrm{DT}}\right) \frac{m_{\mathrm{DT}}}{m_{\mathrm{DD}}}
$$

This yields about $1 / 50 \mathrm{mg} / \mathrm{s}(0.2 \mathrm{Ci} / \mathrm{s})$ for $P=1 \mathrm{GW}, f=0.10, \varepsilon_{\mathrm{th}}=0.50, E_{\mathrm{TN}}=347 \mathrm{MJ} / \mathrm{mg}, \Phi_{\mathrm{DD}}=0.20, \Phi_{\mathrm{DT}}=0.33$, and fuel mass ratio $m \mathrm{DT} / \mathrm{mDD}_{\mathrm{D}}=1 / 1000$ (which is only approximate). There may also be some tritium produced by the $\mathrm{DD}$ reactions that does not burn, and if so, the tritium buildup could be faster than $0.2 \mathrm{Ci} / \mathrm{s}$.

A blanket $1.5 \mathrm{~m}$ thick at a chamber radius $R=4 \mathrm{~m}$ has a mass of 300 metric tons $\left(300 \mathrm{~m}^{3}\right)$, which we will double to 600 metric tons $\left(600 \mathrm{~m}^{3}\right)$ to account for storage and piping volume. Tritiated water is hazardous to humans only if ingested, because it emits a positron that is easily stopped by clothing et cetera. According to 10CFR20 (Appendix B, Table II, Col. 2), tritiated water is safe to spill directly on the ground at a concentration less than $3 \times 10^{-3} \mathrm{Ci} / \mathrm{m}^{3}$. At $0.2 \mathrm{Ci} / \mathrm{s}$, this concentration is reached after only 9 seconds of equivalent averaged operation. Tritium extraction facilities are therefore necessary. The current planning for the LLNL LMF design is to purify the LMF waste stream only to $1 \mathrm{Ci} / \mathrm{m}^{3}$, and this concentration is reached in Hydro*Star after 50 minutes of operation. Methods to extract the tritium for reuse in the targets must of course be developed, and the level to which the tritium should be extracted with such methods must be determined from future investigation. Containment of the tritium in water, however, is not only the safest from the standpoint of a biological hazard, but the easiest for processing.

Of greater concern is the radioactive target debris that will end up in the water. The full impact of this debris must await a description of the target, but about 100 metric tons of substance per gram of target material must be processed per year of plant operation. If some of this is activated and/or toxic, considerable expense could be involved in wastestream processing.

Another potential problem that cannot be ignored is the buildup of contaminants in the water because they are left behind when the water evaporates, as discussed for stress-corrosion cracking in boiling-water reactors in the section above on first-wall stresses. For Hydro*Star, we need to assess whether such buildup is an issue, or whether recirculation of the water will prevent buildup. We also need to establish whether the surface of the blanket is exposed to $x$ rays, and if so, whether the contaminants would also be vaporized, thereby excluding the contaminant issue as a problem for Hydro*Star. 


\section{Vent and Steam Piping}

The interface of the steam vents with the fusion chamber must allow for adequate neutron and $x$-ray shielding of piping and exterior components. In particular, the vents must not be an avenue for the escape of neutrons otherwise contained within the blanket. There should be no problem, however, with the operating temperature being $900 \mathrm{~K}$ and the operating pressure being tens of atm because standard steam turbines operate at this temperature and well above this pressure (e.g., 1000-2000 psig $=68$ to $136 \mathrm{~atm}$ typically and $5000 \mathrm{psig}=340 \mathrm{~atm}$ maximum). ${ }^{7}$ Nevertheless, there could be problems with oxygen corrosion or problems similar to the stress-corrosion cracking difficulties discussed above.

\section{Radioactivity In \& Maintenance Of the Turbines}

Because hydrogen tends to be absorbed by various materials and can cause embrittlement, it is generally not good to operate turbines with hydrogen. It is also not good to have the turbines operating with a mixture of $\mathrm{H}_{2}$ and $\mathrm{O}_{2}$, which may be explosive. Thus, it would be best if the chamber effluent were merely steam upon entering the turbines. Because tritium will be present, some tritium will likely penetrate the turbine blades and require personnel to be suited up during normal turbine maintenance. Although it might be nice to avoid such procedures, there would be no serious threat to personnel as a result of such tritium buildup in the turbines. It might even be possible to flush the turbines with water or some other substance and remove part of the tritium, but other radioactive debris can probably be removed with such procedures more easily than forms of hydrogen. Nevertheless, we do not view the tritium buildup as a problem serious enough to warrant the introduction of a heat exchanger, which would significantly reduce thermal efficiency.

\section{Turbine Temperature \& Plant Availability}

The standard operating temperatures for steam turbines is approximately $625 \mathrm{C}=900 \mathrm{~K}$, although state-of-the-art turbines operate near $1000 \mathrm{~K}$. The higher the turbine temperature, the lower the plant availability because of down time to fix turbine components. Therefore, after making allowance for future improvements in turbine design, a systems study must be conducted to determine the tradeoff between higher operating temperatures at the turbine to obtain high plant thermal efficiencies and the desire to maintain high plant availability.

\section{Tritium Breeding vs Purchase of Tritium}

Only $1 / 3$ of the tritium need be purchased, after tritiated-water recycling begins, and at $0.01 \mathrm{mg} / \mathrm{s}$, this amounts to only 315 grams (1.3 liter) per year of operation. Because the cost of tritium is roughly $\$ 10,000.00$ per gram, the cost of the tritium is $\$ 3$ million per year. Although this is only about $1 \%$ of the gross electricity sales, it is non-negligible. We should therefore study whether some form of breeding is possible in the water. For instance, $\mathrm{LiH}$ or some other compound of lithium might be acceptable if it can be dissolved in the water, and if it does not disrupt the vaporization of the water at the blanket or the condensation of the vapor in the turbines and final heat exchanger, and if it does not cause buildup problems as discussed in the section above on First Wall Stresses. Further investigation is required.

\section{Startup Procedures}

Because the water blanket in Hydro*Star is stabilized dynamically, with the input flow balanced by fusion vaporization, the blanket must be gradually established upon plant startup after any down time. Future studies must address how this can best be accomplished, and to establish the necessary procedures.

\section{HYDRO*STAR REPRATE}

Because Hydro*Star operates by not condensing the vaporized blanket material, contrary to other designs, the reprate of the facility is not as constrained by the time to cool and condense the vaporized wall materials (and its risk is not as affected by the current uncertainties in the physics of condensation). A future study must therefore be conducted to determine what else will limit the reprate. It may be the energy needed to clear channels for the driver beams, because higher reprates correspond to higher chamber pressures (at a given chamber radius). In any case, because the reprate can be increased significantly relative to other ICF fusion-chamber designs, detailed studies are required to determine how much the projected cost of electricity (COE) can be reduced by higher reprate operation. Specifically, previously published generic estimates ${ }^{12}$ showing that the COE is minimum for reprates of 25 to $30 \mathrm{~Hz}$ cannot be used for 
Hydro*Star because of the detailed nonlinear nature of a real driver, especially for a DPSSL. Besides, the generic case has a very broad minimum in which the COE at 5 to $10 \mathrm{~Hz}$ is only about 5 to $10 \%$ higher than at 25 to $30 \mathrm{~Hz}$. In addition, as can be ascertained from the current rise in costs of producing electricity and the results of the next section, just about any reasonable reprate is acceptable for Hydro* Star.

Therefore, the reprate of Hydro*Star can be determined from the consideration of several critical factors. First, the reprate must be fast enough to avoid gravitational distortion of the inner surface of the water blanket. Hydro*Star can hence be reprated as fast as is needed to keep the blanket operation within acceptable requirements. Second, the desired dynamical water-vapor chemistry must be compatible with turbine operation in addition to the vapor pressure and temperature. These dynamical parameters change with reprate. Third, the size of the plant might be affected by fusion economics, especially the desire of utility companies for a certain size of plant. The optimum overall reprate can be determined based on factors such as these.

\section{COST OF ELECTRICITY (COE)}

We report here the results of an initial study of how COE and plant size can vary with reprate for the case of a DPSSL driver. For this purpose, we started by assuming that the reprate for a plant with $1 \mathrm{GWe}$ net electrical output would be identical to that required for the $\mathrm{HI}$ plant having the features assumed in this report. Thus, for Hydro*Star, every $0.826 \mathrm{~Hz}$ corresponds to $1 \mathrm{GWe}$ of electrical power. We hence doubled this basic reprate to obtain a 2-GWe plant, tripled it for a 3-GWe plant, and so forth. Figure 3 displays the results, as obtained using our DPSSL*IFE systems code with COE in constant 1991 dollars. ${ }^{13}$ Note that COEs between 4 and $20 \notin / \mathrm{kWh}$ are obtainable for plant sizes of up 10 GWe for laser efficiencies of 6 to $9 \%$. All cases have 1200 to 1400 laser beams. For $1 \mathrm{GWe}$, it is interesting to note that the simple analysis given by Eq. (1) incorrectly indicates a target gain of 168 and a recycled power fraction of $11.8 \%$ for a driver energy of $16.1 \mathrm{MJ}$.

In contrast to the results in Fig. 3, note that if reprate and driver energy are allowed to vary independently, the minimum COE for $1 \mathrm{GWe}$ occurs for a reprate of $3 \mathrm{~Hz}$, a $30 \%$ recycled power fraction, a target gain $\mathrm{G}$ of 63 , and a COE of $14 \varangle / \mathrm{kWh}$. With this same type of freedom, but using completely DT fuel, the minimum COE of $7 \notin / \mathrm{kWh}$ occurs at $3 \mathrm{~Hz}$ with only 192 beams. Thus, the results depend on the constraints and the way the optimum is obtained.

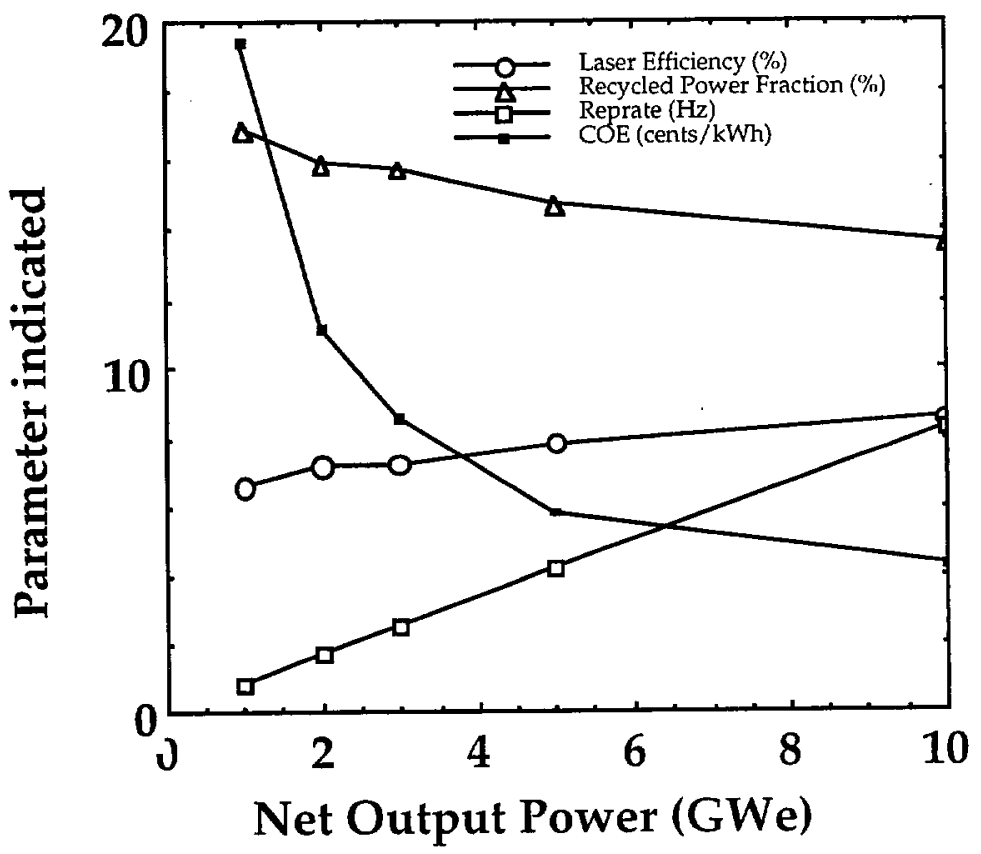

Figure 3 Variation of COE and other plant features with reprate for a DPSSL plantunder the assumption of $0.826 \mathrm{~Hz}$ per GWe of plant net output power 


\section{CONCLUSIONS}

We have described a new IFE fusion-chamber system called Hydro*Star that uses DD targets ignited with DT hot spots, and have described a multitude of physics and engineering issues that must be addressed before Hydro*Star can be considered to be a viable concept. Nevertheless, Hydro*Star has so many significant advantages over other IFE chamber concepts that it seems worthwhile to pursue the additional studies that will address these and other concerns. The result would be a much safer fusion plant with greater appeal to the general public because the main fluid used is simply hot water, which is of course nontoxic, nonradioactive, nonflammable, and safe for the environment.

This work was performed under the auspices of the U.S. Department of Energy by the University of California, Lawrence Livermore National Laboratory under contract W-7405-Eng-48.

\section{REFERENCES}

1. Wayne Meier, W. J. Schafer Associates, Inc., private communication (Jan. 1990).

2. Richard A. Sacks and Roger O. Bangerter, Integrated Gain Curves for Indirectly Driven ICF Laser Targets, IEEE Thirteenth Symposium on Fusion Engineering, Knoxville, TN (2-6 October 1989) paper 16-I-01 (invited oral presentation only).

3. Roger Bangerter, LLNL, private communication (Nov. 1989).

4. E.g., J. D. Lindl, R. O. Bangerter, J. W-K. Mark, and Yu-Li Pan, "Review of Target Studies for Heavy Ion Fusion," Heavy Ion Inertial Fusion, M. Reiser, T. Godlove, and R. Bangerter, eds. (N.Y.: American Institute of Physics, 1986), pp. 89-99 (cf. Fig. 3).

5. Max Tabak, J. Hammer, M. Glinsky, W. Kruer, S. Wilks, J. Woodworth, E. M. Campbell, M. Perry, and R. Mason, "Ignition and High Gain with Ultra-Powerful Lasers," Physics of Plasmas, Vol. 1 \# 5, pp. 1626-1634 (1994); and Max Tabak, private communication (1998).

6. Nate Hoffman, Energy Technology and Engineering Center (ETEC), Rockwell/Rocketdyne, Canoga Park, CA, private communication (1989).

7. . Philip J. Potter, Power Plant Theory and Design (N.Y.: John Wiley, 1959).

8. E.g., C. L. Olson, "Final Transport in Gas and Plasma," Proc. Heavy Ion Fusion Workshop, Berkeley, California, September 1980, Lawrence Berkeley Laboratory, LBL-10301, p. 403 (1980); and S. S. Yu, E. P. Lee, and H. L. Buchanan, "Focal Spot Size Predictions for Beam Transport Through a Gas-Filled Reactor," Proc. Heavy Ion Fusion Workshop, Berkeley, California, September 1980, Lawrence Berkeley Laboratory, LBL-10301, p. 504 (1980).

9. M. Aguilar-Benitez, et al., Particle Properties Data Booklet (Amsterdam: North-Holland, April 1988), p. 95.

10. M. Russell Wehr and James A. Richards, Jr., Physics of the Atom (Reading, Mass.: Addison-Wesley Pub. Co., Inc., 1960), p. 39.

11. Charles D. Orth, Wall Ablation, Gas Dynamics, and the History of Wall Stresses for Inertial Confinement Fusion Target Chambers, University of California, Lawrence Livermore National Laboratory, Internal Report ICFA 89-08 (Nov. 1989).

12. Wayne R. Meier and William J. Hogan, ICF Reactor Economics: Identifying the High Leverage Design Features, University of California, Lawrence Livermore National Laboratory, UCRL-91648 (Feb. 28, 1985).

13. C. D. Orth, S. A. Payne, W. F. Krupke, Nuclear Fusion, 36, 75-116 (1996).

14. M. Tabak, "What is the Role of Tritium-Poor Fuels in ICF?", Nuclear Fusion, 36 (1996) 147 


\section{Laser Optics and Materials Research ACtivity Highlights}

\section{LS\&T Improves $\mathrm{CO}_{2}$-Laser Treatment of NIF Final Optics}

A limitation to the performance of high-fluence laser systems is the growth of small damage sites on exposure to subsequent laser pulses. Even state-of-the-art production methods yield a small number of initiator sites that would be expected to grow on exposure to high fluence. Previous work has shown that treatment with a $\mathrm{CO}_{2}$ laser can prevent damage from growing.

The $\mathrm{CO}_{2}$-laser treatment process has been improved, and significant steps have been taken towards implementing the technique in a production facility (Figure 1). The key to the

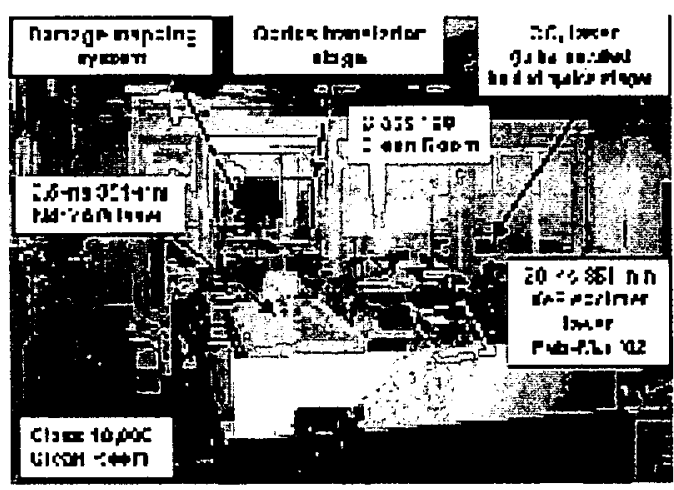

Figure 1. The Phoenix conditioning and mitigation facility.

improvement is the minimal-vaporization technique applied to small initiators before they experience significant growth. A low-power $(\sim 2.5 \mathrm{~W} \mathrm{CW})$, tightly focused $(\sim 125 \mathrm{~mm}$ diameter) $\mathrm{CO}_{2}$ laser beam, with an exposure time of $\sim 10 \mathrm{~ms}$, has been used to treat these small initiators. The resulting pit is only $\sim 75$ $\mathrm{mm}$ diameter and $1 \mathrm{~mm}$ deep. These treated sites do not grow further when exposed to 350 $\mathrm{nm}$ pulses at $-14 \mathrm{~J} / \mathrm{cm}^{2}$ and a few ns duration.

Treatment of optical damage with a $\mathrm{CO}_{2}$ laser is expected to play a central role in the life cycle of National Ignition Facility (NIF) fused-silica optics in the Final Optics Assembly (FOA). Figure 2 shows two separate input streams into the $\mathrm{CO}_{2}$ treatment cycle during the material flow of optics from initial production at the vendor to scrap at the end of the useful life. The upper stream, shown in black, represents the final treatment of optics prior to installation. The $\mathrm{CO}_{2}$ treatment cycle identifies these sites while they are still small and modifies them so they will not grow. This process is the focus of the current work.

The lower stream, shown in red, represents optics with identified damage removed from the system. Some sites eventually damage during operation. Although the mitigation of these larger sites will leave larger and deeper pits, $\mathrm{CO}_{2}$ treatment can halt further growth of these sites.

Key to implementing the mitigation process are four critical components: (1) The required parameters for the $\mathrm{CO}_{2}$ laser treatment must be determined. This includes not only determining how to treat damage sites so that they no longer grow when exposed to high fluence $350-\mathrm{nm}$ light, but also ensuring that the resulting modification of the surface does not lead to damage of downstream optics due to beam modulation. (2) Small damage sites must be located and targeted. (3) A process must be developed that ensures that all damage precursors have been mitigated. (4) The process must be automated to deliver the high throughput required for NIF. Progress has been made in each of these key areas. 


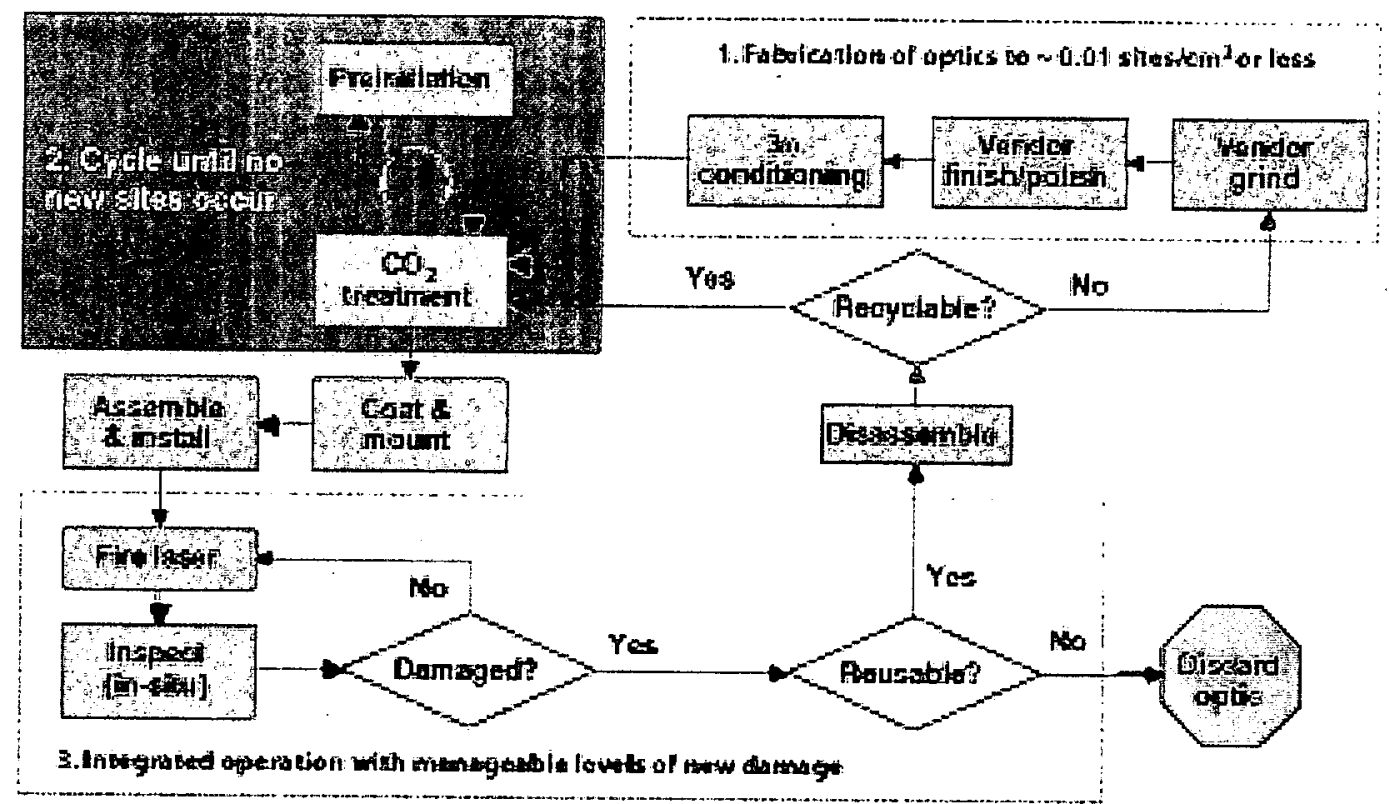

Figure 2. Material flow for NIF FOA fused-silica optics. Shaded region represents $\mathrm{CO}_{2}$ mitigation process.

Figure 3 shows two of the sites that have been successfully treated. Of the approximately 60 naturally initiated sites that have been mitigated, only one grew when exposed to $14-\mathrm{J} / \mathrm{cm}^{2}$ fluence of $350-\mathrm{nm}$ light. This one failure is probably the result of an alignment problem. Measurements of the diffraction patterns of the mitigated sites have shown relatively small peak beam modulation extending over only a small area. The modulation usually peaks within a few centimeters of the treated

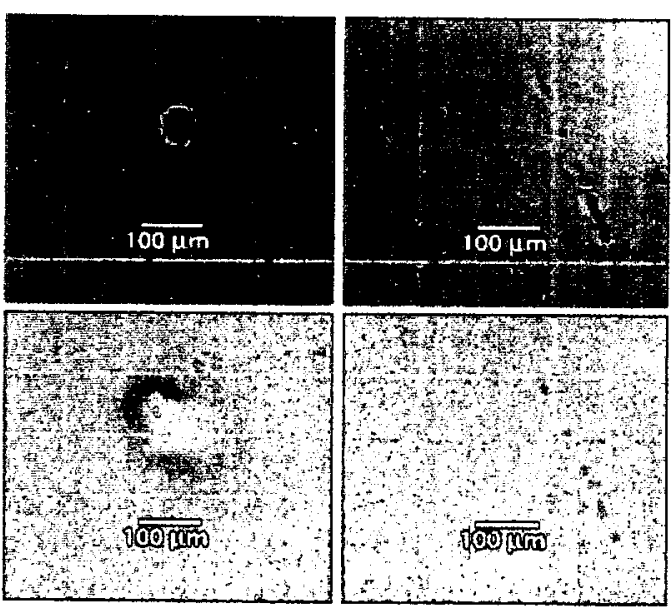

Figure 3. Microscope images of two damage sites before (top) and after (bottom $\mathrm{CO}_{2}$ - laser treatment. These sites were created in a rasterscan at 14 $\mathrm{J} / \mathrm{cm}^{2}$. After mitigation there was no further growth at $14 \mathrm{Jcm}^{2}$ site and then falls. Work is continuing to study the effect of modulation on downstream damage.

Progress on implementation and automation of the process has also been made. Production of very small mitigation sites requires tight alignment tolerances and a short depth-of-focus which increases the complexity in the treatment of curved optical surfaces. An alternative to maintaining tight tolerances is using slightly larger mitigation sites that still have acceptable beam modulation properties. Mitigation sites two or three times larger $(\sim 50 \mathrm{~mm})$ may be an acceptable tradeoff. A new facility will be activated in February to develop the automation and integration capabilities required for production of $3 w$ optics for NIF. This technique will help to ensure reliable operation of NIF final optics.

\section{Continuous Melting Process Produces High-Quality, Meter- Sized Glass Slabs}

A novel, continuous melting process is being used to manufacture meter-sized slabs of laser glass (Figure 4 ) at a rate 20 times faster, five times cheaper, and two to three times better optical quality than with the previous oneat-a-time, "discontinuous" technology process. The glasses manufactured by this new process are Nd-doped phosphate-based glasses and are marketed as LG-770 (Schott Glass Technologies) and LHG-8 (Hoya Corporation 


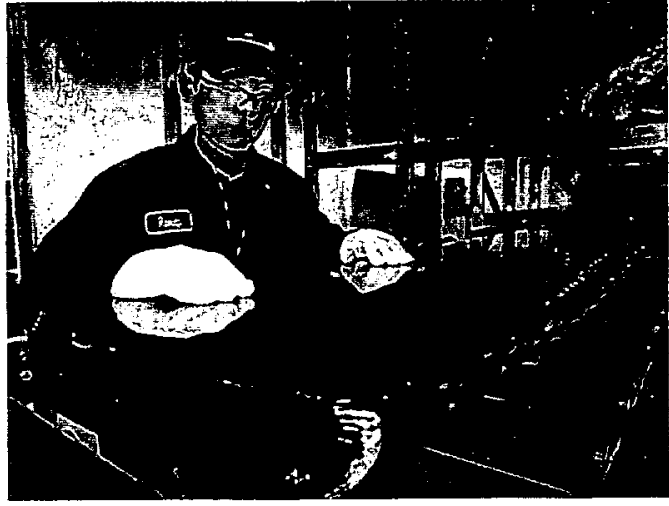

Figure 4. Continuous strip of laser glass coming out of couarse annealing at Hoya Corporation.

USA). After finishing, these amplifier slabs will serve as the gain media on the National Ignition Facility (NIF) and the Laser Megajoule (LMJ) lasers. The combined NIF and LMJ lasers require approximately 75 times the laser glass production capacity used for Nova and OMEGA with glass-part sizes more than two times larger. Thus, almost 8000 laser glass plates will be needed for the two laser systems: this represents a volume in excess of $125 \mathrm{~m} 3$ (330 metric tons) of finished optical quality glass.

Discontinuous processing methods have been used with good results for producing small quantities of laser glass. However, the discontinuous process has a small throughput and a single melting system can, at best, only produce fewer than five glass plates per week. In addition, the quality of the glass produced can vary from one melt to the next simply because of small, but random, run-to-run variations in pro- cessing conditions. Continuous glass melting, on the other hand, has two major advantages: much greater production rates'can be achieved, and once steady state is achieved, there is little if any measurable variation in glass properties from one glass plate to the next. Continuous optical glass melting systems are generally divided into several interconnected zones. Each zone consists of one or more vessels designed to carry out a particular part of the process. In laser glass continuous melters, there are six main interconnected processing zones (Figure 5): (1) raw material mixing and feeding, (2) melting, (3) conditioning, (4) refining, (5) homogenizing, and (6) continuous strip forming.

The manufacture of laser glass by continuous melting is a result of numerous technical advances after a six-year, joint research and development effort between LLNL, Hoya, and Schott:

1. Pt-inclusion removal: Microscopic Pt particles $(<10 \mu \mathrm{m})$ in the laser glass can absorb laser light and cause fracture in the glass (laserinduced damage). Research on their formation and dissolution has led to a redox-controlled process for minimizing the number and size of inclusions in the glass.

2. $\mathrm{OH}$ removal: Hydroxyl $(\mathrm{OH})$ groups in the glass quench the fluorescence of the $\mathrm{Nd}$ and reduce the laser output energy. Research on the chemical mechanism of $\mathrm{OH}$ removal (dehydroxylation) using reactive gas bubbling and the incorporation of this information in process models have led to a 50 times reduction of $\mathrm{OH}$ content in continuously melted glass. Figure 6

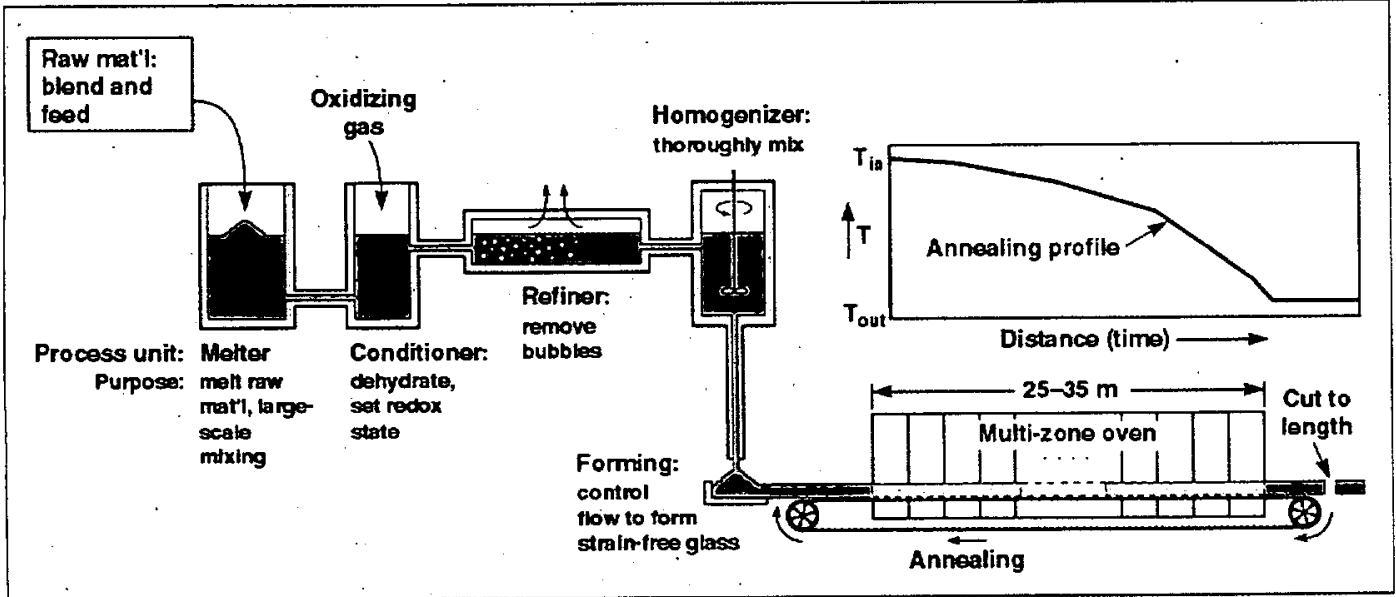

Figure 5. Schematic representation of the continuoous laser glass melting systems. 


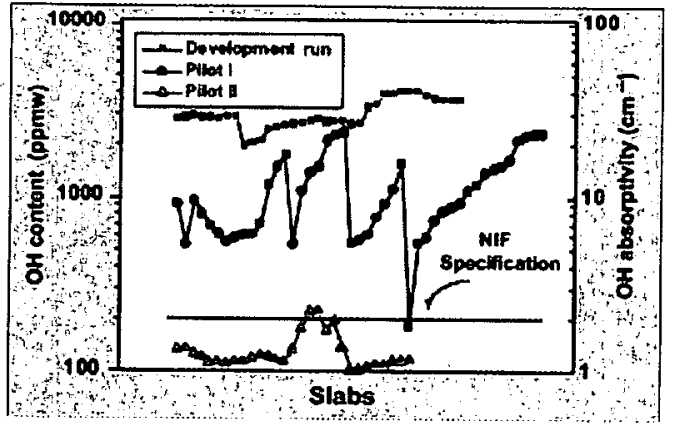

Figure 6. $\mathrm{OH}$ content (in ppmw and $\mathrm{OH}$ absorptivity) in randomly selected laser glass slabs showing the nearly $50 x$ reduction in this contaminant achieved during a recent melt campaign.

illustrates the dramatic improvements in glass $\mathrm{OH}$ content that have been achieved.

3. Fracture prevention: Phosphate laser glasses are prone to fracture due to their low fracture toughness and high thermal expansion. Finite elemental heat transport and stress analyses combined with the identification of various stress sources and research in crack growth have led to improvements in the annealing process to eliminate fracturing.

4. Impurity minimization: Metal ion impurities (such as $\mathrm{Fe}^{2+}$ and $\mathrm{Cu}^{2+}$ ) at 10 parts-permillion level can increase the optical absorption of the glass above acceptable limits. New analytical techniques to quantify impurity levels combined with research on the absorption characteristics of these impurities have led to much improved specifications and quality control procedures for both the laser glass and the raw materials.

5. Homogeneity: Laser glass requires a refractive index uniformity (i.e., optical homogeneity) of about one-part-per-million, requiring advanced forming technologies.

6. Quality assurance: A number of unique quality-assurance tools have been developed to inspect large optical glass plates at a high rate. These tools include large-aperture (24-inch) phase-measuring interferometers and largeaperture laser damage testers.

The recent melting campaigns have been very successful yielding a combined output of $\sim 3400$ NIF/LMJ-quality slab blanks to date. The LLNL Laser Glass Group will continue their effort to further improve glass yield in future campaigns.

\section{Improving Antireflection Coatings for NIF KDP and DKDP Optics}

Antireflection (AR) coatings are required to minimize reflection losses from the fused silica windows, lenses, and the potassium dihydrogen phosphate (KDP) and deuterated KDP (DKDP) switch and conversion crystals on the National Ignition Facility (NIF) laser. Porous sol-gel coatings, first developed for Nova laser optics, have been used on many high-power laser systems such as OMEGA, Helen, Phebus, and Beamlet. These sol-gel coatings are composed of layers of 10- to 20-nm-diam silica spheres that form a film with approximately $50 \%$ porosity. The resultant film has an index of refraction of 1.2, approaching the theoretical optimum index for a single-layer AR coating on fused silica or KDP. Figure 7 shows the coating performance.

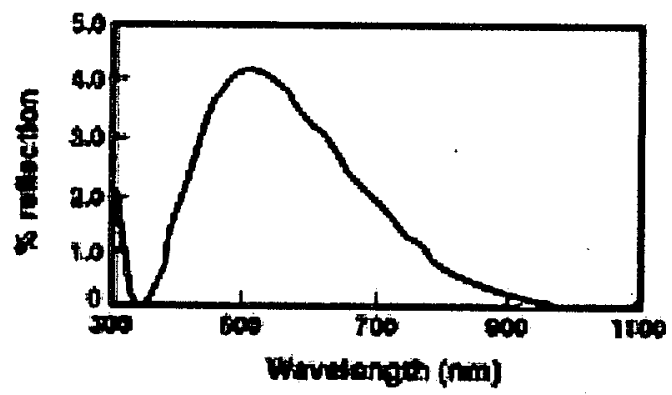

Figure 7: Reflection from the surface of a porous solgel coating on KDP, optimized for 1053-nm laser light.

Porous sol-gel coatings are the only AR coatings that have demonstrated survivability at projected NIF 351-nm operating fluences . Unfortunately, the porosity that gives these films their outstanding optical and damage performance also makes them extremely sensitive to their use environment. As the pores adsorb organic or water vapor from their environment, the films' index of refraction increases and they lose their antireflection properties. For coated $\mathrm{KDP}$ optics, exposure to humidity has a second detrimental impact-it produced etch pits like those shown in Figure 8. These pits scatter laser light, causing both additional loss of energy on target and the potential for increased laser damage to downstream optics. Etch pits, 


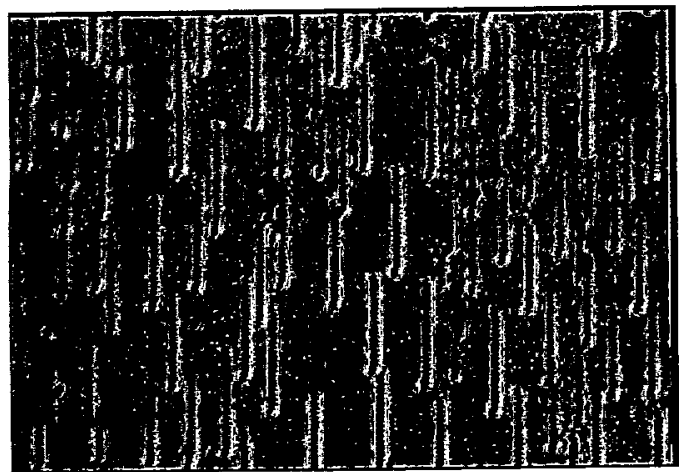

Figure 8: Etch pits on a Beamlet third-harmonicgeneration crystal.

which were first observed during the final Beamlet campaigns, motivated an effort to identify and implement "etch-pit mitigation" methods for NIF crystals.

Surface adsorption of water vapor, subsequent capillary condensation of water into the porous sol film, and the dissolution of the underlying substrate cause etch-pit formation on optics under humid environments. During the past few years, we have developed several methods to effectively retard etch-pit formation on optical crystals: (1) application of a polymer barrier coating, (2) chemical passivation of the KDP surface, and (3) modification of the surface chemistry of sol-gel to eliminate capillary condensation.

The first method, applying a barrier coating of methyl silicone polymer to reduce etch-pits formation, hence improving the optical performance of KDP frequency conversion crystals, was used in the ' $90 \mathrm{~s}$ in Nova experiments. This silicone coating process is now used on the OMEGA conversion crystals and will be used in NIF on frequency conversion crystals.

The second method was developed to protect the KDP crystals used for optical switching. Methyl silicone coatings would be oxidized and destroyed by the oxygen plasma in the NIF Pockels cells. We developed a thermal annealing process that produces a $70-\mathrm{nm}$ thick, water-insoluble, dehydrated-phosphate layer on the surface of finished KDP optics. During the annealing process (three months at $160^{\circ} \mathrm{C}$ ), the $\mathrm{KH}_{2} \mathrm{PO}_{4}$ molecules on the optic surfaces undergo interfacial dehydration reaction and produce a polymeric film with composition of Kurrol's salt $\left(\mathrm{KPO}_{3}\right)_{\mathrm{n}}$. We were able to monitor the development of this chemical passivation layer by Fourier transform

infrared (FT-IR) spectroscopy, electron spectroscopy for chemical analysis (ESCA), and $\mathrm{x}$-ray diffraction.

The deuterated third-harmonic-generation crystal (DKDP) cannot tolerate the temperatures required for either the thermal annealing process or the Nova methyl silicone process. To prevent etch-pit formation on the DKDP, we have modified the surface chemistry of the solgel particles themselves, converting them from a hydrophilic (water-loving) to a hydrophobic (water-hating) surface, as shown in Figure 9. During the surface treatment process, the hydrophilic surface silanols $(\mathrm{Si}-\mathrm{OH})$ are reacted with hexamethyldisilazane (HMDS) to produce hydrophobic trimethylsilyl groups:

$$
\begin{array}{r}
2(\equiv \mathrm{SiOH})+\left(\mathrm{CH}_{3}\right)_{3} \mathrm{Si}-\mathrm{NH}-\mathrm{Si}\left(\mathrm{CH}_{3}\right)_{3} \\
\Rightarrow 2\left(\equiv \mathrm{Si}-\mathrm{O}-\mathrm{Si}\left(\mathrm{CH}_{3}\right)_{3}\right)+\mathrm{NH}_{3}
\end{array}
$$

We were able to control the stability of the hydrophobic surface by optimizing concentration of reactive surface silanol groups prior to introduction of the HMDS. One unexpected benefit of the hydrophobic surface modification is that it appears to reduce the sol's affinity for polar organic contaminants as well as water.

(a)

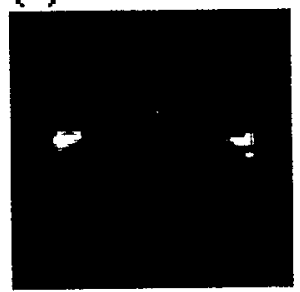

Figure 9: Water droplet on a (a) hydrophilic and (b) hydrophobic porous sol-gel coating.

To date, all three methods-methyl silicone, thermal annealing, and hydrophobic sol-have prevented etch-pit formation on KDP and DKDP surfaces exposed to $75 \%$ relative humidity for over six months. This provides the flexibility to match the etch-pit mitigation method to the use environment and optical requirements for each NIF crystal type.

Future work will focus on the ineraction of laser energy with these coatings to ensure their survivability at projected NIF operating fluences. 


\section{Shoeked and Stressed, Metals Get Stronger}

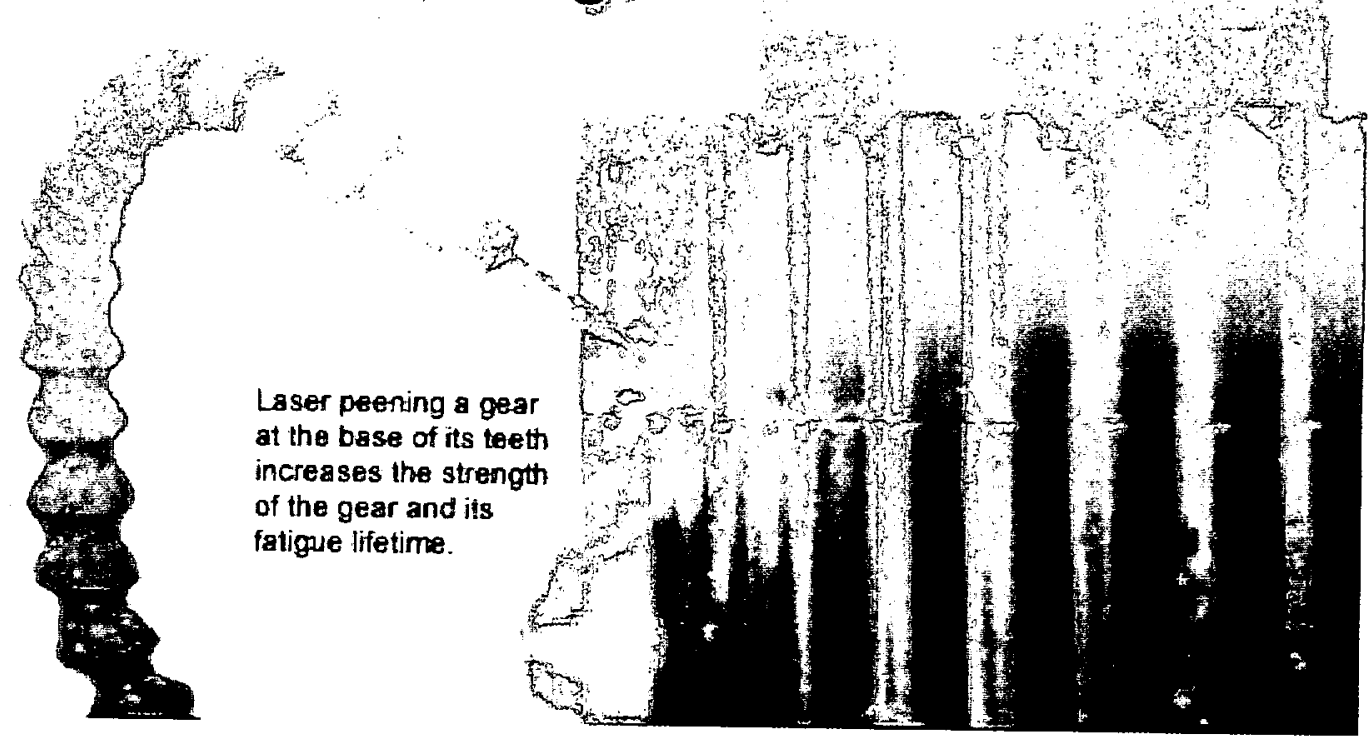

\section{Shocked and Stressed, Metals Get Stronger}

People who know their way around metalworking are no doubt familiar with peeningusing a ball-peen hammer to pound a piece of metal into shape and strengthen it against fatigue failure. For the past 50 years, an industrialized equivalent has been shot peening, in which metal or ceramic beads as large as marbles or as small as salt and pepper grains pneumatically bombard a metal surface. Laser peening, a process based on a superior laser technology developed at Lawrence Livermore, replaces the hammer blows and streams of beads with short blasts of laser light. The end result is a piece of metal with significantly improved performance.

Lawrence Livermore and Metal Improvement Company, Inc., won a coveted R\&D 100 Award for their laser-peening process in 1998 (see $S \& T R$, October 1998, "Blasts of Light to Strengthen Metals"). Since that time, they've been developing uses for the technology with a number of industries, including automotive, medical, and aerospace. They've also developed an offshoot technique-laser peenmarking ${ }^{S M}$ — which provides a way to easily and clearly identify parts with a mark that is extremely difficult to counterfeit. Another outgrowth is a new peen-forming technology that allows complex contouring of problematic thick metal components such as the thick sections of large aircraft wings. There have also been spinback applications to the Department of Energy's programs for stockpile stewardship, fuel-efficient vehicles, and long-term nuclear waste storage.

\section{Peening with Light}

The concept of laser peening is not new, but it took a DOE Cooperative Research and Development Agreement (CRADA) between Livermore and Metal Improvement Company to develop a machine that makes laser peening a cost-effective option. The resultant Lasershot ${ }^{\mathrm{SM}}$ Peening System uses a solid-state, high-energy (50-joule), neodymium-doped glass laser, which pulses at a rate 20 times faster than other available systems and can peen about 1 square meter of metal per hour. With each pulse of the laser, an intense shock wave is created over a roughly 5-millimeter by 5 -millimeter area and drives in a residual compressive stress about 1 to 2 millimeters deep into metal. In conventional peening, this compressed layer is only about 0.25 millimeter deep. The added depth is key to laser peening's superior ability to keep cracks from propagating and extends the life of parts three to five times over that provided by conventional treatments. 


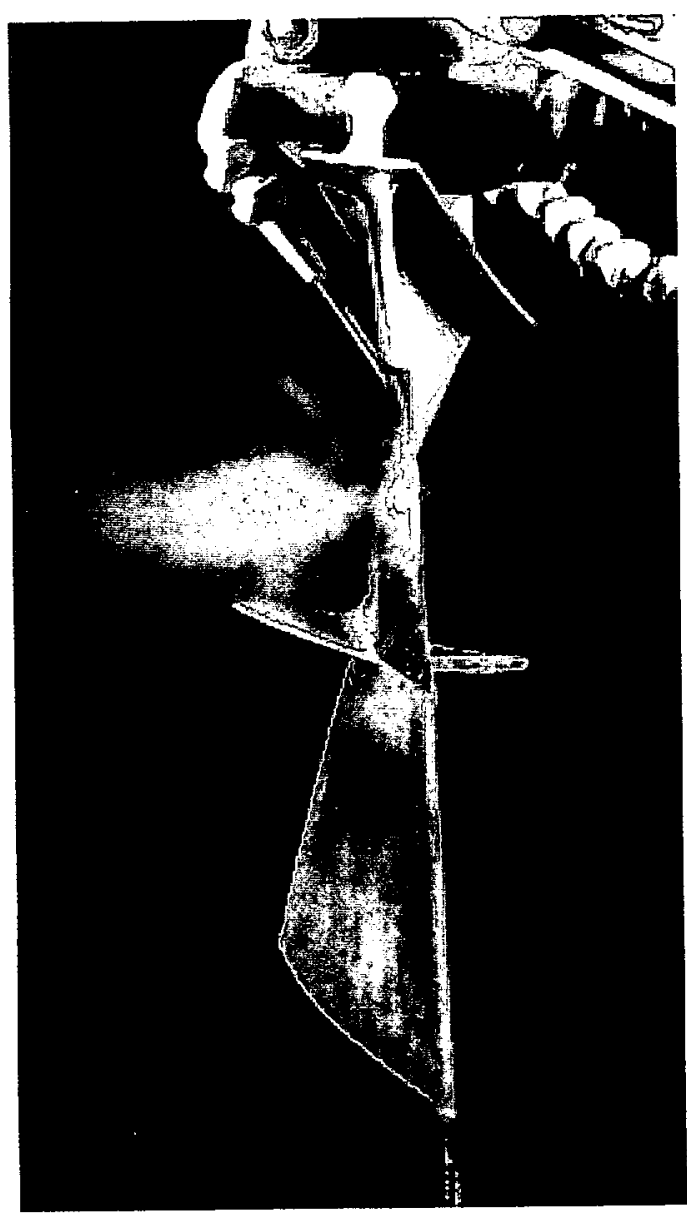

A jet engine fan blade that is being peened by a laser shot.

\section{For Fan Blades and Knee Implants}

Shot peening has long been used on automobile springs and transmissions because the treatment increases resistance to cracks, corrosion, and fatigue. Physicist Lloyd Hackel, who heads the Livermore side of the joint development effort, says that the automotive industry is now interested in applying the depth compression afforded by laser peening to automobile frames.

Traditionally, automakers have added mass to the entire frame structure to achieve the required fatigue lifetime and keep high-stress areas in frames from cracking. Now, laser peening can extend fatigue lifetime and allow manufacturers to cut back on the weight of the frame. By one company's calculations, laser peening would improve the fatigue lifetime of a 200-kilogram frame by a factor of two, allowing them to lessen the frame weight by about 20 kilograms. This 20-kilogram weight savings translates into gas savings as well. Laser peening 8 million automobile frames could save about 285 million liters of gasoline per year. "So this technology has two big benefits: it makes the car lighter and cheaper to build, and it results in more fuel efficiency," says Hackel.

Livermore is also working with the Biomechanics Department of the University of California at Los Angeles to use laser peening for knee implants. "The biggest concern in this area is pediatric knee replacement," says Hackel. "A surgeon puts in a small knee joint, the child grows, so the knee is loaded with more stress, which can lead to joint failure. What do you do? Until now, the answer has been to undertake a painful and risky operation every few years to replace the knee with a larger model." In contrast, a laser-peened metal joint would be strong enough to last nearly a decade. The aerospace industry also sees major applications for laser peening, particularly in jet engines. "If you look at a modern turbo jet engine such as those used in a Boeing 777," says Hackel, "you'll see that it's essentially a giant propeller engine, with the fan blades in the front and the compressor blades inside." These blades get hit by a variety of debris including nuts and bolts, seagulls, sand, and rocks, that can cause cracks and failure. Laser peening adds safety while also lowering the life-cycle cost of each fan blade.

Another use of laser peening for aerospace and other industries involves metal shaping. For instance, the leading edge of an airplane wing is basically a big piece of curved metal. "Aerospace and other industries bend metal all the time, but it's difficult to bend very thick pieces and get certain complex shapes. And when you do bend metal, its surface is under tension-think of the metal as being 'stretched' around that bend. That stretching weakens it and makes it more vulnerable to cracking."

Laser peening just one side of a metal piece will make it naturally bend, which places both peened and unpeened sides under compression and makes the part more resistant to failure. The deep compressive stress and the precise placement of the stress afforded by the laserpeening process allows forming of thick, complex shapes never before possible. 


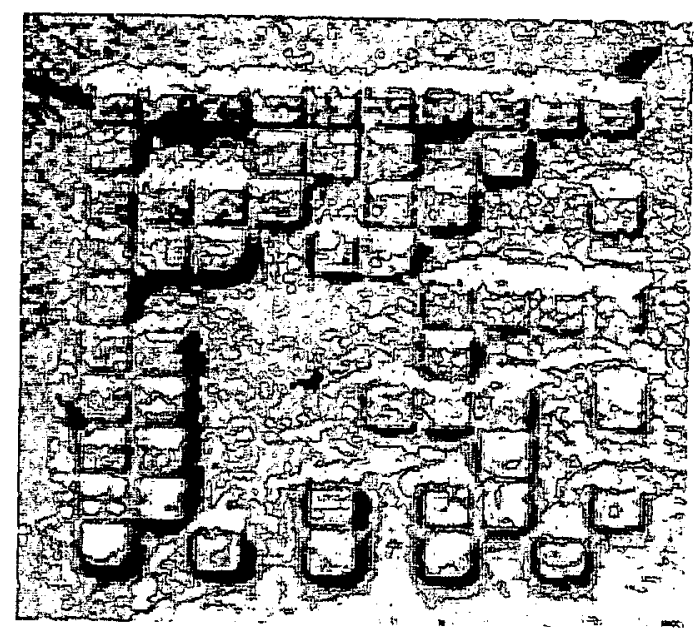

Laser peenmarking prints a complete high-quality, machine-readable matrix mark that could deter the counterfeiting of metal parts. This data matrix represents the number string 123456.

\section{Marking by Laser}

Another recently developed application involving industries using or manufacturing metal parts is laser peenmarking $S M$, in which a high-resolution mark is imprinted into the metal. This identification mark can take any form, for example, as alpha numeric characters, a logo, or a data matrix. This development is particularly timely for aerospace industries facing a new marking requirement from the Aerospace Transportation Association, called the ATA 2000. An ATA mark, in a matrix form that can

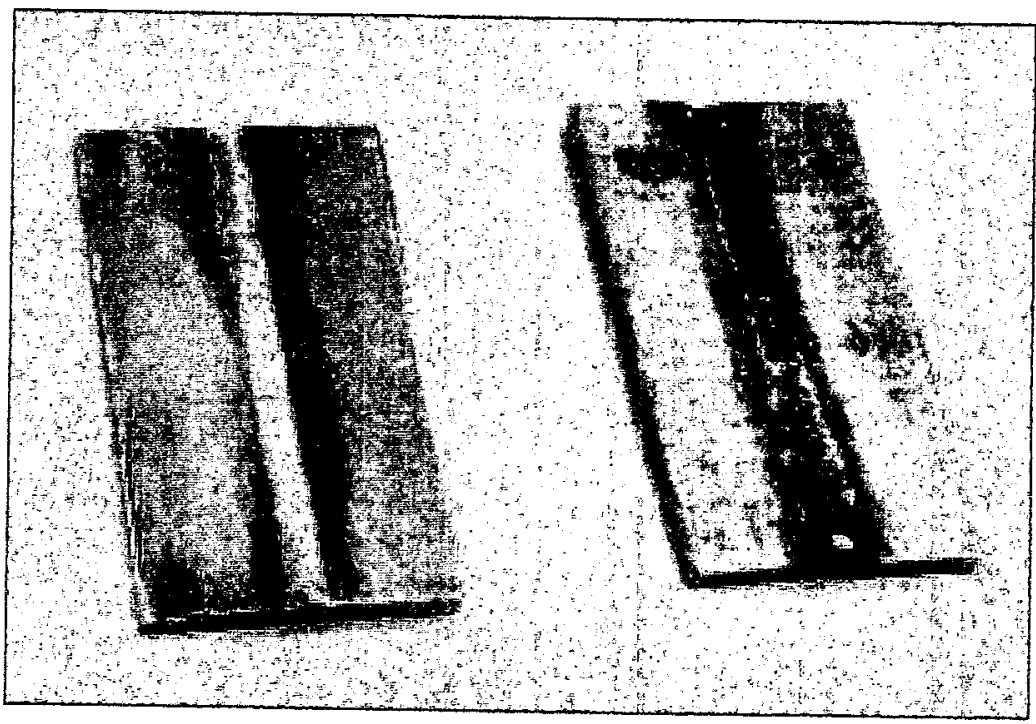

To test the resistance of laser-peened welds to corrosion, the team took two welded pieces of 304 stainless steel and bathed them in a 40-percent solution of magnesium chloride, a highly corrosive salt, at $160 ; \mathrm{C}$. Cracks developed in the unpeened weld within 24 hours, whereas the laser-peened weld showed no observable cracks after a week of exposure. be read by barcode machines, must be set into each part early in the manufacturing process so that the part can be tracked throughout its lifetime.

Normal marking methods-scribing, etching, or stamping - remove material or impart tensile stresses that can leave the part weakened at the marked spot. But laser peenmarking adds a strengthening residual compressive stress. Peenmarks are also of very high resolution, similar to the watermark on currency, and thereby provide a barrier to counterfeiting. Counterfeit, substandard parts are a major concern, notes Hackel. For example, the U.S.

Coast Guard prosecutes approximately 20 cases each year involving the fraudulent use. of counterfeit parts. "Laser peenmarking could be an enormous deterrent to criminals and really put a dent in the counterfeit metal parts racket," says Hackel.

Lawrence Livermore and Metal Improvement Company have been working with other organizations, including the National Aeronautics and Space Administration, to determine the efficiency of laser peenmarking. In June 2001 , three laser peenmarked parts are tentatively scheduled to ride on the NASA shuttle to the international space station. The parts will be bolted onto the space station to face the slipstream solar wind. After three years, they'll be retrieved and examined to see how well they held up in the hostile space environment.

\section{Spinback to DOE}

The laser-peening technology is a spinoff of high-energy lasers developed in the DOE Inertial Confinement Fusion program. Those lasers were brought to high average power with Department of Defense funding. The technology is spinning back home as it becomes clear that peening has relevant applications for DOE's Yucca Mountain Nuclear Waste Disposal and Stockpile Stewardship programs.

For Yucca Mountain, laser peening could be used to prevent stress corrosion cracking in the final closure welds of 6-meter by 1.5 -meter nuclear waste storage canisters. Such canisters must completely contain waste for a minimum of 10,000 years. Analyses show that stress corrosion in some of the canister welds could cause the canisters to fail prematurely. Experiments show that laser peening the welds would keep corrosion and cracking at bay, 


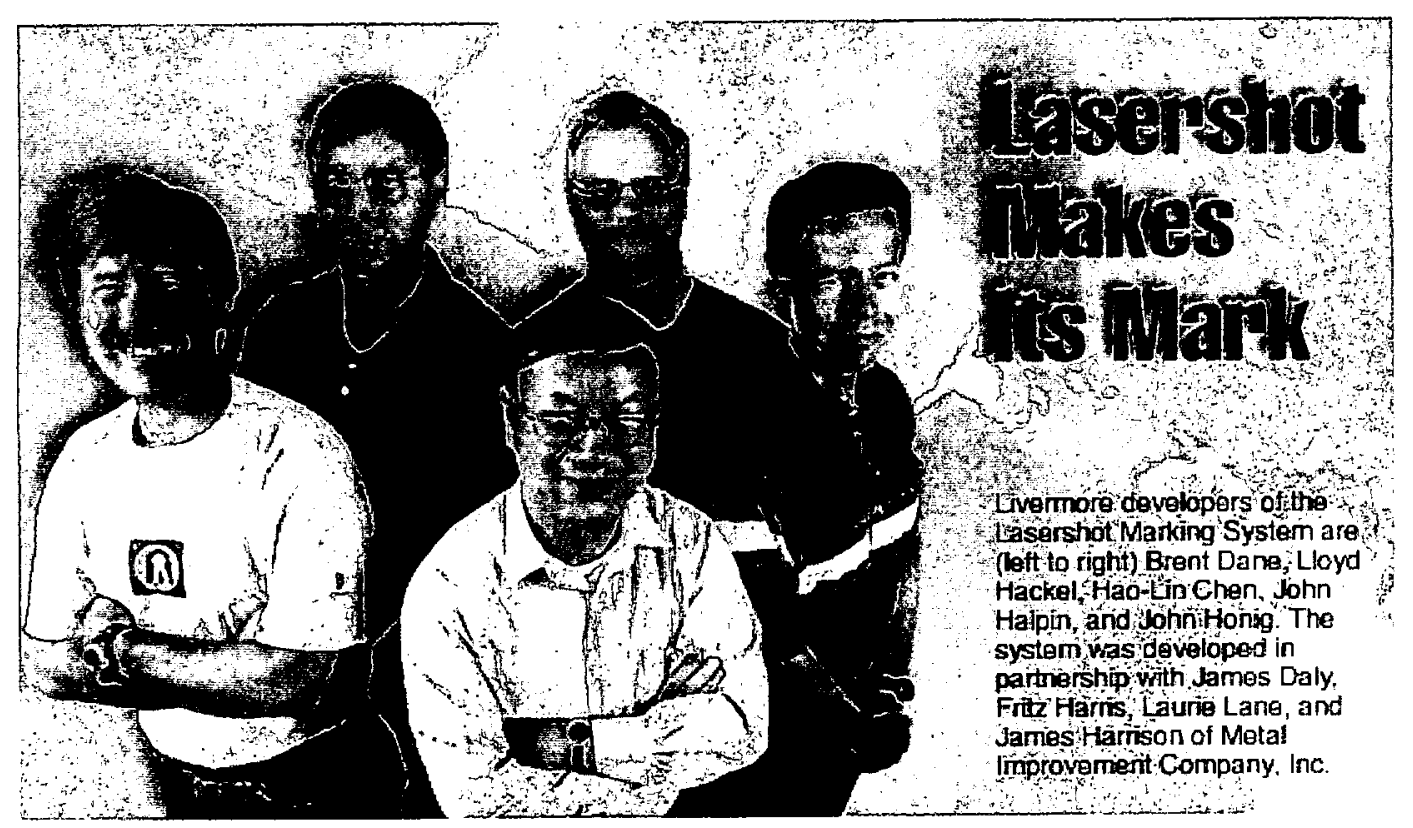

allowing the canister to remain intact for 10,000 years and more.

In the Stockpile Stewardship Program, one research area seeks to determine the effect of intense strain on various materials. The laserpeening team discovered that it could generate meaningful strain rates and effects through shock waves created by the laser-peening process. "We can give stockpile stewardship scientists 10 laser shots a minute, providing them with an enormous amount of data and information," says Hackel. The process, he adds, can give these scientists exquisite control over test parameters, including the intensity, duration, and profile of the desired shock wave.

As for DOE's efforts in promoting fuel efficiency in vehicles, Hackel says, "I see peening as another spinback for the DOE particularly the Office of Transportation Technology in terms of reducing the weight of vehicles. DoD would also benefit, from getting better fuel efficiency in the field and also for airlift capability."

\section{Far-Reaching Technology}

Going from ball-peen hammers to laser light takes a big jump in technology. The applications of laser peening-some known years ago, others newly discovered-are just as farreaching. "What we've come to," says Hackel, "is an active CRADA that's working to field the technology for specific industries and spinning it back with important benefits to Laboratory and DOE work."

\section{Lasershot Makes its Mark}

Anyone getting a hip replacement expects the implant to be certified and last a long time. The same goes for new and replacement parts in aircraft. The assumption is that critical parts-especially those used in applications where safety is paramount-are certified by the manufacturer and the government and are made to exact specifications. Lawrence Livermore and Metal Improvement Company, Inc., have developed a system that helps identify certified and other high-value parts

The Lasershot Marking System imprints permanent, high-resolution identification marks as another spinback for the DOE-particularly the Office of Transportation Technology-in

that are difficult to counterfeit, readable by machine, and strengthen the part at the site of the mark, in contrast to other marking methods, which can actually weaken the part. "Before Lasershot, there was no way to permanently mark parts used in safety-critical applications without inducing the danger of fatigue and stress-crack corrosion," notes Livermore physicist Lloyd Hackel, primary developer of the system.

\section{From Hip Implants to Space Stations}

The Lasershot Marking System has the potential to be of great use not only to makers of medical and aircraft components, but also to aerospace organizations such as the National Aeronautics and Space Administration (NASA). It is a prime candidate for imprinting safety-critical parts with the Air Transport 


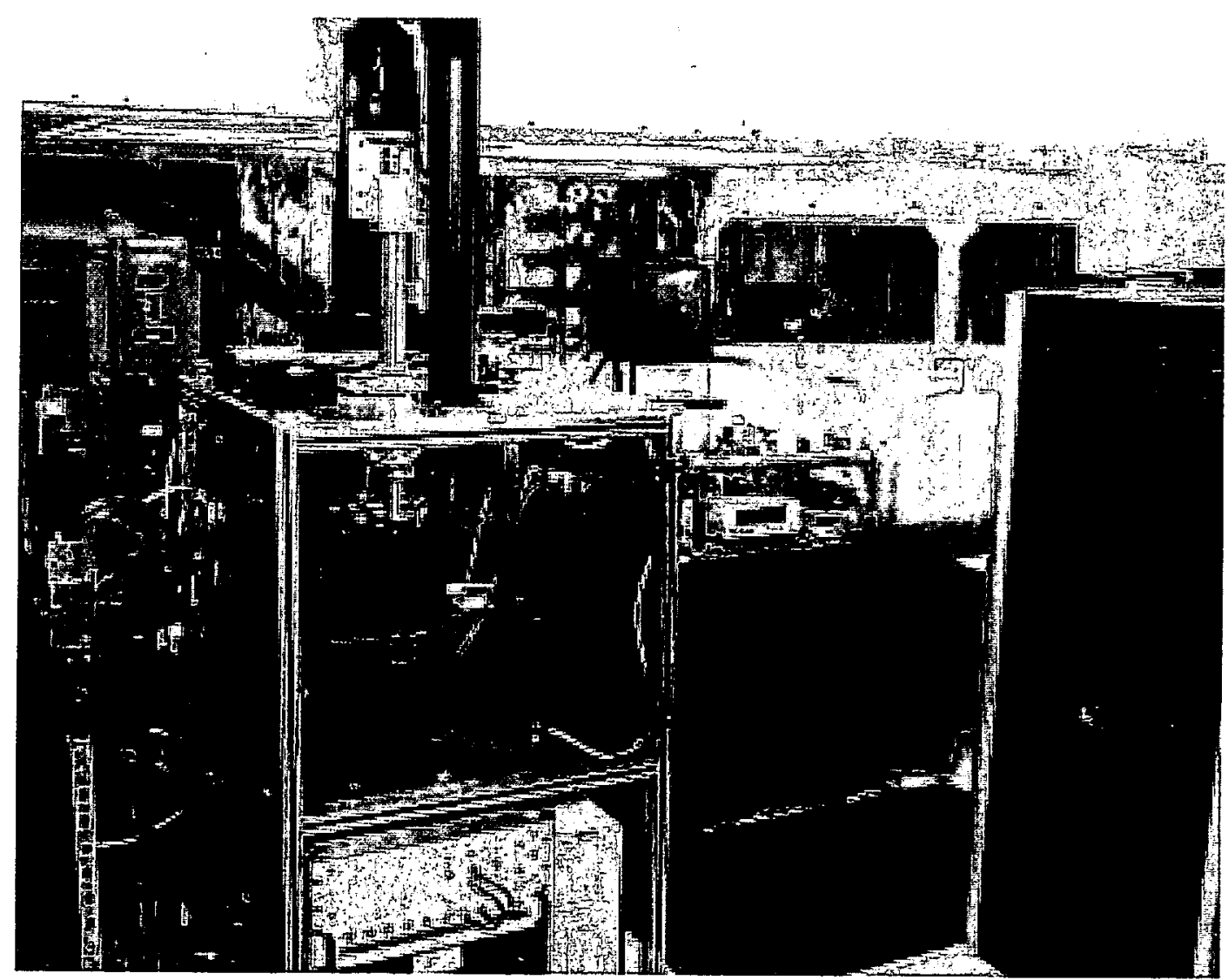

A Lasershot peen-marking station. The Lasershot Marking System (shown in action in the background of the opening image) uses laser pulses to safely and permanently impress identification markings on metal components without weakening them. The system is thus ideal for marking parts used in situations where safety is critical-from hip-joint replacements to commercial airliner components.

Association 2000 Data Matrix, a high-dataintensity, two-dimensional, machine-readable symbol recently adopted by NASA. NASA plans to use this data matrix to identify and track the millions of parts used in the space program. Currently, the matrix is imprinted on the thousands of heat-resistant tiles on the Space Shuttle using a traditional marking technique. However, safety-critical metal parts are not marked at all because of the risk of marking-induced failure. The invention of the Lasershot Marking System means that NASA may soon be able to mark and track these important parts as well.

NASA has added Lasershot marking to its Data Matrix Direct Part Marking Standard and Handbook and included three samples imprinted with the mark in its Materials International Space Station Experiment, which was launched on the STS-105 in August. The sample parts were bolted onto the space station to face the slipstream solar wind. After one year, they will be retrieved and examined to evaluate how well they held up in the hostile space environment. NASA and the Department of Defense are also conducting ground, flight, and in-orbit tests of laser-peened marks to certify Lasershot's use in current and future programs.

Other organizations-the Air Transport Association, the Electronic Industry Association, the Automotive Industry Action Group, and the Semiconductor Equipment Manufacturers' Institute-have chosen the Data Matrix standard as the preferred one for parts marking, thereby extending the potential applications for the Lasershot system. Components that could be marked with this method include fan blades, disks, rotors, and integrated rotor assemblies as well as components in automobiles.

A mark created by the Lasershot system allows manufacturers and users to positively identify each individual part and trace each part from manufacture to retirement. In addition, Lasershot peen marking will be a valuable tool in combating counterfeit parts by providing a 
unique, permanent, and difficult-to-reproduce tracking symbol, one that also strengthens the part at the site. The mark contains fine detail nearly impossible to counterfeit, much like the watermark on modern currency. Part counterfeiting is a growing concern. According to government estimates, as much as $\$ 2$ billion in unapproved parts distributors, airlines, and repair stations.

\section{A Chip off the Laser Peening Block}

Peening-a technique common in metalworking-uses a ball-peen hammer or pneumatically shot small metal balls to pound a piece of metal into shape and strengthen it against fatigue failure. Replace the hammer or metal balls with a laser and the blow of metal on metal with the pressure wave of a laser light pulse on metal, and laser peening results. (See $S \& T R$, March 2001, Shocked and Stressed, Metals Get Stronger.

In the Lasershot peen-marking process, a layer of absorptive material is placed over the area to be peened, and a thin layer of water is flowed over the absorption layer. A high-intensity laser with an energy density (fluence) of about 100 joules per square centimeter illuminates and ablates material from the absorption layer, creating an intense pressure pulse that is initially confined by the water. The absorption layer protects the part surface from material removal or melting. The pressure pulse creates a shock wave that strains the surface in a twodimensional pattern that mirrors the laser's intensity profile. By creating the desired pattern upstream in the light and then imaging this pattern onto the metal, a complete mark can be made with a single laser pulse.

"The laser system projects the pattern on the part in much the same way that a slide projector creates an image on a screen," explains Hackel. "A slide projector without a slide in place projects a light field of uniform intensity on the screen. No image or pattern appears. When a slide is inserted between the projector bulb and lens, the light and dark areas of the slide provide an intensity profile pattern that is imaged onto the screen. With the Lasershot system, we use a laser and a special telescopic system to image the pattern of a mark onto the metal part. The laser fires, and that entire mark is printed on the part in a single pulse."

This single-pulse technique is well suited for high-volume marking applications. For low-volume use, a smaller system-the multi- ple-shot matrix marking system-builds up a two-dimensional mark using multiple laser pulses.

\section{Technology Breakthroughs Make It Possible}

The Lasershot technique was made possible by a patented breakthrough in laser technology developed at Livermore involving a neodymium-doped glass laser and a wavefront correction technology, called phase conjugation. "We can now build laser systems that operate up to six pulses per second, with output energy of greater than 25 joules," says Hackel. "This means we can peen-mark six data matrices per second, using the single-shot pattern marking technique." The phase conjugation provides a high-quality beam that has high, long-term pointing stability for the high repetition rates needed for the smaller multiple-shot marking system. As a result, the Lasershot system can mark parts at a rate comparable to or exceeding that of conventional marking methods.

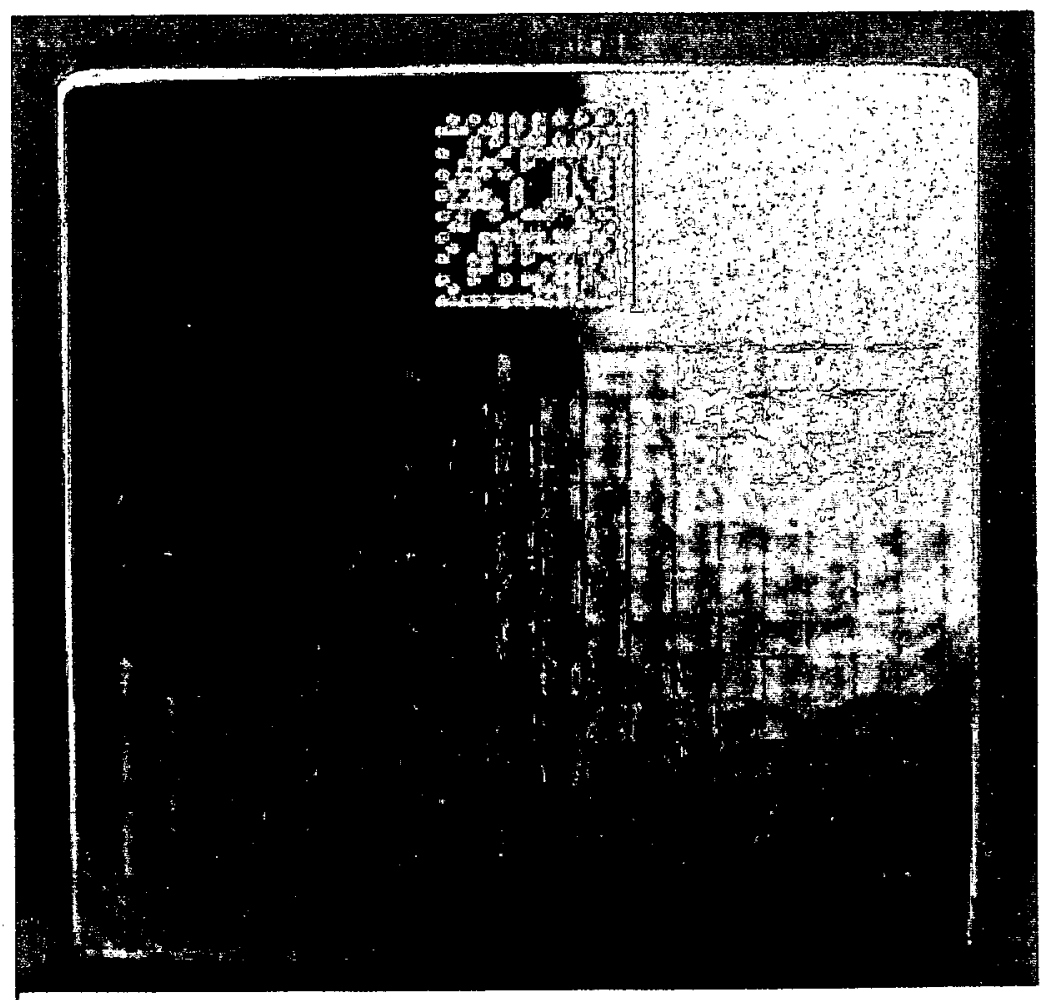

A 10- by 10-character identification mark-approximately 0.3 centimeter on a sie-imprinted into an aviation-grade aluminum alloy using the Lasershot Marking System. Unlike other marking methods, Lasershot increases the marked area's resistance to fatigue and corrosion failure, and the resulting high-resolution mark is difficult to counterfeit. 
The other key to the system is a specially designed and patented telescopic delivery system, which precisely relays the image onto the part surface. "This beam delivery is critical for accurately replicating a two-dimensional marking pattern," notes Hackel. "The resulting mark has uniquely embossed fine detail, making it nearly counterfeit-proof."

\section{Mark Is Stronger, More Durable}

Although other techniques are available for imprinting identification marks on metal parts, none measures up to the Lasershot method. The primary techniques are laser etching, pin stamping, and ink-jet printing. Laser etching systems work by focusing energy directly onto the surface to be marked and etching the parts surface, even vaporizing the surface in some cases. Although this technique has good permanence and generates a clear mark, it modifies the material. In steel, for instance, the high temperature causes carbon to precipitate out in the area hit by the laser beam, ultimately degrading the part's strength. The modification and strength degradation can lead to fatigue or stress-corrosion crack failures. In a scanning electron micrograph study of 10 hip replacement implants that had failed much earlier than they should have, 5 of the 10 showed fatigue fractures that began in the characters that had been laser-etched on the implant surfaces. "These failures could have been prevented by the Lasershot method," notes Hackel

In pin stamping, a conical stylus impacts the surface, with the size of the mark controlled by how deeply the stylus indents the metal. Like peening with conventional ball-peen hammer, pin stamping may leave some residual compressive stress in the part, which would provide some protection against fatigue and mechanical stress. However, this method also roughens the surface and concentrates the stress at the bottom of the sharp indentations. In addition, pin stamping can distort small or thin parts.

Although marking using the ink-jet technique does not affect the surface material, the markings are not necessarily permanent. The permanence of the mark depends on the chemical interaction of ink and part as well as on the environment in which the part is used.

Sandblasting, machining or engraving, chemical etching, and welding also degrade strength and shorten the fatigue lifetime of metals. "Other techniques can cast symbols on parts during manufacturing," notes Hackel.

However, they only work for larger parts and don't address the need to mark parts already manufactured or those produced by noncasting methods such as forging and machining.

Unlike laser etching, Lasershot removes no material, and the marked surface remains chemically unaltered. Unlike pin-stamped marks, Lasershot does not roughen the surface. Plus the compressive layer from laser peening extends as deep as 1 millimeter into the metal, adding strength to this local area.

\section{Mark the Future for Lasershot}

The Lasershot Marking System allows manufacturers for the first time to safely and permanently mark and label metal parts used in situations where failure means big trouble. Donald L. Roxby, director of the Symbol Research Center, an international leader in the development of advanced symbology solutions for industrial, materials handling, and manufacturing environments, notes in a recent letter to Hackel, \&\#147;Our organization was elated when we became aware of your work related to lasershot peening. The lasershot peening process provides the marking fidelity required to apply dense symbols to small parts without injecting risk. The process makes it possible to identify internal engine components such as aircraft turbine blades and a host of other difficult marking applications.

The R\&amp;D 100 Award judges voted their agreement. Lasershot is poised to make its mark in the world of safety-critical parts manufacturing. 


\title{
Improving 351-nm damage performance of large-aperture fused silica and DKDP optics
}

\author{
A. K. Burnham \\ L. Hackel \\ P. Wegner \\ T. Parham \\ L. Hrubesh \\ B. Penetrante \\ P. Whitman \\ S. Demos \\ M. Runkel \\ M. Fluss \\ J. Menapace \\ M. Key \\ T. Biesiada
}

\begin{abstract}
A program to identify and eliminate the causes of UV laser-induced damage and growth in fused silica and DKDP has developed methods to extend optics lifetimes for large-aperture, high-peak-power, UV lasers such as the National Ignition Facility (NIF). Issues included polish-related surface damage initiation and growth on fused silica and DKDP, bulk inclusions in fused silica, pinpoint bulk damage in DKDP, and UV-induced surface degradation in fused silica and DKDP in a vacuum. Approaches included an understanding of the mechanism of the damage, incremental improvements to existing fabrication technology, and feasibility studies of non-traditional fabrication technologies. Status and success of these various approaches are reviewed. Improvements were made in reducing surface damage initiation and eliminating growth for fused silica by improved polishing and post-processing steps, and improved analytical techniques are providing insights into mechanisms of DKDP damage. The NIF final optics hardware has been designed to enable easy retrieval, surface-damage mitigation, and recycling of optics.
\end{abstract}

Keywords: fused silica, DKDP, laser damage, laser damage mitigation, laser damage growth

\section{INTRODUCTION}

The National Ignition Facility (NIF), currently under construction, will contain 7360 large optics of 40-cm size or larger. ${ }^{1}$ The laser consists of 192 beams with a nominal energy of $3 \mathrm{MJ}$ at $1053 \mathrm{~nm}(1 \omega)$ and $1.8 \mathrm{MJ}$ at $351 \mathrm{~nm}(3 \omega)$. The conversion of laser light to $351 \mathrm{~nm}$ is needed for improved coupling to the laser targets in order for the NIF to achieve its primary mission within the Stockpile Stewardship program. A schematic diagram of the final optics assembly, in which frequency conversion, beam smoothing, and focusing occur, is given in Figure 1. Achieving the desired lifetime of the $3 \omega$ optics has been challenging, and their lifetime can have a significant impact on the operating costs of the facility. Consequently, there has been a considerable effort over the past few years to improve the UV optics performance.

UV optics are made of deuterated potassium dihydrogen phosphate (DKDP), which generates the 351-nm light, and fused silica, from which the focus lens, beam sampling grating, and debris shield are made. Laser-induced damage can occur from defects in both the bulk and the surface of the material. A summary of the different types of damage is given in Table 1. Note that there are similarities and differences between the damage characteristics of DKDP and fused silica. For example, both fused silica and DKDP are susceptible to surface damage that grows exponentially with the number of shots at constant fluence. ${ }^{2}$ In contrast, bulk damage in crystals occurs as numerous pinpoints that do not grow significantly, ${ }^{3}$ while bulk damage in fused silica is rare but causes growable rear-surface damage from beam modulation when it does occur. ${ }^{4}$ Both fused silica and DKDP are susceptible to surface degradation in a vacuum, but the details are different. ${ }^{5,6}$

This paper describes the efforts over the past two years to solve these laser-induced damage problems for the NIF laser. These efforts have been extremely successful. The solutions fall into three categories: (1) reduce or eliminate bulk damage by improved material synthesis, (2) eliminate surface degradation by increasing the operating pressure to 10 Torr, and (3) control surface crater damage through a combination of better finishing processes and a pre-initiate/mitigate sequence. The surface damage mitigation methods can also be applied to optics retrieved from service before the surface damage craters 
become too large. The overall strategy is portrayed graphically in Figure 2. The technology developments summarized here should be widely applicable to large-aperture high-peak-power lasers.

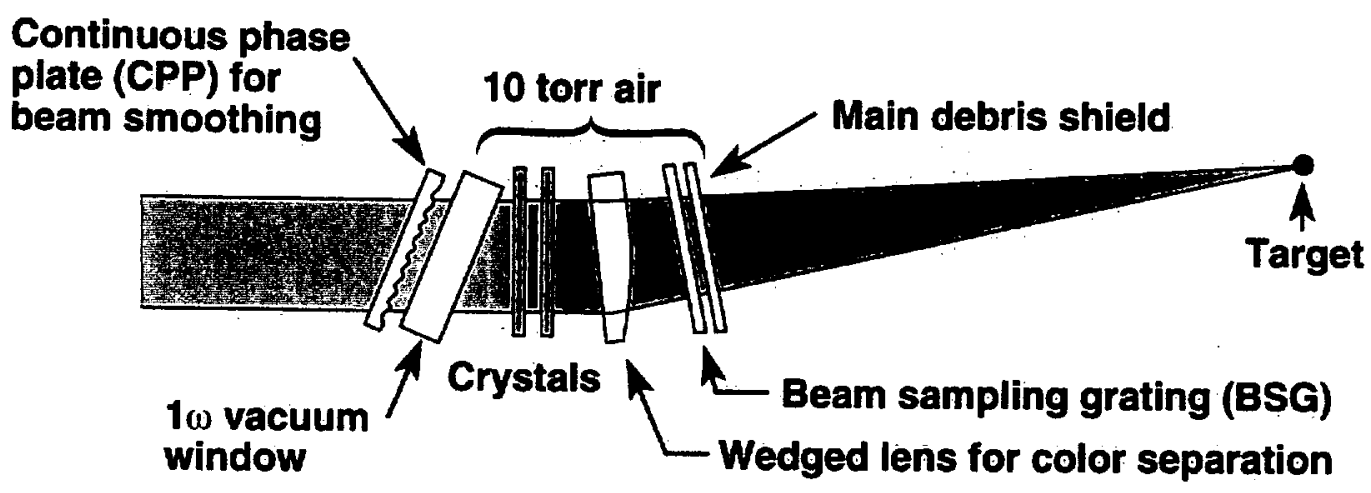

Figure 1. Schematic diagram of the final optics assembly used in the National Ignition Facility to convert infrared laser light (1053 nm) to UV laser light $(351 \mathrm{~nm})$.

Table 1. Summary of types of damage for fused silica and crystal optics typically used in large-aperture, high-peak-power lasers such as the National Ignition Facility.

\begin{tabular}{|c|c|c|}
\hline & DKDP & $\begin{array}{r}\text { Fused Silica } \\
\end{array}$ \\
\hline $\begin{array}{l}\text { Bulk } \\
\text { Damage }\end{array}$ & $\begin{array}{l}\text { Up to a few thousand } \\
\text { pinpoints } / \mathrm{mm}^{3} \\
\text { - nonisotropic }\end{array}$ & $\begin{array}{l}\text { Tens of bulk damage sites per optic for } \\
\text { refractory-furnace based material }\end{array}$ \\
\hline $\begin{array}{l}\text { Surface } \\
\text { Damage }\end{array}$ & $\begin{array}{l}\text { Up to a few near-surface bulk } \\
\text { damage pinpoints } / \mathrm{cm}^{2} \\
\quad<50 \text { surface damage sites of size } \\
50-100 \mu \mathrm{m} \text {; possibly related to } \\
\text { imbedded particles from } \\
\text { fabrication* } \\
\text { - Induced roughness and absorption } \\
\text { upon UV vacuum exposure }\end{array}$ & $\begin{array}{l}\text { Many sub- } \mu \mathrm{m} \text { pinpoints from residual } \\
\text { polishing material (gray haze) } \\
\text { - } 20-100 \mu \mathrm{m} \text { damage initiation craters, with } \\
\text { the number depending on fluence* } \\
\text { - Tens of rear-surface damage sites caused } \\
\text { by bulk inclusions and lenslets* } \\
\text { - Induced absorption upon UV vacuum } \\
\text { exposure }\end{array}$ \\
\hline
\end{tabular}

*Susceptible to growth at NIF fluences

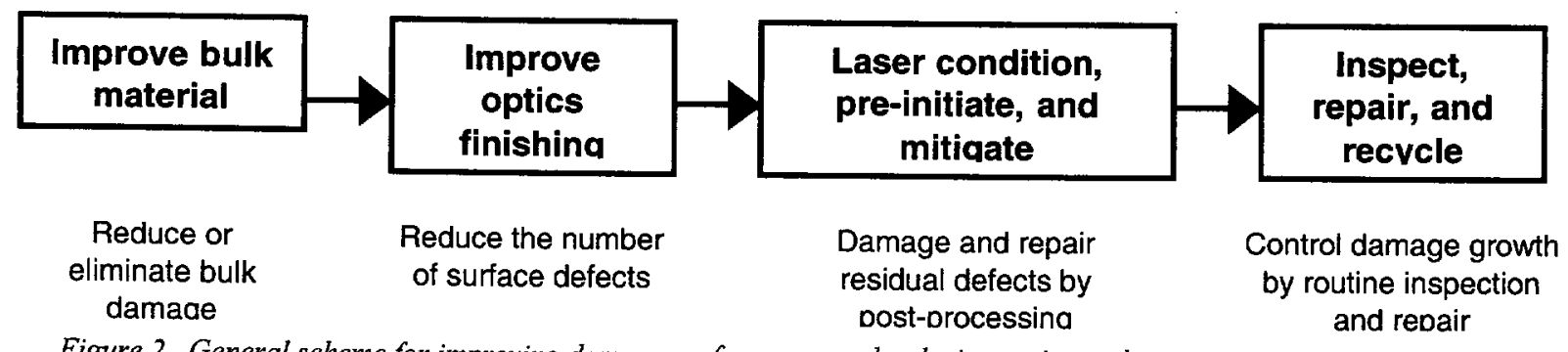

Figure 2. General scheme for improving damage performance and reducing optics replacement costs. 


\section{BASIC DAMAGE STATISTICS}

Laser damage probability or density depends on fluence $F$ according to some function $d(F)$. A large aperture beam consists of a distribution of fluences (i.e., contrast) across the beam, and the damage probability or density $D(<F>$ ) at any given area is given by convolving the damage function $\mathrm{d}(\mathrm{F})$ with the fluence probability function $\mathrm{p}(\mathrm{F}) \mathrm{:}^{7}$

$$
\mathrm{D}(<\mathrm{F}>)=\int_{0}^{\infty} \mathrm{p}(<\mathrm{F}>\mathrm{F}) \mathrm{d}(\mathrm{F}) \mathrm{dF}
$$

where $\langle\mathrm{F}>$ is the most probable fluence. The operational beam is assumed a nominal flattop with intensity fluctuations described by a Rician distribution. ${ }^{8}$ The resulting hot spots are assumed to move randomly shot to shot. The single shot Rician probability distribution $p(f)$ is given by

$$
\mathrm{p}(\mathrm{F},<\mathrm{F}>, \mathrm{c})=\left(1 / 2 \sigma^{2}\right) \exp \left[-\left(\mathrm{F}+<\mathrm{F}>-2 \sigma^{2}\right) / 2 \sigma^{2}\right]^{*} \mathrm{I}_{0}\left\{\left(\mathrm{~F} / \sigma^{2}\right)\left[(\mathrm{F} /<\mathrm{F}>)^{*}\left(1-2 \sigma^{2} /<\mathrm{F}>\right]^{0.5}\right\}\right.
$$

where $\angle \mathrm{F}>$ is the mean fluence, $\sigma$ the standard deviation of the noisy field amplitude, and $\mathrm{I}_{0}$ is a Bessel function of the second kind. The intensity contrast $c$ is twice $\sigma$. Although the functional form looks complicated, the distribution profiles of interest are very nearly Gaussian for beam contrast up to $20 \%$. The contrast in the beam can move spatially from shot to shot, meaning that any given location can see a different fluence for each shot at constant average beam fluence. For a randomly migrating fluence, the distribution of maximum fluences at each location after $\mathbf{n}$ shots is given by

$$
\mathrm{p}_{\mathrm{m}}(\mathrm{F})=\mathrm{np}(\mathrm{F}) *\left[\int_{0}^{\mathrm{F}} \mathrm{p}(\mathrm{F}) \mathrm{dF}\right]^{\mathrm{n}-1}
$$

Plots of the maximum fluence distributions for a Rician distribution after 1 to 1000 shots is given in Figure 3. Note that after many shots for this assumption, most locations have been exposed to a fluence at the high end of the single shot distribution. The cumulative damage probability after $n$ shots is found by replacing $p(F)$ by $p_{m}(F)$ in $E q(1)$.

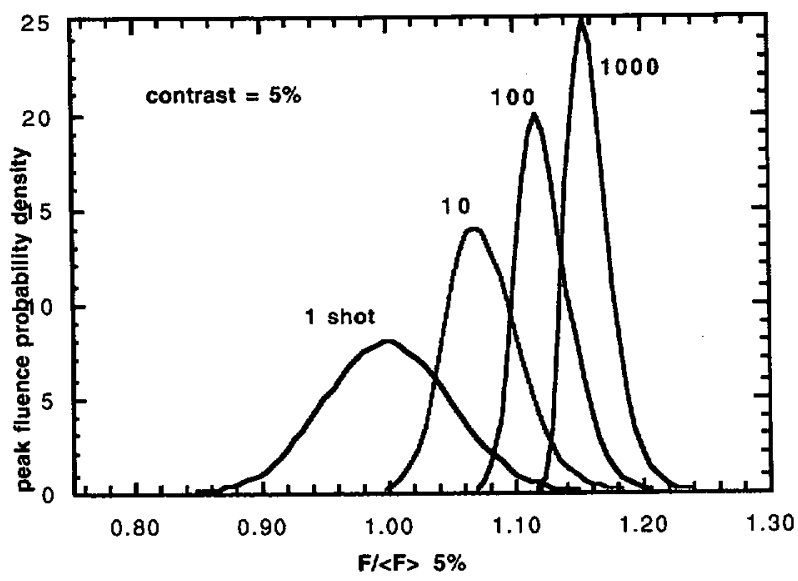

Figure 3. Probability distribution of maximum fluence after $1,10,100$ and 1000 shots for $5 \%$ randomly migrating contrast.

\section{IMPROVING BULK MATERIAL}

\section{Fused silica}

Fused quartz and fused silica are available in a wide range of quality, depending on their method of manufacture. Fused quartz is not suitable for high-performance optics due to the large number of inclusions that lead to bulk damage. Fused silica, formed by oxidizing chemical precursors and collecting the soot on a boule, is much better but still comes in a range of quality depending on its intended use. Corning 7980, and its predecessor Corning 7940, is formed in a refractory furnace in which occasional high-index refractory dust particles are incorporated into the growing boule. ${ }^{4}$ As shown schematically in Figure 4, these particles partially dissolve, forming a high-refractive index lenslet, which focuses the light and causes bulk damage immediately downstream at $351-\mathrm{nm}$ fluences of $2-4 \mathrm{~J} / \mathrm{cm}^{2}(3 \mathrm{~ns})$. Beam modulation continues to propagate to the rear surface, where a damage site appears. Material is preselected to eliminate visible inclusions, and the remaining inclusions are not visible until damage tested. 
Figure 4. Schematic representation of the formation of bulk and rear surface damage from high index inclusions in some fused silicas. From Kozlowski et $a l^{4}$

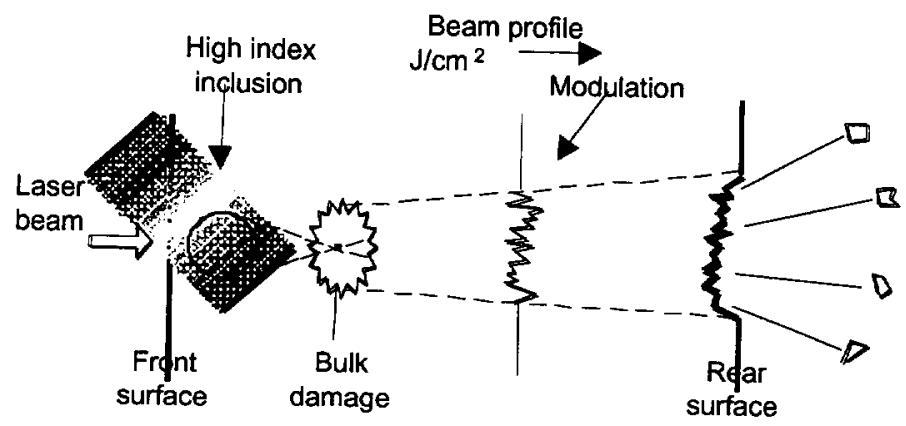

The number of inclusion-induced damage sites in Corning 7980 fused silica is typically a few dozen per 40 -cm square aperture. With the remarkable recent advances in reducing polish-related damage sites and mitigating the growth of surface damage, these bulk inclusions would become the limiting factor for operating above $4 J / \mathrm{cm}^{2}(3 \mathrm{~ns})$ at $351 \mathrm{~nm}$. This is because no method has yet been developed to stop the reinitiation and growth of rear surface damage that they cause. Consequently, fused silicas manufactured without a surrounding refractory as in Figure 5 were pursued. 40-cm scale optics fabricated from Schott Lithosil Q and Heraeus Suprasil 312 were found to be free of inclusion-induced damage for exposure
up to $14 \mathrm{~J} / \mathrm{cm}^{2}$ ( $3 \mathrm{~ns}$ ) of $355-\mathrm{nm}$ laser light.

Figure 5. Formation of fused silica preforms by a method that eliminates bulk inclusions that can lead to laser-induced damage.

\section{Crystals}

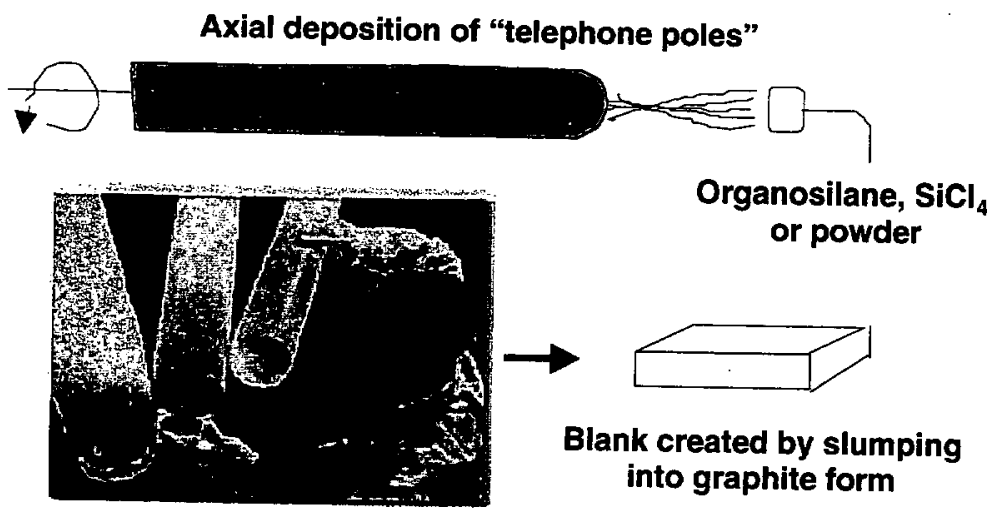

KDP and DKDP can be grown either conventionally or rapidly. Conventional growth rates are about $1 \mathrm{~mm} /$ day, and it takes two years to grow a boule large enough for 40 -cm-scale optics. Pyramidal growth occurs from a plane seed, while prismatic growth is poisoned by trivalent impurities. Rapid growth occurs from a point seed in both the prismatic and pyramidal directions at a rate of about $10 \mathrm{~mm} /$ day and takes less than 2 months to grow a sufficiently large boule.

Unlike fused silica, bulk damage in KDP and DKDP is pervasive, forming thousands of damage pinpoints $/ \mathrm{mm}^{3}$ for fluences significantly above its bulk damage threshold. The pinpoint size depends on pulse length, ranging from a few $\mu \mathrm{m}$ at $1 \mathrm{~ns}$ to
about $100 \mu \mathrm{m}$ at $10 \mathrm{~ns}{ }^{10}$ The pinpoints do not grow significantly, and their primary detriment is scattered light (scattered
light fraction $=\mathrm{f}_{\mathrm{s}}$. Scattered light light fraction $=\mathrm{f}_{\mathrm{s}}$ ). Scattered light causes increased beam contrast, $\mathrm{c}$, according to the relation $\mathrm{c}=\left(2 \mathrm{f}_{\mathrm{s}}\right)^{1 / 2}, 11$ increased stray
light on the neighboring non-optical components, and a decrease in energy on target. Collateral damage from the first two far
outweighs the third.

Attaining 351-nm damage-resistant DKDP has been the greater challenge and required three achievements: a quantitative relationship between routine bulk damage detection methods and scattered light levels, a method for reliably growing crystals with the required damage threshold, and a reliable method for conditioning the crystal prior to installation on the laser.

Four papers published last year provide the basis for first achievement. Damage measurements must be made on test coupons cut at the type-II tripler angle $\left(59^{\circ}\right),{ }^{12} 0.1 \%$ scattered light levels correspond to $\sim 10 \%$ damage probability ${ }^{10}$ conscuration increases exponentially with fluence and scales with pulse length to the 0.25 power, ${ }^{10}$ and raster-scan laser 6). ${ }^{13}$ Using these results, a $10 \% \mathrm{R} / 1$ damage probability fluence of $12.5 \mathrm{~J} / \mathrm{cm}^{2}(7.6 \mathrm{~ns})$ for $\left.355-\mathrm{nm} / 1\right)$ damage test (Figure 
limit for materials to be operated at $8 \mathrm{~J} / \mathrm{cm}^{2}(3 \mathrm{~ns})$ with a $15 \%$ beam contrast. Further, the relationship between fluence and scattering from bulk scattering, $\mathrm{S}$, for an optimally conditioned crystal is estimated as

$$
\mathrm{S}(\mathrm{F})=1.3 \times 10^{-5} \exp \left[11^{*} \mathrm{~F} / \mathrm{F}_{0}\right] \mathrm{cm}^{-1},
$$

where $\mathrm{F}$ is the operating fluence at $3 \mathrm{~ns}$ and $\mathrm{F}_{0}$ is the $10 \%$ damage probability fluence at $7.6 \mathrm{~ns}$. Inserting Eq. (4) into Eq (3) gives the relationship between scattering after $\mathrm{n}$ shots and average fluence given in Table 2, assuming that only the second half of the crystal experiences high $3 \omega$ fluences.

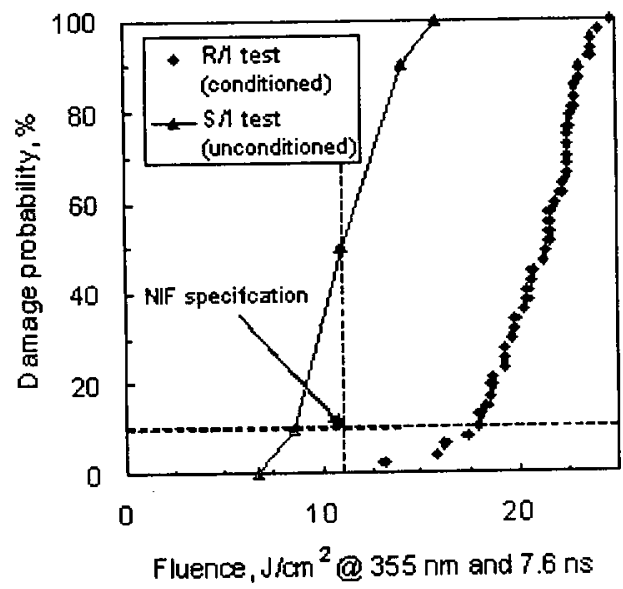

Figure 6. Standard small-beam bulk damage test results for conventional growth material $70 \%$ LLII, the first NIF production boule. The conditioned material ( $R / 1$ test) passes the NIF specification by a considerable margin. The $S / 1$ test exposes each site to only a single fluence (no ramp).

Table 2. Calculated percentage of scattered light from bulk damage for a DKDP sample having a $10 \% \mathrm{R} / 1$ damage probability of $12.5 \mathrm{~J} / \mathrm{cm}^{2}$ for a 3-ns $355-\mathrm{nm}$ laser beam with $15 \%$ randomly migrating contrast. The material can tolerate a single shot at $10 \mathrm{~J} / \mathrm{cm}^{2}$ or at least 100 shots at $8 \mathrm{~J} / \mathrm{cm}^{2}$, since the random migration assumption is conservative. The shaded boxes represent the obscuration limit.

\begin{tabular}{|r|r|r|r|r|r|}
\cline { 2 - 6 } \multicolumn{1}{c|}{} & \multicolumn{5}{c|}{ Average fluence, $\mathrm{J} / \mathrm{cm}^{2}$} \\
\hline \# shots & 7 & 8 & 9 & 10 & 11 \\
\hline 1 & 0.004 & 0.01 & 0.03 & 0.07 & 0.2 \\
\hline 10 & 0.01 & 0.03 & 0.10 & 0.3 & 1.0 \\
\hline 100 & 0.03 & 0.08 & 0.3 & 1.0 & 3.3 \\
\hline 1000 & 0.05 & 0.18 & 0.7 & 2.5 & 9.1 \\
\hline
\end{tabular}

The bulk damage precursors are thought to be absorbing nanoparticles. ${ }^{14}$ Damage threshold improves with continuous filtration ${ }^{15}$ and is lowered by addition of highly absorbing particulates such as iron phosphate (M. Yan, unpublished results, 1999). However, attempts to isolate and identify the specific precursors have been inconclusive, in part because of the low levels of material involved. One thousand $50-\mathrm{nm}$ particles $/ \mathrm{mm}^{3}$ represent only $\sim 0.1 \mathrm{ppb}$ by weight of the crystal. Analysis methods attempted to date include ion milling into a damage site and analyzing it by time-of-flight SIMS, dissolving crystals and analyzing the collected particulate, and transmission electron microscopy (TEM).

In parallel, improvements to the growth procedures have largely solved the problem for both rapid and conventional growth. Satellite boules in NIF conventional growth production tanks yielded $10 \% \mathrm{R} / 1$ damage values ranging from 12 to $17 \mathrm{~J} / \mathrm{cm}^{2}$, with an average of $14.5 \mathrm{~J} / \mathrm{cm}^{2}$. Three test samples from the first recovered production boule (Fig. 6) averaged $18 \mathrm{~J} / \mathrm{cm}^{2}$, suggesting that the satellite boules may underestimate quality. For rapid growth material, we reported last year that material rapidly grown below $45^{\circ} \mathrm{C}$ had a significantly higher damage resistance than material grown above $45^{\circ} \mathrm{C} .{ }^{16}$ However, work over the past year indicates that it is necessary to grow above $45^{\circ} \mathrm{C}$ to meet homogeneity requirements and to prevent crazing due to hydrogen exchange on the surface. Damage results for $20-\mathrm{L}$ growth tanks are summarized in Figure 7 . Similarly, samples across two large DKDP boules grown in 1200 - L polycarbonate tanks have averaged 17.2 and $19.5 \mathrm{~J} / \mathrm{cm}^{2}$, respectively, for their $10 \% \mathrm{R} / 1$ damage probability. The better material was grown exclusively above $45^{\circ} \mathrm{C}$ and slightly exceeds the best sample from a small tank boule at that temperature, indicating that scaleup is not a problem. 


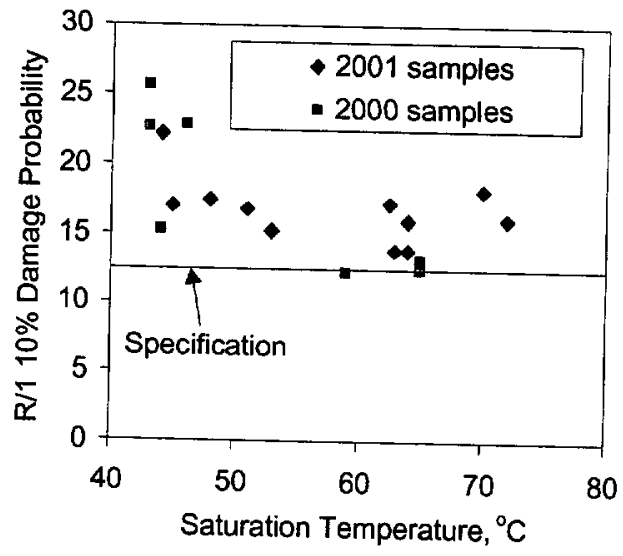

Figure 7. Summary of damage results for 20-L tank $D K D P$ boules showing a general improvement over the past year for those grown above $45^{\circ} \mathrm{C}$. About a $20^{\circ} \mathrm{C}$ temperature drop from the saturation temperature is required to grow a boule in this system The improvement is thought to be due to $a$ combination of improved salt purity and replacement of Pyrex tanks with polycarbonate tanks. Data include unpublished results of R. Floyd and T. Land (2001).

The final element in achieving high-fluence operation is offline conditioning, which allows an optic to be placed immediately into high-fluence operation. Last year we reported success in achieving R/1 damage performance for $S / 1$ tests of DKDP that had been raster scanned by both $355-\mathrm{nm} \mathrm{Nd-YAG}$ and $308-\mathrm{nm} \mathrm{XeCl}$ excimer lasers. ${ }^{13}$ The excimer laser conditioning parameters have been refined over the past year. Two significant results are the shift to a XeF laser at $351 \mathrm{~nm}$ to reduce induced absorption and the exceptional improvement of $1 \omega$-damage resistance of KDP by conditioning at $351 \mathrm{~nm}$. ${ }^{17}$

\section{REDUCING SURFACE DAMAGE INITIATION}

\section{Fused Silica}

Fused silica is an inherently good material with respect to surface damage, but flaws induced during finishing have typically lowered the damage resistance to a small fraction of the dielectric breakdown limit. In order to improve fused silica 351-nm lifetimes significantly, we explored in parallel the basic mechanisms of laser-induced surface damage, pursued improvements in conventional finishing, and explored advanced finishing concepts. A summary of that effort is given in Table 3 . While the scientific efforts helped refine our understanding of the damage process, a combination of magneto-rheological finishing followed by acid etching and $3 \omega$ laser conditioning was most successful at solving the problem.

Table 3. Outline of effort over the past two years to improve the surface damage initiation. Scientific and engineering
approaches were pursued in parallel.

\# Fundamental studies to understand the mechanism of initiation

- Argonne LIMS of engineered defects

- TEM characterization of pilot-production optics

- Spectroscopic evaluation of damage craters

\# Engineering approaches to improved finishing

- Acid etch to completely remove subsurface damage

- 3w laser conditioning

- Repolish after damage initiation

- Magneto-rheological finishing

- $\mathrm{CO}_{2}$ laser polishing

- Surface reflow by ion implantation

- Surface removal by plasma etching

ncreasing

difficulty of

implementation

It has been known for some time that surface damage is caused by some combination of subsurface cracks and imbedded contamination, ${ }^{18}$ and possible mechanisms are summarized in Figure 8 . Damage can be caused by absorbing particulates in University of Rochester ("gel") layer or subsurface cracks from grinding and polishing. Recent experiments at both the $10 \mathrm{~J} / \mathrm{cm}^{2}$ at $355 \mathrm{~nm}$. ${ }^{19,20}$ alt at LLNL find that gold nanoparticles overcoated with a high-quality silica layer damage below optic polished by a potential vendor ${ }^{21}$. scratches and indents also damage below $10 \mathrm{~J} / \mathrm{cm}^{2} 22$ tamination is not required, since both fractured surfaces and clean brittle fracture. $^{20}$ 
Figure 8. Schematic diagram of damage initiation mechanisms. The left shows damage initiated by absorbing particulates either in the redeposition ("gel") layer or in cracks, and the right shows damage initiated by field enhancement to constructive interference of light reflected by cracks.

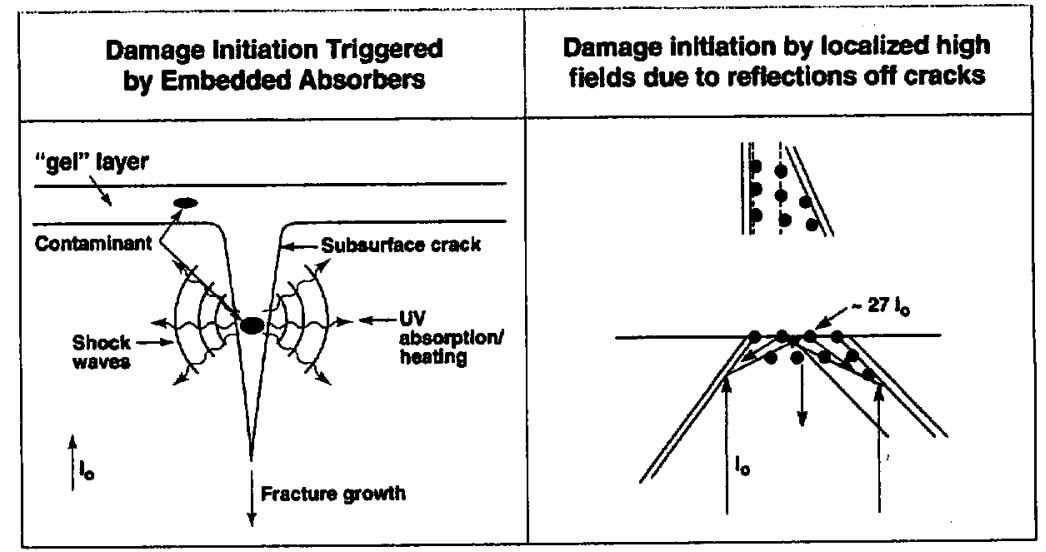

The classic example of surface damage from contaminants in the redeposition layer is the appearance of gray haze, which is thought to occur from residual ceria particles used in the polishing process. ${ }^{23,24}$ This damage mechanism can be eliminated by either use of zirconia in the final polishing steps or by acid etching. This leaves either cracks or contamination in cracks as potential damage mechanisms. Even though most subsurface damage does not lead to surface damage at fluences relevant to operation of large aperture lasers, eliminating cracks would also eliminate the associated contamination, thereby solving the problem regardless of its precise origin. Also, the remaining issue is one of large-area damage density, since even one damage site at high fluence will rapidly destroy an optic.

$\mathrm{CO}_{2}$ annealing of the surface has been reported to increase 1064-nm damage resistance by reducing the amount of subsurface damage as detected by total internal reflection microscopy. ${ }^{25}$ Although our initial efforts also showed a $\sim 10 \mathrm{~J} / \mathrm{cm}^{2}$ increase in the $\mathrm{R} / 1$ damage profile, further work was unsuccessful at finding conditions that would maintain transmitted wavefront quality. ${ }^{26}$ Consequently, large-aperture damage density measurements were not attempted. Similarly, a high-temperature plasma removal process achieved minor increases in the $\mathrm{R} / 1$ damage profile, but thermally induced stress and wavefront issues were not resolved with the modest effort applied. Never the less, the partial success of the $\mathrm{CO}_{2}$ treatment confirmed that further reduction of subsurface damage during polishing would reduce damage densities.

Another approach was to etch away the subsurface damage by a low-temperature method that would not involve material reflow. However, etching with neither $\mathrm{HF} / \mathrm{NH}_{4} \mathrm{~F}$ nor a low-temperature plasma torch showed significant benefit. Both tended to convert existing subsurface damage into increased microroughness when present, although the increase is negligible for high-quality optics. The acid etch technique was explored more completely. However, even when 100-200 $\mu \mathrm{m}$ of material was removed, no significant reduction in damage densities was achieved. Our presumption is that subsurface cracks tend to run ahead of the etching processes.

Although laser conditioning is routinely used to improve damage resistance of many optics, ${ }^{27}$ and there is one report of conditioning at $248 \mathrm{~nm},{ }^{28}$ it was generally thought not to be effective for improving damage resistance at $351 \mathrm{~nm}$, The conclusion of no conditioning may be based on the fact that the $\mathrm{S} / 1$ and $\mathrm{R} / 1$ curves for fused silica, unlike crystals as seen in Figure 6, are basically within experimental precision. However, the principal concern for large aperture lasers is the low fluence $\left(<14 \mathrm{~J} / \mathrm{cm}^{2}\right.$ at $\left.3 \mathrm{~ns}\right)$, large-area damage densities rather than the high-fluence $\left(>25 \mathrm{~J} / \mathrm{cm}^{2}\right)$, small-area damage probabilities measured by an $\mathrm{R} / 1$ test. It is quite important, therefore, that a significant reduction-typically fourfold-in the low fluence damage densities has been observed repeatedly for high-quality polished optics. ${ }^{29}$

Finally, samples finished by magneto-rheological finishing were supplied by Zygo Corporation. As received, the samples had very poor damage performance. However, when residual iron and ceria from the polishing process were removed by acid etching and residual initiators were reduced by $355-\mathrm{nm}$ laser conditioning, a nearly complete elimination of damage below $10 \mathrm{~J} / \mathrm{cm}^{2}$ and a nearly 100 -fold reduction at $14 \mathrm{~J} / \mathrm{cm}^{2}$ were obtained. ${ }^{30}$ The overall progress in damage reduction over the past years is summarized in Figure 9. This last improvement makes practical the mitigation of the pre-initiated damage sites, as outlined in a later section. 

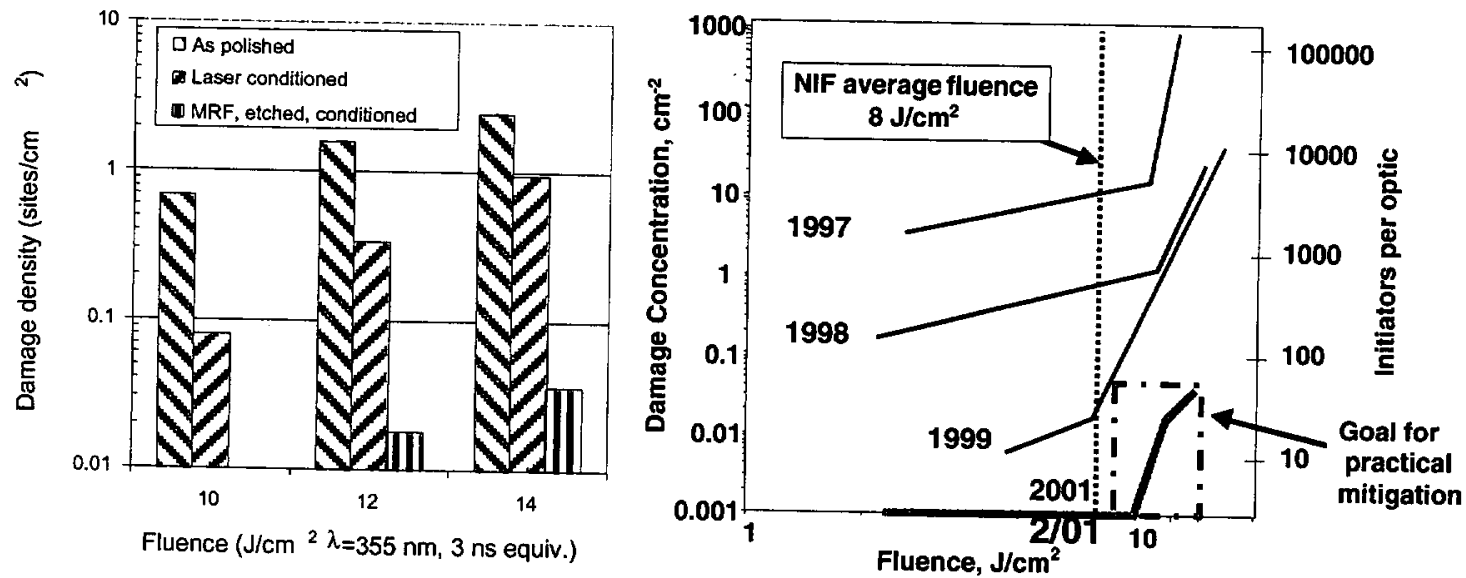

Figure 9. Progressive reduction of initiators in fused silica optics through process improvements. The low damage density values achieved in 2001 were due to a combination of magneto-rheological finishing, acid-etching, and laser conditioning, as seen on the lefthand figure. Earlier improvements were due to improved management of subsurface damage and elimination of ceria from the final polishing steps. The 1999 data on the right are similar to the "as polished" damage densities on the left, which represent the current state
of the art for lenses without MRF.

\section{DKDP}

Surface damage initiation for DKDP is less well understood than for fused silica. Large aperture conversion crystals were operated in air on Beamlet at an average fluence of $8 \mathrm{~J} / \mathrm{cm}^{2}$ with no apparent surface damage. ${ }^{31}$ Surface damage was present on some later Beamlet experiments, but it was not clear whether it was due to fabrication defects, the vacuum environment, or tighter optics spacing. As a result, we embarked on a program to identify defects leading to surface damage on crystals, which is described in detail by Demos et al. ${ }^{32}$

A central feature in this effort was the creation of scattering and flourescence mapping facilities with a resolution on the scale of a few micrometers that can scan and record images of crystals prior to damage testing. Although this work is still in its early stages, a correlation has emerged between the presence of elongated fluorescent bodies and surface damage. The origin and composition of these defects is currently under investigation.

\section{MITIGATION OF SURFACE DAMAGE GROWTH}

Surface damage initiation would not be a serious problem if the damage did not grow. For example, 1000 damage sites 50 $\mu \mathrm{m}$ in diameter over a $1000-\mathrm{cm}^{2}$ clear aperture cover less than $0.01 \%$ of the area. However, surface damage grows exponentially in area above a threshold fluence. The threshold fluence is known fairly well for fused silica and is $5.5 \pm 0.5$ $\mathrm{J} / \mathrm{cm}^{2}$, independent of pulse length to within experimental precision for pulse lengths in the 1-10 ns range. ${ }^{2}$ The threshold seems to be more variable for DKDP, though it is usually higher than for fused silica. Also, DKDP sometimes shows a transition from slow to rapid crater growth.

Surface damage growth requires an absorption mechanism to supply energy for the absorption. For both $\mathrm{SiO}_{2}$ and DKDP, damage craters show UV-induced fluorescence. Upon exposure to $>2 \mathrm{~J} / \mathrm{cm}^{2}$ of UV laser light, localized plasmas are formed and merge into continuous plasma across the damage crater at a fluence approximately equal to the damage growth threshold. ${ }^{33}$ By eliminating the absorption source, damage growth could be stopped. A variety of approaches were pursued, which are described in more detail for fused silica in the following paragraphs. The corresponding effort for DKDP is at an earlier stage and will be reported later.

Parallel investigations for fused silica pursued a basic understanding of the damage characteristics causing absorption and methods to eliminate either the absorption or its effect on growth. A combination of $x$-ray tomography, ${ }^{34}$ Raman
spectroscopy, ${ }^{35}$ and UV-induced fluorescence studies ${ }^{36}$ showed previously that about $10 \mu \mathrm{m}$ of the fused silica in the bottom of the damage crater is densified, thereby forming a variety of absorbing defects without changing the overall stoichiometry of the material. Modeling this year confirmed that the densification and enhanced absorption is a direct result of the shock wave formed by the damage event. ${ }^{37,38}$ Although a detailed model of the damage growth process has not been developed, it 
is also clear that the surrounding crack network both weakens the surrounding material as well as provides the opportunity for additional absorption due to locally enhanced intensity via reflections and scattering. ${ }^{22}$

Consequently, it is clear that damage growth can be stopped only by either removing or remelting the damaged material. An initial attempt at removing the damaged material by whole surface HF etching was reported last year; ${ }^{39}$ this method was only partially successful. During the past year, localized surface removal and modification methods were explored, including acid etching, plasma etching, and $\mathrm{CO}_{2}$ laser treatment. ${ }^{40}$ The latter was the most successful, as well as the easiest to implement. ${ }^{41}$ An important result reported in this proceedings showed that only local melting, not ablation, is needed to stop damage growth on large damage sites formed at $45 \mathrm{~J} / \mathrm{cm}^{2}(355 \mathrm{~nm}, 8 \mathrm{~ns})$ for subsequent exposure up to $14 \mathrm{~J} / \mathrm{cm}^{2}(10 \mathrm{~ns})$. Very recent work has improved the process so that smaller damage sites initiated at $355-\mathrm{nm}$ fluences up to $14 \mathrm{~J} / \mathrm{cm}^{2}$ ( $3 \mathrm{~ns}$ ) can survive subsequent exposure to $14 \mathrm{~J} / \mathrm{cm}^{2}$ (3 ns) (W. Molander, unpublished results, 2001).

An alternate approach of stopping or slowing damage was also explored and is reported here for completeness. It was hypothesized that variations in trace species, such as water or fluorine, in the fused silica might alter its susceptibility to damage growth, either through changes in the propensity to form absorbing defects or by slight changes in mechanical properties. Various specialty grades of fused silica were acquired and polished by a standard process. Damage was then initiated at $45 \mathrm{~J} / \mathrm{cm}^{2}(7.6 \mathrm{~ns})$ and exposed to fluences up to $12 \mathrm{~J} / \mathrm{cm}^{2}(11 \mathrm{~ns})$. As shown in Figure 10, however, there is no difference in damage growth for any of these materials, confirming that the factors leading to damage growth are intrinsic to fused silica.

\section{UV VACUUM EFFECTS}

Previous work showed that the surfaces of both fused silica and DKDP degrade upon prolonged exposure to 355-nm laser light. ${ }^{5,6}$ For fused silica, a thin layer of sub-stoichiometric SiOx is formed, which absorbs some of the 355-nm light and fluoresces in the visible and IR. Operational experience for the spatial filters of the Optical Science and Slab Lab lasers has found that only a few millitorr of air are required to prevent its formation. Similarly, 10 Torr of air eliminates an observed increase in surface crater damage. ${ }^{5}$

During the past year, the effect of gas pressure on UV-induced surface degradation was explored in more detail for DKDP. ${ }^{42}$ DKDP shows two deleterious effects-absorption-fluorescence and roughening-scattering. Although neither are understood in any detail, both can be eliminated by the presence of gas, either air or nitrogen, at the 1-10 Torr level. As a result, the debris shield seal in the NIF final optics assembly was modified so that the final optics can operate at 10 Torr, thereby eliminating the potentially deleterious effects of vacuum.

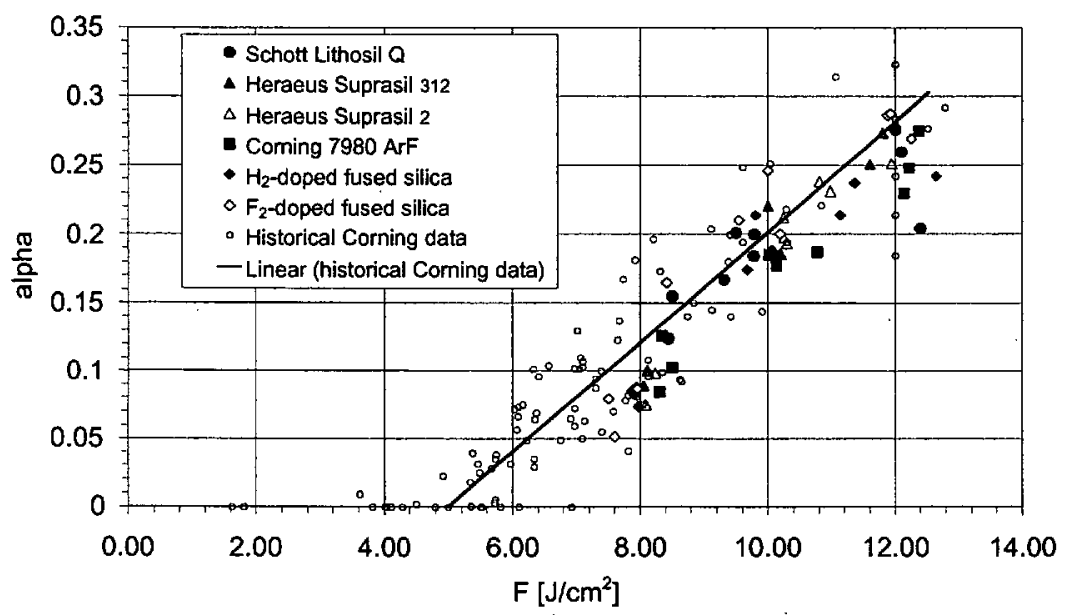

Figure 10. Surface damage growth rates for various commercial grades of fused silica. Growth is governed by the relation $L=L_{0} e^{\alpha N}$, where $L$ is the diameter of the damage crater, $N$ is the number of shots, and $\alpha$ is the growth coefficient. Subtle changes in composition have no detectable affect on growth. 


\section{OPERATIONAL STRATEGY FOR THE NIF FINAL OPTICS}

With these advances in both understanding and control of laser-induced damage, an improved operational strategy for the NIF final optics was devised. The basic elements are given in Figure 2, and an expanded view of the post-

processing/recycling aspects is given in Figure 11. The basic concept is to precondition any optic susceptible to damage, preinitiate and mitigate any surface damage, establish environmental conditions that minimize damage during operations (see preceding section), and then retrieve and repair optics damaged during operations.

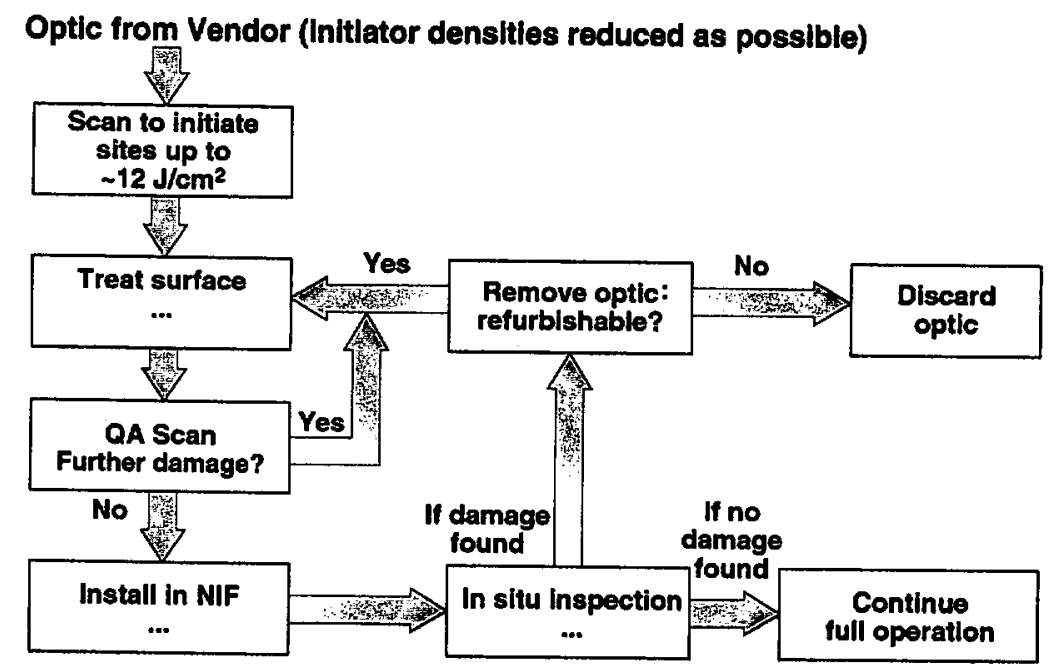

Figure 11. Schematic diagram of the final optics post-processing and recycling scheme.

One of the main issues to be considered for optics lifetime predictions for this strategy is the effect of beam contrast during both post-processing and subsequent operation. The mitigation process is shown schematically in Figure 12 for a surface damage density distribution from an MRF-polished part. The presence of beam contrast means that there is no sharp cut-off fluence for which surface damage sites have and have not been initiated. Multiple passes with a migrating contrast increase the mean exposure fluence and narrow the distribution, as shown in Figure 3. For this particular example, 5 passes at 12 $\mathrm{J} / \mathrm{cm}^{2}$ with a beam having a $15 \%$ contrast, effectively pre-initiates all sites below $11 \mathrm{~J} / \mathrm{cm}^{2}$ and initiates few above $15 \mathrm{~J} / \mathrm{cm}^{2}$. This corresponds to about 50 damage sites for an MRF-polished part.

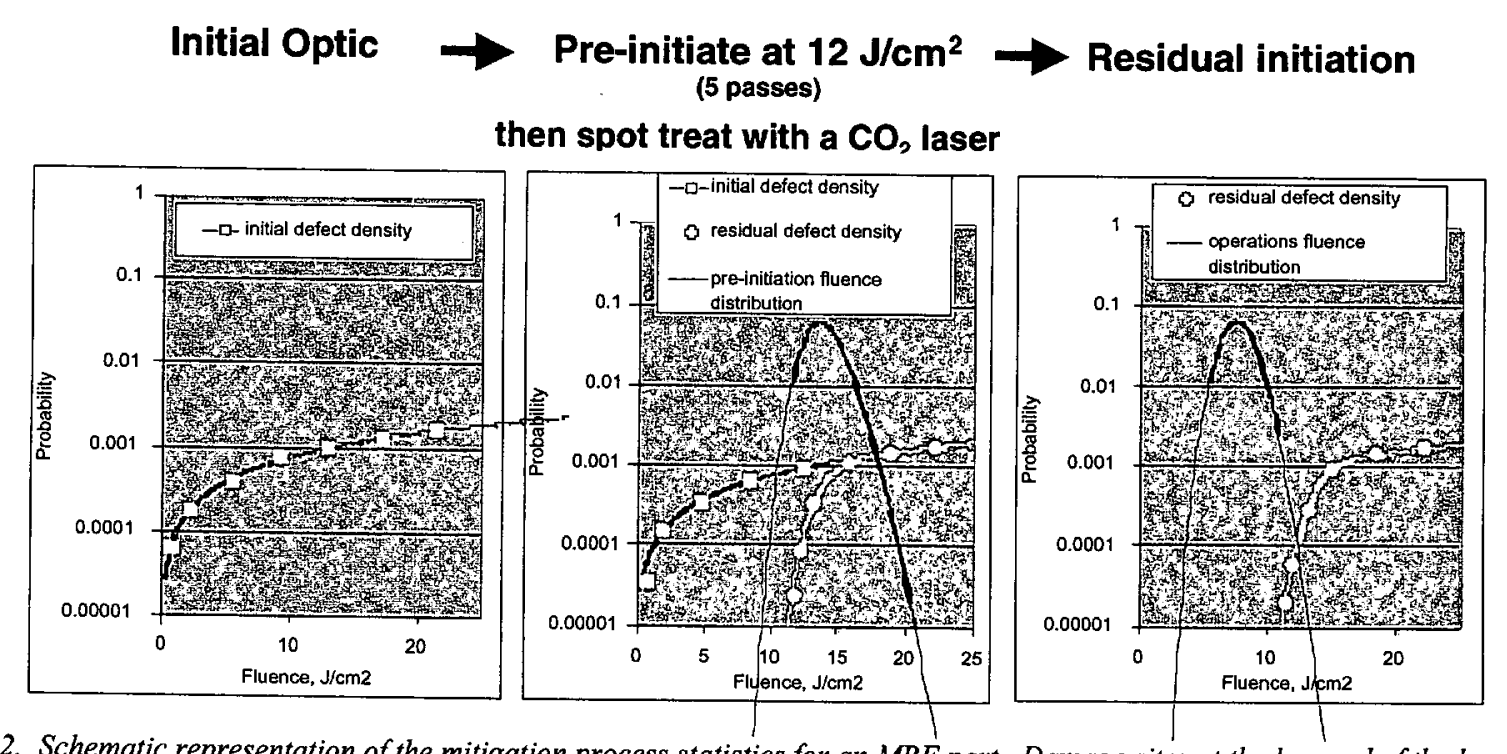

Figure 12. Schematic representation of the mitigation process statistics for an MRF part. Damage sites at the low end of the beam fluence distribution are not fully initiated, while others are initiated at the high end of the fluence distribution that would not initiated on line. 
After installation, there is a finite probability that a residual defect is exposed to a high fluence portion of the beam. Using Equations (1) and (3), one finds that it takes on average $~ 7600$ shots at $8 \mathrm{~J} / \mathrm{cm}^{2}$ with $15 \%$ beam contrast for a hot spot in the beam to find a residual defect. (This is probably an overestimate of the lifetime due to the potential presence of contamination induced damage.) Once initiation has occurred, the damage site grows to unacceptable size in tens of shots at $8 \mathrm{~J} / \mathrm{cm}^{2}$. Consequently, the optic must be retrieved and repaired promptly. To accomplish this, the conversion crystals and final focus lens are now held in a line-replaceable cassette, enabling rapid retrieval, repair, and replacement upon damage detection.

\section{SUMMARY}

A combination of fundamental science and engineering improvements have led to greatly improved damage performance of large-aperture UV optics such as those to be used on the National Ignition Facility. Elimination of bulk inclusions in fused silica and improvements in KDP growth and laser conditioning processes has essentially eliminated those mechanisms for operation at an average fluence of $8 \mathrm{~J} / \mathrm{cm}^{2}$, and modification of the final optics assembly to operate at 10 Torr eliminates vacuum-enhanced surface degradation mechanisms. Advances in fused silica finishing have greatly reduced the subsurface damage and reduced surface contamination, resulting in only $\sim 50$ surface damage initiation sites for $40-\mathrm{cm}$ scale optics. Preinitiating these sites offline and spot treating them with a $\mathrm{CO}_{2}$ laser eliminates the formation and growth of these sites online. Progress has also been made on understanding the origin of surface damage on crystals. These advances have been combined into a new concept of pre-initiating and repairing damage prior to installation, then frequently inspecting the optics during operations to retrieve and repair the optics prior to development of unacceptable levels of damage. This process not only allows the optics to be recycled several times but also reduces the collateral damage on neighboring optics.

\section{ACKNOWLEDGMENTS}

This work was performed under the auspices of the U.S. Department of Energy by University of California Lawrence Livermore National Laboratory under Contract W-7405-Eng-48. The contributions of many other individuals to the various tasks summarized here is greatly appreciated, including Chris Adams, Ray Brusasco, Jeff Carr, Gene Donohue, Randy Floyd, Ruth Hawley-Fedder, Terry Land, Jim McElroy, Bill Molander, Kurt Neeb, Mike Nichols, Mary Norton, John Peterson, Wally Sell, Dave Swift, and Tim Weiland.

\section{REFERENCES}

1. J. R. Murray, "Overview of the National Ignition Facility," Third Annual Intl Conf. of Solid State Lasers for Application to ICF, Suppl. to Proceedings of SPIE, Vol. 3492, pp. 1-10 (1998).

2. M. Norton, L. Hrubesh, Z. Wu, M. Feit, M. Kozlowski, D. Milam, K. Neeb, W. Molander, A. Rubenchik, W. Sell, P. Wegner, "Growth of laser-initiated damage in fused silica at 351 nm," Laser Science and Technology Program Annual Report-2000, LLNL Report UCRL-ID-142744-00 and UCRL-JC-13964 (2001)

3. M. Runkel, J. De Yoreo, W. Sell, D. Milam, "Laser conditioning study of KDP on the Optical Sciences Laser using large area beams," Laser-Induced Damage in Optical Materials: 1997, G. J. Exarhos, ed., Proceedings of SPIE, Vol. 3244, pp. 51-63 (1998).

4. M. R. Kozlowski, R. Mouser, S. Maricle, P. Wegner, and T. Weiland, "Laser damage performance of fused silica optical components measured on the Beamlet laser at $351 \mathrm{~nm}$," Laser-Induced Damage in Optical Materials: 1998, G. J. Exarhos, ed., Proceedings of SPIE, Vol. 3578, pp. 436-443 (1999).

5. A.K. Burnham, M. Runkel, S. G. Demos, M. R. Kozlowski, P. J. Wegner "Effect of vacuum on the occurrence of UVinduced surface photoluminescence, transmission loss, and catastrophic surface damage," Photonics for Space Environments VII, E. W. Taylor, ed., Proceedings of SPIE, Vol. 4134, pp. 243-252, (2000).

6. S. G. Demos, A. Burnham, P. Wegner, M. Norton, L. Zeller, M. Runkel, M. R. Kozlowski, M. Staggs, H. B. Radousky, "Surface defect generation in optical materials under high fluence laser irradiation in vacuum," Electronic Lett. 36, 566$567(2000)$.

7. M. D. Feit, A. M. Rubenchik, M. R. Kozlowski, F. Y. Genin, S. Schwartz, L. M. Sheehan, "Extrapolation of damage test data to predict performance of large-area NIF optics at $355 \mathrm{~nm}$," Laser-Induced Damage in Optical Materials: 1998, G. J. Exarhos, ed., Proceedings of SPIE, Vol. 3578, pp. 226-233 (1999).

8. P. Wegner and M. Henessian, "Mathematical model for describing the intensity statistics of ICF laser beams," LLNL internal memorandum, 1990. 
9. M. S. Dobbins, R. E. McLay, "Method of making fused silica by decomposing siloxanes," U.S. Patent 5,043,002, August 27, 1991.

10. M. Runkel, A. Burnham, D. Milam, W. Sell, M. Feit, A. Rubenchik, "The results of pulse-scaling experiments on rapidgrowth DKDP triplers using the Optical Sciences Laser at $351 \mathrm{~nm}$," Laser-Induced Damage in Optical Materials: 2000, G. J. Exarhos, ed., Proceedings of SPIE, Vol. 4347, pp. 359-368 (2001).

11. J. W. Goodman, "Statistical properties of laser speckle patterns," Laser Speckle and Related Phenomena, Topics in Applied Physics, Vol 9, J. C. Dainty, ed., Springer-Verlag, Berlin, 1975, p. 31.

12. M. Runkel and A. Bumham, "Differences in bulk damage probability distributions between tripler and z-cuts of KDP and DKDP at $355 \mathrm{~nm}$," Laser-Induced Damage in Optical Materials: 2000, G. J. Exarhos, ed., Proceedings of SPIE, Vol. 4347, 408-419 (2001).

13. M. Staggs, M. Yan, M. Runkel, "Laser raster conditioning of KDP and DKDP crystals using XeCl and Nd:YAG lasers," Laser-Induced Damage in Optical Materials: 2000, G. J. Exarhos, ed., Proceedings of SPIE, Vol. 4347, 400-407 (2001).

14. M. D. Feit, A. M. Rubenchik, M. Runkel, "Analysis of bulk DKDP damage distribution, obscuration and pulse length dependence," Laser-Induced Damage in Optical Materials: 2000, G. J. Exarhos, ed., Proceedings of SPIE, Vol. 4347, 383-388 (2001).

15. N. Zaitseva, J. Atherton, R. Rozsa, L. Carman, I. Smolsky, M. Runkel, R. Ryon, L. James, "Design and benefits of continuous filtration in rapid growth of large KDP and DKDP crystals," J. Crystal Growth 197, 911-920 (1999).

16. A. K. Burnham, M.Runkel, R. Hawley-Fedder, L. Carman, R. Torres, P. Whitman, "Low-temperature growth of DKDP for improved 3 $\omega$-damage resistance," Laser-Induced Damage in Optical Materials: 2000, G. J. Exarhos, ed., Proceedings of SPIE, Vol. 4347, 373-381 (2001).

17. M. Runkel, K. Neeb, M. Staggs, J. Auerbach, A. Burnham, "Raster-scan laser conditioning studies on frequency conversion crystals using Nd:YAG and excimer lasers," Laser-Induced Damage in Optical Materials: 2001, this proceedings.

18. L. Sheehan, M. Kozlowski, D. W. Camp, "Application of total internal reflection microscopy for laser damage studies on fused silica," Laser-Induced Damage in Optical Materials: 1997, G. J. Exarhos, ed., Proceedings of SPIE, Vol. 3244, 282-295 (1998).

19. S. Papernov, A. W. Schmid, R. Krishnan, L. Tsybesdov, "Using colloidal gold nanoparticles for studies of laser interaction with defects in thin films," Laser-Induced Damage in Optical Materials: 2000, G. J. Exarhos, ed., Proceedings of SPIE, Vol. 4347, 146-154 (2001).

20. A. V. Hamza, W. J. Seikhaus, A. M. Rubenchik, M. Feit, L. L. Chase, M. Savina, M. J. Pellin, I. D. Hutcheon, M. C. Nostrand, M. Runkel, B. W. Choi, M. Staggs, and M. J. Fluss, "Engineered defects for investigation of laser-induced damage of fused silica at 355nm," Laser-Induced Damage in Optical Materials: 2001, this proceedings.

21. M. Wall, J. Plitzko, M. J. Fluss, "Initiation Identification in Fused Silica 35-nm Optics", Laser-Induced Damage in Optical Materials: 2001 , this proceedings.

22. F. Y. Génin, A. Salleo, T. V. Pistor, L. L. Chase, Role of light intensification by cracks in optical breakdown on surfaces," J. Opt. Soc. Am. A 18, 2607-2616 (2001).

23. M. R. Kozlowski, J. Carr, I. Hutcheon, R. Torres, L. Sheehan, D. Camp, M. Yan, "Depth profiling of polishing-induced contamination on fused silica surfaces," Laser-Induced Damage in Optical Materials: 1997, G. J. Exarhos, ed., Proceedings of SPIE, Vol. 3244, 365-375 (1998).

24. C. L. Battersby, L. M. Sheehan, M. R. Kozlowski, "Effects of wet etch processing on Laser-induced damage of fused silica surfaces," Laser-Induced Damage in Optical Materials: 1998, G. J. Exarhos, ed., Proceedings of SPIE, Vol. 3578, 446-455 (1999).

25. P. A. Temple, D. Milam, and W. H. Lowdermilk, " $\mathrm{CO}_{2}$-laser polishing of fused silica surfaces for increased laser damage resistance at $1.06 \mu \mathrm{m}$," Damage in Laser Materials: 1979, H. E. Bennett, ed., Nat. Bur. Stand. (U.S.) Spec. Publ. 568, pp. 229-236 (1979).

26. R. M. Brusasco, B. M. Penetrante, J. A. Butler, S. M. Maricle, J. E. Peterson, " $\mathrm{CO}_{2}$-laser polishing for reduction of 351nm surface damage initiation in fused silica," Laser-Induced Damage in Optical Materials: 2001, this proceedings.

27. F. Rainer, R. M. Brusasco, J. H. Campbell, F. P. DeMarco, R. P. Gonzales, M. R. Kozlowski, F. P. Milanovich, A. J. Morgan, M. S. Scrivener, M. C. Staggs, I. M. Thomas, S. P. Velsco, C. R. Wolfe, "Damage measurements on optical materials for use in high-peak-power lasers," Laser-Induced Damage in Optical Materials: 1989, H. E. Bennett, ed., NIST Special Publication 801, pp. 74-83 (1990).

28. N. C. Kerr and D. C. Emmony, "The effect of laser annealing of laser induced damage threshold," Laser-Induced Damage in Optical Materials: 1989, H. E. Bennett, ed., NIST Special Publication 801, pp. 164-179 (1990). 
29. R. M. Brusasco, B. M. Penetrante, J. E. Peterson, S. M. Maricle, J. A. Menapace, "UV-laser conditioning for reduction of 351-nm damage initiation in fused silica," Laser-Induced Damage in Optical Materials: 2001, this proceedings.

30. J. A. Menapace, B. Penetrante, D. Golini, A. Slomba, P. E. Miller, T. Parham, M. Nichols, and J. Peterson, "Combined Advanced Finishing and UV Laser Conditioning for Producing UV Damage Resistant Fused Silica Optics," LaserInduced Damage in Optical Materials: 2001, this proceedings.

31. B. M. Van Wonterghem, J. R. Murray, J. H. Campbell, D. R. Speck, C. E. Barker, I. C. Smith, D. F. Browning, W. C. Behrendt, "Performance of a prototype for a large-aperture multipass Nd:glass laser for inertial confinement fusion," Applied Optics 36, 4932-4953.

32. S. G. Demos, M. Nostrand, M. Staggs, C.W. Carr, D. Hahn, A. Burnham," Investigation of fluorescence imaging as a tool for noninvasive detection and imaging of damage precursors at $3 \omega$, , Laser-Induced Damage in Optical Materials: 2001 , this proceedings.

33. S. G. Demos, M. R. Kozlowski, M. Staggs, L. L. Chase, A. Burnham, H. B. Radousky, "Mechanisms to explain damage growth in optical materials," Laser-Induced Damage in Optical Materials: 2000, G. J. Exarhos, ed., Proceedings of SPIE,

Vol. 4347, 277-282 (2001).
34. J. Wong, D. Haupt, J. H. Kinney, M. Stevens-Kalceff, A. Stesmans, J. Ferreira, E. Lindsey, and I. Hutcheon, Morphology, Microstructure and Defects in Fused Silica Induced by High-Power 355-nm Laser Pulses, Lawrence Livermore National Laboratory Report UCRL-JC-142167 (2001).

35. S. G. Demos, L. Sheehan, M. R. Kozlowski, "Spectroscopic investigation of SiO2 surfaces of optical materials for highpower lasers," Laser Applications in Microelectronic and Optoelectronic Manufacturing V, H. Helvajian, ed., Proceedings of SPIE, Vol. 3933, pp. 316-321 (2000).

36. M. R. Kozlowski, C. L. Battersby, S. G. Demos, "Luminescence investigation of SiO2 surfaces damaged by $0.35 \mu \mathrm{m}$ laser illumination," Laser-Induced Damage in Optical Materials: 1999, G. J. Exarhos, ed., Proceedings of SPIE, Vol. 3902, 138-143 (2000).

37. A. Kubota, M. J. Caturla, J. S. Stolken, M.D. Feit, "Densification of fused silica due to shock waves and its implications for 351-nm laser-induced damage," Optics Express 8, 611-616 (2001)

38. A. Kubota, L. Davila, M.J. Caturla, J. S. Stolken, B. Sadigh, A. Quong, A. Rubinchek, M. D. Feit, "Structural modifications in fused silica due to laser damage induced shock compression," Laser-Induced Damage in Optical Materials: 2001, this proceedings.

39. L. W. Hrubesh, M. A. Norton, W. A. Molander, P. J. Wegner, M. Staggs, S. G. Demos, J. A. Britten, L. J. Summers, E. F. Linsey, M. R. Kozlowski, "Chemical etch effects on laser-induced surface damage growth in fused silica," Laser-Induced Damage in Optical Materials: 2000, G. J. Exarhos, ed., Proceedings of SPIE, Vol. 4347, 553-559 (2001).

40. L. W.Hrubesh, M. A.Norton, W. A.Molander, E. E.Donohue, S. M.Maricle, B. M.Penetrante, R. M.Brusasco, W.Grundler, J. A.Butler, J. W.Carr, R. M.Hill, L. J.Summers, M. D.Feit, A.Rubenchik, M. H.Key, P. J.Wegner, A. K.Burnham, L. A. Hackel, M. R.Kozlowski, "Methods for mitigating surface damage growth on NIF final optics," LaserInduced Damage in Optical Materials: 2001, this proceedings.

41. R. M. Brusasco, B. M. Penetrante, J. A. Butler, L. W. Hrubesh, "Localised $\mathrm{CO}_{2}$-laser treatment for mitigation of 351-nm damage growth on fused silica," Laser-Induced Damage in Optical Materials: 2001, this proceedings.

42. P. Whitman, M. Norton, M. Nostrand, W. Molander, A. Nelson, M. Engelhard, D. Gaspar, D. Baer, W. Siekhaus, J. Auerbach, S. Demos, M. Staggs, A. Burnham, "Degradation of bare and sol-gel coated DKDP crystals exposed to multiple 351-nm laser pulses," Laser-Induced Damage in Optical Materials: 2001, this proceedings. 


\title{
Performance of Thin Borosilicate Glass Sheets at 351-nm
}

\author{
P.K.Whitman \\ M. Staggs \\ C. W. Carr \\ S. Dixit \\ W. Sell \\ D. Milam
}

\begin{abstract}
Commercial thin borosilicate glass sheets have been evaluated for use as a single-shot optic "debris shield" to separate the radiation and contamination produced by the inertial confinement fusion (ICF) experiment from the expensive precision laser optics which focus and shape the 351-nm laser beam which irradiates the target. The goal of this work is identification of low cost materials that can deliver acceptable beam energy and focal spots to the target. The two parameters that dominate the transmitted beam quality are the transmitted wave front error and bulk absorption. This paper focuses on the latter. To date, the materials with the lowest linear 351-nm absorption have also generally demonstrated the lowest non-linear absorption. Commercial materials have been identified which approach the beam energy and focus requirements for many ICF missions.
\end{abstract}

\section{INTRODUCTION}

The interaction of high intensity laser light with glass can result in both reversible and permanent changes in optical properties. Excellent treatises on (reversible) two-photon absorption in dielectric materials can be found in Chase ${ }^{1}$ and Smith $^{2}$. The interaction of solar or laser radiation can also produce free electrons or holes which can be trapped in structural or chemical defects in the glass resulting in 'color centers' or 'solarization'. Bishay ${ }^{3}$ and Griscom ${ }^{4,5}$ published detailed discussions of color centers induced in multicomponent glasses including borosilicates. The linear and non-linear optical properties of potential fusion-laser materials were investigated extensively in the early $80^{\prime} \mathrm{s}^{6}$. Non-linear absorption, solarization, bulk damage (platinum inclusions) and nonlinear refractive index all limit the utility of borosilicate optical glasses, such as Schott BK-7, as thick transmissive optics in high-peak-power applications at 351-nm. We propose that thin commercial borosilicate glass sheets could function as low cost optics that can deliver acceptable beam energy for a limited shot sequence on an ICF laser. In order to assess the feasibility of specific commercial thin glass sheets, we have measured their transmittance under irradiance by $351-\mathrm{nm}, 3-\mathrm{ns}$ laser pulses at various fluence levels.

\section{EXPERIMENT}

\section{Materials preparation and measurements}

All materials were tested as-received from the vendor. With the exception of the Hoya NA-35 substrates, all test sample surfaces were as-formed - i.e. drawn or float glass surfaces. The Hoya NA-35 substrates were mechanically polished after forming. All optics were cleaned, but not anti-reflection coated before testing. Material composition is shown in Table 1 .

Table 1: Test matrix materials

\begin{tabular}{|l|l|l|l|l|}
\hline Sample ID & Test Thickness (mm) & Forming process & Type & Major constituents (in addition to SiO2) \\
\hline Schott Borofloat ${ }^{81}$ & 1.1 and 3.3 & float & borosilicate & $\mathrm{B}_{2} \mathrm{O}_{3}, \mathrm{Na}_{2} \mathrm{O}, \mathrm{Al}_{2} \mathrm{O}_{3}$ \\
\hline Schott D263 & 1.1 & drawn & zinc borosilicate & $\mathrm{B}_{2} \mathrm{O}_{3}, \mathrm{Na}_{2} \mathrm{O}, \mathrm{K}_{2} \mathrm{O}, \mathrm{ZnO}_{2} \mathrm{Al}_{2} \mathrm{O}_{3}, \mathrm{TiO}_{2}$ \\
\hline Corning 0211 & 2 & drawn & zinc borosilicate & $\mathrm{B}_{2} \mathrm{O}_{3}, \mathrm{Na}_{2} \mathrm{O}, \mathrm{K}_{2} \mathrm{O}, \mathrm{ZnO}, \mathrm{Al}_{2} \mathrm{O}_{3}, \mathrm{TiO} 2$ \\
\hline Hoya NA-35 & 0.4 & Drawn \& polished & aluminosilicate & $\mathrm{Al}_{2} \mathrm{O}_{3}, \mathrm{~B}_{2} \mathrm{O}_{3}, \mathrm{BaO}, \mathrm{CaO}, \mathrm{SrO}$ \\
\hline Schott BK-7 & 0.9 & cast & borosilicate crown & $\mathrm{B}_{2} \mathrm{O}_{3}, \mathrm{Na}_{2} \mathrm{O}, \mathrm{K}_{2} \mathrm{O}, \mathrm{BaO}$ \\
\hline
\end{tabular}

\section{Test facilities}

Two test facilities were used in these experiments. Initial experiments were conducted on Corning 0211 zinc borosilicate glass sheet on our Optical Sciences Laser (OSL) using a setup similar to that previously described in Whitman ${ }^{8}$. The Qswitched Nd-glass OSL laser produces 1053-nm pulsed output that can be frequency tripled to 351-nm; the output beam has

\footnotetext{
${ }^{1}$ Schott Corporation, Yonkers, NY

${ }^{2}$ Corning Inc., Corning, NY

${ }^{3}$ Hoya Corporation USA, Fremont, CA
} 
an approximate "top-hat" temporal and spatial intensity profile. Figure 1 shows the test system and diagnostics layout. Dichroic mirrors were used to separate the $351-\mathrm{nm}$ beam from the residual light at 1053 and $527 \mathrm{~nm}$. A plane near the KDP crystals was imaged by a single lens onto the sample. Input diagnostics allowed measurement of the pulse energy, temporal waveform, and the fluence distribution in an "equivalent sample plane". Fractions of the output beam were observed by a calorimeter and a photodiode that were used to monitor relative changes in the laser output that occurred when the sample was inserted into the beam and when the fluence in the input beam was increased. The output beam pulse length was 3-ns at 351-nm.

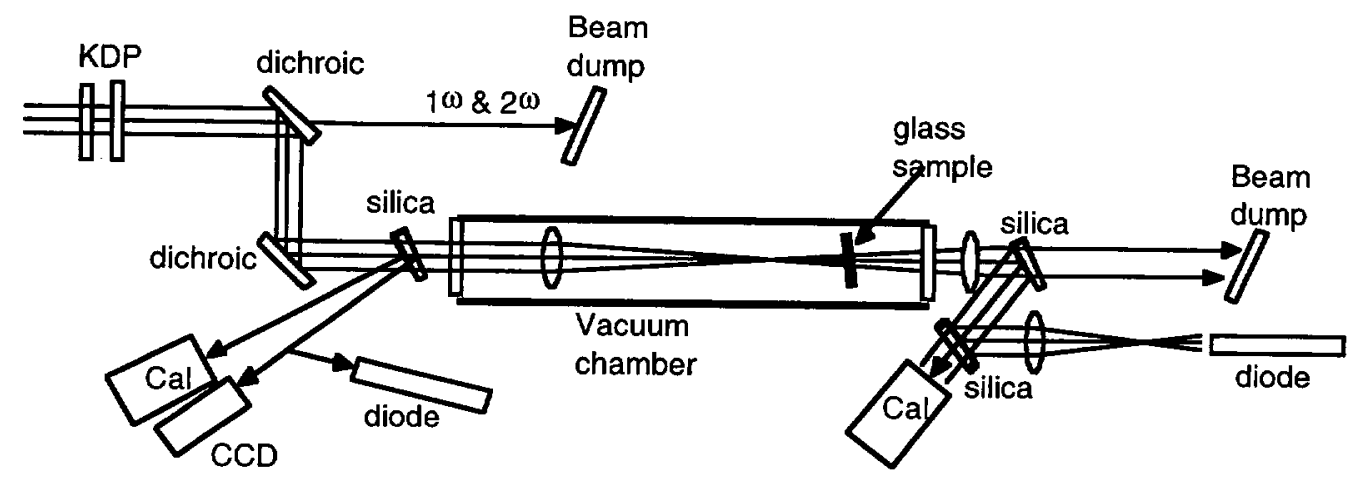

\section{Figure 1. Experimental arrangement for the Optical Sciences Laser (OSL)}

The second test facility was assembled specifically for these experiments. A Coherent Infinity tripled Q-switched Nd:YaG laser provided the 3-ns (FWHM) 355-nm Gaussian (temporal and spatial) test beam. Figure 2 shows the layout for this experiment. Vacuum photodiodes and a boxcar integrator recorded the intensity of each 3-ns, 355-nm pulse as a voltage. The transmittance was determined by the ratio of voltages before and after the sample and normalized by the ratio without a sample in place. All samples were illuminated at a two-degree angle of incidence.

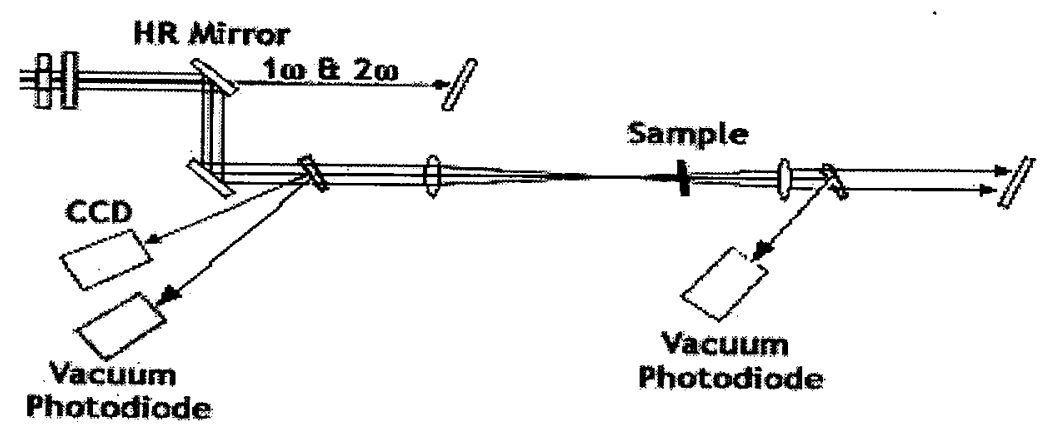

Figure 2: Experimental arrangement for the Coherent Infinity laser experiments

\section{RESULTS}

\section{Transmittance of a 351-nm flat pulse}

Figure 3 gives the results of our measurements of transmittance as a function of incident 351 -nm fluence for the 3-ns flat beam produced in the OSL experimental setup described above. Transmittance was defined to be the ratio of output to input energy that was measured with the sample in the beam, divided by a value of the same ratio that was measured before the sample was installed. From a line that was fitted through the data, we estimate that the transmittance varied from about $88 \%$ to $76 \%$ as the fluence increased from 0 to $10 \mathrm{~J} / \mathrm{cm}^{2}$. The low power measurement of $88 \%$ is close (within error bars) to the spectraphotometer transmission measurement of $90 \%$. The linear variation of the transmission with $351-\mathrm{nm}$ fluence is indicative of a two-photon absorption process whose source has not yet been identified. 
Although there was no visible surface damage to the sample for these single shot irradiation experiments, there was visible sample discoloration ('browning') for incident fluences around $5 \mathrm{~J} / \mathrm{cm}^{2}$ or higher. This is possibly some form of color center formation. The sample discoloration increased with increasing fluence levels. We have not done a systematic study of its causes or tested whether thermal bleaching could recover the original sample transmittance.

For each shot, waveforms were captured by SCD50000 digitizers. Figure 4 shows the waveforms for one shot that was recorded before the sample was loaded, and for all seven shots that were used to determine the transmittance. They were normalized such that voltages within the half-power points have average value of unity. The systematic variation of these waveforms with increase in fluence is slight. At low fluence the output power tended to be slightly low at the first of the pulse and slightly high at the end of the pulse. That trend was reversed for shots at highest fluence, see Fig. 4. However, the power reduction at the end of the pulse was not as large as the $8 \%$ calorimetric deficit. The absence of pulse shape distortion also indicates that the cause of the transmittance loss is most likely a bulk effect (such as a two-photon-induced absorption process) in the glass itself.

\section{Transmittance of a 355-nm Gaussian pulse}

Figure 5 shows the results of our measurements of transmittance as a function of incident 355-nm fluence for the $\sim 3$-ns Gaussian pulse. All of the samples measured exhibited behavior that is characteristic of nonlinear absorption. Several displayed a linear variation in transmittance as a function of fluence that is indicative of two-photon absorption. Of the samples examined, Hoya NA-35, $0.9 \mathrm{~mm}$ in thickness, had the least loss overall. The transmittance varied with intensity from $89 \%$ at $4 \mathrm{~J} / \mathrm{cm}^{2}$ to $87 \%$ at $12 \mathrm{~J} / \mathrm{cm}^{2}$.

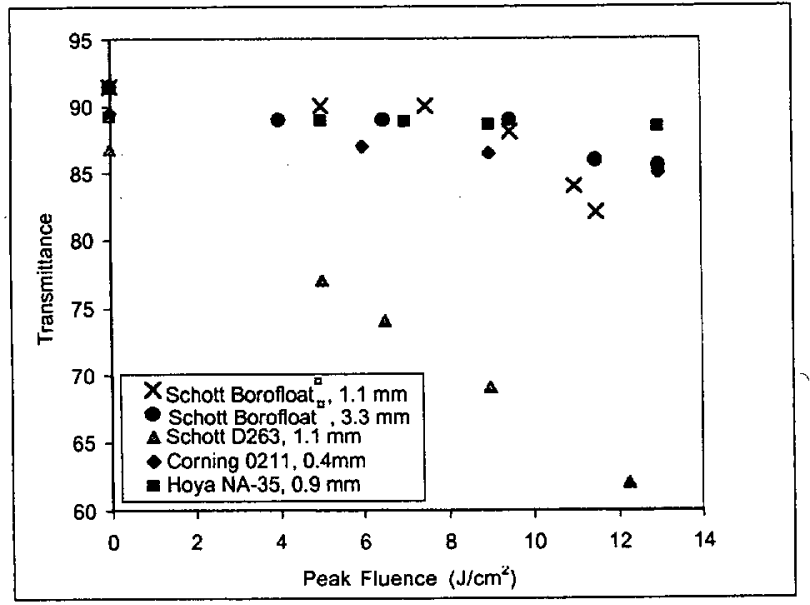

Table 2 summarizes the result of linear least squares regression fits to the data. The extrapolated y-intercept should represent the linear absorption plus the Fresnel reflection losses; for most samples there is good correlation between the transmittance measured on a spectrophotometer and the $y$-intercept. For borosilicate materials (refractive index around 1.53), Fresnel losses limit the maximum transmittance to no more than $\sim 90 \%$. Extrapolated y-intercepts greater than $90 \%$, in combination with the poor linear fit suggest that non-linear absorption cannot fully account for the observed transmittance of the Schott Borofloat $($ samples. In this experimental setup, the beam size was approximately $1-\mathrm{mm}$ FWHM - so small surface damages or 'browning' would most likely be undetectable without magnification.
Figure 5: Comparison of transmittance of thin borosilicate glass sheets as a function of incident fluence in a 3-ns FWHM Gaussian 355-nm pulse.

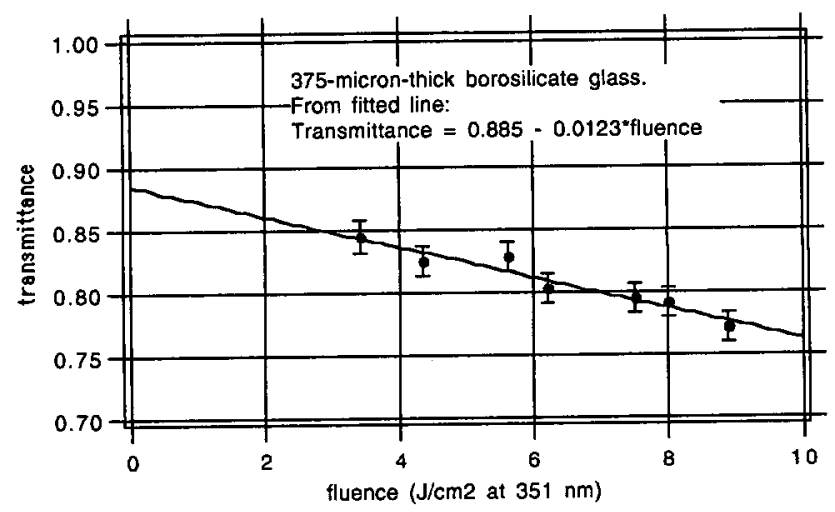

Figure 3. Transmittance of unpolished borosilicate glass sheet (Corning 0211) as a function of incident fluence in a 3-ns, 351-nm pulse. 

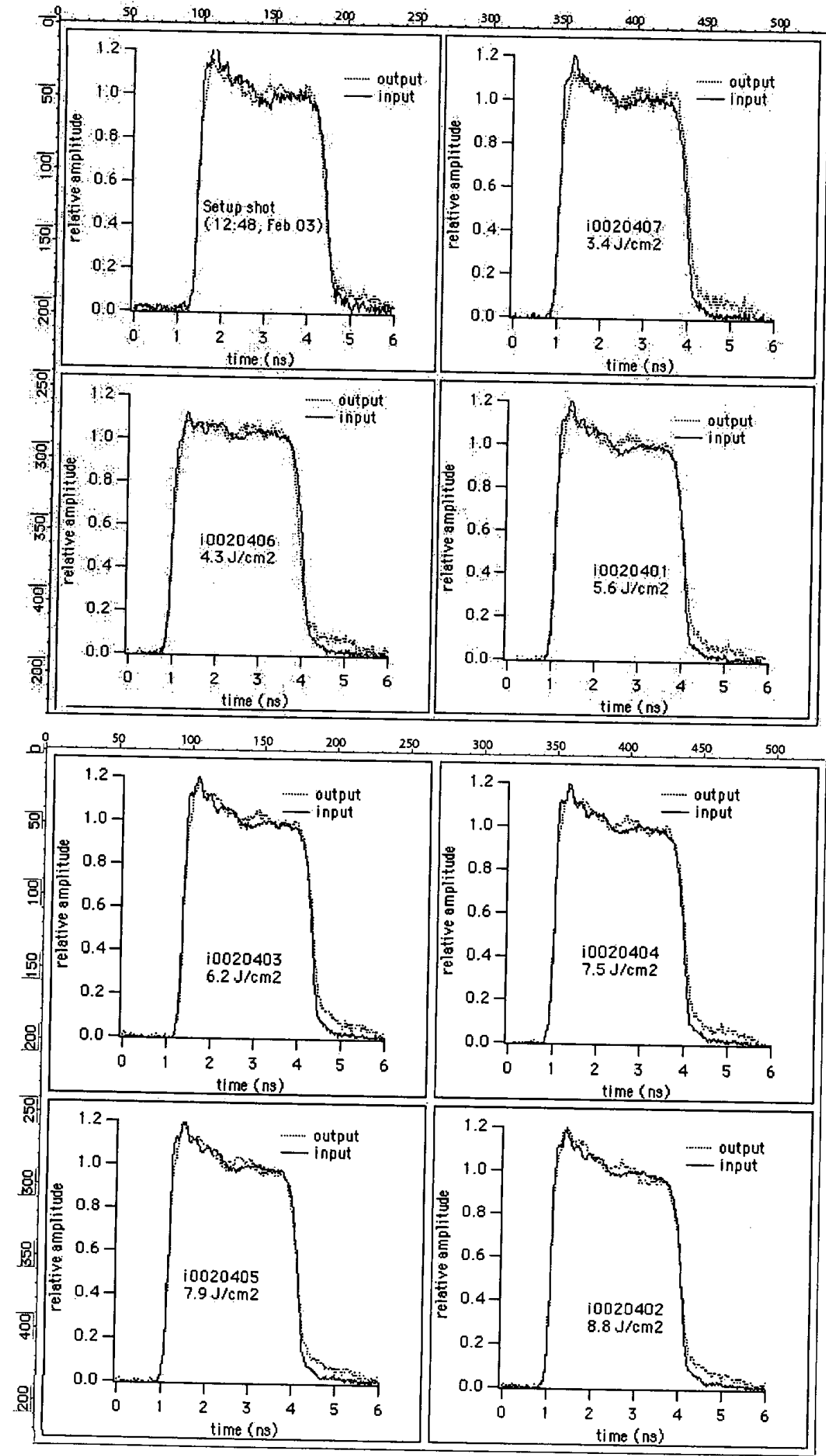

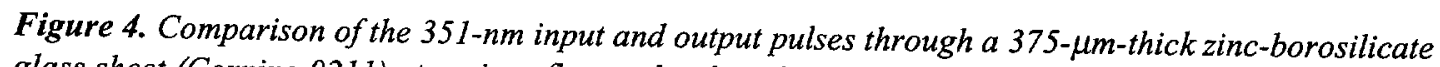
glass sheet (Corning 0211) at various fluence levels. The frame at the upper left is without the sample in the beam path and provides a measure of the errors in the pulsed shape measurement. 
Table 2: Comparison of transmittance parameters for four different commercial borosilicate glasses

\begin{tabular}{|l|c|c|c|c|}
\hline Test sample & $\begin{array}{c}\text { Measured } \\
\text { transmitta } \\
\text { nce }\end{array}$ & $\begin{array}{c}\text { Extrapolated } \\
\text { "zero-fluence" } \\
\text { intercept }\end{array}$ & $\begin{array}{c}\text { Intensity-dependent } \\
\text { absorption loss at 355-nm } \\
\left(\% / \mathbf{J} / \mathbf{c m}^{2}\right)\end{array}$ & $\begin{array}{c}\text { Quality of } \\
\text { linear fit }\left(\mathbf{R}^{\mathbf{2}}\right)\end{array}$ \\
\hline $1.1 \mathrm{~mm}$ Schott Borofloat & $91.5 \%$ & $96.2 \%$ & -1.0 & 0.878 \\
\hline $3.3 \mathrm{~mm}$ Schott Borofloat & $91.7 \%$ & $91.4 \%$ & -0.40 & 0.784 \\
\hline $1.1 \mathrm{~mm}$ Schott D263 & $84.8 \%$ & $86.8 \%$ & -1.97 & 0.996 \\
\hline $0.4 \mathrm{~mm}$ Corning 0211 & $89.8 \%$ & $84.7 \%$ & -0.30 & 0.905 \\
\hline $0.9 \mathrm{~mm}$ Hoya NA-35 & $91.2 \%$ & $89.3 \%$ & -0.02 & 0.797 \\
\hline
\end{tabular}

Extraction of non-linear absorption coefficient

In order to predict material performance for different irradiance pulse shapes, we must extract the non-linear absorption coefficient (_) from the raw data. To do this, both the temporal and spatial profiles of the incident laser pulse must be taken into consideration. Following Smith ${ }^{2}$, the change in intensity, I, propagating in the z-direction through an optic with one- and two-photon absorption coefficients $\alpha$ and , and one-and two-photon-induced solarization $s_{1}$ and $s_{2}$ respectively is described by equation 1 :

$$
d I / d z=-\alpha I-\beta I^{2}-s_{1} I-s_{2} I
$$

Ignoring the solarization terms, the total transmittance through thickness $\mathrm{L}$ is becomes:

$$
T=T_{L} T_{N L}=I(z) / I_{I N C}
$$

where $I_{i n c}$ and $I(z)$ are the incident and transmitted intensity, respectively, and the linear transmission factor, $T_{L}$ and the nonlinear transmission factor, $\mathrm{T}_{\mathrm{NL}}$, are defined as follows:

$$
\begin{gathered}
T_{L}=(1-R)^{2} e^{-a z} \\
T_{N L}=(1+Q)^{-1} \\
Q=\beta(1-R) I_{I N C}\left(1-e^{-\alpha z}\right) / \alpha
\end{gathered}
$$

and

where $\mathrm{R}$ is the Fresnel surface reflectance. Then for a flattop -square pulse incident beam, such as we have on OSL,

$$
1 / T=1+\beta I_{I N C} z
$$

and the slope of a plot of $1 / T$ vs. incident intensity yields the non-linear absorption coefficient, , directly. For a flat-square spatial and temporal profile, the total transmittance, $\mathrm{T}_{\mathrm{F}}$, can be rewritten:

$$
T_{F}=T_{L} \frac{1}{1+Q}
$$

In contrast, the total transmittance for a Gaussian-Gaussian spatial and temporal profile, $T_{G}$, is given by Smith ${ }^{2}$ as:

$$
T_{G}=T_{L} \sum_{n=1}^{\infty}(-Q)^{n-1} n^{-1.5} \quad \mathrm{Q}<1
$$

To convert the Gaussian beam data to the transmittance expected for a flat beam, we must calculate $Q$ and then multiply the measured transmittance by the ratio of $T_{F} / T_{G}$. Figure 6 shows results from such a calculation for the Corning 0211 zinc borosilicate sample. The excellent agreement between the flat beam and the Gaussian beam experiments gives us confidence that the non-linear absorption coefficients extracted using the small-beam Gaussian experiment setup are reliable.

To determine the non-linear absorption coefficient_for the remaining materials, we use the following algorithm. $T_{L}$ is known (measured). Guess, calculate $\mathrm{Q}$ and $\mathrm{T}_{\mathrm{G}}$. Minimize the difference between the measured and calculated transmittances, $\mathrm{T}$. The results from these calculations are shown in Figures $7 \mathrm{a}-\mathrm{c}$, and summarized in Table 3. For comparison, one- and two-photon absorption coefficients for BK7 and BK10 glasses are also included in Table 3.

Two different sample thicknesses were measured for one material (Schott Borofloat ${ }^{\circledR}$ ). Unfortunately, this was the only material which deviated substantially from the bulk absorption model, as is evident from the higher losses observed in the thinner (rather than the thicker) part as well as the deviation from linear fit in Fig. 7c. Although surface damage was not evident with the naked eye, it cannot be ruled out. Alternately, this particular glass is manufactured by a float process and it is possible that a thin surface contamination layer could have strong non-linear absorption. We have reported non-linear absorption coefficients for this material, but one can at best interpret this data as an upper bound. 


\begin{tabular}{|c|c|c|c|}
\hline Test Sample & $\begin{array}{l}\text { UV- } \\
\text { edge }\end{array}$ & $\alpha / \mathrm{cm}$ & $\begin{array}{l}\overline{\mathrm{cm} / \mathrm{G}} \\
\mathrm{W}\end{array}$ \\
\hline Schott Borofloat ${ }^{\mathbb{Q}}, 1.1 \mathrm{~mm}$ thick $^{1}$ & $4.3 \mathrm{eV}$ & 0.016 & 0.435 \\
\hline Schott Borofloat $^{(2)}, 3.3 \mathrm{~mm}$ thick ${ }^{\top}$ & $4.3 \mathrm{eV}$ & 0.032 & 0.110 \\
\hline Hoya NA-35, $0.9 \mathrm{~mm}$ thick & $4.4 \mathrm{eV}$ & 0.118 & 0.360 \\
\hline Corning $0211,0.4 \mathrm{~mm}$ thick ${ }^{2}$ & $3.9 \mathrm{eV}$ & 0.937 & 1.245 \\
\hline Corning $0211,0.4 \mathrm{~mm}$ thick & $3.9 \mathrm{eV}$ & 0.637 & 0.950 \\
\hline Schott D263, $1.1 \mathrm{~mm}$ thick & $3.9 \mathrm{eV}$ & 0.708 & 2.376 \\
\hline Schott $B K 7^{3}$ & $4.4 \mathrm{eV}$ & 0.036 & 0.006 \\
\hline Schott BK10 ${ }^{3}$ & $4.7 \mathrm{eV}$ & 0.007 & 0.0045 \\
\hline Schott BK10, antisolarant ${ }^{3}$ & $4.7 \mathrm{eV}$ & 0.013 & 0.01 \\
\hline
\end{tabular}

Table 3: UV-cutoff edge $\left(5 \mathrm{~cm}^{-1}\right)$, linear and non-linear absorption coefficients for test matrix are compared to literature values for BK optical glasses. 'Calculated from this work, 3-ns Gaussian beam. ${ }^{2}$ Calculated from this work, 3-ns Flat Pulse (OSL). ${ }^{3}$ from Smith ${ }^{6}$.
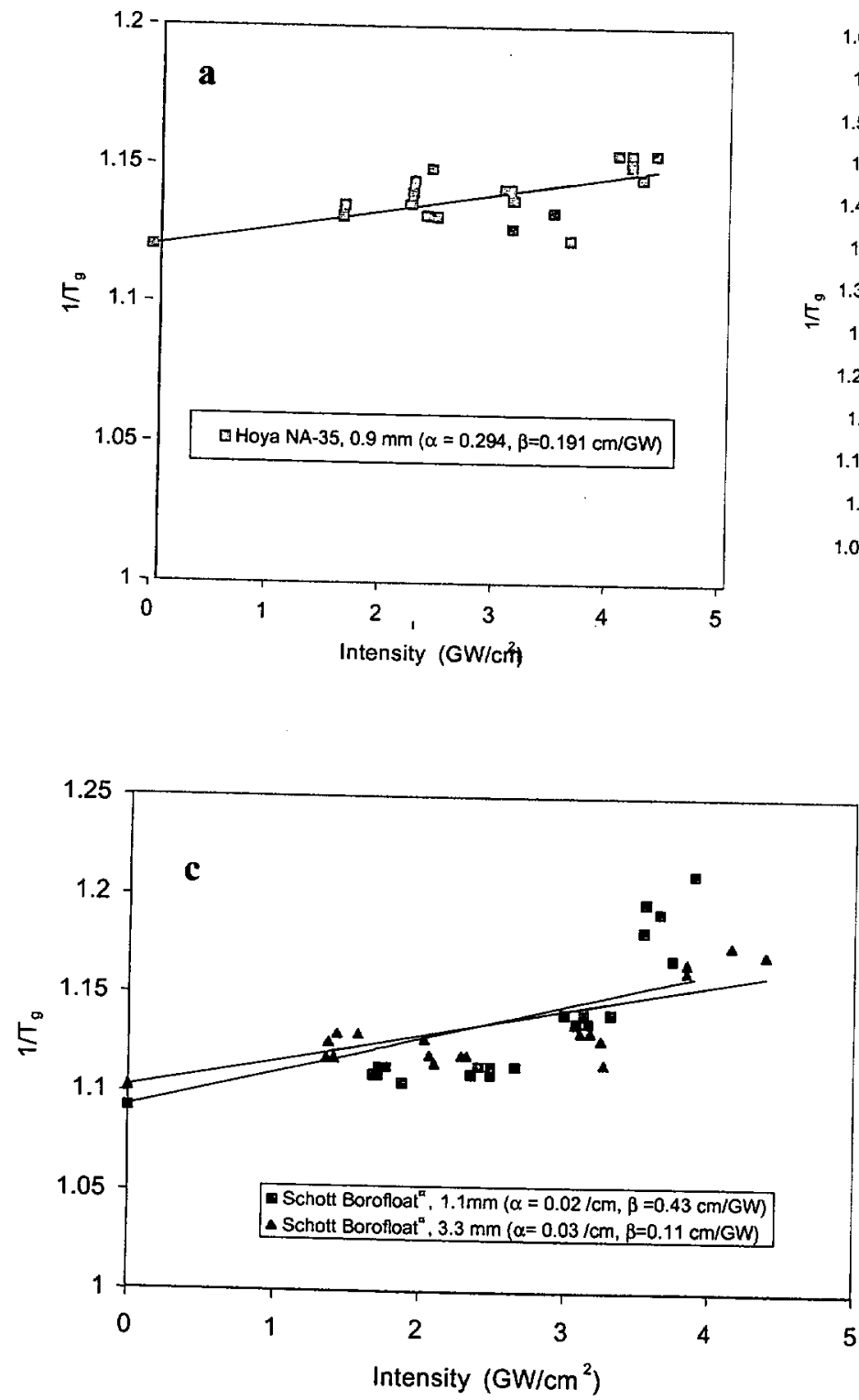

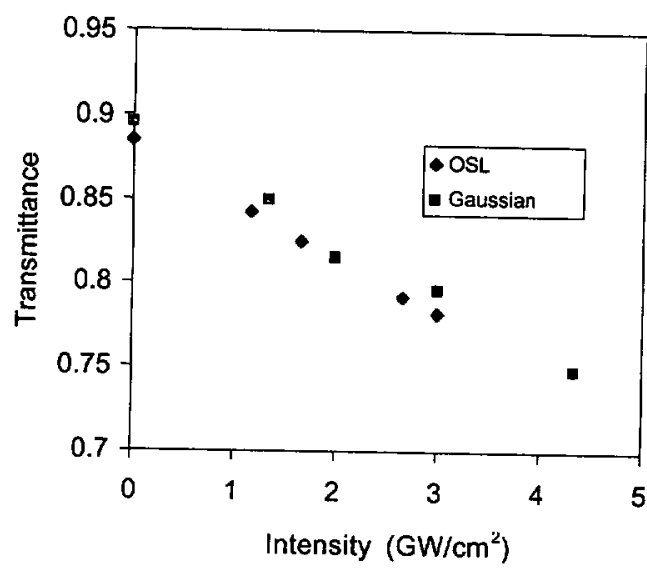

Figure 6. Comparison of Gaussian-Gaussian beam data converted to flat-square beam ( $\alpha=$ $0.94, \beta=1.24$ ) vs. data measured on OSL using flat-square beam for Corning 0211 borosilicate glass sheet.

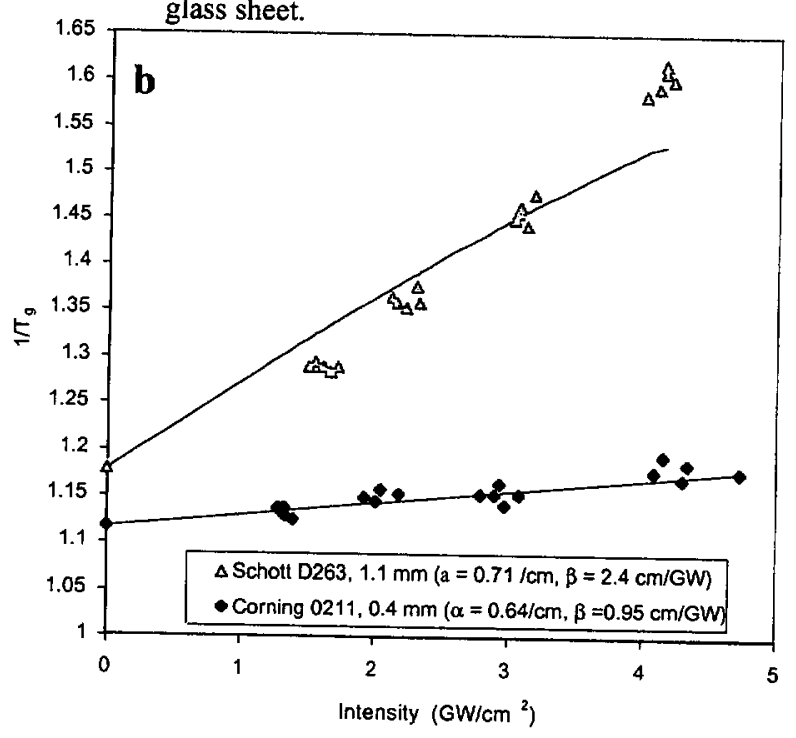

Figure 7: Transmission of 3-ns flat-square pulse through a) $0.9 \mathrm{~mm}$ Hoya NA-35 aluminoborosilicate; b) through $1.1 \mathrm{~mm}$ Schott $D 263$ and $0.4 \mathrm{~mm}$ Corning 0211 zinc borosilicate, and c) $1.1 \mathrm{~mm}$ and $3.0 \mathrm{~mm}$ Schott Borofloat ${ }^{\circledR}$ borosilicate. Lines represent regression fit to the transformed data; slope and intercept are 2-photon and 1photon absorption coefficients 


\section{DISCUSSION}

Published values for two-photon absorption in transparent dielectric materials, such as the borosilicates that were measured in this work, are typically $1-2$ orders of magnitude smaller than our reported non-linear absorption coefficients. In the case of the Schott Borofloat ${ }^{\circledR}$ material, it is clear that linear and non-linear absorption alone cannot explain our observed losses. In this case, it is suspected that surface loss is a dominating influence. But the remaining samples follow a linear fit with $1 / T$, suggesting linear and non-linear absorption is the dominating loss mechanism. Because we have good correlation between the flat and Gaussian beam experiments conducted in two different laboratories, it is unlikely that the measurements themselves are awry. Rather it is more likely that we are measuring a combination of two-photon and other excited-state absorption phenomena that follow the nonlinear absorption. The observation of sample discoloration ('browning') after a single shot is confirmation that at least some long-lived induced absorption is occurring.

To first order, non-linear absorption in our commercial borosilicates appears to be strongly correlated with linear absorption,

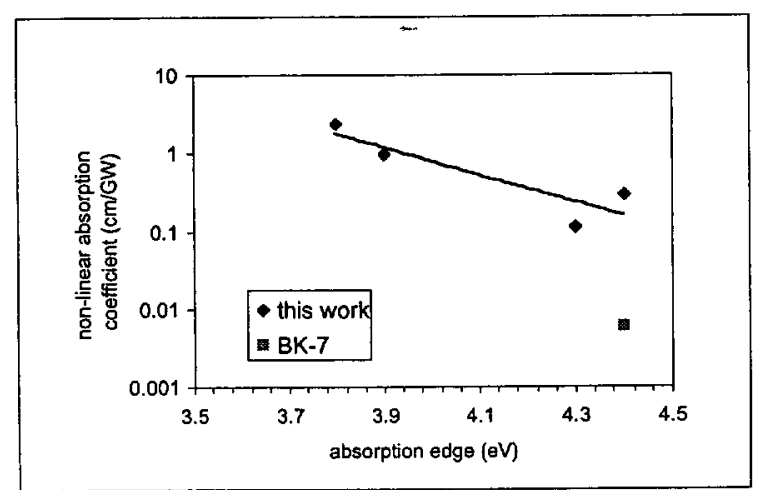

Figure 8. The non-linear absorption coefficient $\beta$ is strongly correlated with the UV absorption edge see Figure 8. Linear absorption is expected to follow the Urbach rule ${ }^{9}$ : the $\log$ of the absorption coefficient is proportional to the difference between the energy of the incident photon and the fundamental band gap. For all materials tested, the two-photon energy $(6.99 \mathrm{eV})$ exceeds the UV-edge $\left(5 \mathrm{~cm}^{-1}\right)$, and two-photon absorption is energetically expected.

The two materials with highest non-linear (and highest linear absorption) are both zinc borosilicates. The complex interaction of melting conditions, photochemical reactions, structure, multivalent additives, and trace impurities which lead to induced absorption make it difficult to predict performance of these multi-component glasses a priori. Efimov ${ }^{10,1}$ investigated the role of iron impurities at concentrations up to $0.1 \%$ in multi-photon absorption and photoinduced changes in crown alkali silicate glasses such as Schott BK-7. The presence of arsenic or antimony can enhance solarization ${ }^{12}$ by

providing an effective trap for the ionized electrons produced for example by photo-ionization of "colorless" $\mathrm{Fe}^{2+}$ to the stronglyabsorbing $\mathrm{Fe}^{3+}$. The materials in this study are all used in optical or flat panel applications where visible absorption must be kept to a minimum. Hence, the concentrations of known colorants such as iron are at the ppm level, and the vendor-to-vendor variation that we see appears to be driven more by base glass composition and forming conditions than impurities per se. Indeed, Figure 9 shows no correlation of absorption with any of the common trace elements. Compared to pure amorphous silica, $\mathrm{B}$ and $\mathrm{Al}$ would be expected to lower the UV cutoff wavelength; $\mathrm{Ti}, \mathrm{Li}, \mathrm{Na}, \mathrm{K}$, and $\mathrm{Ba}$ would be expected to raise the UV cutoff (although much less effectively than $\mathrm{Fe}^{3+}$ ) and $\mathrm{Zn}$ should either raise or have no significant effect on UV-cutoff depending upon forming conditions and other constituents ${ }^{13}$.

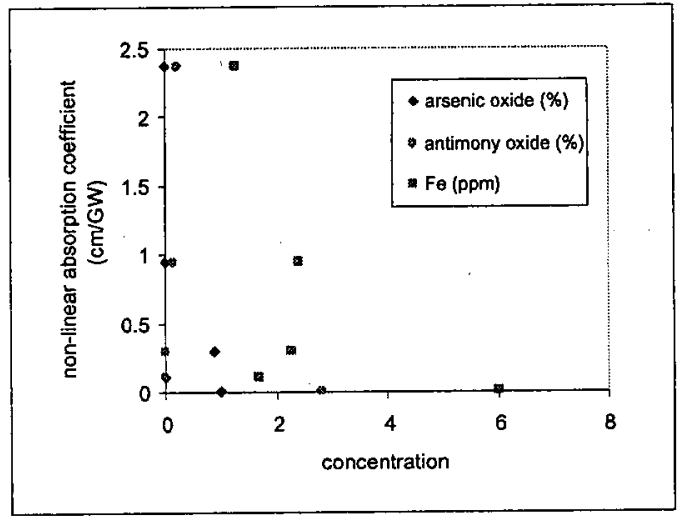

Figure 9. There is no strong correlation between non-linear absorption and $\mathrm{Fe}$ concentration with or without $A s$ and $S b$.

\section{CONCLUSIONS}

We have measured linear and non-linear absorption losses for commercial borosilicate glasses. Observed losses include linear and non-linear absorption as well as scatter from damage during the pulse. A strong correlation was found between linear absorption and transmittance at $351-\mathrm{nm}$ for laser intensities in the $\mathrm{GW} / \mathrm{cm}^{2}$ range. Several promising debris shield candidates have been identified for single (or a few) shot service at these high intensities. 


\section{ACKNOWLEDGEMENTS}

The authors would like to thank Terry Duewer for chemical analyses, Leslie Summers for linear transmission measurements, Greg Rogowski for procuring glass samples, Will House for interferometry measurements.

This work was performed under the auspices of the U. S. Department of Energy by University of California, Lawrence Livermore National Laboratory under Contract No. W-7405-ENG-48

\section{REFERENCES}

1. E. W. Van Stryland and L.L. Chase, "Two-photon absorption in solids," CRC Handbook of Laser Science and Technology Supplement 2: OpticalMaterials, M. Weber (ed.) CRC Press, Boca Raton, FL) pp.299-328, 1995.

2. W. L. Smith, "Two-photon absorption in condensed media," CRC Handbook of Laser Science and Technology Vol. III. Optical Materials Part 1: Nonlinear Optical properties/radiation damage, M. Weber (ed.) CRC Press, Boca Raton, FL) pp. 229-258, 1986.

3. A. Bishay, "Radiation induced color centers in multicomponent glasses," J. Non-Crystalline Solids 3, pp. $54-114$ (1970).

4. E. J. Friebele and D. L. Griscom, Treatise on Mateials Science and Technology, Vol. 17, M. Tomozawa and R. H. Doremus, eds. (Academic Press, New York) p 257 - xx. (1979). 5. D. L. Griscom and E.J. Fiebele, "Effects of ionizing radiation on amorphous insulators," Radiation Effects 65, pp. 63 -
72(303-312), 1982.

6. W. L. Smith, "Nonlinear optical measurements," LLNL Laser Program Annual Report, UCRL-50021-82, pp. 7-34-38, 1982.

7. T. S. Izumitani, Optical Glass, Am. Institute of Physics (New York) 1986.

8. Whitman, P., Milam, D., Norton, M., Sell, W., "Loss of Transmittance in Fluoropolymer Films Due to Laser-induced Damage at 1053 and $351 \mathrm{~nm}$," Laser-Induced Damage in Optical Materials: 1997, SPIE Vol. 3244, 528-534 (1998).

9. F. Urbach, "The long wavelength edge of photographic sensitivity and the electronic absorption of solids," Phys. Rev. 92, pp 1324, 1953.

10. O.M. Efimov, L. B. Glebov, Yu. A. Matveev and A. M Mekryukov, "Photo-induced changes of silicate glasses optical parameters at multi-photon laser radiation absorption," Laser-Induced Damage in Optical Materials: 1945, SPIE Vol. 2428, 34 - 41 (1995).

11. O.M. Efimov, Yu. A. Matveev and A. M Mekryukov, "Influence of impurity ions on nonlinear coloration of alkalisilicate glasses," Laser-Induced Damage in Optical Materials: 1995, SPIE Vol. 2714, 22 - 30 (1996).

12. L. M. Cook and S. E. Stokowski, CRC Handbook of Laser Science and Technology Vol IV: Optical Materials Part 2: Properties, M. Weber (ed.) CRC Press, Boca Raton, FL) pp. 143-146, 1986.

13. K.H. Sun, J. Am. Ceram. Soc., 30 pp. 227, 1947. 


\title{
Initiation, Growth and Mitigation of UV Laser Induced Damage in Fused Silica
}

\author{
A.M. Rubenchik M.D. Feit
}

\begin{abstract}
Laser damage of large fused silica optics initiates at imperfections. Possible initiation mechanisms are considered. We demonstrate that a model based on nanoparticle explosions is consistent with the observed initiation craters. Possible mechanisms for growth upon subsequent laser irradiation, including material modification and laser intensification, are discussed. Large aperture experiments indicate an exponential increase in damage size with number of laser shots. Physical processes associated with this growth and a qualitative explanation of selfaccelerated growth is presented. Rapid growth necessitates damage growth mitigation techniques. Several possible mitigation techniques are mentioned, with special emphasis on $\mathrm{CO}_{2}$ processing. Analysis of material evaporation, crack healing, and thermally induced stress are presented.
\end{abstract}

\section{INTRODUCTION}

The damage of optical elements is one of the main concerns for builders of large laser systems. The problems are especially severe for UV optics due to the more efficient interaction of high-energy photons with optical materials. Powerful laser light initiates a breakdown in fused silica and ionized material effectively absorbs laser radiation. This absorbed energy generates high temperatures and pressures and modifies and destroys the fused silica. The spatial extent of damage increases with repetitive pulses so that finally the optic becomes nonfunctional due to large obscurations or mechanical failure.

In the present paper, we discuss the physical phenomena associated with damage initiation and growth in fused silica, as well as methods to mitigate growth. Typically, laser damage studies deal with damage threshold [1]. However, the useful optics lifetime is determined mainly by the damage growth rate with repetitive pulses. Even the observable damage threshold is strongly influenced by damage growth during the laser pulse. As a result, much of our focus will be on damage growth.

First, we discuss the relations between intrinsic and defect-related damage. What kind of defects can initiate damage and how are they related to the polishing process? Laser absorption in defects and the surrounding matrix generates high pressure and temperature and produces a microexplosion. We will estimate the absorbed energy density and generated pressure and will evaluate the crater size, and extent of damage.

Damage sites continue to grow with repetitive pulses. We will discuss the growth mechanisms and experimental data on growth rate of damage spots. A qualitative explanation of the observed self-accelerated damage growth will be presented.

Optics lifetime extension requires mitigation of damage growth. Among the few mitigation schemes explored, mitigation by $\mathrm{CO}_{2}$ laser radiation looks most promising. We will analyze the physics of this mitigation process and will discuss the optimal mitigation schemes

\section{INTRINSIC AND DEFECT-RELATED DAMAGE}

Optical damage is produced by laser energy deposition in nominally transparent material. The photon energy is smaller than the bandgap and, hence, laser damage is intrinsically a nonlinear process. The physics of damage is evident for the case of ultra-short laser pulses[2]. If the laser pulse duration is shorter than the electron-phonon scattering time of about $10 \mathrm{ps}$, heated electrons have no time to transfer energy to the lattice during the laser pulse. Again because the pulse is so short, a large number of seed electrons is necessary for dielectric breakdown to occur. These can be produced by multiphoton ionization. Oscillating in an intense laser field, the electrons 
collide with atoms and are heated. When the electron energy exceeds the bandgap a new electron is produced by impact ionization and an electron avalanche is initiated. For short pulses, the only factor limiting development of the avalanche is the pulse duration.

To produce macroscopic damage, one must deposit a minimal energy per atom on the order of the lattice binding energy. Practically, one considers the optical material to be at the threshold for damage when the density of free electrons reaches the plasma critical density, $10^{21} \mathrm{~cm}^{-3}$ for $1 \mu \mathrm{m}$ light. At the critical density, laser light absorption becomes very efficient, so the deposited density of laser energy is extremely high -sufficient to produce macroscopic damage. Ultimate manifestations of damage-material melting and ejection, crack formation, etc. takes a much longer time to form. Typically, they are produced well after the USLP pulse termination.

Because the processes determining threshold are intrinsic to the material, the threshold fluence for USLP is well defined, reproducible, and independent of spot size.The physics of damage initiation is much different for pulse durations well above $10 \mathrm{ps}$. In this case the energy electrons gain through collisions with atoms can be balanced by the energy transfer from electrons to the lattice. To keep an electron avalanche going the energy gain must overcome losses giving the criteria for avalanche existence [2]

$$
\sigma \mathrm{E}^{2}>\gamma \mathrm{U}_{\mathrm{ph}}
$$

Here $E$ is the laser electric field and $U_{p h}$ is the typical phonon energy. The electrical conductivity, $\sigma$, and the rate of energy transfer to the lattice, $\gamma$, are taken for electron energy near the bottom of the conduction band. The electron collisional rates and energy transfer rates in fused silica have been measured experimentally [3]; for $1 \mu \mathrm{m}$ light the critical intensity $I_{d}$ to establish the avalanche is estimated as $80 \mathrm{GW} / \mathrm{cm}^{2}$ [2]. If we assume the Drude formula for the conductivity for $355 \mathrm{~nm}$ light, we find $I_{d}=150 \mathrm{GW} / \mathrm{cm}^{2}$. The above estimate is only a lower estimate for the intrinsic damage threshold of long pulses, since other possible energy losses are disregarded.

From Eq.(2.1), it follows that the intrinsic threshold fluence is proportional to pulse duration $\tau$. This result is in strong disagreement with multiple experimental data [4] which demonstrates a scaling $F_{\text {th }}-\tau^{x}, 0.3<x<0.5$. The intrinsic threshold fluence at $355 \mathrm{~nm}$ for a $3 \mathrm{~ns}$ pulse according to the above estimate is about $450 \mathrm{~J} / \mathrm{cm}^{2}$, at least 50 times higher than the typical threshold fluence observed in experiments.

These facts indicate that the threshold for long pulses is actually determined by defects in the nominally transparent material. The occurrence of damage depends on the probability of finding suitable defects so that the damage probability now increases as the laser spot size increases. For small laser spots, the threshold fluence can
be very high, close to the intrinsic threshold.

As a result of the probabilistic nature of defect caused damage, the threshold fluence is not an adequate characteristic of the material. A more useful characteristic, which can be assumed independent of spot size and is a function of the material only is the density of defects damaged at given fluence $n(F)$ [5]. Typically, the dependence on fluence is a power law where $\mathrm{k}$ is typically $\sim 8-12$.

$$
\mathrm{n}(\mathrm{F})=\mathrm{bF}^{\mathrm{k}}
$$

Materials are available for modern large optics in which the optical quality is high enough that bulk damage is either rare or completely absent compared to surface damage. Most of the observed damage is concentrated near the optics surface and, consequently, associated with defects arising from the polishing process. More prevalent and dangerous is the rear (exit) surface damage. For front side damage, plasma produced by the laser pulse shields the material and reduces material destruction. For rear side damage, the absorption zone moves toward the beam and laser energy is deposited inside the optical material. The confinement of the plasma produced increases the generated pressure and increases material destruction [6]. As a result, rear side damage is more
visible and dangerous.

\section{INITIATORS AND POLISHING PROCESS.}

Damage in fused silica is typically localized on the surface and is related to the polishing process. Contributions to the damage initiators potentially include distributed point defects, residual micro-inclusions produced by the polishing process and microcracks generated during the polishing process. For high quality polishing, the thickness of material modified by polishing does not exceed $1 \mu \mathrm{m}$ [7].

First, let us evaluate the role of point defects. To damage material one must heat it up to some high temperature $\mathrm{T}_{0}$. At high temperature, the bandgap collapses and the material starts to absorb laser light, thereby initiating a 
thermal explosion. The exact value of $\mathrm{T}_{0}$ is not known, but the results are not sensitive to this value. We will use, for estimates, $T_{0} \sim 2000^{\circ} \mathrm{C}$. During a nanosecond pulse, thermal conduction is negligible and the local temperature increase can be estimated from the energy balance.

$$
\rho c \mathrm{~T}=\alpha \mathrm{F}
$$

For fluence $F=5 \mathrm{~J} / \mathrm{cm}^{2}$, to increase temperature by $T_{0}$ the absorption coefficient a must be larger than $300 \mathrm{~cm}^{-1}$. For homogeneously distributed defects the susceptive parts of material must have a high absorption and must look black. Fused silica can have a variety of point defects, see e.g. the review [8]. To estimate the number of defects needed we suppose that every defect can absorb $2 \mathrm{eV}$ of energy. This corresponds, for example, to non-bridging oxygen centers (NBOC). It is easy to see that to heat the material up to $T \sim T_{0}$ the density of defects $n$ must be about $10^{22} \mathrm{~cm}^{-3}$. This density is too high to be real, and we can conclude that distributed point defects can not be responsible for the observable damage. It is realistic if the defects are combined into small clusters or if some small inclusions are present in the material. Such an absorber can be, for example, ceria microparticles, which are commonly used in the polishing process. The energy absorbed by a small particle with radius a much smaller than the laser wavelength is $Q=\alpha I \pi a^{2}$. The absorption efficiency $\alpha=\sigma / \pi \mathrm{a}^{2}$ is given by expression [9]

$\alpha=\frac{12 n \omega a}{c} \operatorname{Im}\left[-\frac{n^{2}}{\varepsilon+2 n^{2}}-\frac{1}{6}+\frac{1}{2(k a)^{2}}-\frac{\operatorname{Cot}(k a)}{2 k a}\right] \approx-\frac{12 n \omega a}{c} \operatorname{Im} \frac{n^{2}}{\varepsilon+2 n^{2}}$

Here $n=1.5$ is the refractive index of the glass matrix and $\varepsilon$ is the dielectric constant of the particle. The ceria particle refractive index at wavelength $355 \mathrm{~nm}$ is $\sqrt{\varepsilon}=2+0.2 \mathrm{i}[10]$. For this case $\alpha=0.45 \omega \mathrm{a} / \mathrm{c}=0.008 \mathrm{a} \mathrm{nm} \mathrm{n}^{-1}$ Due to the small particle size the temperature distribution can be treated as stationary. For linear thermal conduction, the temperature $\mathrm{T}_{0}$ near the particle surface is given by the formula

$$
\mathrm{T}_{\mathrm{O}}=\frac{\alpha I a}{4 \kappa}
$$

A plot of the ceria particle temperature as a function of the inclusion radius a is presented in Fig. 1 for laser intensity $3 \mathrm{GW} / \mathrm{cm}^{2}$ and thermal conductivity $\kappa=0.02 \mathrm{~W} / \mathrm{cmK}$. The temperature dependence of the thermal conductivity doesn't change the situation qualitatively even for very small inclusions, and particles a few tens of nanometers in size can initiate damage in fused silica.

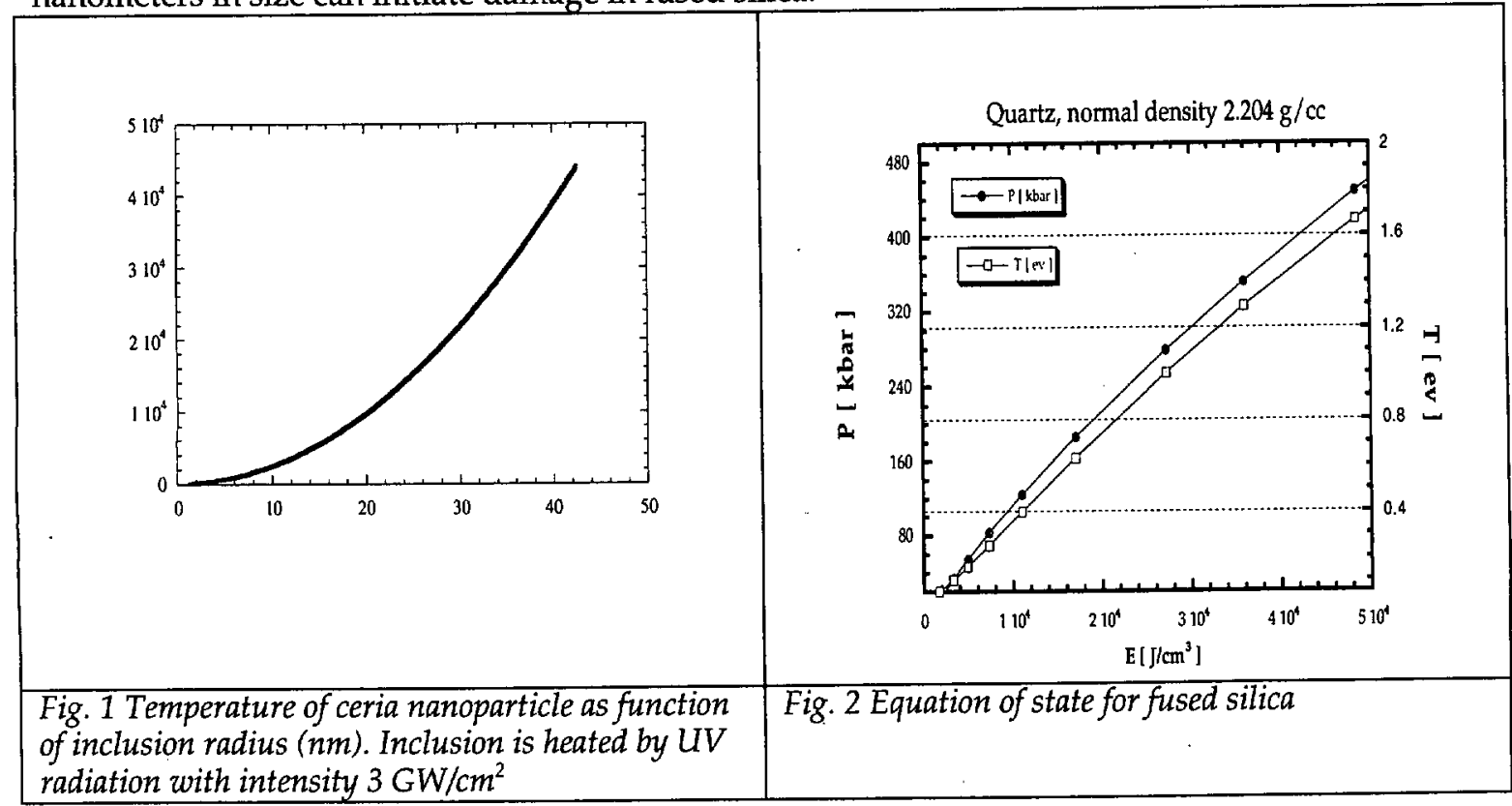


The idea of nanoparticles being the main cause for damage initiations has been discussed in many publications; see e.g.[11]. At first glance, the idea is inconsistent with experiments. From Eq.(3.2) one can see that the threshold fluence for a fixed size particle is expected to be proportional to the pulse duration. As we discussed above, the experimentally observed threshold fluence varies with pulse duration as a fractional power between 0.3 and 0.5 [4]. The contradiction can be resolved if we assume that the material has an ensemble of microinclusions of various sizes. It is possible to show that for a given pulse duration, the most dangerous inclusions are of a size comparable to the thermal diffusion length $1 \sim \sqrt{ } \mathrm{D} \tau[12,13]$. Detailed calculations result in pulselength dependence of threshold consistent with experiment [13].

Damage initiation is often associated with microcracks. We can present several arguments to suggest why this is so. First, microcracks can effectively trap inclusions, thereby protecting them during the cleaning procedure. Second, microcracks can produce field enhancements both as a result of electrostatic enhancement originally pointed out by Blombergen [14] and by interference of light reflected and scattered by cracks [15]. Also, the presence of cracks weakens the material. The deposition of the same amount of energy in the weakened material can produce damage of a greater extent and, hence, more noticeable. Experiments by Hamza et al with artificial cracks demonstrate a direct correlation between the drop in damage threshold and the magnitude of material cracking [16]. Consequently, an improvement in the polishing procedure, e.g., reducing the number of microcracks by gentler polishing, can reduce the initiator density substantially [17].

\section{THERMAL EXPLOSION AND FIREBALL GROWTH}

When the temperature around the inclusion reaches a critical value, a thermal explosion takes place. This involves the rapid expansion of the heated region into the glass, which is then ionized. It occurs because the plasma produced by the initially absorbed light radiates UV, which is strongly absorbed in the matrix resulting in heating and an increase in the absorption coefficient of the glass. The situation is very similar to a laser-supported ionization wave, with the main difference being that for a small inclusion the ionization front is spherically symmetric. In the following, we assume the Drude model for the dielectric coefficient of the plasma:

$$
\varepsilon=1-\frac{n_{e}}{n_{c}\left(1+\frac{i v}{\omega}\right)} \approx-\frac{n_{e}}{n_{c}}\left(1-\frac{i v}{\omega}\right)
$$

where $n_{e}$ is the electron density, $n_{c}$ is the critical density, and $v$ is the electron collision frequency.

Consider the situation having the plasma density in a fireball close to critical and the electron scattering rate smaller than the light frequency. We assume that most of the absorbed energy is spent ionizing the material and only small part of the absorbed energy is spent heating the material. The rate of growth of the plasma ball can be
estimated from energy balance:

$$
n_{e} I_{0} 4 \pi a^{2} \frac{d a}{d t}=\sigma I(t)
$$

Here $I_{0}$ is the ionization potential and $n_{e}$ is the electron density. If one uses only the first term in Eq (3.1) for the $\mathrm{G}$ is given by

$$
G=10 \frac{F \omega}{n I_{0} c} \operatorname{Im} \frac{1}{\varepsilon+2}
$$

The growth factor is independent of pulse duration and is determined by the fluence only. Let us discuss the value of the electron density in the plasma fireball. The plasma temperature in this very dense plasma cannot be
higher than a few eV due to huge radiation losses. Experimental data of Carr et al [18] indicates a plasma temperature below $1 \mathrm{eV}$ for fluences of about $10 \mathrm{~J} / \mathrm{cm}^{2}$. The equilibrium electron density at this temperature, absorption. 
The additional non-equilibrium ionization is produced by laser radiation. Free electrons oscillate in the laser electric field, gain energy due to collisions and produce new free electrons. The ionization rate by electron impact is given by

$$
\frac{d n_{e}}{d t}=\alpha I n_{e}
$$

We will take the coefficient $\alpha$ from ref[2], for fused silica $\alpha \sim 10 \mathrm{~cm}^{2} / \mathrm{nscGW}$. One can see from the arguments presented in ref[2] that $\alpha$ for the dense cold plasma must have the same order of magnitude. For laser intensity $\mathrm{I}=2 \mathrm{GW} / \mathrm{cm}^{2}$, the ionization time is about $50 \mathrm{ps}$. We see that the impact ionization can easily increase the electron concentration during the pulse up to the critical value. Electron density cannot greatly exceed the critical density value. In this case, the absorption depth becomes smaller then the fireball radius and the absorption efficiency drops. For absorption at $3 \omega$, a solid state density even about ten times critical, ionization potential of $10 \mathrm{eV}$, fluence $F$ of $10 \mathrm{~J} / \mathrm{cm}^{2}$, and scattering rate 0.5 of the optical frequency, the growth factor $\mathrm{G}(4.3)$ is about 10 . Taking into account the growth of absorptivity with increase of radius, we can say that if plasma formation starts, the plasma ball will rapidly grow to a size for which $k a>1, k=\frac{\omega}{c} \sqrt{\varepsilon}$. In this case ka $>>1$ while $k_{0} a<<1$ the absorption cross-section is

$$
\sigma=6 \pi a^{2} n \operatorname{Re} \frac{1}{\sqrt{\varepsilon}}
$$

which grows only proportionally to the geometric area. Using Eq. (4.5) for the absorption cross-section, we have

$$
a(t)=3.5 \operatorname{Re} \frac{1}{\sqrt{\varepsilon}} \frac{\int_{-\infty}^{t} I(t) d t}{n I_{0}}
$$

The plasma sphere radius at the end of the pulse is seen to be

$$
a=3.5 \operatorname{Re} \frac{1}{\sqrt{\varepsilon}} \frac{F}{n I_{0}}
$$

where $F$ is the fluence and $I_{0}$ the ionization energy.

For the parameters used above, this radius a is about $500 \mathrm{~nm}$, comparable with the size of wavelength. Now the strongly absorptive plasma ball shields its rear surface. There is no simple description for fireball absorption; Mie theory must be used to calculate it [19]. The plasma can expand toward the laser light from the front surface like the laser-supported detonation wave (LSDW). But the lateral losses due to thermal conduction and light scattering arrest the LSDW propagation. As a result the fireball doesn't grow larger then the laser wavelength.

We argued above that absorption of laser light by small particles can produce a plasma fireball with size comparable to the laser radiation wavelength $\lambda$. Such a fireball of size, a, will absorb almost all incident energy, so the energy of the fireball can be estimated as $\mathrm{E} \sim \mathrm{F} \pi \lambda^{2}$, where $\mathrm{F}$ is the laser fluence. The energy density in the fireball is about $F / 4 / 3 \lambda$. For a fluence of $F=5 \mathrm{~J} / \mathrm{cm}^{2}$ and $\lambda \sim 350 \mathrm{~nm}$, the total deposited energy is about $20 \mathrm{~nJ}$ and the energy density is $110 \mathrm{~kJ} / \mathrm{cm}^{3}$, approximately 10 times larger than the typical evaporation energy density $E_{e}$.

One can obtain a better estimate for the energy density in the fireball from a fireball temperature measurement [18]. It was found that the temperature of the fireball $10 \mathrm{~ns}$ after the pulse is about $0.5 \mathrm{eV}$. Due to the confinement by the surrounding material, the density in fireball is the same as in solid state. From the equation of state for 
fused silica presented in Fig.(2), one can find the energy density in the fireball and the generated pressure. The conse temperature corresponds to a pressure about $150 \mathrm{kbar}$ and energy density $15 \mathrm{~kJ} / \mathrm{cm}^{3}$, which is roughly consistent with estimate presented above taking into account that assumption of total energy absorption in the
fireball is clearly an overestimate.

\section{CRATER FORMATION}

After the pulse termination the absorbed energy is concentrated in a small fireball with energy density well above the evaporation energy. It means that the damage extent is determined by the total energy of the fireball, not the fireball size and structure. The problem of describing laser damage crater formation is similar to that for underground explosions or meteorite impacts, and we will use some of the ideas developed there [20].

The microexplosion creates a strong shock wave. After this shock wave passes, the resulting crushed material can be described as an incompressible liquid. The strength of the material is taken into account by assuming that the region of crushed material is bounded by the point at which the material velocity $v$ becomes smaller than a critical velocity, $c$. This velocity, $c$, can be estimated by $\rho c^{2}=G$ where $G$ is the characteristic "strength" of the material. For example, the compressive strength of fused silica, $\mathrm{G}=1 \mathrm{GPa}$, corresponds to velocity $\mathrm{c}=670 \mathrm{~m} / \mathrm{s}$,
much less than the sound speed $5.8 \mathrm{~km} / \mathrm{s}$.

Before presenting specific results, we make some general comments based on scaling. The radius, $R$, of a crater $\mathrm{G}$ and $\rho$. The most general relation between these parameters is of the form

$$
\mathrm{R}=\mathrm{hf}\left(\mathrm{E} / \mathrm{Gh}^{3}\right)
$$

where $f$ is a function which needs to be determined from modeling or experimental data. Thus, for craters with the same $\mathrm{R} / \mathrm{h}$ ratio, the crater size will be proportional to $\mathrm{E}^{1 / 3}$. The scaling law actually observed in experiments the scaling works over 4 orders of between 1/3 and 1/4 [20,21]. Fig.(3) adapted from [19] demonstrates that craters for high-energy explosion is due to the in explosion energy. The deviation from $\mathrm{E}^{1 / 3}$ scaling in explosion (5.1) indicates also the role of material to the effect of gravity which is insignificant for laser damage. Formula deposit less energy. We recently devere same crater in weaker material one needs to fits well the experimental data (see Fig (3) a simple hydrodynamic description of crater formation [22], which maximal burial depth $h_{d}$ for which a surfat maximum size,

$$
h_{m}=\frac{h_{d}}{3^{3 / 4}} \approx 0.44 h_{d}
$$

Crater radius as function of charge depth is given by the expression [22]

$$
R^{2}=h^{2 / 3}\left(h_{d}^{4 / 3}-h^{4 / 3}\right)
$$

The maximal crater radius is given by

$$
R_{m}=\sqrt{2} h_{m} \approx 0.6 h_{d}
$$

At high explosion energy or for shallow absorbers, the crater radius increases with burial depth as $h^{1 / 3}$

$$
R=\left(h_{d}^{2} h\right)^{1 / 3}
$$

In Fig. 3 we compare the scaling predicted by Eq.(4.3) with experimental data for strong explosions. The value of the maximum depth for which the crater would just open, was used as the only adjustable parameter. We see
good correlation with the experimental data. 


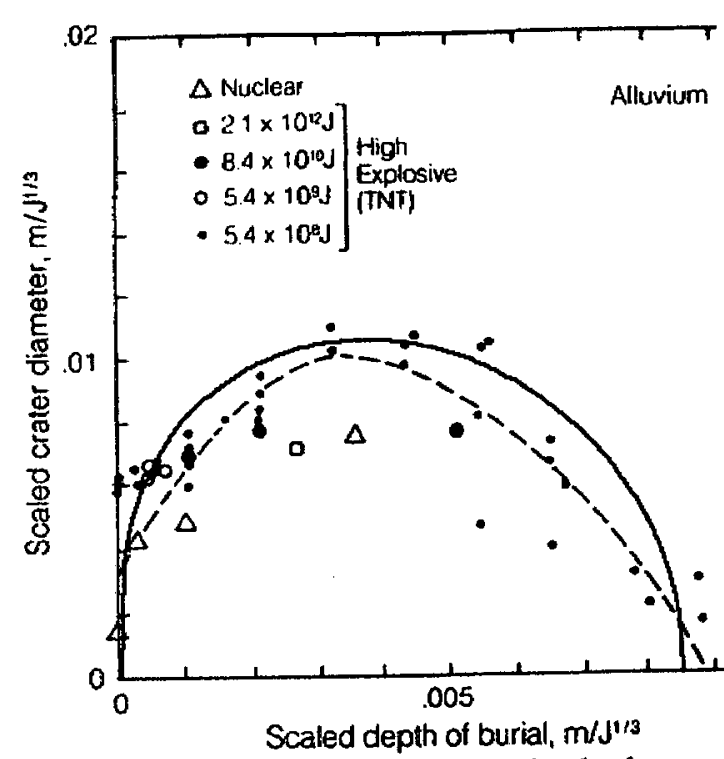

Fig. 3 Explosion crater diameter vs. depth of burial in alluvium at the Nevada Test Site [21]. This figure exemplifies $E^{1 / 3}$ scaling and indicates the importance of the depth of burial for explosions. The dashed line, drawn by the author, is to aid the eye. The solid line is the plot of crater diameter given by theory.

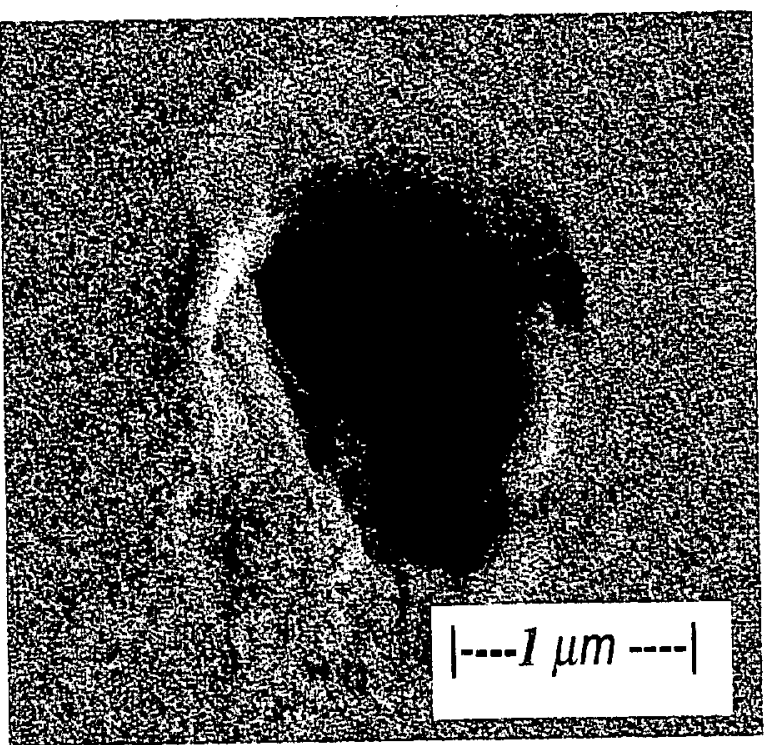

Fig. 4 Damage crater initiated at low fluence

We can derive an idea of the size of the various terms above for the laser damage regime by considering some typical values for fused silica. We take the laser fluence of $5 \mathrm{~J} / \mathrm{cm}^{2}$, and the absorbed energy $\mathrm{F} \pi \lambda^{2} \sim 15 \mathrm{~nJ}$. We can also apply the experimental explosion data from Fig. (3) laser damage regime. To use these data, we must take into account that glass is much stronger then alluvium, with $\mathrm{G} 1 \mathrm{GPa}$, so we rescale the energy according to Eq.(5.1). Taking the experimental value of $2 \mathrm{MPa}$ for the strength of fractured rocks [20], we get $h_{d} \sim 2 \mu \mathrm{m}$ and $R_{m} \sim$ $1.2 \mu \mathrm{m}$. The usual estimate of the thickness of the layer modified by polishing process is $<1 \mu \mathrm{m}$, and it means all craters must be wide open.

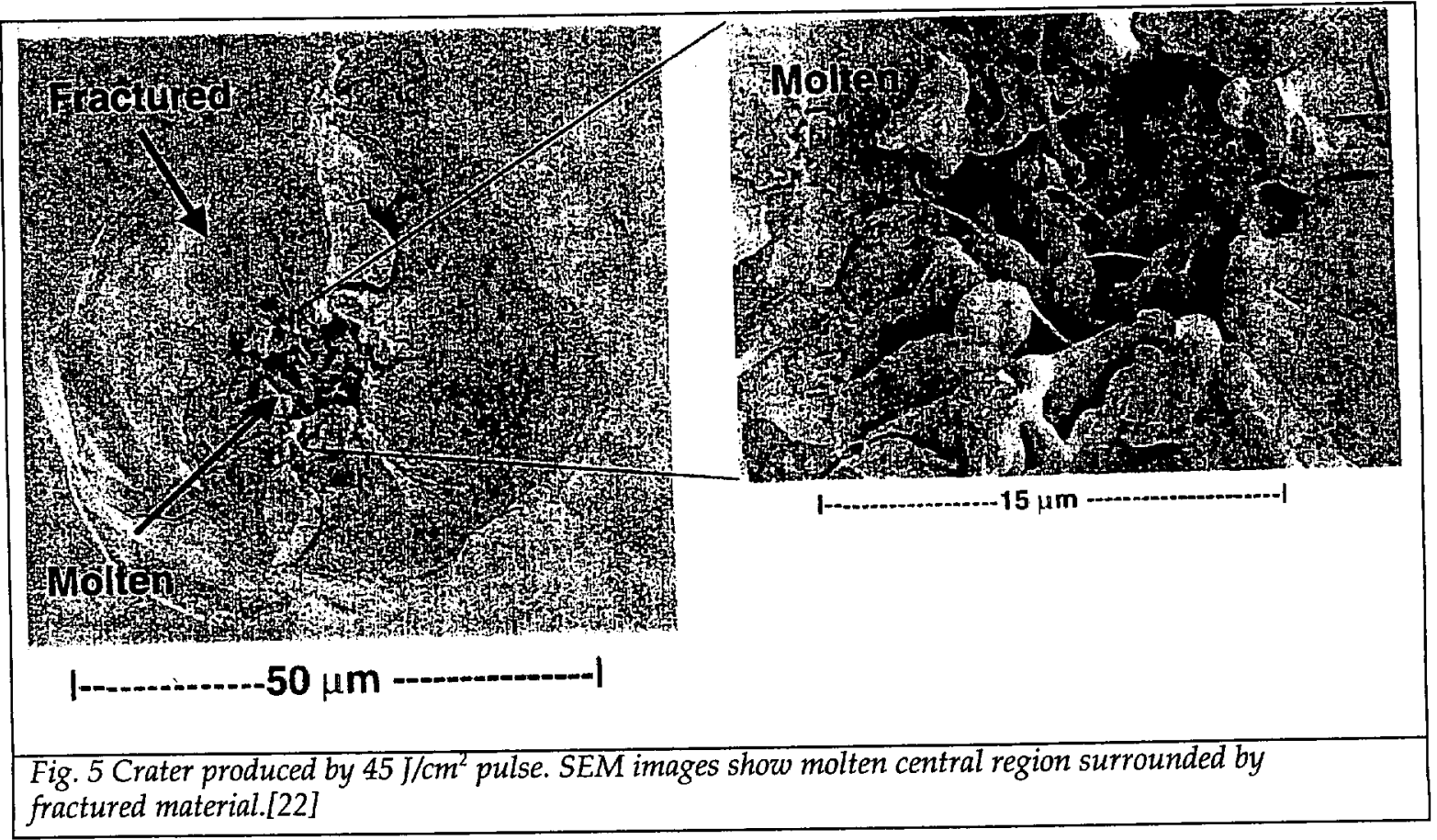


This estimate is consistent with size of crater presented in Fig. 4 , produced by a low fluence shot. The situation is different for damage produced at high fluences. The image of such a crater is presented in Fig. 5 One can see that the size of the crater is 20-30 $\mu \mathrm{m}$, inconsistent with above estimates. Also, the morphology of the craters are different, the material around the crater produced by the powerful shot is cracked and modified. The discrepancy
can be explained by the modification of fused silica by the strong shockwave.

It has been found experimentally that propagation of strong shocks in glass are followed by a slower moving "failure wave" [23]. After passage of the failure wave, the glass, under the effect of high pressure, is densified, modified in structure, lower strength and crushed. This process occurs more rapidly than excavation by MPa in the estimate above. In this case ther intensities it would be natural to use the strength of crushed rock of 2 which agrees with experiment. This suggests a practical formula for estimates of crater size. If we estimate the energy absorbed by the fireball as
$\mathrm{E}=\mathrm{F} \pi \lambda^{2}$ and use the experimental data from Fig.(3), the crater diameter can be given as

$$
d=\left(F \pi \lambda^{2} \frac{G_{a}}{G}\right)^{1 / 3}
$$

Here, all lengths are in $\mathrm{cm}, \mathrm{F}$ is in $\mathrm{J} / \mathrm{cm}^{2}, G$ is the effective strength of silica, and $G_{a}$ is the strength of alluvium. It is not clear now at what fluence the transition from one type of crater to another takes place. Is the boundary sensitive to pulse duration and absorber size? We will argue later that this question is important for estimates of
the damage growth by the repetitive pulses.

Self-consistent, comprehensive modeling of crater formation must include various physical effects including the laser interaction with plasma fireball, fireball expansion, phase transformations in surrounding materials, the flow of crushed material, etc. The existent modeling includes only a part of the important physics $[11,24]$. Nevertheless, the modeling is able to catch some essential features of crater formation.

Experimentally, the possibility of initiating damage at a nanoparticle was demonstrated in experiments [25,16]. In the experiments of ref.[25] tiny gold nanoparticles with diameters about $5 \mathrm{~nm}$ were attached to the surface of $\mathrm{SiO}_{2}$ material and then were coated by $60 \mathrm{~nm}$ of deposited $\mathrm{SiO}_{2}$. The material was irradiated by $351 \mathrm{~nm}$ pulses with $\mathrm{J} / \mathrm{cm}^{2}$. According to our estimate Eq.(3.2) the temperature on the surface of the gold nanoparticle must reach
$3200^{\circ} \mathrm{C}$, a temperature sufficient to start the thermal explosion. The crater Eq.(4.6); for a fluence of $7.2 \mathrm{~J} / \mathrm{cm}^{2}$ the lateral cramal explosion. The crater size is smaller than predicted by depth $(60 \mathrm{~nm})$ can explain it. According to Eq.(4.5), the lateral sizly bigger than $200 \mathrm{~nm}$. Probably the small burial from experiment. An additional factor can be the short pulse duration, which to be about $350 \mathrm{~nm}$, not very far experiments of ref.[16] the nanoparticles were short pulse duration, which can affect the fireball growth. In the were about $1 \mu \mathrm{m}$ in size, in agreement with the many times larger than the initial nanoparticle confirms the parts. The experiments with craters formed which are presented above-formation and growth of a plasma firs the pattern of damage induced by nanoparticles observed crater.

\section{DAMAGE GROWTH}

The density of defects in modern optics is very low. So the appearance of a few small craters doesn't appreciably affect optic performance. Unfortunately, the damage spots grow larger with consequent shots and finally the obscuration exceeds a specification limit or physical integrity will be destroyed. To estimate the useful optics lifetime, one must know the damage growth rate and factors affecting the growth. The key question to answer is
why does the damage spot grow at all?

To have any modification of the damaged site by a new pulse, the absorption of laser radiation must take place. The material around the crater can be crushed, and strongly scatter light, but microscopic pieces of the original material has the same bandgap as the bulk material and are unable to absorb the light. We still are very far from
a full understanding of light interaction with the damaged material. Below we list several mechanisms we think
most likely to explain the absorption in the damage spot. most likely to explain the absorption in the damage spot. 
Heating of fused silica stimulates the loss of oxygen. Oxygen depleted material can efficiently absorb UV radiation. In Fig.(6) we show the absorption coefficient for $\mathrm{SiO}_{x}$ material as a function of oxygen depletion. One can see that oxygen depletion results in high absorption efficiency. For example, the absorption of a $3 \mathrm{~ns} 5 \mathrm{~J} / \mathrm{cm}^{2}$ laser pulse in a $1 \mathrm{~nm}$ film of $\mathrm{SiO}$ embedded in a glass matrix produces a local temperature increase over $5000 \mathrm{~K}$. This effect suggests an explanation for the difference in damage growth in vacuum and air observed in experiments. It is easier for the oxygen to escape in vacuum so the damaged material must be more absorptive. Unfortunately, to date, there is no conclusive evidence that the damage is related to oxygen deficiency in damage spots [26]. The experiment of ref[26] did not find a direct relation between the formation of sub-stoichiometric silica on the surface and an increase in damage vulnerability.

The high pressure generated by laser light absorption modifies fused silica [23]. Material is densified, and the structure changes. For example, the number of 3 and 4 member rings increases. The electronic structure also changes and the modified material can start to absorb UV. A layer of $20 \%$ densified material with thickness of a few microns was found in damaged spots by J.Wong et al [28]. Recently, an attempt to model this effect from first principles was carried out by A. Kubota et al [29]. Modification of fused silica due to the damage-induced shock wave was studied using the molecular dynamics method. Densification on the level of $20 \%$, consistent with experiment, was found. After that the electronic structure of the modified material was modeled and a shift of the absorption edge toward the longwave part of the spectrum was found (see Fig.7). The calculated shift is large enough to greatly enhance two photon absorption. This initial result demonstrates at least the possibility of strong absorption in the shock-modified material.

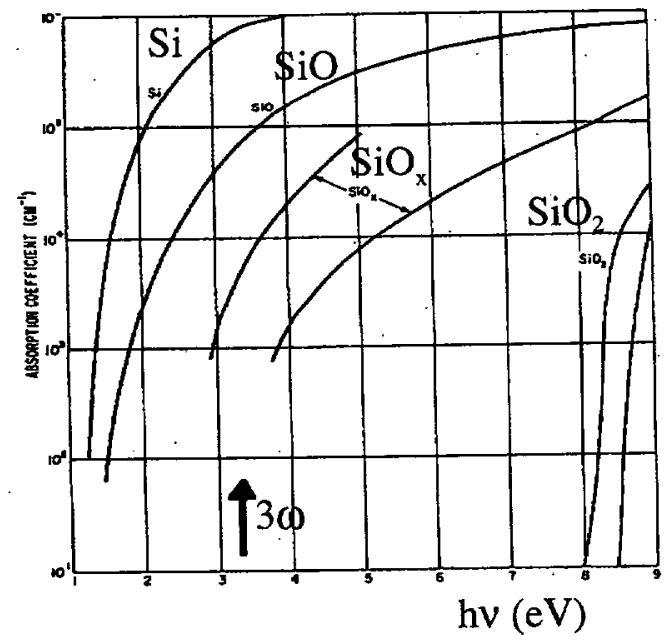

Fig. 6 The absorption in oxygen depleted material [27]. Absorption coefficient vs. photon energy for $\mathrm{Si}, \mathrm{SiO}, \mathrm{SiO}_{x}(x \sim 1.5)$ and $\mathrm{SiO}_{2}$

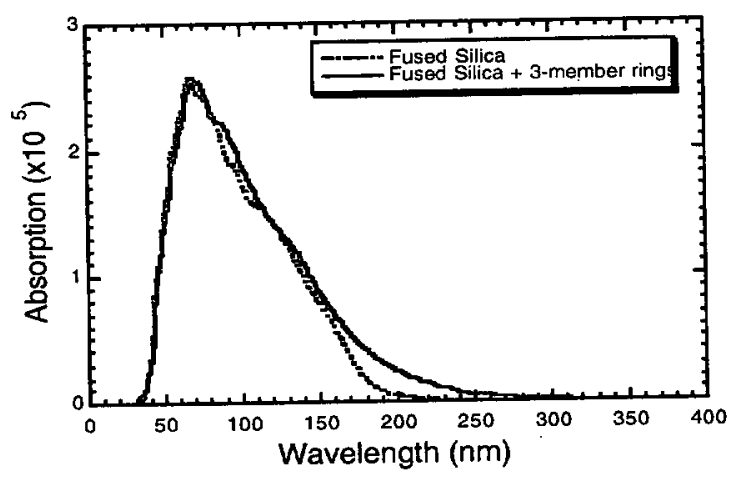

Fig. 7 : Modeling of electronic structure of modified material indicates the absorption increase in comparison with initial fused silica

Finally, small particles in the crushed material in damage spots can strongly scatter light. The interference of the scattered light produces local intensity spikes where breakdown can takes place, igniting plasma and producing the enhanced absorption.

Scattering results in fast randomization of the radiation field. For a random Gaussian wave field with mean intensity $I_{0}$ the probability to have intensity $I$ is

$$
P(I)=\frac{e^{\frac{-I}{I_{0}}}}{I_{0}}
$$

Typically, the density of defects damaged at intensity $\mathrm{I}, \mathrm{d}(\mathrm{I})$ are given by power distribution

$$
\mathrm{d}(\mathrm{I})=\mathrm{AI}^{\mathrm{n}}, \mathrm{n} \sim 10
$$


Averaging density of defects over distribution (6.1) we have

$$
<d(I)>=A<I^{n}>=A n ! I_{0}^{n}=n !<d\left(I_{0}\right)>
$$

We see that the probability to have damage greatly increases ( $n$ ! times) in comparison with coherent radiation at the mean intensity. This means that at high intensities at damage spot we must expect the generation of multiple
absorption spots forming an extended strongly absorbing plasma.

Probably all of these mechanisms are important and manifest, to some degree, in reality.

Damage spots on the rear side of the optic are particularly dangerous. In this case, light absorption in the damage spot creates plasma inside the material. Light absorption in the plasma initiates a laser supported detonation times) the pressure in comparison laser beam. The deposition of energy inside the material greatly increases (3-6 modify and crush the material and ith front surface damage [6] because of confinement. Generated shocks no reliable way at present to theoretically cracks. Cracks continue growing long after pulse termination. There is temporal and spatial scales. So the onclicy describe the variety of physical phenomena operating on very different The experiments on damage growth carried out at LLNL revealed a reproducible and consistent pattern of
damage growth (see Fig.8 and Fig.9) [30].

\section{Typical damage growth history}

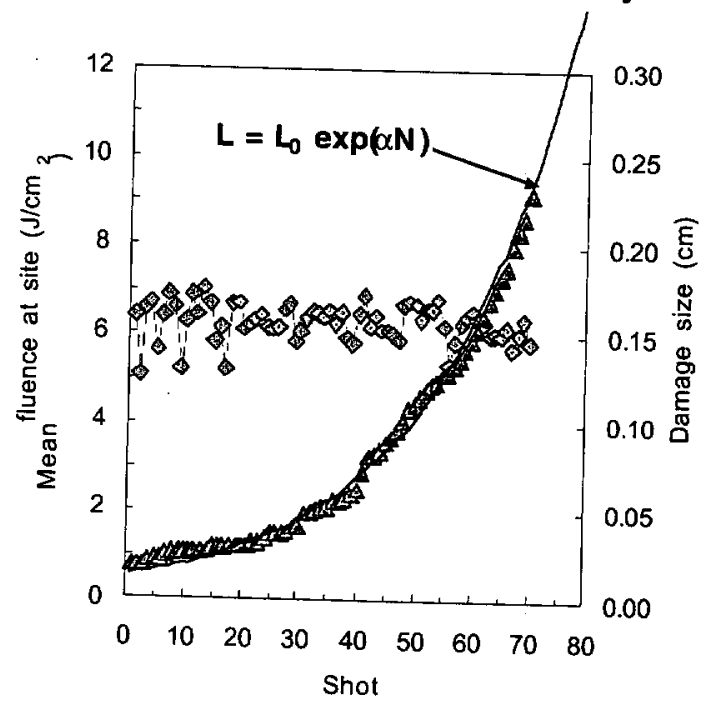

Fig. 8 Growth of damage spot with repetitive pulses

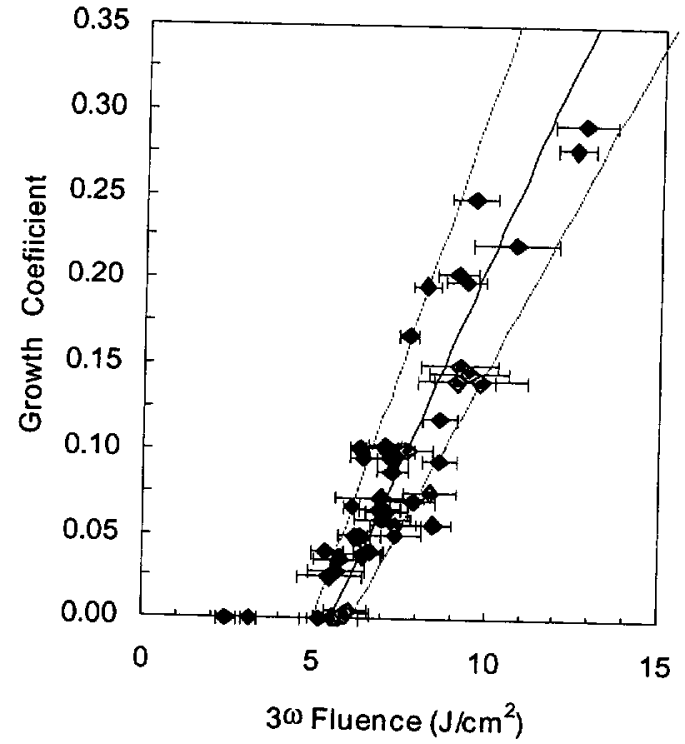

Fig. 9 Growth coefficient $\alpha$ scales linearly with fluence above a threshold The transverse size of the damage spot increases with the number of pulses in an accelerated way very well
approximated by an exponential function

$$
L=L_{0} e^{\alpha N}
$$

Damage growth has a threshold (Fig. 9). No damage growth occurs for fluences below about $5.5 \mathrm{~J} / \mathrm{cm}^{2}$; for higher fluences the growth coefficient scales linearly with laser fluences. This data can be approximated by formula
where the fluence is in $\mathrm{J} / \mathrm{cm}^{2}$.

$$
\alpha=0.047\left(F-F_{t h}\right) ; F_{t h}=5.5 J / \mathrm{cm}^{2}
$$

Let us try to explain, at least, qualitatively, the observed results. Visual observations at the developed stage of growth show that the spot size grows mainly by the development of conical cracks on the periphery of the damage spot. To open a crack of length 1 one must apply the stress $\sigma=K / \sqrt{ } 1$, where $K$ is the toughness of the 
material. $\mathrm{K}=0.75 \mathrm{MPa} \mathrm{m}^{1 / 2}$ for fused silica. The laser energy absorption in the initially damaged zone produces some residual pressure $P$, e.g., due to thermal expansion of the heated material. This pressure produces hoop stresses outside the damage spot that open the crack. The extent of the stress zone increases with the increase of the damage spot size. We see that with increased spot size, it is easier to open the crack and the opened cracks are longer. This is consistent with observed accelerated growth.

If the scale of the damage zone is $\mathrm{R}$ the stresses decay outside as $\mathrm{sP}(\mathrm{R} / \mathrm{a})^{3}$, the crack will grow to the size when stress becomes equal $\mathrm{K} / \mathrm{Va}$ or

$$
\mathrm{a}=\mathrm{R}^{6 / 5}(\mathrm{P} / \mathrm{K})^{2 / 5}
$$

If $\mathrm{a}>\mathrm{R}$ the increase of $\mathrm{R}$ after one shot will be which is close to exponential growth.

$$
\mathrm{dR} / \mathrm{dN}=\mathrm{a}=\mathrm{R}^{6 / 5}(\mathrm{P} / \mathrm{K})^{2 / 5}
$$

The above estimates show that the optics lifetime is extremely sensitive to the structure of the initial crater. If the initial crater is a large and it is surrounded by weakened material, it will be easy to start a new crack and damage will grow exponentially. If the initial crater is small and the material around it is not damaged, it is difficult to create a new crack and rapid damage growth can be postponed.

It must be emphasized again that modern high quality optics have a very low density of defects, and it is very difficult to find and to grow damage site initiated at more typical laser operating fluences of $10 \mathrm{~J} / \mathrm{cm}^{2}$. For this reason, the initial damage in the experiments [30] was initiated at high fluence, typically at $45 \mathrm{~J} / \mathrm{cm}^{2}$. The crater produced at such fluence is shown in Fig. (5) and must be very susceptible to growth. As a result, the initial stage of growth for craters initiated closer to $10 \mathrm{~J} / \mathrm{cm}^{2}$ may be much longer than in these particular experiments. In the real situation, various initiators produce different craters, and not all may grow at $5.5 \mathrm{~J} / \mathrm{cm}^{2}$. But when exponential growth starts, the optics become nonfunctional after $1 / \alpha$ shots.

\section{GROWTH MITIGATION.}

It appears impossible in large-aperture laser systems to escape laser damage. Even initially perfect optics can be damaged due to mishandling or contamination during operation. For a system operating with peak fluences above $5 \mathrm{~J} / \mathrm{cm}^{2}$, some scheme of damage mitigation must be found to extend optics lifetime. From our previous discussion it is clear that for damage mitigation, the modified material must be removed from the damage spot and cracks must be removed or healed.

A few different methods of mitigation were proposed and tested [31]. The most promising now appears to be mitigation by $\mathrm{CO}_{2}$ radiation [31]. Fused silica absorbs $85 \%$ of the incident $\mathrm{CO}_{2}$ radiation on the scale few $\mu \mathrm{m}$. Local heating increases the surface temperature and evaporates the material. Heated up to high temperature material is revitrified and heals the microcracks. Treatment replaces the damage spot with a smooth Gaussianshaped pit.

Let us discuss optimal parameters for damage spot mitigation. We will suppose that at high enough temperature the modified material will be transformed to the initial fused silica. Due to the extremely high viscosity of fused silica the most sensitive part of mitigation process is the crack healing. We will see that the temperature required for crack healing is so high that substantial evaporation is inevitable.

The steady state evaporation rate (velocity of evaporation front) is very sensitive to the surface temperature [32]

$$
V=\frac{d Z}{d t}=V_{0} e^{-\frac{U}{T}}
$$

Here $U$ is the latent heat of evaporation per atom, $3.6 \mathrm{eV}$ for glass, and $T_{s}$ is the surface temperature. The constant $\mathrm{V}_{0}$ is determined by the kinetics of the flow in the Knudsen layer near the surface, but its value is on the order of the sound speed in the condensed phase [32]. For validity of Eq.(7.1), it is clear that the laser pulse duration must be longer than the time for onset of steady temperature distribution: $\mathrm{a}^{2} / 4 \mathrm{D}$, where a is the damage spot radius and $D$ is the thermal diffusivity. If the pulse is shorter than this time, a high evaporation rate takes place only in the end of the pulse and the removal rate will be sensitive to the pulse shape. In the steady state regime the removal rate is less sensitive to the pulse details. 
Also, for the short pulse the high temperature zone has no time to diffuse deep enough to heal the cracks; in the steady state regime temperature is high in a thick enough layer to anneal the damaged material.

Due to the high sensitivity of evaporation rate to the temperature of the surface it is difficult to find the optimal evaporation rate as a function of laser beam material; high power will produce a huge hole. A plot of the evaporation rate as a function of laser beam power is presented in Fig.10.

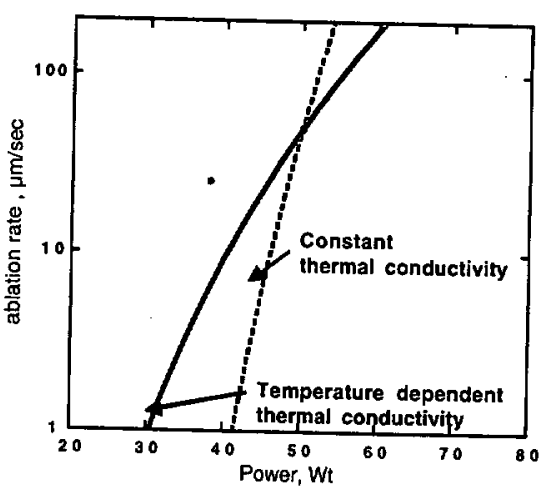

Fig.10 Comparison of ablation rates calculated with linear (dash line) and nonlinear thermal transport. Dots are experimental values.

Laser spot diameter- $2 \mathrm{~mm}$.

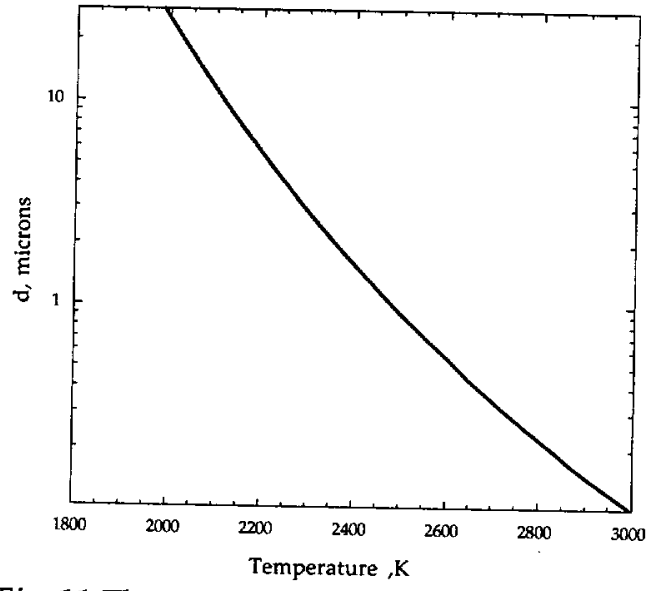

Fig. 11 The amount of material ablated during the crack healing procedure

The dotted line represents calculations done assuming temperature independent thermal conductivity. We see that the power dependence of the ablation rate is very steep and it means that damage spot processing is very sensitive to variation of laser parameters. Fortunately, the thermal transport is strongly nonlinear. The effective glass thermal conductivity increases with temperature and after $\mathrm{T} \sim 800 \mathrm{~K}$ is determined mainly by radiation
transport. For thermal conduction we used the interpolation formula

$$
\chi(T)=\chi_{0}+\beta T^{3}=0.01\left(1+1.7 \cdot 10^{-9} T^{3}\right)(W / \mathrm{cmK})
$$

The coefficient $\beta$ is determined from experimental data, $\kappa \sim 0.04 \mathrm{~W} / \mathrm{cmK}$ at $\mathrm{T}=1200 \mathrm{~K}$ [33]. One can see that nonlinearity of thermal conduction makes the ablation rate more flat and less susceptible to the variation of laser parameters. Including the effect of nonlinearity on thermal transport brings the modeling results closer to experiment, but the difference is still large. The reason is that the ablation rate is extremely sensitive to surface temperature and the absence of detailed information about thermal transport limits modeling accuracy.

The ablation rate observed in an experiment is determined exactly by the surface temperature. For an ablation rate of $50 \mu \mathrm{m} / \mathrm{s}$, the surface temperature $\mathrm{T}_{\mathrm{s}}$ is $2245 \mathrm{~K}$, and even a $5 \%$ change of temperature changes the ablation
rate more than 2 times.

The calculation and experimental data are presented for one value of beam radius a. It is easy to show that the in Fig (10) is is a function of the ratio P/a only for any nonlinearity of thermal conduction. It mean that the curve situation.

The high temperature induced by infrared radiation not only evaporates the material but reduces the viscosity of fused silica so surface tension now is able to close the cracks, thereby annealing the material and increasing the [34]. What is new here is local application to heal spectance after $\mathrm{CO}_{2}$ laser processing has been known since 1980

The typical time for relaxation of a surface disturbance with scale 1 is given by the estimate 


$$
\tau \sim \frac{\eta l}{\sigma}
$$

The surface tension s for glass is about $300 \mathrm{dyne} / \mathrm{cm}$ [35] and not very sensitive to temperature. In the range of $1600-2000^{\circ} \mathrm{C}$, the viscosity $\eta$ is given by the expression [36]

$$
\eta=1.05 * 10^{-9} e^{\frac{E}{T}} \text { poise }
$$

where $\mathrm{E}$ is an activation energy, $\mathrm{E} \sim 6.44 \mathrm{eV}$. For a deep crack, we must use the crack depth for 1 . If we know the crack depth and the acceptable annealing time, we can determine from (7.3) and (7.4) the annealing temperature T.

Let us consider now crack mitigation by local heating. The time for crack healing is given by (7.3). During this time $d=V \tau$ of material will be ablated. The total amount of ablated material is given by the expression

$$
d=\frac{\eta_{0} l V_{0}}{\sigma} e^{\frac{E-U}{T}}=1.75 * 10^{-6} e^{\frac{32944}{T}}
$$

Here $\mathrm{d}$ and $l$ are in microns, $\mathrm{T}$ in Kelvins. The amount of material ablated during the crack healing process as a function of surface temperature is presented in Fig.(11). One can see that for the temperature 2245K used above, ablation of more than $1.5 \mu \mathrm{m}$ guarantees that the cracks in the subsurface layer will be annealed. Hence, in experiments [31] the laser-induced temperature must be high enough to heal the subsurface layer around the damage spot. Due to the nonlinearity of thermal conduction, the temperature distribution around the heated spot is pretty flat and the wide zone around is annealed of microcracks.

\section{CONCLUSION}

We demonstrated that the intrinsic damage threshold in silica for nanosecond pulses is well above the observed values. Damage is initiated at defects, mainly the surface imperfections produced by the polishing process. Improvement of the polishing process has been shown to increase damage threshold.

The model of small, nanoscale initiators is consistent with experimental data. The absorption of laser energy in an inclusion initiates a thermal explosion, formation of plasma fireball, and growth of the fireball up to a size comparable with laser light wavelength. The energy storage in the fireball is released as a microexplosion, forming a crater on the optics surface.

The damage crater is surrounded by mechanically and electronically modified material. The light radiation in this material results in damage spot growth with repeated shots. The damage growth has a fluence threshold. The size of the damage spot increases exponentially with number of shots, and growth takes place via generation of conical cracks on the spot periphery.

Growth of the damage spot can be mitigated by the removal of modified material. $\mathrm{CO}_{2}$ laser annealing can efficiently mitigate local damage sites by removal of modified material and improvement of material structure around the damage spot.

\section{ACKNOWLEDGMENTS}

This work was performed under the auspices of the U.S. Department of Energy by University of California Lawrence Livermore National Laboratory under Contract W-7405-Eng-48. Authors acknowledge the fruitful discussions with A.K. Burnham, R.M. Brusasco, C.W.Carr, S.Demos, M.Fluss, L.A. Hackel, L.W.Hrubesh, M.R.Kozlowski W.A.Molander, M.A.Norton, B.M.Penetrante, P.J.Wegner, 


\section{REFERENCES}

1. Boulder Damage Symposium v.1-33.

2. B. C. Stuart, M. D. Feit, S. Herman, A. M. Rubenchik, B. W. Shore, and M. D. Perry, "Nanosecond-to -femtosecond laser-induced breakdown in dielectrics", Phys. Rev. B 53, 1749 (1996).

3. D.Arnold, E.Cartier, D.DiMaria," Acoustic-phonon runaway and impact ionization by hot electrons in silicon dioxide". Phys.Rev.A 45,1477 (1992).

4 F.Rainer,F.DeMarco, M.Staggs,M.R.Kozlowski,L.J.Atherton, L.M.Sheehan, "A historical perspective on fifteen years of laser damage thresholds in LLNL" SPIE Proc., Vol. 2114, pp 9-22,(1993).

5 Feit M.D., A.M.Rubenchik, M.R.Kozlowski, F.Genin, L.Sheehan, S.Schwartz, "Extrapolation of damage test data to predict performance of large-area NIF optics at $355 \mathrm{~nm}$ ". Proceedings XXX Annual Symposium on Optical Materials for High-Power Lasers, Boulder, CO, Sept.28-Oct.1, 1998,SPIE-3578-48, pp. 226-231

6 M.D.Feit., A.M. Rubenchik, D. Faux, R. Riddle, D. Eder, B. Penetrante, D. Milam, F. Genin,

7 M. Kozlowski, Modeling of Laser Damage Initiated by Surface Contamination", Proceedings in LaserInduced Damage in Optical Materials, Boulder, Co, Oct -7-9, 1996 SPIE, pp.2966-26 (1997).

7 L.M. Sheehan, M.R. Kozlowski ,D.W. Camp, “Application of Total Reflection Microscopy for Laser Damage Studies on Fused Silica", Proceedings in Laser-induced Damage in Optical Materials, Boulder, CO, Oct 6-8, 1997 SPIE 3244 ,pp. 282-295 (1998).

8 L.Skuja, H.Hosono, M.Hirano, "Laser-induced color centers in silica," Proceedings in Laser-Induced Damge in Optical Materials, Boulder, CO. Oct.16-18, 2000, SPIE-4347, pp. 155-168.

9 H.C. van der Hulst, "Light scattering by small particles", John Wiley and Sons, NY (1957).

10 Edward D. Palik, ed., "Handbook of Optical Constants in Solids", Academic Press (1985).

11 M.D. Feit, J.H. Campbell, D.R. Faux, F.Y. Genin, M.R. Kozlowski, A.M. Rubenchik, R.A. Riddle, A. Salleo, J.M. Yoshiyama, "Modeling of laser-induced surface cracks in silica at $355 \mathrm{~nm}$ ", Proceedings in Laser-induced Damage in Optical Materials, Boulder, CO, Oct 6-8, 1997 SPIE 3244, pp. 350-355 (1998).

12 M.R.Lange,J.K.McIver,A.H.Guenther,T.W.Walker, "Pulsed laser induced damage of an U.S. Spec.Publ.669, pp.380-6, (1984).

13 M.D.Feit., A.M. Rubenchik, M.Runkel, "Analysis of bulk DKDP damage distribution ,obscuration and pulse length dependence", Proceedings XXXII Annual Symposium on Optical Materials for High-Power Lasers, Boulder, Co, Oct.16-1 8, 2000,SPIE-4347, pp. 383-388 (2001).

14 N.Blombergen, "Laser induced electric breakdown in solids", IEEE J. of Quantum Electr., 10,375, (1974).

15 F.Genin, A.Salleo,T.Pistor, L.Chase, "Role of light intensification by cracks in optical breakdown on surfaces". JOSA A 18, 2607-2616, (2001).

16. A. V. Hamza, W. J. Siekhaus, A. M. Rubenchik, M. Feit, L.L. Chase, S. Demos, M.Savina, M. J. Pellin, M. J. Fluss, M. C. Nostrand, M. Runkel, B. W. Choi, M. Staggs, nd I.D.Hutcheon, "Engineered defects for investigation of laserinduced damage of fused silica at 355nm", Proceedings in Laser-Induced Damage in Optical Materials, Boulder, CO., Nov.1-2, 2001, SPIE, (2002).

17. J. Menapace. B.Penetrante, D. Golini, A. Slomba, P. Miller, T. Parham, M. Nichols, J. Peterson, Combined advanced finishing and UV laser conditioning for producing UV damage resistant fused silica optics", Proceedings in Laser-Induced Damage on Optical Materials, Boulder, CO., Nov.1-2, 2001, SPIE, (2002).

18. C.W.Carr, H.B.Radousky, M.Staggs, A.M.Rubenchik, S.G.Demos, "Time-resolved spectroscopic investigation of emission observed during damage in the bulk of KDP crystals", Proceedings in Laser-Induced Damage on Optical Materials, Boulder, CO., Nov. 1-2, 2001, SPIE, (2002).

19. P.Grua, H.Bercegol, "Dynamics of electrons in metallic nanoinclusions interacting with an intense laser beam". Proceedings XXXII Annual Symposium on Optical Materials for High-Power Lasers, Boulder, Co, Oct.16-18, 2000,SPIE-4347, pp.579-587

20. H. Melosh. "Impact ejection, spallation and the origin of meteorites". Icarus 59, 234-260,(1984)

21. M.D. Nordyke "An analysis of cratering data from desert alluvium", J. Geophys. Res. 67,1967-1974, (1962)

22. M.D.Feit., L.W.Hrubesh, A.M. Rubenchik and J.Wong, "Scaling relations for laser damage initiation craters", Proceedings in Laser-Induced Damage on Optical Materials, Boulder, CO., Oct.16-18, 2000, SPIE-4347, pp. $316-$
323, (2001).

23. N.Bourne, J.Millet, J.E. Field "On the strength of shocked glasses," Proc.R.Soc.A.455,1275-82, (1999).

24. F.Bonneau,P.Combis,J.Vierne,G.Daval, "Simulations of laser damage of $\mathrm{SiO} 2$ induced by a spherical inclusion", Proceedings in Laser-Induced Damage on Optical Materials, Boulder, CO., Oct.16-18, 2000, SPIE-4347, pp.308-315, (2001). 
25. S.Papernov, A.Schmid, R.Krishnan,L.Tsybeskov,"Using Colloidal Gold nanoparticles for studies of laser interaction with defects in thin films.", Proceedings in Laser-Induced Damage on Optical Materials, Boulder, CO., Oct.16-18, 2000, SPIE-4347, pp.146-154, (2001).

26. A.K. Burnham, M.Runkel, S.Demos, M.R. Kozlowski, P. Wegner, "Effect of vacuum on the occurrence of UVinduced surface photoluminescence, transmission loss and catestrophic urface damage", Photonics for Space Enviroments VII,Proc. SPIE, v.4134 pp.243- 252, (2000).

27. H.Philip,. "Optical properties of non-crystalline Si ,SiO, SiOxand SiO2. J.Phys.Chem.Solids 32.1935-45, (1971).

28. J.Wong, D.Haupt, J.Kinney, J.Ferriere, I.Hutcheon, S.Demos, M.Kozlowski, "Nature of damage in fused silica induced by high-fluence $3 \omega \quad 355-\mathrm{nm}$ laser pulses, a multiscale morphology microstructure and defect chemistry study", Proceedings in Laser-Induced Damage on Optical Materials, Boulder, CO., Oct.16-18, 2000, SPIE-4347, pp. 466, (2001).

29. A. Kubota, L. Davila, M.J. Caturla, J. S. Stolken,B. Sadigh, A. Quong, A. Rubenchik and M. D. Feit, "Structural modifications in fused silica due to laser damage induced shock compression," Proceedings in Laser-Induced Damage on Optical Materials, Boulder, CO., Nov. 1-2, 2001, (2002).

30. M.Norton,L.Hrubesh,Z.Wu,E.Donohue,M.D. Feit,M.Kozlowski,D.Milam, K.Neeb, W.Molander,A.M. Rubenchik,W.Sell,P.Wegner, "Growth of laser initiated damage in fused silica at $351 \mathrm{~nm}$ ", Proceedings in Laser-Induced Damage on Optical Materials, Boulder, CO., Oct.16-18, 2000,S PIE-4347, pp. 468, (2001).

31. L.W.Hrubesh, M.A.Norton, W.A.Molander, E.E.Donohue, S.M.Maricle, B.M.Penetrante, R.M.Brusasco, W.Grundler, J.A.Butler, J.W.Carr, R.M.Hill, L.J.Summers, M.D.Feit, A.Rubenchik, M.H.Key, P.J.Wegner, A.K.Burnham, L.A. Hackel, M.R.Kozlowski. "Methods for mitigating surface damage growth on NIF final optics." Proceedings in Laser-Induced Damage on Optical Materials, Boulder, CO., Nov. 1-2, 2001, (2002).

32. .S.I. Anisimov, V.A.Khokhlov "Instabilities in Laser-matter interaction", CRC Press Boca Raton, (1999).

33. H.Schoize, Glass, Springer- Verlag, (1991).

34. Temple, P.A.; Lowdermilk, W.H.; Milam, D.; "Carbon dioxide laser polishing of fused silica surfaces for increased laser-damage resistance at $1064 \mathrm{~nm}$ ", Appl. Opt. 21, 3249-55 (1982).

35. "The Handbook of Glass Manufacture", 3rd ed.. Vol. 2, p.930 Ashlee Publishing Co. (N.Y. 1984).

36. D.Hewak,Ed., " Properties, processing and applications of glass and rare earth-doped glasses for optical fibres", London, UK: INSPEC, (1998). 


\title{
Methods for mitigating growth of laser-initiated surface damage on fused silica optics at $351 \mathrm{~nm}$
}

\author{
L.W.Hrubesh \\ E.E.Donohue \\ R.M.Brusasco \\ J.W.Carr \\ M.D.Feit \\ P.J.Wegner
}

M.A.Norton

S.M.Maricle

W.Grundler

R.M.Hill

A.Rubenchik

A.K.Burnham

M.R.Kozlowski

\author{
W.A.Molander
}

B.M.Penetrante

J.A.Butler

L.J.Summers

M.H.Key

L.A. Hackel

\begin{abstract}
We report a summary of the surface damage, growth mitigation effort at $351 \mathrm{~nm}$ for polished fused silica optics. The objective was to experimentally validate selected methods that could be applied to pre-initiated or retrievedfrom-service optics, to stop further damage growth. A specific goal was to obtain sufficient data and information on successful methods for fused silica optics to select a single approach for processing large aperture, fused-silica optics used in high-peak-power laser applications.

This paper includes the test results and the evaluation thereof, for several mitigation methods for fused silica surfaces. The mitigation methods tested in this study are wet chemical etching, cold plasma etching, $\mathrm{CW}-\mathrm{CO}_{2}$ laser processing, and micro-flame torch processing. We found that $\mathrm{CW}-\mathrm{CO}_{2}$ laser processing produces the most significant and consistent results to halt laser-induced surface damage growth on fused silica. We recorded successful mitigation of the growth of laser-induced surface damage sites as large as $0.5 \mathrm{~mm}$ diameter, for 1000 shots at $351 \mathrm{~nm}$ and fluences in the range of 8 to $13 \mathrm{~J} / \mathrm{cm}^{2}, \sim 11 \mathrm{~ns}$ pulse length. We obtained sufficient data for elimination of damage growth using $\mathrm{CO}_{2}$ laser processing on sub-aperture representative optics, to proceed with application to large aperture $\left(\sim 40 \times 40 \mathrm{~cm}^{2}\right)$ fused silica.
\end{abstract}

\section{INTRODUCTION}

Surface damage initiated on polished fused silica surfaces during high-peak-power irradiation at $351 \mathrm{~nm}$ encompasses only a fraction of the clear aperture area. However, studies have shown that the damage can grow with the number of shots at $351 \mathrm{~nm}$ at laser fluences above $\sim 5 \mathrm{~J} / \mathrm{cm}^{2}, 11 \mathrm{~ns}$. [1]. A substantial increase in the useful lifetime of the optics can be achieved by stopping damage growth, thus mitigating obscuration caused by growing damage. Ideally this can be accomplished by eliminating the damages while they are small and returning the surface to its undamaged state. We explored several methods to mitigate the growth of UV-laser-induced damage on fused silica. Other studies at this Laboratory have focussed on elucidating the mechanisms for initiation [2] and growth [3] of surface damage on silica, whereas this effort seeks to identify and validate a leading method to successfully terminate the growth of such damage.

The following sections describe the experiments and results of damage growth tests for the four mitigation methods considered in this study; wet chemical etching, cold plasma etching, $\mathrm{CW}-\mathrm{CO}_{2}$ laser processing, and micro-flame torch processing. The results show that $\mathrm{CO}_{2}$ laser processing is consistent in mitigating damage and successfully stopping damage growth. 


\section{EXPERIMENTAL}

In our experimental program, we tested both the intrinsic growth behavior of the mitigation pits and the growth of mitigated laser-initiated damage pits. It was necessary to determine that the pits formed by the mitigation methods themselves did not cause damage or grow with repeated illumination. In fact, this was the case for those methods that tended to contaminate the surface of the fused silica. All of the experiments used the same sample type; Corning 7980 fused silica, $50 \mathrm{~mm}$ diameter, $10 \mathrm{~mm}$ thick, and polished by SESO. For testing the pits produced by the mitigation methods on bare surface, the pits were arranged in an array of 6-9 spots spaced by $10 \mathrm{~mm}$. For the laser-initiated pits, we chose a common set of experimental conditions to place an array of 4-9 nominally uniform, equally spaced damage pits on the output (exit) surface of fused silica samples. Each laser-initiated damage site was produced by a single Gaussian-shaped pulse from a frequency-tripled Nd:YAG laser at $355 \mathrm{~nm}$, with a $0.9 \mathrm{~mm}$ beam diameter, an average fluence of $\sim 45 \mathrm{~J} / \mathrm{cm}^{2}$ and a pulse length $\sim 7.5 \mathrm{~ns}$.

The typical surface damage produced by a single pulse at $\sim 45 \mathrm{~J} / \mathrm{cm}^{2}$ consists of a cluster of 3 to 15 pits within about $0.3 \mathrm{~mm}$ diameter; each pit in the group has a diameter in the range from $0.01-0.05 \mathrm{~mm}$ (see figure 7). Although such surface damage is heavier than what is expected for typical damage on NIF optics, it was used as a worst-case condition for proof-of-principle mitigation testing. Thus, a successful demonstration of methods to mitigate the growth of such sites gives confidence to mitigate NIF related damage.

The growth tests were all carried out in a vacuum chamber operating at $\sim 10^{-5}$ torr, in the slab laser facility at LLNL [1]. The sites were tested for growth using the frequency-tripled Nd-glass laser output, producing a $4 \mathrm{~mm} x$ $6 \mathrm{~mm}$ flat-top beam at $351 \mathrm{~nm}$, with $\sim 11 \mathrm{~ns}$ pulse width at $6-12 \mathrm{~J} / \mathrm{cm}^{2}$. The damage sites are illuminated by the laser at a rate of 1 pulse per 2 seconds. If the tested site grows, the growth rate is determined by measuring the occluded area as a function of the number of laser shots on a site. It is known from other LLNL work that the typical damage area grows at an exponential rate with the number of laser shots above a threshold $3 \omega$ fluence of about $5 \mathrm{~J} / \mathrm{cm}^{2}$ [1]. In some cases, mitigated damage sites had a higher threshold for growth or the growth rate was not typical. Such results were duly noted, but the growth behavior itself was not explored since the primary objective was to study the efficacy of the mitigation methods.

\section{WET-CHEMICAL ETCHING EXPERIMENTS AND RESULTS}

Chemical etching with a hydrofluoric acid solution is a common method for dissolving silica and it is an accepted way to remove damage-affected material on silica surfaces. Ideally, this process will return the damage area to the state of the undamaged bulk material. This method was explored in previous work at LLNL to determine if mild etching (i.e., to a depth of $\sim 600 \mathrm{~nm}$ ) would remove precursors to laser-initiated damage [4]. The results showed a minor improvement over non-etched surfaces. Also, in previous work at LLNL, mild chemical etching was studied as a possible means to raise the growth threshold of UV-laser-induced damage [5]. The prior work used $2 \%$ HF solution to etch up to about 2 micrometers depth from the damage pits. The etched samples were damage growth tested in the slab laser facility at $351 \mathrm{~nm}, 5-6 \mathrm{~J} / \mathrm{cm}^{2}(\sim 11 \mathrm{~ns})$. The results showed a slight increase of the growth threshold for fused silica.

Since some change in the growth threshold was observed in the earlier studies, we decided to explore deeper etching for growth mitigation. We used $2 \% \mathrm{HF}$ solutions to etch the entire surface of some damaged silica samples by dipping or we applied the solution directly to the damage pit using a fountain design [6], in other cases. Etch depths ranged from $0.5 \mu \mathrm{m}$ to $20 \mu \mathrm{m}$. Damage growth tests were performed at $351 \mathrm{~nm}(\sim 11 \mathrm{~ns})$. A summary of the results of mitigation by etching at different etch depths is shown in Table 1.

Our measurements show that damage sites that have been etched to depths greater than about $9 \mu \mathrm{m}$ have about a $40 \%$ chance for zero growth with 1000 shots at fluences of $6.8-9.4 \mathrm{~J} / \mathrm{cm}^{2}$ and $\sim 1 \mathrm{lns}$. For the etched sites that grow in this fluence range, the growth rates are consistent with those $f$ for un-etched sites. Contamination of the sample surfaces was observed for both the dipped and fountain etched cases, which could affect the variable results. 
Figure 1 shows the growth coefficient vs. fluence data for samples that were etched to depths of $10 \mu \mathrm{m}$ and $20 \mu \mathrm{m}$, as they compare to the statistical range of data for un-etched damage sites. These results are encouraging for possible mitigation of surface damage. More data is needed for smaller damage sites to determine if the statistics for complete mitigation of growth improve for such sites.

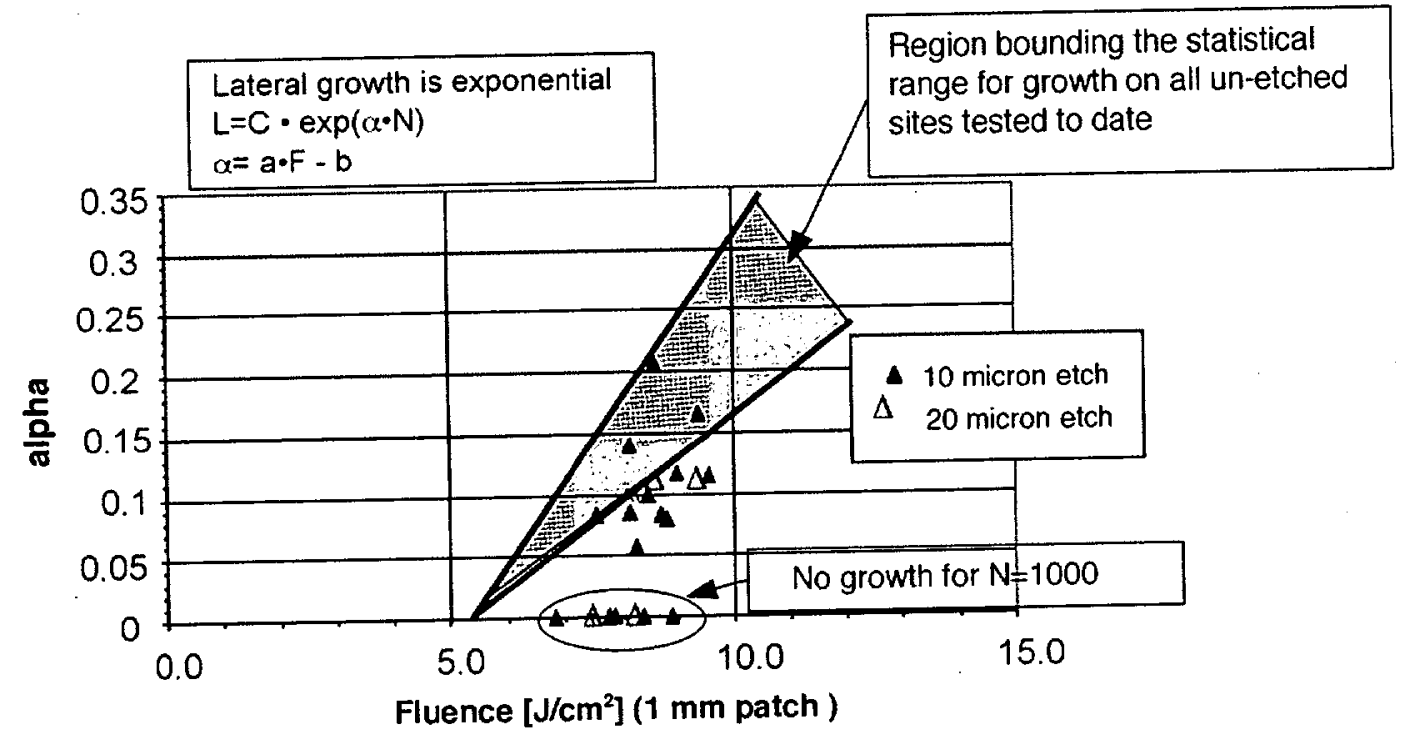

Figure 1. Plot showing results of damage growth experiments for wet chemical etched damage sites relative to the range of data for un-etched sites. About $40 \%$ of the etched sites did not grow for 1000 shots at the given fluences.

Table 1. Tabulated results for polished fused silica, surface damage growth tests at $351 \mathrm{~nm}, 6.8-9.4 \mathrm{~J} / \mathrm{cm}^{2}$, $\sim 11 \mathrm{~ns}$, of sites treated with a $2 \% \mathrm{HF}$ solution etch.

\begin{tabular}{|c|c|c|c|c|}
\hline Sample & Site & Etch depth( $(\mu \mathrm{m})$ & Test fluence $\left(\mathrm{J} / \mathrm{cm}^{2}\right)$ & Shots to cause growth \\
\hline \multirow[t]{6}{*}{ SC40021 } & $\mathrm{G}$ & 10 & 7.8 & $1000+$ \\
\hline & D & 10 & 6.8 & $1000+$ \\
\hline & I & 10 & 8.9 & $1000+$ \\
\hline & $\mathrm{F}$ & 10 & 9.4 & 40 \\
\hline & $\mathrm{H}$ & 10 & 8.4 & $1000+$ \\
\hline & E & 10 & 8.4 & $1000+$ \\
\hline \multirow[t]{5}{*}{ SC40023 } & C & 0.5 & 7.8 & 3 \\
\hline & A & 1 & 7.5 & 1 \\
\hline & I & 3 & 8.4 & 2 \\
\hline & G & 5 & 8.1 & 3 \\
\hline & E & 10 & 8.1 & 1 \\
\hline \multirow[t]{6}{*}{ SC40026 } & A & 10 & 8.2 & 24 \\
\hline & B & 10 & 8.1 & 17 \\
\hline & $\mathrm{C}$ & 10 & 8.1 & 830 \\
\hline & D & 10 & 7.8 & $1000+$ \\
\hline & $\mathrm{E}$ & 10 & 8.5 & 19 \\
\hline & $\mathrm{F}$ & 10 & 8.4 & 23 \\
\hline \multirow[t]{4}{*}{ SC40027 } & $\mathrm{F}$ & 20 & 8.4 & 10 \\
\hline & $\mathrm{E}$ & 20 & 8.2 & $1000+$ \\
\hline & $\mathrm{H}$ & 20 & 8.5 & 3 \\
\hline & G & 20 & 7.4 & $1000+$ \\
\hline
\end{tabular}




\section{PLASMA-ETCHING EXPERIMENTS AND RESULTS}

A radio-frequency (RF) plasma, spiked with fluorine-containing molecules, is commonly used to etch silica for integrated circuit applications. Fluorine atoms generated in the plasma, chemically attack the silicon-oxygen bonds of silica, forming a gas, $\mathrm{SiF}_{4}$, thus effectively etching the material. With this approach to mitigation in mind, two types of plasma torches were investigated for damage growth mitigation. One type was a miniature version of a RF argon plasma torch (micro-plasma), fed by carbon tetrafluoride $\left(\mathrm{CF}_{4}\right)$ gas, which etched small sites on fused silica. Figure 2 is a photo of the micro-plasma torch. The plasma diameter is about $1.5 \mathrm{~mm}$ at the tip. When the torch is applied directly to the surface of the silica, it produces a $\sim 2 \mathrm{~mm}$ diameter pit; the pit depth $(\sim 1$ $5 \mu \mathrm{m})$ depends on the exposure time. A typical pit profile is shown in Fig. 3. The second type of torch was a microwave plasma torch (nitrogen gas) also fed by $\mathrm{CF}_{4}$ gas, to produce fluorine atoms for reactive-atom plasma processing (RAPP). The large RAPP torch shown in Fig.4, etches silica at a fast rate $(\sim 0.2 \mu \mathrm{m} / \mathrm{min})$ over a diameter of $10 \mathrm{~mm}$. The depth $(\sim 10-100 \mu \mathrm{m})$ depends on the exposure time.

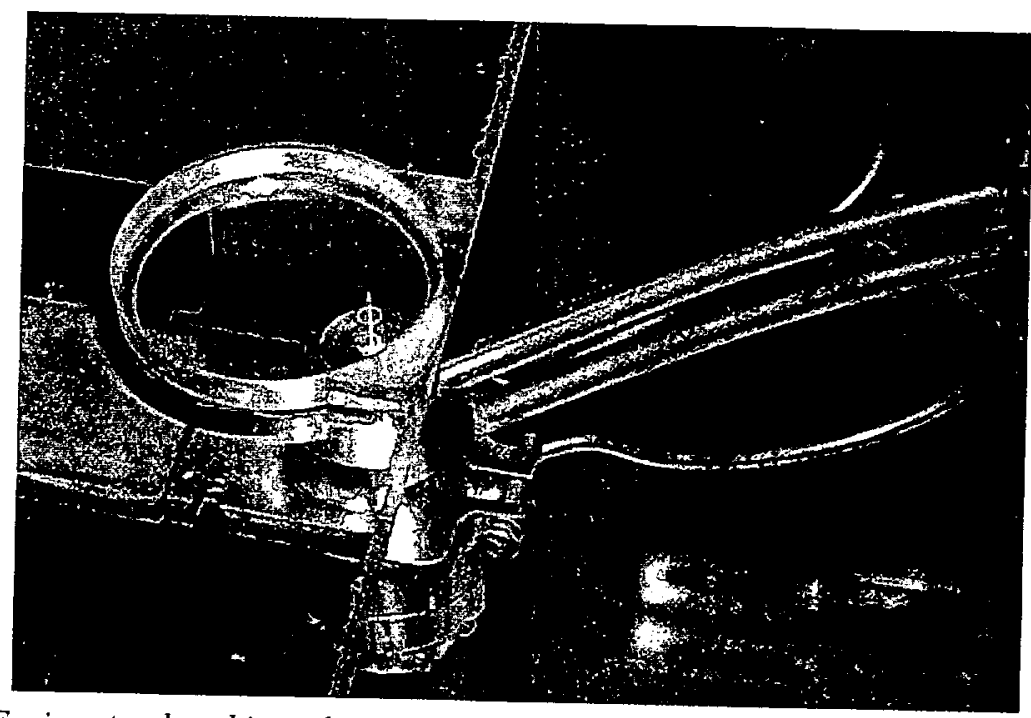

Figure 2. Photo of a RF micro-torch etching a local site on fused silica.

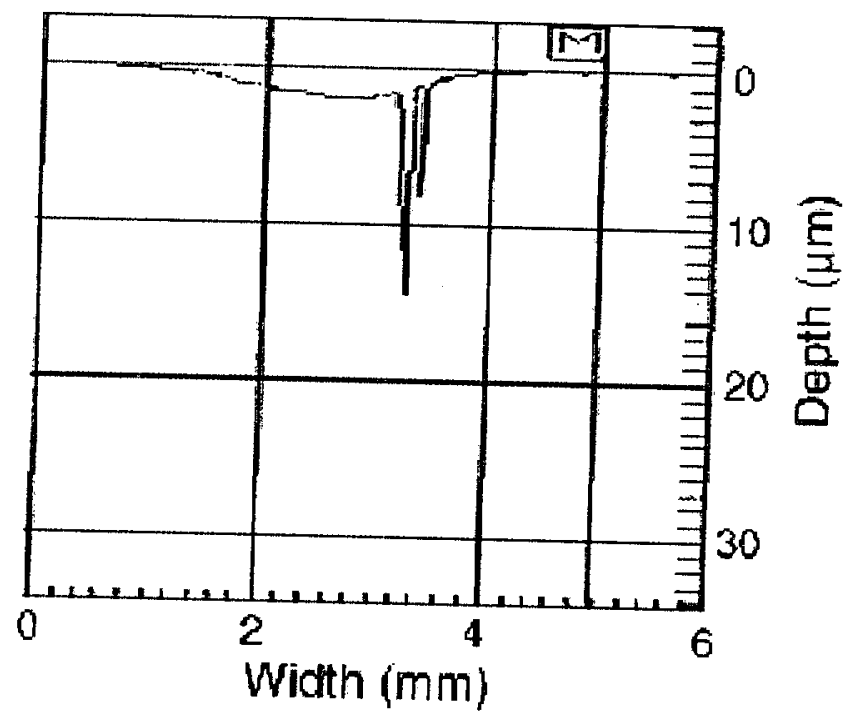

Figure 3. Profile of a $2 \mu \mathrm{m}$ deep pit micro-plasma etched over a 10-15um deep laser-initiated damage site in fused silica. 


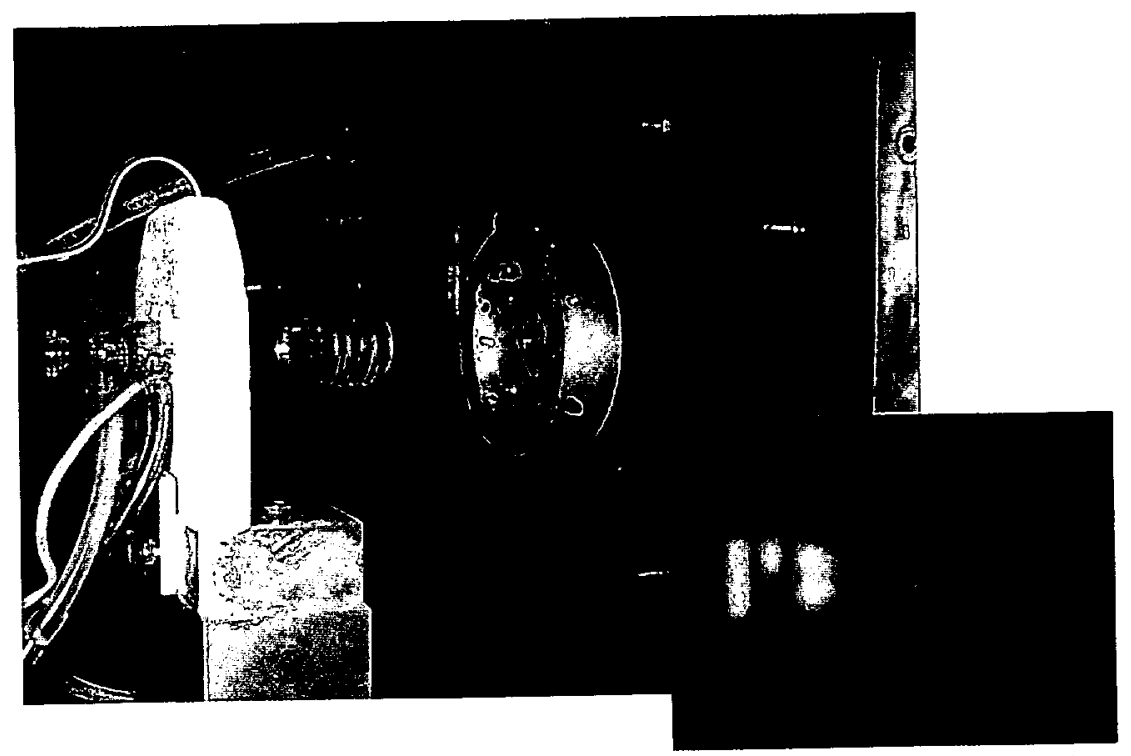

Figure 4. A microwave plasma torch (nitrogen gas) fed by $C F_{4}$ gas, to produced fluorine atoms for reactive atom plasma processing (RAPP).

The initial work with the micro-plasma torch exposed several problems; 1$)$ the etch rate is very slow $(\sim 2 \mu \mathrm{m} / \mathrm{hr})$, 2) the etching does not rapidly smooth the damage pit, and 3) the plasma produces contamination residue in the treated area. Evidence for 2) is seen in the profile shown in Fig. 3, where the original damage pit has receded into the substrate without smoothing as the etching takes place. Apparently, etching by the micro-torch is not isotropic as is typically the case for chemical solution etching. The initial experiments to apply the micro-plasma torch to etch laser-damage sites resulted in the damage receding into the site rather than being smoothed. These sites were not tested for growth because there was not a significant change in the damage structure after etching.

One sample was prepared for growth testing micro-plasma-produced pits on bare silica. All the pits were shallow $(<3 \mu \mathrm{m})$ due to low etch rates, and wide $(\sim 1.8 \mathrm{~mm})$. Microscopy of the pits before the tests disclosed some deposits located within each pit (see Fig.5). Analysis of the deposits showed that they contained carbon, which is believed to be residue from dissociation of the $\mathrm{CF}_{4}$ in the plasma. The laser fluence range for testing the growth at $351 \mathrm{~nm}$ was $6-8 \mathrm{~J} / \mathrm{cm}^{2}$. All of the tested sites that had contamination developed a 'stain' within the first 20 shots, as they were illuminated at $351 \mathrm{~nm}$. These stains spread in the next 20-50 laser shots, to totally cover the original plasmaproduced pit surface. The stains appeared to originate generally at or near visible contaminants within the pits.

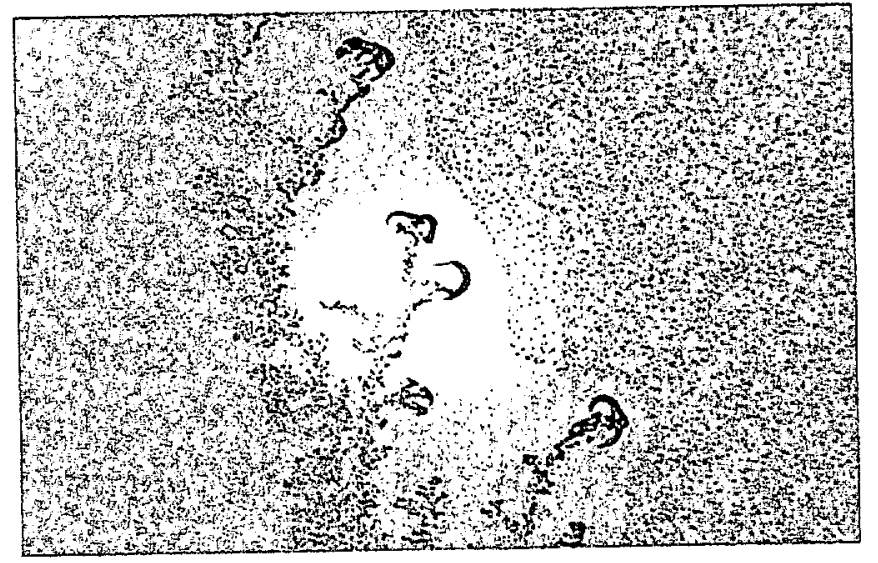

Figure 5. Photomicrograph of carbon containing deposits on the fused silica surface produced by decomposition of carbontetrafluoride gas from the plasma torch. 
Another sample was prepared for growth testing pits produced by the RAPP torch. Each site was about $10 \mathrm{~mm}$ diameter, and the depths ranged from $20-50 \mu \mathrm{m}$. Microscopy disclosed deposits located within 3 of the 4 pits. When tested for growth, one site developed a 'stain' that grew, similar to the micro-plasma torch case. Two of the sites having visible contamination developed catastrophic growth (i.e., rapidly expanding damage) within 17 shots. The fourth site, a 'clean' site, had no visible change after 26 shots.

The results of tests for both of the plasma torch processes show that contamination in the mitigation pit tended to be unstable at the test fluences. These processes need additional development using non-carbon, fluorine containing compounds. Also, development is needed to speed the slow etch rate of the micro-plasma torch. Due to the failure of the bare mitigation pits to survive laser illumination without contamination induced growth, and because of the success with alternative methods, we did not proceed to test mitigated damage sites by either of the plasma torch methods.

\section{$\mathrm{CO}_{2}$ LASER EXPERIMENTS AND RESULTS}

$\mathrm{CO}_{2}$ lasers have been used successfully by others for reducing damage initiation on fused silica at $1054 \mathrm{~nm}[7,8]$ but we were not aware of any attempts to use $\mathrm{CO}_{2}$ laser heating to treat existing damage on fused silica. We explored the use of $\mathrm{CO}_{2}$ lasers for mitigating $351 \mathrm{~nm}$ laser-induced damage growth on fused silica. Preliminary experiments were done using an industrial size $\mathrm{CO}_{2}$ laser (Rofin-Sinar RS-1000,1KW CW), primarily designed for cutting and welding. This laser was operated at reduced power $(<100 \mathrm{~W} \mathrm{CW})$, with a focussed, Gaussianshaped, $1 / \mathrm{e}^{2}$ beam diameter of $\sim 5 \mathrm{~mm}$, for one-second duration. The laser beam locally melted and evaporated the

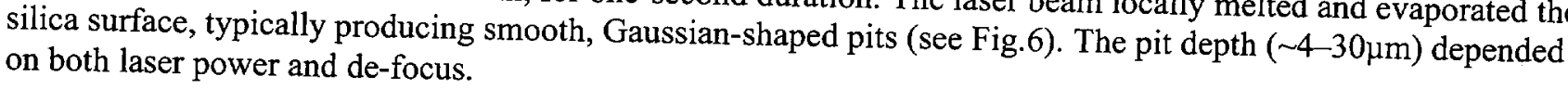
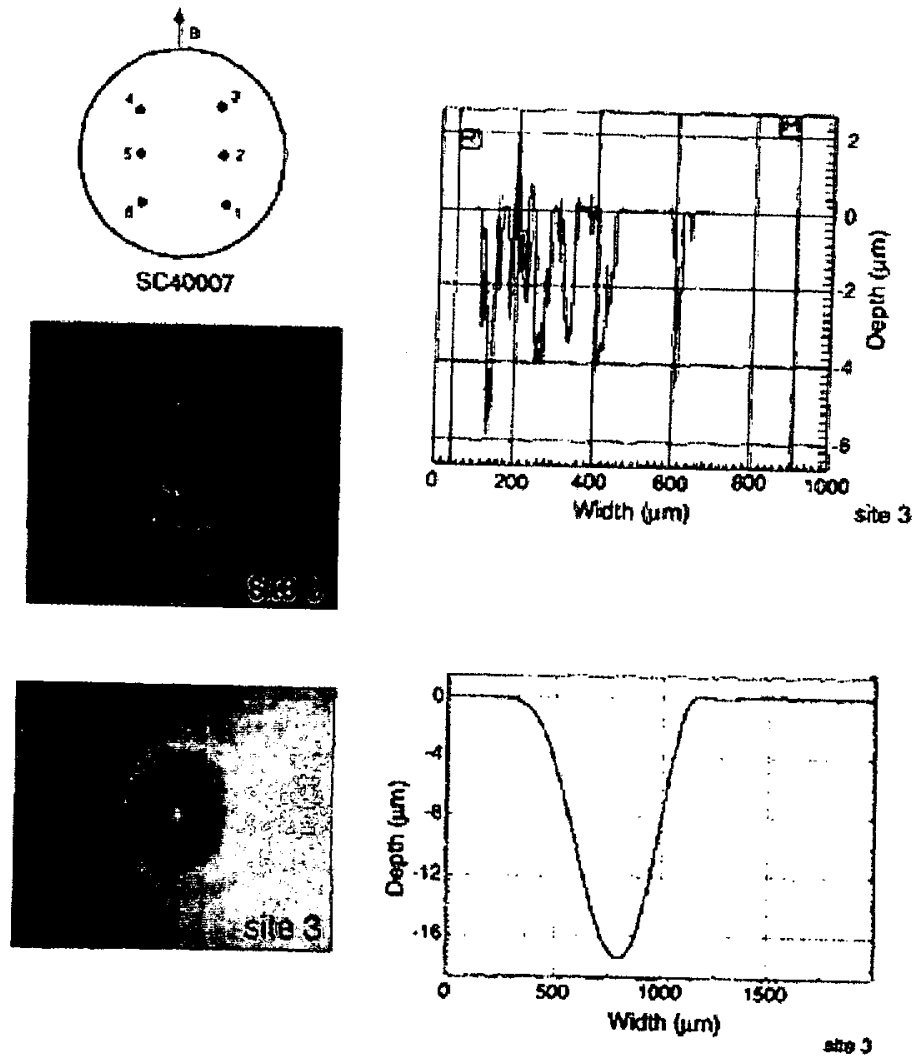

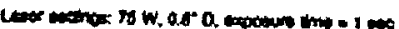

Figure 6. Example of surface damage mitigation by $\mathrm{CO}_{2}$ laser processing. Many rough pits (top photo and graph) are transformed to a smooth Gaussian-shaped pit (bottom photo and graph) by a single $50 \mathrm{~W}$ pulse in 1 second. 
The first damage tests at $351 \mathrm{~nm}$ and $\sim 11 \mathrm{~ns}$ pulse width, showed that the $\mathrm{CO}_{2}$ laser-induced pits on undamaged silica, did not damage at fluences up to $\sim 8 \mathrm{~J} / \mathrm{cm}^{2}$. An extensive set of mitigation experiments was done for $\mathrm{CO}_{2}$ laser-treated, UV-laser-induced damage pits. Figure 6 shows examples of the UV-laser-induced damage and the pit produced after a one-second exposure of the $\mathrm{CO}_{2}$ laser at $50 \mathrm{~W}$. All of the $\mathrm{CO}_{2}$ laser-treated sites which were tested at $351 \mathrm{~nm}, \sim 11 \mathrm{~ns}$, in the fluence range from $\sim 6.7-12 \mathrm{~J} / \mathrm{cm}^{2}$, survived 1000 shots. An example of the typical results for mitigating surface damage on fused silica is shown in Fig. 7.

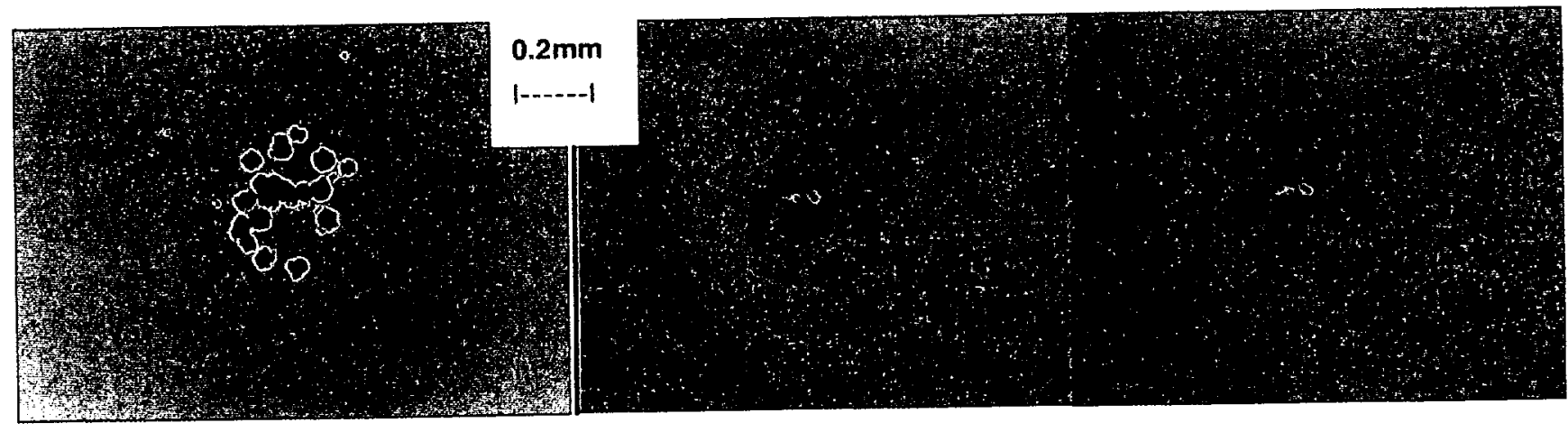

\section{Before $\mathrm{CO}_{2}$ laser}

After 1s pulse $\mathrm{CO}_{2} @ 27.5 \mathrm{~W}$

After 1000 shots@ $351 \mathrm{~nm}, 12 \mathrm{~J} / \mathrm{cm}^{2}$

Figure 7. Optical micrographs of a laser-initiated damage site before (left) and after $\mathrm{CO}_{2}$ processing (middle), and after exposure of 1000 shots at $35 \mathrm{lnm}, 11 \mathrm{~ns}$ (right), showing that damage growth is completely stopped.

After the initial successes with the $\mathrm{CO}_{2}$ laser processing, three issues were identified for further consideration; 1) what is the mechanism for removal of material and what parameters determine the pit size and shape, 2) what is the affect on the wavefront propagation by the pit geometry and, 3) would simple thermal annealing by the $\mathrm{CO}_{2}$ laser be sufficient to mitigate growth? All of these issues were addressed by modeling efforts and an experiment was also done to address the thermal annealing question.

A summary of the tests that were done for $\mathrm{CO}_{2}$ mitigation is shown in Table 2. One site (SC40031-B) that was shot multiple times during initiation, which had very deep cracks associated with it, grew immediately. Four other sites on sample SC40031 were treated with laser conditions designed to test thermal annealing; all grew immediately when tested at $11-12 \mathrm{~J} / \mathrm{cm}^{2}$. All of the other 16 sites tested in the range of 6.7 to $12 \mathrm{~J} / \mathrm{cm}^{2}$, did not grow in 1000 or more shots, exhibiting complete mitigation of damage growth. 
Table 2. Tabulated results for growth tests at $351 \mathrm{~nm}, \sim 1 \mathrm{lns}$, of damage sites treated with a $\mathrm{CW}-\mathrm{CO}_{2}$ laser.

\begin{tabular}{|c|c|c|c|c|}
\hline Sample & Site & $\mathrm{CO}_{2}$ treatment & Shots@Test fluence (J/cm & Comments \\
\hline SC40036 & $\begin{array}{l}\text { A } \\
\text { B } \\
\text { C } \\
\text { D }\end{array}$ & $\begin{array}{l}50 \mathrm{~W}, 1 \mathrm{sec} \\
50 \mathrm{~W}, 1 \mathrm{sec} \\
\text { none } \\
50 \mathrm{~W}, 1 \mathrm{sec}+\mu \text { plasma }\end{array}$ & $\begin{array}{l}1000 @ \sim 6.7 \\
1000 @ \sim 6.7 \\
35 @ \sim 6.7 \\
1000 @ \sim 6.7\end{array}$ & $\begin{array}{l}\text { no growth } \\
\text { no growth } \\
\text { control, normal growth } \\
\text { darken, no growth }\end{array}$ \\
\hline SC 40037 & $\begin{array}{l}\text { A } \\
\text { B } \\
\text { C }\end{array}$ & $\begin{array}{l}37.5 \mathrm{~W}, 1 \mathrm{sec} \\
37.5 \mathrm{~W}, 1 \mathrm{sec} \\
37.5 \mathrm{~W}, 1 \mathrm{sec}\end{array}$ & $\begin{array}{l}1000 @ \sim 8.0 \\
1000 @ \sim 8.0 \\
1000 @ \sim 8.0\end{array}$ & $\begin{array}{l}\text { no growth } \\
\text { no growth } \\
\text { no growth }\end{array}$ \\
\hline SC40029 & $\begin{array}{l}\text { B } \\
C \\
D \\
E \\
G \\
H \\
I\end{array}$ & $\begin{array}{l}37.5 \mathrm{~W}, 1 \mathrm{sec}+\mu \text { plasma } \\
37.5 \mathrm{~W}, 1 \mathrm{sec} \\
37.5 \mathrm{~W}, 1 \mathrm{sec}+\mu \text { plasma } \\
\text { none } \\
37.5 \mathrm{~W}, 1 \mathrm{sec} \\
37.5 \mathrm{~W}, 1 \mathrm{sec}+\mu \text { plasma } \\
37.5 \mathrm{~W}, 1 \mathrm{sec} \\
37.5 \mathrm{~W}, 1 \mathrm{sec}+\mu \text { plasm }\end{array}$ & $\begin{array}{l}400 @ \sim 8.3 \\
1000 @ \sim 12.0 \\
1000 @ \sim 8.3 \\
1000 @ \sim 8.3 \\
20 @ \sim 8.3 \\
1000 @ \sim 12.0 \\
1000 @ \sim 8.3 \\
1000 @ \sim 8.3 \\
1000 @ \sim 8.3\end{array}$ & $\begin{array}{l}\text { no growth } \\
\text { darken, no growth } \\
\text { no growth } \\
\text { no growth } \\
\text { control, normal growth } \\
\text { no growth } \\
\text { darken, no growth } \\
\text { no growth } \\
\text { darken, no growth }\end{array}$ \\
\hline SC40031 & $\begin{array}{l}A \\
B \\
C \\
D \\
E \\
F \\
G \\
H \\
I\end{array}$ & $\begin{array}{l}27.5 \mathrm{~W}, 1 \mathrm{sec} \\
27.5 \mathrm{~W}, 1 \mathrm{sec} \\
27.5 \mathrm{~W}, 1 \mathrm{sec} \\
27.5 \mathrm{~W}, 1 \mathrm{sec} \\
17.7 \mathrm{~W}, 60 \mathrm{sec} \\
\text { none } \\
17.5 \mathrm{~W}, 60 \mathrm{sec} \\
17.5 \mathrm{~W}, 60 \mathrm{sec} \\
17.5 \mathrm{~W}, 60 \mathrm{sec}\end{array}$ & 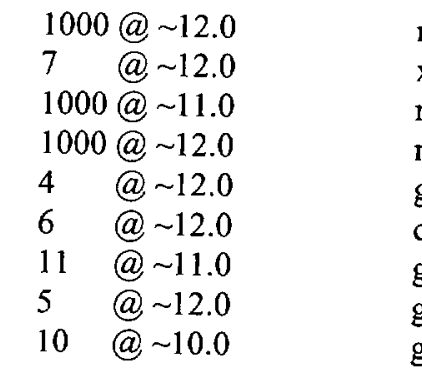 & $\begin{array}{l}\text { no growth } \\
\text { xtra heavy damage } \\
\text { no growth } \\
\text { no growth } \\
\text { grew on first shot } \\
\text { control, grew first shot } \\
\text { grew first shot } \\
\text { grew first shot } \\
\text { grew first shot }\end{array}$ \\
\hline
\end{tabular}

These results led us to conclude that $\mathrm{CO}_{2}$ laser treatment can stop the growth of large $(\sim 0.3 \mathrm{~mm}$ dia. $)$ damage on polished fused silica surfaces at $351 \mathrm{~nm}$ and $\sim 11 \mathrm{~ns}$ pulse length. It remains to be proven that mitigation works at shorter pulse lengths (eg., $3 \mathrm{~ns}$ ) and comparable frequencies, and that the mitigated sites do not re-initiate damage. The $\mathrm{CO}_{2}$ laser process is not complex and it is amenable to rapid processing of a large number of sites on a given optic. It may also be possible to achieve growth mitigation by treating the entire optic surface, rather than pointby-point; this would eliminate the difficult, tedious task to locate specific damage sites. On the other hand, maintaining surface figure for whole-surface treatment is clearly an issue. Additional details of the experiments to test the $\mathrm{CO}_{2}$ laser treatment for mitigation are published elsewhere in this
Proceedings [9].

\section{MICRO-FLAME TORCH EXPERIMENTS AND RESULTS}

A hydrogen flame torch is another method to locally anneal a small damage site on fused silica. A miniature version of such a torch was developed and tested. The torch was fed by $\mathrm{CF}_{4}$ to produce a hot flame with fluorine atoms that etch the silica surface. A schematic of the micro-flame torch system is shown in Fig.8. This torch represents an improvement over the RF plasma-torch for mitigation because it provides a combination of sufficient heat to soften the silica and fluorine atoms to etch the silica. It operates as an atomization source, wherein the hot flame dissociates the $\mathrm{CF}_{4}$ molecules, producing $\mathrm{F}$ atoms, which attack the silica. The flame temperature can be varied to control the degree of dissociation. The torch produces a smooth, Gaussian shaped 
pit, approximately $1.5 \mathrm{~mm}$ dia. and $\sim 2$ to $4 \mu \mathrm{m}$ deep, with no apparent lip. This torch was applied to treat 3 sites on a fused silica substrate.

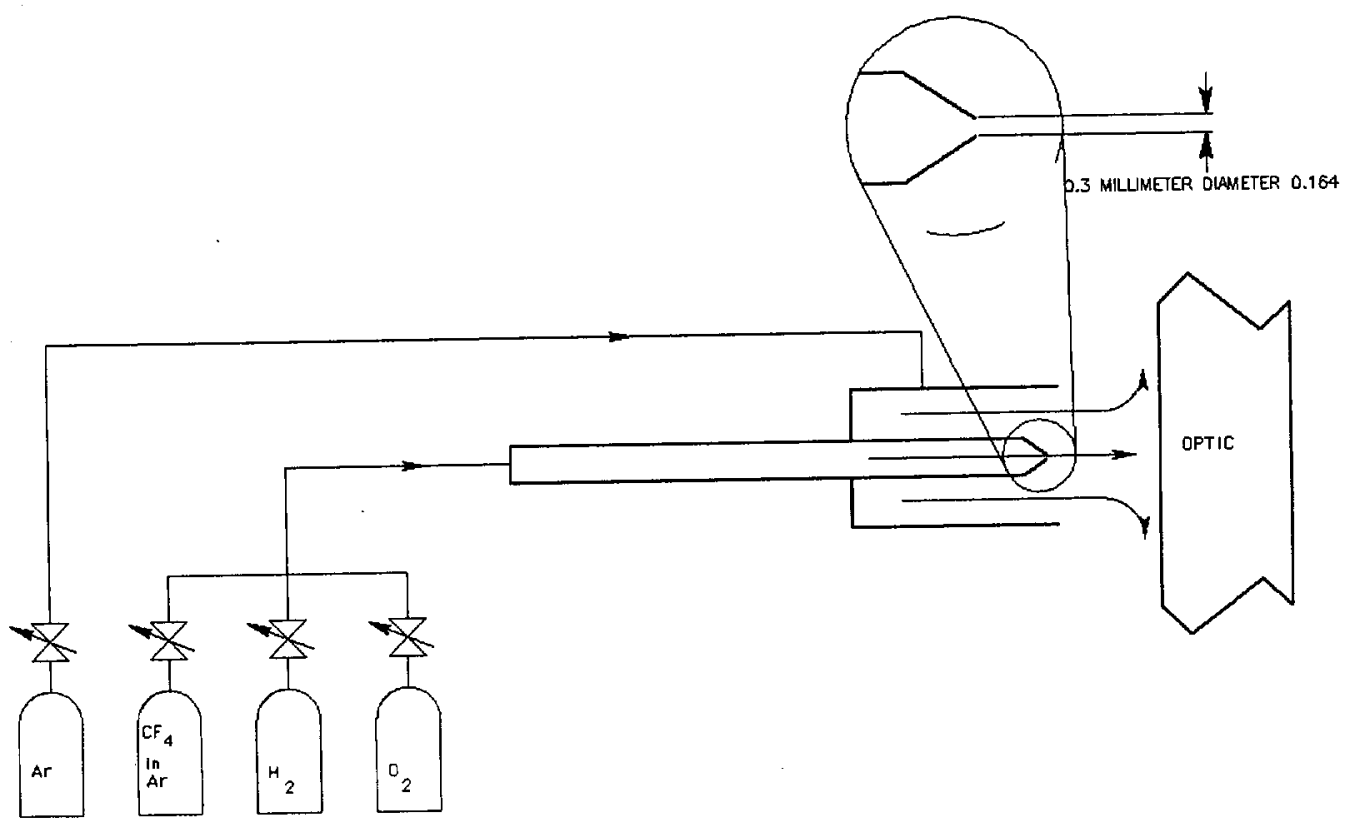

Figure 8. A schematic of the micro-flame torch system.

A single sample was tested for the degree of mitigation at $351 \mathrm{~nm}$ and $\sim 11 \mathrm{~ns}$. The sample had 4 large damage sites (produced at $\sim 45 \mathrm{~J} / \mathrm{cm}^{2}, 355 \mathrm{~nm}, 7.5 \mathrm{~ns}$ ), three of which were treated by the flame torch; one control site was not treated. One of the treated sites was totally mitigated against growth, i.e., it survived 1000 shots at $8 \mathrm{~J} / \mathrm{cm}^{2}$. Another one of the treated sites survived 200 shots at each of the test fluences, 8,12 and $14 \mathrm{~J} / \mathrm{cm}^{2}$, but other surface damage appeared near the test site, and grew. The third treated site, which was not completely removed by the flame torch process, grew after 20 shots at $8 J / \mathrm{cm}^{2}$. The untreated control site grew immediately at $8 \mathrm{~J} / \mathrm{cm}^{2}$. We speculate that the other surface damage appearing during the tests was caused by contamination, possibly carbon residue from the dissociation of $\mathrm{CF}_{4}$.

Although only a small set of sites were tested, the flame torch shows promise to mitigate growth of heavy laserinitiated damage. Contamination by carbon deposits is also a problem with this method. The hardware is simple and inexpensive, and it could be configured to rapidly process multiple sites on large optics. The method needs additional development, but it could be a reasonable back-up method to the $\mathrm{CO}_{2}$ process.

\section{CONCLUSIONS}

All of the tested methods, except the plasma torches, gave promising results for mitigating the growth of surface damage sites on polished fused silica. Clearly the $\mathrm{CO}_{2}$ processing gives the most consistent, convincing results. Moreover, the $\mathrm{CO}_{2}$ laser processing method should be relatively inexpensive and straightforward to apply, for mitigating sites on large aperture optics. Chemical etching the surface with HF solution also exhibits promise to mitigate laser-initiated damage. However, deep chemical etching would not be acceptable if it aberrates the transmitted wavefront beyond user specifications. Nevertheless, since it is potentially a relatively inexpensive method also, it should be considered as a primary backup to the $\mathrm{CO}_{2}$ method, for mitigating surface damage on polished silica optics.

One can speculate about any advantage of the flame torch mitigation compared with $\mathrm{CO}_{2}$ mitigation process. One advantage of the flame torch may be the ability to produce a smoother, shallower pit, which is less disruptive to 
the optic surface. Also, it may require less capital cost than the $\mathrm{CO}_{2}$ method. While there may be a cost advantage for implementing the flame torch method, it must be considered as an alternative to the $\mathrm{CO}_{2}$ process because more tests are needed to determine its reliability. Unless further testing of the $\mathrm{CO}_{2}$ mitigation process uncovers difficulties, the flame torch approach would not be developed further.

Site-by-site processing of laser damage with a $\mathrm{CO}_{2}$ laser is feasible and straightforward to apply to large optics (eg., $0.5-1.0 \mathrm{~m}$ scale). We are developing it as the primary mitigation method for fused silica optics used in highpeak-power applications at $351 \mathrm{~nm}$. Furthermore, it is reasonable to envisage a system that does the initiation, identification, and $\mathrm{CO}_{2}$ processing steps, all at the same time.

\section{ACKNOWLEDGEMENTS}

We wish to acknowledge the help of many LLNL associates who contributed to this effort. Those who performed the growth tests in the slab laser facility; K.Neeb and E.Donohue. Those who provided samples, analysis and interpretation; S.Maricle, L.Sheehan, J.Wong, S.Demos, M.Staggs, J.Ferreira, E.Lindsey and R.Torres. Those who performed the chemical etching; L.Summers and J.Britten, and others who contributed advice; D.Milam, L.Chase, W.Siekhaus and F.Genin.

\section{REFERENCES}

1. M.A. Norton, L.W. Hrubesh, Z. Wu, E. Donohue, M.D. Feit, M.R.Kozlowski, D.Milam, K.P. Neeb, W.A. Molander, A.M. Rubenchik, W.D. Sell, P.Wegner, "Growth of Laser Initiated Damage in Fused Silica at $351 \mathrm{~nm}$," Laser-Induced Damage I n Optical Material:2000, SPIE Vol. 4347, p.468, October 2000. LLNL Report UCRL-JC-139649.

2. M.D. Feit, L.W. Hrubesh, A.M. Rubenchik, J. Wong, "Scaling relations for laser damage initiation craters," Laser-Induced Damage I n Optical Materials-2000, SPIE Vol. 4347, pp.316-323, October 2000.

3. S.G. Demos, M.R. Kozlowski, M.C. Staggs, L.L. Chase, A.K. Burnham, H.B. Radousky, "Mechanisms to explain damage growth in optical materials," Laser-Induced Damage In Optical Materials-2000, SPIE Vol. 4347, pp.277-284, October 2000.

4. J.M. Yoshiyama, F.Y. Genin, A. Salleo, I.M. Thomas, M.R. Kozlowski, L.M. Sheehan, I.D. Hutcheson, D.W. Camp, "Effects of polishing, etching, cleaving, and water leaching on UV laser damage of fused silica," Laser-Induced Damage In Optical Materials-1997, SPIE Vol. 3244, pp.331-340, October 1997.

5. C.L. Battersby, L.M. Sheehan and M.R. Kozlowski, "Effects of Wet Etch Processing on LaserInduced Damage of Fused Silica Surfaces," Laser Induced Damage in Optical Materials - 1998, SPIE Vol. 3578, pp. 392-400, October 1998.

6. L.W. Hrubesh, M.A.Norton, W.A.Molander, P.J.Wegner, M.Staggs, S.G.Demos, J.A.Britten, L.J.Summers, E.F.Lindsey, and M.R.Kozlowski, "Chemical etch effects on laser-induced surface damage growth in fused silica," Laser-Induced Damage In Optical Materials:2000, SPIE Vol. 4347, pp.553-559, October 2000.

7. P.A.Temple, D. Milam and H. Lowdermilk, " $\mathrm{CO}_{2}$-Laser polishing of fused silica surfaces for increased laser damage resistance at $1.06 \mu \mathrm{m}$," Proceedings of Laser Induced Damage in Optical Materials: NBS Publ. 568, pp.229-235 (1979). 
8. P.A.Temple, S.C.Seitel and D.L.Cate, " $\mathrm{CO}_{2}$-Laser polishing of fused silica: recent progress," Proceedings of Laser Induced Damage in Optical Materials: NBS Publ. 669, pp.130-137 (1982).

9. R.M. Brusasco, B.M. Penetrante, J.A. Butler and L.W. Hrubesh, "Localized $\mathrm{CO}_{2}$ Laser Treatment for Mitigation of $3 \omega$ Damage Growth in Fused Silica," these Proceedings, October 2001. 


\title{
Investigation of fluorescence microscopy as a tool for noninvasive detection and imaging of damage precursors at $351 \mathrm{~nm}$
}

\author{
S. G. Demos $(1)$ \\ M. C. Nostrand ${ }^{(1)}$ \\ M. Staggs ${ }^{(1)}$ \\ C. W. Carr $^{(1)}$ \\ D. $\mathrm{Hahn}^{(l)}$ \\ M. R. Kozlowski (2) \\ L. Sheehan ${ }^{(3)}$ \\ C. Battersby ${ }^{(4)}$ \\ A. Burnham ${ }^{(1)}$ \\ (1) Lawrence Livermore National Laboratory, PO Box 808, L-411, Livermore, CA 94551 \\ ${ }^{(2)}$ Currently with Cierra Photonics, 3640 Westwind Blvd., Santa Rosa, CA 95403 \\ ${ }^{(3)}$ Currently with Therma-Wave, 1250 Reliance Way, Fremont, CA 94539 \\ ${ }^{(4)}$ Currently with Terabeam, 12413 Willows Rd. NE, Kirkland, WA 98034
}

\begin{abstract}
This work is an experimental investigation to evaluate the potential of fluorescence microscopy as a tool to detect surface contamination as well as reveal surface damage precursors on DKDP and $\mathrm{SIO}_{2}$ optics. To achieve these technical objectives, microscopic imaging systems were built that also incorporated in-situ damage testing capabilities. Fluorescence imaging experiments were performed using 351-nm laser excitation while damage testing was performed at relatively high laser fluences. The experimental results demonstrated the potential of this technique to address the aforementioned technical issues.
\end{abstract}

\section{INTRODUCTION}

The detection and characterization of surface defects in various materials in which high purity is critical for their performance has led to a great deal of research and development. This problem was recognized soon after the invention of the laser when laser induced damage initiation at very low fluences was attributed to the presence of mechanical and chemical defects ${ }^{1,2}$. In the fields of high-power laser development and applications, surface quality of the various optical components is of critical importance. Various methods have been explored to remove much of the contamination that led to an increase in the damage threshold ${ }^{3-6}$. However, the damage threshold is still substantially below the intrinsic material limit, especially in large aperture optical components where downselection and/or replacement of optical elements significantly increase the cost of operation ${ }^{7.8}$. To manufacture high damage threshold optics, elimination of damage initiating defect structures located on their surface represents a major challenge. Noninvasive methods to characterize the quality of the surface with respect to its resistance to damage under $355-\mathrm{nm}$ irradiation and pinpoint the location of damage precursors may be the key to address laser induced damage issues.

Fluorescence microscopy utilizes the absorption and emission characteristics of the sample. The most common applications of fluorescence imaging is found in the biomedical field ${ }^{9}$ whereby tissues or cells are "tagged" with particular fluorescing bio-molecules which in turn are used to record images indicating their incorporation into the cells. Most recently, fluorescence microscopy was used to image defect nanostructures located in the bulk of dielectric materials ${ }^{10}$. The presence of emission by a system under laser excitation is associated with the presence of absorption. In wide band-gap optical materials, such as $\mathrm{SiO}_{2}$ and $\mathrm{KDP}$, there should be no absorption, and therefore emission, at $355-\mathrm{nm}$. However, the observed laser induced damage at relatively low fluence may be suggestive of the presence of absorbing defects such as foreign particles, etc. This indicates that fluorescence microscopy may be a suitable tool for the detection of absorbing defect structures in optical materials that may be the cause of laserinduced damage.

The objective of this work was to investigate fluorescence microscopy as a tool that could provide information regarding the quality of optical surfaces by detecting the presence of foreign substances. The observed defect structures could then be exposed to high power laser irradiation to reveal their potential to initiate damage. In this report, we will describe our preliminary effort that involved fluorescence imaging of $\mathrm{SiO}_{2}$ and $\mathrm{KDP}$ surfaces and in situ damage testing experiments. 


\section{EXPERIMENTAL SET-UP}

A detailed description of the experimental apparatus utilized in this work can be found elsewhere ${ }^{11}$. The experiments were performed on polished fused silica and diamond-turned, conventionally and rapidly grown DKDP crystals. All sample surfaces were flat. The experiments were performed with the samples held at room temperature.

\section{FLUORESCENCE IMAGING ON $\mathrm{SiO}_{2}$ SURFACES}

Fluorescence images of $\mathrm{SiO}_{2}$ surfaces were recorded and compared. The various samples tested had undergone different polishing processes culminating in a wide range of surface damage thresholds the results of which are listed in Table 1 along with the information regarding the polishing process and the corresponding descriptive damage threshold. In addition, Figure 1 is the Ramped-on-1 (R/1) probability damage plot versus fluence for each sample listed in Table 1. Thus, Figure 1 shows how the descriptive average damage thresholds for Table 1 were formulated (i.e., very low for sample L-120-1 to high for sample 6K03).

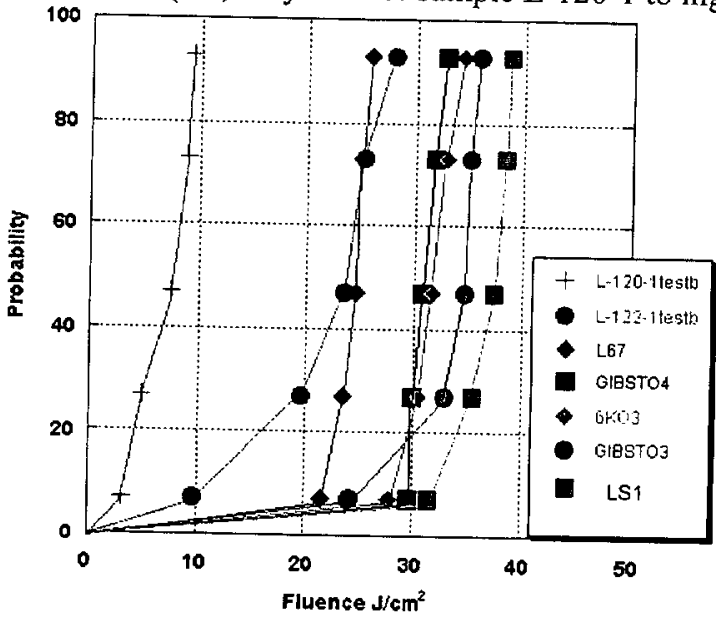

\begin{tabular}{llcl} 
Part \# & Polish & $\begin{array}{c}\text { Subsurface } \\
\text { damage }\end{array}$ & Average DT \\
\hline L-120-1 & Ce & High & Very low \\
L-122-1 & Ce & High & Low \\
L67 & Ce & Med & Low \\
GIBST04 & Ce + & None & High \\
GIBST03 & Zr & None & High \\
LS1 3/97 & unknown & None & High \\
6K03 & Ce+lon Mill & None & High
\end{tabular}

Table 1: $\mathrm{SiO}_{2}$ samples utilized to obtain fluorescence images of their surface.

Figure 1: R/I probability damage plot versus

fluence for each sample listed in Table 1.

Figure 2 shows microscopic fluorescence images (MFI) of the surface of the samples (10x objective with a $5 x$ zoom). The contrast on these images has been adjusted to better show the existing variation on the emission intensity from the different points of the surface. The average emission intensity from the surface of each sample was different between samples and it was found to depend on the polishing process. The Ce polished samples exhibited maximum emission that appeared to scale with the damage threshold of the material. The highest damage threshold samples exhibit very low emission with relatively uniform distribution while features on the surface that exhibit significantly higher intensity are rare. Dark scratches and points are observed on sample 6K03. Few features with some dark scratches are observed on sample GIBST03. Sample LS13/97 shows also a clean surface exhibiting low emission. Sample GIBST04 shows the presence of emissive lines and points with somewhat higher intensity. The above samples have relatively high damage thresholds. On the other hand, low damage threshold samples exhibit a different behavior. Sample L122-1 exhibits a speckled background and bright points having diameters of the order of a few microns. Sample L67 shows the presence of a high concentration of bright points. Finally, the image of the surface of sample L120 indicates the presence of a web of cracks that are filled with an emissive material. The images of the surfaces in Figure 2 are arranged according to their measured damage threshold (high to low, left to right, top to bottom). An examination of the surface morphology as depicted by the fluorescence images suggests a reasonable correlation of the presence of emissive features on the surface with a lowering of the damage threshold.

Comparison of the fluorescence images with the light scattering images of the same section of the samples indicate that the observed features using fluorescence microscopy are not always visible in the corresponding light scattering image. This is demonstrated in Figures 3 and 4. Figure 3 indicates that the fluorescence image contains a much smaller number of features than the corresponding light scattering image. Some of these features can be observed using light scattering. Two lines observed in the fluorescence image are presumably cracks or scratches filled with an emissive material. These features are not visible using light scattering. 

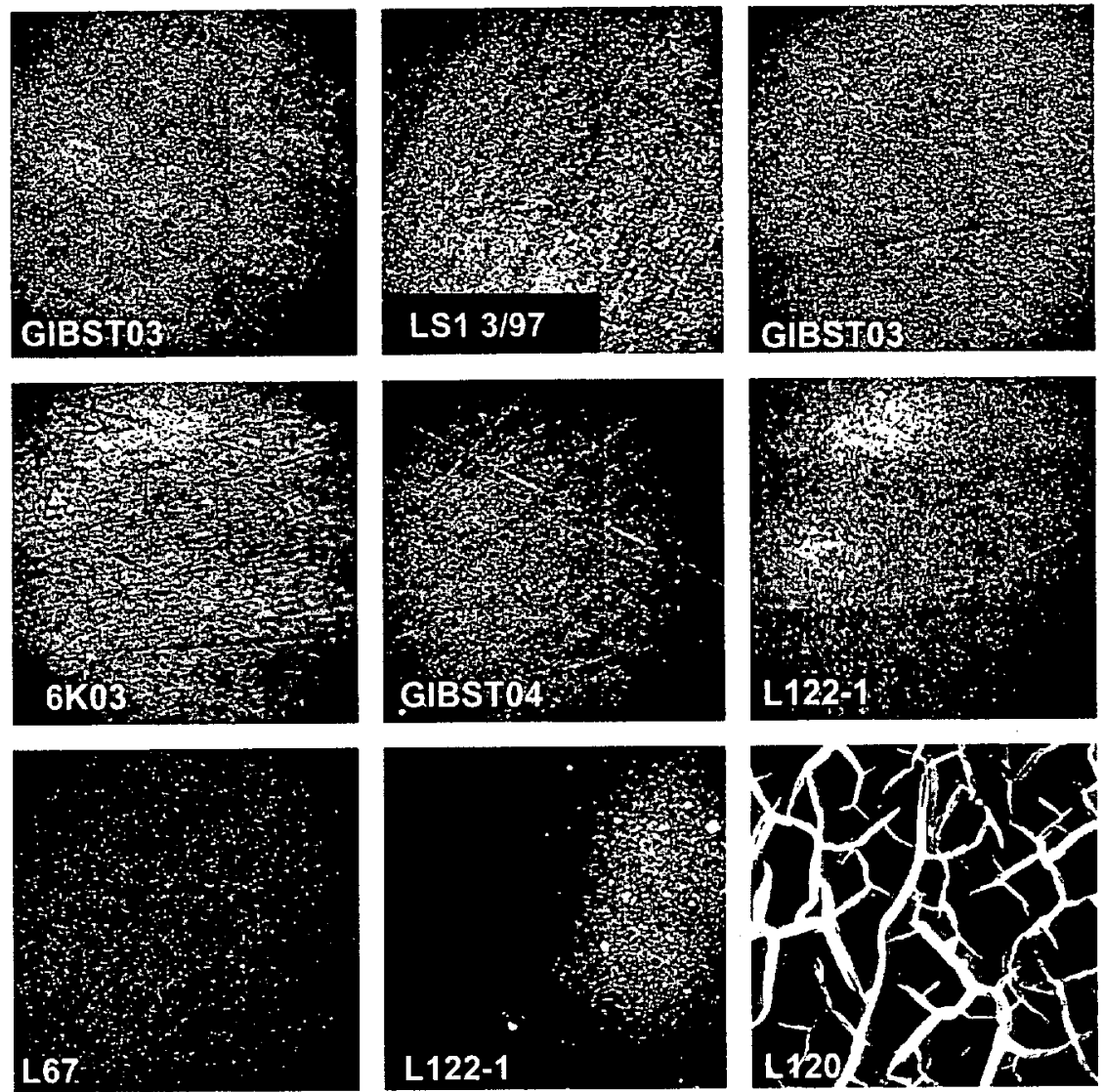

Figure 2: Microscopic fluorescence images of the surface of $\mathrm{SiO}_{2}$ samples under $351 \mathrm{~nm}$ excitation. Each image measures $1.2 \mathrm{~mm}$ per side.
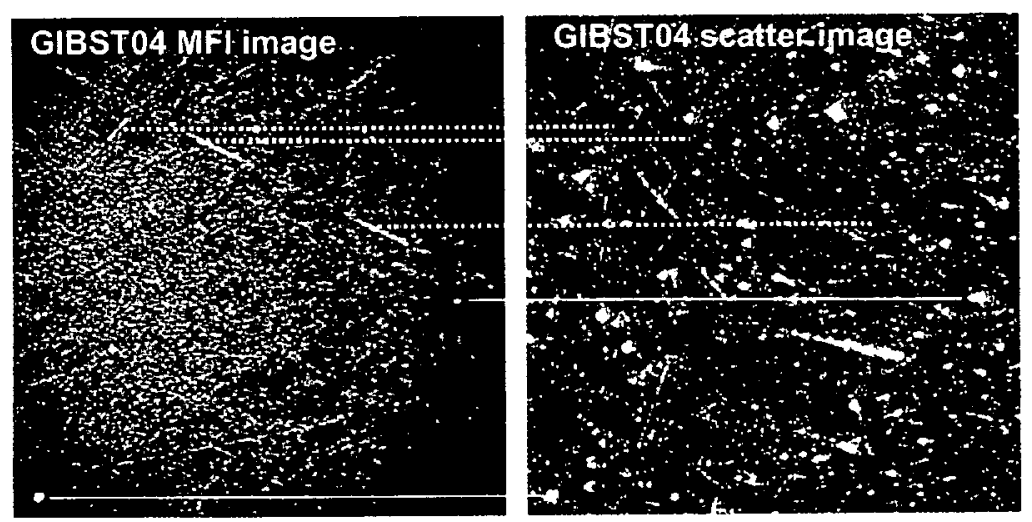

Figure 3: Fluorescence and light scattering images of the same section of the surface of $\mathrm{SiO}_{2}$ sample GIBST04. Each image measures $1.2 \mathrm{~mm}$ per side.
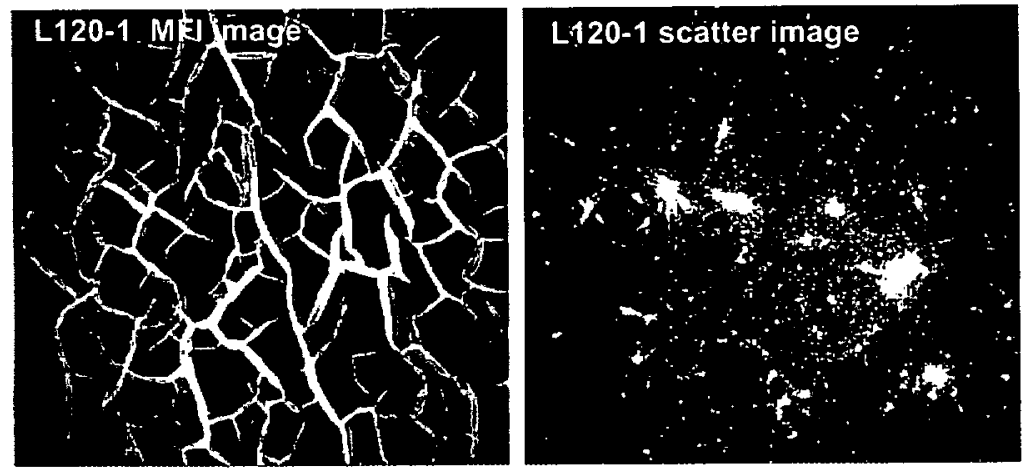

Figure 4: Fluorescence and light scattering images of the same section of the surface of $\mathrm{SiO}_{2}$ sample L1201. Each image measures 1.2 mm per side. 
Similarly, Figure 4 shows that the web of fluorescing cracks in sample L-120-1 is totally invisible in the corresponding light scattering image.

An obvious question arising from these experimental results is if the observed fluorescing features are responsible for damage initiation. To address this issue, experiments were performed using a Spectra-Physics commercial laser operating at $355-\mathrm{nm}, 7.5-\mathrm{ns}$, with fluences up to $-40 \mathrm{~J} / \mathrm{cm}^{2}$. Figure 5 shows a typical example of the experimental data. The fluorescence image of the sample prior and following irradiation with the $355-\mathrm{nm}$ laser pulses is shown. Damage sites are clearly visible due to the emission from the modified material. An examination of the location where damage was initiated with respect to the location of preexisting fluorescing features indicates reasonable correlation. There are damage sites that were initiated at the exact location where fluorescing features were observed prior to irradiation. However, there are a number of damage sites that were initiated at locations where there were no dominant features in the fluorescence image. It must be pointed out that all experiments on $\mathrm{SiO}_{2}$ samples were performed at the early stages of this effort when instrumentation and methodology were still not optimized. It is therefore difficult to conclude if the initiators of the damage sites that do not correlate with the fluorescence map is not visible using fluorescence microscopy or if their sizes are so small that their emission signals are below the detection limit of the system. In any case, the correlation of damage initiation (at 7.5-ns, 40 $\mathrm{J} / \mathrm{cm}^{2}$ ) with preexisting features was much better for the fluorescence microscopy approach than for light scattering at the time that the experiments were performed.
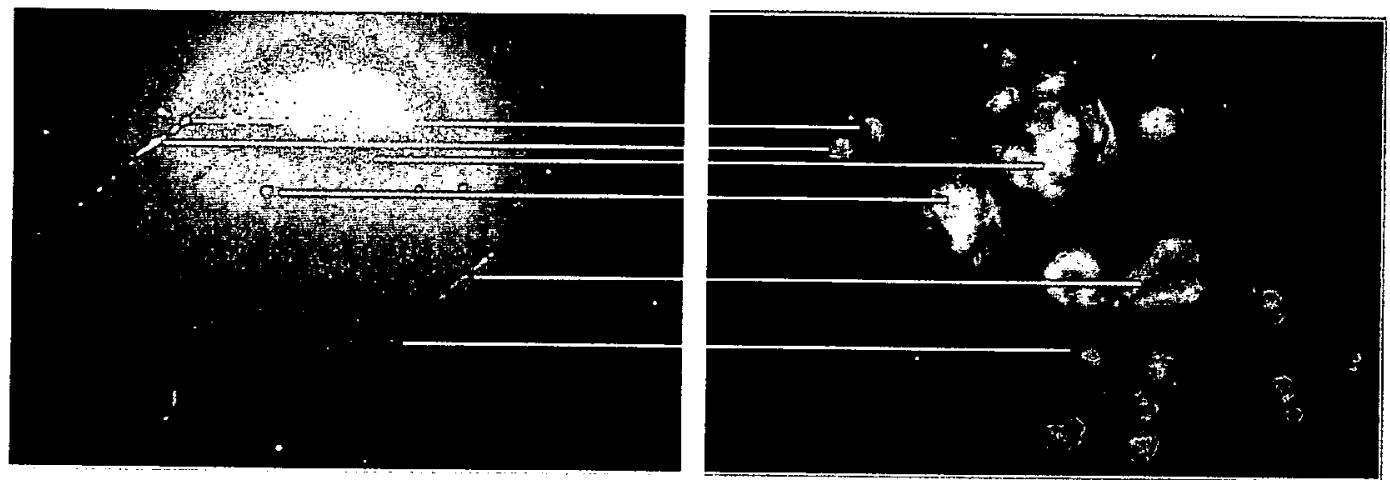

Figure 5: Fluorescence images of the surface of the $\mathrm{SiO}_{2}$ sample prior and following irradiation with a 355-nm, 7.5 ns laser pulse at $\sim 40 \mathrm{~J} / \mathrm{cm}^{2}$. The horizontal dimension in these images is $1.2 \mathrm{~mm}$.

\section{FLUORESCENCE IMAGING OF DKDP SURFACES}

Two deuterated KDP (DKDP) samples with characteristically different surface damage behavior were studied using fluorescence imaging. These were samples RG8A, a third-harmonic-generation crystal with greater than $80 \%$ deuteration, and LL3-LG, a crystal cut normal to the extraordinary axis with $70 \%$ deuteration, which are rapid and conventional growth, respectively. The $R / 1$ probability damage plot versus fluence for the above samples are shown in Figure 6. Figure 7 shows typical fluorescence and light scattering images from DKDP sample RG8A. This sample has a very low surface damage threshold. The main features in the fluorescence image are not present in the light scattering image. This indicates that these fluorescing objects are located below the top layer of the surface. Using maximum optical magnification (X100 microscope objective) the estimated dimension of these features was $\sim 100 \mu \mathrm{m}$ in length, $\sim 8 \mu \mathrm{m}$ in width and, $\sim 50 \mu \mathrm{m}$ in depth.

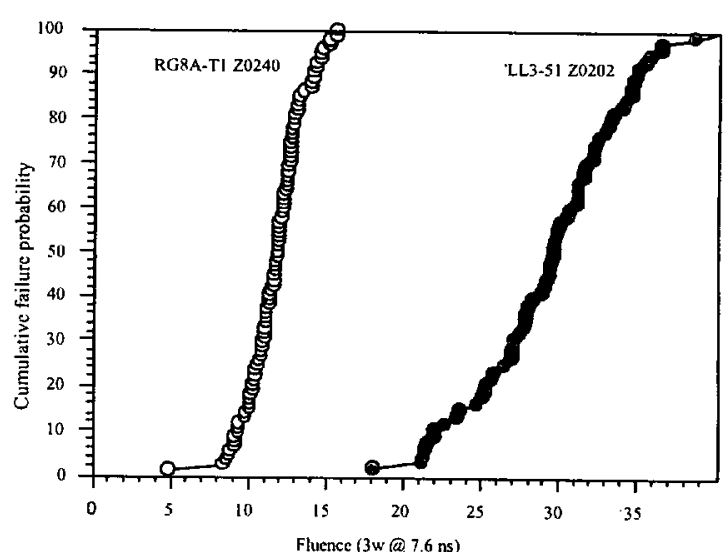

Figure 6: $R / 1$ probability damage plot versus fluence for DKDP samples RG8A and LL3-LG 
Sample LL3-LG has a high surface damage threshold. Typical fluorescence and light scattering images from this sample are shown in Figure 8. Fluorescing spots are observed on the surface of this sample that do not correlate with the observed features in the light scattering image. Comparison of the light scattering images from the two samples indicates that sample LL3-LG has a much cleaner surface. In addition, the fluorescing objects in sample RG8A are much bigger and brighter compared to those observed in sample LL3-LG. The background emission observed in the fluorescence images is due to the nearly uniform emission from the surface of the samples while the structure observed is due to the nonuniformity of the $351-\mathrm{nm}$ illumination beam. Examination of the surface of these samples indicated that it is rather common to observe features that are visible to both imaging approaches. This is attributed to the presence of contamination particles that are on the surface of the samples that are also emissive under 351-nm laser photoexcitation. The presence of emissive structures embedded into the surface of the material (not visible with light scattering) suggests the incorporation of contaminants during the polishing process and/or subsequent handling. These results suggest that fluorescence microscopy may provide a way of monitoring the degree of contamination. Furthermore, the observation of large size, highly emissive features on the surface of sample RG8A is consistent with its low surface damage threshold.
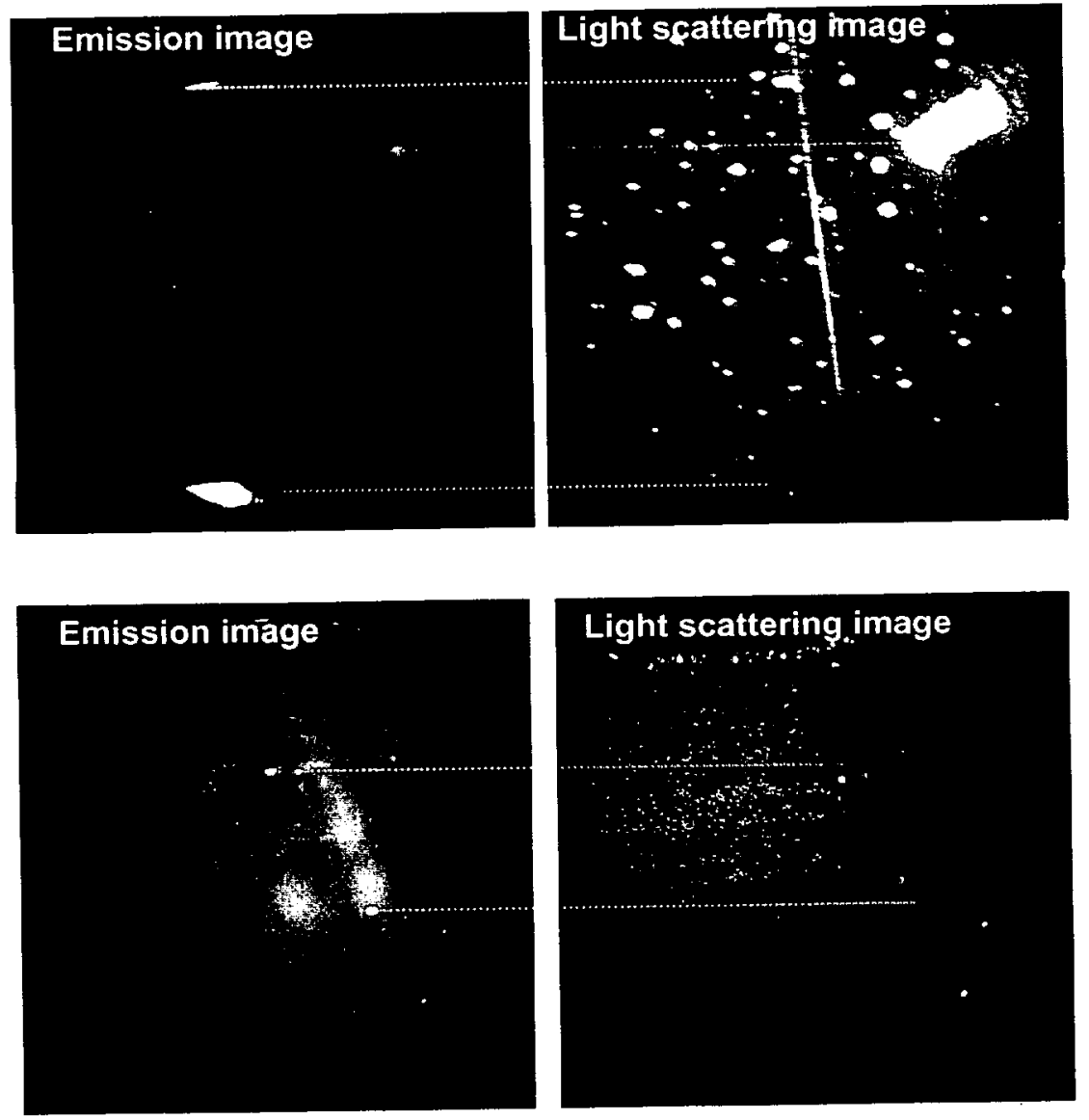

Figure 7: Fluorescence and light scattering images of the same $1025 X 1025 \mathrm{\mu m}^{2}$ section of the surface of DKDP sample RG8A.

Figure 8: Images of the same $1025 X 1025 \mathrm{um}^{2}$ section of the surface of $D K D P$ sample $L L 3-L G$.

\section{IN-SITU DAMAGE TESTING OF DKDP SURFACES}

In-situ damage testing experiments were performed on samples RG8A and LL3-LG. The objective was to irradiate objects observed using fluorescence microscopy with $355-\mathrm{nm}$ laser pulses and examine their susceptibility to laser induced damage initiation.

Figure 9-1 shows a light scattering image from the surface of sample RG8A before irradiation. The corresponding fluorescence image under $351-\mathrm{nm}, \mathrm{CW}$ excitation is shown in Figure 9-2. These images were obtained before the sample was irradiated with the high fluence, $355-\mathrm{nm}$ laser pulse. Figure $9-3$ shows the captured image during irradiation with a single $3-\mathrm{ns}, 355-\mathrm{nm}$ pulse at $15 \pm 3 \mathrm{~J} / \mathrm{cm}^{2}$. The light scattering image after irradiation with the 355-nm pulse shown in Figure 9-4 demonstrates the formation of a damage site as a result of the exposure 
of the sample to the laser pulse. The location of the damage site is identical to the location where light was observed during irradiation (see Figure 9-3) and the location of the preexisting large fluorescing object (see Figure 9-2). This experiment showed the correlation of the preexisting feature visible only using fluorescence microscopy with damage initiation. The detected light during irradiation (Figure 9-3) is presumably due to plasma formation during damage. The same experiment was repeated using the same sample and four additional fluorescing features, similar to those shown in Figures 9-2 and 8, were examined. In total, 5 sites were chosen and in all cases, damage was initiated within the area where the observed features were located. All experiments were performed using $\sim 15 \mathrm{~J} / \mathrm{cm}^{2}$
laser fluence at $3 \mathrm{~ns}$.
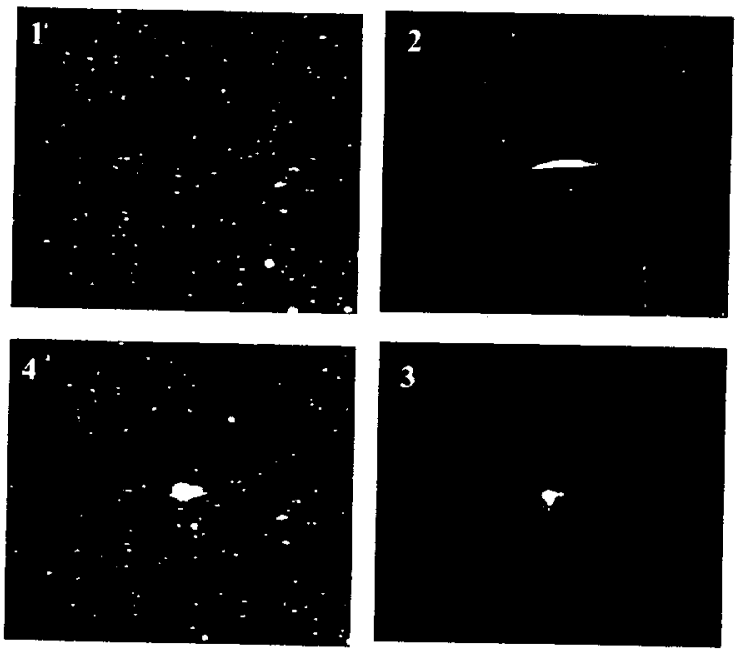

Figure 9: Images from same 600X520 $\mu^{2}$ section of the surface of DKDP sample RG8A.
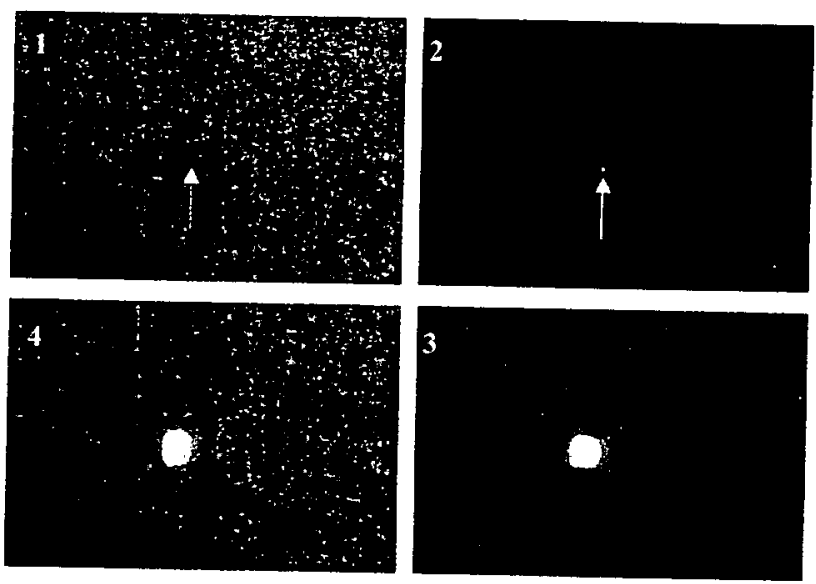

Figure 10: Images from same $420 X 280 \mathrm{\mu m}^{2}$ section of the surface of DKDP sample LL3-LG.

1) Light scattering image before irradiation. 2) Fluorescence image under 351-nm, excitation. 3) Plasma emission image during irradiation with a single $3-\mathrm{ns}, 355-\mathrm{nm}$ pulse at $\sim 15 \mathrm{~J} / \mathrm{cm}^{2} .4$ ) Light scattering image after irradiation
with a single $355-\mathrm{nm}$ pulse.

Similar experiments were performed on sample LL3-LG. Fluorescence features were irradiated with $\sim 15 \mathrm{~J} / \mathrm{cm}^{2}$, 3-ns, 355-nm pulses while the behavior was monitored during this process. More than 40 sites over a two-week period were targeted and tested at $\sim 15 \mathrm{~J} / \mathrm{cm}^{2}$. In all but one case, fluorescence features did not correlate with an observable reaction due to the $355-\mathrm{nm}$ laser irradiation that can be classified as laser induced damage. In most of the cases, the intensity of the fluorescence feature was observed to be reduced after laser irradiation. The only location fluorescing damage that was clearly visible using light scattering is shown in Figure 10. Typical reactions of other fluorescing features at other locations to laser irradiation will be shown in later figures.

Figures 10-1 and 10-2 show a light scattering image and the corresponding fluorescence image, respectively, from the surface of sample LL3-LG before irradiation. Note that the fluorescence object is not visible in the light scattering image. Figure 10-3 shows the plasma image recorded during irradiation with a $355-\mathrm{nm}$ pulse at $\sim 15 \mathrm{~J} / \mathrm{cm}^{2}$. The light scattering image after irradiation with the $355-\mathrm{nm}$ pulse (Figure 10-4) indicates the presence of damage and a correlation with the pre-existing fluorescence "object". Thus, the only damage initiation we were able to record during the course of this experiment on sample LL3-LG is suggesting that the fluorescing defect was the
damage initiator.

The presence of emission is evidence for absorption. It has been suggested that an absorbing particle can heat up to thousands of degrees and cause damage initiation. ${ }^{12}$ In this case, it is obvious that the absorbing particle needs to be appropriately coupled in to the host material so that its excess energy is efficiently transferred in the form of can respond in a wave into the host. It was one objective of this preliminary work to prove that fluorescing objects damage of the host maversible and dynamic manner to exposure to high fluence laser irradiation without leading to damage of the host material. Experimental evidence of this effect was recorded during our study of sample LL3-LG. 
Figure 11-1 shows the light scattering image of the surface of the sample with an object of interest indicated with an arrow. Figure 11-2 shows the fluorescence image of the same area where the object of interest is clearly visible. During irradiation with a single laser pulse, formation of plasma was recorded as shown in Figure 11-3. The light scattering image after irradiation (Figure 11-4) suggests no damage to the host material as a result of plasma formation. The only clear difference is that the size of the object has been reduced. However, the fluorescence image shown in Figure 11-5 is noticeably different from that shown in Figure 11-2. A contaminated fluorescing area around the particle that led to plasma formation is observed. This is indicative of a micro-explosion that was associated with the generation of mobile ionic and/or molecular species that were spattered into the surrounding area. Subsequent 355-nm irradiation led to no additional formation of plasma or change in the fluorescence images. This is demonstrated in Figure 11-6 where the fluorescence image following irradiation with 10 pulses is shown. A long arrow shaped feature fluorescing adjacent to the object of interest did not initiate damage. The only noticeable change of this object as a result to laser irradiation was the reduction of its intensity as demonstrated in figs. 11-2, and 11-5.
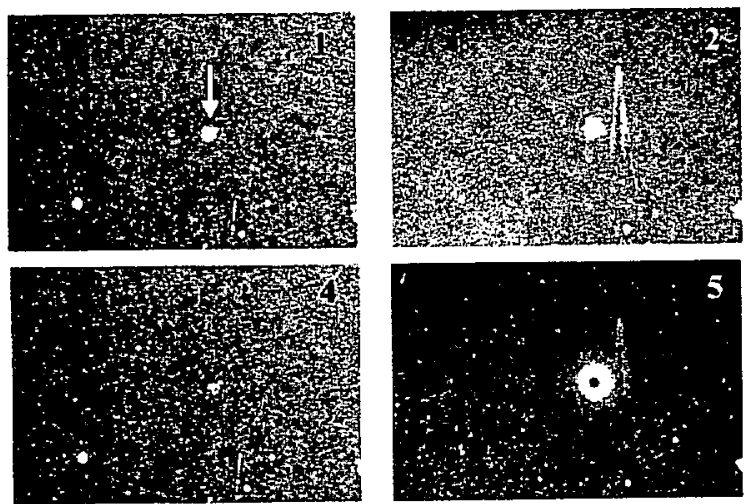
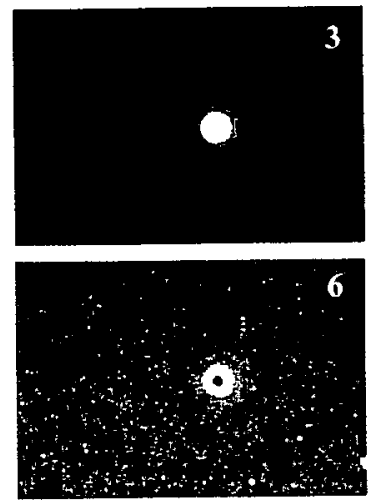

Figure 11: Images from same $450 \times 310 \mathrm{\mu m}^{2}$ section of the surface of sample $L L 3-L G$ showing the reaction of surface defects from contamination to $355 \mathrm{~nm}, 3 \mathrm{~ns}$ laser pulses at $\sim 15 \mathrm{~J} / \mathrm{cm}^{2}$. Details are provided in the text.
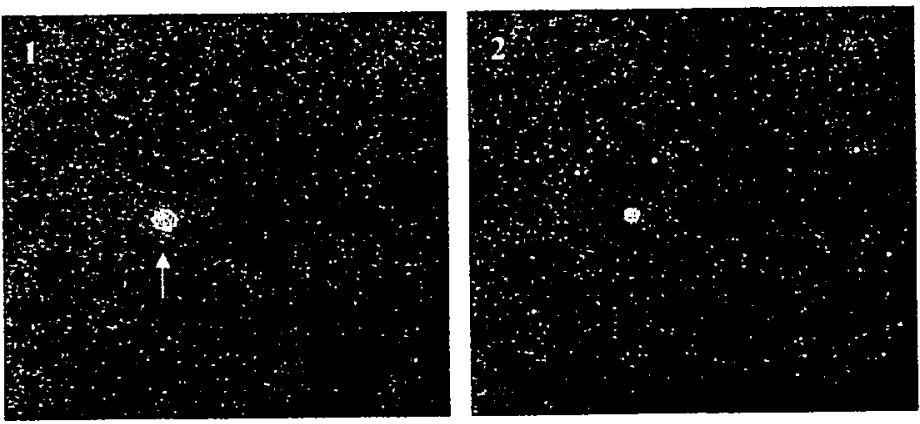

Figure 12: Light scattering images from same $290 X 260 \mathrm{\mu m}^{2}$ section of the surface of sample $L L 3-L G$ (1) before and (2) after irradiation with a single laser pulse at ${ }^{\sim} 15$ $\mathrm{J} / \mathrm{cm}^{2}$.

A similar effect is demonstrated in Figure 12. The object of interest is denoted with an arrow in the fluorescence image (Figure 12-1) acquired before irradiation of the sample. This object was very bright with a maximum pixel intensity of $\sim 6000$ counts and it was not visible in the light scattering image. Figure 12-2 shows the fluorescence image after irradiation. The intensity of the object has now been reduced to $\sim 200$ counts/pixel while a number of new particles are observed on the image. This suggests the original particle "exploded" creating a number of smaller ones. Post irradiation examination of the sample with light scattering does not indicate the presence of damage. 


\section{FLUORESCENCE IMAGING AND DAMAGE TESTING OF A 15-cm DKDP CRYSTAL}

Recently, higher quality $15-\mathrm{cm}$ DKDP ( $80 \%$ deuteration, to be used for 351-nm harmonic generation) became available for experimentation. These materials were considered to be from the best production and handling methods available at the time. Such material is desirable to avoid unnecessary contamination and/or irrelevant damage precursors. One crystal of this set, taken from rapid-growth production boule BD8-10 and finished with a single-point diamond turning machine, is the subject of this section. The fluorescence imaging technique was used on this sample in an attempt to identify and correlate surface damage precursors.

The experimental plan consisted of constructing a fluorescence image map of the sample before and after exposure to $351-\mathrm{nm}$ laser pulses, then correlate the surface damage features in the 'after' map to the fluorescent features in the 'before' map. The laser beam was that of the Optical Sciences Laser (OSL), consisting of 3-cm diameter, 3-ns flattop pulses. A total of eight OSL footprints were applied to the sample. In order to test the environmental effects of surface damage, four of these footprints were associated with an environment of 760 torr (air), while the other four were at 1 torr. Figure 13 shows the laser footprint configuration.

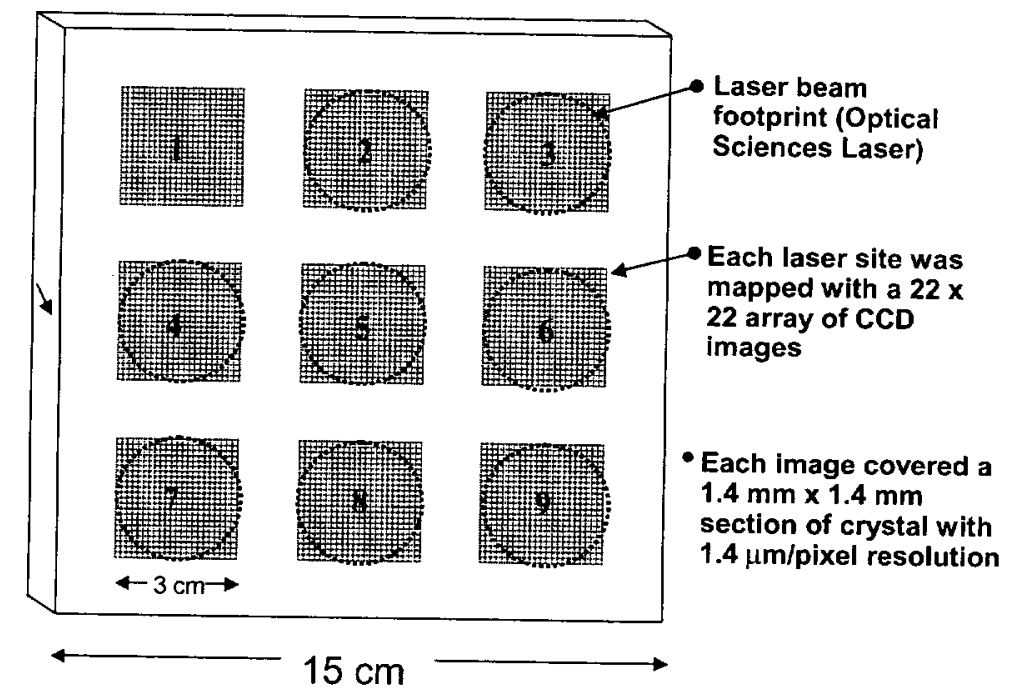

Figure 13: The crystal was mapped at each of the 9 sites shown above. The laser irradiated sites 2-9. Site 1 was left un-irradiated for reference.

Since bulk damage needed to be avoided for our surface damage experiments, it was first laser-conditioned. ${ }^{13,14} \mathrm{~A}$ five-shot ramp to $7 \mathrm{~J} / \mathrm{cm}^{2}$ was performed (nominally $1,3,5,6,7 \mathrm{~J} / \mathrm{cm}^{2}$ ) at each footprint. The actual fluence history for each footprint is shown in Table 2.

Table 2: Average fluence for each shot of a five-shot ramp (351-nm, 3-ns) at each of the eight OSL beam footprints shown in Figure 13. The peak-to-valley intensity variation was about $\pm 15 \%$.

\begin{tabular}{|c|c|c|c|c|c|}
\cline { 2 - 6 } \multicolumn{1}{c|}{} & \multicolumn{5}{c|}{ Average Fluence $\left(\mathrm{J} / \mathrm{cm}^{2}\right)$} \\
\hline Test Site \# & Shot 1 & Shot 2 & Shot 3 & Shot 4 & Shot 5 \\
\hline $\mathbf{2}$ & 1.5 & 3.0 & 4.7 & 6.5 & 7.5 \\
\hline $\mathbf{3}$ & 1.4 & 3.3 & 5.3 & 6.3 & 7.3 \\
\hline $\mathbf{4}$ & 1.3 & 3.2 & 5.8 & 6.6 & 7.4 \\
\hline $\mathbf{5}$ & 1.5 & 3.6 & 5.5 & 6.2 & 7.5 \\
\hline $\mathbf{6}$ & 1.4 & 3.2 & 5.8 & 6.4 & 7.5 \\
\hline $\mathbf{7}$ & 1.5 & 3.1 & 5.6 & 6.2 & 7.6 \\
\hline $\mathbf{8}$ & 1.2 & 4.1 & 5.8 & 6.2 & 7.1 \\
\hline $\mathbf{9}$ & 1.7 & 3.4 & 5.6 & 5.8 & 7.1 \\
\hline
\end{tabular}

The fluorescence mapping was performed using a high-resolution imaging system, where a single image consists of a $1.4 \times 1.4 \mathrm{~mm}^{2}$ section of the sample surface. With the (liquid nitrogen-cooled) $1024 \times 1024$ CCD array, this gives 
$\sim 1.4 \mu \mathrm{m} /$ pixel resolution. To cover the $3-\mathrm{cm}$ diameter laser beam footprint, a $22 \times 22$ square array of these fluorescence images were taken at each OSL footprint (see Figure 13). The exposure time for each image was 35 seconds. The laser used as the excitation source was a Q-switched Nd:YLF (Photonics Industries model DS10-351), operating at $3 \mathrm{kHz}$ with an average power of about $750 \mathrm{~mW}$ and pulse width of $20 \mathrm{~ns}$. The angle of incidence is near grazing, and the illumination area on the sample was much larger than the $1.96 \mathrm{~mm}^{2}$ contained in each image. An identical array of images was also taken with a white-light illumination source (fiber-coupled ring light) instead of the 351-nm laser. These scatter images indicate whether a fluorescing object also scatters light. An exposure time of 0.5 seconds was used to collect these images.

Figure 14 shows a large-area scatter map of the crystal after exposure to $351-\mathrm{nm}$ pulses from the OSL. This image was taken with a 45 mega-pixel scanning CCD camera. The sample was illuminated from the side with 6 " light bars. This light is transmitted through the bulk of the sample by total internal reflection, and is scattered by defects at the surface or in the bulk. The location of the defect relative to the surface of the crystal is lost in the image since the depth of focus of the imaging system is long compared to the crystal thickness.

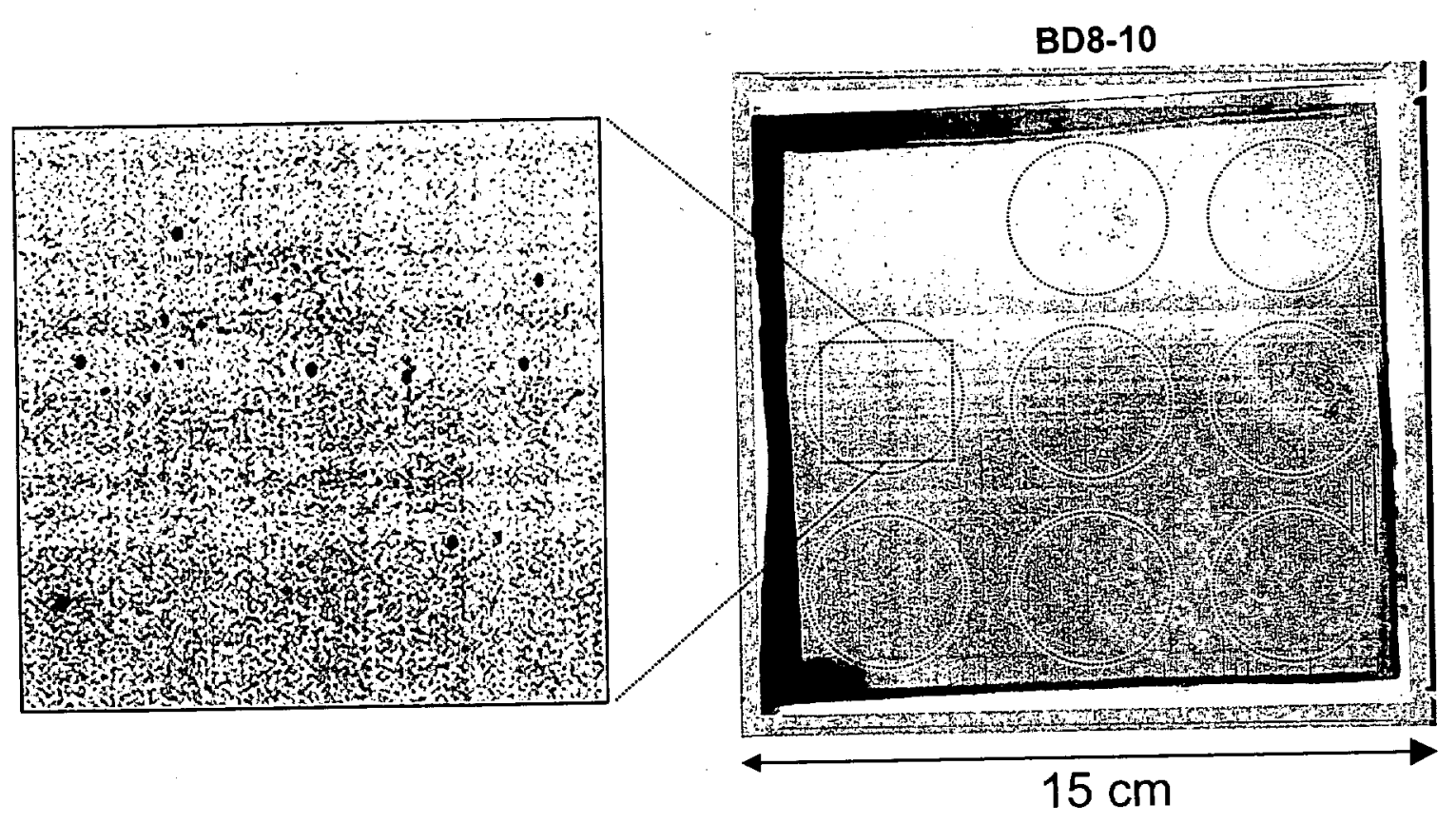

Figure 14: A large-area scatter map reveals output-surface damage (black areas) after exposure of sample BD8-10 to laser irradiation (351-nm, 3-ns) with successive shots up to $7 \mathrm{~J} / \mathrm{cm}^{2}$. Although input-surface, bulk, and outputsurface features are not distinguishable in these images, much of the damage shown at left is on the output surface. Site 6 shows the greatest amount of bulk damage. The vertical features between sites 6 and 9 are due to marks left over from the diamond turning process. These are removed with minimal laser fluence, for example by the first shot of the laser ramp. Most of the other features are artifacts of the imaging process.

The inset of Figure 14 shows a typical damage density in one 351-nm laser irradiation footprint. Most of the defects in this image are pits on the output surface. This data indicates an output-surface damage density on the order $2 / \mathrm{cm}^{2}$. Figure 15 shows brightfield microscope images of typical defect sites. These defect sites were typically pits $\sim 10$ microns in radius and $\sim 1$ micron in depth. 

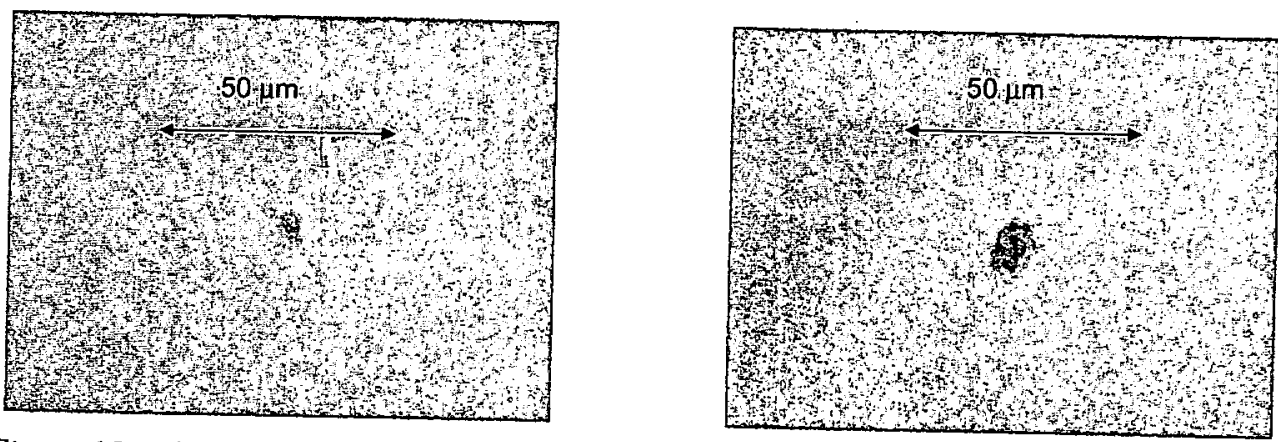

Figure 15: These microscope images show typical output surface pits after exposure to ramped fluences up to $7 \mathrm{~J} / \mathrm{cm}^{2}$ (351-nm, 3-ns) in sample BD8-10.

The correlation of the surface damage pits to precursors recorded in the fluorescence and scatter maps is displayed in the Venn diagram of Figure 16. Of the 54 pits identified, 38 had both a scatter and fluorescence precursor, 8 had only a fluorescence precursor, and another 8 had no precursor. These uncorrelated sites could be due to near-surface bulk damage, or by precursors that appeared after the maps were obtained but before the laser was fired, for example during the handling of the sample between the mapping and laser facilities.

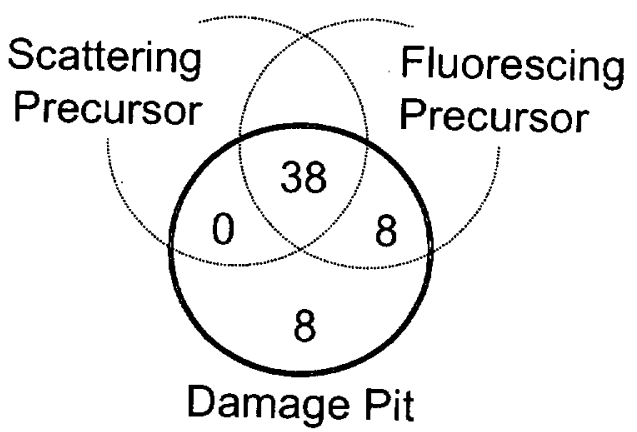

Figure 16: Venn diagram showing the correlation of output-surface damage pits to fluorescence and scatter precursors. A total of 54 damage pits were counted.

Of the 46 damage pits that were correlated to a precursor, 41 of these precursors (90\%) had a unique geometrical fluorescence signature. While most of these precursors also had a scatter signal as well, it was not nearly as $\mathrm{J} / \mathrm{cm}^{2}$, and the scatter image signal. Figure 17 shows the scatter and fluorescence images before irradiation up to 7 unique elliptical shape. They ranged from with the OSL laser. A magnified view of the precursor reveals its size typical of those seen in general on these samples, did not lead to damage. 


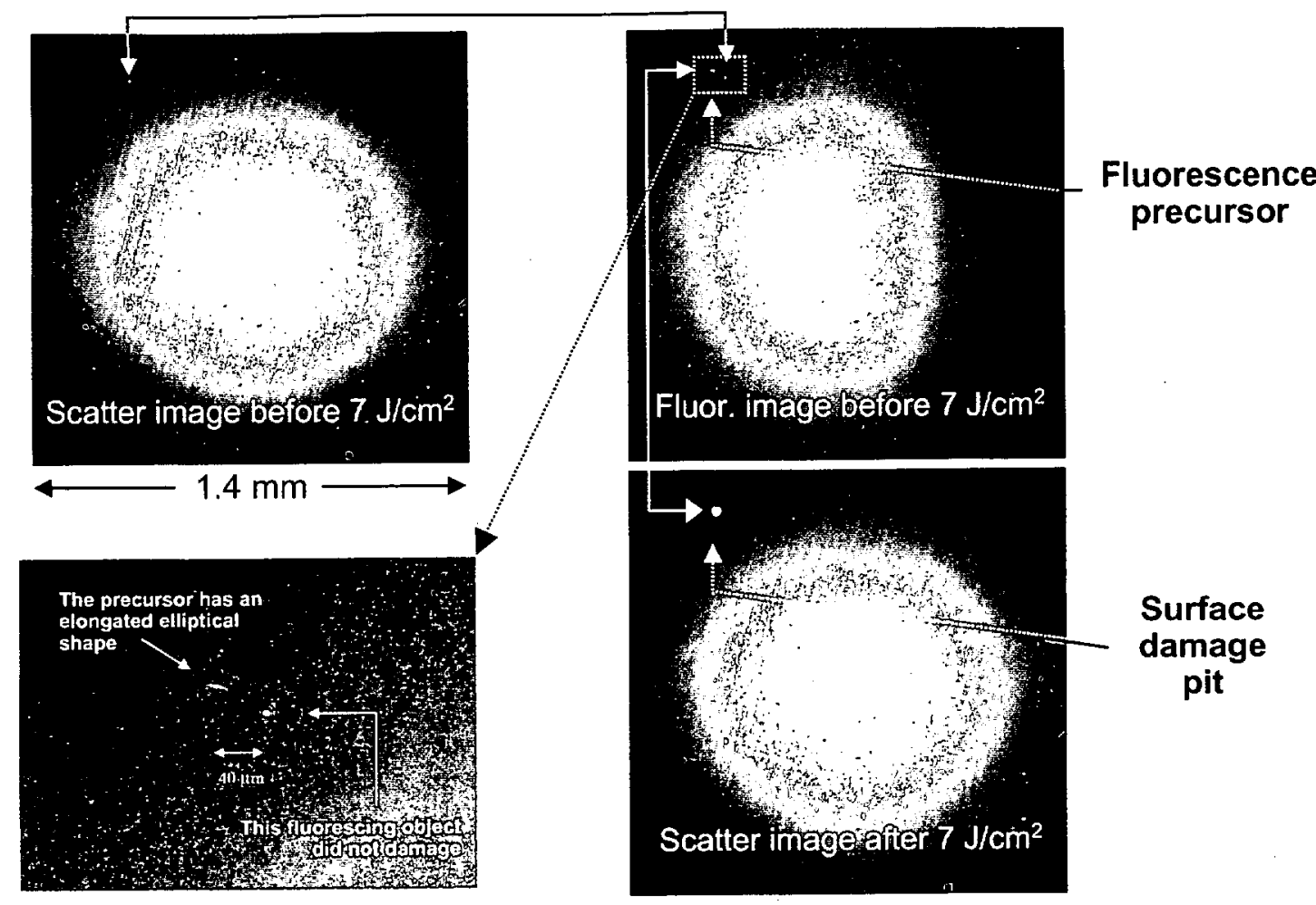

Figure 17: Before and after images showing a fluorescence precursor that led to damage after 351-nm exposure up to $7 \mathrm{~J} / \mathrm{cm}^{2}$. This fluorescence precursor had no scatter signal. A nearby feature both scattered and fluoresced. A magnified view of both features is shown in the lower left image.

In addition, most of these precursors were aligned perpendicular to the extraordinary axis of the crystal. We are currently investigating the nature and origin of these features. It is unclear whether they were incorporated during the growth of the crystal, or were created by mechanical means during finishing or handling. We are also investigating whether this phenomena is unique to this particular rapid-growth boule, or occurs more generally in all or most crystals.

\section{CONCLUSION}

The fluorescence images of $\mathrm{SiO}_{2}$ and DKDP samples demonstrate that this technique can detect the presence of contaminants embedded into the surface during the polishing process. There may be particular contaminants that are not emissive and therefore visible using fluorescence imaging. However, if the polishing process is such that contamination of the surface is a problem, fluorescence imaging may be a useful quality control tool.

Results from in-situ damage testing experiments suggest a correlation between visible fluorescing features and damage initiation. Furthermore, fluorescing objects located on the surface of the sample were shown to violently interact with the high fluence laser irradiation. In the high damage threshold sample, most of the fluorescing objects did not initiate damage at $\sim 15 \mathrm{~J} / \mathrm{cm}^{2}$ at $351-\mathrm{nm}, 3-\mathrm{ns}$. This indicates that this technique cannot be used as a uniquely identify damage precursors. The damage initiators may be visible with fluorescence imaging but not all observed features will initiate damage. Thus, damage initiation may be the result not only of absorption but also a set of other parameters such as electronic structure of the absorbent which can be critical for the initiation of subsequent multiphoton or multi-step electron excitation which can lead to cascade photo-ionization and plasma formation. The shape and thickness of the absorbing contaminant and its physical coupling with the host material are probably also important parameters.

The experimental results shown in Figures 11 and 12 demonstrate a conditioning effect where absorbing contaminants are removed from the surface without causing damage. This may be suggesting that sub-damage threshold illumination of the surface can remove contaminants that could initiate damage at a higher fluence. The mechanism for such effect may be that if the energy absorbed by some contaminants is barely sufficient to cause a micro-explosion associated with plasma formation and/or heating under 351-nm irradiation, the energy density 
(within the contaminant) may not be sufficient to cause significant energy transfer into the host that can lead to damage initiation.

The experiments on the $15-\mathrm{cm}$ DKDP crystal validated the usefulness of the fluorescence imaging technique for precursor detection. With this method, fluorescence features were identified and correlated to output surface damage pits after exposure up to $7 \mathrm{~J} / \mathrm{cm}^{2}$ at $351-\mathrm{nm}$ and $3-\mathrm{ns}$. $85 \%$ of all damage pits correlated to fluorescence precursors, and $90 \%$ of these exhibited an elliptically shaped unique fluorescence signal. The (15\%) uncorrelated pits could be due to near surface bulk damage or contaminants that appeared after the 'before' images were taken. Investigation is currently underway to chemically identify the unique fluorescence precursors, so that their origin can be determined. Removal of these features from the crystal (e.g. from the growth or finishing process) could lead to a significant decrease in the surface damage density of DKDP triplers.

\section{ACKNOWLEDGEMENTS}

This work was performed under the auspices of the U.S. Department of Energy by the University of California, Lawrence Livermore National Laboratory under contract No. W-7405-Eng-48.

\section{REFERENCES}

1. N. Bloembergen, "Role of cracks, pores, and absorbing inclusions on laser induced damage threshold at surfaces of transparent dielectrics", Applied Optics 12, 661-664 (1973).

2. J. Glass and A. H. Guenther, "Laser induced damage of optical elements-a status report", Applied Optics 12, 637-649 (1973)

3. J. A. Ringlien, N. L. Boling, and G. Dube, "An acid treatment for raising the surface damage threshold of laser glass", Applied Physics Letters 25, 598-600 (1974).

4. R. A. House, II, J. R. Bettis, and A. H. Guenther, "Efficacy of ion polishing optical surfaces", Applied Optics 16, 1486-1488 (1977).

5. P. A. Temple, W. H. Lowdermilk, and D. Milam, "Carbon dioxide laser polishing of fused silica surfaces for increased laser-damage resistance at 1064 nm", Applied Optics 21, 3249-3255 (1982).

6. F.Y. Genin, M.D. Feit, M. R. Kozlowski, A.M. Rubenchik, A. Salleo, J. Yoshiyama, "Rear-surface laser damage on 355-nm silica optics owing to Fresnel diffraction on front-surface contamination particles", Appl. Opt., 39, 3654, 2000

7. M. D. Feit, A. M. Rubenchik D. Faux , R. Riddle, A. Shapiro, D. C. Eder, B. M. Penetrante, D. Milam, F. Y. Genin, M. R. Kozlowski, Modeling of laser damage initiated by surface contamination, Optical materials for high power lasers, A. H. Guenther, Ed., SPIE, 3966, 417 (1996).

8. M. R. Kozlowski, J. Carr, I. D. Hutcheon, R. Torres, L. M. Sheehan, D. W. Camp, and M. Yan, "Depth profiling of polishing-induced contamination on fused silica surfaces", Laser-Induced Damage in Optical Materials, SPIE vol. 3244, 365-375, (1998).

9. G. J. Brakenhoff, H. T. M. van der Voort, E. A. van Spronsen, and N. Nanninga, "Three-dimensional imaging in fluorescence in confocal scanning microscopy," Journal of Microscopy, 153, 2, 151-159 (1989).

10. S. G. Demos, M. Staggs, M. Yan, H. B. Radousky and J. J. De Yoreo, "Microscopic fluorescence imaging of bulk defect clusters in $\mathrm{KH}_{2} \mathrm{PO}_{4}$ crystals", Opt. Lett., 24, 268 (1999).

11. S. G. Demos, M. R. Kozlowski, M. Staggs, L. L. Chase, A. Burnham, H. B. Radousky, "Mechanisms to explain damage growth in optical materials", Laser-Induced Damage in Optical Materials, G. J. Exarhos, A. H. Guenther, M. R. Kozlowski, K. L. Lewis, M. J. Soileau, Eds., Proc. SPIE, 4347, 277, (2000).

12. M. D. Feit, A. M. Rubenchik, M. Runkel, "Analysis of bulk DKDP damage distribution, obscuration and pulse length dependence," Laser-Induced Damage in Optical Materials, G. J. Exarhos; A. H. Guenther, M. R. Kozlowski; K. L. Lewis; M. J. Soileau; Eds., Proc. SPIE Vol. 4347, 383, (2000).

13. L.J. Atherton, F. Rainer, J.J. De Yoroe, I.M. Thomas, N. Zaitseva and F. De Marco, "Thermal and Laser conditioning of production and rapid-growth KDP and KD*P crystals," Laser-Induced Damage in Optical Materials, SPIE Proceedings, Vol. 2114, pp. 36-45, 1993.

14. M. Runkel, J.J. De Yoreo, W.D. Sell, D. Milam, "Laser conditioning study of KDP on the Optical Sciences Laser using large area beams," Laser-Induced Damage in Optical Materials, SPIE Proceedings, Vol. 3244, pp. 51-63, 1997. 


\section{Short Pulse Lasers, Applications and TECHNOLOGY Resarch ACtivity Highlights}

\section{High-Bandwidth Technology for High-Power Short-Pulse Lasers}

With support from the Laboratory Directed Research and Development (LDRD) Program, LS\&T has recently developed a front-end technology utilizing optical parametric chirped pulse amplification (OPCPA) to replace the regenerative amplifier previously used in the Nova Petawatt laser system. This method obtained a gain of $6 \times 107$ with broad bandwidth without the use of electro-optic modulators or multipass amplification. Preliminary experiments have demonstrated $6 \%$ overall pump-to-signal conversion with excellent bandwidth fidelity. The wavelength and pulse energy level obtained from this OPCPA is ideal for seeding $\mathrm{kJ}$-class $\mathrm{Nd}$ :glass amplifiers (such as the National Ignition Facility) and other high-average-power, ultrashort-pulse laser systems.

Previously high-energy lasers employing chirped pulse amplification (CPA) were built on a hybrid (Ti:sapphire-Nd:glass) laser technology. Ti:sapphire regenerative amplifiers were used as preamplifiers because of their intrinsic large gain bandwidth. One difficulty with the hybrid laser system is its low pulse contrast and generation of prepulses by the regenerative amplifier. In target experiments where focused intensities near $10^{21} \mathrm{~W} / \mathrm{cm}^{2}$ are possible, a $10^{-2}$ to $10^{-3}$ level of prepulse can significantly perturb the target prior to the arrival of the main pulse. The contributing factors to low pulse contrast include amplified spontaneous emission (ASE), leakage from regenerative amplifiers, and hard edge spectral clipping on diffraction gratings and other optical components. The gain coefficient of Ti:sap- phire at $1054 \mathrm{~nm}$ (laser wavelength) is low, necessitating a large number of passes in the regenerative amplifier. Each time the pulse completes one round-trip in the cavity, a small fraction $(\sim 1 \%)$ leaks out of the cavity, producing prepulses spaced one cavity round-trip time ahead of the main pulse. While spectral clipping can be eliminated by using large-aperture gratings, the pulse contrast remains fundamentally limited by ASE and leakage from regenerative amplifiers. Single-pass OPCPA eliminates prepulses originating from regenerative amplifiers and reduces the ASE by approximately one order of magnitude compared to Ti:sapphire.

OPCPA offers additional advantages when compared to regenerative chirped pulse amplification: low thermal aberration, greater wavelength flexibility, higher gain, high beam quality, and lower B-integral as a result of short beam path through the gain medium.

Figure 1 shows the compact OPCPA pumped by a commercial Q-switched Nd:YAG

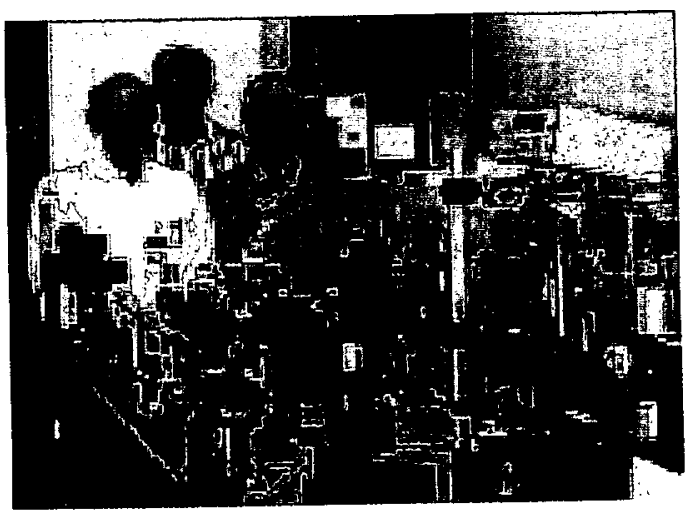

Figure 1. Optical parametric chirped pulse amplifier pumped by a commercial Q-switched Nd:YAG pump laser. 
laser. It consists of three $\beta$-barium borate (BBO) crystals, utilizing type I angular phase matching. A commercial frequency-doubled Q-switched Nd:YAG laser is used to pump the OPCPA. The first two crystals are configured as a preamplifier and pumped by $90 \mathrm{~mJ}$, while $420 \mathrm{~mJ}$ is used to pump the power amplifier crystal. We obtained $1.5 \mathrm{~mJ}$ of amplified signal from the preamplifier, which was seeded by $0.5-n J$ stretched oscillator pulses. A saturated gain of 20 was obtained from the power amplifier BBO crystal, which amplifies the signal beam up to $31 \mathrm{~mJ}$. A pump-to-signal conversion efficiency of $6 \%$ was achieved. This is the highest overall extraction efficiency demonstrated from an OPCPA to date. The input stretched pulse is $3 \mathrm{~ns}$ in length, interacting with the 8.5-ns (FWHM) pump pulse. The fraction of pump energy extracted during the temporal window defined by the seed pulse is estimated to be $>20 \%$.

Figure 2 shows the measured spectra of the stretched seed, the amplified signal from the preamplifier, and the output from the power amplifier. In these experiments, we saturate the OPCPA in order to obtain large extracted energy. The amplified signal spectrum is modified by strong saturation in the power amplifier and resembles a top-hat shape near the point of maximum conversion efficiency. The intensity

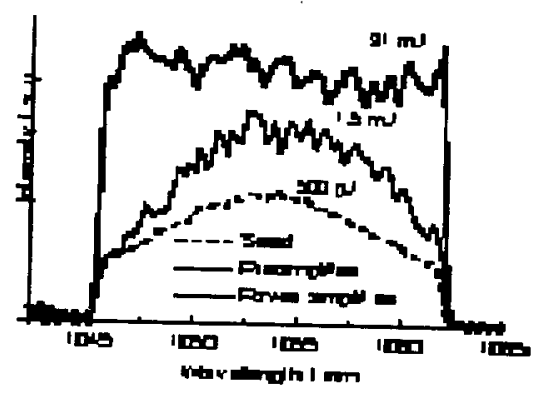

Figure 2. Seed and amplified signal spectra in OPCPA.

autocorrelation of the recompressed pulse at energy of $31 \mathrm{~mJ}$ is shown in Figure 3. The recompressed amplified pulse duration is measured to be $310 \mathrm{fs}, 10 \%$ longer compared to the best-achieved seed recompression before amplification in OPCPA. The pulse width is limited by the spherical and chromatic aberrations occurring in the pulse stretcher. Our calculations show negligible self-phase modulation due to short beam path through the gain medium.
We have successfully demonstrated high gain, high conversion efficiency, and good amplified pulse beam quality $\left(M^{2}<2\right)$ in OPCPA. We anticipate widespread use of OPCPA as front-end technology for glass-based Petawatt laser systems. In the future, it may be possible to exploit the full bandwidth capability of OPCPA to produce $\mathrm{kJ}$-class pulses with durations of several tens of femtoseconds. This would potentially allow focused intensities of $10^{24} \mathrm{~W} / \mathrm{cm}^{2}$ or higher for experiments in the extreme field science.

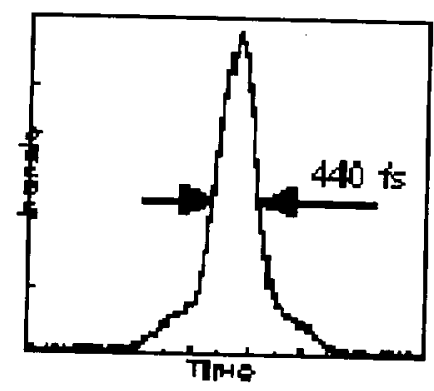

Figure 3. Autocorrelation trace of the recompressed pulse. Deconvolved FWHM pulse width is $310 \mathrm{fs}$, assuming a Gaussian pulse shape.

\section{Meter-Scale Gratings Delivered to Rutherford for Petawatt Laser Upgrade}

LLNL recently completed the fabrication of meter-scale diffraction gratings for Rutherford Appleton Laboratory's (RAL) Vulcan Laser Petawatt Upgrade. RAL in the United Kingdom has been operating Vulcan at $100 \mathrm{TW}$, providing sub-picosecond pulses at intensities of $10^{19}$ $\mathrm{W} / \mathrm{cm}^{2}$ for the study of high-intensity laser/matter interactions. The upgrade (which is based on LLNL's Petawatt laser and scheduled for completion in 2002) will enable the compression of 500-J, 500-picosecond pulses for 1 petawatt $\left(10^{15} \mathrm{~W}\right)$ of power and $10^{21} \mathrm{~W} / \mathrm{cm}^{2}$ at target.

The enabling components for achieving this ultrahigh power density are large-aperture diffraction gratings, which are only obtainable from LLNL. These gratings are optimized for high-efficiency and flat wavefront at the nominal 1053-nm use wavelength. These gold-overcoated plane gratings are patterned in photoresist using laser interference lithography, on optically polished substrates $94 \mathrm{~cm}$ in diam- 
eter with the top and bottom chords removed for a vertical aperture of $75 \mathrm{~cm}$. The grating pattern is written on LLNL's large holographic exposure station, which utilizes two $110-\mathrm{cm}-$ diameter F6 fused silica aspheric collimating lenses to provide a flat-wavefront exposure field at this aperture. Figure 4 shows LS\&T's large-aperture diffractive optics production chamber, where the meter-scale diffractive gratings are produced.

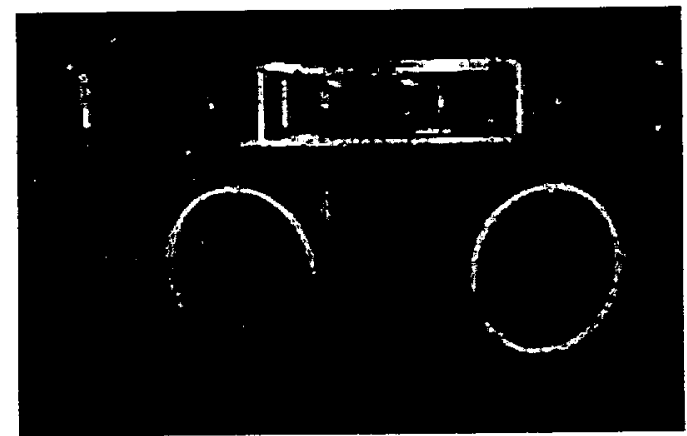

Figure 4. Large-aperture diffractive optics production chamber, where the meter-scale diffractive gratings are produced.

RAL has contracted with LLNL for several gratings over the years. The fabrication procedure is basically the same as that used for the original LLNL Petawatt gratings (see March 2000 LS\&T Program Update), with the exception of recent improvements made to the exposure system to improve fringe stability during patterning of the grating. Briefly, the substrate is cleaned and then a layer of material is vacuum-deposited as an adhesion layer for the photoresist. A film of photoresist, $\sim 250 \mathrm{~nm}$, is applied by meniscus coating, and the substrate baked in a large convection oven to dry the resist. The blank is then mounted on the large interferometer table and allowed to stabilize for at least $24 \mathrm{~h}$. The grating pattern is then written by exposing the resist film to interfering plane waves of 413-nm light from a $\mathrm{Kr}$-ion laser. Exposure times are typically 10 minutes. During this time, the spatial location of the fringe pattern generated at 1480 lines/mm (675$\mathrm{nm}$ period) must be held to within a small fraction of this period to maintain the intensity contrast in the exposure plane. Various methods are utilized to accomplish this, including vibration isolation, isothermal environmental control, and active fringe stabilization using a por-
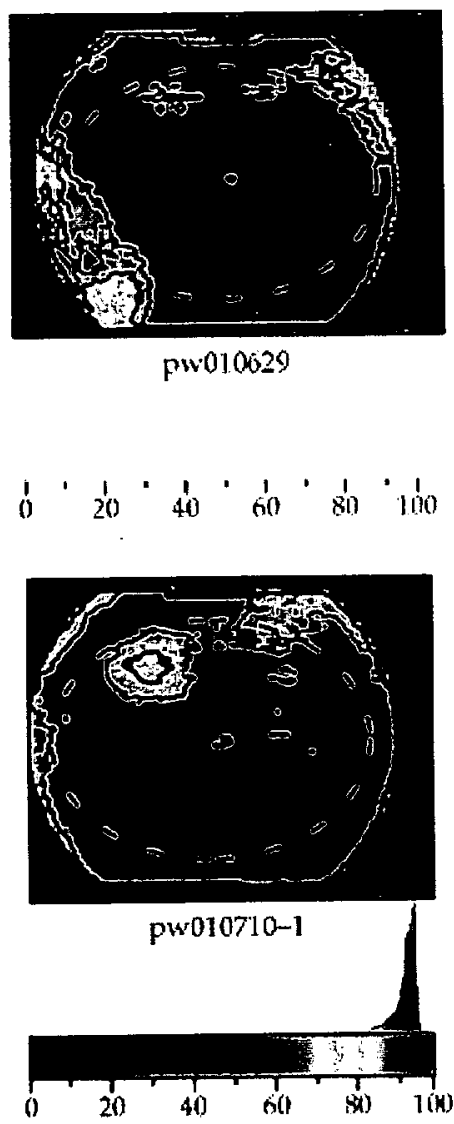

Figure 5. Full-aperture diffraction efficiency of two compressor gratings. Dashed lines show footprint of the compressed beam.

tion of the two exposure beams to provide a secondary interference pattern. A fringe detection/piezoelectric mirror system is used to control the path length of one of the beams to compensate for vibration drift. Following exposure, the latent image in the photoresist film is developed by contact with a base solution to convert it into a surface relief image.

The grating structure evolution is monitored with a probe laser during development to control the details of the groove shape. Following the development step, visual inspection for defects is done, and measurements of the diffraction efficiency of the photoresist grating are made to determine the spatial uniformity of the pattern. The grating is subsequently hardbaked, and then $\sim 500 \mathrm{~nm}$ of gold is applied by vacuum e-beam evaporation. The grating is then subjected to full-aperture diffraction efficiency measurements at the use wavelength as well as wavefront measurements. If at any time of the process, specifications are not met or 
defects in the grating surface are identified, the gold and photoresist layers can be stripped off and the grating blank reprocessed without the need to repolish.

Figure 5 shows the full-aperture diffraction efficiency of two $94-\times 75$-cm gold-overcoated petawatt compressor gratings (measured at $1064 \mathrm{~nm}, 54^{\circ}$ incidence angle) that meet specifications for delivery. Also shown is the footprint of the beam RAL researchers are to compress; 80 and $90 \%$ contours are also shown. The average diffraction efficiency in the beam footprint for these gratings is $92.8 \%$ and $93.8 \%$, respectively, which represent the best uniformity we have been able to achieve to date at this scale. Figure 6 shows one of the gratings during wavefront testing at LLNL. The two large gratings, along with four smaller ones for the pulse stretcher and diagnostics, have been shipped to RAL.

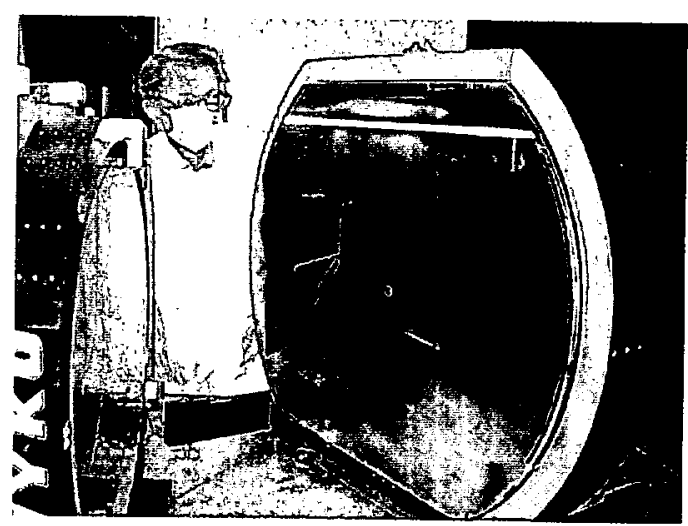

Figure 6. A meter-scale grating undergoing a wavefront test at LLNL.

\section{New Wet-Etching Process Figures Large-Aperture and Ultrathin Optics}

With support from the Laboratory Directed Research and Development (LDRD) Program, LS\&T has recently developed a low-cost wetetching tool for precision optical figuring and finishing of large-aperture and ultrathin optical components (see Figure 7). This wet-etch figuring (WEF) method uses applicator geometry and surface tension gradients (the Marangoni effect) to confine the footprint of the flowing etchant on the surface. In contrast to the conventional methods using abrasive slurries or ion milling, no mechanical or thermal stresses or

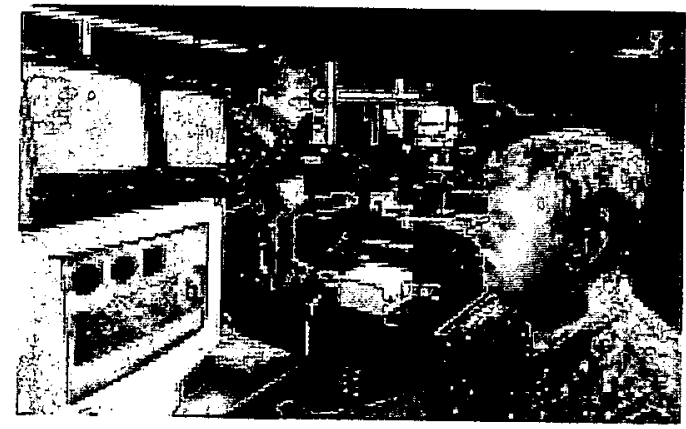

Figure 7. LLNL-developed wet-etching tool (operated by authors).

residues are applied to the optic by this process. WEF also uses a real-time interferometry to measure the thickness of the optical material while surfacing and figuring, which then controls the placement and dwell time of the wetted zone.

Traditionally, small-tool finishing or figuring of optical surfaces has involved moving a small, abrasive polishing tool in a controlled manner to shape the surface of an optic. Recent advances in finishing involve flowing jets of fine abrasive slurries, magneto-rheological control of the viscosity of abrasive slurry, or the use of ion-beam milling. All of the abovementioned techniques rely on calibrated removal rates and are, therefore, iterative processes: the workpiece must be dismounted from the machine and measured, reworked and remeasured, until specifications are met. These polishing techniques cannot easily be used to figure thin optics since the local mechanical stresses involved cause workpiece deformations that impact removal control and can even cause breakage.

We have employed the Marangoni effect to confine the wetted zone of a hydrofluoric acid etchant on the surface of an optic. Figure 8 shows circular and linear etching toolheads developed for two- and one-dimensional (2- and 1-D) figuring. For the case of a circular toolhead, the wetted zone size can be controlled between $\sim 1$ to $10 \mathrm{~cm}$ by flow pulsation and wicking. The width of the wetted zone for 1-D figuring can be similarly controlled, and the length can be made arbitrarily large. An interferometer that measures the local glass thickness from above is used in feedback control for closed-loop figuring.

We have made several custom phase correction and beam-shaping optics using the 2-D WEF tool. An example is shown in Figure 9. 

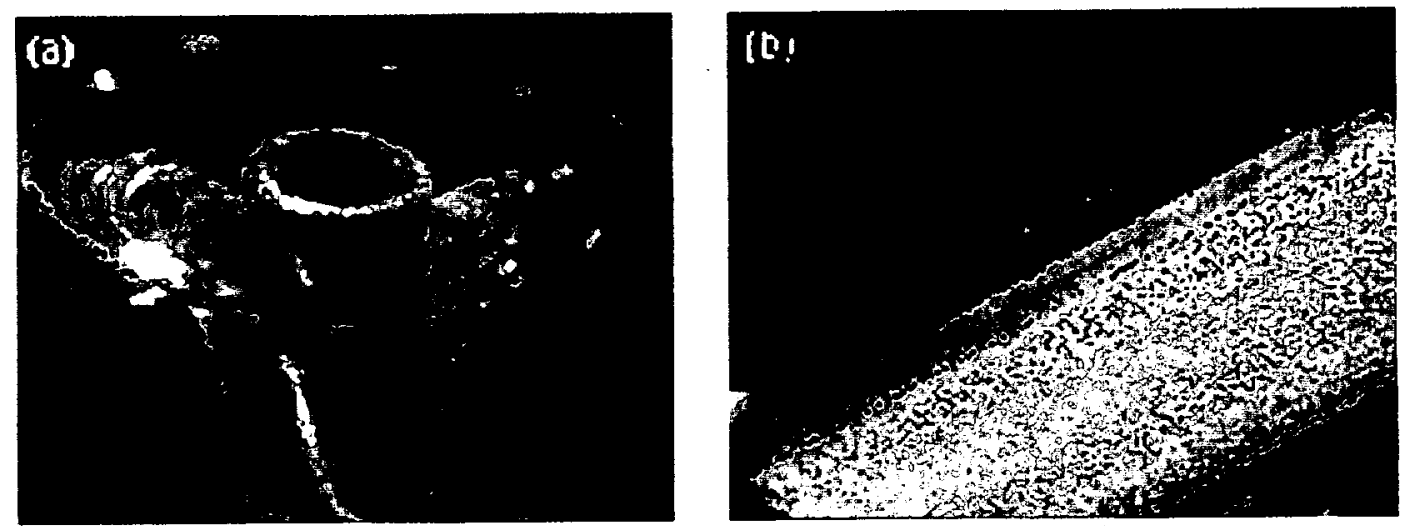

Figure 8. (a) Looking downwards through the thin glass being processed, left is a drop of etchant solution $\sim 10$ $\mathrm{mm}$ in diameter adhering to the underside of a glass sheet. The solution is flowing up through and then down the outside of the tube. (b) A $150-\times 10-\mathrm{mm}$ rectangular wetted zone in which liquid flows up out of a slit and down the inclined plane on either side. In both cases, a blue line has been drawn on the three-phase contact line as a visual aid.

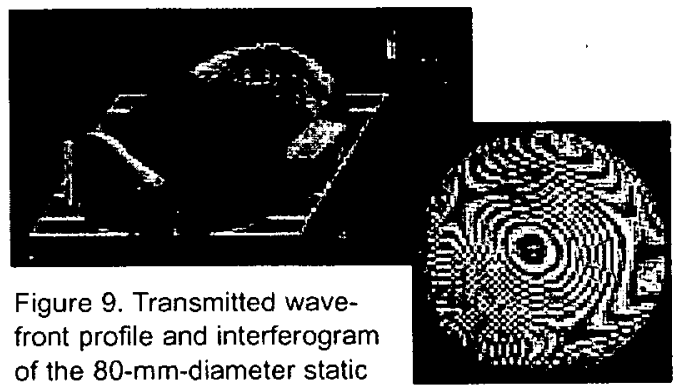
wave-front corrector fabricated by the WEF process. The optic is $380-\mathrm{m}$ thick.

We are presently constructing a 1-D WEF machine that will take the 1-D thickness ripple out of commercially extruded, $850-\times 1150-\times$ $0.7-\mathrm{mm}$ glass sheets.

We have also fabricated static wavefront correctors for high-power laser application. Figure 10 shows an 80 -mm-aperture corrective optics recently fabricated for the Atomic Weapons Establishment (AWE), U.K., Helen laser, based on specifications given by AWE researchers. The optic is to be installed upstream in the laser chain to precorrect for wavefront aberrations due to amplifiers and other optics. The optic surface contour specified by AWE requires a maximum excursion of $12 \mu \mathrm{m}$ over this aperture. The final transmitted wavefront profile and interferogram of the 80$\mathrm{mm}$-diameter wavefront corrector are shown in Figure 3 . The $380-\mu \mathrm{m}$-thick optic was completed within one machine setup step, using the circular 2-D WEF tool. The surface profile is within $100 \mathrm{~nm}$ of the target goal. The residual

error of the part is equivalent to a Strehl of 0.26 .

WEF is an ideal technology for the fabrication of large-aperture phase-modifying optics and optically flat optics. It is particularly useful for precision figuring of very thin ( $<<1 \mathrm{~mm}$ ), lightweight optics for use in space and astronomy, and high-power laser systems in which nonlinear effects due to bulk transmissive optics

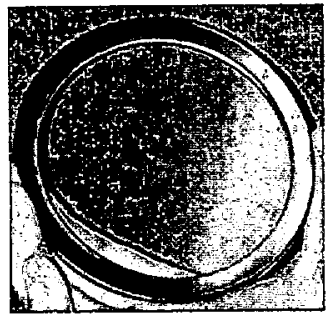

Figure 10. Photograph of finished part. need to be minimized.

We will continue to improve the precision of the WEF machine and to use it to demonstrate figuring of continuous phase plate for NIF. We are also trying to form partnerships with private companies to commercialize this process.

\section{Segmented Fesnel Lens Developed for the Eyeglass Space Telescope}

Examination of distant astronomical bodies, such as galactic nuclei and extrasolar planets, requires telescopes with apertures in the 25 - to 100-m range to achieve greater resolution and signal-to-noise ratio than currently available. Manufacture and deployment of such telescopes are well beyond the scope of current technological capabilities. Large-aperture space telescopes will have to be lightweight and space deployable. We have recently proposed a 


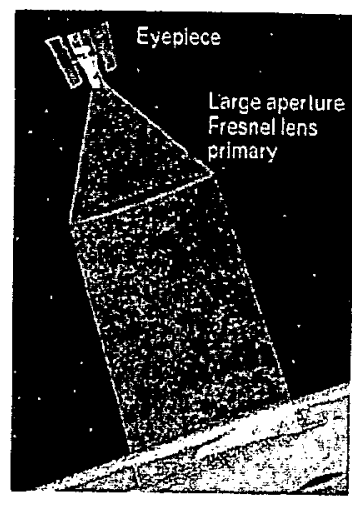

Figure 11. The Eyeglass space telescope concept new type of space telescope, called Eyeglass, which consists of a large-aperture primary lens (magnifying glass) for collecting the light and a secondary lens (eyepiece) moving along the focal surface for imaging (Figure 11).

As a first step in the technology development, we have built small-aperture $(20-\mathrm{cm}$ and $50-\mathrm{cm}$ ) diffractive telescopes and have obtained fully color-corrected, broadband (470to 700$\mathrm{nm}$ ) images of the lunar surface, Jupiter, and Saturn (see LS\&T Program Update, November 1999).

The next step is to demonstrate that the technology can be scaled up to much larger size and engineered for deployment in space. In this context, we are developing technologies to fabricate a 5-m-diam Fresnel lens. The schematic of such a lens is shown in Figure 12. The Fresnel lens is composed of 81 panels, approximately 1 in wide and $0.7 \mathrm{~mm}$ thick, that are patterned lithographically and subsequently joined together using metallic tabs.

We have identified vendors that produce such large, thin glass sheets for flat-panel displays. The wavefront of such glass sheets supplied by vendors is not adequate for Eyeglass applications. We are currently developing finishing technologies for flattening such large, thin glass sheets.

Before building the 5-m. Fresnel lens, we wanted to demonstrate that a seamed lens performs as well as a monolithic lens and also to investigate the effect of folding and unfolding such a seamed lens. For this purpose, we patterned and assembled a six-segment, foldable Fresnel lens (shown in Figure 13) on 75-cmdiam, 1-mm-thick fused silica plates and examined its focus quality when illuminated by a monochromatic, collimated beam. The result, shown in Figure 4a, demonstrates a nearly diffraction-limited focal-spot shape.

Subsequent to this measurement removed the lens from the mount, folded it (Figure 3), unfolded it, placed it in the mount and measured its focal spot again.

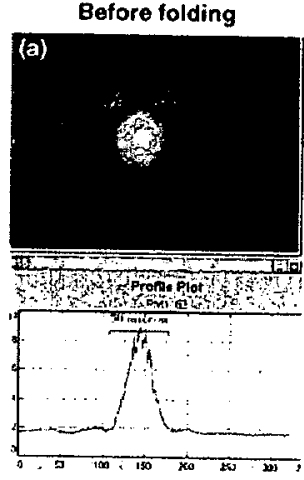

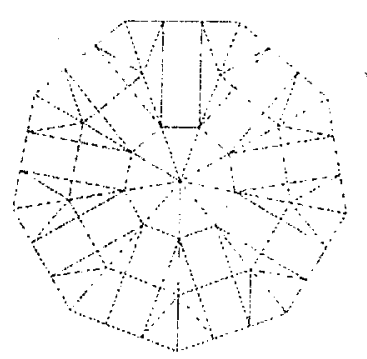

Figure 12.

Schematic of a

5-m segmented

Fresnel lens.

This spot, shown in Figure $4 \mathrm{~b}$ verifies that the folding/ unfolding process, if done carefully, preserves the high quality and tight focus of the segmented lens. These results lend strong credibility to the segmented, folding lens approach for the large-aperture Fresnel lenses for space telescopes. We plan to build the 5-m segmented Fresnel lens with metrologically

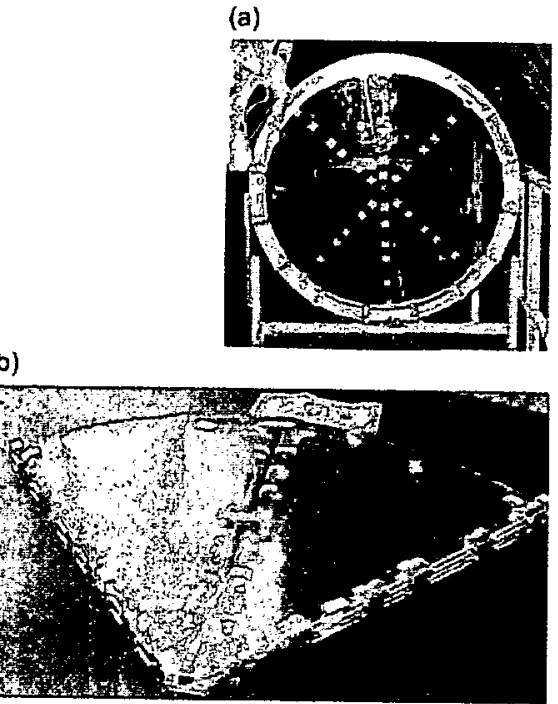

(b)

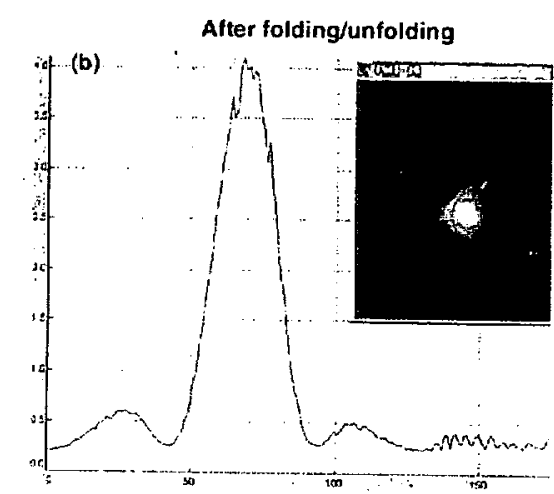

Figure 13. (a) The foldable Fresnel lens before folding demonstrates a nearly diffraction-limited focal spot shape. (b) The measured focal spot of the foldable Fresnel lens after folding shows the same quality and focusability. Figure 4. (a) The foldable Fresnel lens before folding demonstrates a nearly diffraction-limited focal spot shape. (b) The measured focal spot of the foldable Fresnel lens after folding shows the same quality and focusability. 


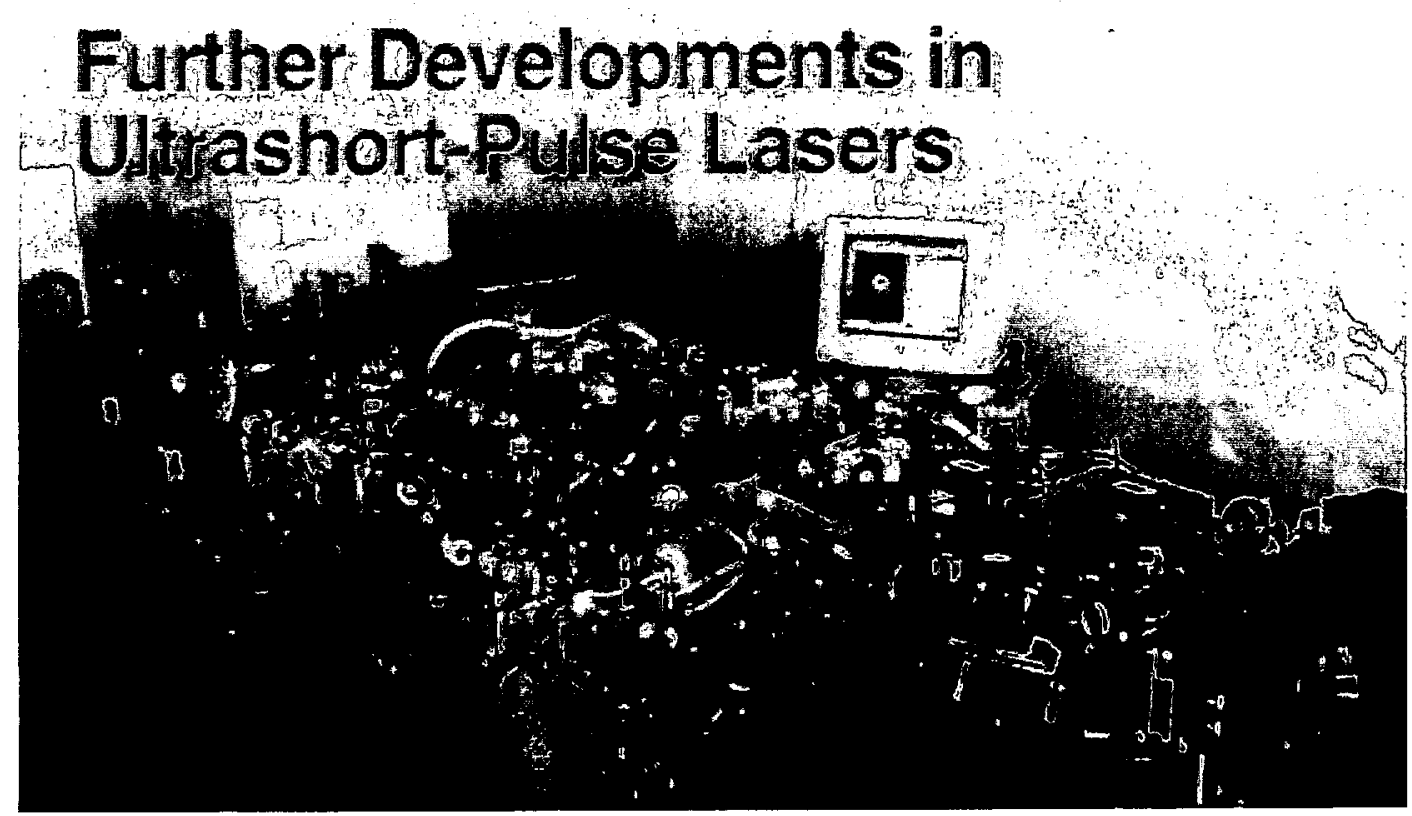

\section{Further Developments in Ultrashort-Pulse Lasers}

Livermore continues to push the frontiers of laser science. Researchers have taken the ultrashort pulses of the record-shattering Petawatt laser and found new uses for them. They are also applying a novel technology to create extremely short laser pulses with high average power and high energy to access and investigate extreme-field conditions. The technology improvements will benefit the Stockpile Stewardship Program as well as national defense and manufacturing.

The Petawatt laser operated for three years and routinely produced more than 500 -joule laser pulses lasting 500 femtoseconds--less than a trillionth of a second. Experiments with the Petawatt evaluated the fast ignitor method of achieving inertial confinement fusion, generated powerful electrons or $x$-rays for radiography research, and produced short, powerful gamma rays for nuclear physics experiments. (See S\&TR, March 2000, The Amazing Power of the Petawatt.) In addition, the discovery of intense, high-energy, collimated proton beams emitted from the rear surface of Petawatt laser targets has opened the way to new applications such as proton radiography. The Petawatt laser still holds the world's record for the highest peak power ever achieved by a laser.

The Petawatt operated on one of the 10 beam lines of Livermore's Nova laser. When the Nova laser was decommissioned in 1999 , the Petawatt went with it. But work on shortpulse lasers by no means stopped, notes physicist Mark Hermann, associate program lẹader for Livermore's Short-Pulse Lasers, Applications, and Technology program, known as SPLAT, which is a part of the National Ignition Facility (NIF) Programs Directorate. His team of about 30 people is advancing the science of short-pulse lasers and applications, developing new laser components, fielding advanced laser systems, and developing new optical components and optical fabrication technologies. There is also an active program in short-pulse technology in the Physics and Advanced Technology (PAT) Directorate. This research stems from the need to develop hightemperature plasma sources and accurate plasma probes for high-energy-density materials research.

In SPLAT, a diverse set of challenging projects focused on developing high-average-power, short-pulse lasers for a variety of customers is under way. Current SPLAT-developed laser systems use conventional titanium-doped sapphire (Ti:sapphire) amplifiers, but now the team is developing new chirped-pulse amplifier technologies geared toward high average power. One is a direct, diode-pumped, chirpedpulse amplifier laser crystal that promises efficient, compact, and robust picosecond-pulse laser systems. Another is an optical-parametric chirped-pulse amplification (OPCPA) technique, described in more detail below.

The SPLAT team is using a short-pulse laser 
to create unique nanocrystals and gain knowledge about the novel properties of nanostructures. This knowledge affects the basic sciences, from solid-state physics to biology. Being able to synthesize nanocrystals of specific size and properties, at an industrial rate, may revolutionize the field of nanotechnology and enable a broad sector of manufacturing, from semiconductors to pharmacology.

The team is collaborating on a project that integrates a short-pulse laser with a Livermore linear accelerator for stockpile stewardship applications. Supporting the team's efforts, Livermore's Diffractive Optics Group is developing new optical technologies and fabricating new optics for petawatt-class lasers around the world, for the National Ignition Facility, and for the National Aeronautics and Space Administration to use in space-based telescopes. The group currently produces the world's largest diffraction gratings.
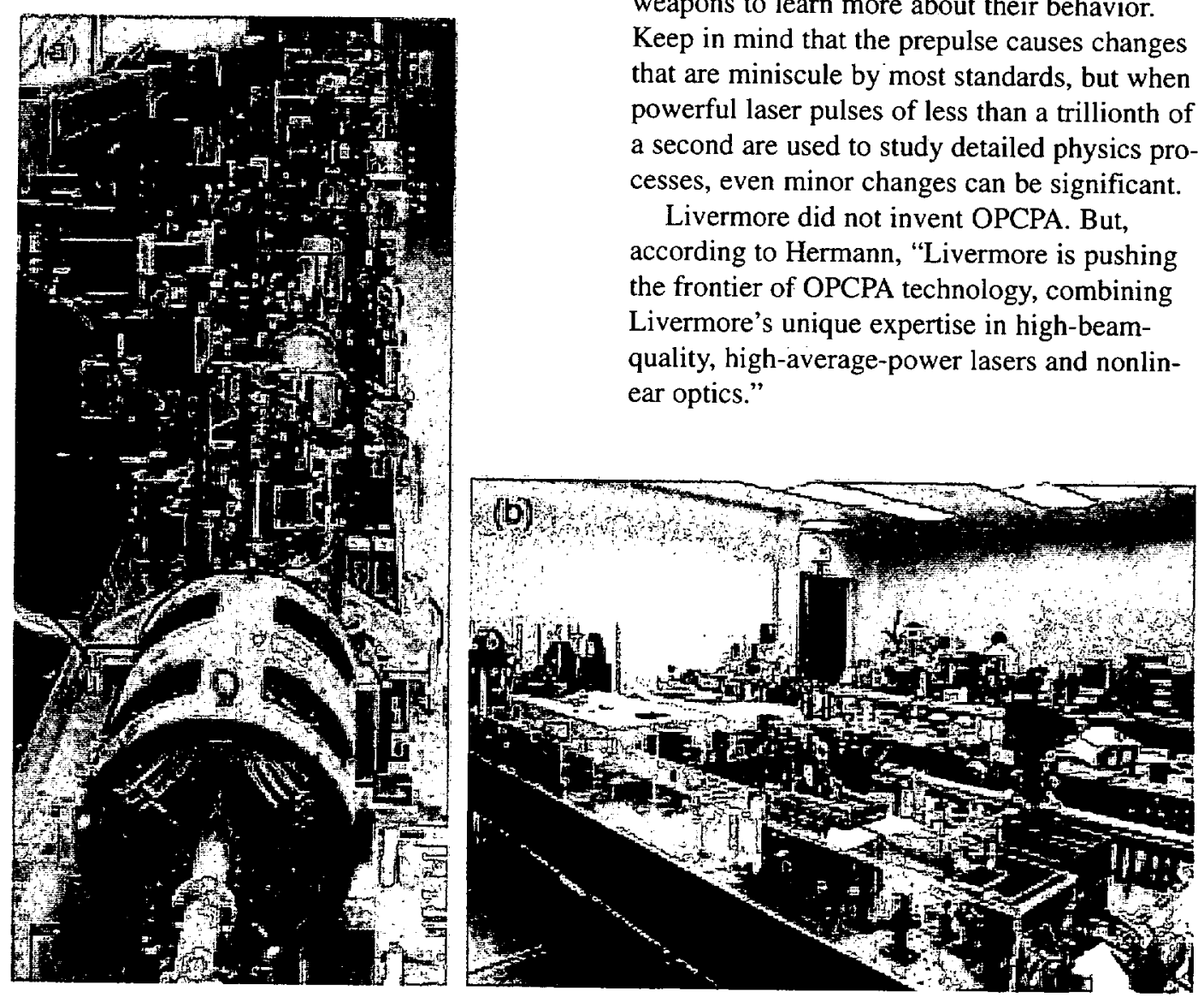

(a) The100-megaelectronvolt linear accelerator and (b) the Falcon ultrashort-pulse laser are being integrated to produce a short-pulse $x$-ray source. Livermore scientists will use the $x$ rays to probe the dynamics of materials under shock conditions.

\section{New Path to a Short Pulse}

An interesting new development in shortpulse laser technology has been the emergence of OPCPA. Laser-pumped nonlinear crystals made of beta-barium borate (BBO) would replace the Ti:sapphire used in the Petawatt and other conventional lasers as the preamplifier. In a Ti:sapphire regenerative power amplifier, a pulse passes 10 to 100 times through a regenerative cavity, increasing in energy with each pass. By the time it leaves the amplifier, its 1 nanojoule to approximately 10 millijoules. In contrast, a pair of BBO crystals can produce the same energy gain with a single pass of the light pulse.

With a regenerative amplifier, a tiny bit of energy leaks out with each round trip of the laser pulse. If this leak, or prepulse, is not attenuated, it may cause a preplasma, which changes the coupling of the laser to the target. Many stockpile stewardship experiments use lasers to probe materials essential to nuclear weapons to learn more about their behavior. Keep in mind that the prepulse causes changes that are miniscule by most standards, but when powerful laser pulses of less than a trillionth of a second are used to study detailed physics processes, even minor changes can be significant.

Livermore did not invent OPCPA. But, according to Hermann, "Livermore is pushing the frontier of OPCPA technology, combining Livermore's unique expertise in high-beamquality, high-average-power lasers and nonlinear optics." energy has increased by 10 million, from about 

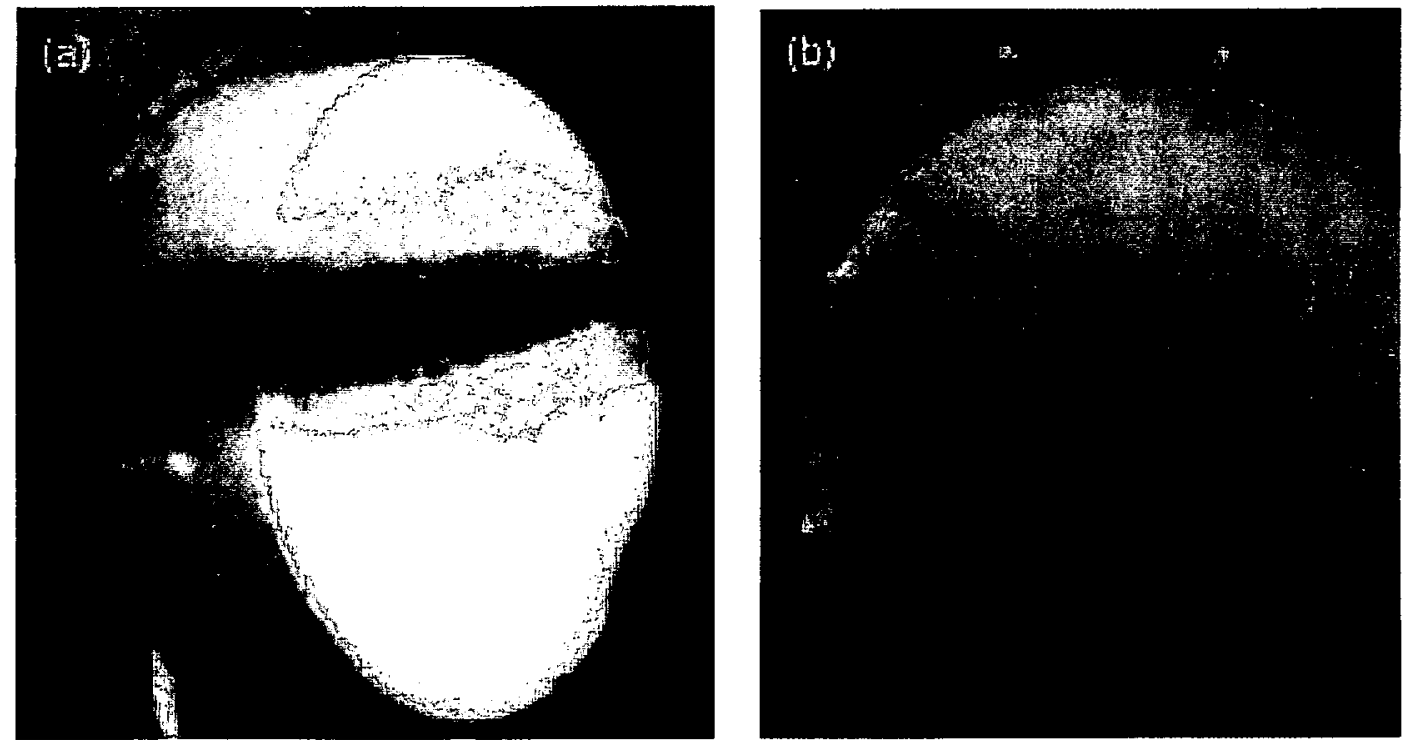

(a) A laser with 500-picosecond pulses caused the high explosive $L X-16$ to burn during cutting. (b) In Livermore s system, the 150-femtosecond laser pulses are so short and fast that they deliver virtually no head to the area being cut.

A major application for OPCPA will likely be in laser machining, which requires high average power, high beam quality, and ultrashort pulses (about 20 to 1,000 femtoseconds). Unlike other chirped-pulse amplification approaches, OPCPA produces negligible thermal aberrations that in turn cause degradation of the laser beam. Although not yet demonstrated at high average powers, an OPCPA laser should be able to produce hundreds of watts or even kilowatts of average power with high beam quality. In contrast, most conventional Ti:sapphire chirped-pulse lasers, including Livermore's systems, have operated at 20 watts or less. Higher power should translate into faster production and better process control during machining.

Much of the SPLAT program's work is related to stockpile stewardship and improving Livermore's ability to verify the safety and reliability of the nation's aging nuclear weapons stockpile. Before the arrival of OPCPA technology, the team built the Falcon, a 3-terawatt, 35femtosecond laser facility with a moderate repetition rate, to use as a material probe. A joint team of SPLAT and PAT personnel recently began to integrate the output from Falcon with the electron beam generated by Livermore's 100-megaelectronvolt linear accel- erator. Together, the laser and the accelerator will be an advanced light source whose ultrafast and ultrabright pulsed $x$-rays will be used as probes for dynamic studies of solid-state and chemical systems.

Hermann notes that the Falcon and other short-pulse lasers at Livermore may benefit from being upgraded with the OPCPA system. "Controlling and in some cases eliminating the prepulse is desirable," says Hermann. "It means that researchers will be able to control the experimental initial conditions of the laser material dynamics."

\section{Benefits Abound}

Emerging technologies for optical-parametric chirped-pulse amplification and diffractive optics will soon find their way into several Livermore lasers, from small high-averagepower systems for manufacturing to high-energy systems such as NIF, the 192-beam laser being built to support stockpile stewardship science research. NIF and SPLAT personnel are assessing the potential for adding these technologies to produce short pulses on NIF. Combining ultrashort pulses with the powerful, multimedgajoule capacity of NIF would result in a unique stockpile stewardship capabilities, and investigate new areas of extreme-field science. 
4-1d Laser Science and TeChnolocy 


\title{
Hybrid chirped pulse amplification
}

\author{
Igor Jovanovic Christopher A. Ebbers $\quad$ C. P. J. Barty
}

\begin{abstract}
Conversion efficiency in optical parametric chirped pulse amplification is limited by spatio-temporal characteristics of the pump pulse. We have demonstrated a novel hybrid chirped pulse amplification scheme that uses a single pump pulse and combines optical parametric amplification and laser amplification to achieve high gain, high conversion efficiency, and high prepulse contrast without utilization of electro-optic modulators. We achieved an overall conversion efficiency of $37 \%$ from the hybrid amplification system. Generation of multi-terawatt pulses is possible by using this simple method and commercial Q-switched pump lasers.
\end{abstract}

\section{INTRODUCTION}

In the past decade, chirped pulse amplification (CPA) ${ }^{1}$ revolutionized the research in high-field science by allowing unprecedented peak power and focused intensity to be achieved. Despite the multitude of technological advances, amplification of chirped optical pulses remains challenging. Expansion and recompression of ultrashort pulses to their transform limit over many orders of magnitude, combined with compensation of group delay due to dispersion and nonlinear phase accumulated in the system (B-integral), is dependent on the particular design of the laser system. Amplification of pulses over many orders of magnitude in laser amplifiers also results in bandwidth narrowing. ${ }^{2}$ Most sub-100-fs CPA systems to date are based on Ti:sapphire, ${ }^{3-9}$ many of which utilize regenerative amplification and electro-optic switching to produce high gain. However, regenerative amplifiers can produce prepulses at integer multiples of the cavity roundtrip time prior to the main pulse. Without further isolation, the resulting pulse contrast can be incompatible with desired experimental conditions in high-field target experiments.

Optical parametric chirped pulse amplification (OPCPA) ${ }^{10-11}$ has been suggested as an alternative high-gain amplifier technology with potential to simplify CPA systems. OPCPA operating in the nanosecond pump regime has favorable characteristics: a gain of 6-7 orders of magnitude can be readily obtained in a single pass through only $\sim 30 \mathrm{~mm}$ of nonlinear solid-state optical parametric amplifier $(\mathrm{OPA})^{12}$ such as $\beta$-barium borate (BBO). This can be achieved without bandwidth narrowing at multiple center wavelengths, dependent on the amplifier material, pump-signal geometry, and pump wavelength. Reduced optical path through the gain medium results in reduced B-integral. Other favorable characteristics of OPCPA include a reduction of amplified spontaneous emission (ASE) level of one order of magnitude compared to Ti:sapphire, ${ }^{11}$ and a negligible heat load associated with perfectly elastic parametric amplification process. The most problematic aspect of OPCPA, however, is the relatively poor conversion efficiency when simple, commercial, Q-switched pump lasers are used. The most significant obstacle to high conversion efficiency in OPCPA is the mismatch between the pump pulse width and the pulse width of the stretched broadband signal pulse. Pump beam quality and spatio-temporal evolution are additional considerations due to finite angular acceptance of OPA. ${ }^{13}$ As a result, the highest reported conversion efficiency to date in OPCPA pumped by a commercial Q-switched pump laser is $6 \%{ }^{14}$ While a perfect $(100 \%)$ conversion efficiency to signal plus idler is theoretically possible in OPCPA, development of suitable ns-pump lasers is expensive and impractical for low-cost tabletop applications. On the other hand, amplification in broadband laser materials such as Ti:sapphire has been demonstrated at up to $50 \%$ pumpto-signal conversion efficiency, which is $90 \%$ of the theoretical quantum efficiency in that material. ${ }^{8,15}$ In this Letter, we present a novel technique of hybrid chirped pulse amplification, which combines the advantages of high-gain, single-pass, optical parametric amplification with the superior conversion efficiency of a laser amplifier, when pumped by a simple, commercial, Q-switched pump laser.

\section{PUMP ABSORPTION}

A unique characteristic of optical parametric amplification that distinguishes it from amplification in conventional laser gain media is the absence of pump absorption. Since optical parametric amplification is 
an instantaneous nonlinear process, unconverted pump energy leaves the amplifier with losses limited to surface reflections. This opens the possibility of using the unconverted pump energy to pump a laser amplifier. Typically, pulses produced by commercially available pump lasers are much longer than the signal chirped pulses, allowing only a small temporal slice of the pump energy to be converted in optical parametric amplification. Since a laser amplifier acts as a temporal integrator of the pump energy, it is insensitive to this temporal modulation of the pump. In our experiment we used the residual pump energy after an OPA to pump a Ti:sapphire multipass amplifier and thus obtain high overall conversion efficiency.

\section{HYBRID CHIRPED PULSE AMPLIFICATION SYSTEM}

The hybrid chirped pulse amplification system is shown in Fig. 1. A mode-locked Ti:sapphire oscillator produced a train of 5-nJ, 30-fs pulses at the center wavelength of $820 \mathrm{~nm}$, with a 26-nm FWHM bandwidth. Oscillator pulses were stretched to $600 \mathrm{ps}$ prior to amplification. ${ }^{16}$ We used a seeded, commercial, Q-switched, Nd:YAG laser (Spectra-Physics GCR Pro 350-10) as a pump. The laser operated in a single longitudinal mode and produced $1.5 \mathrm{~J}$ of $532-\mathrm{nm}$ output in $6.9-\mathrm{ns}$ FWHM pulses, at a $10 \mathrm{~Hz}$ repetition rate. The pump laser was timed to the oscillator output, with a characteristic timing jitter of $\sim 0.5$ ns. Since we were limited by the available aperture of our OPA crystals $(5 \mathrm{~mm} \times 5 \mathrm{~mm})$, we used only 225 $\mathrm{mJ}$ of pump energy to pump the hybrid CPA system.

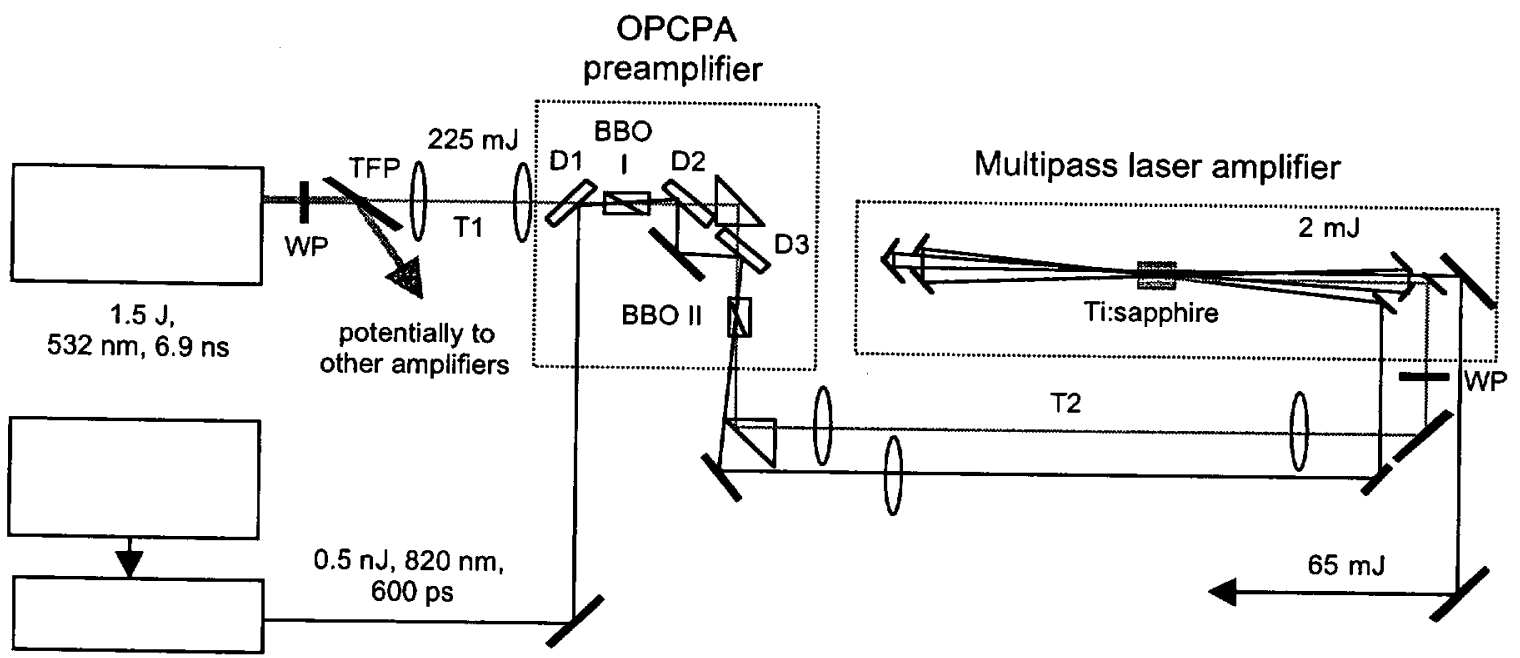

FIG 1. Experimental setup for hybrid chirped pulse amplification. D-dichroic, TFP-thin film polarizer, WP-waveplate, T-telescope.

The OPA ${ }^{17}$ was comprised of two BBO crystals. The lengths of the first and the second crystal were $20 \mathrm{~mm}$ and $15 \mathrm{~mm}$, respectively. The crystals were cut at $23.8^{\circ}$ for type I noncollinear angular phase matching. The external noncollinear angle between the signal and the pump was selected to be $3.7^{\circ}$, which maximizes the gain bandwidth in BBO at this combination of pump and signal wavelengths. The plane of noncollinearity was selected perpendicular to the principal plane of the first (longer) crystal to increase the effective interaction length. In the second crystal, the plane of noncollinearity was chosen parallel to the principal plane, which allowed signal and pump beams to emerge out of the OPA at the same height. Crystals had a $2^{\circ}$ wedge on their output faces to prevent intra-crystal parasitic oscillation.

The near-field pump beam profile was imaged from the doubling crystal of the pump laser to a plane between the two BBO crystals. The diameter of the collimated pump beam in the crystals was $3 \mathrm{~mm}$ FWHM, and produced a peak intensity of $330 \mathrm{MW} / \mathrm{cm}^{2}$. The size of the seed beam was $1 \mathrm{~mm}$ in the first crystal, and the beam was slightly divergent throughout the system to reach the size of $2 \mathrm{~mm}$ in the second BBO crystal. In this way, better spatial overlap of pump and signal was achieved in the first (longer) crystal, while better conversion efficiency resulted in the second (shorter) crystal, where local pump depletion occurred.

The signal beam was separated from the pump after amplification in the first crystal using a 
dichroic beamsplitter. The signal was subsequently coupled into the next crystal using another dichroic beamsplitter. Separation of signal and pump between the two crystals was necessary to produce good spatial overlap and the desired noncollinear angle in each crystal. We obtained a maximum gain of $6 \times 10^{6}$ from the two-stage OPA. The maximum amplified signal pulse energy in the OPA was $3 \mathrm{~mJ}$, for a conversion efficiency to signal and idler of $38 \%$ in the temporal window defined by the FWHM stretched seed pulse width, or $2 \%$ overall conversion efficiency. Fig. 2 shows the output energy from the OPA as a function of pump energy, together with the results of our numerical model. ${ }^{14}$ Subsequent optimization of the beam profile of the amplified signal was obtained by adjusting the spatial overlap and displacement of pump and signal and resulted in an output energy of $2 \mathrm{~mJ}$. Following the OPA, only $195 \mathrm{~mJ}$ of the unconverted pump energy was available due to the energy lost to nonlinear conversion and losses on unoptimized optical coatings.

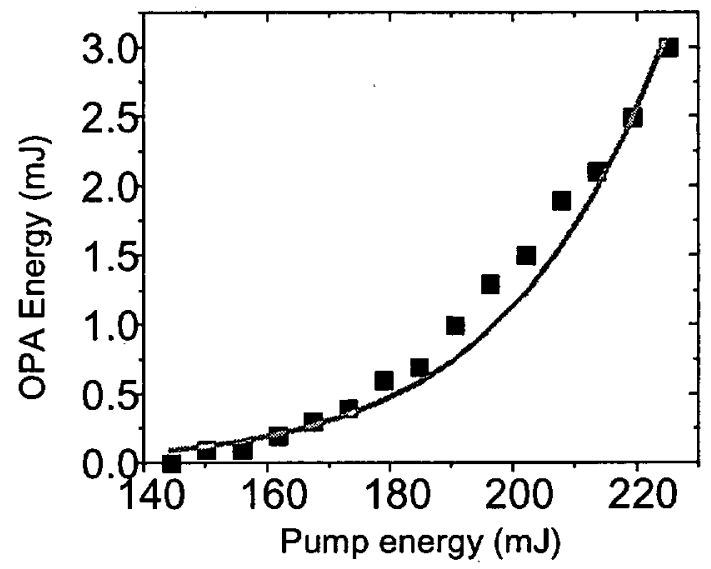

FIG 2. Output energy from the optical parametric amplifier as a function of input pump energy. The lines represent the results of calculations using our numerical model.

After amplification in the OPA, the residual pump was relay imaged onto a $10-\mathrm{mm}$ long Ti:sapphire crystal, which was a part of a bow-tie four-pass amplifier. The pump beam fluence on the Ti:sapphire crystal was $3 \mathrm{~J} / \mathrm{cm}^{2}$, and $175 \mathrm{~mJ}$ of pump energy was absorbed in Ti:sapphire. The signal was spatially separated from pump after $10 \mathrm{~cm}$ of propagation out of the second BBO crystal and collimated to FWHM beam diameter of $3 \mathrm{~mm}$, slightly overfilling the pump beam in the Ti:sapphire crystal. A 1-meter delay was introduced between the signal and the pump after the OPA to allow full absorption of pump energy in Ti:sapphire crystal before the arrival of the signal pulse. The maximum obtained signal energy from the four-pass amplifier was $65 \mathrm{~mJ}$, which is $37 \%$ of the absorbed pump energy.

In Fig. 3 we present the stretched seed and amplified signal spectra from the OPA and the fourpass amplifier. The measured spectral bandwidth of the OPA output increased to $49 \mathrm{~nm}$ FWHM due to pump depletion. Additionally, a small spectral shift to longer center wavelengths occurred as a result of nonuniform gain profile in the nondegenerate BBO OPA. Some bandwidth narrowing (44.5 nm FWHM output vs. $49 \mathrm{~nm}$ FWHM input) occurred in the four-pass Ti:sapphire amplifier, and the center wavelength was pushed by saturation to even longer wavelengths. The Fourier transform limit of the amplified output spectrum is $30 \mathrm{fs}$. It has been shown previously ${ }^{11}$ that the additional, phase-mismatch-induced, spectral phase in OPCPA can be readily compensated using conventional techniques to produce pulses shorter than $10 \mathrm{fs}$.

The near field output beam profile after amplification in OPA and four-pass amplifier is shown in Fig. 4. The beam quality of the amplified signal was measured ${ }^{18}$ to be $M^{2}=1.4$ in the horizontal direction, and $M^{2}=1.7$ in the vertical direction, and was limited primarily by the use of available low wavefront quality reflectors in the four-pass amplifier. With pump depletion in the OPA and onset of saturation in the four-pass amplifier, our measured energy stability was $3 \%$ (one standard deviation of 100 shots). Pump depletion in OPA greatly improves the signal energy stability compared to unsaturated OPA. ${ }^{19}$

We measured the prepulse contrast from our system using a diode and a set of calibrated filters to be better than $10^{8}$, despite the fact that we do not utilize any electro-optic modulators in the system. To first order, prepulse contrast is determined by the total gain in the system. A 1-meter delay between the signal and the pump after amplification in the OPA ensured that essentially all the energy stored in the Ti:sapphire 
amplifier is transferred to the oscillator pulse that is amplified in the OPA. The pulses arriving prior to the main pulse did not experience any gain, either in the OPA or in the four-pass amplifier. In effect, OPA acted as a pulse selector in the system.

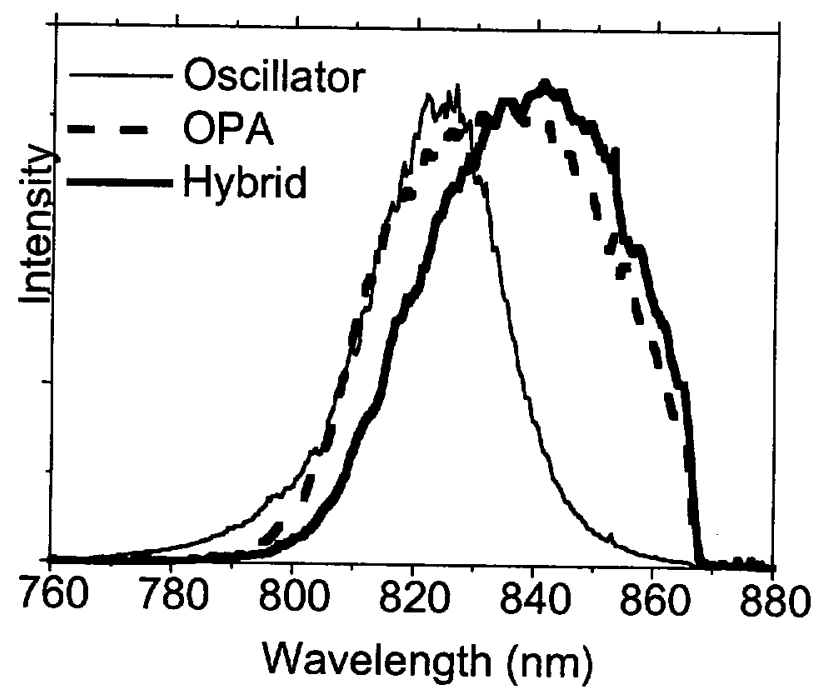

FIG 3. Seed and amplified signal spectra from OPA and four-pass amplifier.

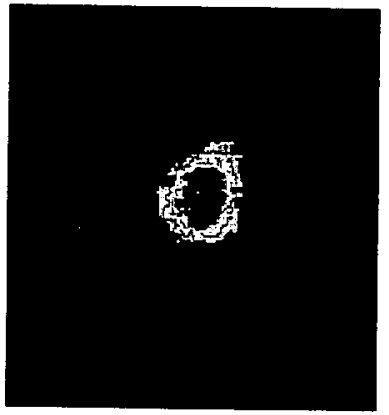

FIG 4. Transverse near-field beam profile of the output of the hybrid CPA system

\section{SUMMARY}

In summary, we have demonstrated for the first time the use of a hybrid architecture of OPAs and laser amplifiers to simultaneously achieve with a single pump pulse large gain and high conversion efficiency without any electro-optic switching. The output energy and operating wavelength of this source makes it suitable for energy scaling by subsequent amplification in large Ti:sapphire amplifiers. The system exhibits a good beam quality and superior prepulse contrast compared to systems based on Ti:sapphire alone. With 1-J pump pulses, peak powers in excess of $10 \mathrm{TW}$ can be produced using this simple technique. The idea of hybrid chirped pulse amplification can also be extended to different combinations of OPA nonlinear crystals and laser gain materials, e.g. Nd:glass amplifiers.

The authors wish to acknowledge technical input and assistance of Rick Cross, John Crane, Mark Hermann, Paul Springer, and David Gibson.

This work was performed under the auspices of the U. S. Department of Energy by the University of California, Lawrence Livermore National Laboratory under Contract No. W-7405-Eng-48. 


\section{REFERENCES}

1. D. Strickland and G. Mourou, Opt. Commun. 56, 219 (1985).

2. F. G. Patterson, R. Gonzales, and M. D. Perry, Opt. Lett. 16, 1107 (1991).

3. J. D. Kmetec, J. J. Macklin, and J. F. Young, Opt. Lett. 16, 1001 (1991).

4. W. E. White, J. R. Hunter, L. Van Woerkom, T. Ditmire, and M. D. Perry, Opt. Lett. 17, 1067 (1992).

5. C. P. J. Barty, C. L. Gordon III, and B. E. Lemoff, Opt. Lett. 19, 1442 (1994).

6. P. F. Curley, C. Le Blanc, G. Cheriaux, G. Darpentigny, P. Rousseau, F. Salin, J. P. Chambaret, and A. Antonetti, Opt. Commun. 131, 72 (1996).

7. S. Backus, C. G. Durfee III, M. M. Murnane, and H. C. Kapteyn, Rev. Sci. Instrum. 69, 1207 (1998).

8. K. Yamakawa, M. Aoyama, S. Matsuoka, H. Takuma, C. P. J. Barty, and D. Fittinghoff, Opt. Lett. 23, 525 (1998).

9. J. D. Bonlie, F. Patterson, D. Price, B. White, and P. Springer, Appl. Phys. B 70, 155 (2000).

10. A. Dubietis, G. Jonasauskas, and A. Piskarskas, Opt. Commun. 88, 437 (1992).

11. I. N. Ross, P. Matousek, M. Towrie, A .J. Langley, J. L. Collier, Opt. Commun. 144, 125 (1997).

12. R. A. Baumgartner and R. Byer, IEEE J. Quantum Electron. QE-15, 432 (1979).

13. I. Jovanovic, B. J. Comaskey, and D. M. Pennington, J. Appl. Phys. 90, 4328 (2001).

14. I. Jovanovic, B. J. Comaskey, C. A. Ebbers, D. M. Pennington, R. A. Bonner, and E. C. Morse, Appl. Opt. (to be published).

15. B. Walker, C. Toth, D. N. Fittinghoff, T. Guo, D.-E. Kim, C. Rose-Petruck, J. A. Squier, K. Yamakawa, K. R. Wilson, and C. P. J. Barty, Opt. Exp. 5, 196 (1999).

16. P. S. Banks, M. D. Perry, V. Yanovsky, S. N. Fochs, B. C. Stuart, and J. Zweiback, IEEE J. Quantum Electron. 36, 268 (2000).

17. I. Jovanovic, C. A. Ebbers, B. C. Stuart, M. R. Herman, and E. C. Morse, accepted for presentation at the Conference on Lasers and Electro-Optics, Long Beach, Calif., 19-24 May 2002.

18. A. E. Siegman, in Optical Resonators, D. A. Holmes, ed., Proc. SPIE 1224, 2 (1990).

19. S. K. Zhang, M. Fujita, M. Yamanaka, M. Nakatsuka, Y. Izawa, and C. Yamanaka, Opt. Commun. 184, 451 (2000). 


\title{
Optical parametric chirped pulse amplifier as an alternative to Ti:sapphire regenerative amplifiers
}

\author{
Igor Jovanovic ${ }^{1,2}$ \\ Brian J. Comaskey ${ }^{I}$ \\ Chris A. Ebbers ${ }^{1}$ \\ Randal A. Bonner ${ }^{1}$ \\ Deanna M. Pennington ${ }^{1}$ \\ Edward C. Morse ${ }^{2}$ \\ ${ }^{\prime}$ Lawrence Livermore National Laboratory \\ ${ }^{2}$ Department of Nuclear Engineering, University of California at Berkeley,
}

\begin{abstract}
We demonstrated a high pulse energy, femtosecond pulse source based on optical parametric chirped pulse amplification. We successfully amplified $1 \mu \mathrm{m}$ broadband oscillator pulses to $31 \mathrm{~mJ}$ and recompressed them to $310 \mathrm{fs}$ pulse duration, at a $10 \mathrm{~Hz}$ repetition rate. The gain in our system is $6 \times 10^{7}$, achieved by single passing only $40 \mathrm{~mm}$ of gain material pumped by a commercial Q-switched Nd:YAG laser. This relatively simple system replaces a more complex Ti:sapphire regenerative amplifier based chirped pulse amplification system. Numerous features in design and performance of optical parametric chirped pulse amplifiers make them a preferred alternative to regenerative amplifiers based on Ti:sapphire in the front end of high peak power lasers.
\end{abstract}

\section{INTRODUCTION}

Chirped pulse amplification ${ }^{1}$ (CPA) is now routinely used to generate gigawatt, terawatt, and even petawatt ${ }^{2}$ peak powers for applications such as short-pulse precision machining ${ }^{3}$, high harmonic generation and time resolved spectroscopy ${ }^{4}$, and fast ignitor driver for inertial confinement fusion ${ }^{5}$, respectively. Typically, these sources consist of a short-pulse oscillator, stretcher, regenerative amplifier, a series of high-energy amplifiers and a compressor. Bandwidth limitations of common laser gain media are partially circumvented by using high-bandwidth materials such as Ti:sapphire in the system front end, combined with high energy storage materials such as Nd:glass in the final amplifiers. Different applications require different combinations of pulse energy and pulse width: spectroscopic applications require $\mu \mathrm{J}$ to $\mathrm{mJ}$ pulse energies with 65$100 \mathrm{fs}$ pulse widths, machining applications require pulse energies in the range of $0.1-50 \mathrm{~mJ}$ with pulse widths between $0.5-2$ $\mathrm{ps}$, and fast ignitor drivers require relatively large pulse energies (1-10 kJ) with fairly long pulse widths (1-10 ps).

The existing technologies for short pulse generation and amplification rely upon laser hosts such as Cr:LiSAF, Ti:sapphire, or Yb:YAG. Yb:YAG is used where direct diode pumping of the source is desired. Otherwise, green pumped Ti:sapphire is utilized. Nd:glass amplifiers find application when large pulse energies with relatively long pulse widths (hundreds of fs) are sought. Optical parametric amplification ${ }^{6}$ has been recently identified as an attractive amplification technology, and its use in CPA systems is referred to as optical parametric chirped pulse amplification ${ }^{7-9}$ (OPCPA). Optical parametric amplification is a nonlinear process which involves a signal wave, a pump wave and an idler wave. In OPAs, intense pulses essentially instantaneously interact in a nonlinear material through nonlinear polarization wave generated at the difference frequency. Parametric gain is achieved over the coherence length, defined as the length over which the phase relationship among the three waves departs from the ideal condition $\phi_{p}-\phi_{s}-\phi_{i}=-\pi / 2$ by $\Delta \phi=\pi$. An underappreciated feature of optical parametric amplification is that it results in a minimal heat deposition in the OPA crystal, allowing a different set of material constraints to limit the final energy. Combined with the demonstrated performance of existing doubled pump lasers, OPCPA appears capable of extending CPA into the kilowatt average power regime ${ }^{10}$.

OPCPA has the broad bandwidth capability similar to Ti:sapphire, but offers the flexibility of operating at an arbitrary wavelength, making OPAs more flexible than solid state lasers. Broad bandwidth can be achieved in a collinear OPA operating near degeneracy, or in a noncollinear nondegenerate OPA. High gain can be obtained from nanosecond OPCPA by single-passing a relatively short length (several $\mathrm{cm}$ ) of gain material, which eliminates the need for complicated regenerative multi-pass amplification and electro-optic switching. Since the total length of the gain material is short, the accumulated nonlinear phase (B-integral) is substantially reduced, enabling good amplified pulse recompression without additional compensation for B-integral. A substantial reduction of the prepulse from a CPA system can be achieved by eliminating the pulse leakage originating from the regenerative multipass cavity. In addition to the mentioned advantages of OPCPA when used to replace regenerative amplifiers, OPCPA also allows large energy scaling through large available apertures of potassium dihydrogen phosphate (KDP) crystals. 
Another feature of an OPA makes it even more attractive for applications where the reduction of pulse aberrations is crucial. Optical parametric amplification is inherently a low-noise amplification process, allowing possible pump phase aberrations to be transferred to the idler wave, while introducing a negligible modulation to the signal wave. The only noise that is introduced in the process is due to parametric superfluorescence ${ }^{11}$, a process analogous to ASE in lasers, and is typically less intense than ASE for similar gain ${ }^{8}$. The condition for signal amplification to occur with high fidelity is a good overlap between the signal and the idler waves in space and time. A reduced transverse beam overlap and pump beam nonuniformity lead to spatial modulation of the signal, while a reduced temporal overlap can produce a modified chirped pulse spectrum.

In this paper we report on an efficient chirped pulse OPA pumped by a commercial Nd:YAG laser. We present a numerical model and design for an optical parametric chirped pulse amplifier as a replacement for a regenerative amplifier. We evaluate the performance of our OPCPA system designed for the front end of a Nd:glass high energy CPA system. In section 2 we present the experimental setup. This is followed by a description of our numerical modeling in section 3 . Finally, we show the obtained results in section 4 and follow with a discussion of these results and a conclusion. Our result represents the most efficient OPCPA to date pumped by a commercial Q-switched pump laser.

\section{EXPERIMENTAL SETUP}

The design of our OPCPA system is presented in Fig. 1. A mode-locked Ti:sapphire oscillator (Spectra-Physics Tsunami) with a center wavelength of $1054 \mathrm{~nm}$ produces $100 \mathrm{fs}$ transform limited pulses. The pulse train consists of $2.5 \mathrm{~nJ}$ pulses at $84 \mathrm{MHz}$. A single pulse is selected from the oscillator using a Pockels cell and a pair of polarizers. The pulse is stretched in a four-pass stretcher which contains a 1480 lines $/ \mathrm{mm}$ diffraction grating and a plano-convex lens, with the effective grating separation of $8 \mathrm{~m}$. The stretcher is configured to clip the spectrum at its FWHM, with the bandwidth of 16.5 $\mathrm{nm}$. The stretched pulse width is $3 \mathrm{~ns}$, and the stretched pulse energy is $0.5 \mathrm{~nJ}$. As noted in section 4 , spherical and cromatic aberrations in the stretcher limit the recompression of the seed pulse to $280 \mathrm{fs}$.

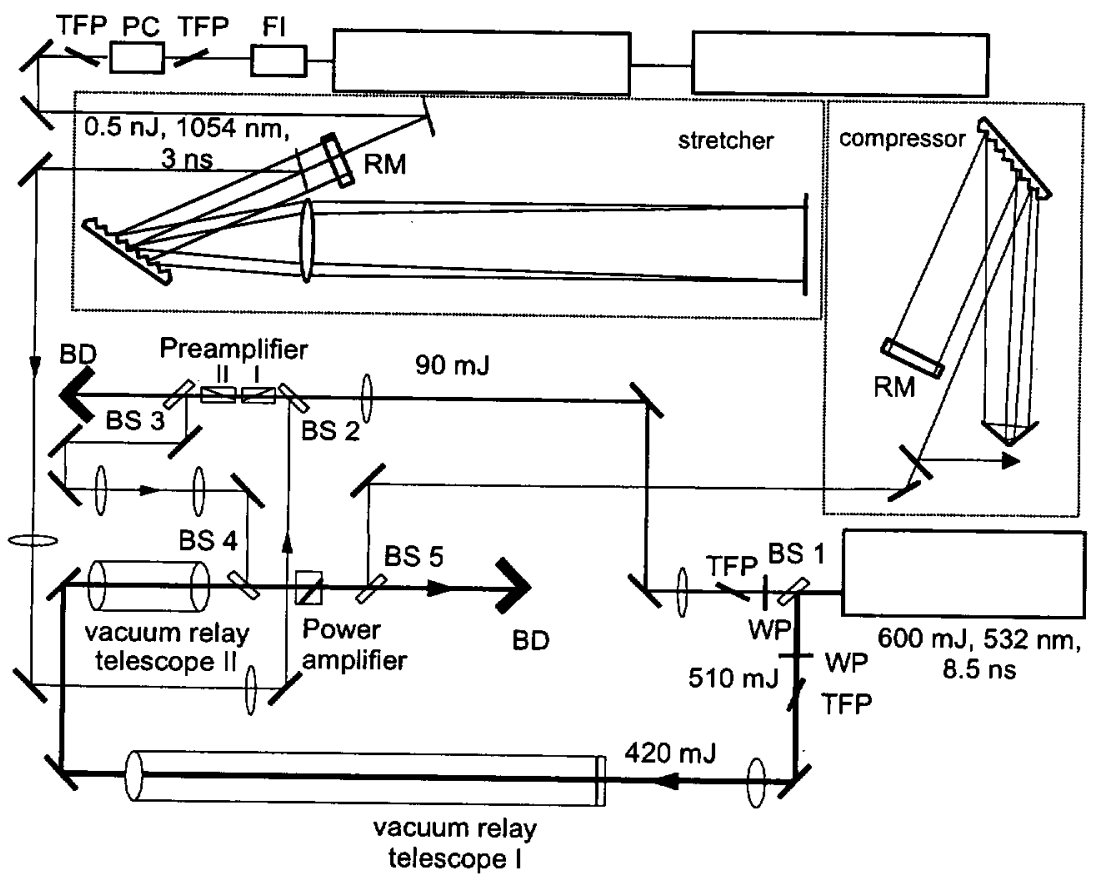

Fig. 1. Experimental OPCPA setup. BS-beamsplitter, TFP-thin film polarizer, WP-waveplate, BD-beam dump, FI-Faraday isolator, PC-Pockels cell, RM-roof mirror.

The stretched seed pulse is then relay imaged to the OPA. The OPA consist of three $\beta$-barium borate (BBO) crystals. The size of the first two crystals is $4 \times 4 \times 15 \mathrm{~mm}^{3}$, while the third crystal is $10 \times 10 \times 10 \mathrm{~mm}^{3}$, permitting scaling to large incident pump pulse energy by increasing the pump beam diameter. The crystals are cut at $22.8^{\circ}$ to allow type $\mathrm{I}$ angular phase matching, and they have a $2^{\circ}$ wedge on their output faces to prevent intra-crystal parasitic oscillation.

The pump source for the OPA is a Spectra-Physics GCR 270-10 Nd:YAG laser, operated in single longitudinal mode, producing $600 \mathrm{~mJ}$ of $532 \mathrm{~nm}$ output. The pulse width of Q-switched pulses is $8.5 \mathrm{~ns}$, at a $10 \mathrm{~Hz}$ repetition rate. The transverse 
intensity profile of the pump beam is a supergaussian, while the temporal profile is gaussian, with a characteristic pulse spatiotemporal evolution for an unstable resonator ${ }^{12}$. To maximize the gain, the relative temporal position of the signal and pump pulse is adjusted so that the maximum intensity of the signal coincides with the maximum intensity of the pump transverse center.

The first two BBO crystals are configured as preamplifiers and separated by $2 \mathrm{~mm}$. Walk-off compensation ${ }^{13}$ is used in order to reduce the effect of the intrinsic $3.2^{\circ}$ extraordinary beam Poynting vector walk-off in BBO. The pump beam is split by a $15 \%$ beamsplitter BS1 into a $90 \mathrm{~mJ}$ beam and a $510 \mathrm{~mJ}$ beam. The supergaussian transverse intensity profile of the pump laser is relay imaged to a plane between the first two BBO crystals by means of a telescope, which simultaneously adjusts the pump beam diameter in the first two crystals to $1.6 \mathrm{~mm}$. The proximity of the two walk-off compensated crystals in the preamplifier eliminates the need for separate imaging of the pump beam on each crystal, which is a significant simplification when compared to previous designs. ${ }^{8,9}$ In addition, dephasing in the small air gap is calculated to produce only a small $(<0.1 \%)$ modification of gain in the preamplifier. The peak intensity of the pump beam in the first and second BBO crystal is $450 \mathrm{MW} / \mathrm{cm}^{2}$. The pump beam is collimated in order to minimize dephasing due to the small angular acceptance of $\mathrm{BBO}(0.4 \mathrm{mrad} \mathrm{cm})$.

An additional source of dephasing is the beam quality from the pump source (we measured the pump beam quality to be 1.6xDL in the sensitive phase matching plane and $1.8 \times \mathrm{XL}$ in the insensitive phase matching plane). The measured deviation of the pump beam from ideal beam quality has negligible impact on parametric gain with the selected pump beam diameter.

The size of the seed beam is dictated by the requirement to avoid spatial modulation on the signal beam due to transverse walk-off of the extraordinary polarized pump beam, and is set to $0.4 \mathrm{~mm}$ in the first two BBO crystals. The center of the seed beam is displaced from the center of the pump beam by $0.4 \mathrm{~mm}$ in the sensitive phase matching direction on the front face of the first BBO crystal, maximizing the spatial overlap of the beams in the preamplifier. We treat our first two crystals as large-gain preamplifiers and neglect the low conversion efficiency resulting from the small spatiotemporal overlap of the seed and the pump pulses. The seed beam is introduced into the crystal at a $1^{\circ}$ external angle with respect to the pump beam, perpendicular to the crystal principal plane. The amplified signal and the idler beam are spatially separated after propagating $30 \mathrm{~cm}$ out of the preamplifier, and the idler is subsequently blocked, leaving only the amplified signal.

We use the beam transmitted through the $15 \%$ beamsplitter BS1 and relay image the supergaussian spatial profile of the pump beam onto the final BBO crystal (power amplifier). The pump imaging telescope consists of two vacuum image relays. The use of two vacuum relay telescopes in our system is necessary because of spatial constraints and the requirement for imaging, beam sizing and temporal matching of the seed and pump between the preamplifier and the power amplifier. The pump beam diameter in the power amplifier is $3.5 \mathrm{~mm}$, with peak intensity of $430 \mathrm{MW} / \mathrm{cm}^{2}$ on the crystal with $410 \mathrm{~mJ}$ incident pump energy used from the available $510 \mathrm{~mJ}$. Operating at intensity near $0.5 \mathrm{GW} / \mathrm{cm}^{2}$ is a compromise between the requirement for strong nonlinear drive and the reduction of the risk of crystal damage, leading to greater system reliability. At operating intensities near $1 \mathrm{GW} / \mathrm{cm}^{2}$ we periodically observed crystal damage on antireflection-coated surfaces, which is unacceptable in a system with appreciable repetition rate. The signal beam is imaged from the preamplifier and introduced into the power amplifier using the beamsplitter BS4. The signal and idler beams are separated spatially after 2 meters of propagation out of the power amplifier crystal, given the larger diameter of the signal and idler beam in the power amplifier.

The amplified signal pulse is compressed using a single-grating, double-pass compressor. The compressor contains a 1480 lines $/ \mathrm{mm}$ grating, set at a diffracted angle of $55.33^{\circ}$ to match the stretcher angle. No spectral clipping occurs in the compressor as a result of the large size of the associated optics. The spectral range is limited by the hard spectral clip in the stretcher, which limits the size of the spectrally dispersed beam in the compressor. The measured compressor efficiency is $50 \%$. The recompressed pulse width measurement is performed using a scanning intensity autocorrelator for the recompressed seed, and a single-shot intensity autocorrelator for the amplified signal pulse at $10 \mathrm{~Hz}$.

\section{NUMERICAL MODELING}

Numerical modeling was found to be an important step for optimizing the performance of the preamplifier and subsequent power amplifier. Our OPCPA model is based on a numerical solution for the system of coupled differential equations for difference frequency generation. The system that describes traveling waves can be written in a concise form ${ }^{6}$ as

$$
\begin{aligned}
& \frac{d A_{1}}{d z}=i \frac{2 \omega_{1}}{n_{1} c} d_{e f f} A_{2}^{*} A_{3} \exp (i \Delta k z), \\
& \frac{d A_{2}}{d z}=i \frac{2 \omega_{2}}{n_{2} c} d_{e f f} A_{1}^{*} A_{3} \exp (i \Delta k z),
\end{aligned}
$$




$$
\frac{d A_{3}}{d z}=i \frac{2 \omega_{3}}{n_{3} c} d_{e f f} A_{1} A_{2} \exp (-i \Delta k z)
$$

where $A_{1}, A_{2}$ and $A_{3}$ are the amplitudes of the electric field of the signal, idler and pump, respectively, $d_{e f f}$ is effective nonlinearity, and $\Delta k$ is the wave vector mismatch. The temporal walk-off due to group velocity dispersion (GVD) is neglected because we are concerned with long (nanosecond) pulses. GVD introduces negligible dephasing, since the total spectral walk-off of signal, idler and pump is very small over the length of the nonlinear crystal. Diffraction effects were not included in the model explicitly due to their weak relative impact compared with the temporal and spatial modulation. In particular, two effects arising from diffraction can influence gain and conversion efficiency in the optical parametric amplification. First, a changing beam diameter creates a nonuniform spatial intensity along the crystal. Second, angular dephasing leads to reduced gain due to finite angular acceptance of the nonlinear process. Both of these effects are insignificant with the weakly focused beams used in the nanosecond OPCPA, and do not play an important role in the
amplification process.

Eqs. (1) model the interaction of plane waves and have to be suitably modified to describe the situation when the beam diameter is finite and the beam has a nonuniform transverse intensity profile. In addition, temporal and spectral modification of the signal pulse occurs in OPCPA when the pump pulse intensity is not a constant with respect to time. Finally, the effect of Poynting vector walk-off for extraordinary waves in nonlinear crystals presents an additional effect that requires inclusion in the model. This is particularly important when the beam diameter is comparable to the total transverse walk-off over the nonlinear crystal length.

We evaluate those effects by solving Eqs. (1) numerically on a 3-dimensional grid in space and time. We start with the assumed intensity distribution of the original waves at a point:

$$
I_{i}(x, y, t)=2 n_{i} \sqrt{\frac{\varepsilon_{0}}{\mu_{0}}}\left|A_{i}(x, y, t)\right|^{2}, \quad i=1,2,3,
$$

where $I$ is the beam intensity, $x$ and $y$ are the two transverse coordinates, and $t$ is the temporal coordinate. The intensity distribution is normalized in the following fashion:

$$
\int_{-\infty}^{\infty} \int_{-\infty-\infty}^{\infty} \int_{i}^{\infty} I_{i}(x, y, t) d x d y d t=E_{i}, \quad i=1,2,3,
$$

where $E$ is the pulse energy. We assign a zero phase to the electric field of the seed and pump at the input face of the crystal $(z=0)$. This is consistent with the insensitivity of optical parametric amplification with respect to the initial phase relationship between the seed and the pump when an idler is not initially present. Additionally, we introduce the effect of pump beam walk-off angle, $\rho$, in one direction $(x)$ and the noncollinear angle in two directions for the signal $\left(\Omega_{1 x}, \Omega_{1 y}\right)$ and idler beam $\left(\Omega_{2 x}, \Omega_{2 y}\right)$. We account for the appropriate transverse part the signal, idler and pump at the point $z$ in the crystal by calculating the corrected transverse displacement:

$$
\begin{aligned}
& x_{1}{ }^{\prime}=x_{1}+\Omega_{1 x} z, y_{1}{ }^{\prime}=y_{1}+\Omega_{1 y} z, \\
& x_{2}{ }^{\prime}=x_{2}+\Omega_{2 x} z, y_{2}{ }^{\prime}=y_{2}+\Omega_{2 y} z, \\
& x_{3}{ }^{\prime}=x_{3}+\rho z,
\end{aligned}
$$

where $\left(x_{1}{ }^{\prime}, y_{1}{ }^{\prime}\right),\left(x_{2}{ }^{\prime}, y_{2}{ }^{\prime}\right)$ and $x_{3}{ }^{\prime}$ represent the corrected transverse coordinates for the signal, idler and pump, respectively.

In CPA the spectrum is linearly chirped in time, so the phase mismatch due to spectral bandwidth can be included by assigning the appropriate value for wave vector mismatch and its projection in the direction normal to crystal surface:

$$
\begin{aligned}
& \Delta \mathbf{k}(t)=\mathbf{k}_{3}-\mathbf{k}_{1}(t)-\mathbf{k}_{2}(t) \\
& \Delta k_{z}(t) \approx 2 \pi\left(\frac{n_{3}}{\lambda_{3}}-\frac{n_{1}(t)}{\lambda_{1}(t)}-\frac{n_{2}(t)}{\lambda_{2}(t)}\right)
\end{aligned}
$$

Finally, the system of differential equations can be written as

$$
\begin{aligned}
& \frac{d A_{1}\left(x_{1}{ }^{\prime}, y_{1}{ }^{\prime}, t\right)}{d z}=i \frac{2 \omega_{1}(t)}{n_{1}(t) c} d_{e f f} A_{2}^{*}\left(x_{2}{ }^{\prime}, y_{2}{ }^{\prime}, t\right) A_{3}\left(x_{3}{ }^{\prime}, y, t\right) \exp (i \Delta k(t) z), \\
& \frac{d A_{2}\left(x_{2}{ }^{\prime}, y_{2}{ }^{\prime}, t\right)}{d z}=i \frac{2 \omega_{2}(t)}{n_{2}(t) c} d_{e f f} A_{1}^{*}\left(x_{1}{ }^{\prime}, y_{1}{ }^{\prime}, t\right) A_{3}\left(x_{3}{ }^{\prime}, y, t\right) \exp (i \Delta k(t) z),
\end{aligned}
$$




$$
\frac{d A_{3}\left(x_{3}{ }^{\prime}, y, t\right)}{d z}=i \frac{2 \omega_{3}(t)}{n_{3}(t) c} d_{e f f} A_{1}\left(x_{1}{ }^{\prime}, y_{1}{ }^{\prime}, t\right) A_{2}\left(x_{2}{ }^{\prime}, y_{2}{ }^{\prime}, t\right) \exp (-i \Delta k(t) z) .
$$

We perform a discretization of the electric field on the grid of $(D X, D Y, D T)$ points with a spatial and temporal window size ( $W X, W Y, W T$ ), such that the following normalization applies:

$$
2 n_{i} \sqrt{\frac{\varepsilon_{0}}{\mu_{0}}} \frac{W X}{D X} \frac{W Y}{D Y} \frac{W T}{D T} \sum_{j=1}^{D X} \sum_{k=1}^{D Y} \sum_{l=1}^{D Y}\left|A_{i}(j, k, l)\right|^{2}=E_{i}, \quad i=1,2,3 .
$$

The system (9) in discrete form is solved numerically using a 4th order Runge-Kutta integration routine, yielding solutions for electric field amplitude and phase at the crystal output in a spatially and temporally resolved form. It is important to note that the model allows initial misalignment of the seed and pump beam at the input face of the nonlinear crystal. This is a frequent experimental optimization procedure required to achieve best overlap of the beams inside a crystal which exhibits walk-off for one of the beams.

Our system uses a pump laser based on an unstable resonator, with a complicated spatiotemporal pulse evolution ${ }^{12}$. We recorded the pulse shape using a scanning pinhole in the image plane of the pump pulse. A numerical fit has been obtained for the pump pulse shape, as shown in Fig. 2 in a 4 ns temporal window centered at the peak of the pump pulse intensity. We use the obtained pump spatial and temporal pulse shape in our numerical model.

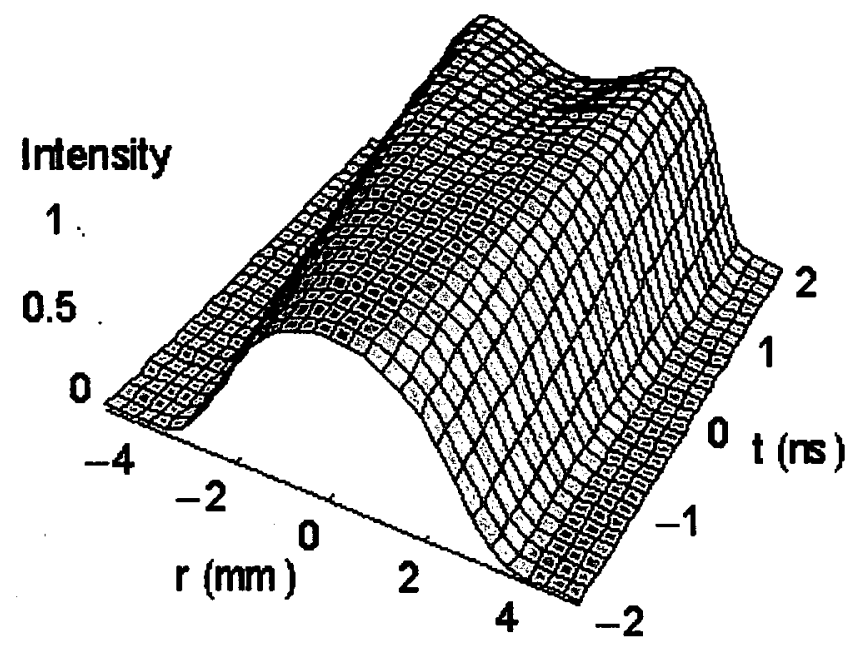

Fig. 2. Spatiotemporal evolution of the pump pulse originating in an unstable laser resonator. Shown is the normalized intensity as a function of the radial position $r$ and time $t$, in a 4 ns time window.

\section{RESULTS}

With the injected signal of $0.5 \mathrm{~nJ}$, the measured output from the first BBO crystal was $1.8 \mu \mathrm{J},($ Gain $=3700)$, and from the second BBO crystal we obtained $1.5 \mathrm{~mJ}($ Gain $=830)$. Gain was optimized by adjusting the transverse displacement between the seed and the pump on the front face of the first preamplifier crystal. The measured amplified signal pulse width, after passing through the preamplifier, was $3 \mathrm{~ns}$ at the clip points in the temporal domain. This is identical to the original stretched pulse width at its clip points in the temporal domain. Through adjustment of the waveplate, we could vary the amplified signal energy exiting the preamplifier by changing the pump intensity.

The expected small signal gain in the power amplifier is 160 . We measured a maximum gain of 20 from the power amplifier operating in the regime of strong pump depletion. The signal beam is amplified up to $31 \mathrm{~mJ}$ with $1.5 \mathrm{~mJ}$ of injected signal from the preamplifier. In Fig. 3 we show the extracted energy for several preamplifier outputs. The maximum pump to signal conversion efficiency in the power amplifier crystal is achieved when the preamplifier output is set to $1.5 \mathrm{~mJ}$. The conversion efficiency of the pump to signal is $25 \%$, by including only the pump energy contained in the temporal overlap of the pump with the signal. The overall pump to signal conversion efficiency in our OPCPA system is $6 \%$. Our numerical model was applied to calculate the energy extraction in the final amplifier. We used the measured spatiotemporal characteristics of the pump and the signal in our calculation. The integration was performed on a $5 \mathrm{~mm}^{2}$ spatial and $3 \mathrm{~ns}$ 
temporal window, on a grid of $50 \times 50 \times 1000$ points. The maximum intensity of the seed corresponded to the maximum intensity of the pump. The diameter of the signal in the final amplifier was $2.5 \mathrm{~mm}$ at its FWHM. As shown in Fig. 3, we observe fairly good agreement between the numerical model and the experimental results.

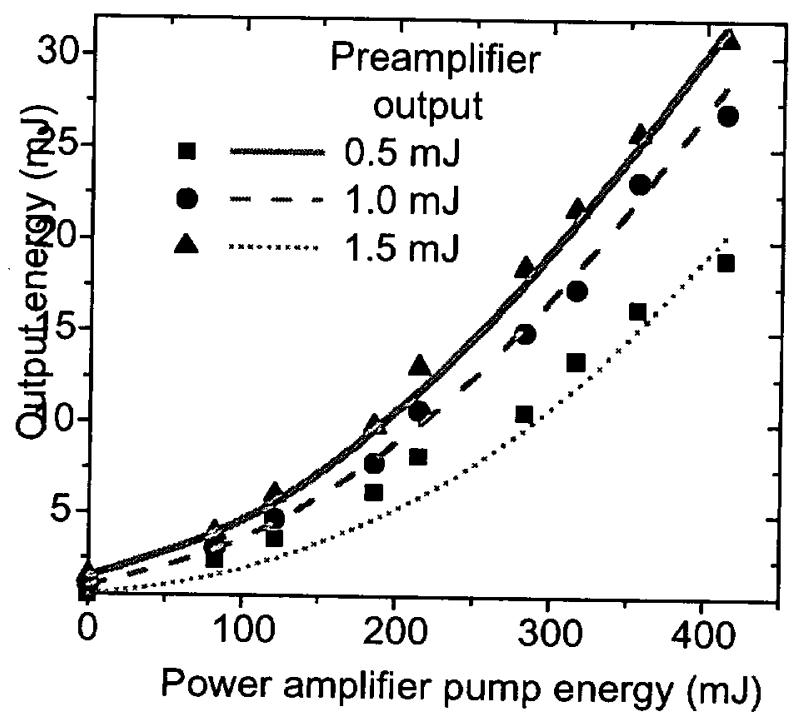

Fig. 3. Seed and amplified signal spectra from preamplifier and power amplifier. A spectral modification occurs in both preamplifier and power amplifier.

Fig. 4 shows the measured spectra for stretched seed, amplified signal from the preamplifier and the amplified signal from the power amplifier. The measured bandwidth of the pulse is $16.5 \mathrm{~nm}$ at the spectral clip points in both the preamplifier and the power amplifier. This is consistent with the absence of bandwidth narrowing due to intrinsic OPA bandwidth. In our configuration we operate the power amplifier of the OPA in the depleted pump regime in order to obtain a large extracted energy. This is done at the expense of the recompressed pulse contrast level, which remains limited by the hard clip on the spectrum occurring in the pulse stretcher. The amplified signal spectral shape is modified by strong pump depletion in the power amplifier and resembles a top-hat shape in the central $70 \%$ of the beam diameter. The measured output stability of the signal is greatly improved in the regime of strong pump depletion ${ }^{14}$, reducing the effect of pump energy variation and temporal jitter. Since the stretched signal pulse width is relatively long, a timing jitter in excess of $\sim 1$ ns produces a significant modification of the amplified spectrum.

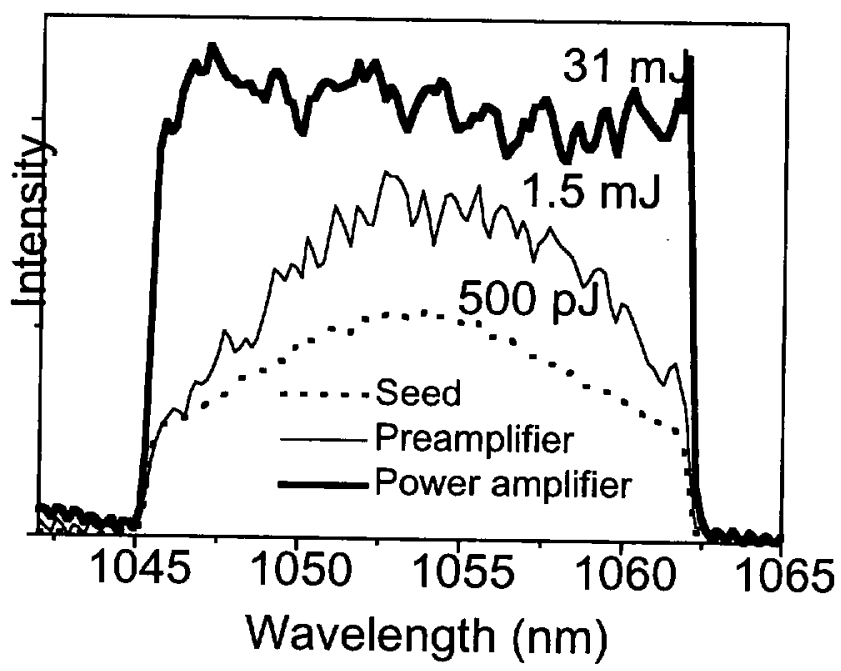

Fig. 4. Experimental extraction data from the final $B B O$ amplifier. The lines are obtained from the numerical model. 
The near-field transverse intensity profiles for pump, seed and amplified signal is shown in Fig. 5 . The incident seed beam quality was $M^{2}=1.6 \pm 0.2$ in the sensitive direction, and $M^{2}=1.2 \pm 0.2$ in the insensitive direction. The source of the measured imperfect beam quality of the seed is the hard beam clip that occurs on the stretcher lens and spherical and chromatic aberrations in the stretcher. We measured the beam quality of the signal and obtained $M^{2}=2 \pm 0.2$ in the sensitive direction and $M^{2}=1.2 \pm 0.2$ in the insensitive direction. Optical parametric amplification is a process which conserves the signal beam quality to a very high degree. However, the small diameter of the pump beam in the preamplifier leads to a strong effect of pump beam walk-off in the crystal sensitive direction. It is believed that this spatial modulation gives rise to the increase in the observed $M^{2}$ in the sensitive direction of the crystal.
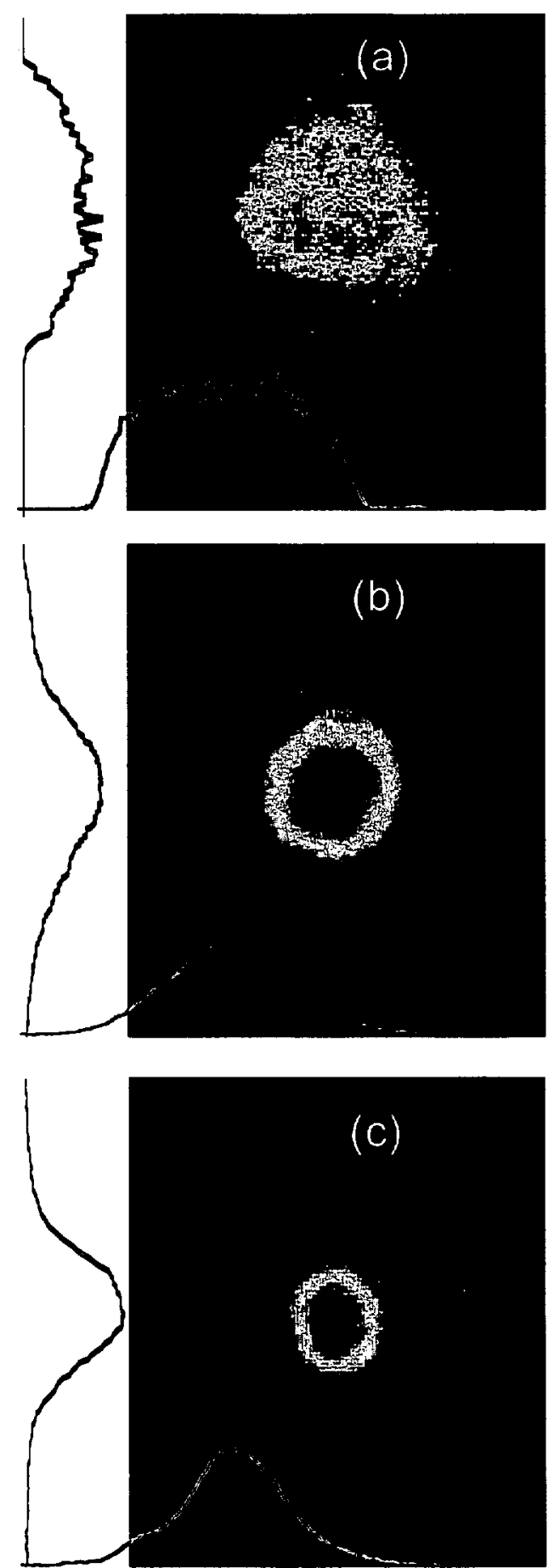

Fig. 5. Intensity profiles in the near field for (a) pump, (b) seed, and (c) amplified signal. 
As mentioned previously, we initially compressed the stretched $0.5 \mathrm{~nJ}$ seed pulses to $280 \mathrm{fs}$ FWHM (Fig. 6a), limited by the spherical and chromatic aberrations occurring in the pulse stretcher, which contains a lens-based refractive telescope. The intensity autocorrelation of the recompressed pulse at an energy of $31 \mathrm{~mJ}$ is shown in Fig. $6 \mathrm{~b}$. The recompressed amplified pulse duration is measured to be $310 \mathrm{fs}$ FWHM, a $10 \%$ increase compared to the best achieved seed recompression before amplification in OPA. The recompressed pulse pedestal is decreased after amplification. This is probably the result of the modification of amplified spectrum in OPCPA operating in the regime of strong pump depletion. Since the exact impact of stretcher aberrations on the recompressed pulse shape is unknown, we used a conservative gaussian deconvolution factor to calculate the recompressed pulse width.
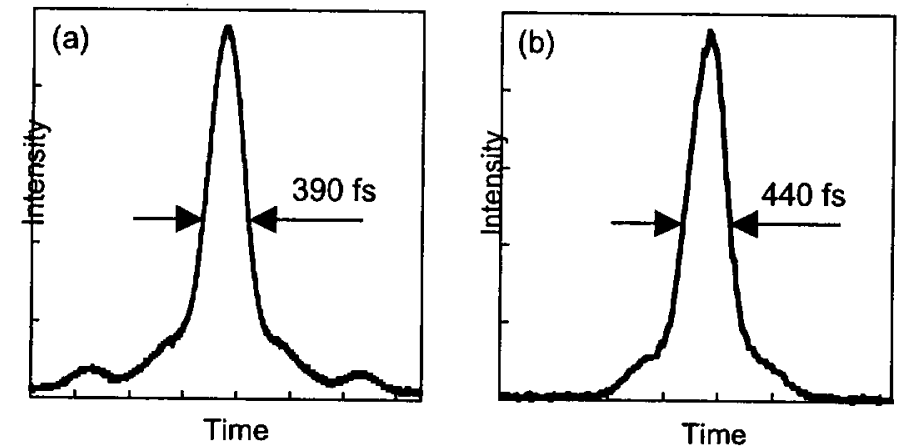

Fig 6. Recompressed pulse intensity autocorrelation for (a) seed pulses, and (b) amplified pulses. Indicated is the FWHM of the autocorrelation trace. Deconvolved pulse width is (a) $280 \mathrm{fs}$ and (b) $310 \mathrm{fs}$.

\section{CONCLUSION}

In summary, we demonstrated a successful use of optical parametric amplifiers for broadband, high fidelity, chirped pulse amplification. Our OPCPA produced a 50-fold increase in amplified pulse energy and a 10 -fold improvement in efficiency compared to previous systems pumped by commercial Q-switched pump lasers ${ }^{15,16}$. Our improved efficiency is a result of several factors. Asymmetric splitting of the pump pulse is utilized to obtain a high gain from the preamplifier with small fraction of the pump energy discarded. This is followed by the efficient power amplifier with large pump beam and short crystal, resulting in good spatial overlap between the pump and the signal. Walk-off compensated double-crystal preamplifier design allows a relatively small pump beam to be used in the preamplifier. Pump beam is collimated to reduce the angular dephasing in the type I process in BBO. The increased stretched seed pulse width allows a greater fraction of the pump energy to be transferred to signal.

The wavelength and pulse energy level from the demonstrated OPCPA is ideal for seeding high energy Nd:glass amplifiers of high power lasers. The peak power of $50 \mathrm{GW}$ was obtained from a relatively compact and simple setup which does not utilize multipass amplification. We expect the focused intensity from our system to be on the order of $10^{16} \mathrm{~W} / \mathrm{cm}^{2}$. Although our system did not exploit the full bandwidth capabilities of type I nearly degenerate OPAs, we demonstrated high gain, conversion efficiency and amplified pulse fidelity in OPCPA, which gives a viable cause for an all OPA-based kilojoule level ultrashort pulse source.

We foresee several improvements that can lead to better performance of our system. It is expected that a nondegenerate OPCPA at $800 \mathrm{~nm}$ will produce better efficiency because of the favorable splitting of the pump energy to the higher frequency field. In addition, the availability of ultrashort pulse sources will also enable the examination of the capability of OPCPA to produce pulses significantly shorter than those possible with our source. Finally, the development of appropriate pump lasers with uniform spatial and temporal intensity profile and pulse width matched to the stretched pulse can produce better conversion efficiency. We are currently upgrading our system with an aberration-free, all-reflective stretcher, eliminating the bottleneck in our design that limits the recompressed pulse width. With a suitable high energy pump laser and current compression grating technology, powers in excess of $10^{16} \mathrm{~W}$ are feasible using OPCPA ${ }^{8}$.

This work was performed under the auspices of the U. S. Department of Energy by Lawrence Livermore National Laboratory under Contract No. W-7405-Eng-48. 


\section{REFERENCES}

1. D. Strickland and G. Mourou, "Compression of amplified chirped optical pulses," Opt. Commun. 56, 219-221 (1985).

2. D. M. Pennington, C. G. Brown, T. E. Cowan, S. P. Hatchett, E. Henry, S. Herman, M. Kartz, M. Key, J. Koch, A. J. MacKinnon, M. D. Perry, T. W. Phillips, M. Roth, T. C. Sangster, M. Singh, R. A. Snavely, M. Stoyer, B. C. Stuart, S. C. Wilks, "Petawatt laser system and experiments," IEEE J. of Sel. Top. in Quant. Electron. 6, 676-688 (2000).

3. M. D. Perry, B. C. Stuart, P. S. Banks, M. D. Feit, V. Yanovsky, A. M. Rubenchik, "Ultrashort-pulse laser machining of dielectric materials," J. of Appl. Phys. 85, 6803-6810 (1999).

4. D. Riedel, J. L. Hernandez-Pozos, R. E. Palmer, S. Baggott, K. W. Kolasinski, J. S. Foord, "Tunable pulsed vacuum ultraviolet light source for surface science and materials spectroscopy based on high order harmonic generation," Rev. of Sci. Instrum. 72, 1977-1983 (2001).

5. M. Tabak, J. Hammer, M. E. Glinsky, W. L. Kruer, S. C. Wilks, J. Woodworth, E. M. Campbell, M. D. Perry, R. J. Mason, "Ignition and high gain with ultrapowerful lasers," Phys. Plasmas 1, 1626-1634 (1994).

6. R. A. Baumgartner and R. Byer, "Optical parametric amplification," IEEE J. Quantum Electron. QE-15, 432-444 (1979).

7. A. Dubietis, G. Jonu_auskas, and. A. Piskarskas, "Powerful femtosecond pulse generation by chirped and stretched pulse parametric amplification in BBO crystal," Opt. Commun. 88, 437-440 (1992).

8. I. N. Ross, P. Matousek, M. Towrie, A. J. Langley, J. L. Collier, "The prospects for ultrashort pulse duration and ultrahigh intensity using optical parametric chirped pulse amplifiers," Opt. Commun. 144, 125-133 (1997).

9. I. N. Ross, J. L. Collier, P. Matousek, C. N. Danson, D. Neely, R. M. Allott, D. A. Pepler, C. Hernandez-Gomez, and K. Osvay, "Generation of terawatt pulses by use of optical parametric chirped pulse amplification," Appl. Opt. 39, 2422$2427(2000)$.

10. S. P. Velsko and W. F. Krupke, "Applications of high-average power nonlinear optics," Proc. SPIE, M. C. Gupta, W. J. Kozlowski, and D. C. MacPherson, eds. 2700, 6-17 (1996).

11. D. A. Kleinman, "Theory of Optical Parametric Noise," Phys. Rev. 174, 1027-1040 (1968).

12. A. A. Isaev, M. A. Kazaryan, G. G. Petrash, S. G. Rautian, and A. M. Shalagin, "Evolution of Gaussian beams and pulse stimulated emission from lasers with unstable resonators," Sov. J. Quant. Electron. 5, 607-614 (1975).

13. D. J. Armstrong, W. J. Alford, T. D. Raymond, and A. V. Smith, "Parametric amplification and oscillation with walkoffcompensating crystals," J. Opt. Soc. Am. B 14, 460-474 (1997).

14. S. K. Zhang, M. Fujita, M. Yamanaka, M. Nakatsuka, Y. Izawa, C. Yamanaka, "Study of the stability of optical parametric amplification," Opt. Commun. 184, 451-455 (2000).

15. J. Collier, C. Hernandez-Gomez, I. N. Ross, P. Matousek, C. N. Danson, J. Walczak, "Evaluation of an ultrabroadband high-gain amplification technique for chirped pulse amplification facilities," Appl. Opt. 38, 7486-7493 (1999).

16. S. K. Zhang, M. Fujita, H. Yoshida, R. Kodama, H. Fujita, M. Nakatsuka, Y. lzawa, and C. Yamanaka, "Study on high gain broadband optical parametric chirped pulse amplification," Proc. SPIE, K. Mima, G. L. Kulcinski, and W. Hogan, eds. 3886, 588-595 (2000). 


\title{
Angular effects and beam quality in optical parametric amplification
}

\author{
Igor Jovanovic, ${ }^{\text {a) }}$ Brian J. Comaskey, and Deanna M. Pennington \\ Lawrence Livermore National Laboratory, Mail Code L-399, 7000 East Avenue, Livermore, California 94550
}

Advances in optical parametric devices, in particular those requiring high conversion efficiency, rely on pump laser and gain medium properties. We describe and theoretically model the source of dephasing due to angular deviation from ideal phase matching in optical parametric amplification. Real laser beams have angular content, which is described by their spatial frequency spectrum. Such beams cannot be treated as single plane waves in nonlinear interactions. Our mathematical model is based on a plane wave decomposition of Gaussian and top-hat beams into their components in spatial frequencies. Several popular nonlinear materials (beta-barium borate, lithium borate, and potassium dihydrogen phosphate) are examined for phase matching angles and dephasing is rigorously calculated. The impact of the beam angular content on small signal gain and on conversion efficiency in the strongly depleted regime is evaluated numerically. In addition, a criterion is formulated for beam quality tolerance in optical parametric amplifiers, for critical and noncritical phase matching. The impact of initial conditions in optical parametric amplification is considered. Our calculations are intended primarily for devices pumped with long (nanosecond) pulses. (1) 2001 American Institute of Physics. [DOI: 10.1063/1.1407312]

\section{INTRODUCTION}

Since their first demonstration in $1965,{ }^{1}$ optical parametric amplifiers (OPAs) have received significant attention due to their intrinsic design simplicity, large gain, and broad tunability. Devices were demonstrated with femtosecond, ${ }^{2}$ picosecond, ${ }^{3}$ and nanosecond ${ }^{4}$ pulses. These pulse widths allow large beam intensities required to drive the nonlinear amplification process efficiently. Recently, a scheme has been demonstrated which allows high energy extraction in a short pulse from an OPA. The technique, termed optical parametric chirped pulse amplification, ${ }^{5}$ has been theoretically shown to support up to $15 \mathrm{PW}$ peak powers with the current compression gratings technology, ${ }^{6,7}$ and a potential to completely replace Ti:sapphire in broadband amplification. ${ }^{8}$

Optical parametric amplification is a three-wave mixing process in which pump, signal, and idler waves are involved. A low intensity signal wave (seed) is incident on a nonlinear medium together with a high intensity pump wave. Through the difference frequency generation process, the idler wave is generated at difference frequency between the pump and the signal wave. Simultaneously, the signal wave is coherently amplified. The system of coupled differential equations for the amplitudes of three waves, which was derived by Armstrong et al. ${ }^{9}$ is

$$
\begin{aligned}
& \frac{d E_{1}}{d z}=i \frac{2 \omega_{1}}{n_{1} c} d_{\mathrm{eff}} E_{2}^{*} E_{3} \exp (i \Delta k z), \\
& \frac{d E_{2}}{d z}=i \frac{2 \omega_{2}}{n_{2} c} d_{\mathrm{eff}} E_{1}^{*} E_{3} \exp (i \Delta k z),
\end{aligned}
$$

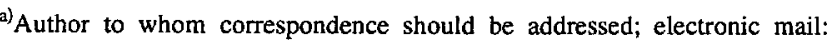
jovanovic1@llnl.gov
}

$$
\frac{d E_{3}}{d z}=i \frac{2 \omega_{3}}{n_{3} c} d_{\mathrm{eff}} E_{1} E_{2} \exp (-i \Delta k z),
$$

where 1,2 , and 3 denote the signal, idler, and pump waves, respectively, $d_{\text {eff }}$ is the effective nonlinearity, and $\Delta k$ $=|\Delta \mathbf{k}|$ is the wave vector mismatch. The intensity $I$ is given in terms of the electric field amplitude $E$ by

$$
I=2 \epsilon_{0} n c|E|^{2} .
$$

The amount of dephasing among the waves is described by the wave vector mismatch $\Delta \mathbf{k}$. In general, dephasing is due to departure from the ideal phase matching condition in parametric three-wave interactions: ${ }^{10}$

$$
\Delta k=k_{p}-k_{s}-k_{i}
$$

In optical parametric amplification, efficient energy transfer from the pump wave to signal and idler waves occurs when a constant phase relationship among the three waves is maintained:

$$
\phi_{p}-\phi_{s}-\phi_{i}=-\pi / 2 \text {. }
$$

A nonvanishing wave vector mismatch $\Delta k$ causes the waves to fall out of phase, with characteristic coherence length $L_{c}$ :

$$
L_{c}=\frac{\pi}{\Delta k} .
$$

Careful maximization of efficiency is particularly important for high repetition rate OPAs, where the available pump pulse energy is relatively small. The conversion efficiency depends on the overlap between the pump and signal pulses in space and time. ${ }^{11,12}$ When nanosecond pump pulses are used, longer interaction lengths in the crystals are required. Under these conditions, dephasing due to angular, spectral, and temperature bandwidth and crystal nonuniformity becomes important. In this article we theoretically investigate 
the impact of the angular spectrum of the pump and signal on conversion efficiency in optical parametric amplification, and we show results of our calculations for several nonlinear crystals commonly used as OPAs.

Boyd and Kleinman ${ }^{13}$ performed the first analytical calculations of coupled wave interactions with focused Gaussian beams. Their calculations included effects of rapidly variable intensity in tight focusing conditions, walkoff, and diffraction. However, their analysis was limited to small conversion efficiency and Gaussian beams. More recently, Wong et al. ${ }^{14}$ investigated conversion efficiency for second harmonic generation. In their model they used a beam with Gaussian distribution of divergence and included depletion effects. We extend the calculation of Wong et al. to predict the limits of performance for OPAs, using a plane wave decomposition model for Gaussian and top-hat beams. We derive phase matching curves for several common nonlinear crystals and apply them to the specific case of optical parametric amplification. Additionally, we establish a relationship between laser beam quality and conversion in OPAs. We include the impact of initial conditions in the process of optical parametric amplification. Finally, we derive a criterion for beam quality that allows large conversion efficiency in OPAs, for critical and noncritical phase matching.

\section{MODELING OF ANGULAR DIVERGENCE EFFECTS}

In optical parametric devices, nonlinear crystals are commonly placed in the laser beam waist, which allows the laser intensity to rise to the level required for efficient parametric interactions and ensures best possible collimation. A perfect Gaussian beam propagates according to the following equation:

$$
w^{2}(z)=w_{0}^{2}\left(1+\left(\frac{\lambda\left(z-z_{0}\right)}{\pi w_{0}^{2}}\right)^{2}\right),
$$

where $w$ is the beam radius, $w_{0}$ is the beam waist radius, and $z$ is the longitudinal position ( $z_{0}$ being the waist position). In the beam waist, we can obtain the one-dimensional distribution of the electric field in spatial frequencies by taking the Fourier transform of the transverse electric field distribution:

$$
E(s)=\int_{-\infty}^{\infty} E(x) e^{-i s x} d x
$$

where $s$ is the transverse spatial frequency. The Fraunhofer diffraction pattern in the far field is equivalent to the nearfield distribution in spatial frequencies $(s=\theta / \lambda)$. Therefore, any real laser beam consists of a superposition of plane waves, with a distribution of divergence as indicated by the spatial frequency distribution. This divergence spread contributes to dephasing in optical parametric amplification.

We use the $M^{2}$ formalism ${ }^{15}$ to describe beam propagation when transverse beam quality differs from the ideal transform limit:

$$
w^{2}(z)=w_{0}^{2}\left(1+\left(M^{2} \frac{\lambda\left(z-z_{0}\right)}{\pi w_{0}^{2}}\right)^{2}\right) .
$$

Imperfect beam quality can be treated as an increase in the minimum beam waist-spatial frequency bandwidth product

$$
w_{0} \sigma_{s 0}=\frac{M^{2}}{2 \pi}
$$

where the beam waist $w_{0}$ and spatial frequency bandwidth $\sigma_{s 0}$ are defined as variances of the beam intensity distribution in configuration and spatial frequency space, respectively. In our analysis we consider two ideal beam profiles: Gaussian, a common beam profile obtainable from laser resonators, and top-hat, favored for nonlinear conversion processes due to reduced spatial variation of gain and reduced Poynting vector walkoff effects. While most real beams used in optical parametric amplification have beam profiles intermediate between the two ideal cases, this analysis still gives a quantitative assessment of the expected conversion from real beams.

Perfect Gaussian beam intensity distribution can be written in terms of the electric field amplitude:

$$
E(r)=E_{0} \exp \left(-\frac{r^{2}}{w^{2}}\right)
$$

The corresponding distribution in spatial frequencies is obtained by taking the Fourier transform of Eq. (10):

$$
|E(s)|=\pi E_{0} w^{2} \exp \left(-\pi^{2} s^{2} w^{2}\right),
$$

or, for a beam that is not diffraction limited,

$$
|E(s)|=\frac{\pi E_{0} w^{2}}{M^{4}} \exp \left(-\frac{\pi^{2} s^{2} w^{2}}{M^{4}}\right)
$$

We choose a uniphase distribution for the pump: $E(s)$ $=|E(s)|$. This arbitrary choice is justified by the phase insensitivity of the difference frequency generation. For every pair of angular components of the signal and pump, an idler is generated with the phase given by Eq. (4). Therefore, any initial phase difference among the angular components is projected to the idler as idler phase content.

Spatial frequency spectrum of the ideal top-hat beam can be calculated by taking the Hankel transform of the nearfield electric field distribution:

$$
E(s)=E_{0} w^{2} \frac{J_{1}(2 s w)}{s w},
$$

where $J_{1}$ denotes the first order Bessel function. For a tophat beam with a beam quality parameter $M^{2}$, we define the spatial frequency spectrum in analogy to the Gaussian case as

$$
E(s)=\frac{E_{0} w^{2}}{M^{4}} \frac{J_{1}\left(2 \pi s w / M^{2}\right)}{s w / M^{2}} .
$$

The minimum value of the angular divergence $\alpha$ corresponds to $M^{2}=1$.

In Fig. 1 we show normalized intensity distributions in divergence angles for a Gaussian TEMOO and a top-hat radially symmetric beam of equal radial intensity variance. We note that the spatial frequency spectrum for a top-hat beam is broader than for the Gaussian beam for the same radial intensity variance. While the spatial frequency variance cannot be evaluated for a top-hat beam, ${ }^{15}$ the calculated distribution for the top-hat beam nevertheless allows quantitative analysis in OPAs. An OPA acts as a spatial filter on the spatial 


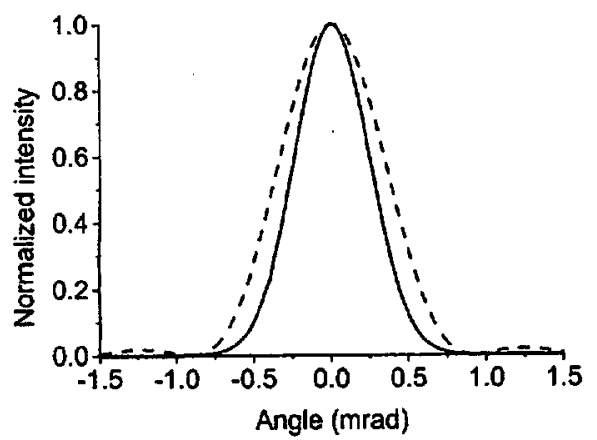

FIG. 1. Normalized intensity distributions in divergence angles. The solid curve represents a Gaussian and the dashed curve represents a top-hat beam, $w_{0}=0.5 \mathrm{~mm}, \lambda=1054 \mathrm{~nm}$.

frequency distributions Eqs. (12) and (14) due to its finite angular phase matching bandwidth. This limits the spatial frequency range that needs to be evaluated. In our test cases the relevant spatial frequencies are limited to $3 \sigma_{s 0}$ for Gaussian beams, and to the secondary zero of the intensity distribution in spatial frequencies for top-hat beams.

When the beam is not propagating along one of the crystal axes, the change of the index of refraction is more sensitive to angular deviation in the principal plane of the crystal (critical phase matching). In Fig. 2 we calculate small signal gain for a $1054 \mathrm{~nm}$ signal in $15 \mathrm{~mm}$ of beta-barium borate (BBO) pumped by a $532 \mathrm{~nm}$ pump. The signal and the pump are collinear plane waves. We show the dependence of gain on angular detuning of the pump beam, in the principal plane and perpendicular to the principal plane of the crystal. We observe that detuning in the principal plane is the dominant contribution to total angular detuning. Our general twodimensional analysis for uniaxial crystals can therefore be reduced to a computationally less demanding onedimensional case, where only detuning in the principal plane is considered. In biaxial crystals, critical phase matching is generally achieved in one of the principal planes of the crystal. In that case we can apply the same analysis to biaxial crystals, taking advantage of the large difference in angular sensitivity in the principal plane and perpendicular to the principal plane. We calculate the one-dimensional field distribution as

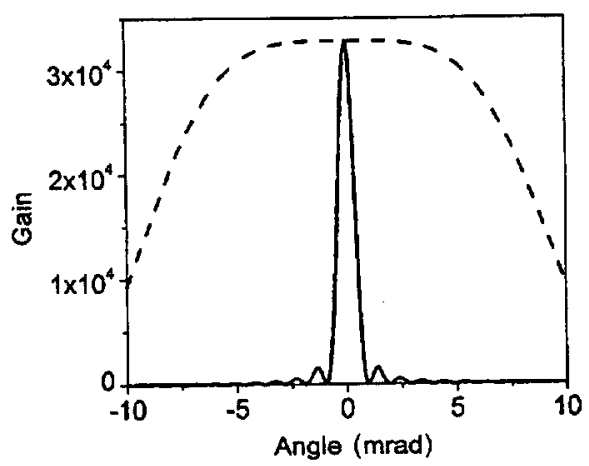

FIG. 2. OPA gain in $15 \mathrm{~mm}$ of $\mathrm{BBO}$ as a function of angular detuning, $\lambda_{s}$ $=1054 \mathrm{~nm}, \lambda_{p}=532 \mathrm{~nm}$, and $I_{p}=500 \mathrm{MW} / \mathrm{cm}^{2}$. The solid curve represents the detuning in the sensitive direction, and the dashed curve represents the detuning in the insensitive direction. The phase-matching angle $\theta=22.8^{\circ}$.

$$
\left|E_{1}\left(s_{x}\right)\right|^{2}=C \int_{-\infty}^{\infty}\left|E\left(\sqrt{s_{x}^{2}+s_{y}^{2}}\right)\right|^{2} d s_{y}
$$

where $s_{x}$ and $s_{y}$ are the spatial frequencies in two principal directions, $E_{1}\left(s_{x}\right)$ is the one-dimensional field distribution, $E(s)$ is the radial field distribution in spatial frequencies, and $C$ is the normalization constant, such that

$$
\int_{-\infty}^{\infty}\left|E_{1}\left(s_{x}\right)\right|^{2} d s_{x}=\int_{0}^{\infty}|E(s)|^{2} 2 \pi s d s
$$

A notable exception to this simplified treatment is the analysis of noncritically phase-matched processes, where beam propagation is achieved along one of the principal axes. In that case, angular sensitivity has similar magnitude in both directions, requiring a full two-dimensional analysis. A common example for a noncritically phase-matched crystal is lithium triborate (LBO), with its sensitivity curve plotted in Fig. 13 of the Appendix.

In this study we focus on dephasing due to angular deviation from the ideal phase matching in optical parametric amplification. For this model we assume a single frequency pump and signal and negligible group velocity dispersion. Without parametric interaction, we assume the transverse pump beam intensity distribution constant along the length of the crystal $z$. We evaluate the parametric interaction at an arbitrary transverse point $(x, y)$ on the crystal. We perform our calculation from $\left(x, y, z_{i}\right)$ to $\left(x, y, z_{f}\right)$, where $z_{i}$ is at the input face and $z_{f}$ is at the exit face of the crystal. Aside from depletion effects, the initial angular distribution of the pump chosen at $z_{i}$ is maintained throughout the length of the crystal. We use the spatial frequency distribution of the electric field as the angular distribution for the plane wave decomposition at the input face of the crystal. We are neglecting the intensity variations, as we are mostly interested in nanosecond OPAs, with relatively weak focusing and long depth of focus compared to the OPA length. Also, the Guoy phase shift that occurs on relatively short scale when tightly focused beams are used is not relevant to our calculation with weakly focused beams and short crystals.

We represent the electric field for the incident signal and pump as a sum of plane waves with angular distribution identical to the one-dimensional electric field distribution in angles Eq. (15):

$$
\mathbf{E}_{l}=\sum_{i=1}^{N} A_{l i} \mathbf{e}_{\mathbf{l i}} \exp \left[-i\left(\omega_{l} t-\mathbf{k}_{l i} \mathbf{r}\right)\right], \quad l=1,3,
$$

where $\mathbf{e}_{\mathbf{l i}}$ represents the unit vector perpendicular to the propagation direction of the corresponding plane wave, and $A_{l i}$ represents the amplitude of an individual plane wave, normalized to the intensity $l_{l}$ of the corresponding beam:

$$
\sum_{i=1}^{N} A_{l i}^{2}=\frac{\mu_{0}}{\epsilon_{0}} \frac{I_{l}}{2 n_{l}}, \quad l=1,3 .
$$

The limited width of angles about the phase matching angle for the signal and pump is uniformly divided into $N$ angular components. We use the finite angular ranges of signal and pump as described above. We note that an approximation can be made: $\mathbf{k}_{1 \mathrm{i}} \mathbf{r}=k_{l i} z$, as a consequence of paraxial beam 
propagation. We can now solve the coupled differential equations that govern the difference frequency generation:

$$
\begin{aligned}
& \frac{d A_{1 i}}{d z}=i \frac{2 \omega_{1} d_{\mathrm{eff}}}{n_{1} c} \sum_{j=1}^{N} A_{2 i j}^{*} A_{3 j} \exp \left(i \Delta k_{i j} z\right), \quad i=1, \ldots N, \\
& \frac{d A_{2 i j}}{d z}=i \frac{2 \omega_{2} d_{\mathrm{eff}}}{n_{2} c} A_{1 i} A_{3 j}^{*} \exp \left(i \Delta k_{i j} z\right), \quad i=1, \ldots N, \\
& j=1, \ldots, N, \\
& \frac{d A_{3 j}}{d z}=i \frac{2 \omega_{3} d_{\mathrm{eff}}}{n_{3} c} \sum_{j=1}^{N} A_{1 i} A_{2 j} \exp \left(-i \Delta k_{i j} z\right), \\
& \quad j=1, \ldots N,
\end{aligned}
$$

where $k_{i j}$ is the wave vector mismatch among the signal angular component $A_{1 i}$, idler angular component $A_{2 i j}$, and pump angular component $A_{3 j}$. The wave vector mismatch $k_{i j}$ is given by Eq. (A8) for uniaxial crystals, and by Eq. (A15) for biaxial crystals. Note that an idler angular component $A_{2 i j}$ is assigned to each combination of the angular components of the signal $A_{1 i}$ and pump $A_{3 j}$. The direction of the generated idler angular component $A_{2 i j}$ is defined by the nonlinear Snell's law for the incident signal and pump angular components $A_{1 i}$ and $A_{3 j}$, respectively. This direction is explained in more detail in the Appendix.

We solve the system of coupled differential equations by the fourth order Runge-Kutta method for numerical integration. The required number of the integration steps and the transverse mesh size are determined by testing the resultant convergence with increasing grid finesse. Our typical calculations were carried out with $N=50$ in the spatial frequency domain and 1000 steps in the numerical integration per $\mathrm{cm}$ of crystal length. Our analysis tracks the electric field amplitude and phase throughout the interaction region, followed by the calculation of total beam intensity and gain.

\section{RESULTS OF MODEL CALCULATIONS}

We now proceed to calculate the influence of the angular divergence on small signal gain in an OPA. In order to quantify the beam divergence in our calculations, we introduce the pump beam divergence parameter $\alpha$ :

$$
\alpha=\frac{M^{2} \lambda}{w} .
$$

The divergence parameter takes into account beam size, wavelength, and beam quality, and is proportional to the farfield diffracted angle. We choose the simulation parameters: $I_{p}=500 \mathrm{MW} / \mathrm{cm}^{2}, I_{\text {seed }}=10^{-3} \mathrm{~W} / \mathrm{cm}^{2}, \lambda_{p}=532 \mathrm{~nm}$, and $\lambda_{s}$ $=1054 \mathrm{~nm}$. The difference frequency mixing process used is $1054 \mathrm{~nm}(o)+1074 \mathrm{~nm}(o)=532 \mathrm{~nm}(e)$. The minimum radial intensity variance-spatial frequency bandwidth product is assumed for the incident seed beam. This corresponds to the experimentally relevant case of amplification of a high quality seed beam using an energetic pump beam of less than ideal beam quality.

Figure 3(a) shows the small signal gain in $\mathrm{BBO}$ as a function of interaction length in the crystal, for several divergence parameters $\alpha$ with Gaussian beams. Indicated in
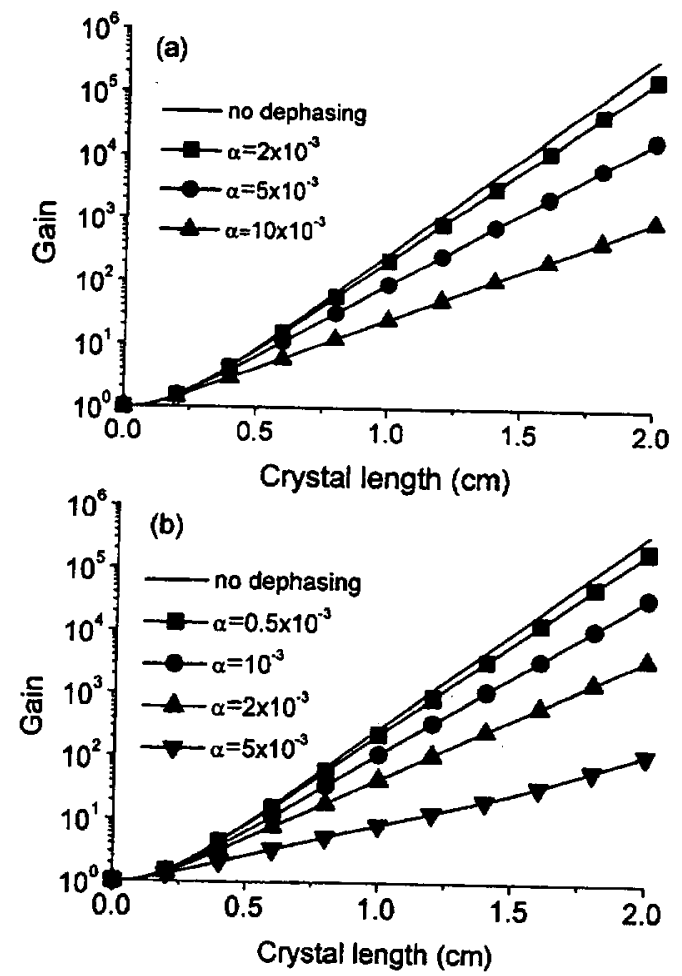

FIG. 3. Small signal gain in BBO for different divergence parameters for: (a) Gaussian beams and (b) top-hat beams. Simulation parameters: $\lambda_{s}$ $=1054 \mathrm{~nm}, \lambda_{p}=532 \mathrm{~nm}$, and $I_{p}=500 \mathrm{MW} / \mathrm{cm}^{2}$.

Fig. 3(a) is the result of our model calculation when zero dephasing $(\Delta k=0)$ is assumed. It is in agreement with a simple calculation based on a single, perfectly phasematched plane wave. An identical calculation is performed with a top-hat beam, with results shown in Fig. 3(b). It is apparent that a reduction of gain occurs even for modest values of divergence parameter $\alpha$, consistent with the relatively narrow angular acceptance of $\mathrm{BBO}$. We also observe that the gain reduction is more severe for a top-hat beam than for a Gaussian beam with the same divergence parameter $\alpha$, resulting from the broader spatial frequency distribution for a top-hat beam compared to a Gaussian beam with the same radial intensity variance $w$. Identical behavior is observed in potassium dihydrogen phosphate (KDP). This crystal is also a uniaxial crystal, but it has a much lower nonlinearity than $\mathrm{BBO}$. KDP is available in large apertures and it is therefore attractive for high energy nonlinear applications. Results are shown in Fig. 4, with the same interaction parameters, except that longer crystal length was observed due to relatively small gain. Finally, we analyze LBO in a noncritically phasematched configuration. Noncritical phase matching in LBO for the type I process $1054 \mathrm{~nm}(o)+1074 \mathrm{~nm}(o)$ $=532 \mathrm{~nm}(e)$ can be achieved by heating the crystal to $\sim 150^{\circ} \mathrm{C}$. This configuration is characterized by large angular tolerance. The result of small signal gain calculations in noncritically phase-matched LBO is shown in Fig. 5. We note the same qualitative behavior for both noncritically phase-matched and critically phase-matched crystals. Noncritically phase-matched LBO exhibits 1 order of magnitude greater divergence tolerance than $\mathrm{BBO}$ and $\mathrm{KDP}$. 

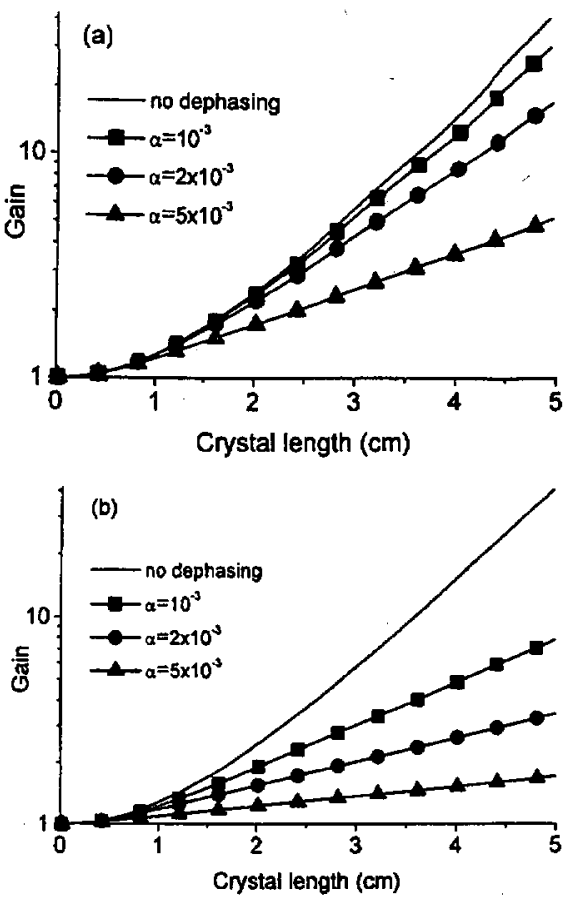

FIG. 4. Small signal gain in KDP for different divergence parameters for: (a) Gaussian beams and (b) top-hat beams. Simulation parameters: $\lambda_{s}$ $=1054 \mathrm{~nm}, \lambda_{p}=532 \mathrm{~nm}$, and $I_{p}=500 \mathrm{MW} / \mathrm{cm}^{2}$.

To evaluate maximum conversion efficiency in an OPA we need to analyze large signal gain configurations. We calculate gain when Power $_{\text {pump }}=5$ Power $_{\text {seed }}$. This leads to strong depletion of the pump wave and back conversion.
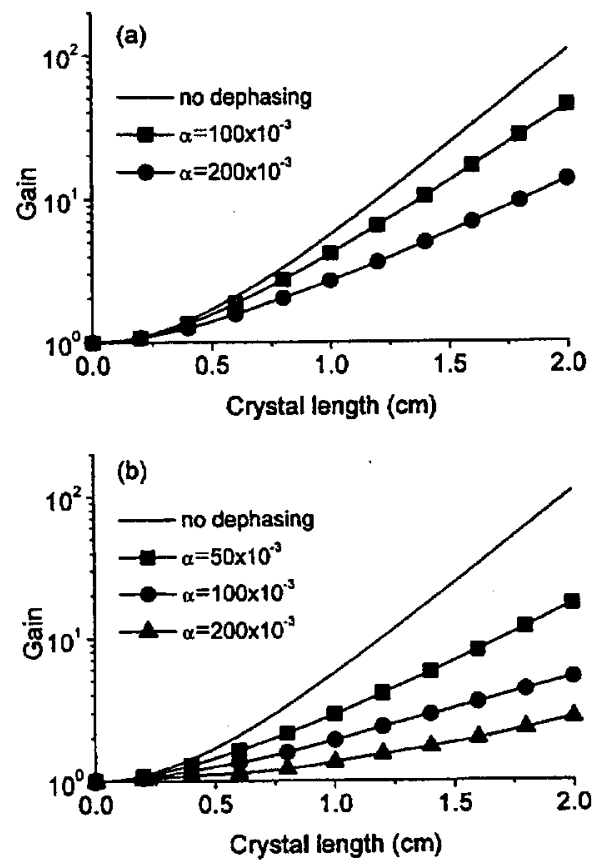

FIG. 5. Small signal gain in noncritically phase-matched LBO for different divergence parameters for: (a) Gaussian beams and (b) top-hat beams. Simulation parameters: $\lambda_{s}=1054 \mathrm{~nm}, \lambda_{p}=532 \mathrm{~nm}$, and $I_{p}=500 \mathrm{MW} / \mathrm{cm}^{2}$. Note that the divergence parameter scale is an order of magnitude greater than for $\mathrm{BBO}$ and KDP.
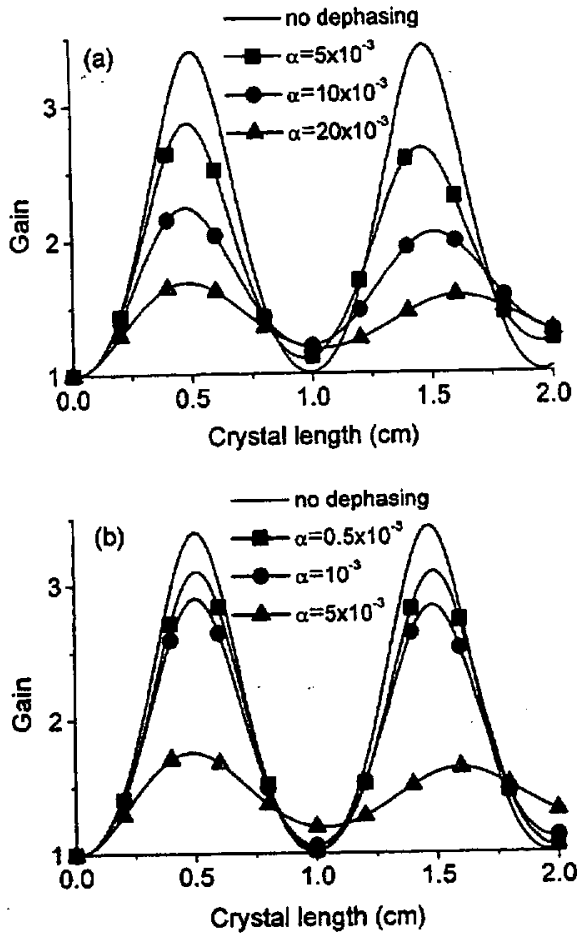

FIG. 6. Large signal gain in BBO for different divergence parameters for: (a) Gaussian beams and (b) top-hat beams. Simulation parameters: $\lambda_{s}$ $=1054 \mathrm{~nm}, \lambda_{p}=532 \mathrm{~nm}, I_{p}=500 \mathrm{MW} / \mathrm{cm}^{2}, 50 \mathrm{~mJ}$ pump, and $10 \mathrm{~mJ}$ seed.

Results for BBO, KDP, and LBO are shown in Figs. 6-8 for Gaussian and top-hat beam profiles. Note an important difference between our results and the results of simple calculations in which a single plane wave with a single averaged
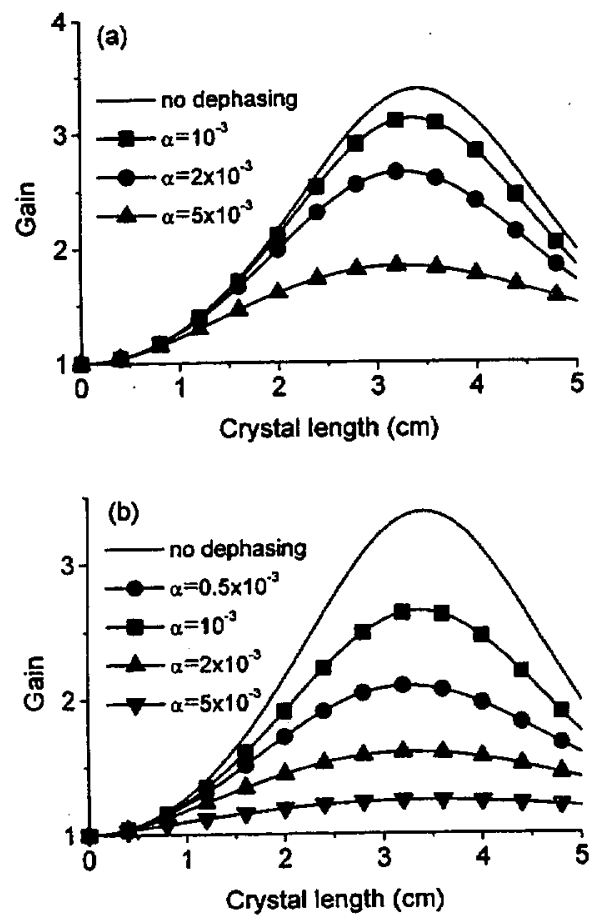

FIG. 7. Large signal gain in KDP for different divergence parameters for: (a) Gaussian beams and (b) top-hat beams. Simulation parameters: $\lambda_{s}$ $=1054 \mathrm{~nm}, \lambda_{p}=532 \mathrm{~nm}, I_{p}=500 \mathrm{MW} / \mathrm{cm}^{2}, 50 \mathrm{~mJ}$ pump, and $10 \mathrm{~mJ}$ seed. 

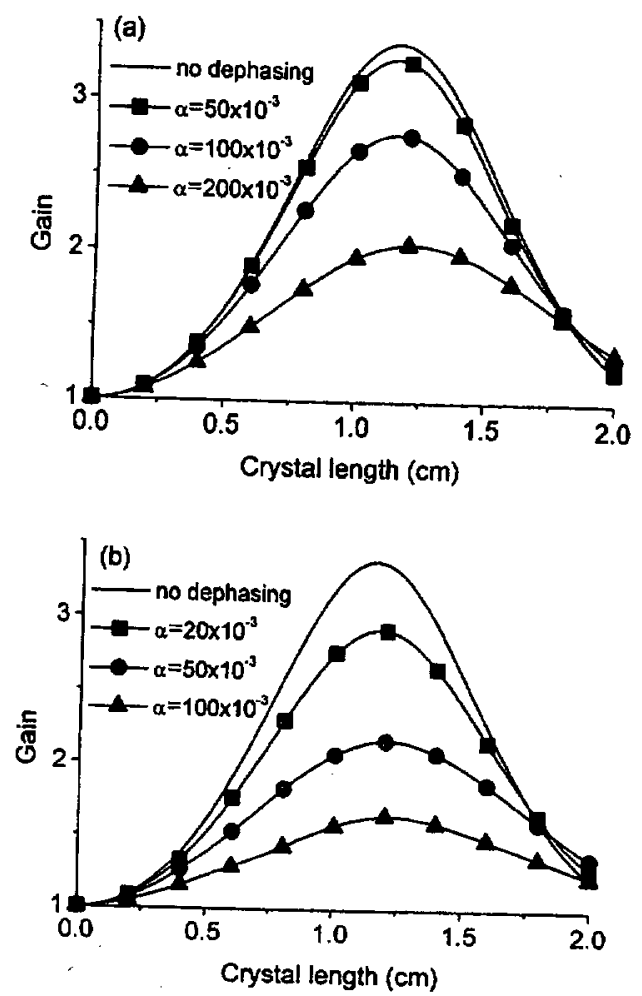

FIG. 8. Large signal gain in noncritically phase-matched LBO for different divergence parameters for: (a) Gaussian beams and (b) top-hat beams. Simulation parameters: $\lambda_{s}=1054 \mathrm{~nm}, \lambda_{p}=532 \mathrm{~nm}$, and $I_{p}=500 \mathrm{MW} / \mathrm{cm}^{2}$.

value of phase mismatch is considered. A plane wave analysis without angular distribution results in cycles of conversion and back conversion that occur with specific frequency and consistently attain the same maxima and minima. Our results show conversion behavior similar to the behavior of a damped oscillator, where successive oscillations become less pronounced. This is the result of a large number of plane waves interacting with different wave vector mismatch and subsequent different conversion and back-conversion rates for plane wave components across the spatial frequency spectrum. The damped oscillatory conversion behavior is consistent with the previous calculation ${ }^{14}$ for second harmonic generation.

Top-hat beams are preferred in optical parametric processes due to uniform conversion. However, for the same radial intensity variance, we find that Gaussian beams are less sensitive to angular divergence than top-hat beams in optical parametric amplification. When we compare the spatial frequency spectrum of Gaussian and top-hat beams, we find that the Gaussian's spectrum has greater energy content in its central lobe than the top-hat's. This can be shown by considering a spatial frequency window defined by the first zero of the Bessel function for the spatial frequency spectrum of the top-hat beam. While the top-hat beam has only $84 \%$, the Gaussian beam with the same radial intensity variance has $99.9 \%$ of its energy concentrated in the same enclosed area. We argue that this difference is responsible for the observed variation in the angular sensitivity. Common nonlinear crystals in critically phase-matched configurations typically exhibit narrow angular tolerances. Frequently, only the central lobe of the spatial frequency spectrum of a top-hat beam is included in the angular bandwidth for optical parametric amplification. Since the rate of difference frequency generation has a strong nonlinear dependence on beam intensity, a significant drop in gain is expected with reduced effective beam intensity: In an alternative view, the high spatial frequencies of the pump experience rapid cycles of conversion and back conversion, not contributing significantly to the overall conversion.

\section{HIGH CONVERSION CRITERIA}

Eimerl ${ }^{16}$ has previously studied second harmonic generation and concluded that the conversion efficiency in second harmonic generation is determined exclusively by the beam peak power and beam quality in critically phasematched crystals. While increasing the beam intensity by telescoping the beam decreases the required crystal length, there is no impact on the overall conversion efficiency. This result can be extended to optical parametric amplification. For this case, the simplified expression for small signal gain is

$$
G=\frac{1}{4} \exp \left(2 g_{0} L\right) \operatorname{sinc}^{2}\left(\frac{\Delta k L}{2}\right),
$$

where $g_{0}=K \sqrt{I_{p}}$ is the gain coefficient, $I_{p}$ is the intensity of the pump, $L$ is the crystal length, and $K$ $=4 \pi d_{\text {eff }} / \sqrt{2 \varepsilon_{0} n_{p} n_{s} n_{i} c \lambda_{s} \lambda_{p}}$. In critical phase matching, the wave vector mismatch $\Delta k$ can be approximated by the linear function of divergence angle for the small angle:

$$
\Delta k L=\beta_{\theta} \Delta \theta L=\frac{\beta_{\theta}}{4} \frac{M^{2} \lambda}{\pi w} L,
$$

where $\beta_{\theta}=d(\Delta k) / d \theta$ is the angular sensitivity for a critically phase-matched nonlinear crystal. We can now rewrite the expression for small signal gain for radially symmetric beams as

$$
G=\frac{1}{4} \exp \left(\frac{2 K}{\sqrt{\pi}} \sqrt{P_{p}} \frac{L}{w}\right) \operatorname{sinc}^{2}\left(\frac{\beta_{\theta}}{8} \frac{M^{2} \lambda}{\pi} \frac{L}{w}\right),
$$

where $P_{p}$ is the peak power of the pump beam. We notice that while telescoping the beam changes beam radius $w$, and the ratio $L / w$ remains invariant for the same gain. The problem of obtaining maximum gain reduces to selecting the proper ratio of crystal length $L$ to beam radius $w$ for a particular pump peak power.

Dephasing can be reduced in uniaxial crystals by using elliptical beams, elongated in the principal plane of the crystal. Elliptical beams take advantage of the anisotropy in angular sensitivity, reducing dephasing while enabling the same pump intensity. Compared to a radially symmetric beam of the same intensity, dephasing is reduced by a factor of $\sqrt{A}$, where $A$ is the beam aspect ratio. As a consequence, the beam quality parameter $M^{2}$ can be $\sqrt{A}$ times greater compared to a radially symmetric beam. The limit of usability of this scheme in uniaxial crystals is determined by dephasing due to divergence in the insensitive direction. This dephasing in the insensitive direction starts to compete with dephasing due to divergence in the sensitive direction at large aspect 
ratios. We calculate this aspect ratio in $\mathrm{BBO}$ to be $\sim 20$. Another advantage of using elliptical beams in uniaxial crystals is the reduction of the impact of walkoff. Walkoff occurs for the extraordinary polarized beam and is directed in the crystal sensitive direction. Using elliptical beams increases the overlap of the ordinary and extraordinary beams. This results in better conversion efficiency, particularly when intersecting beams are small and interaction lengths are long.

In noncritical phase matching, phase mismatch can be approximated by a quadratic dependence on the divergence angle

$$
\Delta k L=\gamma_{\theta} \Delta \theta^{2} L=\frac{\gamma_{\theta}}{16} \frac{M^{4} \lambda^{2}}{\pi^{2} w} \frac{L}{w},
$$

where $\gamma_{\theta}=d^{2}(\Delta k) / d \theta^{2}$ is the angular sensitivity for noncritical phase matching. Increasing the beam size can increase the conversion efficiency in noncritically phasematched crystals. Contrary to intuition, weaker beam focusing enables higher conversion in noncritically phasematched crystals. The obvious limit of practicality of this result is the availability of large crystals of sufficient quality. Otherwise, the limit is set by the dephasing sources that are directly proportional to crystal length, such as spectral and temperature bandwidths.

In a simplified analysis, we now derive the criterion for beam quality that allows high conversion efficiency in OPAs. By Eq. (21), if we neglect dephasing, the desired small signal gain can be obtained for a given value of pump peak power by selecting the appropriate ratio of crystal length and beam radius

$$
L / w=\frac{\sqrt{\pi}}{2 K \sqrt{P}} \ln (4 G) .
$$

The dephasing term for the selected ratio $L / w$ is

$$
\begin{aligned}
& \operatorname{sinc}^{2}\left(\frac{\Delta k L}{2}\right)=\operatorname{sinc}^{2}\left(\frac{\beta_{\theta}}{16 \sqrt{\pi}} \frac{M^{2} \lambda \ln (4 G)}{K \sqrt{P}}\right), \\
& \operatorname{sinc}^{2}\left(\frac{\Delta k L}{2}\right)=\operatorname{sinc}^{2}\left(\frac{\gamma_{\theta}}{32 \pi^{3 / 2}} \frac{M^{4} \lambda^{2} \ln (4 G)}{K w \sqrt{P}}\right),
\end{aligned}
$$

for critically and noncritically phase-matched crystals, respectively. If we define the requirement for high conversion efficiency as $\operatorname{sinc}^{2}(\Delta k L / 2)>0.5$, or $\Delta k L / 2<1.39$, we now have a criterion for required beam quality for lasers pumping OPAs:

$$
\begin{aligned}
& M^{2}<1.39 \times 16 \sqrt{\pi} \frac{K \sqrt{P}}{\beta_{\theta} \lambda \ln (4 G)}, \\
& M^{4}<1.39 \times 32 \pi^{3 / 2} \frac{K w \sqrt{P}}{\gamma_{\theta} \lambda^{2} \ln (4 G)},
\end{aligned}
$$

for critically and noncritically phase-matched crystals, respectively. Note that for critical phase-matched operation the required beam quality $M^{2}$ does not depend on the beam radius. For noncritical phase matching, the required $M^{2}$ scales as the square root of the beam radius.

An important consideration in OPA design concerns a simple difference between harmonic generation and seeded
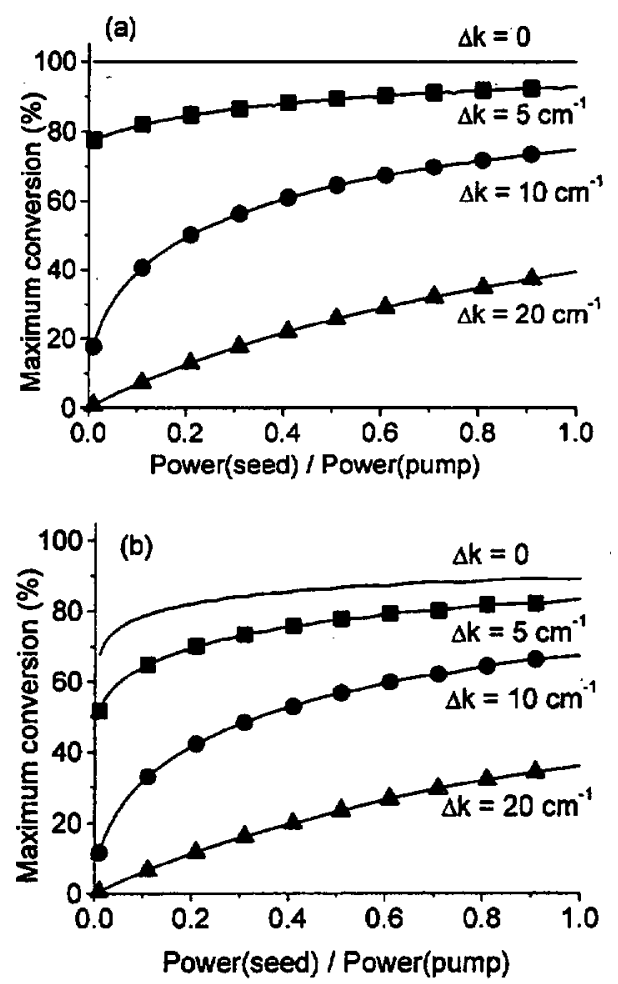

FIG. 9. Maximum conversion in BBO as a function of the ratio of seed and pump input for: (a) no walkoff included and (b) walkoff included. Simulation parameters: $\lambda_{s}=1054 \mathrm{~nm}, \lambda_{p}=532 \mathrm{~nm}$, and $I_{p}=1 \mathrm{GW} / \mathrm{cm}^{2}$.

OPAs. While second harmonic generators build up without an incident harmonic beam, seeded OPAs have radically different boundary conditions, where seed beam intensity is typically many orders of magnitude greater than the zeropoint field fluctuation. Seed power influences the overall conversion efficiency in the OPA. We performed a simple calculation to determine maximum conversion in an OPA as a function of seed power. Our results are shown in Fig. 9(a), without walkoff, and in Fig. 9(b), with walkoff. We plot the maximum conversion of the pump beam to signal and idler as a function of the ratio of seed and pump peak power, for different values of wave vector mismatch $\Delta k$. A single plane wave with wave vector mismatch $\Delta k$ is assumed. The evaluated mixing process is $1054 \mathrm{~nm}(o)+1074 \mathrm{~nm}(o)$ $=532 \mathrm{~nm}(e)$, with pump waist size $2 \mathrm{~mm}$ (top hat), and pump intensity of $1 \mathrm{GW} / \mathrm{cm}^{2}$. Maximum conversion is found by varying the crystal length with constant input intensity. With $\Delta k=0$ and in the absence of walkoff, maximum conversion is $100 \%$ and it is independent of the seed power. As the wave vector mismatch increases, we note that higher conversion efficiency is possible when the input seed intensity increases. When we include walkoff, even zero wave vector mismatch leads to a maximum conversion of $\sim 85 \%$ when Power $_{\text {seed }} /$ Power $_{\text {pump }}=1$. The efficiency can be improved by

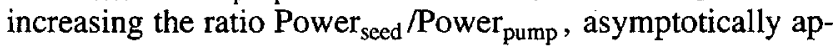
proaching $100 \%$ when Power seed $/$ Power $_{\text {pump }} \rightarrow \infty$.

\section{CONCLUSION}

We explored the angular effects in optical parametric amplification using a numerical model of difference fre- 
quency generation. Our model is based on plane wave decomposition. The model can be used to isolate and evaluate the influence of spatial frequency content of real laser beams on the performance of devices based on optical parametric amplification. We found that top-hat beams exhibit greater sensitivity to angular dephasing than Gaussian beams with the same radial intensity variance. This result is attributed to the more broadly distributed angular spectrum of the top-hat beam.

We derived the criteria for required beam quality in critically and noncritically phase-matched optical parametric amplification. It was shown that the required beam quality is invariant with respect to the beam diameter in critical phase matching. In noncritical phase matching, the beam quality requirements are more relaxed as the beam radius increases. We also determined practical tolerances on beam quality for Gaussian and top-hat beams in several commonly used nonlinear crystals.

We also studied the conversion efficiency sensitivities with respect to the beam ellipticity and seed power. Beam quality requirements can be relaxed by using elliptical beams in critically phase-matched crystals by taking advantage of the large anisotropy in the angular sensitivity. The maximum conversion in an OPA is increased for greater incident seed power, for all values of wave vector mismatch.

At an arbitrary point, our angular decomposition model for optical parametric amplification describes the input beams more realistically than single plane wave models. A complete description would include a variable intensity profile in the spatial and temporal domains. Such an inclusion would necessitate considerably more computational power than our simple model. As a stand-alone calculation, the angular decomposition model nevertheless provides insight into angular effects in OPAs and in estimating their relative magnitude.

\section{ACKNOWLEDGMENTS}

The authors acknowledge useful discussions with M. D. Feit, A. Rubenchik, and I. P. Mercer. This work was performed under the auspices of the U.S. Department of Energy by the University of California, Lawrence Livermore National Laboratory under Contract No. W-7405-Eng-48.

\section{APPENDIX: DETERMINATION OF WAVE VECTOR MISMATCH IN UNIAXIAL AND BIAXIAL CRYSTALS}

We wish to determine the wave vector mismatch that is a result of the angular deviation from the ideal phase matching in difference frequency generation. We first consider only the angular deviation in the principal plane of the crystal for a uniaxial critically phase-matched crystal. This deviation is commonly referred to as a deviation in the sensitive direction. The angular deviation is radially symmetric in both the sensitive and the insensitive direction for radially symmetric beams. Since the dependence of the wave vector mismatch on the deviation in the insensitive direction is weak (second order), the wave vector mismatch in the sensitive direction is the dominant factor affecting the phase matching in our study.

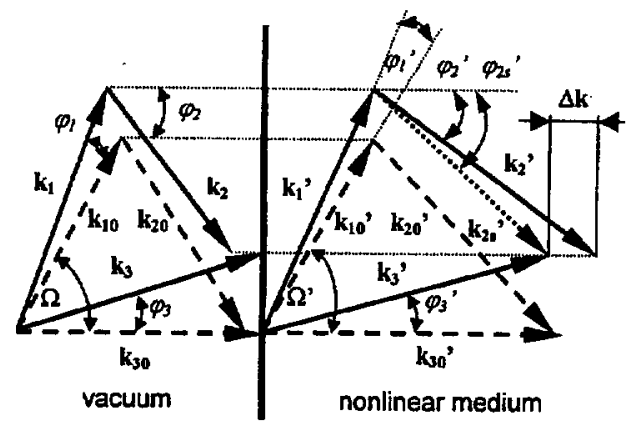

FIG. 10. Signal, idler, and pump wave vector interaction on a boundary between vacuum and a nonlinear medium. The wave vector mismatch arises from the difference between the transmitted idler $\mathbf{k}_{\mathbf{2}}^{\prime}$ and the driven idler $\mathbf{k}_{\mathbf{2}}^{\prime}$.

In Fig. 10 we present the interacting waves on the boundary between the vacuum and a nonlinear medium. A pump wave with wave vector $\mathbf{k}_{\mathbf{3 0}}$ and a signal wave with wave vector $\mathbf{k}_{10}$ are incident on the boundary. We choose $\mathbf{k}_{\mathbf{3 0}}$ perpendicular to the boundary, and the signal with an external noncollinear angle $\Omega$. A perfect phase matching is achieved in the crystal for the combination of transmitted signal $\mathbf{k}_{10}^{\prime}$ and transmitted pump $\mathbf{k}_{\mathbf{3 0}}^{\prime}$, with the internal noncollinear angle $\Omega^{\prime}$. An incident idler with wave vector $\mathbf{k}_{\mathbf{2 0}}$ conserves the nonlinear Snell's law ${ }^{17}$ on the boundary and is transmitted as $\mathbf{k}_{\mathbf{2 0}}^{\prime}$. The amplitude of the incident idler is zero, which is consistent with the continuity of the idler wave on the boundary. We do not include the waves reflected on the boundary, as they do not have relevance to our discussion of optical parametric amplification in a nonlinear medium.

We now consider the wave vectors of the incident signal $\mathbf{k}_{1}$ and pump $\mathbf{k}_{\mathbf{3}}$, with the associated transmitted signal wave vector $\mathbf{k}_{\mathbf{1}}^{\prime}$ and transmitted pump wave vector $\mathbf{k}_{\mathbf{3}}^{\prime}$. The wave vectors $\mathbf{k}_{\mathbf{1}}$ and $\mathbf{k}_{\mathbf{3}}$ exhibit a small angular deviation from the ideally phase-matched waves by the external angles $\varphi_{1}$ and $\varphi_{3}$, respectively. The refracted field angles and wave vectors $\left(\varphi_{1}^{\prime}, \varphi_{3}^{\prime}, \mathbf{k}_{1}^{\prime}\right.$, and $\left.\mathbf{k}_{3}^{\prime}\right)$ are calculated from the incident angles and wave vectors:

$$
\begin{aligned}
& \sin \left(\Omega^{\prime}+\varphi_{1}^{\prime}\right)=\frac{1}{n_{1}} \sin \left(\Omega+\varphi_{1}\right), \\
& \sin \varphi_{3}^{\prime}=\frac{1}{n_{3}} \sin \varphi_{3}, \\
& k_{1}^{\prime}=k_{1} n_{1}, \quad k_{2}^{\prime}=k_{2} n_{2}, \quad k_{3}^{\prime}=k_{3} n_{3},
\end{aligned}
$$

where $n_{1}, n_{2}$, and $n_{3}$ are the refractive indices of the transmitted signal, idler, and pump. The solution for the idler wave in a nonlinear medium consists of a homogeneous and a particular solution. ${ }^{17}$ The driven idler wave $\mathbf{k}_{2 \mathrm{~s}}^{\prime}$ corresponds to the homogeneous solution, while the transmitted idler $\mathbf{k}_{2}^{\prime}$ corresponds to the particular solution. The transmitted idler $\mathbf{k}_{\mathbf{2}}^{\prime}$ is generated in the interaction. The incident idler angle $\varphi_{2}$, the driven idler angle $\varphi_{2 s}^{\prime}$, and the transmitted idler angle $\varphi_{2}^{\prime}$ can be extracted from the boundary conditions:

$$
\begin{aligned}
& k_{2} \sin \varphi_{2}=k_{1} \sin \left(\Omega+\varphi_{1}\right)-k_{3} \sin \varphi_{3}, \\
& k_{2 s}^{\prime} \cos \varphi_{2 s}^{\prime}=k_{3}^{\prime} \cos \varphi_{3}^{\prime}-k_{1}^{\prime} \cos \left(\Omega^{\prime}+\varphi_{1}^{\prime}\right),
\end{aligned}
$$


4336

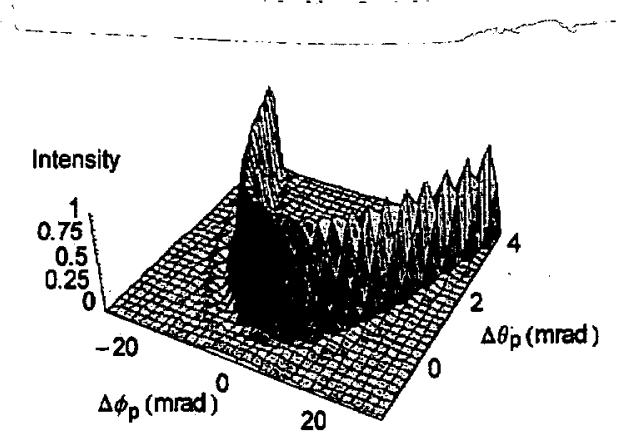

FIG. 11. Relative gain in $1 \mathrm{~cm}$ of $\mathrm{BBO}$ as a function of angular detuning in two angular directions.

$$
\sin \varphi_{2}^{\prime}=\frac{1}{n_{2}} \sin \varphi_{2} .
$$

Finally, the phase mismatch is given by the difference between the transmitted idler wave and the driven idler wave

$$
\Delta k=k_{2}^{\prime} \cos \varphi_{2}^{\prime}-k_{2 s}^{\prime} \cos \varphi_{2 s}^{\prime} .
$$

In the paraxial limit, the exact expressions Eqs. (A1)-(A7) give a simple equation for wave vector mismatch:

$$
\begin{aligned}
\Delta k= & n_{2} k_{2}\left(1-\frac{1}{2}\left(\frac{k_{1}\left(\Omega+\varphi_{1}\right)-k_{3} \varphi_{3}}{k_{2} n_{2}}\right)^{2}\right)-k_{3} n_{3} \\
& \times\left(1-\frac{1}{2}\left(\frac{\varphi_{3}}{n_{3}}\right)^{2}\right)+k_{1} n_{1}\left(1-\frac{1}{2}\left(\frac{\Omega+\varphi_{1}}{n_{1}}\right)^{2}\right) .
\end{aligned}
$$

The expression Eq. (A8) for the wave vector mismatch is more convenient for numerical evaluation. We find it sufficiently accurate for numerical modeling of wave vector mismatch between laser beams, where angular deviations are relatively small.

We now evaluate the wave vector mismatch in biaxial crystals. Three distinct crystallographic axes $(X, Y, Z)$ can be defined for biaxial crystals: The refractive indices for fast and slow waves are the solutions of the general Fresnel equation:

$$
\frac{\sin ^{2} \theta \cos ^{2} \phi}{1 / n^{2}-1 / n_{X}^{2}}+\frac{\sin ^{2} \theta \sin ^{2} \phi}{1 / n^{2}-1 / n_{Y}^{2}}+\frac{\cos ^{2} \theta}{1 / n^{2}-1 / n_{Z}^{2}}=0,
$$

where $\theta$ and $\phi$ are the usual polar angles, and $n_{X}, n_{Y}$, and $n_{Z}$ are the refractive indices of the waves propagating along the respective crystallographic axes. The index of refraction in the direction defined by the angles $(\theta, \phi)$ is $n$. We consider the departures from ideal phase matching by tilting the

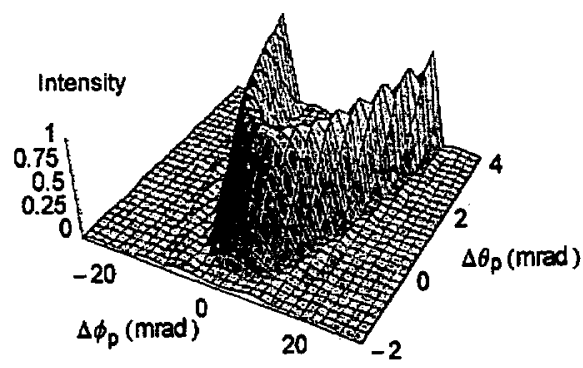

FIG. 12. Relative gain in $1 \mathrm{~cm}$ of KDP as a function of angular detuning in two angular directions.

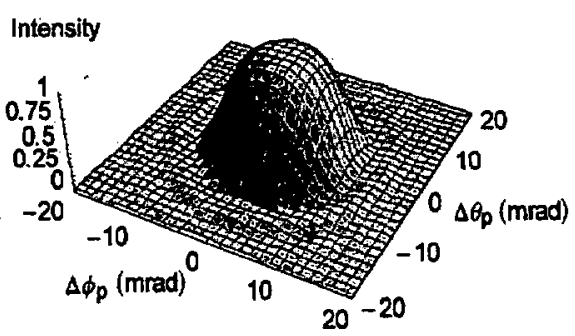

FIG. 13. Relative gain in $1 \mathrm{~cm}$ of noncritically phase-matched LBO as a function of angular detuning. A broad angular tolerance is the result of noncritical phase matching.

beams in both angular directions. In the paraxial limit, the transmitted signal and pump wave angles are

$$
\begin{array}{cc}
\varphi_{1}^{\prime}=\frac{\varphi_{1}}{n_{1}}, & \theta_{1}^{\prime}=\frac{\theta_{1}}{n_{1}}, \\
\varphi_{3}^{\prime}=\frac{\varphi_{3}}{n_{3}}, & \theta_{3}^{\prime}=\frac{\theta_{3}}{n_{3}} .
\end{array}
$$

The incident and the transmitted idler wave angles are

$$
\begin{aligned}
& \varphi_{2}=\frac{k_{3} \varphi_{3}-k_{1} \varphi_{1}}{k_{2}}, \quad \theta_{2}=\frac{k_{3} \theta_{3}-k_{1} \theta_{1}}{k_{2}}, \\
& \varphi_{2}^{\prime}=\frac{\varphi_{2}}{n_{2}}, \quad \theta_{2}^{\prime}=\frac{\theta_{2}}{n_{2}} .
\end{aligned}
$$

We perform a self-consistent calculation to obtain the transmitted angles from the incident angles. This is important because the refractive indices are the functions of transmitted angles. The driven idler wave is

$$
k_{2 s}^{\prime} \cos \varphi_{2 s}^{\prime} \cos \theta_{2 s}^{\prime}=k_{3}^{\prime} \cos \varphi_{3}^{\prime} \cos \theta_{3}^{\prime}-k_{1}^{\prime} \cos \varphi_{1}^{\prime} \cos \theta_{1}^{\prime} .
$$

The wave vector mismatch is

$$
\Delta k=k_{2}^{\prime} \cos \varphi_{2}^{\prime} \cos \theta_{2}^{\prime}-k_{2 s}^{\prime} \cos \varphi_{2 s}^{\prime} \cos \theta_{2 s}^{\prime} .
$$

In Figs. 11-13 we show the result of our calculation for critically phase-matched $\mathrm{BBO}$ and $\mathrm{KDP}$, and for noncritically phase-matched LBO. The mixing process is $1054 \mathrm{~nm}(o)+1074 \mathrm{~nm}(o)=532 \mathrm{~nm}(e)$. The curves represent relative small signal gain as a function of the departure from the ideal phase matching angle in two orthogonal angular directions. Note the existence of the curves of perfectly phase-matched points in critically phase-matched crystals. This enables angular multiplexing of the laser beams in optical parametric amplification.

${ }^{1}$ C. C. Wang and G. W. Racette, Appl. Phys. Lett. 6, 169 (1965).

${ }^{2}$ A. Shirakawa and T. Kobayashi, Appl. Phys. Lett. 72, 147 (1998).

${ }^{3}$ F. Huang and L. Huang, IEEE J. Quantum Electron. QE-30, 2601 (1994).

${ }^{4}$ S. Wu, V. A. Kapinus, and G. A. Blake, Opt. Commun. 159, 74 (1999).

${ }^{5}$ A. Dubietis, G. Jonusauskas, and A. Piskarskas, Opt. Commun. 88, 437 (1992).

${ }^{6}$ I. N. Ross, P. Matousek, M. Towrie, A. J. Langley, and J. L. Collier, Opt. Commun. 144, 125 (1997).

${ }^{7}$ P. Matousek, B. Rus, and I. N. Ross, IEEE J. Quantum Electron. QE-36, $158(2000)$

${ }^{8}$ S. K. Zhang, M. Fujita, H. Yoshida, R. Kodama, H. Fujita, M. Nakatsuka, Y. Izawa, and C. Yamanaka, Proc. SPIE 3886, 588 (1999). 
${ }^{9}$ J. A. Armstrong, N. Bloembergen, J. Ducuing, and P. S. Pershan, Phys. Rev. 127, 1918 (1962).

${ }^{10}$ R. A. Baumgartner and R. Byer, IEEE J. Quantum Electron. QE-15, 432 (1979).

${ }^{11}$ I. A. Begishev, A. A. Gulamov, E. A. Erofeev, E. A. Ibragimov, S. R. Kamalov, T. Usmanov, and A. D. Khadzhaev, Sov. J. Quantum Electron. 20, 1100 (1990).

${ }^{12}$ I. A. Begishev, A. A. Gulamov, E. A. Erofeev, E. A. Ibragimov, S. R.
Kamalov, T. Usmanov, and A. D. Khadzhaev, Sov. J. Quantum Electron. 20, 1104 (1990).

${ }^{13}$ G. D. Boyd and D. A. Kleinman, J. Appl. Phys. 39, 3597 (1968).

${ }^{14}$ S. K. Wong, G. Fournier, P. Mathieu, and P. Pace, J. Appl. Phys. 71, 1091 (1992).

${ }^{15}$ A. E. Siegman, Proc. SPIE 1224, 2 (1990).

${ }^{16}$ D. Eimerl, IEEE J. Quantum Electron. QE-23, 575 (1987).

${ }^{17}$ N. Bloembergen and P. S. Pershan, Phys. Rev. 128, 606 (1962). 


\title{
Manufacture of large-aperture diffractive optics and ultrathin refractive optics for high-power laser and space applications
}

\author{
J. A. Britten
}

\begin{abstract}
We have developed equipment and technology for fabricating submicron pitch, high-efficiency diffraction gratings over meter-scale apertures that are used for pulse compression in ultrafast systems around the world. We have also developed wet-etch figuring (WEF) to generate arbitrary continuous contours on ultrathin glass substrates in a closed loop process. The current and future states of these technologies will be discussed.
\end{abstract}

\section{LARGE-APERTURE DIFFRACTION GRATINGS}

LLNL has been fabricating large-aperture submicron pitch diffraction gratings for pulse compression since the mid 1990 's, when much of the facilitization and process development was done in support of LLNL's original Petawatt laser [1]. Presently, we are fabricating wet-etched low efficiency sampling gratings at $40 \mathrm{~cm}$ square aperture for LLNL's NIF laser [2], as well as supplying large-aperture, gold-overcoated high-efficiency gratings for a variety of internal and external users. Some of the unique capabilities existing at LLNL to do this work include rigorous codes for grating design, meniscus coating systems for precision photoresist application on flat substrates up to $1 \times 2 \mathrm{~m}$ wide, three laser interference lithography systems including one with collimating optics $1.1 \mathrm{~m}$ in diameter capable of printing $1-\mathrm{m}$ diameter submicron-pitch gratings with $\sim 10^{\text {th }}$ wave flatness in diffracted wavefront, vacuum coating systems for application of metal and dielectric layers at $1 \mathrm{~m}$ aperture, a reactive ion beam etcher capable of uniformly patterning optics at $\sim 60 \mathrm{~cm}$ aperture, and a repertoire of processing techniques for control and tailoring of grating profiles. A detailed description of capabilities can be found on our website [3].

There has been considerable activity recently in the construction of Petawatt-class lasers worldwide. We have provided compressor gratings in the last year to the Institute for Laser Engineering, University of Osaka, Japan, and to Rutherford Appleton Laboratory in the U.K. These optics, based on LLNL's original Petawatt technology, were $94 \mathrm{~cm}$ diameter gold-overcoated master gratings, $1480 \mathrm{l} / \mathrm{mm}$ and optimized for high efficiency at $1.053 \mu \mathrm{m}$ at nearLittrow mount. Figure 1 shows a full-aperture diffraction efficiency scan of one of the gratings produced.
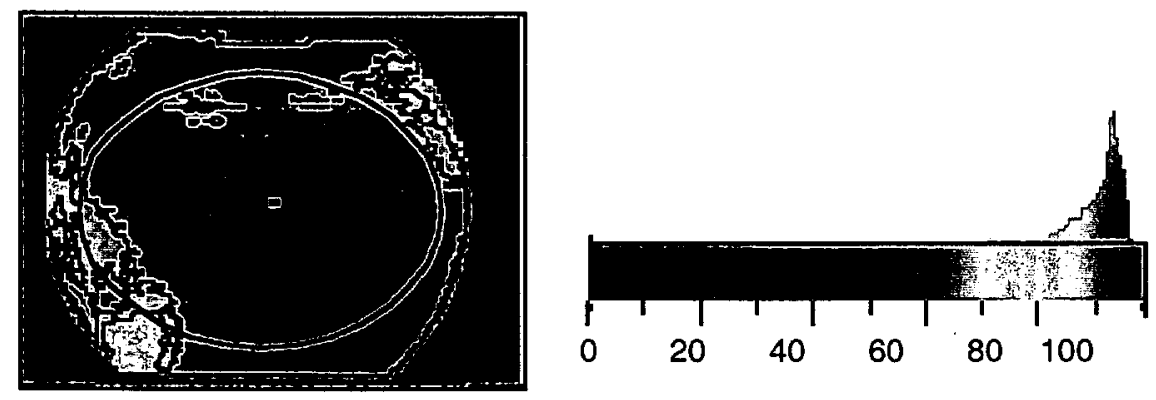

Fig. 1. -1 order \% diffraction efficiency (in reflection) of $94 \mathrm{~cm}$ diameter, $1480 \mathrm{l} / \mathrm{mm}$ grating measured at $1.064 \mu \mathrm{m}$, $54^{\circ}$ incidence angle. Efficiency $93.8 \%$ within beam footprint shown. 80 and $90 \%$ efficiency contours also shown.

We are also continuing the development of multilayer dielectric diffraction gratings $[4,5]$. We have recently fabricated a $355 \times 150 \mathrm{~mm}$ grating consisting of a 18-layer $\mathrm{Ta}_{2} \mathrm{O}_{5} / \mathrm{SiO}_{2}$ multilayer stack with an $1800 \mathrm{l} / \mathrm{mm}$ grating ion-beam etched into the top $\mathrm{SiO}_{2}$ layer, that exhibits $>99 \%$ diffraction efficiency at the use conditions of $1.030 \mu \mathrm{m}$, $64^{\circ}$ incidence angle (see Figure 2). This grating is being used in a very high average-power, $4 \mathrm{kHz}$ rep-rate shortpulse machining laser at LLNL. Use of this grating has increased energy throughput at the compressor by $50 \%$ compared with the best available gold-overcoated gratings. 

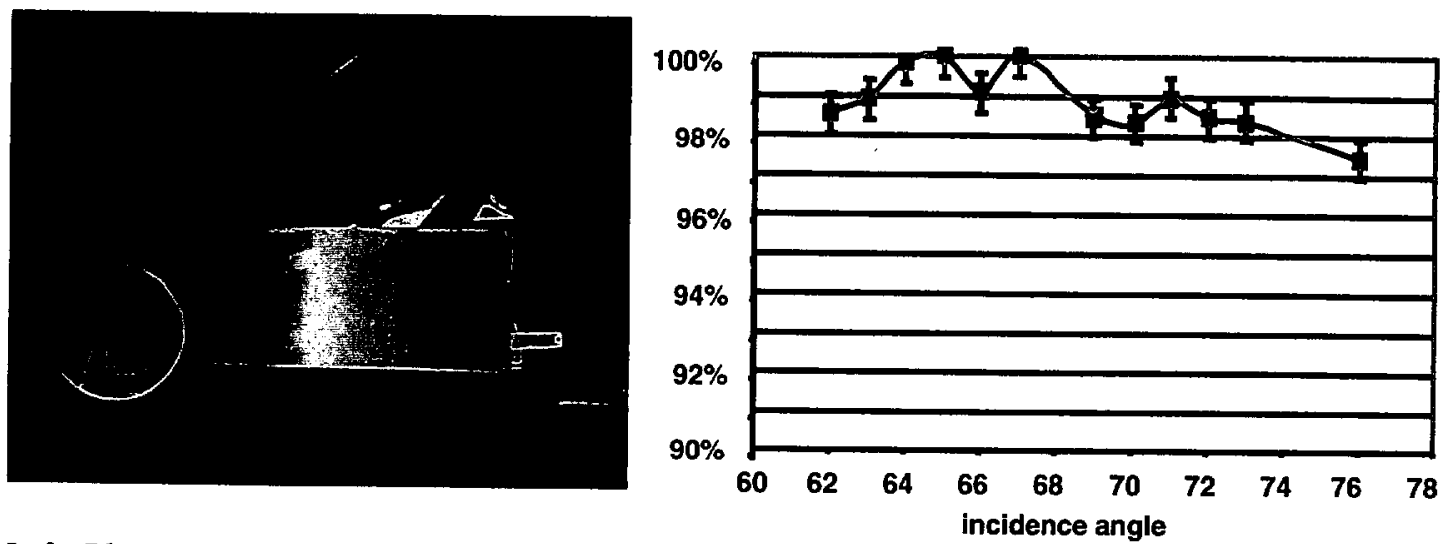

Fig. 2. Left: Photograph of $355 \times 150 \mathrm{~mm}$ multilayer dielectric diffraction grating also shown with $150 \mathrm{~mm}$ round grating of same design. Right: -1 order diffraction efficiency (in reflection) as function of angle at $1.03 \mu \mathrm{m}$ for this optic.

Multi-Petawatt laser systems are now on the horizon. Laser damage threshold of the optics will always limit fluence on the optics. The next generation of gratings will be transmission gratings etched into very thin fused silica or high-damage multilayer dielectric gratings, at apertures greater than our current capability. We are in the process of upgrading our ion-beam etching capability to process 2-meter class optics, and also beginning efforts to pattern phased gratings on monolithic substrates in a multi-exposure process.

\section{WET-ETCH FIGURING (WEF)}

Minimization of nonlinear self-focusing effects in high-intensity laser systems, and weight constraints of spacedeployed optical systems, represent compelling reasons to use ultrathin optics whenever feasible. Finishing or figuring of submillimeter thickness optics by conventional means is problematic and very expensive. Inexpensive, thin float or extruded sheet glasses possess excellent specular and microroughness properties as manufactured, but suffer from larger-scale thickness nonuniformities that make this commodity unsuitable for most applications where a precise optical thickness control is required.

We have developed a method, known as wet-etch figuring (WEF) for precision optical figuring of ultrathin glass based on confinement of an etchant solution attached to the underside of an optic [6]. A schematic of this geometry is shown Figure 3. Consider a situation wherein aqueous etchant solution issues at a slow rate from the end of a tube facing up, and flows down the outside of the tube. When a glass sheet or other hydrophilic surface is placed near the tube end, capillary forces will attach a liquid droplet to the underside of the glass. If the glass is moved laterally, a thin film of water is left behind the trailing edge of the drop. Introduction of a very small amount of volatile organic carbon (VOC) such as isopropanol vapors in the atmosphere surrounding the drop will result in absorption of this VOC into the liquid film. The concentration of absorbed VOC will be higher in the relatively static zone of the liquid meniscus (labeled A in Fig. 3), than in the region B which is continuously renewed by the falling film flow. This results in a surface tension gradient between A and B sufficiently strong to pull the liquid film off of the surface and confine it to a constant, stable size as the surface is moved laterally with respect to the attached drop. This confinement enables a 'small-tool' figuring process. Simultaneously, the local optic thickness can be measured interferometrically by a laser looking down from above. The local thickness measurements can be used to control the local dwell time of the droplet in a closed loop process using computer-controlled $x-y$ stage motion to converge on the desired figure with no iteration. This process imparts no mechanical or thermal stresses to the workpiece. It requires only a specular surface to begin with since etching alone cannot reduce microroughness. Figure 4 shows the transmitted wavefront of an optic made using WEF to pre-correct for static aberrations in a large laser system. This optic was figured over a $90 \mathrm{~mm}$ aperture on 700 micron-thick extruded sheet glass. 


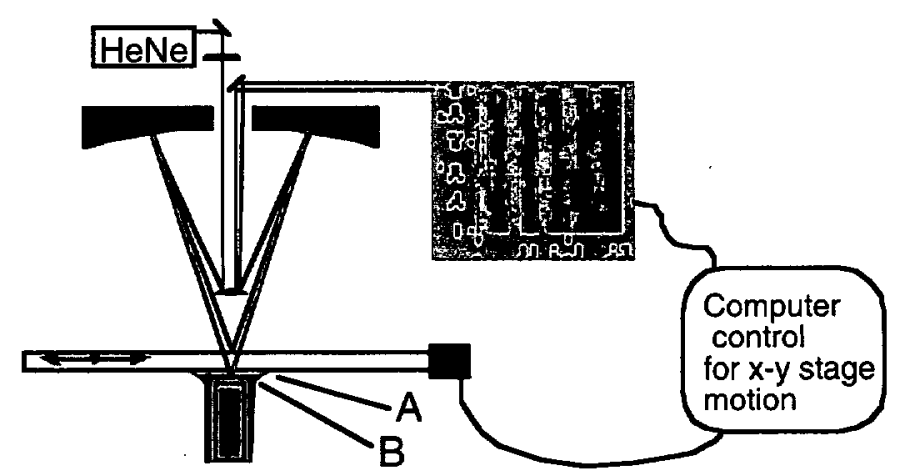

Fig. 3. Schematic of the wet etch figuring (WEF) process

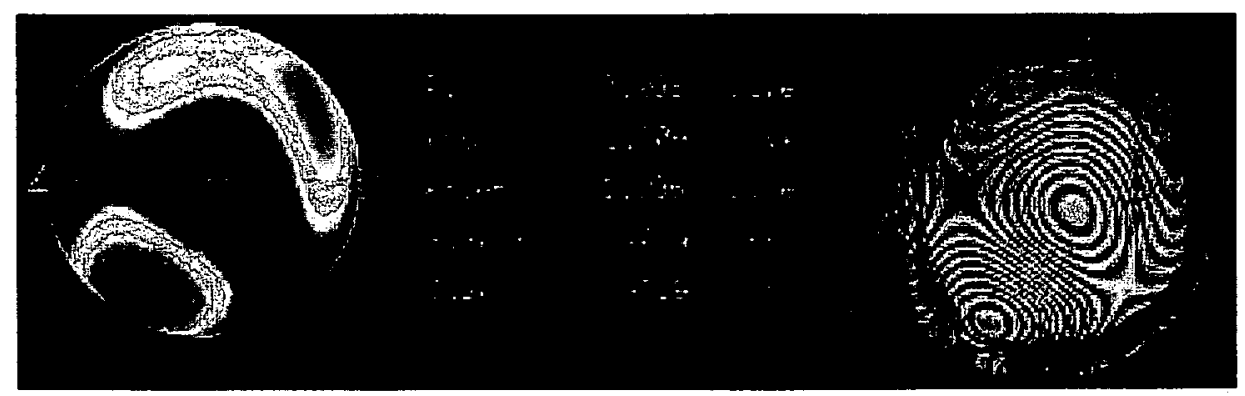

Fig. 4. Transmission interferogram of wavefront correction optic fabricated out of 700 micron-thick sheet glass using WEF.

Commercial extruded thin sheet glass typically exhibits largely one-dimensional thickness variations orthogonal to the draw direction. We have applied the WEF process as a one-dimensional line source to remove the bulk of the thickness variation, leaving a smaller amplitude, larger spatial-scale 2-D residual behind. Figure 5 shows a $150 \mathrm{x}$ $250 \mathrm{~mm}$ section of a glass plate flattened by this method. Sheets flattened in this manner have applications as lowcost disposable debris shields for LLNL's NIF laser. Applications requiring flatter glass need to be further figured using a 2-D tool as described above. We have built and currently are using a 1-D WEF tool to flatten $1150 \times 850 \times$ $0.7 \mathrm{~mm}$ glass sheets for a project whose goal is to deploy a thin segmented Fresnel lens in space [7].

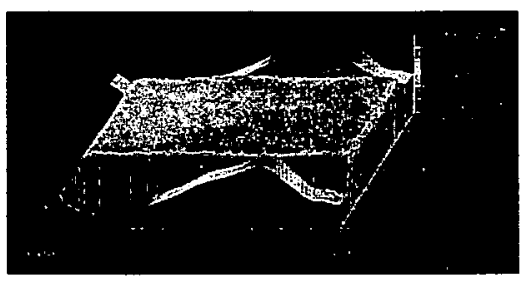

Fig. 5. Transmitted wavefront of a $150 \times 200 \mathrm{~mm}$ section of 340 micron-thick borosilicate glass sheet flattened using a $150 \mathrm{~mm}$ wide one-dimensional WEF tool. Approximately 9 waves of distortion was removed, leaving about 0.5 waves residual error.

\section{REFERENCES}

1. M.D. Perry, D. Pennington, B.C. Stuart, J.A. Britten, C. Brown, S. Herman, B. Golic, M. Kartz, J. Miller, H.T. Powell, M. Vergino and V. Yanovsky, 'Petawatt Laser Pulses, Optics Letters, 24, 160-162, (1999)

2. Exhaustive information on the NIF laser can be found at the URL http://nif.llnl.gov.

3. Detailed information on LLNL's Diffractive Optics Group can be found at http://asers.llnl.gov/diffractiveoptics/index.html 
4. M.D. Perry, R.D. Boyd, J.A. Britten, D. Decker, B.W. Shore, C. Shannon, E. Shults, and L. Li, 'High Efficiency Multilayer Dielectric Diffraction Gratings' Optics Letters, 20(8), 940-942, (1995)

5. M.D. Perry, J.A. Britten, H.T. Nguyen, R. D. Boyd and B.W. Shore, 'Multilayer Dielectric Diffraction Gratings, U.S. Patent no. 5907436, (1999).

6. M.C. Rushford, J.A. Britten, C.R. Hoaglan, I.M. Thomas, L.J. Summers and S.N. Dixit, 'Wet-Etch Figuring: Optical Surfacing by Controlled Application of Etchant Solution Using the Marangoni Effect', H.P. Stahl, ed., Proc SPIE 0277, 249-258 (2001)

7. I.M. Barton, J. A. Britten, S. N. Dixit, L. J. Summers, I. M. Thomas, M. C. Rushford, K. Lu, R. A. Hyde, and Michael D. Perry, 'Fabrication of large-aperture lightweight diffractive lenses for use in space, Applied Optics -OT, 40(4) 447-451, (2001)

\section{ACKNOWLEDGMENTS}

The success of the work detailed here was the result of outstanding efforts by many current and former colleagues, including but not limited to Mike Rushford, Leslie Summers, Sham Dixit, Curly Hoaglan, Hoang Nguyen, Mike Perry, Steve Herman, Bruce Shore, and Bob Boyd. This work was performed under the auspices of the United States Department of Energy by the Lawrence Livermore National Laboratory under contract no. W-7405-Eng-48. 


\title{
Wet-Etch Figuring: Optical Surfacing by Controlled Application of Etchant Solution Using the Marangoni Effect
}

\author{
M. C. Rushford, J. A. Britten, C.R. Hoaglan, I.M. Thomas, L. J. Summers, S.N. Dixit \\ Lawrence Livermore National Laboratory \\ Livermore, CA 94550
}

\begin{abstract}
Wet-etch figuring (WEF), a computer-controlled method for generating arbitrarily shaped optical surfaces using wet chemical etching, has been developed. This method uses applicator geometry and surface tension gradients (the Marangoni Effect) to define and confine the footprint of a wetted etchant zone on the surface. Capillary forces attach the flowing etchant solution to the underside of the optic being figured. No mechanical or thermal stresses or residues are applied to the optic by this process. This enables interferometric measurement of the glass thickness while surfacing, which then controls the placement and dwell time of the wetted zone. The result is a truly deterministic, closed-loop figuring process with a high degree of optical precision. This process can figure sub-millimeter thickness, large-aperture plates or sheets that are very difficult to finish by conventional methods. Automated linear and circular spot etching tools were used to demonstrate surfacing on 380 micron-thick glass sheets, to Strehl better than 0.8 , as specified by data array or Zernike polynomials.
\end{abstract}

\section{KEYWORDS}

Small tool figuring, surfacing, polishing, Marangoni, wet etching, aspheric, phase correction, phase mask, interferometry, surface tension

\section{INTRODUCTION}

Small-tool finishing or figuring of optical surfaces basically involves moving a small polishing tool in a controlled manner to shape the surface of an optic. It is a critical technology for producing optics for applications ranging from camera lenses for the consumer market, to large-aperture optics for inertial confinement fusion and space telescope systems. Examples of optics figured by these techniques include aspheric lenses, continuous-contour phase plates, wavefront correction optics, Alvarez lenses, Schmidt corrector plates and optics requiring local figure correction after processing via traditional lap polishing. Small-tool finishing is simultaneously a mature technology and one undergoing continuous development driven by the high cost and reproducibility problems of processes when applied to ever-tightening figure tolerances.

Traditional small-tool optical figuring techniques employ rotary polishing pads. Recent developments (1-4) utilize directed flow fields to impinge fine abrasive slurries to the optical surface. Magneto-rheological finishing (5-7), recently implemented on a commercial scale, extends this concept by controlling the viscosity of a specially formulated abrasive slurry by application of magnetic fields. Ion-beam milling techniques $(8,9)$ have been commercialized as well. These are alternative, fundamentally different methods for high-accuracy optical figuring.

All of the above-mentioned techniques suffer from fundamental limitations. They rely on calibrated removal rates, and are therefore iterative processes: the workpiece must be dismounted from the machine and measured, reworked and remeasured, until specifications are met. Ion beam milling techniques require large, expensive vacuum processing chambers and are not applicable to all materials. Abrasive small-tool polishing techniques cannot be used to figure very thin optics since the local mechanical stresses involved cause workpiece deformations that impact removal control and can even cause breakage.

Material removal on optical surfaces can be accomplished by etching or dissolution methods (e.g. silicate glasses are soluble in hydrofluoric acid solutions), but until now, wet etching has not been employed to figure optics. The problem has been largely how to confine the wetted zone of etchant solution to a specific stable geometry. A surface being etched is hydrophilic to the etching solution. If a bolus of etchant solution is moved along the surface of a workpiece, a thin liquid film will be left behind that will continue to etch the surface. In ramant ranm .....en ension gradient driven flow (the 
Marangoni effect) has been shown to be effective in causing this thin entrained film to flow off the surface of a workpiece back into the bulk liquid if said liquid is applied in the appropriate $\cdot$ manner (10-14). This phenomenon has been commercialized in the semiconductor processing industry for critical wafer drying applications.

We have employed the Marangoni effect to confine the wetted zone of an etchant solution on the surface of an optic. The wetted zone size and shape remains fixed as it is moved around on the workpiece surface. This allows the fluid to act on the surface of the workpiece only in this wetted zone with no mechanical contact or induced mechanical stresses applied to the workpiece. This in turn allows for real-time local metrology of the processing, and enables the processing of very thin $(\ll<$ $1 \mathrm{~mm}$ thickness) large-aperture plates that cannot be surfaced by other means. This is an enabling technology for the precision figuring of very thin optics that can be used in lightweight, space and astronomy applications, high-power laser systems in which nonlinear effects due to bulk transmissive optics need to be minimized, display applications, pellicles, etc.

\section{PROCESS DESCRIPTION}

Liquid flows induced by surface tension gradients are generally known as Marangoni flows in honor of a 19th century Italian physicist who is credited with their elucidation (cf. 15). Thermal or concentration gradients can establish surface tension gradients. We have employed concentration gradients of absorbed low-molecular weight volatile organic compounds (VOC's, e.g. isopropanol) in a geometry in which the optic surface is etched from below (see Figure 1). The aqueous etchant flows slowly up through a small tube (or slot, in a one-dimensional tool) and flows out and down the outside of the tube or slot. Capillary forces cause the liquid to attach to the underside of an optic when it is placed in close proximity to the exit orifice. In the absence of surface tension gradients, a liquid film remains behind on the surface when it is translated with respect to the liquid applicator. VOC's introduced in the volume surrounding the fountain, either by natural evaporation from a pool, or convection in a carrier gas, absorb into the liquid. Greater concentrations of absorbed VOC build up near where the liquid meniscus attaches to the glass than lower down the meniscus where the falling film flow on the outside of the applicator refreshes the surface. This concentration gradient results in a surface tension gradient strong enough to pull the liquid film off of the surface as is moves laterally, resulting in a wetted zone of constant, stable size. The shape of this zone is fixed by the applicator geometry and to a lesser extent by the flow rate and the spacing between the orifice and the workpiece. Figure 2 shows a circular wetted zone for a two-dimensional figuring tool, and a linear wetted zone in a line-source geometry used to take the one-dimensional phase ripple out of commercially extruded thin sheet glass.
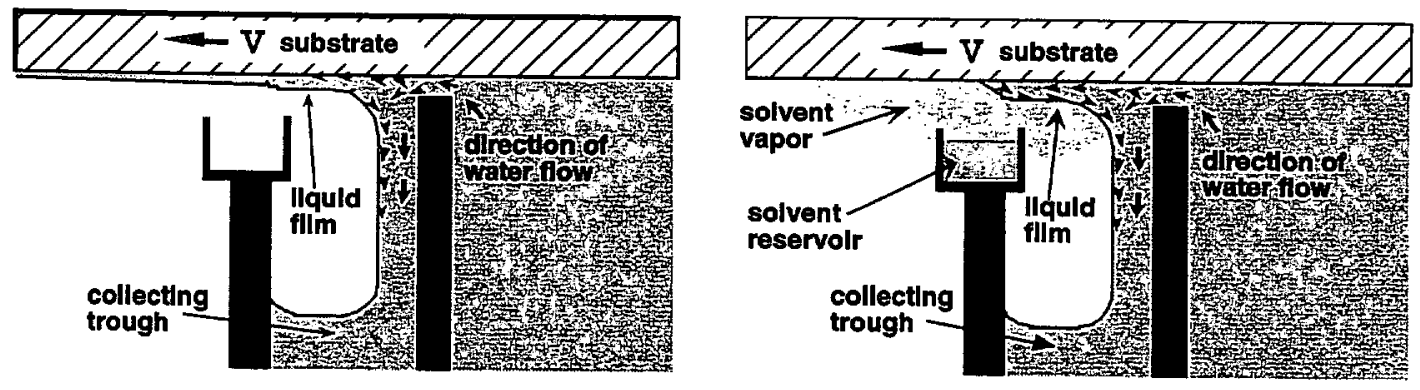

Figure 1. Schematic of Marangoni effect applied to aqueous etchant confinement. Left: water flowing over a dam with a glass sheet just over the dam being wetted as the glass is translated from right to left. Water in this case will adhere to the hydrophilic glass surface and remain behind as a film. Right: In the presence of VOC vapors (e.g. isopropanol) that absorb into the water film and reduce its surface tension, a surface tension gradient is established, lowest in the relatively quiescent thin film flow on the glass and highest in the continuously refreshed falling film zone. This gradient is strong enough to pull the liquid film off of the surface and maintain a wetted zone of constant size as the substrate is translated. 


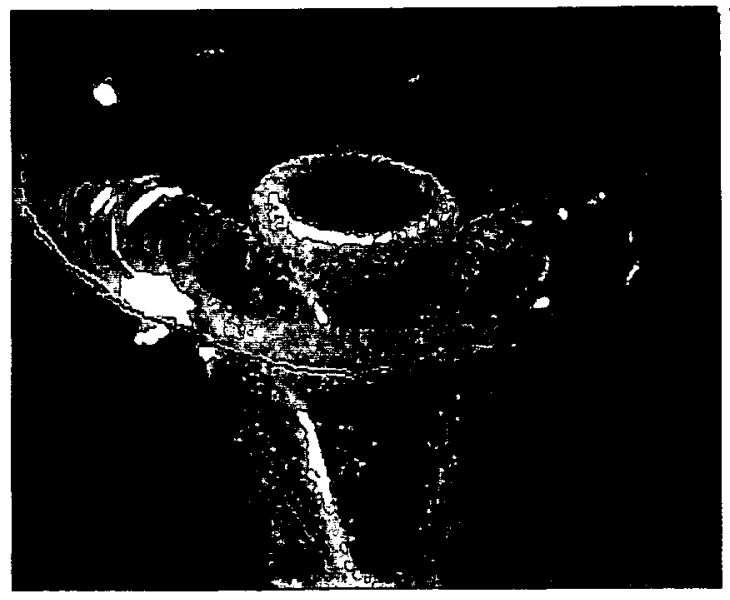

A

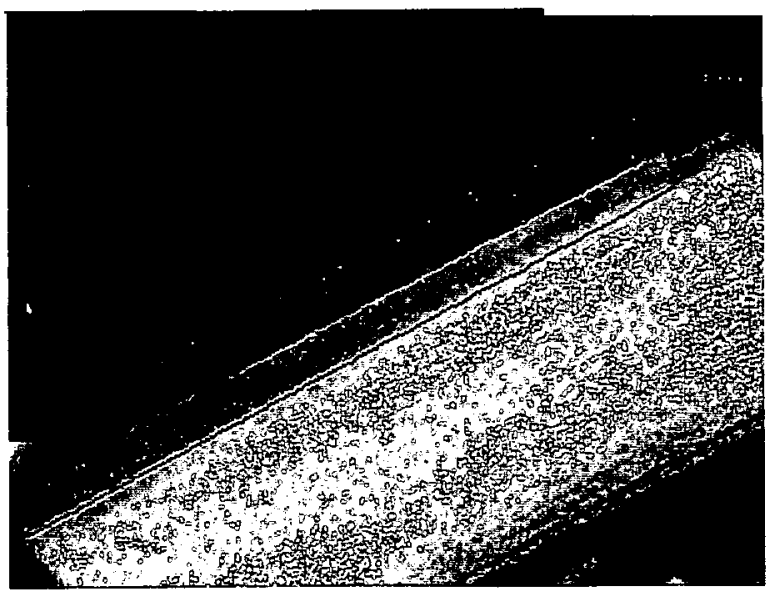

B

Figure 2. (A) Looking downwards through the thin glass being processed, left is a drop of etchant solution $\sim 10$ mm in diameter adhering to the underside of a glass sheet. The solution is flowing up through and then down the outside of the tube. (B) A 150x10-mm rectangular wetted zone in which liquid flows up out of a slit and down the inclined plane on either side. In both cases, a blue line has been drawn on the three-phase contact line as a visual aid.

A key element to the technology is the ability to measure while figuring in a feedback control loop that makes the process truly closed-loop controllable. In situ real-time interferometry is used to measure the surface shape as shown in Figure 3.
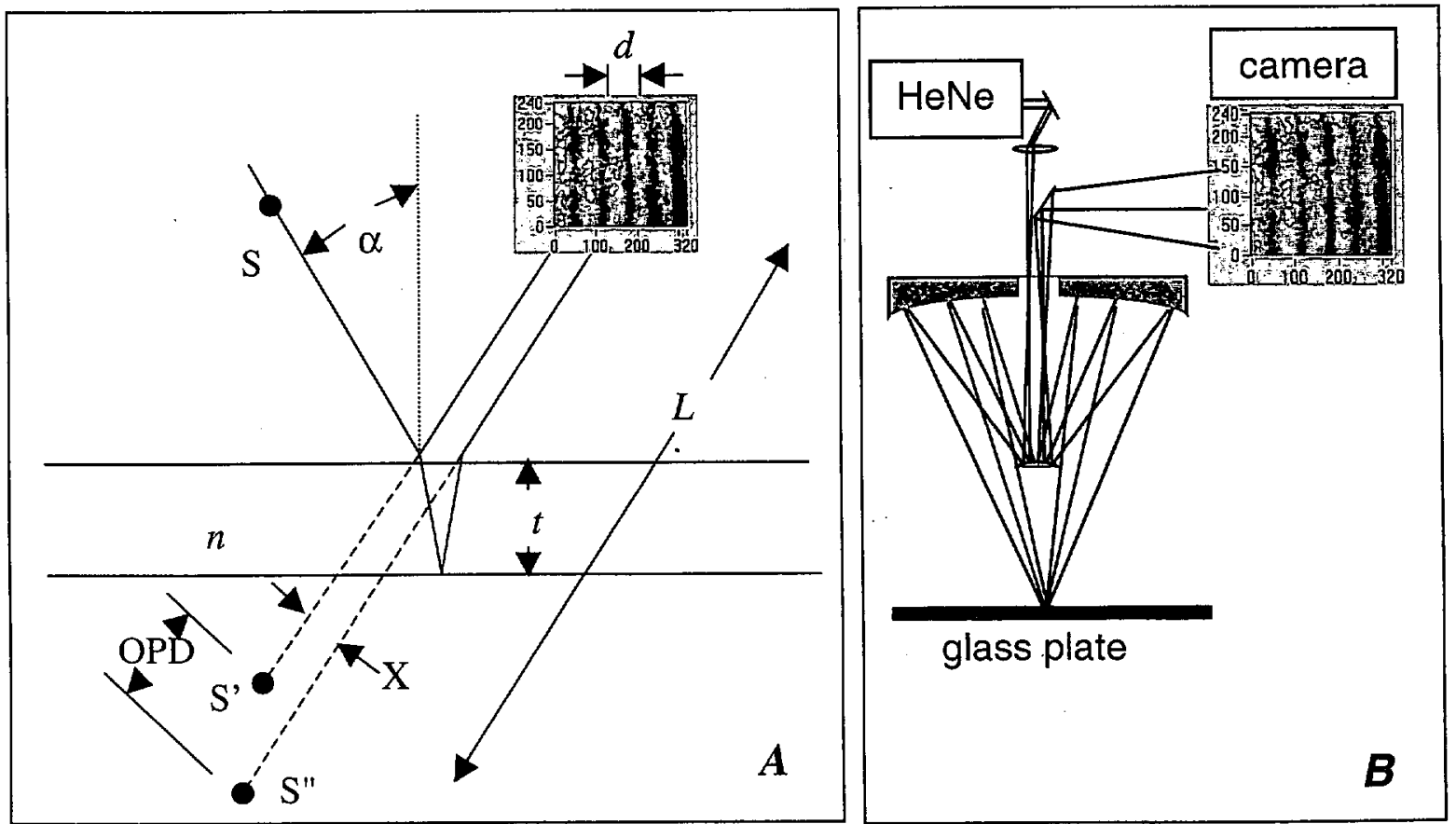

Figure 3. (A) Is a schematic layout showing the creation and relative displacement between two divergent light source S' and $S^{\prime \prime}$ which give rise to uniformly spaced fringes. (B) Shows how the source $S$ can be created while also projecting the fringes to a camera. 
A point source $(S)$ of coherent light illuminates the glass sheet from above directly over the etching head and at a slight angle. Two virtual point sources $\left(S^{\prime}\right)$ and $\left(S^{\prime \prime}\right)$ appear in reflection from this glass plate and produce Interference fringes. At a given glass thickness $(t)$ and angle of incidence $(\alpha)$ the apparent source shear separation $(X)$ is given by:

$$
X=2 t \sin (\alpha)
$$

The two sources are further separated in Optical Path Difference $(O P D)$ :

$$
O P D=2 n t \cos \alpha
$$

where $(n)$ is the glass index of refraction. These two-beam interference fringes translate in phase as the glass thickness $(t)$ changes. The fringe phase and thus the relative change of the OPD is measured and scaled to control the tool dwell time. The fringe period $d$ is given by:

$$
d=L \lambda / X
$$

where $(L)$ is the distance from the source to the fringe projection plane and $(\lambda)$ is the laser wavelength. In practice, a $633-\mathrm{nm}$ $\mathrm{HeNe}$ laser source (S) is focused within a few $\mathrm{mm}$ of the glass surface using the geometry shown in Figure $3 \mathrm{~B}$. The interferometer fringe stability is very robust to machine vibrations due to its common-path design.

The glass relative thickness is measured from relative phase of the fringes on the camera. While in closed loop control the glass bottom side reflectivity is reduced because this interface being contacted with etchant, reducing fringe visibility to 0.1 . The two dimensional digitized raw fringe intensities are seen in Figure 4 (A). A single one-dimensional array is made by summing column pixels along the fringe length to increase the fringe signal-to-noise. This one-dimensional sinusoidal array is FFT band-pass filtered to further improve the signal-to-noise ratio and generate the sinusoidal intensity pattern. This data $I_{3}$ are sums of 4 data zones $R_{0}-R_{3}$ spanning a fringe period from PK1 to PK2, as shown in Figure 4B. The scalar values $I_{0^{-}}$ $I_{3}$ are sums of these data regions. These values are used to calculate the fringe phase $\Phi$ according to the algorithm developed by Schwider et al. (16), relative to these fixed regions:

$$
\tan (\Phi+\pi / 4)=\frac{2\left(I_{0}-I_{2}\right)}{I_{0}-\left(I_{1}+I_{2}\right)+I_{3}}
$$
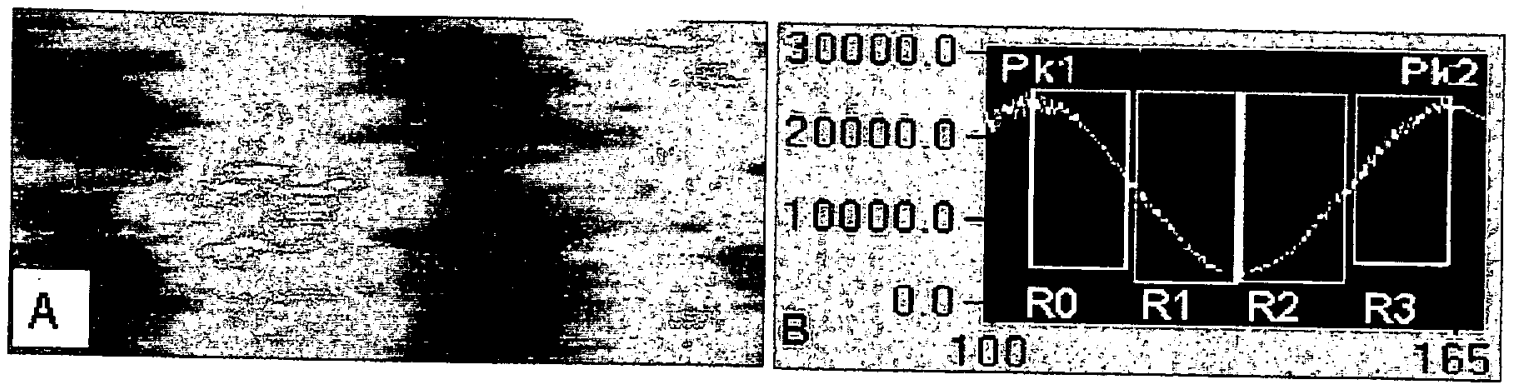

Figure 4 (A) Raw interference-fringes seen in reflection off a 380-micron thickness glass plate when wet by water on its bottom side. The fringes are exhibit speckle from the coherent source at $633 \mathrm{~nm}$ and imperfections in the optics. (B) Summed and filtered intensity profile binned into 4-zones for phase-stepping algorithm.

The area to be figured is divided into $m$ by $n$ pixels. The dwell time per pixel is scaled from real time relative thickness measurements as described above. The pixel size is chosen to be smaller than the tool size to ensure smooth figuring. The dwell time is a velocity of passage over a pixel. The dwell algorithm is to move the tool as rapidly as possible over areas that do not require surface removal. This top speed limit is set by the surface tension confinement strength of the wetted zone, and has comfortably demonstrated $1000 \mathrm{~mm} / \mathrm{min}$, The slowest speed, used where material removal rates are to be maximized, is typically 10-20 times less than the top speed. If the rate becomes too slow, etching of patterns on the scale of
the tool size begins to become a problem. 


\section{DEMONSTATIONS OF THE PROCESS}

\section{One Dimensional Smoothing of Sheet Glass}

Many types of commercial extruded thin glass sheet products exhibit a reasonable transmitted wavefront in the draw direction, but suffer from several waves of optical distortion perpendicular to the draw direction. These multi-component glass sheets are available in dimensions greater than one meter and thickness ranging from 100 microns to a few millimeters. Until this time, there has been no method to flatten such large thin sheets to optical tolerances at a reasonable cost.

As a proof-of-principle experiment, we constructed a 1-D etcher wherein a stepper-motor stage moved a glass sheet over a slotted orifice $150 \mathrm{~mm}$ long, creating a wetted zone of this length and about $2-10 \mathrm{~mm}$ wide. Interferometric measurements were taken along one line, and used to control the wetted zone dwell time. A $150-\mathrm{mm}$ wide by $200-\mathrm{mm}$ long section of Corning 0211 glass, 380 microns thick, was processed using a buffered HF solution over a continuous 96 -hour period. The transmitted wavefront of the result is shown in Figure 5. Over 12 microns PV were removed. There is about 1 wave residual 2-dimensional error remaining on the flattened section. A second demonstration using a higher etchant concentration and $10-30$-mm wide by $400-\mathrm{mm}$ long wetness footprint was used to flattened 6.5 microns of excursion on a $400 \times 400-\mathrm{mm}$-square section of $0.75-\mathrm{mm}$ thick Corning 1737 glass in 24 hours. Profiles of the plate thickness measured at the control line at the start and end of the process are shown in Figure 6.

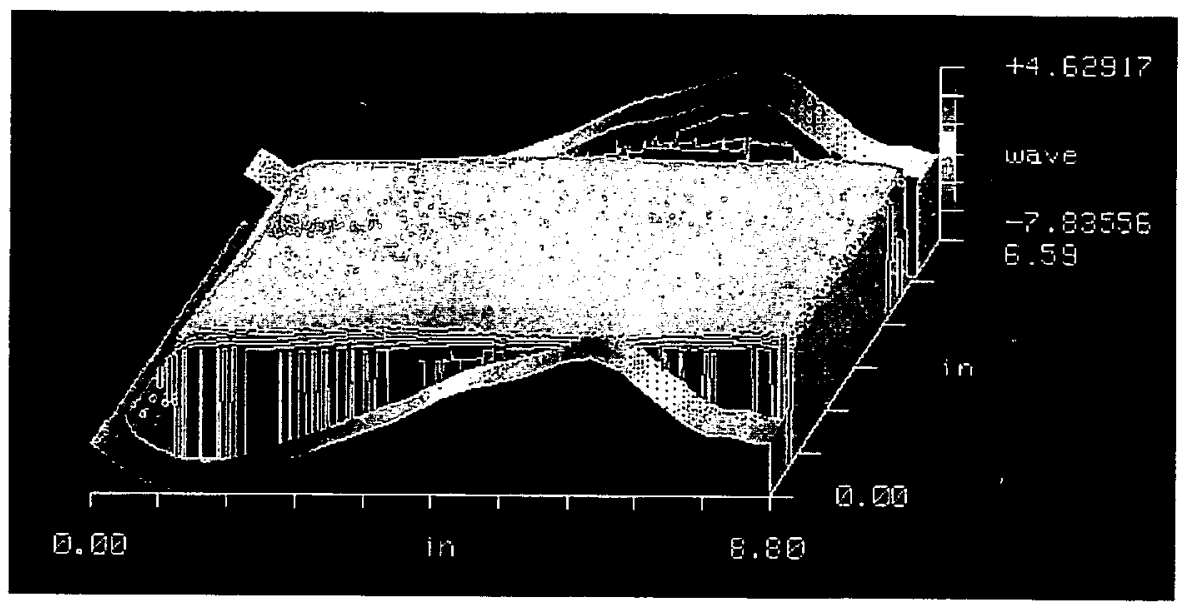

Figure 5. Transmitted wavefront of $150 \times 200-\mathrm{mm}$ section of 380 micron-thick glass plate flattened using linear etching tool. Apparent discontinuities in profiles along edges caused by fringe dropouts at high-gradient zone along edges. Approximately 9 waves of distortion in transmitted wavefront taken out, leaving $\sim 1 / 2$ wave of residual 2-dimensional error across the part.

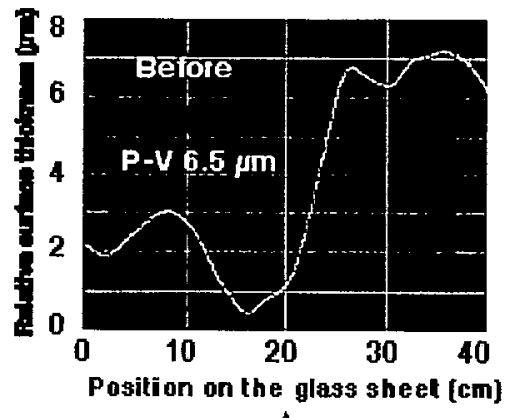

A

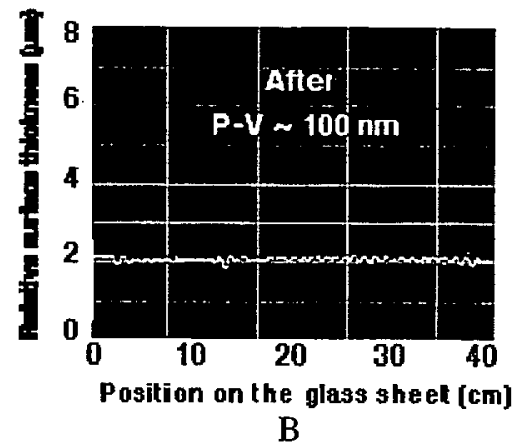

B

Figure 6. Sheet thickness lineout used for process control for figuring Corning $1737400 \times 400 \times 0.7 \mathrm{~mm}$ thick sheet. (A) At start and (B) and after 24 hrs figuring with one-dimensional WEF tool. 


\section{Fabrication of a Beam-Shaping Phase plate}

An ultra-short pulse laser-cutting application at LLNL (17) can improve upon hole-drilling performance by use of a top-hat beam as opposed to a gaussian beam at focus. We designed a phase plate to transform a far field gaussian beam at $1030 \mathrm{~nm}$ into a flatter intensity profile before and after focus. It is essentially a lens of weak power but has spherical aberration. We fabricated this design using a circular 2-D WEF tool to figure this design out of 380 micron-thick, Corning 0211 glass, at $2.3-\mathrm{cm}$ aperture. The substrate was mounted on a $\mathrm{x}-\mathrm{y}$ stepper motor stage and glass thickness was mapped in-place interferometrically. This map and subsequent updates were used to control the dwell time of a circular WEF spot that varied from $\sim 5-\mathrm{mm}$ diameter at the start and shrinking to $\sim 1 \mathrm{~mm}$ in diameter near the end of the working time. The final residual surfacing error was used to calculate the Strehl ratio. This criterion was used to determine when the part was finished. Several phase-plates of this design have been manufactured. Figure 7 shows the transmitted wavefront of one fabricated phaseplate that exhibited a residual surface error Strehl ratio of better than 0.85 . The optic was finished in about 18 hours of continuous, automatic operation. This optic is now being used in routine laser-cutting operations.
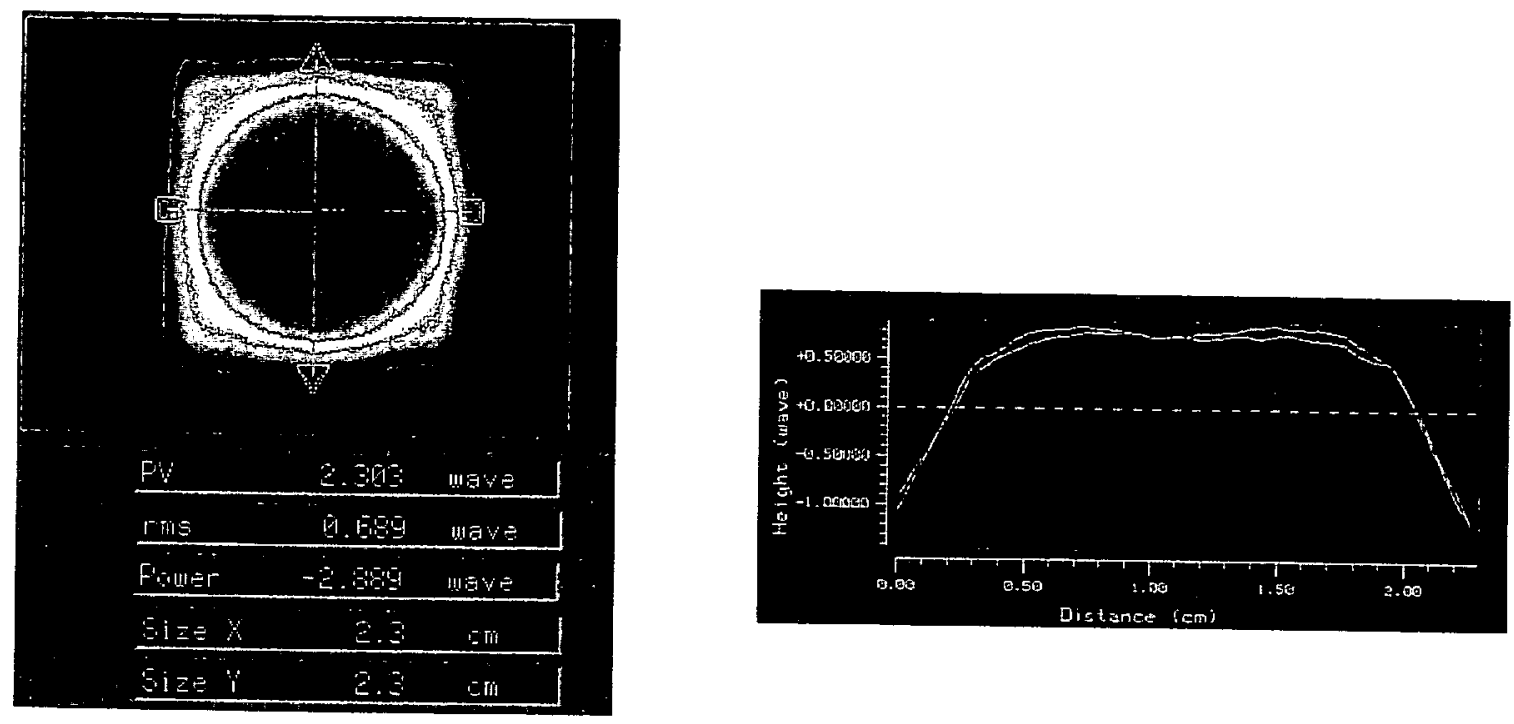

Figure 7: Transmitted wavefront of phase-plate figured with automated 2-dimensional WEF tool - to convert $1030 \mathrm{~mm}$ gaussian profile at focus to top-hat profile. Surface was contoured in 380-micron thick Corning 0211 glass with 23 -mm aperture. Its residual error Strehl was 0.85.

\section{Static Wave front Corrector for a High-Power Laser System.}

Most high power laser systems suffer from wavefront aberrations that are both static and dynamic. Adaptive optical elements such as deformable mirrors are typically employed to compensate for most aberrations. However, it is highly desirable to use a static wavefront 'pre-corrector' in the optical chain to correct for static aberrations and reduce the dynamic range required for the active wavefront control device. Researchers at the AWE facility in the United Kingdom have designed a static wavefront corrector for their purposes that calls for a complex surface with 12 microns PV departure over a $80 \mathrm{~mm}$ circular aperture (18). Attempts at fabricating this corrector by a gray-scale photo-resist profile exposure followed by reactive ion etching to transfer the continuous profile into fused silica were of limited success, due to the lack of active process control to converge on the desired profile. Multilevel binary approximations to the continuous profile are less desirable due to intensity modulations that are known to occur at sharp edges that are capable of damaging downstream optics.

We have fabricated a corrective optic to specifications given by Zernike polynomials by the WEF technique, using the circular 2-D tool described above. This optic profile was a considerable challenge to fabricate and led to many process and 
control improvements. Processing of data at the edges of the part where steep gradients are etched over time was a significant problem. We employed a "corona guard band" strategy to overcome this. This zone spanned the distance from the circularly defined optic to a rectangular region $1 \mathrm{~cm}$ away from the optic. Whatever the dwell time was supposed to be at the edge of the optic was radially carried outward to the outer guard band frame edge. The final transmitted wavefront profile at $80-\mathrm{mm}$ aperture shown in Figure 8 . The part was completed when the surfacing was within $100 \mathrm{~nm}$ of target. The residual error of the part is equivalent to a Strehl of 0.26 . Since the process is controlled to the final figure, this optic was made in one step.
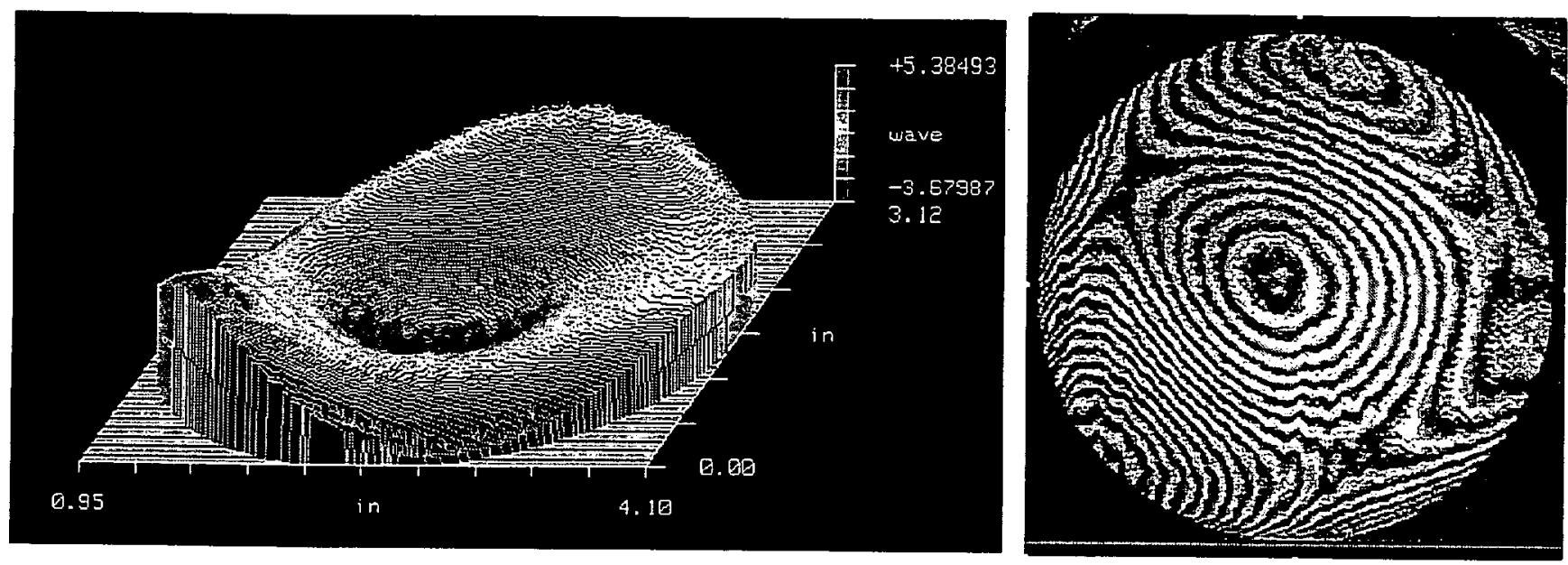

Figure 8. Interferogram of static wavefront corrector for AWE's Helen laser, fabricated with automated 2-dimensional WEF tool. $80 \mathrm{~mm}$ aperture. PV of this figure is 12 microns. It achieved a residual Strehl of 0.26 .

\section{DISCUSSION}

Conventional polishing processes require more mechanical force as the tool size increases. The complexity and power requirements in ion-beam figuring increase substantially as the ion source size is increased. In contrast, it is quite easy to tailor the tool size in wet-etch figuring. The wetted zone can be made arbitrarily large and arbitrarily shaped without distorting the optical surface being figured. Furthermore, under conditions in which the surface removal rate is kinetically controlled, the etch rate is independent of local flow conditions and is constant everywhere in the wetted zone. Since the tool area is flexible, it is possible to rapidly work large area of low spatial frequency and later shrink the wetted zone size to finish off the higher frequency components of a surface shape. We have made applicators varying in wetted-zone size from $1 \mathrm{~mm}$ to $15 \mathrm{~mm}$ in diameter with a circular shape and $400 \mathrm{~mm}$ long fixed by $1-30 \mathrm{~mm}$ variable width for doing $1 \mathrm{D}$ profiles. The size of the wetted zone is easily customized to the particulars of a surface contour.

To date we have done the majority of our work on Corning 0211 (zinc borosilicate) sheets. We have used HF concentration of $0.5 \%-10 \%$ buffered with $\mathrm{NH}_{4} \mathrm{~F}$, or in combination with a mineral acid (nitric or hydrochloric) added to solubilize the non-silicate components of this glass. We have also etched pure fused silica and Corning 1737 (an alkaline-earth aluminosilicate). The etching rate is dependent on the glass solubility, etchant composition, temperature and concentration. This process is operated under ambient temperature and for concentration conditions that ensure that the etching rate is controlled by chemical kinetics and not influenced by mass transport phenomena. Of course, the exact etch rate need not be known, since the removal rates are measured in real time. On 0211 glass we have worked etching rates from 10 to 900 $\mathrm{nm} / \mathrm{minute}$. The time to finish a profile is the ratio of the volume of material to remove divided by the volume removed per unit of time by the tool. We never have to stop production to inspect progress, as it is measured in real-time. Since the tool area can be increased to arbitrary size and shape it becomes possible to decrease the working time by customizing the wet foot print tool area for some routinely similar work.

If the wetted zone size is constant over the course of the etching, the surface profile gradually develops discontinuities. Pulsating the etchant flow to cycle the size of the wetted zone alleviates this effect. This imparts a more Gaussian etch rate 
profile on average and minimizes sharp gradients at the tool edges. High-frequency tool-generated surface ripples were also minimized by reducing the tool size at later times in the figuring process and by keeping the ratio of high-to-low dwell times no greater than 5:1. We have also found it advantageous to provide a wicking effect on the outside of the applicator, to reduce the effective tool size to on the order of the orifice dimension, for final high-frequency figuring work. Glass fogging was observed if we were not careful to completely flush acid vapors from the vicinity of the etch head, by supplying an adequate flow of carrier gas to serve a dual purpose as a flushing gas as well as to supply isopropanol vapors.

Since WEF is a figuring process and not a polishing process, the micro-roughness of the surface is not improved upon figuring, and in fact could be worsened, depending on the degree of subsurface damage left by prior polishing processes, scatter loss is of primary inhomogeniety of the glass. This may limit its application in some critical applications where conventional polishing concern. However, this figuring method may alleviate some other problems often associated with been shown to significantly degrade the lands at the sub-micron scale left behind in the gel layer upon final polish have The WEF process leaves no residues behind amage threshold of optics used for high-intensity UV laser applications (19). damage characteristics of optical surfaces. Since WEF imparts nove residues, so in principle it can improve the laser strong.

\title{
FUTURE APLICATIONS
}

We are presently building an apparatus to remove the primary one-dimensional ripple from drawn glass sheets $1160 \times 850$ $\mathrm{mm}$ by $0.7 \mathrm{~mm}$ thick. This same tool will be outfitted with an adjustable wetted-zone width capability along its length, to remove the residual two-dimensional random errors left in these sheets. A 2D image-wise interferometer has been developed and its data output will be used to control the WEF width variations. The goal is to produce $800 \times 800 \times 0.7 \mathrm{~mm}$ sheets that are optically flat to less than $1 / 8$ wave in transmission. These will be used as segments in a 5 -meter diameter foldable Fresnel lens for space applications $(20,21)$. We are also investigating the manufacture of $400 \mathrm{~mm}$ square, $10 \mathrm{~mm}$ thick random continuous contour phase plates for beam homogenizing on LLNL's NIF laser system (22) as well as wavefront corrective optics. We will look into making continuously varying gray scale apodizers having embedded phase correction, in which an initially uniform gray scale is selectively tailored by etching. We are exploring the utility of this process for figuring of fused silica pellicles for deep UV lithographic imaging systems (23). It is also possible to use a shearing interferometer (24) to measure the front surface contour of opaque substrates to control WEF processing of these
surfaces.

\section{CONCLUSIONS}

A process for closed-loop computer-controlled figuring of optical surfaces by Marangoni-confined wet-etch figuring has been demonstrated. Unlike other figuring techniques, no mechanical or thermal stresses are applied to the workpiece in this method. No residue is left on the optic. This allows for in-situ interferometric metrology of the workpiece during figuring, to control the placement and dwell time of the wetted zone locally on the workpiece. Thus, figuring is truly deterministic, very thin sub-millimetmeasure iterative cycle. The lack of stress experienced by the substrate also allows for the figuring of expense using existing technicks optical glasses to precise optical tolerances, something that is achievable only at great requires no elaborate or expensive calibration or tooling.

Acknowledgements: The authors thank J.Toeppen and R. Shuttlesworth for helpful assistance during this project. This work was performed under the auspices of the U.S. Department of Energy under contract no. W-7405-Eng-48.

\section{REFERENCES}

\author{
1. Taylor, J.S., 'Precision Non-Contact Polishing Tool', U.S. Patent 5,591,068 \\ 2. Baker, P.C., 'Advanced Flow Polishing of Exotic Optical Materials', SPIE V. 1160, 263-270, (1989) \\ 3. Fahnle, O.W., van Brug, H., Frankena, H.J., , 'Fluid Jet Polishing of Optical Surfaces', Applied Optics, 37, 6771-6773,
(1998)
}


4. Fahnle, O.W., van Brug, H., 'Fluid Jet Polishing: Removal Process Analysis', Proc. SPIE, V. 3739, 68-77, (1999)

5. Jacobs, S.D., Kordonski, W., Prokhorev, I.V., Gollini, D., Grodkin, G.R., Strafford, T.D., 'Deterministic Magnetorheological Finishing', U.S. Patent 5,795,212 (1998)

6. Golini, D., Kordonski, W.I., Dumas, P., Hogan, S.J., 'Magnetorheological finishing (MRF) in Commercial Precision Optics Manufacturing', Proc SPIE, V. 3782, 80-91, (1999)

7. Jacobs, S.D., Arrasmith, S.R., Kozhinova, I.A., Gregg, L.L., Shorey, A.B., Romanofsky, H.J., Golini, D., Kordonski, W.I., Dumas, P., Hogan, S., 'MRF: Computer-Controlled Optics Manufacturing', Amer. Ceramic Soc. Bull., 78, 42-48, (1999)

8. Lynn N. Allen and Robert E. Keim: "An ion figuring system for large optic fabrication" pp. 348-363;

Lynn N. Allen and Henry W. Romig: "Demonstration of an ion figuring process" pp. 364-373; SPIE VMS40,

Selected Papers on Computer-Controlled optical Surfacing, ed. B. J. Thompson (1991)

9. Drueding, T.W., Fawcett, S.C., Wilson, S.R., Bifano, T.G., 'Ion Beam Figuring of Small Optical Components', Optical Engineering, 34, 3565-3571, (1995)

10. Leenaars, A.F.M., Huethorst, J.A.M. and van Oekel, J.J., 'Marangoni Drying: A New Extremely Clean Drying Process',

11. Marra, J. and Huethorst, J.A.M., 'Physical Principles of Marangoni Drying', Langmuir, 7, 2748-2755, 1991

12. Wolke, K, Eitel, B., Schenkl, M., Rummelin, S., and Schild, R., 'Marangoni wafer Drying Avoids Disadvantages' Solid State Technology, 39, 87-90, 1996

13. Britten, J.A., 'Moving-Zone Marangoni Drying of Wet Objects Using Naturally Evaporated Solvent Vapor', U.S. Patent
$5,660,642$ (1997)

14. Britten, J.A., 'A Moving-Zone Marangoni Drying Process for Critical Cleaning and Wet Processing', Solid State Technology, 40, 143-148, (1997)

15. Scriven, L.E. C.V. Sternling, C.V., 'The Marangoni Effects', Nature 187 186-188, 1960

16. Schwider, J., Falkenstorfer, O., Schreiber, H., Zoller, A., Streibl, N., 'New Compensating 4-Phase Algorithm for Phase Shift Interferometry', equation (6) Optical Engineering, 32, 183-1885, (1993)

17. B.C. Stuart, M.D. Perry, M.D. Feit, L.B. Da Silva, A.M. Rubenchik, J. Neev, "Machining of biological materials, dielectrics, and metals with femtosecond lasers", OSA Trends in Optics and Photonics Vol. 9, Lasers and Optics for Manufacturing, Andrew C. Tam, ed. (Optical Society of America, Washington D.C. 1997), pp. 94-98.

18. Bett, T.H., Barnes, A.R., Hopps, N.W., Jinks, P.M.R., Norman, M.J., ‘ 41 Application of Static Phase Control Elements to High Power Solid State Lasers', SPIE Photonics West, Paper 4270, San Jose, CA, January 25, (2001)

19. Battersby, C.L., Sheehan, L.M., Kozlowski, M.R., 'Effects of Wet-Etch Processing on Laser-Induced Damage of Fused Silica Surfaces', Proc. SPIE, V3578, 446-455, (1998)

20. Hyde, R.A., "Eyeglass 1: Very Large Aperture Telescopes," Appl. Opt 38 4198-4212 (1999)

21. Barton, I.M., Britten, J.A., Dixit, S.N., Summers, L.J., Thomas, I.M, Rushford, M.C., Lu, K., Hyde, R.A., Perry, M.D., 'Fabrication of Large-Aperture Lightweight Diffractive Lenses for Use in Space', Applied Optics, 40, 447-451 (2001)

22. Dixit, S.N., Feit, M. D., Perry, M.D. and Powell, H.T., "Designing fully continuous phase screens for tailoring focalplane irradiance profiles", Opt. Lett. (21),1715-1717 (1996) 
23. Gordon, J., 'Navigating the Road Map progress on materials helps advance 157-nm mask substrate efforts, but pellicles remain a major challenge', OEMagazine, 24-25 (March, 2001)

24. Kohno, T., Matsumoto, D., Yazawa, T., Uda, Y., 'Radial shearing interferometer for in-process measurement of diamond turning' Optical Engineering, 39(10), 2696-2699, (2000) 


\title{
Development of static phase control elements for high power solid state lasers
}

\author{
THBett \\ A R Barnes ${ }^{a}$ \\ P Rudman ${ }^{b}$ \\ $N W$ Hopps $^{a}$ \\ $M$ Thompson $^{b}$ \\ $M R$ Taghizadeh ${ }^{b}$ \\ A $J$ Waddie $^{b}$ \\ C. R. Hoaglan ${ }^{c}$ \\ M. C. Rushford ${ }^{c}$ \\ $J$ A. Britten ${ }^{c}$ \\ S. N. Dixit ${ }^{c}$ \\ ${ }^{a}$ AWE plc; ${ }^{b}$ Heriot Watt University; ${ }^{\mathrm{c}}$ LLNL Livermore
}

\begin{abstract}
We describe development of passive phase correction elements to compensate for static phase errors and prompt thermally induced aberrations in the HELEN laser at AWE. Partial compensation of cooling effects is also included in the design. Phase elements have been fabricated through two processes, an indirect write lithographic process using amplitude masks generated from measured laser wavefronts and a direct write method using a novel wet etch figuring
tool.
\end{abstract}

Keywords: Phase correction, phase plates, high power solid state lasers

\section{INTRODUCTION}

Multi-stage glass laser systems used in high temperature plasma generation, for applications such as inertial confinement fusion, ICF, require very well defined laser pulses to be propagated and focused on target. These optical systems can comprise over one hundred components through which the beam propagates, resulting in significant passive aberration on the beam. Flashlamp pumping of the laser amplifier discs produces thermal energy deposition in the laser glass gain medium further degrading the wavefront. Furthermore, there are dynamic effects resulting from firing subsequent shots before the laser glass has cooled to the cold thermal equilibrium state. Gas turbulence in the beam tubes is another dynamic source of phase errors. Multi-passing the beam through the laser system exacerbates the effects.

We are developing surface relief phase elements as static phase correction components to compensate for phase errors. The objective of the work is to obtain an improvement in the focal spot of the laser but diffraction limited performance is not essential. ${ }^{1}$ Phase elements have been fabricated through two processes, an indirect write lithographic process using amplitude masks generated from measured laser wavefronts and a direct write method using a novel wet etch figuring tool. ${ }^{2}$ A Zernike circle polynomial fit to wavefront data, averaged over a number of laser shots, is used as the basis for the design of the correcting elements. Some compensation for shot to shot variations is therefore included.

Grey scale and 64 level elements have been fabricated using lithographic techniques and a grey scale element has been made using the wet etch figuring tool.

\section{HELEN LASER}

A schematic of the HELEN laser ${ }^{3}$ is shown in Figure 1. Four potential locations of a static phase corrector are shown.

The HELEN laser is a flashlamp pumped Nd doped glass laser comprising three beams with a common oscillator pulse generator. The output from the ring oscillator is split into two amplitude modulators, one modulator generating a shaped temporal pulse in the ns regime to be delivered by fibre to the two main beams, the other generating a 100ps FWHM pulse delivered to the third beam. The two main beams are used to generate a hot dense plasma at focus and the third beam is used to generate an $\mathrm{x}$ ray source for flash radiography diagnostics of the target irradiated by the other beams. Each beam comprises three amplification stages, a $6 \mathrm{~mm}$ aperture ring regenerative amplifier amplifying the pulse energy to the $\mathrm{mJ}$ regime after around 25 passes, a $50 \mathrm{~mm}$ rod amplifier stage producing around $100 \mathrm{~mJ}$ after 4 passes and finally a $208 \mathrm{~mm}$ amplifier stage boosting the output to around $1 \mathrm{~kJ}$ after 4 passes. Isolation of the high overall gain is achieved with strategically placed Pockels cell isolators and angular offsets are used to separate the beams in the 4 pass stages. 
Beam transport is controlled throughout the system by confocal optical telescopes providing spatial filtration in the focal plane and image relaying. This architecture is based on that employed on the $\mathrm{LMJ}^{4}$ and NIF ${ }^{5} \mathrm{MJ}$ class lasers under construction.

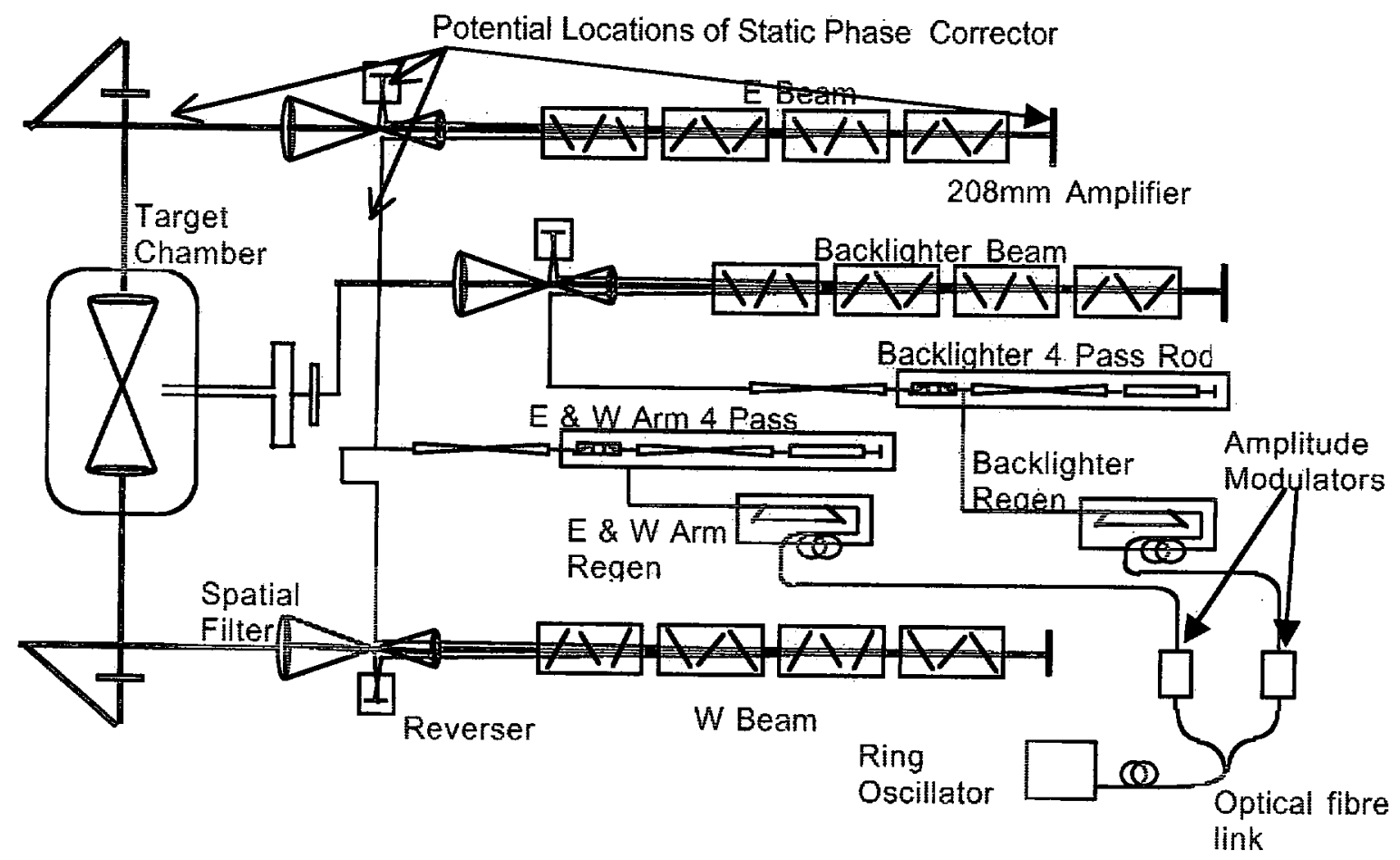

Fig.1

\section{Corrector plate positioning}

Four potential locations for the insertion of phase corrector plates are shown.

1. Injection to the large aperture amplifier stage. This is the smallest component but is used in single pass with full correction resulting in the steepest surface gradients on the part. There is also an issue with propagation of a predistorted beam through the laser.

2. At the end mirror in the 4 pass large aperture amplifier stage. This only needs a quarter of the phase depth with least phase gradients and the beam in any section sees least distortion but is large aperture and more difficult to fabricate.

3. An element at the output of the transport spatial filter can correct the full distortion at the end. This is the largest aperture element with full phase depth.

4. The section of the laser in the middle of the 4 pass $208 \mathrm{~mm}$ stage. After two passes of the amplifiers, the beam is re-collimated at $40 \mathrm{~mm}$ aperture, propagated through an isolator stage and reflected back along a different path for injection for the final two passes through the large amplifiers. This is the preferred location because

a) the element is of a reasonable size for fabrication, approx. $40 \mathrm{~mm}$ diameter.

b) the phase depth is halved since it is used in double pass

c) it sits in an image plane at the reflecting mirror for ease of alignment

d) it is halfway through the system so limits the aberration in any section of the laser system

\section{LASER WAVEFRONT MEASUREMENT AND RECONSTRUCTION}

The wavefront measurements at the output of the East beam were made using a self-referencing radial shear interferometer in which the central $1 / 9^{\text {th }}$ of the beam is expanded as the reference beam. The resultant fringe pattern is imaged onto a computer coupled CCD camera. The beam splitter is tilted to produce a high fringe density for a Fourier Transform analysis technique ${ }^{6}$. 
An example interferogram is shown below, Figure 2. This was taken on Shot 117 on the East arm of the HELEN laser. The fringe analysis yields a far field spot and phase map.

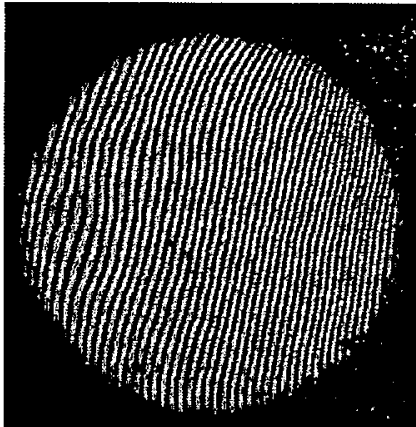

Intereferogram

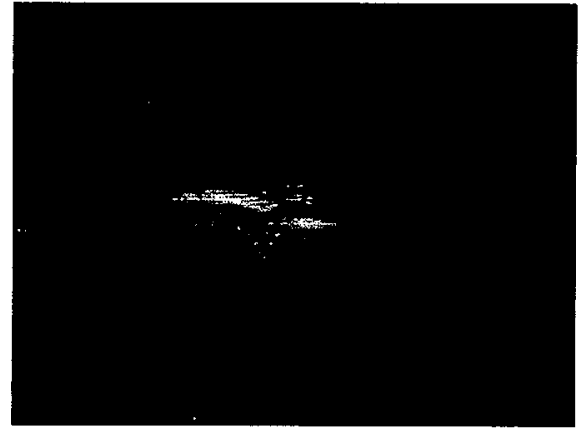

Focal Spot

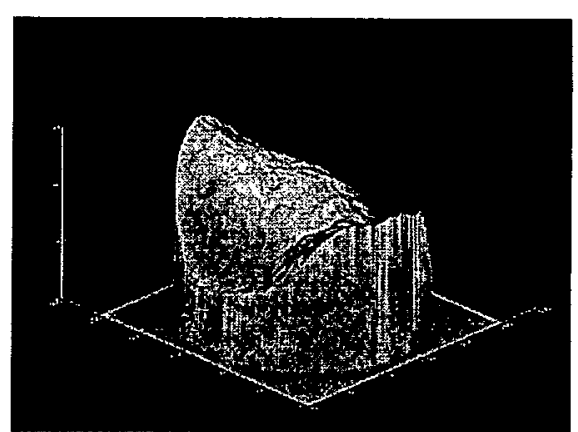

Phase Surface Plot

Fig. 2

For this particular shot, the focal spot diameter enclosing $90 \%$ of the energy is approximately $8.5 \mathrm{x}$ the diffraction limited $90 \%$ energy diameter, with a Strehl ratio of 0.03 . The Peak to Valley phase error is around $5.4 \lambda$.

A convenient basis set of functions to describe the wavefront is the Zernike circle polynomials of generalised form ${ }^{7}$

$$
\phi(\rho, \theta)=\Sigma_{j} a_{j} Z_{j}(\rho, \theta)
$$

where $\phi$ is the phase value with coordinates $\rho$, normalised radius, and $\theta . Z_{j}$ are the polynomial forms with the coefficient $a_{j}$ being the weighting factors. These functions have wide application in circularly symmetric optical systems and may be related to the Seidel aberrations. They are used here to

a) allow removal of tilt from the interferogram

b) provide a simple prescription of the wavefront for reconstruction and mask generation

c) to average out the effects of shot to shot variations

d) to extrapolate the wavefront beyond the beam aperture to enable a clear aperture for the fabricated optic to be larger than the beam size 
Table 1 shows the shot averaged polynomial fits for the examples of a main beam and a backlighter with tilt removed.

Table 1

\begin{tabular}{|c|c|c|c|}
\hline & & Main Beam & Backlighter \\
\hline$j$ & Zernike polynomial $Z_{j}(\rho, \theta)$ & $\left.a_{j}{ }^{\prime} \lambda\right)$ & $a_{j}(\lambda)$ \\
\hline 1 & 1 & & \\
\hline & $2 \rho \cos \theta$ & & \\
\hline & $2 \rho \sin \theta$ & $\cdot$ & 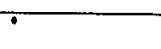 \\
\hline & $\sqrt{3}\left(2 \rho^{2}-1\right)$ & .045 & -1006 \\
\hline & $\sqrt{6} \rho^{2} \cos 2 \theta$ & A.w. & tass \\
\hline & $\sqrt{6} \rho^{2} \sin 2 \theta$ & somis? & sem \\
\hline & $\sqrt{8}\left(3 \rho^{3}-2 \rho\right) \cos \theta$ & sins & 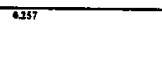 \\
\hline & $\sqrt{8}\left(3 \rho^{3}-2 \rho\right) \sin \theta$ & . & דינד \\
\hline & $\sqrt{8} \rho^{3} \cos 3 \theta$ & sams & 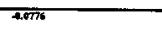 \\
\hline & $\sqrt{8} \rho^{3} \sin 3 \theta$ & \$7/4 & Ams \\
\hline & $\sqrt{5}\left(6 \rho^{4}-6 \rho^{2}+1\right)$ & & wis \\
\hline & $\sqrt{10}\left(4 \rho^{4}-3 \rho^{2}\right) \cos 2 \theta$ & & wom \\
\hline & $\sqrt{10}\left(4 \rho^{4}-3 \rho^{2}\right) \sin 2 \theta$ & & Tind7 \\
\hline & $\sqrt{10} \rho^{4} \cos 4 \theta$ & & .inent \\
\hline & $\sqrt{10} \rho^{4} \sin 4 \theta$ & & .jine \\
\hline & $\sqrt{12}\left(10 \rho^{5}-12 \rho^{3}+3 \rho\right) \cos \theta$ & & .0 .08519 \\
\hline & $\sqrt{12}\left(10 \rho^{5}-12 \rho^{3}+3 \rho\right) \sin \theta$ & & Silm \\
\hline & $\sqrt{12}\left(5 \rho^{5}-4 \rho^{3}\right) \cos 3 \theta$ & & .000 \\
\hline & $\sqrt{12}\left(5 \rho^{5}-4 \rho^{3}\right) \sin 3 \theta$ & & 0.150 \\
\hline & $\sqrt{12} \rho^{5} \cos 5 \theta$ & & solis \\
\hline & $\sqrt{12} \rho^{5} \sin 5 \theta$ & & solses \\
\hline $\bar{y}$ & $\sqrt{7}\left(20 \rho^{6}-30 \rho^{4}+12 \rho^{2}-1\right)$ & & s.0115 \\
\hline
\end{tabular}

These functions are plotted below. Fig 3 a shows the main beam surface plot, phase map and contour plot. Fig $3 \mathrm{~b}$ shows the same information for the backlighter.
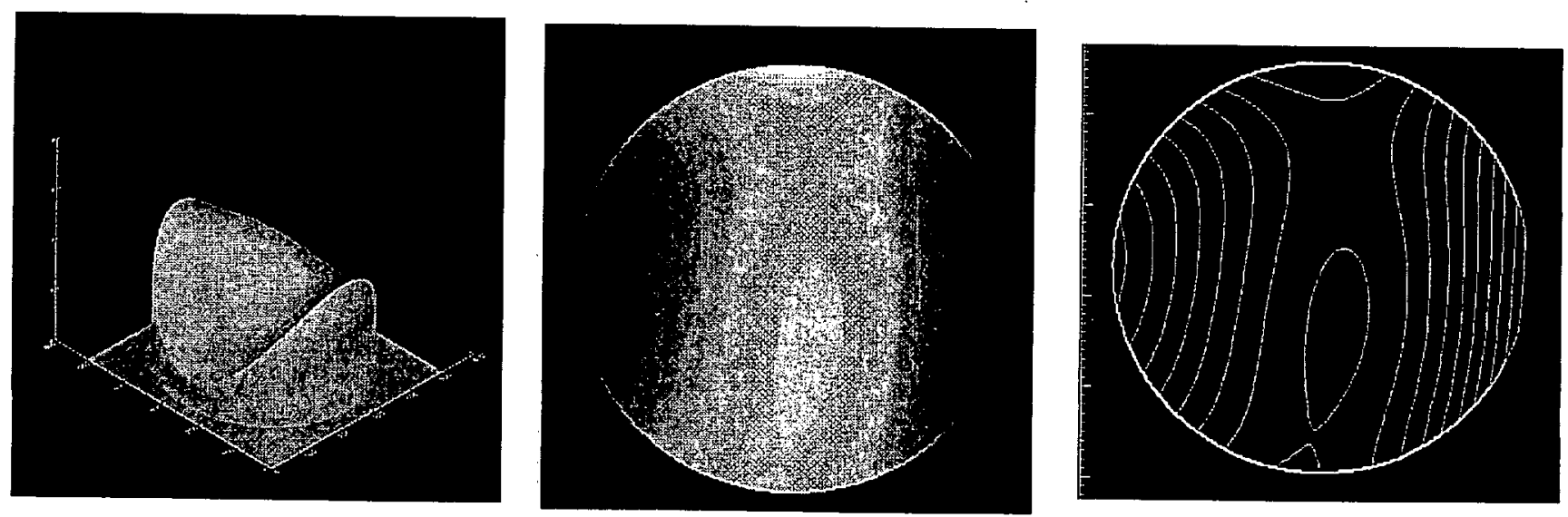

Fig 3a 

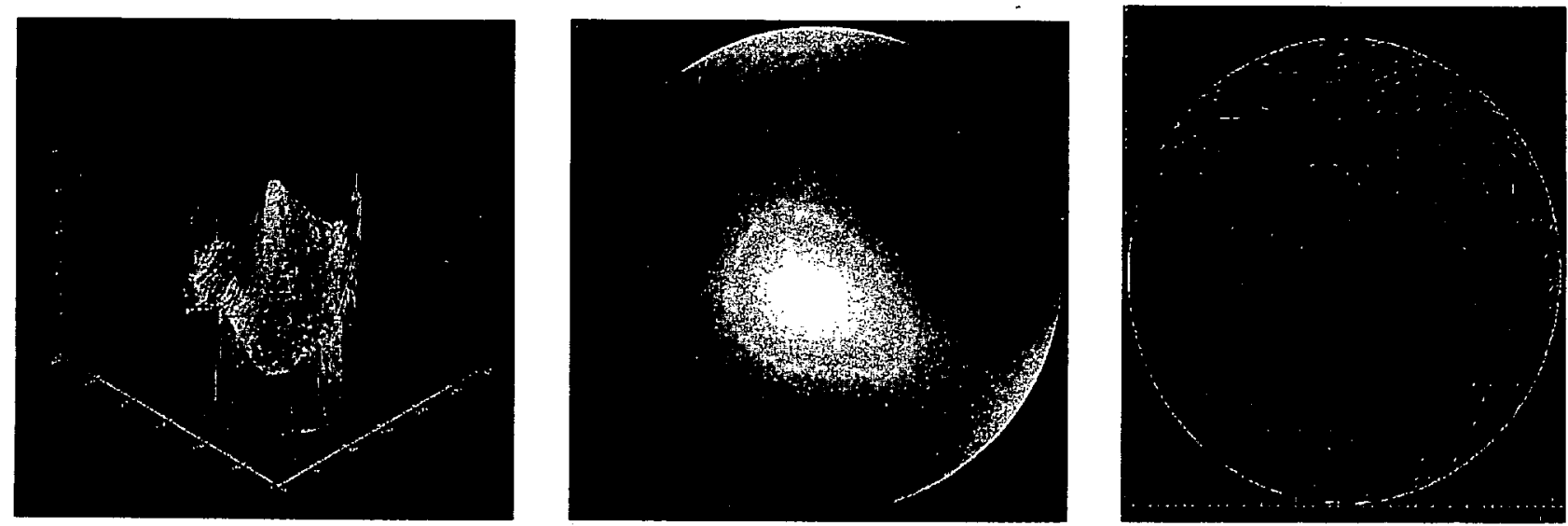

Fig 3b

The P-V correction for the main beam is $5.4 \lambda$ with a gradient of $2.5 \lambda / \mathrm{cm}$. In double pass this is an etch depth of $6.3 \mu \mathrm{m}$ for fused silica with $n=1.45$ at a wavelength of $1054 \mathrm{~nm}$. For the backlighter, the equivalent figures are $9 \lambda \mathrm{P}-\mathrm{V}$ with maximum gradient of $4.5 \lambda / \mathrm{cm}$ and etch depth of in double pass of $10.5 \mu \mathrm{m}$.

\section{Continuous Elements}

\section{INDIRECT WRITE FABRICATION}

The steps involved in production of the main beam correction elements are shown pictorially in Fig. 4 below. Grey scale amplitude masks were laser printed on high quality drafting paper from the phase map described by the mean primary Zernike correction. The mask profile was transferred into a photo-resist layer (Shipley Microposit 1813) on a silica substrate through UV exposure and processing.

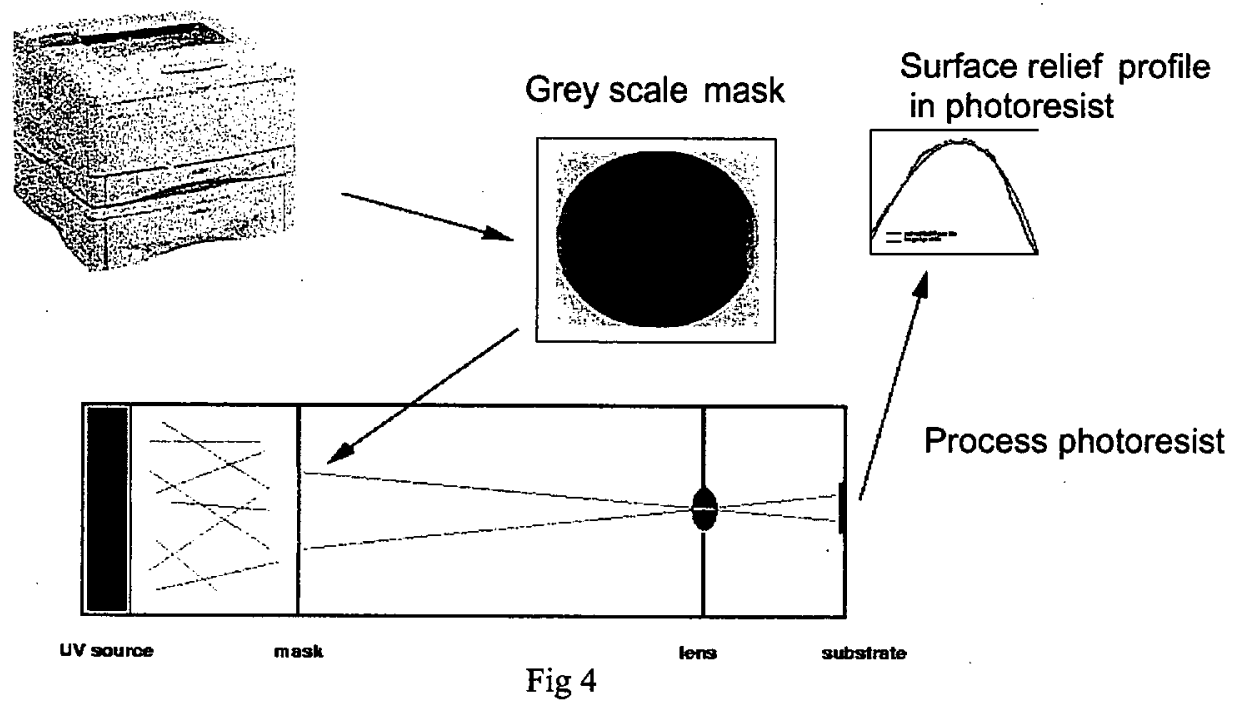

A reactive ion etch process then transferred the relief in the photo-resist layer into the substrate.

High fidelity reproduction requires careful calibration of all the transfer processes to get the required shape and depth. In addition there are issues with production of defect free masks and uniformity of photoresist layers.

An example of a component in photoresist is shown in Fig 5 below. High fidelity reproduction of the desired profile is not achieved in this case. However, an improved mask generation technique using high resolution photographic film and better process calibration is anticipated to yield improved fidelity. 

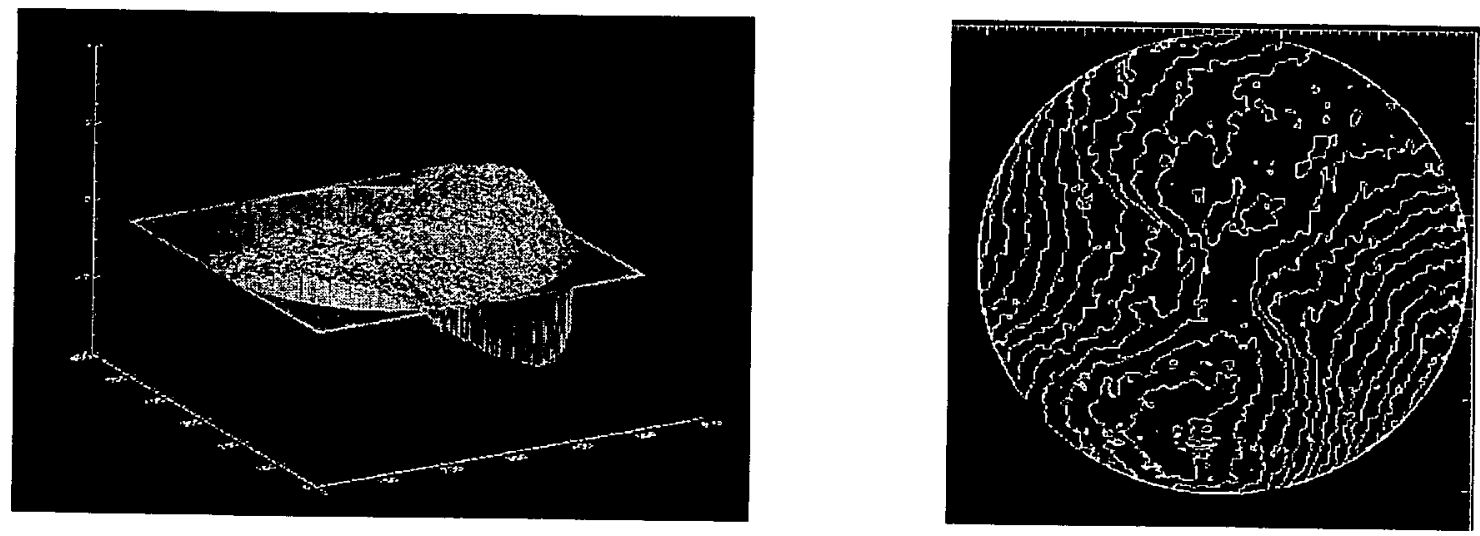

Fig 5

The use of the photoresist coated component as the correcting element in the laser was considered. As there is only one transfer process involved in production it would be easier to fabricate. However, in tests, the photoresist layer was found to damage at the $\mathrm{GW} / \mathrm{cm}^{2}$ intensity levels to which it would be subjected in the beam.

The reactive ion etch step is therefore a necessity to fabricate a bare silica element suitable for AR coating and insertion in the beam. The deep etches led to thermal loading issues on the substrate surface. Fig 6 shows a profile of a silica element. The glitches seen on the line scan are real and due to non-uniformities in the photo-resist and thermal effects in the reactive ion etch phase. These preclude the components use in a high power laser beam.

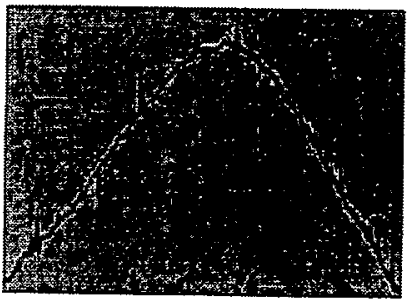

\section{Multi-level elements}

Fig 6

Multi-level elements offer better control over the processes but require more steps to be carried out. The 64 level element involves 6 binary masks. These are e-beam written chrome masks and although the lateral feature size is relatively large, approx. $300 \mu \mathrm{m}$ per step, the mask alignment tolerances are considerably more stringent. Several elements have been fabricated of the desired size. One example is shown below in Fig 7 in which a reasonable approximation to the design as indicated in Fig $3 \mathrm{a}$ is achieved. This is a contour plot of the transmitted wavefront of the element. The generated surface relief profile did not include any compensation for the transmitted wavefront of the substrate, which has been found to be the order of $0.5 \lambda$. This is believed to account for a large part of the difference to the design.

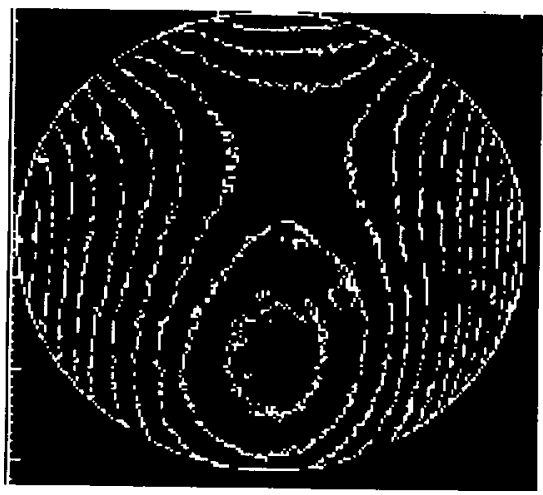

Fig 7

Unfortunately, it is not possible to field these components in a high power beam because enhanced intensity modulation on the beam is initiated by the multi-step structure. '. 


\section{DIRECT WRITE FABRICATION}

A novel wet etch figuring tool has been employed to directly write the surface relief profile on thin glass substrates ${ }^{2}$. The small tool technique uses the controlled application of a flowing liquid chemical etchant HF solution. The tool footprint is defined by the applicator and confined by surface tension. The tool moves relative to the surface being worked. This is shown schematically in Fig 8.
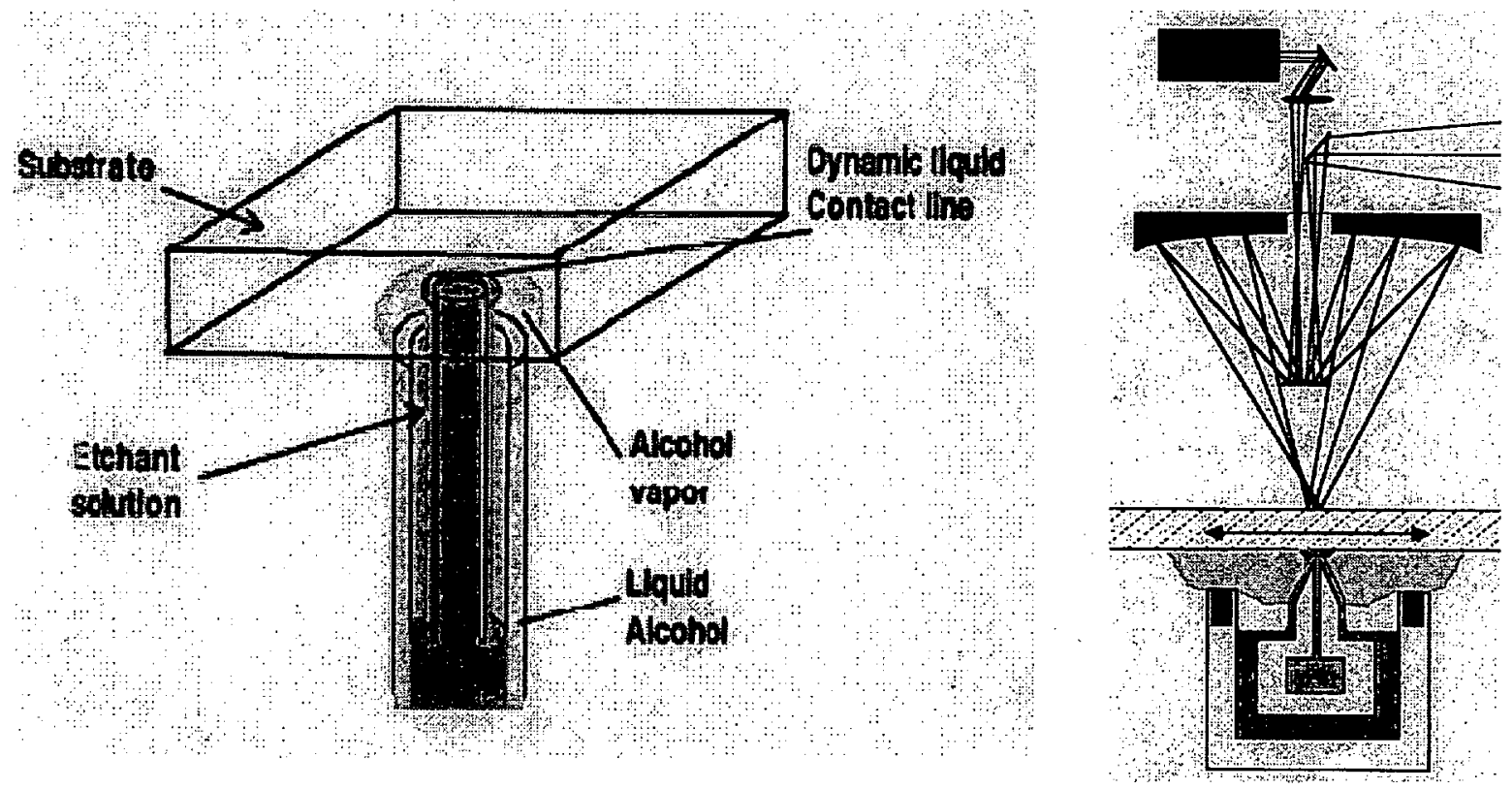

Fig 8

A great advantage of this technique is that direct feedback is used to control the process. A simple interferometer and correction algorithm is used to measure optical thickness change between the front and back surface of the glass plate at the process point. This allows real-time process control and removes any substrate wavefront error.

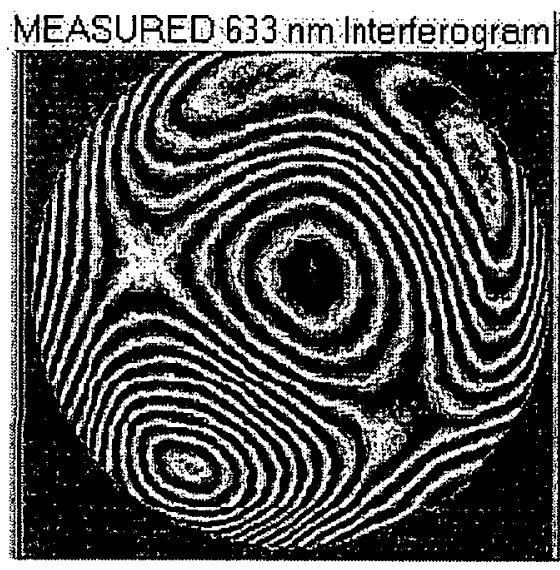

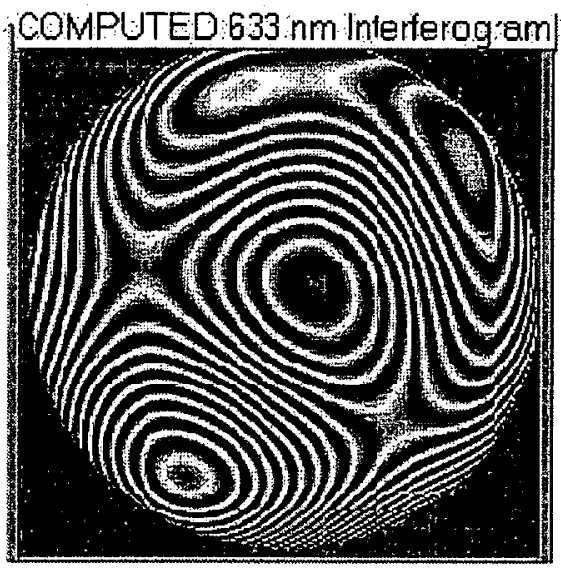

Fig 9

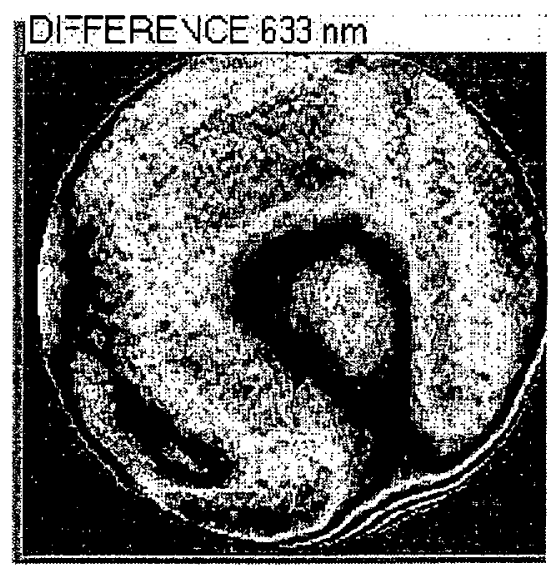

This method has been successfully used to fabricate a part for the backlighter beam. Test data from the tool's built in interferometer is shown in Fig 9. A good match to the desired profile is obtained. 
Further tests in from an off-line interferometer, shown below in Fig 10, confirm the match.
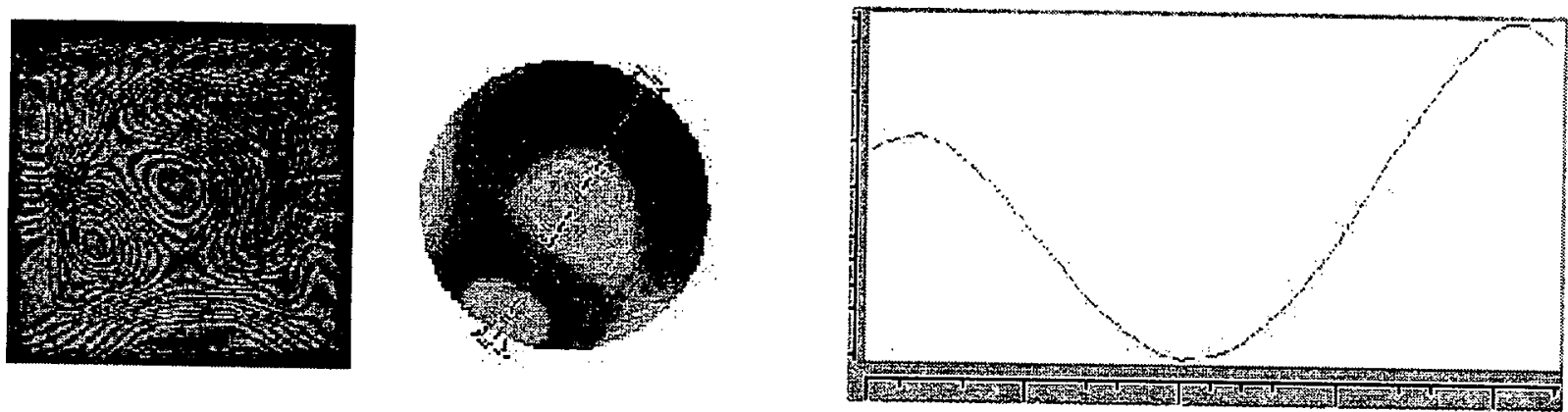

Fig 10

\section{SUMMARY}

The measured output wavefronts of the HELEN laser beams have been shown. Designs for correcting elements have been determined from averaging over multiple shots using Zernike polynomial reconstruction. A Zernike prescription has also been used to specify the element surface relief. Continuous profile surface relief elements have been fabricated using photolithographic techniques but deviations from the design introduced in the fabrication stages have limited the performance as detailed by the match to contour plots. The surface roughness of the final dry etched elements has precluded their use in a high power laser. Work has started to obtain better control the processes involved. Multi-level elements have also been made. They provide a better match to the desired profile but still limited, largely due to failure to compensate for the wavefront error of the substrates. Furthermore, interference induced intensity modulation from the steps precludes their use in a high power laser. An element has been successfully made by a novel direct write method. The wet etch tool features a number of advantages. Feedback during fabrication ensures real-time control and an excellent match to the desired profile with better surface quality has been achieved. It remains to field the element made by the wet etch tool in the laser to assess the value of this technique to improve the
focal performance of the laser.

\section{REFERENCES}

1. Proc SPIE Vol 4270 paper 41,p177 Jan 2001

Bett et al

2. SPIE Vol 4451 paper 28, August 2001

Rushford, Britten, Hoaglan, Summers

3. Paper FB1, Lasers 2000, Albuquerque, NM, USA, December 2000

A Multi-pass Rebuild of the HELEN High Power Glass Laser at AWE

$M J$ Norman et al

4. SPIE Vol 3047 paper 3, pp38-41Oct 96

LMJ

Andre et al

5. SPIE Vol 3492 Supplement

NIF Laser System Performance Ratings

6. SPIE Vol 3492 pp564-572 June 1998

A Combined Phase, Near field and Far Field Diagnostic for Large Aperture Laser Systems

AR Barnes, IC Smith

7. Principle of Optics, Born and Wolf, Chapter 9 


\title{
Fabrication and Applications of Large Aperture Diffractive Optics
}

\author{
Sham Dixit \\ Curly Hoaglan \\ Jerry Britten \\ Mike Rushford \\ Rod Hyde \\ John Toeppen \\ Leslie Summers
}

\begin{abstract}
Large aperture diffractive optics are needed in high power laser applications to protect against laser damage during operation and in space applications to increase the light gathering power and consequently the signal to noise. We describe the facilities we have built for fabricating meter scale diffractive optics and discuss several examples of these.
\end{abstract}

Keywords: Diffraction gratings, Fresnel lenses, Computer generated holograms, large aperture diffractive optics, kinoforms, high power lasers, space telescopes

\section{INTRODUCTION}

In recent years, diffractive optics are finding an increasing number of applications. While diffraction gratings have been extensively used in spectrometers for many years, spot array generators in optics interconnects, fiber Bragg gratings, laser beams shapers, and computer generated holograms are examples of more recent diffractive optics applications. A majority of these applications, especially the more recent commercial ones, require diffractive optics at small aperture sizes (in the $\mathrm{mm}$ to a few $\mathrm{cm}$ size). Large aperture diffractive optics, on the other hand, find applications in high power lasers and in space optics.

In high power lasers, large aperture optics are required in order to minimize the optical damage to the optic. The area of the optic is often set by the laser damage threshold fluence and the maximum amount of the laser energy incident on the optic. For example, in the Nova laser system at the Lawrence Livermore Laboratory (presently nonoperational), the largest optic had to be approximately $70 \mathrm{~cm}$ in diameter in order to propagate $\sim 4 \mathrm{~kJ}$ of laser energy in a $3 \mathrm{~ns}$ pulse at $351 \mathrm{~nm}$. The estimated laser damage fluence at this pulse length was about $2-4 \mathrm{~J} / \mathrm{cm} 2$. In the National Ignition Facility presently under construction at our Laboratory, the laser damage fluence of the optics at $351 \mathrm{~nm}$ is expected to be around $15 \mathrm{~J} / \mathrm{cm} 2$ for the $3 \mathrm{~ns}$ pulse because of the improvements in optical finishing processes. Because of this increased laser damage threshold, more laser energy can be propagated through a given optical aperture. Thus, nearly $11 \mathrm{~kJ}$ of laser energy in a $3 \mathrm{~ns}$ pulse at $351 \mathrm{~nm}$ is expected to be propagated through a $40 \mathrm{~cm}$ square aperture optic. The NIF laser system will have 192 laser beams which together will deliver approximately $1.5 \mathrm{MJ}$ of $351 \mathrm{~nm}$ light to the target [1]. In the current baseline, each of the 192 beams is expected to have a shallow diffraction grating for sampling the laser beam energy and a continuous phase plate for homogenizing the focal spot. These diffractive optic are at the full $40-\mathrm{cm}$ square aperture size.

Another high power application that requires large diffractive optics is the Petawatt laser. Here the chirped pulse amplification (CPA) concept is used to generate ultra high peak powers. In CPA, a short, low energy pulse is 'stretched' in time using a pair of small aperture diffraction gratings. This stretched pulse is amplified through a conventional laser amplifier chain such as the Nova laser. Finally, the amplified, stretched pulse is recompressed in time using another pair of diffraction gratings. The gratings in the compression stage have to be at large apertures in order to be able to withstand the high fluences. The Petawat demonstration at the Lawrence Livermore Laboratory [2] used 94-cm diameter diffraction gratings for pulse compression. These were submicron-pitch, holographically ruled plane gratings. Similar gratings are currently being employed at other Petawatt laser facilities under construction. The size of these gratings is again set principally by the amount of the laser energy generated and the laser damage fluence of the gratings. 
In space applications, large aperture optics are required to increase the light gathering ability and consequently the signal to noise ratio. Examples here are the large aperture primary mirrors in space telescopes. The largest ground based telescope, the Keck telescope in Hawaii, has a $10 \mathrm{~m}$ diameter primary mirror comprising of 36 $1.8 \mathrm{~m}$ wide (comer to corner) hexagonal shaped mirror segments. The largest space based telescope, the Hubble has a monolithic primary mirror of $2.4 \mathrm{~m}$ diameter. The resolution as well as the signal strength are directly related to the aperture size and get better with increasing apertures. In the proposed Gossammer space telescope initiative by NASA [3], the apertures are envisioned to be in the 50-100 m diameter range.

\section{DIFFRACTIVE OPTICS FABRICATION FACILITIES}

Fabrication of large aperture diffractive optics poses many unique challenges. A common way of fabricating such optics is by using lithographic techniques. Here the substrate is coated with a uniform layer of photoresist which then is exposed either to an interference pattern (for fabricating diffraction gratings) or to an intensity pattern transmitted through a mask (for making kinoforms or continuous phase plates). Following the exposure, the photoresist is developed to create a surface relief pattern. At this stage, a gold overcoat can be applied to the relief pattern in resist leading to a reflective grating (for example). On the other hand, for transmissive diffractive optics, the surface relief pattern can be transferred into a glass substrate (often fused silica) by either wetetching or by dry etching. These are fairly standard methods in diffractive optics fabrication and are widely used by the researchers in the field. The uniqueness of our work lies in the application of these techniques to large aperture $(\sim 1 \mathrm{~m}$ size) diffractive optics fabrication.

In conventional lithography, the photoresist coating is applied to the substrate using a spin coater. However, this technology does not easily scale up to larger aperture sizes. There are several reasons for this. The large substrates are often heavy (especially those fabricated for reflective optic) and these can not be spun at the high speeds required for coating. If one uses a dip coating technique, a large amount of photoresist as well as a large dip tank are needed for this. The uniformity of the coating for large apertures produced by the spin coating method is also not very good. For these reasons, we have chosen to apply the photoresist coating to large aperture substrates using a meniscus coating method. A picture of our meniscus coater is shown in figure 1 . In the meniscus coating method, the photoresist is pumped through a precisely machined slot and the optic to be coated is moved at a constant speed slightly above this slot. As the part moves over the flowing photoresist, a thin resist layer is deposited over the optic. The thickness of the deposited layer is proportional to the $2 / 3$ power of the velocity. The meniscus coater is used to apply photoresist and other dielctric layers to substrates up to $1 \mathrm{~m} \times 2 \mathrm{~m}$. For thin layers $(<1 \mu \mathrm{m})$ the flatness is better than $\lambda / 10$ at $633 \mathrm{~nm}$. We have also applied up to $30 \mu \mathrm{m}$ thick coatings with about $5 \%$ uniformity over $\sim 1 \mathrm{~m}$ size substrates.

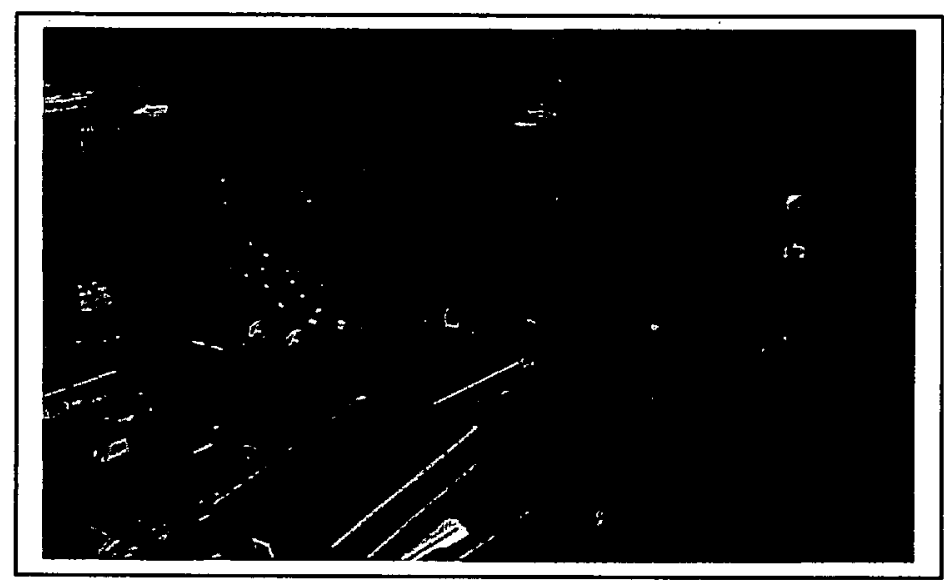

Figure 1. The meniscus coater used for depositing photoresist coatings on large aperture sustrates.

Methods of fabrication for diffraction gratings consist of ruling the grooves with a mechanical tool or an electron beam and interference lithography. Conventional ruling using a mechanical tool becomes impractical for high groove densities. The tool wear also becomes an issue for large sizes. E-beam writing, while possible for a wide 
range of groove densities, quickly becomes cost-prohibitive for large apertures. Interference lithography, on the other hand, is a complementary tool to these methods. In interference lithography a layer of photoresist is exposed to a sinusoidal intensity pattern produced by the interference of two coherent beams. A picture of the large aperture exposure facility is shown in figure 2. A laser beam from a $\mathrm{Kr}$-ion laser is split into two sub-beams and two beams are collimated using custom fabricated $1.1 \mathrm{~m}$ diameter aspheric fused silica lenses. The two collimated beams interfere at an angle and the interference pattern exposes a photoresist layer deposited on over the optic. The exposure area is $\sim 1 \mathrm{~m}$ diameter circle. The groove densities can be up to 2000 lines $/ \mathrm{mm}$ with this facility. Through a careful control of the photoresist type, the exposure and development steps, groove profiles from sinusoidal to lamellar can be produced on up to $1-\mathrm{m}$ wide apertures.

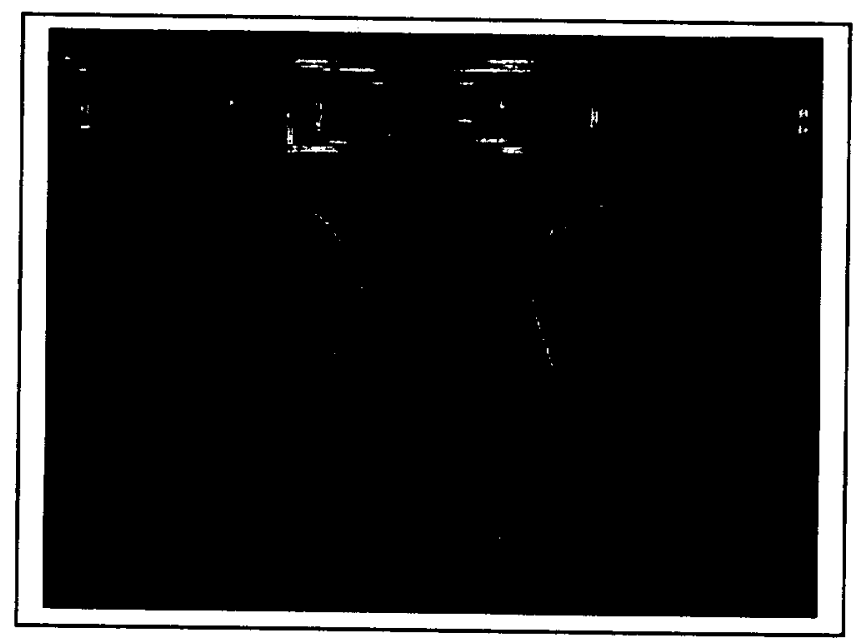

Figure 2. The large aperture interference lithography exposure station. Shown in this picture are the 80-cm fused silica lenses for collimating the $414 \mathrm{~nm}$ laser beams. The current set up uses $1.1 \mathrm{~m}$ diameter lenses.

In addition to the meniscus coater and the holographic exposure facility, we also have mask making and mask-alignment machines, baking, wet processing (for cleaning, developing, HF acid etching etc), vacuum processing (for deposition of metallic and dielectric coatings) and ion-etching facilities for handling up to 1-m size apertures. Finally, the fabrication infrastructure is complemented with a suite of the state of the art optical and diffractive optic design codes.

\section{EXAMPLES}

In this section we discuss some of the large aperture diffractive optics fabricated at our facility. Figure 3 shows a picture of a 94-cm diffraction grating for use in the pulse compression stage of a Petawatt laser. We have made several such gratings for various Petawatt laser facilities under development through out the world. The grating substrate used was a $12 \mathrm{~cm}$ thick BK7. We have also fabricated such gratings on fused silica substrates. The groove density was 1480 lines per $\mathrm{mm}$. The grating structure was first formed in photoresist using interference lithography using two collimated beams and subsequently overcoating it with a thin gold layer. We measured the diffraction efficiency of this grating in a reflecting -1 order in a Litro configuration. A spatial map of the efficiency is shown in figure 4 . We see that the grating efficiency is greater than $92 \%$ over the aperture with good uniformity. To the best of our knowledge, our facility is the only one that can fabricate gratings of such quality and aperture sizes. 


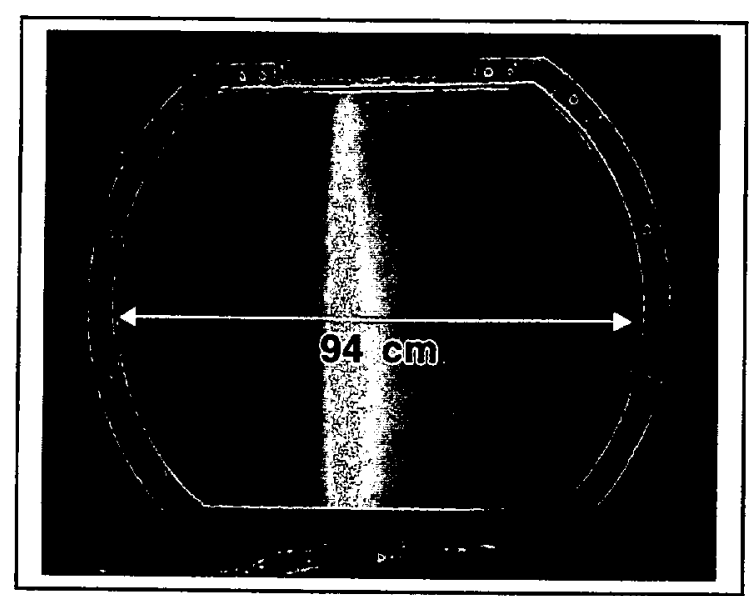

Figure 3 (above). Picture of a 94-cm diameter diffraction grating fabricated using interference lithography.

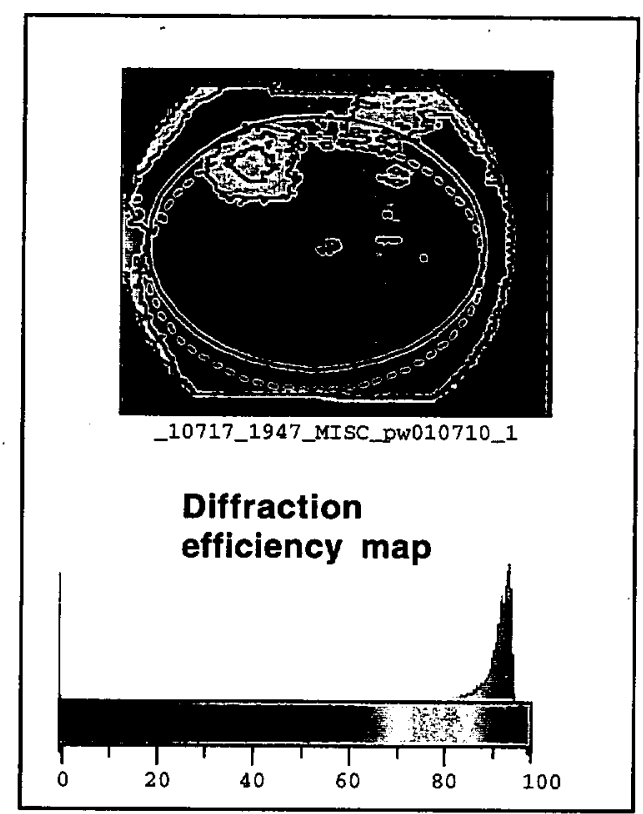

Figure 4 (right). Spatial map of the diffraction efficiency of the 94-cm grating.

Next example we present is a transmission grating in a $40 \times 40 \mathrm{~cm}$ wide, $1-\mathrm{cm}$ thick fused silica substrate fabricated using interference lithography and wet etching. This grating is a prototype of the gratings that will be used for sampling the high power beam on NIF. The requirements are that the grating diffract a small fraction $(\sim 0.3 \%)$ of the incident high power laser beam and bring it to focus at a distance of approximately $1.5 \mathrm{~m}$ from the optic. The sampled beam also needs to clear the main (zeroth order) beam. These implies the grating structure to have shallow $(\sim 20 \mathrm{~nm}$ deep) concentric grooves as shown schematically in figure 5 . We used interference lithography with diverging beams to fabricate the grating structure in photoresist. At the end of the lithography step, the photoresist grooves were separated by clear areas. At this stage we transferred the grating pattern into the fused silica substrate by etching clear areas using a buffered hydrofluoric acid solution. Finally the remaining resist as washed off leaving a clear fused silica optic with $\sim 20 \mathrm{~nm}$ deep grating profile. Figure 6 illustrates the operation of such a beam sampling grating. Here the grating is illuminated with a whitelight source (hidden behind the screen)and the converging and the diverging diffracted orders are seen on the screen past the grating. In the coming years we will be producing several such gratings for the NIF laser beams.

Final example we will discuss here is the development of Fresnel lenses for space telescopes. Other examples such as ion etched fused silica gratings, dielectric multiplayer gratings and kinoform phase plates will not be discussed here because of space limitations. Recently we have proposed a large aperture diffractive telescope concept for imaging of exo-solar planets [4]. Such telescopes consist of a large aperture (25-100 m diameter), long f-number $(50-100)$ transmissive Fresnel lens primary and a much smaller secondary system which is physically separated from the primary but is optically connected to it. Long f-number lenses offer significant advantages over the mirrors by being less sensitive to surface deformities during their operation. Space deployment of such systems requires that the lens be light weight and hence a Fresnel lens. The chromatic effects inherent to a diffractive Fresnel lens can be completely compensated for in the secondary part of the telescope by using a correcting secondary Fresnel lens. We have recently demonstrated the broad-band $(470-700 \mathrm{~nm}$ ), near diffration limited operation of such a telescope in the Laboratory using monolithic Fresnel lenses fabricated in $1-\mathrm{cm}$ thick monolithic fused silica substrates [5]. Figure 7 illustrates a binary mask produced for fabricating a $50-\mathrm{cm}$ diameter $\mathrm{f} / 100$ Fresnel lens. The lens was fabricated in 1$\mathrm{cm}$ thick fused silica substrates using a 2-mask lithography process and wet etching. 


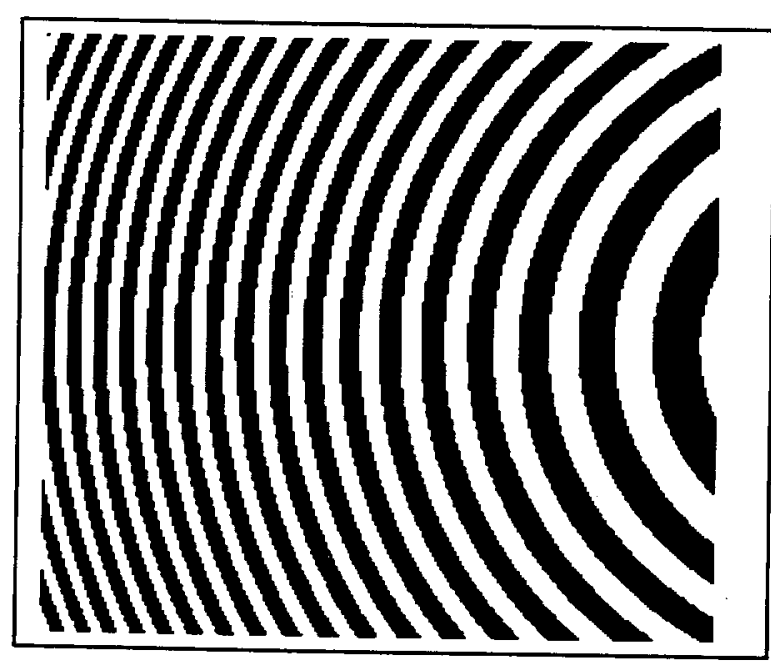

Figure 5. Schematic of the beam sampling grating groove profile.

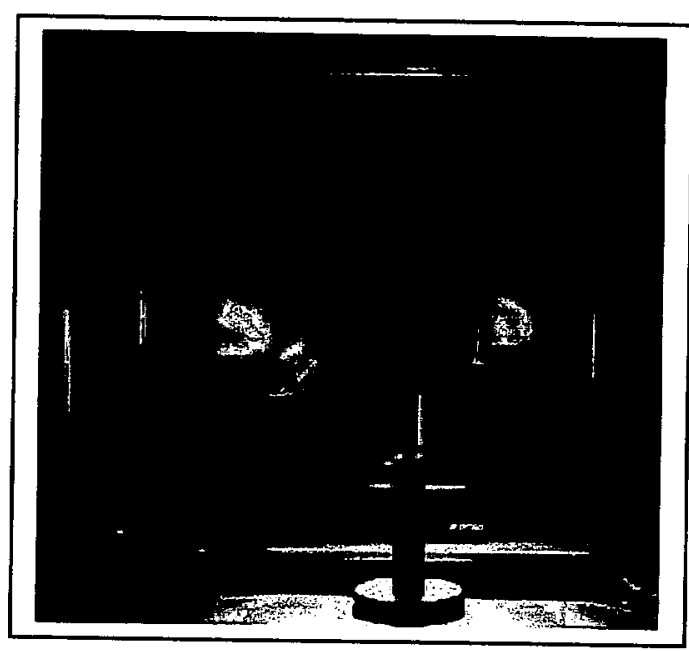

Figure 6. A 40-cm aperture beam sampling grating in operation. A white light source located behind the black circle in the center of the picture illuminates the grating. The converging and the diverging diffracted orders are seen at the screen located behind the optic.

Scale up of such Fresnel lenses to space telescope applications poses some unique challenges. First of all the substrate material has to survive in a space environment during its operational life time. Second, the lens material has to be light weight, packageable and deployable in space. Give these requirements, we have selected $0.7 \mathrm{~mm}$ thin glass sheets as a baseline material for fabricating a 5-m diameter foldable Fresnel lens. These glass sheets are produced in high volume for use in flat-panel displays. The schematic of our 5-m diameter Fresnel lens is shown in figure 8. It consists of 81 panels each approximately $1 \mathrm{~m}$ wide that are patterned with the Fresnel lens pattern and are seamed together using metallic tabs. The particular pattern chosen is so that it can be folded into a 'hat-box' like shape for launch. Some of the folding sequence is also shown in figure 8 .

Figure 7. Picture of a $50-\mathrm{cm}$ diameter Fresnel lens binary mask

Figure 8. Schematic of a 5-m diameter foldable Fresnel lens. Some of the steps in the folding sequence are shown below.
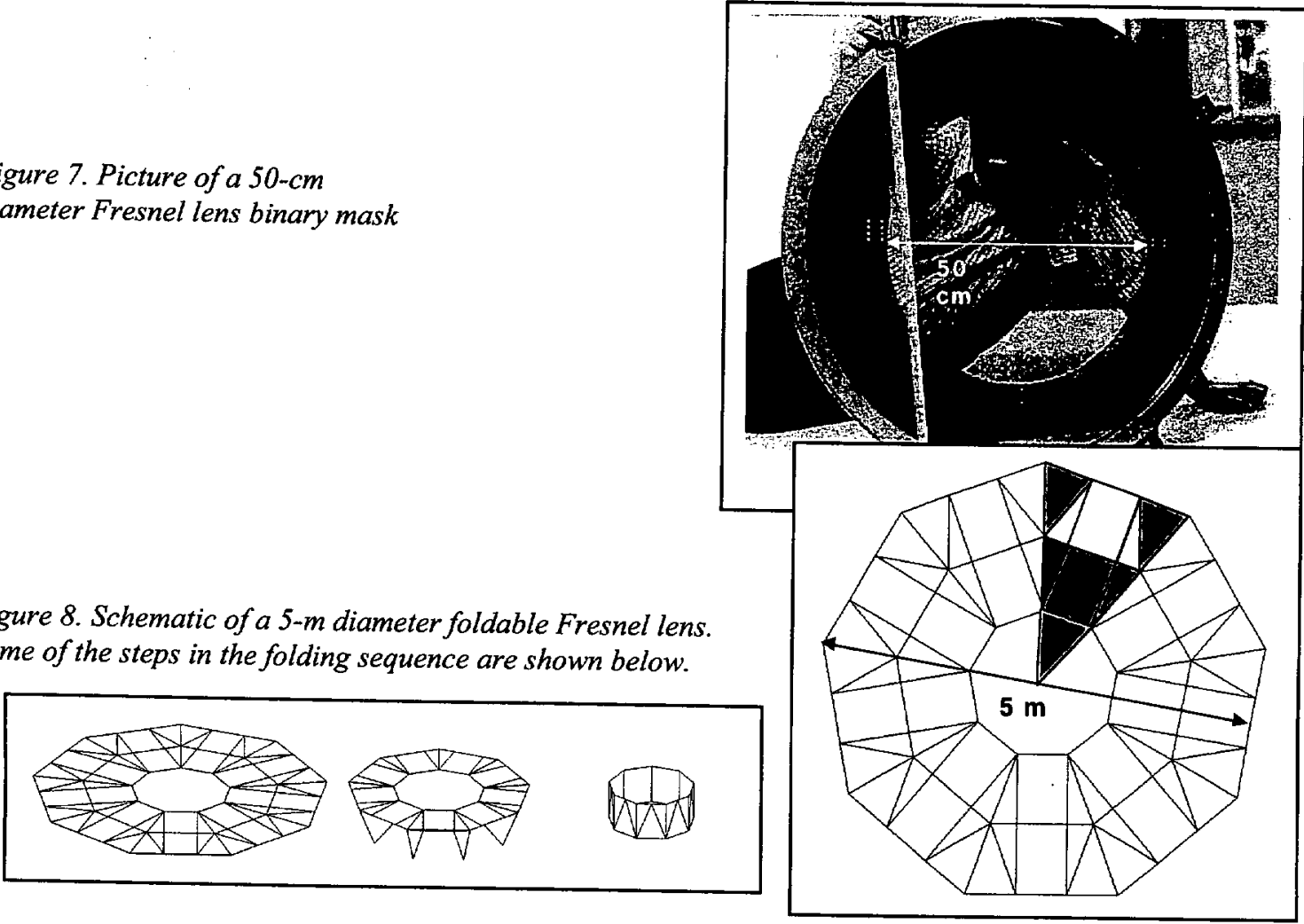
In order to demonstrate the concept of a seamed lens, we built a 6 segmented $75 \mathrm{~cm}$ diameter lens and measured its focusing properties. The glass substrates consisted of $1 \mathrm{~mm}$ thick fused silica polished to $1 / 10$ th wave transmission wavefront. The Fresnel

lens was patterned on each panel separately and the panels were aligned to each other using fiducial marks and seamed together using metal tabs. The seamed lens is shown in figure 9. The nearly diffraction limited focal spot produced by this lens when illuminated by a collimated beam is shown in figure 10 . This confirms that a seamled lens can act as a monolithic lens as long as it is precisely assembled. Following this measurement, we removed the lens from the mount, folded it, unfolded it and placed it back in the mount and measured the focal spot again. This image is also shown in figure 10 . The similarity of the focal spots before and after the folding confirms the validity of the concept of a folding lens for space telescopes. We are currently building a 5-m diameter segmented Fresnel lens.

\section{SUMMARY}

In summary, we have briefly discussed the need for large aperture diffractive optics in high-power laser and space applications. In responding to these needs we have developed over the last several years unique fabrication capabilities for manufacturing diffractive optics at $\sim 1$-m sizes. Finally we illustrated these capabilities with selective examples of diffraction gratings and Fresnel lenses. Currently we are applying this technology for fabricating even larger aperture diffractive optics.

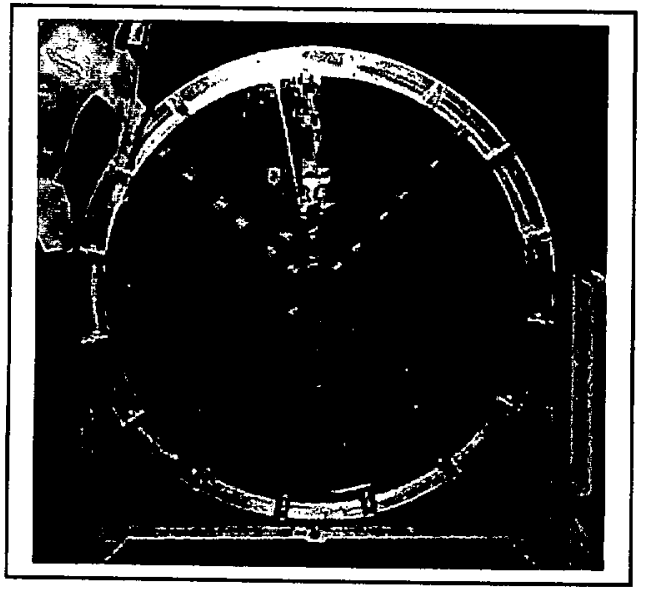

Figure 9. Picture of the 6-segmented seamed Fresnel lens. The radially distributed shiny squares are the metallic seaming tabs.

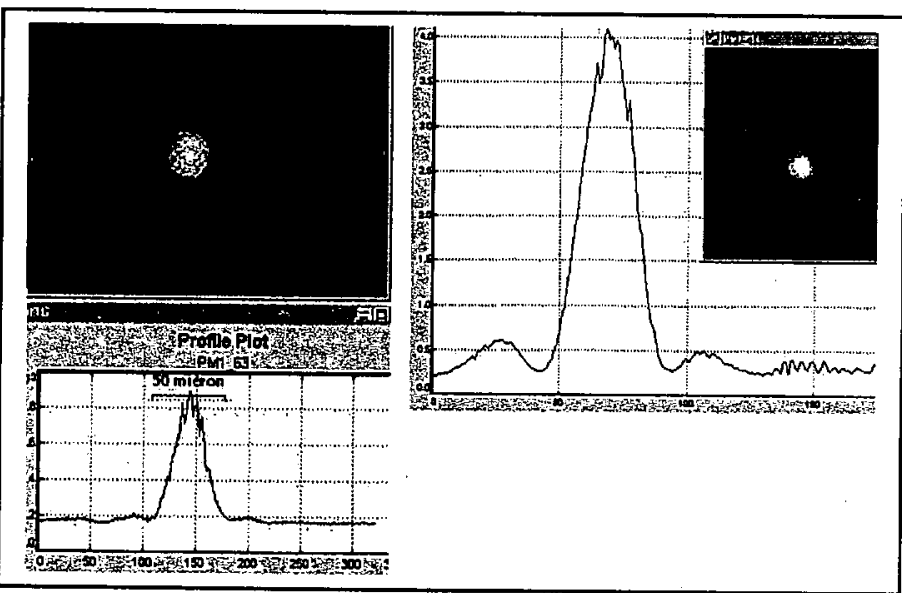

Figure 10. The focal spots produced by the segmented Fresnel lens in the initial assembly shown in figure 9 before folding (left image) and after a folding and an unfolding cycle (right image)

This work was performed under the auspices of the U.S. Department of Energy by the University of California, Lawrence Livermore National Laboratory under contract No. W-7405-Eng-48.

\section{REFERENCES}

[1] "NIF Laser system performance ratings," Supplement to Proceedings of SPIE 3492, 1998

[2] "Petawatt Laser Report," M. D. Perry and B. W. Shore Scientific eds, Lawrence LivermoreNational Laboratory report,UCRL - ID - 124933, December 1996.

[3] "Gossamer Spacecraft Exploratory Research and Technology," NASA Research Announcement, September 14, 2000, NRA 00-OSS-06.

[4] "Eyeglass 1: Very Large Aperture Diffractive Telescopes," R. A. Hyde, Applied Optics $384198-4212,1999$.

[5] "Fabrication of large-aperture lightweight diffractive lenses for use in space," I. M. Barton et. al. Appl. Opt. 40 447-451, 2001. 


\title{
Effects of nonionizing prepulses in high-intensity laser-solid interactions
}

\author{
K. B. Wharton, C. D. Boley, A. M. Komashko, A. M. Rubenchik, J. Zweiback, J. Crane, G. Hays, \\ T. E. Cowan, and T. Ditmire \\ Lawrence Livermore National Laboratory, University of Californin PO. Box 808, Livermore, California 94550 \\ $(-1)$

\begin{abstract}
We present theoretical and experimental evidence that nonionizing prepulses with intensities as low as $10^{8}-10^{9} \mathrm{~W} / \mathrm{cm}^{2}$ can substantially alter high intensity laser-solid interactions. We show that prepulse-heating and vaporization of the target can lead to a preformed plasma once the vapor is ionized by the rising edge of the high-intensity pulse. Our results indicate that peak prepulse intensity is not the only important parameter to duration and the target material is required.
\end{abstract} \\ consider in determining preformed plasma thresholds, and that a more comprehensive analysis of the prepulse
}

The physics of high-intensity laser interactions with solid targets is of wide interest, as near-solid-density plasmas have parameters relevant to inertial confinement fusion as well as being sources of short-pulse $\mathrm{x}$ rays $[1,2]$. At laser intensities above $10^{15} \mathrm{~W} / \mathrm{cm}^{2}$, collisionless processes such as resonance absorption [3] and vacuum heating [4] begin to dominate the laser absorption mechanisms. Both of these mechanisms are highly sensitive to the electron density scale length $L$ $=\left[\left(1 / n_{e}\right)\left(d n_{e} / d x\right)\right]^{-1}$ at the interaction surface, and therefore a prepulse can strongly affect the primary interaction if it creates a preformed plasma. Often the plasma scale length has been experimentally varied with a short-duration, intentional prepulse $[2,5,6]$.

Apart from intentional prepulses, short-pulse lasers are often superimposed on a longer duration, lower-intensity pedestal of amplified spontaneous emission (ASE). This ASE prepulse has also been observed to affect the interaction of the main laser pulse [7-11], and in some recent ultrahigh intensity experiments it is assumed that the ASE produces a substantial preformed plasma in front of the solid target [1214]. However, the conditions where ASE becomes relevant have not been well investigated. Preplasma formation threshold intensities quoted in recent literature, on the order of $10^{11} \mathrm{~W} / \mathrm{cm}^{2}$ [15], arise from the assumption that ASE cannot affect the main interaction unless the field intensity is suffcient to cause plasma breakdown on the solid target. Earlier work has shown that ASE in the $10^{9} \mathrm{~W} / \mathrm{cm}^{2}$ range affects reflection [8], transmission [9], and $x$-ray yield [10], although the latter result used uv laser radiation where singlephoton ionization effects were present. Laser-solid interactions below both the field- and single-photon-ionization thresholds are well documented in the fields of material processing and analytical chemistry [16-18].

In this Rapid Communication we show that peak ASE intensity is not the only appropriate parameter, and a more comprehensive analysis of the prepulse duration and the target material is required to understand the effect of the ASE. High-intensity experiments are presented to support this conclusion, and puzzling results from some previous experiments are reexamined.

For a nonionizing ASE prepulse incident upon a solid target, the primary effect is the thermal heating of the surface. If the surface reaches temperatures on the order of the boiling point, significant densities of neutral vapor can be outgassed. Although this vapor is not yet ionized, the rising edge of the subsequent high intensity pulse will quickly ionize the atoms, turning a preformed vapor into a preformed plasma. Therefore, the vapor profile is directly related to the electron density profile that will in turn affect resonance absorption (and other absorption mechanisms) of the primary laser pulse.

In this context, prepulse intensity and laser "contrast" (the intensity ratio between the ASE and main pulse) are not the most appropriate parameters for ascertaining the preplasma formation. Instead, a more appropriate parameter is an estimate of the energy density at the surface of the target at the time of the main pulse arrival $\left(U_{\text {surf }}\right)$ :

$$
\begin{gathered}
U_{\text {surf }}=\frac{\eta I_{\mathrm{avg}} \sqrt{\tau}}{\sqrt{\alpha}} \quad(\text { for } d>\sqrt{2 \alpha \tau}), \\
U_{\text {surf }}=\frac{\eta I_{\mathrm{avg}} \tau}{d} \quad(\text { for } d \ll \sqrt{2 \alpha \tau}) .
\end{gathered}
$$

Here $\eta$ is the low-intensity surface absorptivity of the target material, $\tau$ is the duration of the ASE, $I_{\text {avg }}$ is the average ASE intensity, $\alpha$ is the thermal diffusivity of the target, and $d$ is the target thickness. For targets thicker than the thermal diffusion depth, the first equation applies; for very thin targets one can assume that the heat is deposited evenly. Although Eq. (1) makes the poor assumption that $\eta$ and $\alpha$ are not functions of temperature, as well as neglecting energy lost to the vapor, $U_{\text {surf }}$ yields a more relevant measure of ASE than the usual "contrast" ratio (or $I_{\text {avg }}$ ). The main predictive feature of $\mathrm{Eq}$. (1) is that similar ASE intensities should have very different effects for target materials with different absorptive and thermal properties.

We experimentally explored the effect of ASE on highintensity interactions using a $35 \mathrm{fs}, \leqslant 130 \mathrm{~mJ}$ Ti:sapphire laser (wavelength $=820 \mathrm{~nm}$ ), defocused to a $500 \mu \mathrm{m}$ spot (full diameter). All shots used $p$-polarization and a $45^{\circ}$ angle of incidence. The targets used were $25 \mu \mathrm{m}$ Ti foils, some overcoated with thin layers of vapor-deposited Al or CH. In order to measure the hot electrons generated at the plasma surface, the primary diagnostic was a time-integrated mea- 


\section{K. B. WHARTON et al.}

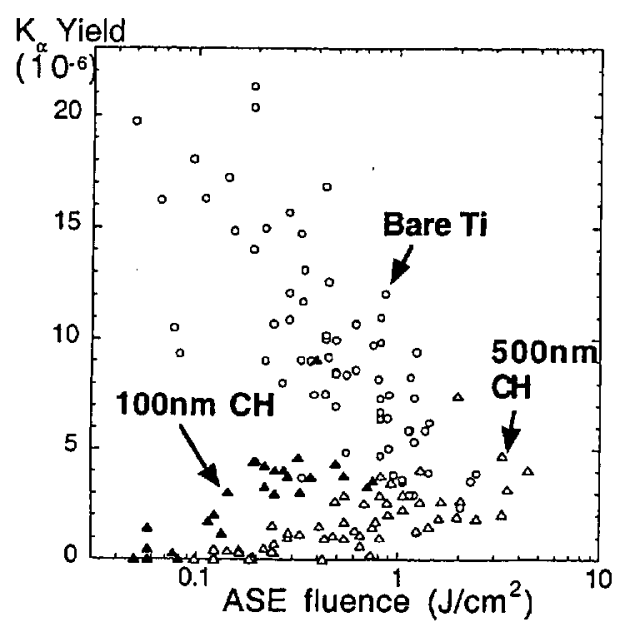

FIG. 1. Conversion efficiency from incident laser energy into outgoing Ti $K_{\alpha}$ x-ray energy is plotted against the total fluence in the prepulse of the laser. Results are shown for $25-\mu \mathrm{m}$-thick bare $\mathrm{Ti}$ foil targets (circles), as well as the same targets overcoated with 100 $\mathrm{nm} \mathrm{CH}$ (closed triangles) and $500 \mathrm{~nm} \mathrm{CH}$ (open triangles).

sure of the Ti $K_{\alpha} \mathrm{x}$ rays produced by the electrons, observed $22 \mathrm{~cm}$ behind the target with a backside illuminated $\mathrm{x}$ ray charge-coupled-device camera. The camera was filtered to operate in a single-photon counting regime so that background $x$ rays could be distinguished from the spectral peak [14]. This technique is "blind" to any low energy electrons produced by the laser, as only electrons over $5 \mathrm{keV}$ can produce a $4.5 \mathrm{keV}$ Ti $K_{\alpha}$ photon. The duration and intensity profile of the ASE were measured on every shot with a 200 ps rise-time fast photodiode; a typical ASE profile rose linearly in time until the arrival of the main pulse.

The ASE level preceding the main pulse was varied in three ways. First, the regenerative amplifier of the laser was pumped below saturation so that shot-to-shot variations shifted the ASE intensity between roughly $10^{-6}$ and $10^{-7}$ of the peak laser intensity. The duration of the ASE was also systematically varied between 0.5 and $2.0 \mathrm{~ns}$ by changing the timing of a fast-slicing Pockels cell in the laser chain. Finally, the total laser energy was varied, always keeping the peak intensity on target between $2 \times 10^{15}$ and $10^{16} \mathrm{~W} / \mathrm{cm}^{2}$. Because the optimum intensity for Ti $K_{\alpha}$ production is 4 $\times 10^{15} \mathrm{~W} / \mathrm{cm}^{2}[19]$, we do not expect a strong $K_{\alpha}$ yield variation over this intensity range.

The measured conversion efficiencies from incident laser energy into outgoing $\mathrm{Ti} K_{\alpha}$ x-ray energy (assuming a uniform distribution of $x$ rays into $4 \pi$ steradians) are plotted in Fig. 1 against total ASE fluence. The results are shown for bare $25-\mu \mathrm{m}$-thick Ti foils, as well as $25 \mu \mathrm{m}$ Ti foils overcoated with a layer of 100 or $500 \mathrm{~nm} \mathrm{CH}$. The bare Ti foils show a strong decrease in $K_{\alpha}$ yield for increasing ASE despite the low absolute ASE levels; the lowest fluence shots on this graph correspond to an average intensity of only $10^{8} \mathrm{~W} / \mathrm{cm}^{2}$ over $0.5 \mathrm{~ns}$. This strongly indicates that some process in the nonionizing $10^{8}-10^{9} \mathrm{~W} / \mathrm{cm}^{2}$ range affects the absorption of the high intensity laser. The neutral-vapor hypothesis presented above is supported by the differences between bare- $\mathrm{Ti}$ and $\mathrm{CH}$-coated targets in Fig. 1. Unlike the

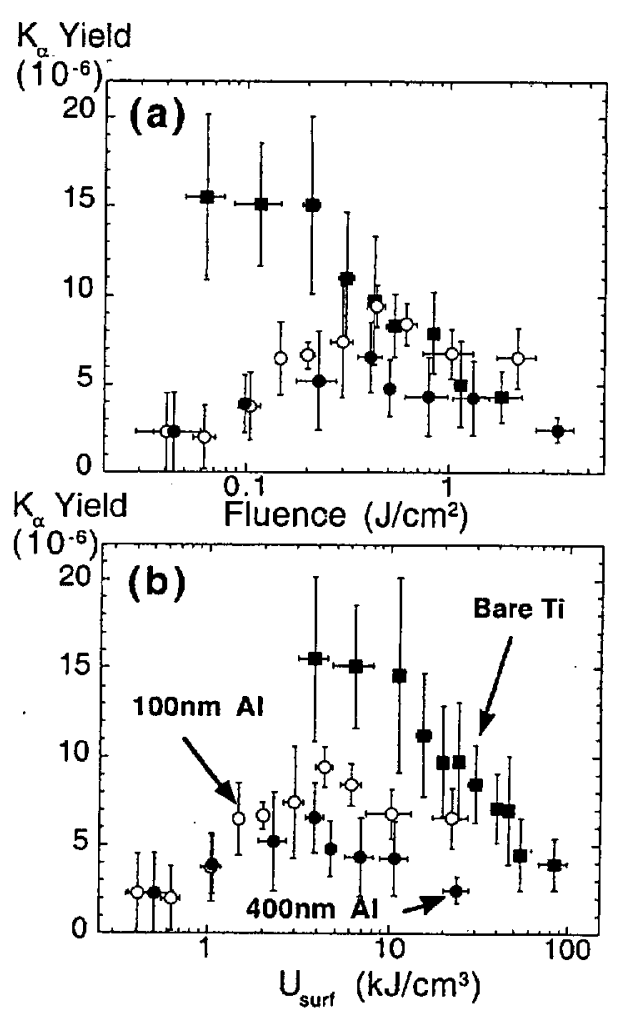

FIG. 2. (a) shows the conversion efficiency from incident laser energy into outgoing Ti $K_{\alpha}$ x-ray energy plotted against total ASE fluence. Results are shown for $25-\mu \mathrm{m}$-thick bare Ti foil targets (squares), as well as the same targets overcoated with $100 \mathrm{~nm} A$ (open circles) and $400 \mathrm{~nm} \mathrm{CH}$ (closed circles). (b) shows the same data plotted against the prepulse parameter $U_{\text {surf }}$ as defined in Eq. (1), which scales as the energy density in the surface of the target.

bare targets, the yield from the coated targets increases with larger ASE fluences. This is consistent with the above model; the thin $\mathrm{CH}$ layer will be transparent to the low intensity ASE and will limit vapor outgassing from the heated Ti foil. However, at some point the heated Ti can melt back through the $\mathrm{CH}$ layer, producing $\mathrm{CH}$ vapor for larger ASE levels.

For a comparison of opaque targets, we deposited a layer of $\mathrm{Al}$ onto the Ti foils in place of the transparent $\mathrm{CH}$, so that the ASE energy would be absorbed on the surface. Figure 2(a) shows the conversion efficiency into $K_{\alpha} \mathrm{x}$ rays as a function of the ASE fluence. The results are shown for bare $25 \mu \mathrm{m}$ Ti foils (the same data set as Fig. 1, binned for clarity), as well as $25 \mu \mathrm{m}$ Ti foils coated with a vapor-deposited layer of 100 or $400 \mathrm{~nm} \mathrm{Al}$. These data seem to indicate quite different optimal fluence levels for the different materials: on bare Ti the optimum yield lies near the lowest ASE fluence $\left(0.05 \mathrm{~J} / \mathrm{cm}^{2}\right)$ while the yield from the Al-coated targets peaks at nearly an order of magnitude higher ASE fluence (near 0.4 $\mathrm{J} / \mathrm{cm}^{2}$ ).

The importance of the deposited surface energy density, however, becomes apparent when these yields are plotted against $U_{\text {surf }}$ [as determined from Eq. (1)] in Fig. 2(b). For bare Ti targets $U_{\text {surf }}$ is calculated using the low-intensity value for $\eta(40 \%)$ and the melting-point value for $\alpha(0.07$ $\mathrm{cm}^{2} / \mathrm{s}$ ). The $400 \mathrm{~nm}$ Al-coated targets used the corresponding 
aluminum parameters of $\eta=20 \%$ and $\alpha=0.5 \mathrm{~cm}^{2} / \mathrm{s}$. The $100 \mathrm{~nm}$ Al-coated targets were sufficiently thin to apply Eq. (1b), which assumes the absorbed ASE energy was evenly deposited throughout the Al layer (and minimal thermal conduction into the much lower thermal conductivity $\mathrm{Ti}$ substrate). In Fig. 2(b) the qualitative scaling is now similar between targets: both Al-coated targets show a peak in the $K_{\alpha}$ yield at a surface energy density of $4-5 \mathrm{~kJ} / \mathrm{cm}^{3}$, which is also consistent with the available data from the bare Ti targets. This implies that this energy density corresponds to a vapor scale length that optimizes the production of fast electrons.

In order to estimate the density and scale length of this vapor plume, we simulated the heat deposition with a $1 \mathrm{D}$ hydrodynamics and vaporization code [20] designed to model material drilling by laser intensities between $10^{8}$ and $10^{11} \mathrm{~W} / \mathrm{cm}^{2}$. This code (THALES) uses temperaturedependent material properties (absorptivity, diffusivity, etc.) and calculates heat transfer in the target, vaporization, and hydrodynamics of the expanding plume. For a given laser intensity profile, the code is believed to provide a good estimate of the target surface temperature. However, the mean free path in the outgassed vapor is on the order of a micron, which is comparable to the vapor scale length. For this reason we analytically computed the vapor density profile from the temperature history of the target, assuming the velocity distribution of the vapor at the surface is a one-directional Maxwellian [21]. From the vapor density, a plasma density is then calculated by noting that the intensity of our main laser pulse would be expected to triply ionize the $\mathrm{Al}$ vapor and $4 x$-ionize the Ti vapor.

The final plasma density profile from these simulations and calculations are shown in Fig. 3 for both Ti and Al targets and various ASE intensities assuming a 1.0 ns ASE ramp. There is a clear material difference; $T i$ targets require less of an ASE prepulse than Al targets to produce similar plasma densities. Also, it is apparent that ASE intensities on the order of $10^{9} \mathrm{~W} / \mathrm{cm}^{2}$ can easily produce overcritical plasmas via the vaporization mechanism described above. This is typically two orders of magnitude smaller than previous estimates of relevant ASE levels.

For both overdense plasma density profiles shown in Fig. 3 , the scale length $L$ is very close to $0.2 \mu \mathrm{m}$. This is near to the optimum plasma scale length for resonance absorption for our laser parameters [3]. However, there is a quantitative difference between the $U_{\text {surf }}=12-24 \mathrm{~kJ} / \mathrm{cm}^{3}$ which yield the optimum plasma profiles and the $U_{\text {surf }}=4-5 \mathrm{~kJ} / \mathrm{cm}^{3}$ seen to optimize the $K_{\alpha} \mathrm{x}$-ray yield in our experiments. Indeed, the lower-density profiles shown in Fig. 3 are closer to the latter numbers, but those underdense plasmas would not be expected to enhance resonance absorption. A number of factors could give rise to this quantitative difference, including inaccurate estimates for the liquid state properties used in THALES, which are not well established. Another possibility is that an underdense plasma shelf might optimize the hot electron production not through resonance absorption, but through vacuum heating $[4,22]$. (We also note that ASEinduced corrugations of the target surface has been proposed as an absorption-altering mechanism [8].) Nonetheless, the

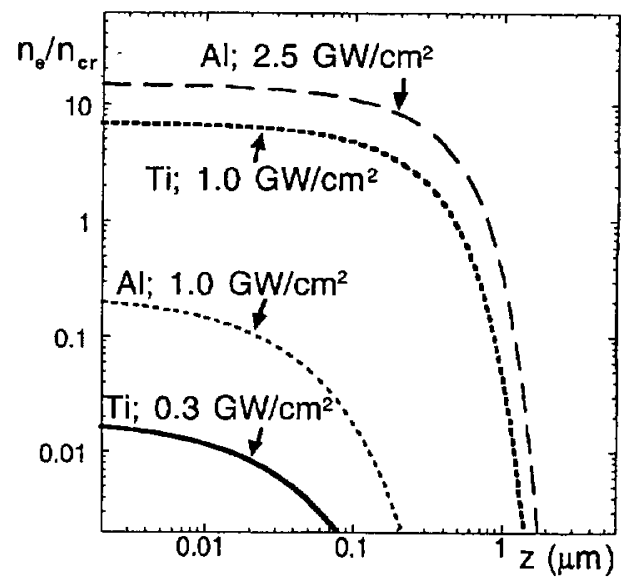

FIG. 3. Calculated plasma density profiles are plotted in units of electron density (normalized to the critical density for $820 \mathrm{~nm}, n_{\mathrm{cr}}$ $=1.66 \times 10^{21} \mathrm{~cm}^{-3}$ ), as a function of distance from the surface of the target. Results are shown for $400 \mathrm{~nm} \mathrm{Al}$ targets (thin lines) and $25 \mu \mathrm{m} \mathrm{Ti}$ targets (thick lines), for peak ASE intensities of 3 $\times 10^{8} \mathrm{~W} / \mathrm{cm}^{2}$ (solid line), $10^{9} \mathrm{~W} / \mathrm{cm}^{9}$ (short-dashed lines), and 2.5 $\times 10^{9} \mathrm{~W} / \mathrm{cm}^{-2}$ (long-dashed line). All ASE profiles are $1 \mathrm{~ns}$ in duration, and have a linearly rising profile; i.e., the average intensity is half of the peak intensity. Equivalent values of $U_{\text {surf }}$ [from Eq. (1)] are as follows: $4.5 \mathrm{~kJ} / \mathrm{cm}^{3}$ (lower intensity $\mathrm{Al}$ ), $11.5 \mathrm{~kJ} / \mathrm{cm}^{3}$ (higher intensity $\mathrm{Al}$ ), $7 \mathrm{~kJ} / \mathrm{cm}^{3}$ (lower intensity Ti), and $24 \mathrm{~kJ} / \mathrm{cm}^{3}$ (higher intensity Ti).

THALES simulation confirms the qualitative material scaling seen in the experiments, and also demonstrates that overcritical plasmas can be generated by target outgassing.

Further evidence for the relevance of these ASE levels can be found in previously published high-intensity lasersolid experiments. Several groups have observed a dramatic difference between high-intensity laser absorption in opaque and transparent targets $[11,14,23]$, which can likely be explained in terms of ASE effects. In particular, Ref. [11] noted that a difference in $\mathrm{X}$-ray yield from $\mathrm{Al}$ and glass targets was strongly dependent on the duration of the ASE before the arrival of the main pulse, yet an interferometric diagnostic saw no preformed plasma on either target at standard ASE levels. Neutral vapor outgassing may explain these results; the surface absorption would be much higher in Al than in glass, and therefore the ASE could cause neutral vapor outgassing on only the $\mathrm{Al}$ targets. However, a plume of neutral vapor would be invisible to standard interferometric diagnostics before the arrival of the main pulse.

Other results in the literature also support this model. Jiang et al. [24] measured the density of x-ray-emitting regions from laser-solid interactions, and found very different results between $\mathrm{Ti}, \mathrm{Al}$, and $\mathrm{NaCl}$ targets. Although these experiments were performed at intensities of $10^{19} \mathrm{~W} / \mathrm{cm}^{2}$, their stated contrast ratio of $10^{-10}: 1$ indicates that their ASE was $\sim 10^{9} \mathrm{~W} / \mathrm{cm}^{2}$. The authors consider the possibility of a prepulse, but observe that in transparent targets $(\mathrm{NaCl})$ the $\mathrm{x}$-ray emission comes from a solid density plasma, and so they rule out a relevant prepulse which might pre-expand their target. However, the lower plasma density measured in their Al targets (and the lack of signal from Ti targets) hint 


\section{K. B. WHARTON et al.}

that ASE may have been affecting these opaque targets, sparing only the transparent $\mathrm{NaCl}$.

This target vaporization analysis should take into account not only changes in target material, but also thickness variations of thin foil targets [where Eq. 1(b) applies]. Feurer et al. [25] report evidence of resistive inhibition of hot electron transport through very thin $(400-700 \mathrm{~nm}) \mathrm{Al}$ layers on a $\mathrm{Cu}$ substrate. This is at odds with high levels of hot electrons that have been seen to propagate through much thicker $(\sim 500 \mu \mathrm{m})$ layers of $\mathrm{Al}$ in other experiments [14]. Another interpretation of these results is that increasing the thickness of the Al layer from 400 to $700 \mathrm{~nm}$ allowed the prepulse heat to diffuse into the target, preventing surface vaporization. Note that the thermal diffusion depth for a $3 \mathrm{~ns}$ ASE pulse in warm Al is $(2 \alpha \tau)^{1 / 2}=550 \mathrm{~nm}$. The experiments presented here are not exactly applicable to this previous work, as our substrate (Ti) has a lower thermal conductivity than $\mathrm{Cu}$, so further experiments would be needed to test this interpretation.

With the experiments presented above, we show that ASE-induced target vaporization and subsequent formation of preformed plasmas is an important effect in laser-solid interactions. This conclusion has implications for a wide range of experiments, not only in setting relevant goals for ASE-supression in high power laser systems, but also in target design, data interpretation, and comparison between various high-intensity experiments.

The authors gratefully thank D. Munro and S. Hatchett for crucial contributions as well as R. A. Smith for useful discussions. This work was conducted under the auspices of the U.S. Department of Energy by Lawrence Livermore National Laboratory under Contract No. W-7405-ENG-48.
[1] D. Kühlke, U. Herpes, and D. Von der Linde, Appl. Phys. Lett. 50, 1785 (1987).

[2] M. M. Murnane, H. C. Kapteyn, and R. W. Falcone, Phys. Rev. Lett. 62, 155 (1989).

[3] V. L. Ginzburg, The Propagation of Electromagnetic Waves in Plasmas (Pergamon, New York, 1964), p. 260.

[4] F. Brunel, Phys. Rev. Lett. 59, 52 (1987).

[5] H. W. K. Tom and O. R. Wood, Appl. Phys. Lett. 54, 517 (1989).

[6] S. Bastiani et al., Phys. Rev. E 56, 7179 (1997); Th. Schlegel et al., ibid. 60, 2209 (1999).

[7] O. Willi et al., Europhys. Lett. 10, 141 (1989).

[8] J.-C. Gauthier et al., in Laser Interactions with Atoms, Solids, and Plasmas, NATO ASI Series, edited by R. M. More (Plenum, New York, 1994), p. 357.

[9] T. R. Boehly et al., Phys. Plasmas 8, 231 (2001).

[10] J. A. Cobble et al., J. Appl. Phys. 69, 3369 (1991).

[11] R. Benattar et al., Opt. Commun. 88, 376 (1992).

[12] U. Teubner et al., Phys. Rev. E 54, 4167 (1996).

[13] F. N. Beg et al., Phys. Plasmas 4, 447 (1997).

[14] K. B. Wharton et al., Phys. Rev. Lett. 81, 822 (1998).
[15] B. F. K. Young, B. G. Wilson, D. F. Price, and R. E. Stewart, Phys. Rev. E 58, 4929 (1998).

[16] Yu. V. Afanasyev, O. N. Krokhin, and G. V. Sklizkov, IEEE J. Quantum Electron, QE-2, 483 (1966).

[17] V. A. Batanov, F. V. Bunkin, A. M. Prokhorov, and V. B. Fedorov, Sov. Phys. JETP 36, 311 (1973).

[18] B. N. Chichov et al., Appl. Phys. A: Mater. Sci. Process. 63, 109 (1996).

[19] Ch. Reich, P. Gibbon, I. Uschmann, and E. Förster, Phys. Rev. Lett. 84, 4846 (2000).

[20] C. D. Boley and J. T. Early, Proceedings of the International Congress on Applications of Lasers and Electro-Optics (ICALEO) (Laser Institute of America, Orlando, FL, 1994), Vol. 2500 , p. 499.

[21] S. I. Anisimov et al., Effects of High-Power Radiation on Metals (NTIS, Springfield, VA, 1971).

[22] P. Gibbon and A. R. Bell, Phys. Rev. Lett. 68, 1535 (1992).

[23] A. Saemann and K. Eidmann, Appl. Phys. Lett. 73, 1334 (1998).

[24] Z. Jiang et al., Phys. Plasmas 2, 1702 (1995).

[25] T. Feurer et al., Phys. Rev. E 56, 4608 (1997). 


\title{
Space-charge effects in high brightness electron beam emittance measurements
}

\author{
S. G. Anderson and J. B. Rosenzweig \\ UCLA Department of Physics and Astronomy, 405 Hilgard Avenue, Los Angeles, California 90095
}

G. P. LeSage and J. K. Crane

Lawrence Livermore National Laboratorv. R.iuermnro Califnrnia 94550

\begin{abstract}
The measurement of emittance in space-charge dominated, high brightness beam systems is investigated from conceptual, computational, and experimental viewpoints. As the self-field-induced collective motion in the low energy, high brightness beams emitted from photoinjector if guns are more important in determining the macroscopic beam evolution than thermal spreads in transverse velocity; traditional methods for phase space diagnosis fail in these systems. We discuss the role of space charge forces in a traditional measurement of transverse emittance, the quadrupole scan. The mitigation of these effects by use of multislit- or pepper-pot-based techniques is explained. The results of a direct experimental comparison between quadrupole scanning and slit-based determination of the emittance of a $5 \mathrm{MeV}$ high brightness electron beam are presented. These data are interpreted with the aid of both envelope and multiparticle simulation codes. It is shown that the ratio of the beam's $\beta$ function to its transverse plasma wavelength plays a central role in the quadrupole scan results. Methods of determining the presence of systematic errors in quadrupole scan data are discussed.
\end{abstract}

\section{INTRODUCTION}

There exists an emerging class of very high brightness electron beams, which are created through photoemission from cathodes embedded in high field radio frequency guns, and driven by picosecond lasers. These systems, termed rf photoinjectors [1,2], produce high brightness beams by creating high charge beams with the time structure of the driving laser, and preserving both the temporal extent and the transverse phase space quality, despite the presence of extremely large space-charge forces. The preservation of the electron beam pulse length is accomplished by application of large amplitude rf accelerating fields, while the transverse phase space quality is controlled and optimized by judicious balancing of space charge defocusing and externally applied focusing forces. Perhaps one of the more remarkable aspects of the beam's evolution is that the transverse emittance can be greatly expanded and subsequently diminished during one plasma oscillation occurring under optimized beam transport conditions. This process, termed space charge emittance compensation, has been studied in detail in theoretical, computational, and experimental investigations [3-6].

As a result of the advancement in the understanding of the emittance compensation process in these beams, new photoinjectors are designed specifically to use external focusing and acceleration to balance the extreme spacecharge forces in the bunch [7]. The same care must be taken in the implementation of diagnostics for photoinjector beams. In particular, measurement schemes that deduce a beam's properties from its behavior in a drift region need to take into account the space-charge forces in the beam, as these forces usually control the beam's behavior. In addition, space charge can change the property one is attempting to measure in the region of measurement. For example, the emittance compensation process relies on the fact that the emittance changes (oscillates) in a drift region after a solenoid. Another issue in the measurement of photoinjector beams is the stability of the photocathode drive laser. Fluctuations in the energy and size of the drive laser cause shot-to-shot fluctuations in the beam size and emittance both directly and indirectly (by changing the space-charge forces and the emittance compensation process.) For this reason, in photoinjectors single shot measurements are preferable to those requiring many shots.

In the remainder of this paper, we review and examine the effects of space charge in two different emittance measurement techniques - the multislit-based and quadrupole (quad) scan systems. This paper builds on previous work on the subject of emittance measurement systems [8] and measuring the emittance of photoinjector beams [9]. We discuss here some of the relevant issues in Refs. [8] and [9] and clarify some key differences between this work and those. We then describe an experiment performed at the LLNL Thomson source photoinjector where these two techniques were used to measure the emittance of a highly space-charge dominated beam. The results of this experiment, which show that the emittance found with the quadrupole scan is in disagreement with that measured with the multislits (the quad scan results are consistently higher), are compared with simulation and analytical models. 


\section{MULTISLIT-BASED EMITTANCE MEASUREMENT}

The first purpose of a phase space measurement system based on multislit collimation [10] is to slice up the beam into physically separated linelike sources, or beamlets, as shown in Fig. 1. This slicing combined with a drift, which reveals the spread in velocities as spatial information at an intensity-sensitive detector, allows a full reconstruction of one of the beam's transverse phase planes, e.g., $\left(x, p_{x}\right)$. This technique is especially useful when implemented as a single-shot diagnostic in photoinjectors, where shot-byshot beam parameter fluctuations may dominate multishot measurements. Multishot variants of the slit-based method involving one or two slits, as well as the pepper-pot method (the 2D analog of the multislit system, which can give the four-dimensional transverse phase space), are discussed in detail in Ref. [8].

The method of determining the phase space from the multislit technique is reviewed below. After introduction of the technique, we discuss the use of the multislit array to mitigate collective effects in space-charge dominated beams which can be large enough to invalidate the measurement. While these effects have been mentioned in previous analyses [8-10], in this paper they are the focus of our concern. They are therefore examined in depth, not only in the context of the multislit method, but also in the quadrupole scan measurement.

The beamlets are created by the multislit mask at a constant spacing $w$, which is much larger than the slit width $d$. The beamlet distribution is then detected downstream of the multislit mask, and the beamlets resolved. The width of each beamlet gives a measure of the width of the transverse momentum distribution at each slit, and the centroid of the beamlets gives the correlated offset of the momentum distribution at each slit. Assuming a drift length $L$ between the multislit mask and the detecting plane, the measured trace space distribution is approximated by a series ( $m=1, \ldots, N)$ of distinct angular distributions at regularly spaced spatial (we take this dimension to be $x$ ) intervals. The beamlets yield the correlated beam divergences

$$
x_{m, c}^{\prime}=\left\langle x_{m}-x_{m, c}\right\rangle / L=\left\langle x_{m}-m w\right\rangle / L,
$$

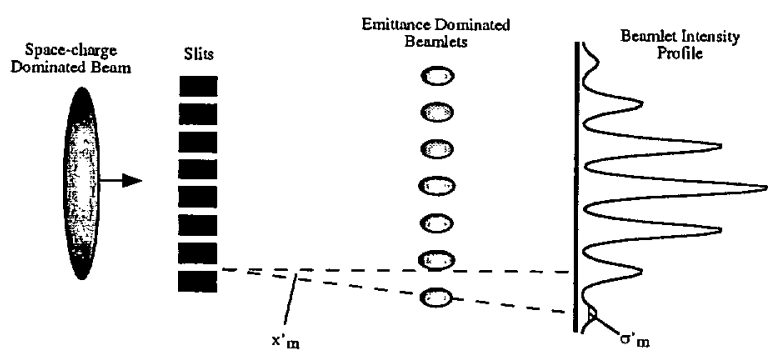

FIG. 1. (Color) Illustration of the multislit-based emittance measurement scheme. and the rms spreads in divergence

$$
\sigma_{m}^{\prime}=\sqrt{\left\langle x_{m}^{2}\right\rangle / L^{2}-\left(x_{m, c}^{\prime}\right)^{2}},
$$

where the average \langle\rangle is performed over the distribution in the $m$ th beamlet. Here we have assumed that the final spread in detected beamlet size is much greater than the slit width. Once these parameters are extracted from the data, a graphical trace space distribution can be constructed, as illustrated in Fig. 2.

Note that the trace space distribution is centered in both $x$ and $x^{\prime}$, by subtracting off the overall value of the centroids,

$$
x_{m, c}=\left.\left(x_{m, c}-x_{c}\right)\right|_{\text {old }}=m w-\frac{\sum_{m=1}^{N} I_{m} m w}{\sum_{m=1}^{N} I_{m}},
$$
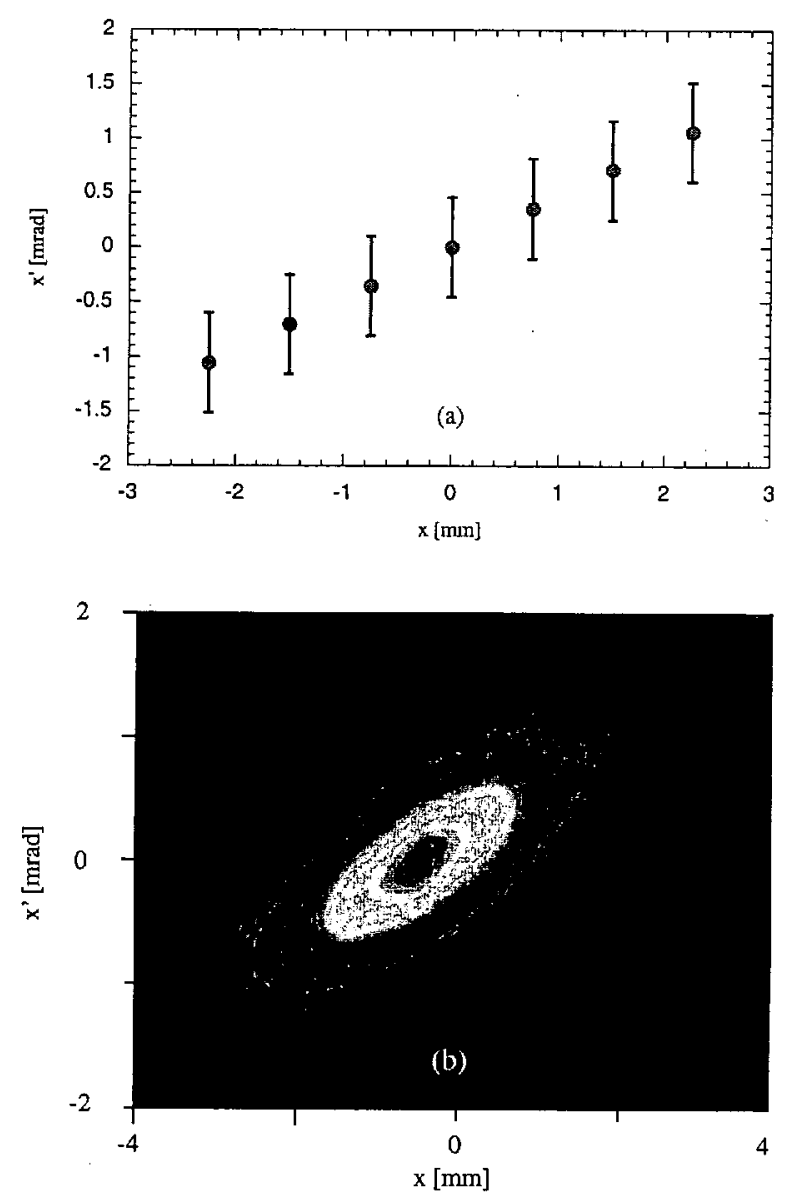

FIG. 2. (Color) (a) Beam trace space constructed from the beamlet intensity profile illustrated in Fig. 1. Each point represents the position of a beamlet in trace space and the error bars indicate the thermal spread of the beamlets. (b) Contour plot representation of the same data. Here the relative weights of the beamlets are resolved. 
and

$$
x_{m, c}^{\prime}=\left.\left(x_{m, c}^{\prime}-x_{c}^{\prime}\right)\right|_{\mathrm{old}}=\left.\left(x_{m, c}^{\prime}\right)\right|_{\text {old }}-\frac{\sum_{m=1}^{N} I_{m} x_{m, c}^{\prime}}{\sum_{m=1}^{N} I_{m}},
$$

where $I_{m}$ is the integrated detected intensity of the $m$ th beamlet.

The second moments of the trace space distribution are obtained from the above determined parameters as

$$
\begin{gathered}
\left\langle x^{2}\right\rangle=\frac{\sum_{m=1}^{N} I_{m} x_{m, c}^{2}}{\sum_{m=1}^{N} I_{m}}, \\
\left\langle x^{\prime 2}\right\rangle=\frac{\sum_{m=1}^{N} I_{m}\left(x_{m, c}^{\prime 2}+\sigma_{m}^{\prime 2}\right)}{\sum_{m=1}^{N} I_{m}},
\end{gathered}
$$

and

$$
\left\langle x x^{\prime}\right\rangle=\frac{\sum_{m=1}^{N} I_{m} x_{m, c} x_{m, c}^{\prime}}{\sum_{m=1}^{N} I_{m}},
$$

where in obtaining the moment $\left\langle x^{\prime 2}\right\rangle$ we have assumed that the beamlet distributions are symmetric about their centroids. From the second moments, we can construct the rms emittance

$$
\varepsilon_{x} \equiv \sqrt{\left\langle x^{2}\right\rangle\left\langle x^{\prime 2}\right\rangle-\left\langle x x^{\prime}\right\rangle^{2}},
$$

as well as the other rms Twiss parameters [11],

$$
\begin{gathered}
\beta_{x} \equiv \frac{\left\langle x^{2}\right\rangle}{\varepsilon_{x}}, \quad \alpha_{x} \equiv-\frac{\left\langle x x^{\prime}\right\rangle}{\varepsilon_{x}}, \\
\gamma_{x} \equiv \frac{\left\langle x^{\prime 2}\right\rangle}{\varepsilon_{x}}=\frac{1+\alpha_{x}^{2}}{\beta_{x}} .
\end{gathered}
$$

The slits serve a secondary purpose which is of central importance in the discussion of beams emitted from rf photocathode guns since these beams are space-charge dominated for almost all relevant energies and beam sizes. The notion of space-charge dominated flow is quantified by comparing the space charge and emittance terms in the rms beam envelope equation for an ultrarelativistic beam $(\gamma \gg 1, \beta=\nu / c \approx 1)$ in a drift space [12]

$$
\sigma_{x}^{\prime \prime}=\frac{\varepsilon_{n}^{2}}{\gamma^{2} \sigma_{x}^{3}}+\frac{I}{\gamma^{3} I_{0}\left(\sigma_{x}+\sigma_{y}\right)},
$$

where $I$ is the peak beam current, $I_{0}=e c / r_{e}$ is the characteristic current, $\varepsilon_{n}=\beta \gamma \varepsilon_{x} \cong \gamma \varepsilon_{x}$ is the normalized rms emittance, and, of course, an analogous equation exists for $\sigma_{y}$. Now, taking the ratio of the second to the first terms on the right-hand side of the envelope equation, and assuming a round beam ( $\sigma_{x}=\sigma_{y} \equiv \sigma_{0}$ ), we have a measure of the degree of space-charge dominance over emittance in driving the evolution of the beam envelope,

$$
R_{0}=\frac{I \sigma_{0}^{2}}{2 I_{0} \gamma \varepsilon_{n}^{2}}=2 k_{p}^{2} \beta_{x}^{2} .
$$

In order to illustrate the relationship between the two effects driving the beam envelope, we have rewritten this ratio in terms of the plasma wave number associated with the beam density $n_{b}, k_{p}=\omega_{p} / c \cong \sqrt{4 \pi r_{e} n_{b} / \gamma^{3}}$, and the rms beta function $\beta_{x}$.

As an example, we take parameters typical of the present experiments: a $5 \mathrm{MeV}$ electron beam with current of $100 \mathrm{~A}, \mathrm{rms}$ beam size of $1 \mathrm{~mm}$, and normalized rms emittance of $4 \mathrm{~mm}$ mrad. This yields a ratio of $R_{0} \cong 75$, and one can see that this beam cannot be emittance dominated until it is focused down to small sizes, $\sigma_{0}<100 \mu \mathrm{m}$. Thus linear transport theory cannot be used to measure the emittance with this type of beam, as we will discuss in detail in our evaluation of the quadrupole scanning technique. Collimation with slits mitigates this situation, however, by creating low current, small $\sigma_{x}$ beamlets that have the same uncorrelated transverse momentum spread as the original beam. Noting that the rms size of a uniform beamlet created by a vertical slit of width $d$ is $\sigma_{x}=d / \sqrt{12}$, and assuming $\sigma_{x} \gg \sigma_{y}$, we have a space-charge dominance ratio for the beamlets,

$$
R_{b}=\sqrt{\frac{2}{3 \pi}} \frac{I}{\gamma I_{0}}\left(\frac{d}{\varepsilon_{n}}\right)^{2}
$$

For the beam parameters of our example, and the choice in our experiments of $d=50 \mu \mathrm{m}$, we see that $R_{b}=$ $0.042 \ll 1$, implying that the beamlets which pass out of the slits are emittance dominated. This process can also be understood in terms of the plasma frequency of the beam and the beta function. Since the beamlets have the same density as the beam before the slits, $k_{p}$ is unchanged by collimation. On the other hand, the beta function of each beamlet is much smaller than that of the beam before collimation, $\beta_{x} \cong \sigma_{x} / \sigma_{x^{\prime}} \Rightarrow(d / \sqrt{12}) / \sigma_{x^{\prime}}$, and for the beamlets the ratio $R_{b}=2 k_{p}^{2} \beta_{x, b}^{2}$ can therefore be made much less than unity.

There are many further technical and physical considerations which must be taken into account in order to arrive at an optimized design for a slit-based phase space measurement system. Since the main point of this article is the overall effect of space charge on the results of emittance measurements, these ancillary concerns are discussed in the Appendix, which is specific to the multislit system implemented in these experiments. Further, more general material on the optimization of this type of instrument is found in the comprehensive review of emittance measurement techniques given in Ref. [8].

\section{QUADRUPOLE SCANNING EMITTANCE MEASUREMENTS}

The quadrupole scanning technique for measuring emittance is well known and widely used in the accelerator physics community [13]. A brief description of the process is given here in order to show its limitations when applied to space-charge dominated beams. One can understand the measurement by considering the evolution of the 
beam's rms Twiss parameters by differentiating Eqs. (9) in a drift length after a thin focusing lens (of focal length $f$ )

$$
\begin{aligned}
& \beta_{x}^{\prime}=2 \frac{\left\langle x x^{\prime}\right\rangle}{\varepsilon_{x}}=-2 \alpha_{x}, \\
& \alpha_{x}^{\prime}=-\frac{\left\langle x^{\prime 2}\right\rangle+\left\langle x x^{\prime \prime}\right\rangle}{\varepsilon_{x}}=-\gamma_{x}, \\
& \gamma_{x}^{\prime}=2 \frac{\left\langle x^{\prime} x^{\prime \prime}\right\rangle}{\varepsilon_{x}}=0,
\end{aligned}
$$

where we used the fact that there are no external forces and ignored the space charge force to set $x^{\prime \prime}=0$. Using Eqs. (13) and applying the thin lens ( $\alpha_{x 0}$ becomes $\alpha_{x 0}+$ $\beta_{x 0} / f$ ) gives

$$
\begin{aligned}
\beta_{x}(z)= & \beta_{x 0}-2\left(\alpha_{x 0}+\frac{\beta_{x 0}}{f}\right)\left(z-z_{0}\right) \\
& +\left[\frac{1+\left(\alpha_{x 0}+\frac{\beta_{x 0}}{f}\right)^{2}}{\beta_{x 0}}\right]\left(z-z_{0}\right)^{2} .
\end{aligned}
$$

We notice that the $\beta$ function is quadratic in $z$ as expected. Upon rearranging this equation in terms of $1 / f$ and multiplying it by the emittance, we obtain an equation for the square of the beam size as a function of the focusing strength of the lens,

$$
\begin{aligned}
\sigma_{x}^{2}(z)= & {\left[\sigma_{x 0}^{2}-2 \alpha_{x 0} \varepsilon_{x}\left(z-z_{0}\right)+\gamma_{x 0}\left(z-z_{0}\right)^{2}\right] } \\
& +\frac{2 \sigma_{x 0}^{2}}{f}\left[\frac{\alpha_{x 0}}{\beta_{x 0}}\left(z-z_{0}\right)^{2}-\left(z-z_{0}\right)\right] \\
& +\frac{\sigma_{x 0}^{2}}{f^{2}}\left(z-z_{0}\right)^{2} .
\end{aligned}
$$

If we then identify the coefficients of $\left(\frac{1}{f}\right)^{i}$ with the letters $m_{i}$, the emittance can be extracted as

$$
\varepsilon_{x}^{2}\left(z-z_{0}\right)^{4}=m_{0} m_{2}-\frac{m_{1}^{2}}{4} .
$$

With this analysis in mind, the emittance can be obtained by measuring the beam size at a given drift length $\left(L_{d}\right)$ after a quadrupole magnet, scanning through a range of focusing strengths. The same result could have been derived by solving the envelope equation for a drifting beam without space charge:

$$
\sigma_{x}^{\prime \prime}=\frac{\varepsilon_{x}^{2}}{\sigma_{x}^{3}}
$$

It is important to stress here that the quad scan formalism is based on rms quantities. That is, Eq. (15) holds (without space charge) for arbitrary, evolving beam distributions provided that we are measuring the rms value of the beam size. When considering the effect of space charge in the remainder of this paper, we are looking for gross differences in the rms beam size. This point is seen also in the envelope equations including space charge. In these equations the space-charge term is derived assuming linear self-fields, but extends to other distributions in the rms sense using the concept of equivalent beams $[14,15]$. This work expands on that of Ref. [9], which points out that the assumption of a Gaussian transverse profile and measurement of full width at half maximum (FWHM) spot sizes is incorrect for nonthermalized, photoinjector beams. For these reasons, all experimental spot sizes quoted in this paper are determined using rms measurements of the distribution, thus avoiding potential problems Gaussian fitting and FWHM-based analyses of the data.

We can now examine how a quadrupole scan behaves in the other extreme, namely the one where we assume the beam has space charge but no emittance. In that case, the envelope equations in $x$ and $y$ become

$$
\begin{aligned}
& \sigma_{x}^{\prime \prime}=\frac{I}{\gamma^{3} I_{0}\left(\sigma_{x}+\sigma_{y}\right)}, \\
& \sigma_{y}^{\prime \prime}=\frac{I}{\gamma^{3} I_{0}\left(\sigma_{y}+\sigma_{x}\right)} .
\end{aligned}
$$

These equations have no exact solution, but we may do an approximate analysis by making the assumption $\sigma_{x}+\sigma_{y} \approx$ const. This approximation is acceptable while $k_{p} L_{d}<1$. In that case we get, through a change of variables $\left(\Sigma=\sigma_{x}+\sigma_{y}, \Delta=\sigma_{x}-\sigma_{y}\right)$,

$$
\begin{aligned}
& \Sigma^{\prime \prime}=\frac{2 I}{\gamma^{3} I_{0} \Sigma} \approx 2 k_{p}^{2} \Sigma_{i} \approx \text { const }, \\
& \Delta^{\prime \prime} \approx 0 .
\end{aligned}
$$

This set of equations is easily solvable and we find

$$
\begin{aligned}
& \Sigma_{f}=\Sigma_{i}+\Sigma_{i}^{\prime} z+k_{p}^{2} \Sigma_{i} z^{2}, \\
& \Delta_{f}=\Delta_{i}^{\prime} z .
\end{aligned}
$$

Using $\sigma_{x}=\frac{1}{2}(\Sigma+\Delta)$, and assuming an initially axisymmetric beam $\left(\sigma_{x, i}=\sigma_{y, i}\right)$, allows a solution of the $x$ envelope equation,

$$
\sigma_{x, f}=\sigma_{x, i}+\left(\sigma_{x, i}^{\prime}-\frac{\sigma_{x, i}}{f}\right) z+k_{p}^{2} z^{2} \sigma_{x, i} .
$$

We see from this that the rms beam size is linear with the focusing strength of the lens, and so the square is quadratic in $1 / f$, just as in the case where space charge is ignored. If one attempts to compute the emittance using the coefficients of powers of $1 / f$, as in the emittance dominated case, the result is zero. This is understandable in the sense that one would expect the beam size to remain linear with $1 / f$ all the way down to zero thickness for a beam with no emittance.

For the measurements described below, neither of these limiting cases is applicable as both the emittance and space charge terms are important in the evolution of the rms beam size. What we have shown here, however, is that one can expect the qualitative outcome of the quadrupole scan to be the same in either regime. In particular, it is notable that, even in the case of space-charge dominated beam dynamics, the algorithm used to extract the emittance from a quadrupole scan [based on Eq. (16)] gives a well-behaved result, and there is no a priori reason that data from these 
scans would be rejected as unphysical. The experimental data shown below demonstrate quad scans that indeed yield good fits to Eq. (16), but which have systematic errors in the resulting calculated emittance. The basis for these errors, which is dependent on the interplay between space charge and emittance forcing of the beam envelope, is discussed below, in the context of the experimental data and modeling.

\section{EXPERIMENTAL SETUP AND PROCEDURE}

The quadrupole scan and multislit-based emittance measurement techniques were compared using the Thomson scattering photoinjector at Lawrence Livermore National Laboratory. The beam line configuration used for these measurements is shown in Fig. 3. The accelerator in this setup was a 1.6 cell, $S$ band, BNL-SLAC-UCLA-LLNL if photocathode gun [16]. The gun produced a $5 \mathrm{MeV}$ beam whose charge, transverse, and longitudinal spot sizes varied as given in Table I and described below. An emittance compensating solenoid after the gun was used to control the rf defocusing at the gun exit and allowed us to select a reasonable beam size at the emittance slits and quadrupole. The magnetic field at the cathode was nulled with an identical bucking solenoid placed upstream of the cathode. The charge was measured using an integrating current transformer. The laser injection phase was monitored during the experiments by mixing the low level rf derived from the laser oscillator with that from a probe in the gun full cell. Long time scale drifts in the rf phase were corrected for all measurements with a manual phase shifter.

A Ti:sapphire-based laser system was used to produce the UV pulses necessary for photoelectron emission in the gun. As the injector is intended for integration with an rf linear accelerator (linac) and a multi-Joule short-pulse laser system $[17,18]$, the beam produced by the photoinjector must be synchronized with the high power laser pulses. The photoinjector laser system (PLS) is seeded by stretched pulses from the Falcon laser system so that the linac can be synchronized to the Falcon master oscillator. The seed light is introduced into the regenerative amplifier
TABLE 1. Electron beam parameter range used for emittance measurements.

\begin{tabular}{lc}
\hline \multicolumn{1}{c}{ Parameter } & Range \\
\hline Electron beam charge & $50-300 \mathrm{pC}$ \\
rms laser spot size & $0.5-2 \mathrm{~mm}$ \\
rms laser pulse length & $2.5-6 \mathrm{psec}$ \\
\hline \hline
\end{tabular}

(regen) of the photoinjector system via single-mode, polarization-preserving fiber that runs from the Falcon stretcher output to the basement laboratory containing the PLS. The timing for the Falcon, linac, and PLS derive from the Falcon master oscillator. In addition to the light pulse, timing signals from the Falcon timing rack are sent to the PLS to trigger the yttrium-aluminum-garnet (YAG) pump laser and regenerative amplifier $Q$ switch. The PLS consists of a fiber-seeded regenerative amplifier, a multipass power amplifier, a pulse compressor, and frequency conversion crystals for frequency tripling the $800 \mathrm{~nm}$ laser pulse. The two laser amplifiers, regen and multipass, are pumped by a single, frequency-doubled, $Q$ switched YAG laser that puts out $300 \mathrm{~mJ}$ of $532 \mathrm{~nm}$ light in an $8 \mathrm{nsec}$ pulse. The output beam of the multipass amplifier is expanded and sent to the grating compressor.

We measured the pulse width at the output of the grating compressor using a single-shot autocorrelator. The desired pulse length of the UV pulse is a few psec, which can be obtained by varying the distance between the gratings in the compressor. We attempted to directly measure pulse width vs grating separation but found that the autocorrelator gives good results for only pulses $\sim 1$ psec or less. Consequently, we estimate the pulse width for $\Delta T>1$ psec using various techniques. For pulse lengths $<1 \mathrm{psec}$ the autocorrelator appears to give accurate results. The shortest pulse we measured was $150 \mathrm{fsec}$, which corresponds to a time-bandwidth-limited pulse.

By varying the difference in grating separation from the minimum pulse length position $\Delta b$ between the gratings in the pulse compressor we can continuously adjust the pulse length in the IR from $184 \mathrm{psec}$ to $150 \mathrm{fsec}$. This variation

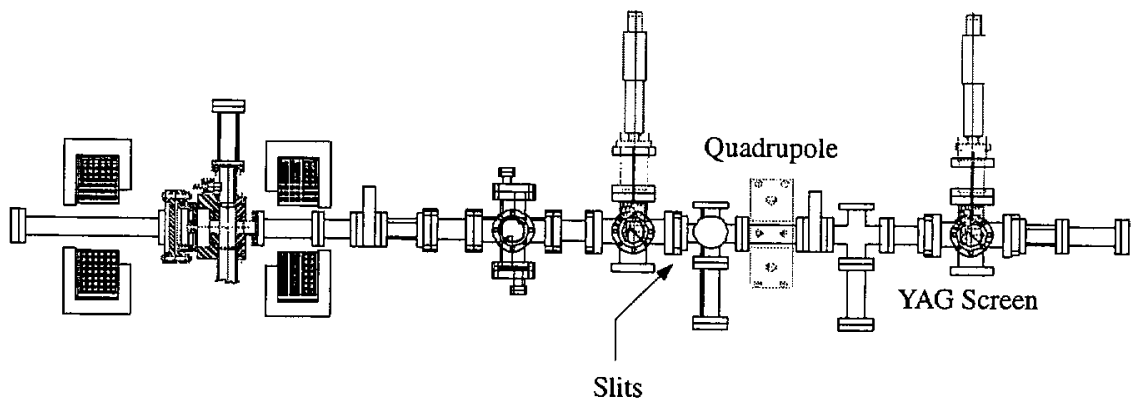

FIG. 3. (Color) Schematic of the LLNL Thomson scattering beam line used in the emittance measurements. 
was used to change the beam intensity as it was launched at the photocathode, thus also varying the plasma frequency of the beam. Three separate methods of modeling the compressor all give the same result, which is that the grating separation tuning $\Delta T / \Delta b \sim 0.2 \mathrm{psec} / \mathrm{mm}$, at $1 \omega$. The $3 \omega$ intensity is proportional to the $1 \omega$ intensity cubed, and, as a result, a Gaussian-shaped pulse narrows up in time, i.e.,

$$
\Delta T_{3 \omega}=\frac{\Delta T_{1 \omega}}{\sqrt{3}} .
$$

Based on this calculation, we expect the pulse width of the $3 \omega$ light to be given by

$$
\Delta T_{3 \omega}=(0.11 \mathrm{psec} / \mathrm{mm}) \times \Delta b(\mathrm{~mm}) .
$$

For the multislit-based emittance measurements we chose, based on the arguments given above and in the Appendix, a set of stainless steel collimating slits with a $50 \mu \mathrm{m}$ slit width, $0.75 \mathrm{~mm}$ separation, and $5 \mathrm{~mm}$ depth. The drift length from the slits to the measurement screen was approximately $50 \mathrm{~cm}$. For a beam size of $1-2 \mathrm{~mm}$ on the slits and at $5 \mathrm{MeV}$, this allowed us to measure a maximum normalized emittance of $12 \mathrm{~mm}$ mrad using the slits. The slits were mounted on an insertable, rotatable actuator powered by stepper motors. This allowed us to align the slits to the beam, insuring the proper angle for maximum acceptance of the slits. A $0.5 \mathrm{~mm}$ thick YAG:Ce crystal was used as the intercepting screen for both types of measurements. The beam images produced at the crystal were captured by a video camera and digitized by a computer controlled frame grabber. Once captured, the beam images were analyzed on-line in the case of the multislit measurements, and saved for latter analysis in the case of the quadrupole scan.

Given the apparatus described above, the experimental procedure was to set the compressor gratings to a given separation distance and measure the dimensions of the laser and electron beams as well as the charge and injection phase. This was important because it allowed us to calculate the plasma frequency of the beam in order to compare our measurements with simulation and analytical models. Once these parameters were known, the beam emittance was measured using both the multislit and the quad scanning techniques. Table I lists the range in beam parameters over which the emittance measurements were done.

\section{EXPERIMENTAL RESULTS}

The quad scan and multislit measurements were performed for seven different electron beam pulse lengths. Figure 4 shows one image of a beam horizontally focused by the quadrupole. The horizontal rms size of the beam is $140 \mu \mathrm{m}$. The figure also shows a representative result of one of the quadrupole scans. Figure 5 shows the
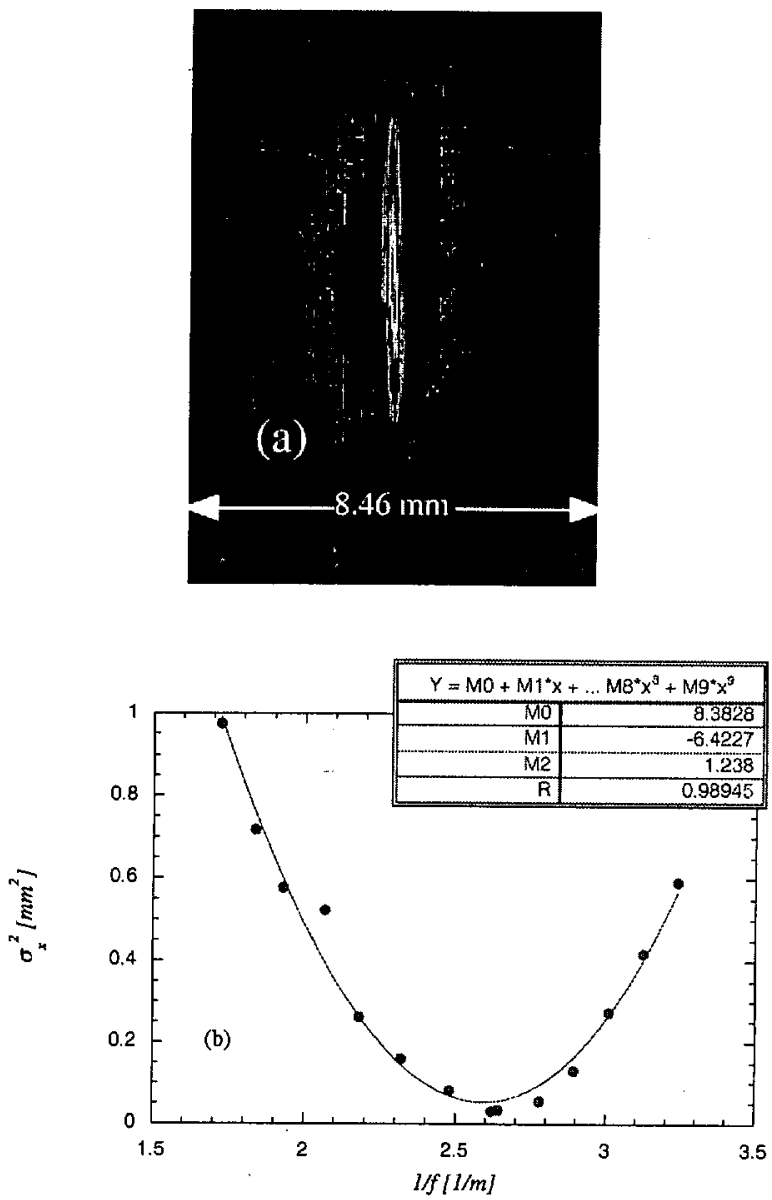

FIG. 4. (Color) (a) False color image of a beam image used in a quadrupole scan. (b) Result of one quad scan. The normalized horizontal emittance found from the curve fit is $9.6 \pm 1.1 \mathrm{~mm}$ mrad.

data found using the slits for the same grating separation as used in Fig. 4. The emittance calculated via the quad scan measurement is higher than with the emittance slits. This pattern was repeated in all of the measurements we performed.

As we argued above, we expect the result of a quadrupole scan to depend not only on the emittance but also the strength of the space-charge defocusing forces encountered by the beam through the drift region. For this reason we plot in Fig. 6 the results of the measurements as a function of the plasma wave number of the beam at the quadrupole multiplied by the drift length between the quadrupole and the screen. The plasma wave number is determined through simulation, using the measured properties of the laser and the total charge launched, as discussed further in the following section [5]. For this range of beam parameters, the quad scan gives consistently higher values for the emittance than the multislit measurement. 

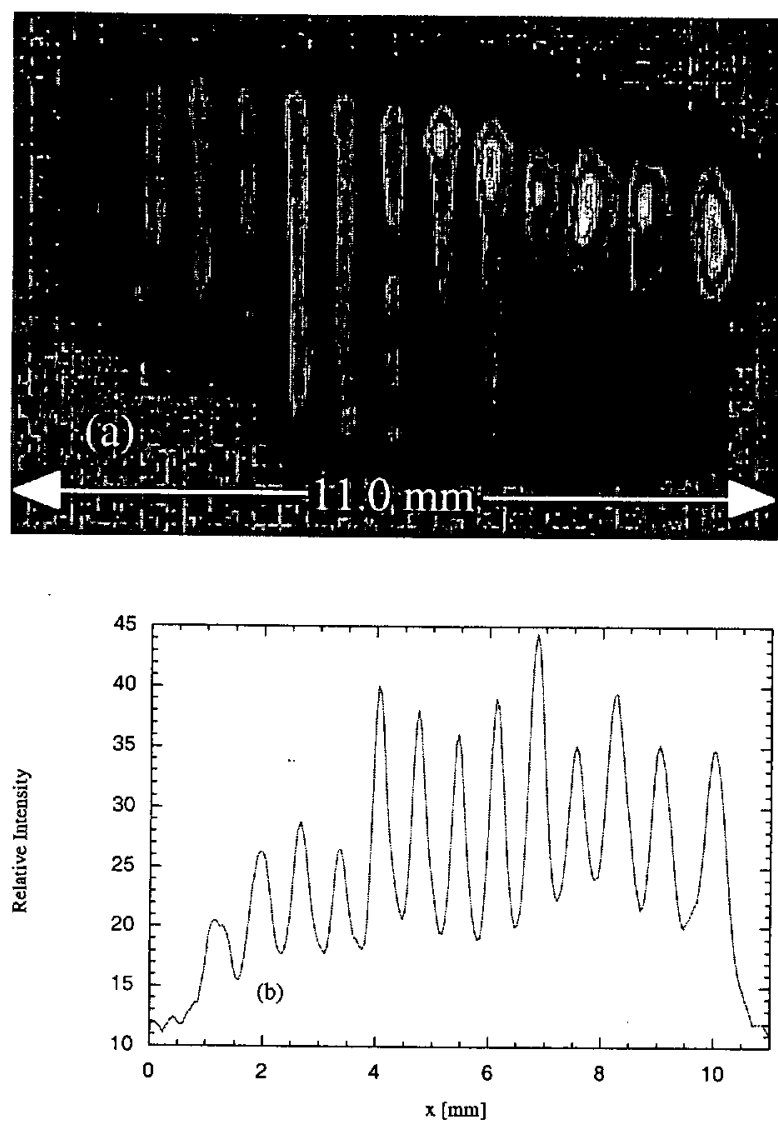

FIG. 5. (Color) (a) False color image of the beam passing through the collimating slits. (b) Intensity graph found by summing the vertical pixel values at a given horizontal position. The normalized horizontal emittance calculated from this plot is $6.9 \pm 0.7 \mathrm{~mm} \mathrm{mrad}$.

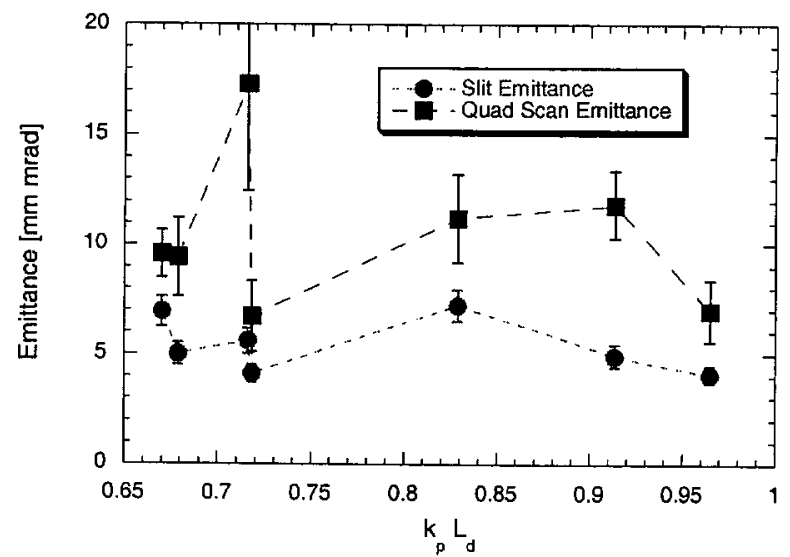

FIG. 6. (Color) Plot comparing the different emittance measurement techniques for varying beam intensities. The strength of the space-charge forces is parameterized in the scan by the product of the drift length between the quadrupole and detector and the plasma wave number at the quad.

\section{ANALYSIS}

The recording of laser spot size, length, and energy for each set of emittance measurements allowed us to accurately simulate the acceleration and focusing of the beam through the 1.625 cell gun. These simulations were done using PARMELA [19], a multiparticle code which includes collective (space-charge) forces. In these simulations, a beam derived from injection of a Gaussian longitudinal and transverse laser pulse shape was used. The purpose of these simulations was both to get a base line expectation value for the emittance of the beam and to find the spatial dimensions of the beam at the position of the quadrupole. The results of the PARMELA simulations are shown in Fig. 7. We see from the figure that the emittance predicted by PARMELA is generally slightly lower than that of the multislit measurements, but follows the same trend from measurement to measurement. This indicates that the small variation in the multislit emittance values at different beam densities is due, at least in part, to changes in the emittance compensation process caused by different beam densities at the cathode, as could be expected.

At this point we simulated the quadrupole scan procedure with space charge included in the simulation. This was done in three different ways. The first was to use the PARMELA beam distributions from the previous simulations at the position of the quadrupole and iteratively simulate their propagation in the drift region between the quad and the measurement screen for different quad strengths. In these PARMELA runs, we changed the space-charge routine to a point-to-point Coulomb method, which is much less accurate than the standard radial mesh algorithm, but was needed to model the beam when it becomes highly asymmetric after quad focusing. In order to avoid the noise inherent in the point-to-point calculations, we also employed HOMDYN, which is an envelope code that has been successfully benchmarked against multiparticle codes [20]. Finally, we also simulated the quad scans by numerically

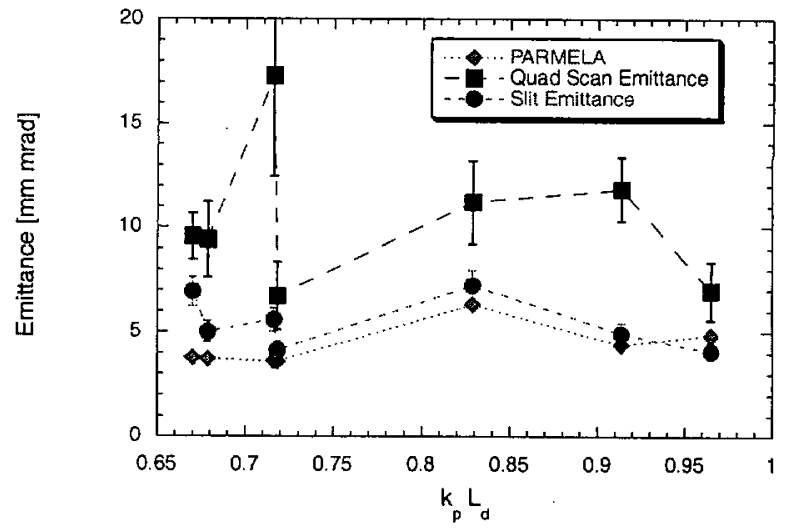

FIG. 7. (Color) PARMELA simulations of the emittance at the quadrupole and the measured emittance using both measurement methods. 
integrating the 2D rms envelope equations for the beam including space charge,

$$
\begin{aligned}
& \sigma_{x}^{\prime \prime}=\frac{\varepsilon_{n}^{2}}{\gamma^{2} \sigma_{x}^{3}}+\frac{I}{\gamma^{3} I_{0}\left(\sigma_{x}+\sigma_{y}\right)}, \\
& \sigma_{y}^{\prime \prime}=\frac{\varepsilon_{n}^{2}}{\gamma^{2} \sigma_{y}^{3}}+\frac{I}{\gamma^{3} I_{0}\left(\sigma_{x}+\sigma_{y}\right)} .
\end{aligned}
$$

In the case of both the HOMDYN and envelope equation simulations, the emittances $\varepsilon_{n}$ [cf. Eq. (24)] were taken to be that measured by the slits, the initial beam sizes were taken from PARMELA, and the beam currents taken from measurement data. In all three cases the simulations gave similar results, and the less noisy (but still three dimensional, and sensitive to differential slice dynamics $[4,5]$ ) HOMDYN results are shown in Fig. 8. In order to avoid confusion about the meaning of the emittance term in Eq. (24), we specify at this point that the symbol $\varepsilon_{n}$ will indicate the normalized thermal emittance. The thermal component to a full measured emittance is due to uncorrelated motion, as opposed to the apparent emittance observed from residual correlations between longitudinal and transverse phase space in poorly compensated beams. Thus we are assuming in the integration of Eq. (23) that the measured multislit emittance is roughly equal to the so-called slice emittance. This point is physically meaningful, as an emittance which was entirely based on longitudinal-transverse phase space correlations, with vanishing slice emittance, would not give rise to effects we have observed.

As we see from Fig. 8, the emittances derived from the simulated quad scans are indeed higher than the actual emittances used in the simulations, as expected. In addition, they agree reasonably well with the results of the quad scan measurements.

The parameter $k_{p} L_{d}$ was chosen to plot against the emittance results because it describes the degree to which the plasma nature of the beam influences its motion in the experiments. In cases where the beam evolves in a purely space-charge dominated way (such as the emittance com-

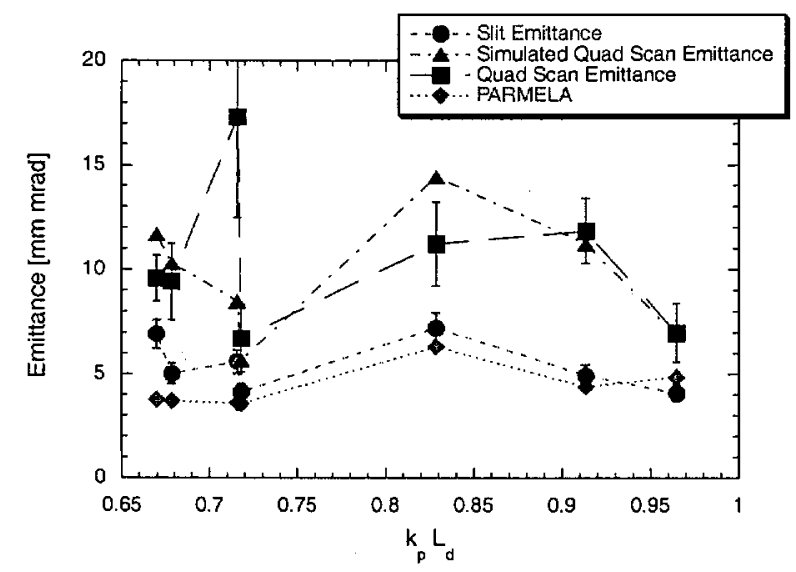

FIG. 8. (Color) Simulation of the quadrupole scan plotted with the PARMELA simulations and the measured emittance data. pensation process), $k_{p} L_{d} / 2 \pi$ would be the number of plasma oscillations that the beam undergoes. However, in the case of the quad scan, the beam's size at the end of the drift is also affected by emittance, especially at points where the beam has gone through a tight waist. In that case, the relative strength of the emittance and spacecharge terms $\left(R_{0}\right)$ in the envelope equation are of interest. The ratio of these forcing terms can be characterized by the beta function times the plasma wave number, and Eq. (11) shows that $k_{p} \beta=\sqrt{\frac{R_{0}}{2}}$. In Fig. 9 we plot the path of the experimental data in the plane defined by $k_{p} \beta$ and $k_{p} L_{d}$. The background of the plane is a contour plot of simulated quad scan emittances using the envelope integration model, with a constant thermal emittance for each point in the plane. We see from the figure that some data points that happen to be close together in $k_{p} L_{d}$ have significantly different values of $k_{p} \beta$. This effect explains in part the drastic difference in emittance between close data points in Fig. 8. Note also that the lower right-hand corner of the graph has values of emittance that are lower than the value that was used to integrate the envelope equations. This point will be discussed further below.

It is instructive at this point to look again at the quad scan procedure to see why the data shows and simulations predict erroneous numbers for the emittance. In particular, we can see both in the data and in simulations that there is an asymmetry in the curves about their minima. In Fig. 10 the quad scan data from Fig. 4 is replotted, and the data points on either side of the minimum are fitted to different curves. We see from the figure that the points before the minimum spot size follow a path with less curvature than the points occurring after the focus. The second part of the figure shows this effect more dramatically. In that case the plot points come from a simulation of a quad scan assuming conditions similar to the experiment, but with a higher beam current of several hundred amps.

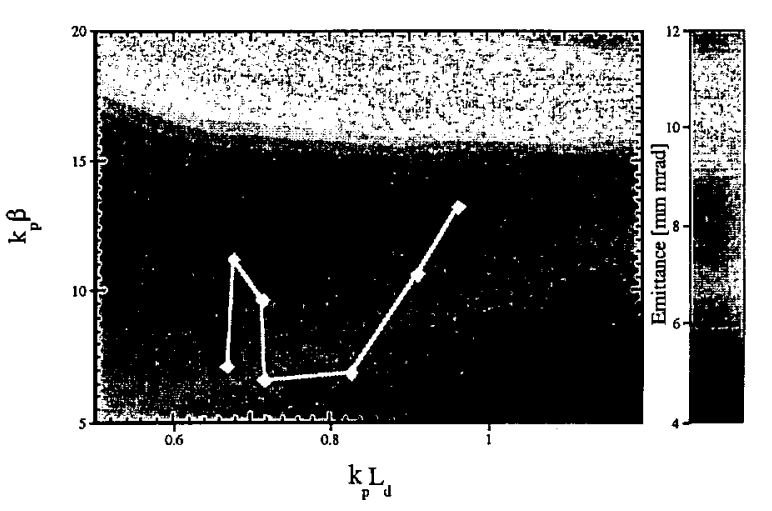

FIG. 9. (Color) Contour plot of the simulated quad scan emittance over a range of both $k_{p} L_{d}$ and $k_{p} \beta$. The white plot points locate the positions of the experimental data. The normalized emittance used as input to the simulations was $5 \mathrm{~mm} \mathrm{mrad}$, while the output emittance values range from 4 (deep blue) to $12 \mathrm{~mm}$ mrad (red). 

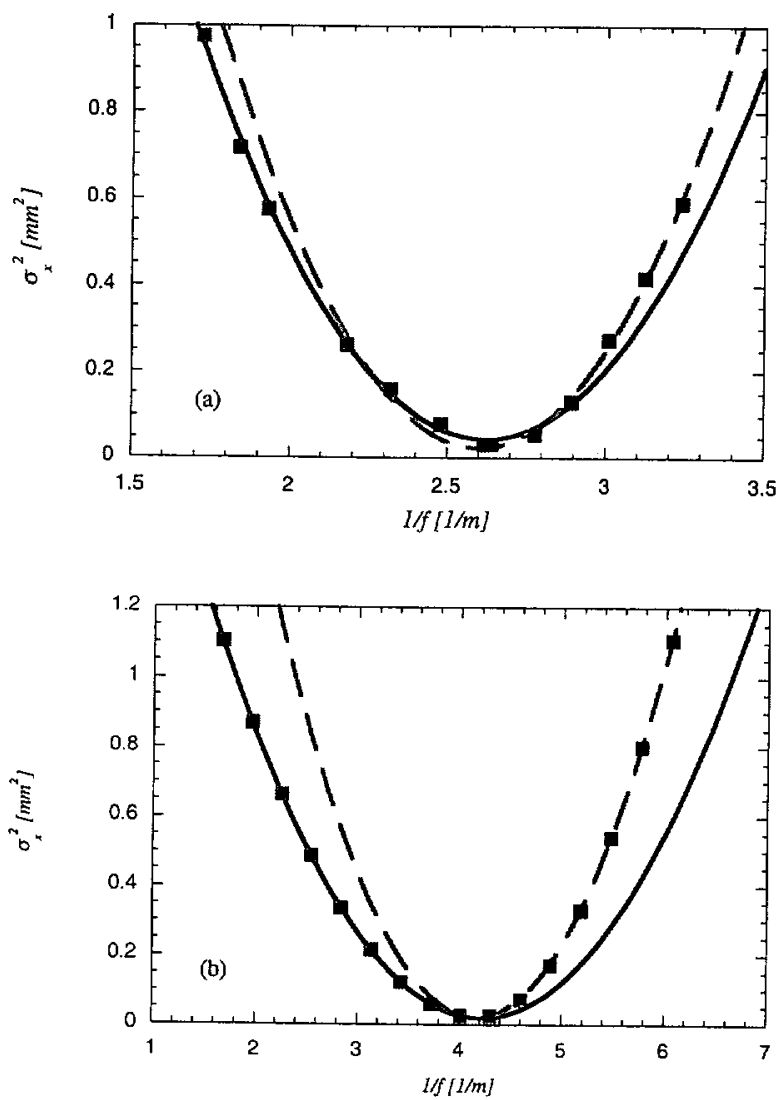

FIG. 10. (Color) (a) Quad scan data as shown in Fig. 4(b). Here we apply different fits to the data before and after the focus. (b) Simulation of a quad scan with an extremely space-charge dominated beam. Again, we show the two fits on either side of the beam waist to illustrate the asymmetry in the simulation points.

This asymmetry in the data about the minimum spot size is a manifestation of the fact that the evolution of the beam through the drift is very different for data points on opposite sides of the minimum. For points on the right-hand side of the curve (weaker focusing) the beam size is deflected appreciably only by space charge. For points in the curve at and to the left of the waist (stronger focusing) there occurred at some position a thermal emittance dominated beam waist. In the region of that waist, the emittance force "turns on" and applies an extra kick to the beam size that deflects it away from the path it would take due only to space charge. The stronger the quadrupole focuses (larger $1 / f$ ), the stronger this thermal emittance kick will be and the further upstream of the measurement screen it occurs. Figure 11 illustrates this behavior, by showing the results of a simulation with and without the emittance term included in the envelope equations. We see here that for points on the left-hand side of the minimum, the simulations with and without emittance agree very well, indicating that the integrated motion of the beam is indeed purely dominated by space charge. However, the points at and after the minimum diverge rapidly from the path that the

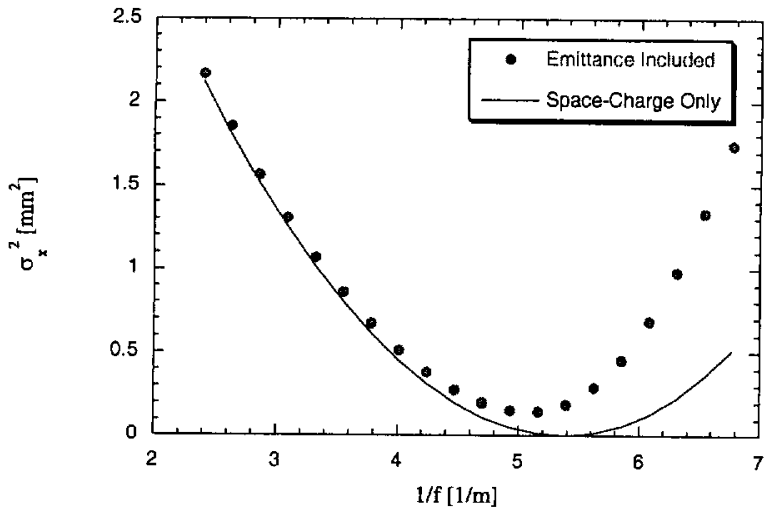

FIG. 11. (Color) Simulations of a quad scan with and without emittance. The solid line represents a simulation without the emittance forces, while the data points show a simulation of the full envelope equations.

simulation with space charge alone predicts even though the beam is space-charge dominated over most, but not all, of its trajectory.

It is interesting to note that both a purely thermal emittance dominated and a purely space-charge dominated scan would produce a $\left(\sigma_{x}^{2}, 1 / f\right)$ curve which is symmetric about the minimum $\sigma_{x}$. The asymmetry observed in the mostly space-charge dominated quadrupole scan is a result of both space-charge and thermal emittance effects asserting themselves in the measurement.

Given this problem, in which points on one side of the curve are space-charge dominated, while on the other side they are affected by emittance, it is certainly no surprise that the emittance computed from these curves is not correct. In addition, the asymmetry of the curve introduces a problem in the consistency of the result. That is, since the computation of the emittance requires us to fit a parabola to a curve that is of higher order, the fit parameters will depend on the portion of the curve used for the fit. To illustrate this point, consider a single quad scan simulation as shown in Fig. 12(a). The range of focal lengths we use for the fit is in principle arbitrary; however, it is reasonable to impose the conditions that the simulation points are equally spaced in $1 / f$ and that the end points give the same beam size, which is about $1 \mathrm{~mm}$. These are the conditions we used for simulations in this paper. We can see how the computed emittance varies when we change the start and stop point of the fit. Figure 12(b) shows the emittances calculated from the simulation data of Fig. 12(a) with the horizontal and vertical axes representing the starting and stopping points of the fit, respectively.

There are two noteworthy features in Fig. 12(b). The first is that it shows both regions above and below the input value of the emittance. In fact, the violet regions in the upper half of the plot represent points where the computed emittance is imaginary [this happens if the right-hand side as determined from the scan parameters of Eq. (16) is negative]. The other point is that the upper left corner of 

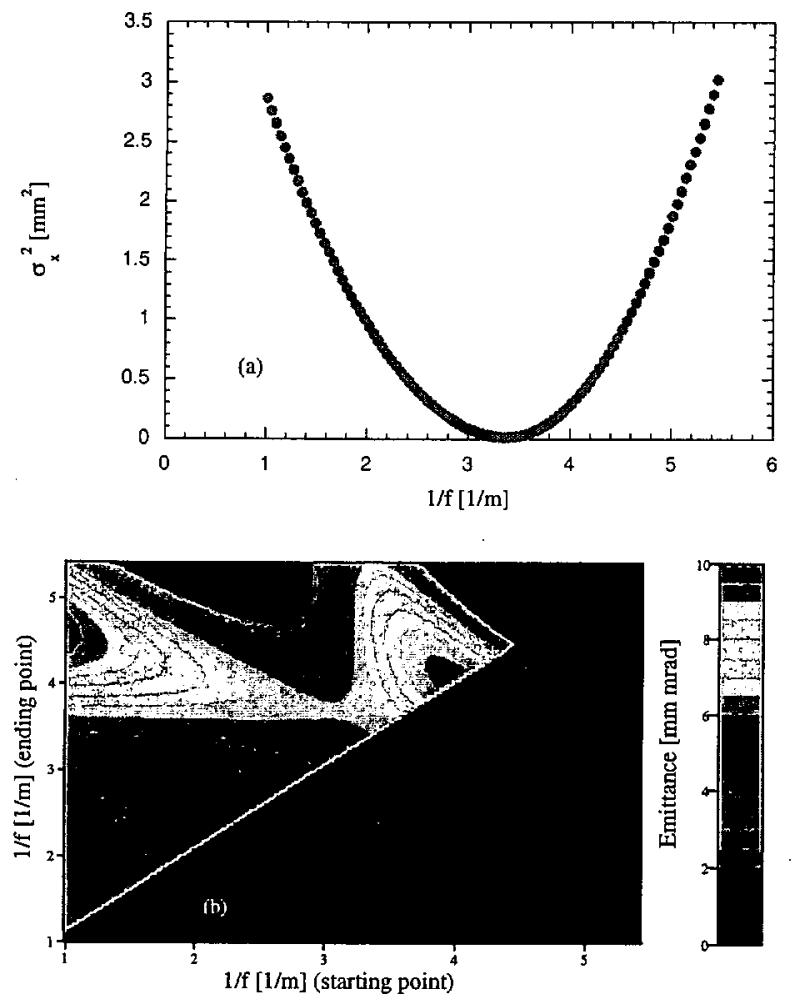

FIG. 12. (Color) (a) Quad scan simulation used to perform the fitting in (b). The emittance used in the simulation is $4 \mathrm{~mm} \mathrm{mrad}$. (b) Contour plot of the emittance calculated by fitting the data in (a). The portion of the graph below the diagonal is unused (this half would be the mirror image of the upper half.) Here, red represents an emittance of $10 \mathrm{~mm}$ mrad and violet is imaginary (the square of the derived emittance is negative).

the graph is the region where the gradient in emittance values is the steepest. This is also the region that we initially assumed to be the most reasonable. The lower left-hand corner of the plot seems to be a stable region, but it is not an accurate solution. Points in this corner fit only the first part of the curve, and that part gives only information about space charge, as shown in Fig. 11. This strict dependence of the computed emittance on the range of fit points is another factor that may help to explain the inconsistency in the quad scan measurements. This effect was not considered at the time the measurements were performed, and for the majority of them slightly more data points were taken before the minimum of the curve than after.

\section{CONCLUSIONS}

Two different emittance measurement techniques were discussed in this paper: the multislit-based measurement and the quadrupole scan. For the highly space-charge dominated beams of interest here, we reviewed how the slit collimation of the beam into beamlets reduces the quantity $k_{p} \beta$ sufficiently to allow the beamlets to expand under the influence of emittance and not space charge. In the ex- periment preformed at the Thomson source photoinjector at LLNL, we found that the emittances measured with the multislit system were reasonably independent of the beam intensity, and agreed well with the PARMELA simulations.

In contrast, we found that the quadrupole scanning procedure was ill-suited to measuring the emittance of these beams. In addition to the issues associated with multishot measurements in systems with notable shot-to-shot charge fluctuations, the fundamental problem with the quadrupole scan is that the beam evolves under the influence of both space-charge and emittance effects. In the Livermore experiments we found that the emittance measured with the quad scan was consistently higher than the multislit measurements and PARMELA predictions. In addition, simulations of the quad scan for the beam parameters of the measurements also show higher values for the emittance, and reproduce the systematic dependence of the measured emittance on the quantity $k_{p} L_{d}$.

Simulations show that when $k_{p} L_{d}$ is of the order of unity or greater, space-charge forces are large enough to significantly alter the evolution of the beam in the drift region of the scan. Further, when the quantity $k_{p} \beta$ is larger than unity (indicating the degree to which the spacecharge forces are dominant over the emittance effects), the quadrupole measurement of the emittance will have notable errors. For $k_{p} \beta \gg 1$, the emittance measurement is no longer valid at all, but is really only a measure of the intricate interplay between the space-charge and emittance effects during the focused trajectory. Thus the conditions $k_{p} L_{d}>1$ and $k_{p} \beta>1$ are theoretical tools for determining the range of parameters over which the validity of the quadrupole scan is no longer guaranteed. Perhaps an even more useful method that can test for possible problems in a quadrupole scan is the examination of the scan data itself. When the $\left(\sigma_{x}^{2}, 1 / f\right)$ curve is no longer symmetric in $1 / f$ about the minimum in $\sigma_{x}^{2}$, this is a clear signature that the combined space-charge/emittance dominated beam evolution produces an unreliable emittance measurement. It is both inaccurate and imprecise, as the exact value of the emittance derived from the $\left(\sigma_{x}^{2}, 1 / f\right)$ curve depends on the number of points on either side of the minimum that are used in the fit.

It may be noted that, as experimental progress is made in lowering the beam emittance obtained from if photoinjectors, the problems noted here with quadrupole scans will be exacerbated. In recognition of such difficulties, most laboratories use slit-based measurements for low energy $(<10 \mathrm{MeV})$ emittance diagnosis. The present results imply that quadrupole scans may be problematic at energies much higher than $10 \mathrm{MeV}$.

\section{ACKNOWLEDGMENT}

This work was performed under the auspices of the U.S. Department of Energy by the Lawrence Livermore National Laboratory under Contract No. W-7405-Eng-48. 


\section{APPENDIX}

We survey in this Appendix a series of design considerations which impact the choice of slit parameters one deploys in the phase space measurement system, and which dictate the resolution of the device. Many of the issues discussed here are also examined in Ref. [8], but since Lejeune and Aubert do not analyze the slit array in particular, a review of the technical considerations one needs to implement the slit-based system is given here. We begin by examining those parameters that have to do with the angular acceptance of the slits. The depth of the material used to intercept the beam is dictated by our desire to either stop the beam or scatter it sufficiently so that it does not affect the measurement of the nonintercepted beamlets. The stopping distance of the beam is approximately

$$
L_{s}=\frac{E}{\frac{d E}{d x}} \approx \frac{E(\mathrm{MeV})}{1.5\left(\mathrm{MeV} \mathrm{cm}^{2} \mathrm{~g}^{-1}\right) \rho\left(\mathrm{g} \mathrm{cm}^{-3}\right)}
$$

for an initially minimum ionizing particle. It is straightforward to stop a $5 \mathrm{MeV}$ beam, such as is encountered in this paper, but with an energy much above $10 \mathrm{MeV}$, the length of the slits for total stopping may become impractical. In order to deal with this we rely on multiple scattering effects in the slit material. The beam scatters off of nuclei as it slows down from ionization losses, and the final rms angle associated with the beam after propagating a distance $L$ in the stopping material is approximately

$$
\begin{aligned}
\theta_{\mathrm{sc}} & \cong \frac{21}{E(\mathrm{MeV})} \sqrt{\frac{L_{s}}{L_{r}}\left(\frac{1}{L_{s}-L}-1\right)} \\
& \cong \frac{21}{E(\mathrm{MeV})} \sqrt{\frac{L}{L_{r}}},
\end{aligned}
$$

where $L_{r}$ is the radiation length in the material, and we assumed the stopping distance is much larger than the material thickness. In order to reduce the background signal from scattered (but not stopped) electrons, it is useful to conservatively require a multiple scattering angle in excess of 0.5 , or approximately,

$$
L_{s} \cong L_{r}\left(\frac{E(\mathrm{MeV})}{42}\right)^{2} .
$$

Once the slit depth $L$ has been chosen, one can examine the angular acceptance of the slits. The first step is to specify an rms beam angle associated with the finite beam emittance which, assuming we place the slits at a waist, is

$$
\phi=\frac{\varepsilon_{n}}{\gamma \sigma_{0}} .
$$

The assumption of a beam waist is invariably a good approximation, as one must be careful not to introduce a large correlated beam angle in order to make sure that all offsets $x$ in the beam pass the slit aperture well. Thus we are left to consider the uncorrelated angles given by Eq. (A4), which must be chosen to be less than one-fourth the angular aperture of the slits, $\phi<d / 4 L$, and preferably even smaller.

The slit separation $w$ is chosen to be much larger than the slit width $d$ and smaller than the beam size to ensure that we can resolve the beam. In our case, the slit width is taken to be $0.75 \mathrm{~mm}$. This width must also be consistent with not allowing the beamlets to overlap at the detection plane, a condition that depends on the distance of the drift to the screen $L_{d}$. The ratio to the beamlet widths to their separation, which should be smaller than unity, is

$$
R_{w s}=2 \frac{L_{d} \phi}{w},
$$

while the ratio of the beamlet rms size at the phosphor to its size at the slit, which should be larger than 1 to achieve resolution of the uncorrelated angular spread in the beam, is

$$
R_{s p}=\sqrt{12} \frac{L_{d} \phi}{d} .
$$

Since one of these ratios should be small and the other large compared to unity, if we set their geometric average equal to unity $\left(R_{s p} R_{w s}=1\right)$, we can optimize the drift length to be

$$
L_{d} \cong \frac{\sqrt{d w}}{3^{1 / 4} 2 \phi},
$$

which for the example parameters given above yields $L_{d} \approx$ $50 \mathrm{~cm}$. This optimum is of course quite broad, so one is free to choose a more convenient value if one desires to measure larger or smaller emittances, as discussed below.

Once the drift length is specified, there is another criterion which should be examined for the diagnostic to give unambiguous results: the contribution to the measured emittance from the residual space-charge forces between beamlets is smaller than that due to the true uncorrelated angular distribution at the slits. Again assuming the slits are at a waist (this gives the highest estimate of the space-charge effect), we have

$$
R_{b}^{\prime}=\frac{2 I}{\gamma^{2} I_{0}} \frac{d L_{d}}{w \varepsilon_{n}}
$$

Again, this quantity must be much smaller than 1 . For our present design it is about 0.25 , but it should be noted that for Hartman's measurements it was in fact greater than 1 .

The subject of slit scattering is a bit complicated, but a detailed calculation using EGS [21] is not necessary if estimates that the signal-to-noise due to slit scatter is not of the order of 100 or less. Theoretical guidelines in this calculation have been developed by Courant [22] and Burge and Smith [23]. Modifying Courant's criterion for energy discrimination, we pick the effective depth of the maximum of the relevant slit scattered flux to occur when the multiple scattering angle is equal to the acceptance half-angle of the slits, 


$$
l_{\mathrm{eff}}=L_{r}\left(\frac{21 d}{E(\mathrm{MeV}) 2 l}\right)^{2}
$$

and the increase in effective slit width is given by

$$
d_{\mathrm{eff}}=\frac{2}{\sqrt{3 \pi}} \frac{l_{\mathrm{eff}}^{3 / 2}}{w_{c}}
$$

where

$$
w_{c}^{2}=\frac{A}{Z^{2} \pi N_{A} \rho}\left(\frac{E}{2 e^{2}}\right)^{2} \ln \left(181 Z^{1 / 3}\right)^{-1},
$$

and $N_{A}$ is Avogadro's number. The minimum signal-tonoise for the detected beam intensity at the phosphor is therefore

$$
\frac{S}{N} \geq \frac{\sqrt{3 \pi} d w_{c}}{2 d_{\mathrm{eff}} l_{\mathrm{eff}}^{3 / 2}} .
$$

For most cases of interest, Eq. (A12) gives a value much in excess of unity. It should be noted, however, that a misalignment of the slits can generate anomalously large slit scattering effects, and thus care must be taken to avoid this situation.

Once the general layout of the slit system is specified by the above-listed optimization, one may examine the resolvable emittances in the measurement device. The maximum emittance one may measure is obtained in the situation where the angular aperture of the slits is entirely filled (assuming that the requirement of the ratio $R_{s p}<1$ is satisfied), so that

$$
\varepsilon_{n, \max }=\frac{\gamma \sigma_{0} w}{\sqrt{12} L_{s}}
$$

Note that this maximum emittance is proportional to the beam size, and thus the total number of slits in the mask $N_{s}$. On the other hand, the minimum emittance which can be resolved is given by

$$
\varepsilon_{n, \min }=\frac{\gamma \sigma_{0} d}{\sqrt{12} L_{s}} .
$$

As a practical matter, it should be noted that great care needs to be taken in the machining specifications for the slits, to ensure that they are flat over the entire surface parallel to the beam propagation. This is accomplished in the present experiments by electron discharge machining. The slits are mounted on a precision insertable-rotatable (2000 steps per rotation) actuator which is driven by a stepping motor. This level of precision is needed to eliminate unwanted angles between the beam centroid propagation and the slit normal directions.

[1] J. S. Fraser and R. L. Sheffield, IEEE J. Quantum Electron. 23, 1489 (1987).

[2] K. J. Kim, Nucl. Instrum. Methods Phys. Res., Sect. A 275, 201 (1989).

[3] B. E. Carlsten, Nucl. Instrum. Methods Phys. Res., Sect. A 285, 313 (1989).

[4] Luca Serafini and J. B. Rosenzweig, Phys. Rev. E 55, 7565 (1997).

[5] S. G. Anderson and J. B. Rosenzweig, Phys. Rev. ST Accel. Beams 3, 094201 (2000).

[6] X. Qiu et al., Phys. Rev. Lett. 76, 3723 (1996).

[7] J. B. Rosenzweig et al., Nucl. Instrum. Methods Phys. Res., Sect. A 410, 437 (1998).

[8] Claude Lejeune and Jean Aubert, Adv. Electron. Electron Phys., Suppl. 13A, 159 (1980).

[9] B.E. Carlsten et al., Nucl. Instrum. Methods Phys. Res., Sect. A 331, 791 (1993).

[10] S. Hartman et al., Nucl. Instrum. Methods Phys. Res., Sect. A 340, 219 (1994); J. Rosenzweig et al., Nucl. Instrum. Methods Phys. Res., Sect. A 341, 379 (1994).

[11] R.Q. Twiss and N.H. Frank, Rev. Sci. Instrum. 20, 1 (1949).

[12] Martin Reiser, Theory and Design of Charged Particle Beams (Wiley, New York, 1994).

[13] Helmut Wiedemann, Particle Accelerator Physics: Basic Principles and Linear Beam Dynamics (Springer-Verlag, New York, 1993).

[14] P. M. Lapostolle, IEEE Trans. Nucl. Sci. 18, 1101 (1971).

[15] F. J. Sacherer, IEEE Trans. Nucl. Sci. 18, 1105 (1971).

[16] D. T. Palmer, Ph.D. thesis, Stanford University, 1998.

[17] G.P. LeSage et al., in Advanced Accelerator Concepts. Ninth Workshop, edited by P.L. Colestock and S. Kelley, AIP Conf. Proc. No. 569 (AIP, New York, 2001).

[18] G.R. Hays et al.; in Proceedings of the Conference on Lasers and Electro-optics, San Francisco, 2000 (Optical Society of America, Washington, DC, 2000), p. 293.

[19] L. Young and J. Billen, Los Alamos National Laboratory Report No. LA-UR-96-1835, 1996.

[20] M. Ferrario et al., in Proceedings of the ICFA Advanced Accelerator Workshop on the Physics of High Brightness Beams, Los Angeles, 1999 (World Scientific, Singapore, 2000).

[21] A.F. Bielajew et al., SLAC Report No. SLAC-PUB-6499, 1994.

[22] E. D. Courant, Rev. Sci. Instrum. 22, 1003 (1951).

[23] E. J. Burge and D. A. Simith, Rev. Sci. Instrum. 33, 1371 (1962). 


\title{
Pleiades: A Sub-picosecond Tunable X-ray Source at the LLNL Electron Linac
}

\author{
Dennis Slaughter ${ }^{1}$ \\ John. Crane ${ }^{I}$ \\ Paul Springer ${ }^{1}$ \\ Todd Ditmire ${ }^{2}$ \\ Greg Le Sage $e^{I}$ \\ Tom Cowan ${ }^{3}$ \\ Scott G. Anderson ${ }^{4}$ \\ James B. Rosenzweig ${ }^{4}$ \\ ${ }^{1}$ LLNL, ${ }^{2}$ Univeristy of Texas, ${ }^{3}$ GA Technologies, ${ }^{4}$ UCLA
}

\begin{abstract}
The use of ultra fast laser pulses to generate very high brightness, ultra short (fs to ps) pulses of $\mathrm{x}$-rays is a topic of great interest to the $x$-ray user community. In principle, femtosecond-scale pump-probe experiments can be used to temporally resolve structural dynamics of materials on the time scale of atomic motion. The development of sub-ps x-ray pulses will make possible a wide range of materials and plasma physics studies with unprecedented time resolution. A current project at LLNL will provide such a novel x-ray source based on Thomson scattering of high power, short laser pulses with a high peak brightness, relativistic electron bunch. The system is based on a $5 \mathrm{~mm}$-mrad normalized emittance photoinjector, a $100 \mathrm{MeV}$ electron RF linac, and a $300 \mathrm{~mJ}$, $35 \mathrm{fs}$ solid-state laser system. The Thomson $\mathrm{x}$-ray source produces ultra fast pulses with $\mathrm{x}$-ray energies capable of probing into high-Z metals, and a high flux per pulse enabling single shot experiments. The system will also operate at a high repetition rate $(\sim 10 \mathrm{~Hz})$.
\end{abstract}

\section{INTRODUCTION}

The use of ultra fast laser pulses to generate very high brightness, ultra short $\left(10^{-14}\right.$ to $\left.10^{-12} \mathrm{~s}\right)$ pulses of $\mathrm{x}$-rays is a topic of great interest to the x-ray user community. In principle, femtosecond-scale pump-probe experiments can be used to temporally resolve structural dynamics of materials on the time scale of atomic motion. The development of sub-ps x-ray pulses will make possible a wide range of materials and plasma physics studies with unprecedented time resolution. The goal of this work is to develop such a novel $\mathrm{x}$-ray source that will exhibit some very important features:

- Ultra fast pulses for probing dynamics on the time scale of atomic motion

- Hard $x$-rays capable of probing into high- $Z$ metals

- High $x$-ray flux per pulse enabling single shot experiments

- Widely tunable $x$-ray wavelength

Bragg and Laue diffraction provide detailed information about the long-range order of a material. Short pulse length and high single-shot flux will allow these techniques to be applied on the time scale of phase transitions, or melt driven by fast heating or compression. In addition, information on the short-range order in a material (such as nearest neighbor distances and coordination numbers) may be obtained using extended $\mathrm{x}$-ray absorption fine structure (EXAFS) spectroscopy ${ }^{1}$. The concept is illustrated in Figure 1.

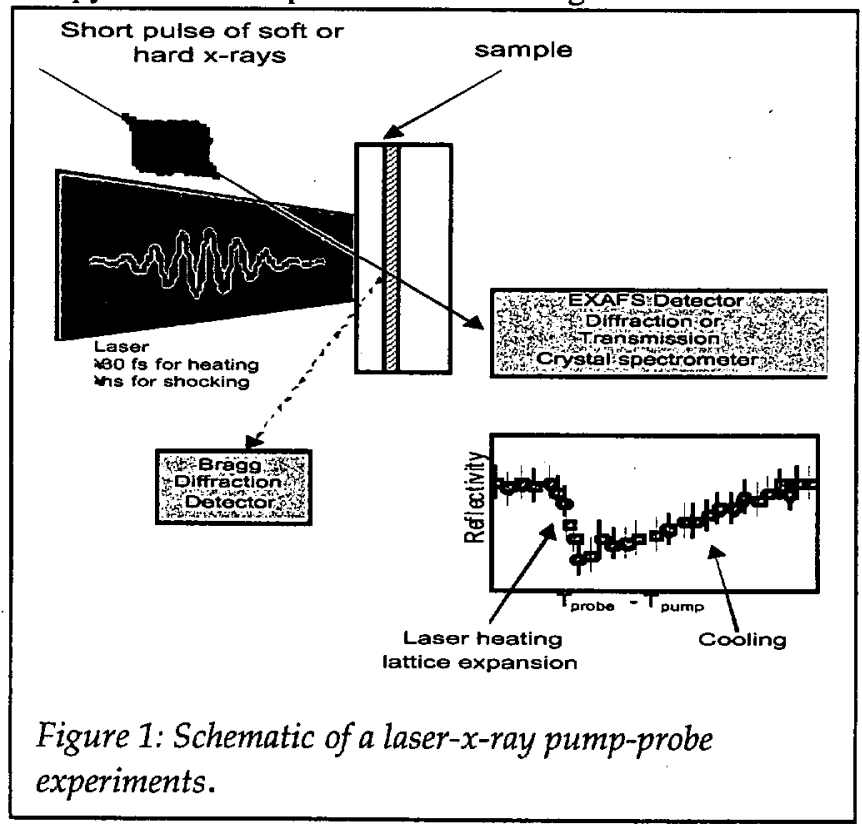


Initial ultra fast dynamic studies of laser excited materials have been pursued in recent years using timeresolved $\mathrm{x}$-ray detectors at synchrotron sources and with laser-plasma $\mathrm{x}$-ray sources in pump-probe experiments. Third generation synchrotrons have been critical sources for $x$-ray studies in materials, but they have a major limitation: although they probe structure on the atomic length scale, their time resolution $(\sim 100 \mathrm{ps})$ is not wellmatched to the natural dynamics of elemental processes in solids, such as the time scale for atomic motion ( $\sim 10-50$ fs). Laser melting in semiconductors may involve excitation, electron-phonon coupling, melt front motion and shock waves, all with $<30 \mathrm{ps}$ relevant timescales. Notable recent successes have included the measurement of the internal strain profile in InSb following laser heating ${ }^{2}$, using Bragg scattering to detect the 1-D compression of a crystal following laser irradiation. The experiments accumulated data averaged over a very large number of shots and could effectively utilize the x-ray flux available from synchrotron sources. However, the time resolution of these experiments was limited to greater than a few picoseconds.

Rose-Petruck, et. al. ${ }^{3}$ recently characterized the propagation of femtosecond laser driven coherent phonons in a $\mathrm{GaAs}$ crystal using synchronized, laser plasma produced $\mathrm{K}$-alpha radiation and later used a $\mathrm{K}_{\alpha}$ source to time resolve dynamics in laser heated thin Ge layers ${ }^{4}$ where they observed melting. Rischel et al. ${ }^{5}$ used a similar technique to time resolve dynamics in an organic Langmuir-Blodgett film heated by a femtosecond laser. These studies were limited to time resolution of 1 to $100 \mathrm{ps}$ and were, like the synchrotron experiments, limited to repetitively pulsed data collection.

The time resolved Bragg diffraction technique has been successfully applied on large-scale lasers with shock pressures of $\geq 1 \mathrm{Mbar}$. Wark et al. ${ }^{6}$ performed some of the first, groundbreaking diffraction experiments on the Janus laser about ten years ago. More recently Remington and Kalantar et al. ${ }^{7}$ have applied this technique to experiments on the Nova laser at LLNL.

We have undertaken the development of an $\mathrm{x}$-ray source based on laser scattering off a relativistic electron bunch. This Thomson scattering approach offers the potential to produce $x$-rays in a unique regime. A temporally compressed laser pulse is focused onto a short relativistic electron pulse as shown in Figure 2 below.

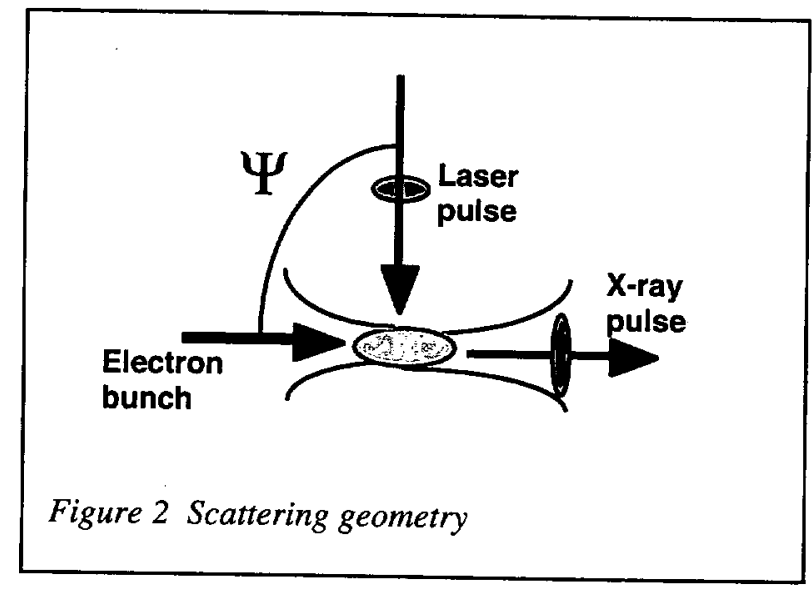

DESIGN AND PERFORMANCE

bunitra fast $\mathrm{X}$-ray pulses are generated by scattering a high power, ultra fast, $800 \mathrm{~nm}$ laser pulse from a beam bunch of relativistic electrons at the LLNL $100 \mathrm{MeV}$ electron Linac. Beam characteristics in several operational modes are summarized in Table 1 below. The scattered laser photons are relativistically up shifted in frequency into the hard x-ray range, and are emitted in a narrow cone about the electron beam direction. Leemans, Schoenlein and coworkers working at the LBNL Advanced Light Source injector Linac have previously demonstrated generation ofsub-ps pulses of hard $x$-rays by Thomson scattering in $1996^{8,9}$. They were able to achieve an $x$-ray beam flux of $\sim 10^{5}$ photons in a $\sim 300 \mathrm{fs}$ pulse, at $\sim 30 \mathrm{keV}$. However, the limited $\mathrm{x}$-ray flux available in that first demonstration required the averaging of several thousand shots at each pump-delay time point. The LLNL Thomson source presently under development is expected to achieve an x-ray beam flux some four to five orders of magnitude larger, enabling the accumulation of sufficient data for a high quality Bragg diffraction spectrum on each shot.

As viewed in the frame of the moving electrons, the incident laser pulse train appears as an electromagnetic undulator of wavelength $\lambda_{u}=\lambda_{\mathrm{I}} / \gamma(1-\cos \Psi)$, where $\gamma=\mathrm{E} / \mathrm{m}_{0} \mathrm{c}^{2}$ and $\Psi$ is the incident angle between electron and laser beams as shown in Figure 2 . The electrons radiate photons, which are up shifted back into the laboratory frame by a second factor of $2 \gamma$. The $x$-ray wavelength is therefore related to the initial laser wavelength by 


$$
\lambda_{x}=\lambda_{\mathrm{J}} / 2 \gamma^{2}(1-\cos \Psi)
$$

where $\lambda_{\mathrm{L}}$ is the laser wavelength. In the laboratory frame, the up shifted $\mathrm{x}$-rays are confined to a narrow cone with opening angle $\sim 1 / \gamma$, and their energy varies with observation angle in the laboratory due to the kinematics of the Lorentz transformation. The laboratory $\mathrm{x}$-ray energy (for scattering of the fundamental) is:

$\mathrm{E}_{\mathrm{x}}=\mathrm{E}_{\mathrm{L}} 2 \gamma^{2}(1-\cos \Psi) /\left(1+\gamma^{2} \theta^{2}+\mathrm{a}_{0}{ }^{2}\right)$

where $E_{L}$ is the laser photon energy. Here, $a_{0}$ is the usual normalized vector potential of the laser field, which is analogous to the $\mathrm{K}$ parameter of a static field undulator. For our parameters, the $\mathrm{x}$-ray energy should be tunable over a range from 10 to $200 \mathrm{keV}$ in the laboratory depending on the incident angle $\Psi$ and initial electron beam energy. The number of $\mathrm{x}$-ray photons produced is proportional to the strength of the effective optical undulator field (for $a_{0}<1$ ) and the number of optical cycles $\left(\mathrm{N}_{\mathrm{o}}\right)$, by

$$
\mathrm{N}_{\mathrm{x}}=(\pi / 3) \alpha_{\mathrm{i}} \mathrm{N}_{0} \mathrm{~N}_{\mathrm{e}} \mathrm{a}_{0}{ }^{2}
$$

where $\alpha_{\mathrm{f}}$ is the fine structure constant and $\mathrm{N}_{\mathrm{e}}$ is the number of electrons in the beam bunch.

In comparison to the $10^{5} \mathrm{x}$-rays per pulse observed by Leemans \& Shchoenlien ${ }^{8,9}$, we expect to produce up to $10^{8} \mathrm{x}$-ray photons in a $\sim 100 \mathrm{fs}$ pulse, and up to $10^{10} \mathrm{x}$-rays in a $1-10 \mathrm{ps}$ pulse, by scattering the laser respectively either across the electron beam $\left(\Psi=90^{\circ}\right)$ which minimizes the temporal overlap, or in a head-on $\left(\Psi=180^{\circ}\right)$ geometry, which maximizes the interaction of the electrons and photons. To achieve the predicted enhancements over the demonstrated LBNL Thomson yield, we are making two straightforward but critical improvements:

- Low emittance electron beam produced from a photoinjector

- High power $10 \mathrm{TW}$ Ti:sapphire laser (with planned upgrade to $100 \mathrm{TW}$ )

Predicted beam characteristics are shown in Table 1 below.

Table 1: Predicted Characteristics of the LLNL Thomson Scatter X-ray Source (assuming $100 \mathrm{TW}$ operation of Falcon)

\begin{tabular}{|l|l|l|}
\hline & Short-pulse mode & Long-pulse mode \\
\hline X-ray flux & $10^{9}$ photons per pulse & $10^{11}$ photons per pulse \\
\hline X-ray pulse duration & $50-100 \mathrm{fs}$ & $<10 \mathrm{ps}$ \\
\hline X-ray energy & $10-100 \mathrm{keV}$ & $20-200 \mathrm{keV}$ (head-on) \\
\hline Bandwidth & $<10 \%$ & $<10 \%$ \\
\hline Divergence Angle & $30-10 \mathrm{mrad}$ & $30-10 \mathrm{mrad}$ \\
\hline Peak Spectral Brightness & $10^{21}\left(\mathrm{ph} / \mathrm{s} / \mathrm{mm}^{2} / \mathrm{mrad}^{2} / 0.1 \% \mathrm{BW}\right.$ & $10^{21}\left(\mathrm{ph} / \mathrm{s} / \mathrm{mm}^{2} / \mathrm{mrad}^{2} / 0.1 \% \mathrm{BW}\right.$ \\
\hline Electron bunch & $1 \mathrm{nC}$ in $1 \mathrm{ps}$ & $10 \mathrm{nC} \mathrm{in} 10 \mathrm{ps}$ \\
\hline Electron energy & $30-100 \mathrm{MeV}$ & $30-100 \mathrm{MeV}$ \\
\hline Laser pulse & $4 \mathrm{~J}$ in $35 \mathrm{fs}(\text { currently } 300 \mathrm{~mJ})^{\#}$ & $4 \mathrm{~J}$ in $1 \mathrm{ps}(\mathrm{currently} 300 \mathrm{~mJ})$ \\
\hline Laser Intensity & $\sim 10^{18} \mathrm{~W} / \mathrm{cm}^{2}$ & $\sim 10^{18} \mathrm{~W} / \mathrm{cm}^{2}$ \\
\hline
\end{tabular}

The LLNL Falcon laser 10,11 currently produces $300 \mathrm{~mJ}$ pulses at repetition rate $10 \mathrm{~Hz}$ that are $5 \mathrm{X}$ more energetic than the $60 \mathrm{~mJ}$ pulses used by the LBNL group, and with their duration as short as $35 \mathrm{fs}$ are nearly three decades brighter without further improvement. Anticipated laser upgrade to $4 \mathrm{~J}$ coupled with increases in electron bunch charge will extend this advantage to 4-5 decades.

The Falcon laser is a Ti:sapphire laser based on chirped pulse amplification. To date, we have integrated the Falcon laser with the Linac and made preparations to transport the laser pulses to the electron beam. Initial characterization of this source using $20 \mathrm{~mJ}$ laser pulses and $5 \mathrm{MeV}$ electron bunches from the photoinjector at $10 \mathrm{~Hz}$ successfully demonstrated $0.6 \mathrm{keV}$ x-ray production. This demonstrated the ability to focus the laser and electron beams to small spots $(<10 \mu \mathrm{m})$, and to synchronize them $(<10 \mathrm{ps})$.

The estimated fluxes, summarized in Table 1, were predicted using the treatment of Esarey et al ${ }^{12}$ assuming that the Falcon laser is upgraded to $100 \mathrm{TW}$ operation. We have designed an interaction geometry in which roughly

\footnotetext{
\#Falcon laser currently generates $300 \mathrm{~mJ}$ pulses, is capable of $600 \mathrm{~mJ}$, and a planned upgrade will increase the pulse energy to $4 \mathrm{~J}$
} 
one $\mathrm{x}$-ray photon is emitted per electron, and therefore predict $10^{9}$ to $10^{11} \mathrm{x}$-ray photons per pulse, depending on the bunch charge and the interaction angle. In fact, the estimates in Table 1 are based on a somewhat idealized laser pulse that is assumed to be uniform (both spatially and temporally) over the laser-electron overlap region. With mping laser-electron overlap with, for example, a moving focus, we should be able to achieve this level of x-ray generation for different interaction geometries. For example, by injecting the laser pulse at $\Psi=90^{\circ}$ to the electron beam direction, we will attempt to achieve x-ray pulses as short as $\sim 100 \mathrm{fs}$ with as many as $10^{9}$ photons per pulse scattering off of a $1 \mathrm{nC}$ bunch. These parameters are most relevant to experiments on ultra fast dynamics in solid-state systems, for example the electron-phonon relaxation process which governs the transfer of energy from $x$-ray or optical pulses to crystals, or for chemical reaction studies, in which the bond breaking and formation times are of order $\sim 10 \mathrm{fs}$.

This is a significant improvement over existing $3^{\text {rd }}$ generation light sources where the pulse widths attainable are usually long, typically greater than $100 \mathrm{ps.} \mathrm{For} \mathrm{fast} \mathrm{dynamics,} \mathrm{these} \mathrm{pulses} \mathrm{are} \mathrm{of} \mathrm{limited} \mathrm{utility.} \mathrm{On} \mathrm{the} \mathrm{other} \mathrm{hand,}$ laser produced $x$-rays via hot plasma production offer the potential for producing sub-picosecond x-rays. However, achieving very high peak brightness is difficult through this means. The Thomson scattering source we are developing at LLNL will yield peak brightness that exceeds those of $3^{\text {rd }}$ generation synchrotrons while delivering $x$ ray pulses with pulse width below 1 ps. The comparison is illustrated in Figure 3 below.

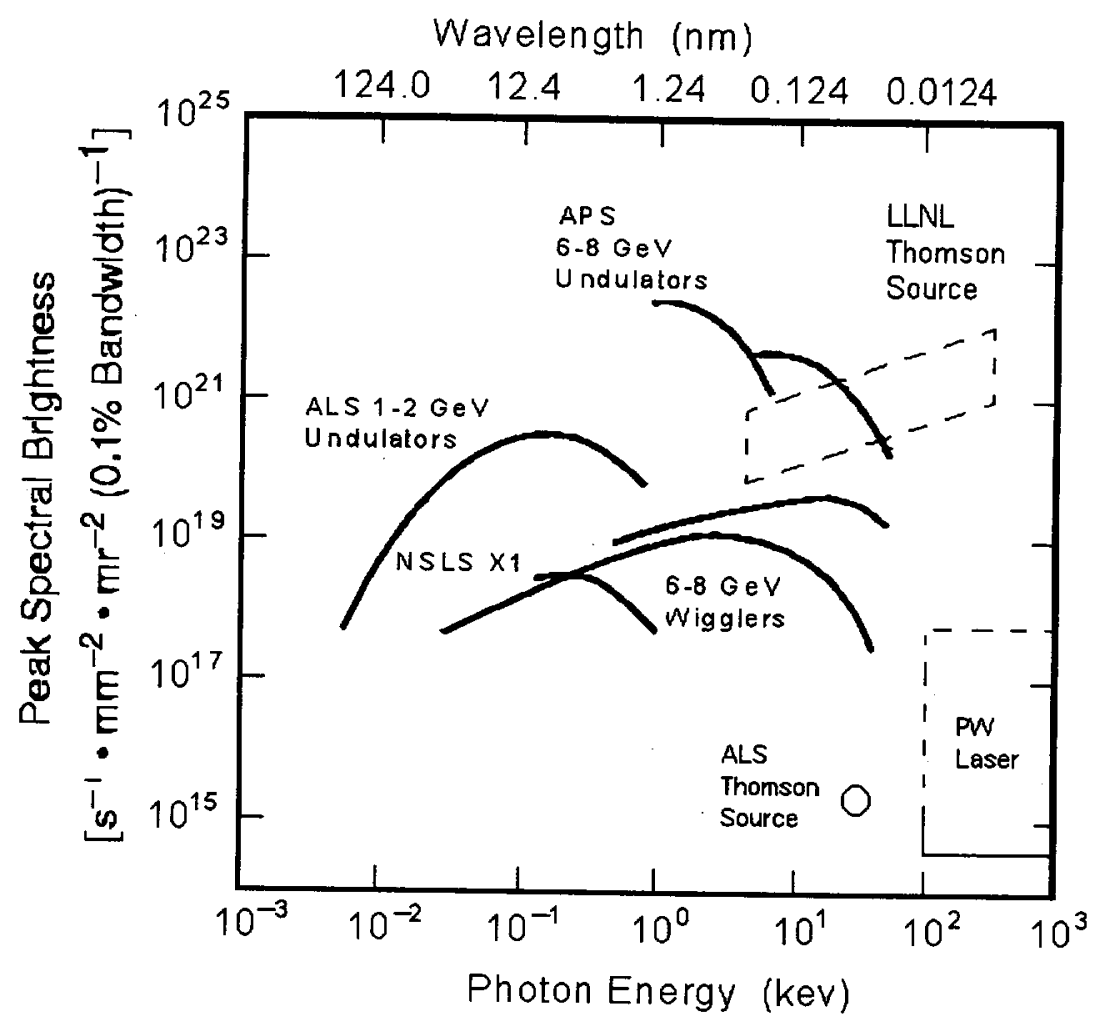

Figure 3: Comparison of the predicted peak brightness of the LLNL Thomson source with current $3^{\text {rd }}$ generation synchrotrons.

Our design is based on a RF photocathode electron injector as the key element for production of a high peak current, low emittance, short pulse, well synchronized electron beam. This photoinjector, shown schematically in Figure 4 below, has been designed, constructed, and characterized.

A pulse of S-band $(2.86 \mathrm{GHz}) \mathrm{RF}$ input with $7 \mathrm{MW}$ peak power and $3 \mu$ s pulse length produces a standing wave electric field with peak gradient greater than $100 \mathrm{MV} / \mathrm{m}$. It accelerates electrons to $5 \mathrm{MeV}$ over a distance of less than $10 \mathrm{~cm}$. A laser pulse, split from the Falcon laser oscillator is amplified and frequency tripled before striking a copper photocathode near the peak RF field to produce the electron injector beam with 1-10 $\mathrm{nC}$ of charge per pulse in a $<10$ ps pulse length. A strong magnetic field matches the beam envelope into the Linac and preserves the low transverse emittance inherent in the high-gradient photoemission process. 


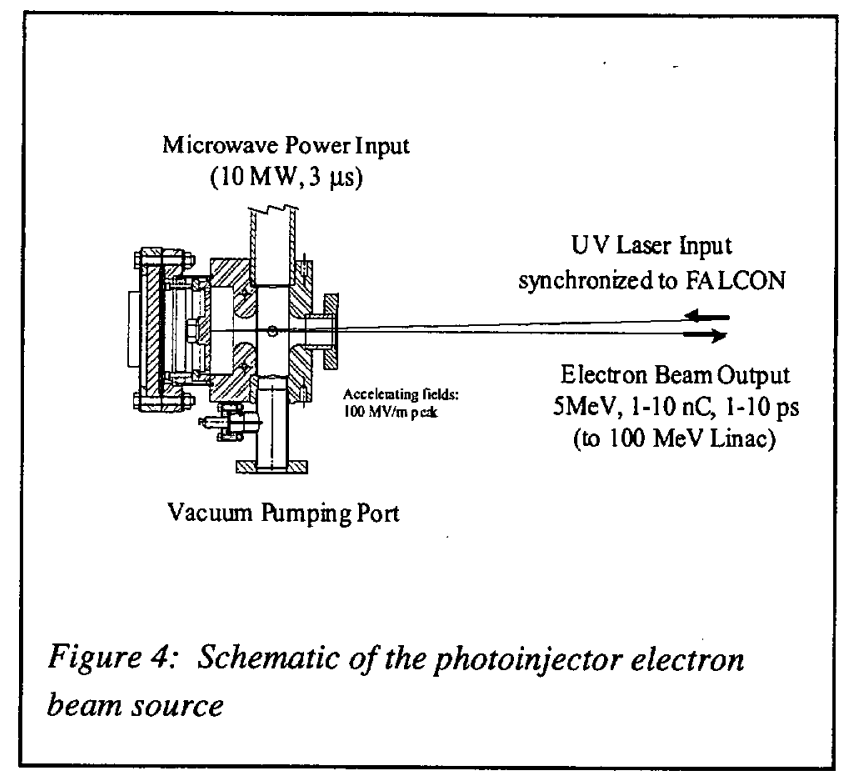

Incorporation of the photoinjector into the Linac provides the needed low emittance, high charge electron bunches, and importantly, facilitates synchronization of these bunches with the compressed laser pulse. This essential to attaining the performance predicted in Table 1.

\section{ELECTRON PULSE COMPRESSION}

At high energy $(100 \mathrm{MeV})$ the minimum focal spot obtainable for the electron beam is emittance dominated for bunch charge in the $\mathrm{nC}$ range. Simulations of the electron beam emittance have been carried out using the PARMELLA code and results have been used to predict beam performance for the Thomson scattering experiments. The results predict a low emittance beam that can be focused to a $10 \mu \mathrm{m}$ spot with a convergence angle of $5 \mathrm{mrad}$. To accomplish this the required normalized transverse emittance is $10 \mathrm{~mm}-\mathrm{mrad}$. This requirement is well within the measured emittance of the photoinjector for a charge of a few $\mathrm{nC}$. In a strong focusing field, low energy spread is also important to maintain a short longitudinal width at focus. The photoinjector can produce an energy spread of a few percent.

The pulse energy will be chirped by a dephased Linac section so that magnetic dispersion can be used to shorten the pulse. A complementary dephased Linac section removes the energy chirp after compression for maximum beam peak current. Achieving a very high peak current is most important for the transverse interaction of the Falcon laser pulse and electron beam. Ultimately, the goal is to place as many monoenergetic, collimated electrons as possible into the laser focus for the production of up-shifted $x$-ray photons.

We will install a magnetic chicane electron bunch compression system after TW section 4 of the Linac, as shown in Figure 5. This will enable shorter, more intense $x$-ray pulses. We expect to achieve an approximately ten-fold longitudinal compression of the electron bunches, with a corresponding ten-fold increase in peak current. This translates into a ten-fold increase in the x-ray flux for the short-pulse, $90^{\circ}$ scattering geometry. For the $180^{\circ}$ geometry, the $x$-ray flux will remain the same (determined by total bunch charge), but the $x$-ray pulse length will be reduced from 10 ps to $\sim 1 \mathrm{ps.}$

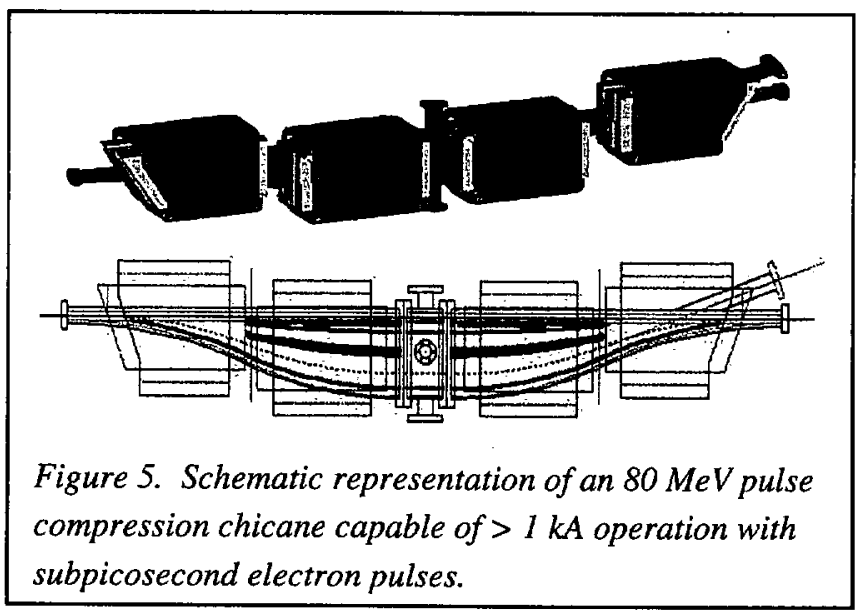


Hartemann, et.al. ${ }^{13}$ have developed a 3-D model for the beam, its interaction with the laser, and subsequent $\mathrm{X}$ ray production. It has been used to predict the $\mathrm{x}$-ray beam characteristics in this system and one result is shown in Figure 6 below. The predicted $x$-ray output is very intense and relatively narrow in wavelength $(\sim 10 \%)$.

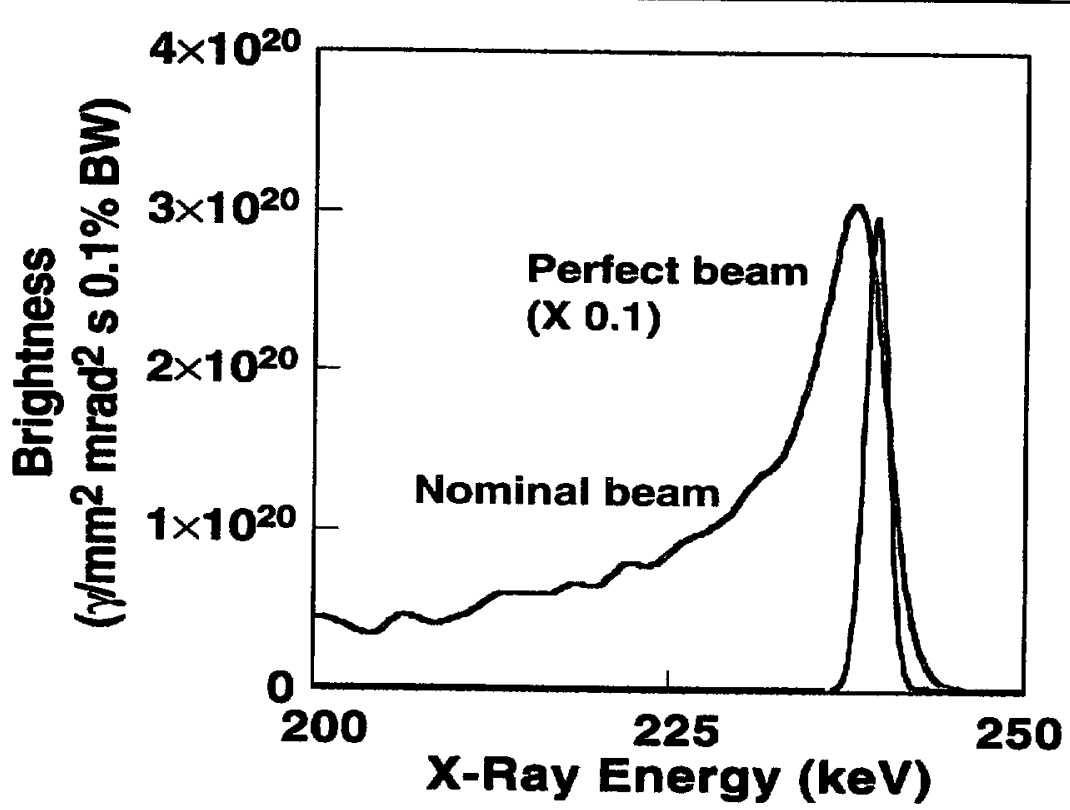

Figure 6. The spectral brightness of the LLNL Thomson source is modeled using the $3 D$ electron/laser interaction model of Hartemann. Showing the expected beam pattern and the peak spectral brightness.

Integration of the laser and electron beams has been demonstrated and we will soon commission the photoinjector on the main Linac. Simulations predict a very bright source of $\mathrm{x}$-rays, but that has not yet been demonstrated.

We propose to extend the experiments previously conducted with low time resolution on large-scale systems to the LLNL Thomson source. For the experiments proposed, we intend to use some of the energy of the Falcon laser pulse to drive a shock wave (blast wave). With the Falcon at the $4 \mathrm{~J}$ level, we will split off $1 \mathrm{~J}$ of energy and focus it to $1 \mathrm{~mm}$ on the sample to be shocked. Simulations using the Hyades hydrodynamics code of the compression and pressure as a function of time have been conducted for both Si and Al targets. These simulations indicate that we should be able to produce shocks of pressure $>100 \mathrm{kBar}$. This is adequate to access the Hugoniot-Elastic Limit (HEL) in materials like $\mathrm{Si}$ (which has an HEL at $54 \mathrm{kBar}$ ). Furthermore, this shock-driving beam is a single spatial mode supergaussian pulse, which will aid in achieving good shock uniformity.

We will perform a simple EXAFS experiment in a mid-Z element such as $\mathrm{Ag}$ (K-edge at $26 \mathrm{keV}$ ) with a similar, follow-on experiment in a high $\mathrm{Z}$ material such as $U$ (with the L-edge at $21 \mathrm{keV}$ ). We will start by conducting static EXAFS on unexcited materials. This will allow us to ascertain the brightness needed. These measurements will be followed by simple experiments in which the sample is mildly shocked; to look for shock induced shifts of the EXAFS features.

\section{SUMMARY}

A high power ( $10 \mathrm{TW}$ ) short pulse ( $35 \mathrm{fs}$ ) laser has been integrated with a $100 \mathrm{MeV}$ electron Linac to provide a Thomson scattering source of $x$-rays tunable in wavelength over the range 10-200 keV. By using a photoinjector for the Linac driven by the same short pulse laser it is possible to provide well synchronized 1-10 nC electron bunches only 1-2 ps in duration with very low emittance so that very small focal spots may be obtained. When properly focused the scattered beams are predicted to provide an unprecedented high brightness (up to $10^{11} \mathrm{x}$-rays per pulse) in durations as short as 1-2 ps, and slightly lower intensity in durations as short as $100 \mathrm{fs}$.

The $10 \%$ bandwidth, tunable wavelength range, and high per-pulse flux of the Thomson source will make it well suited for performing absorption edge spectroscopy of metals at a variety of wavelengths. The $10 \%$ bandwidth will be ideal to backlight an edge and the spectral features near it (spectra within a few hundred eV are necessary for EXAFS). Analysis of EXAFS spectra potentially achievable on the Thomson source indicate that, under the best conditions, EXAFS spectra will be possible on a single shot (if the Thomson source is operated in the high flux, 
longer pulse $180^{\circ}$ mode). Even if the highest $\mathrm{x}$-ray yields are not achieved; multi-shot experiments in which a few shots are averaged will be possible (with the added difficulty of maintaining a nearly uniform shock on every shot).

The goal of this work is to develop a short pulse high intensity $x$-ray source and demonstrate that EXAFS is indeed possible with the Thomson source.

\section{ACKNOWLEDGEMENT}

This work was performed under the auspices of the U.S. Department of Energy by the University of California, Lawrence Livermore National Laboratory under Contract No. W-7405-Eng-48.

\section{REFERENCES}

1. P. A. Lee, P. H. Citrin, P. Eisenberger, B. M. Kincaid, "Extended X-ray absorption fine structure- its strengths and limitations as a structural tool", Rev. Mod. Phys 53, 769-806 (1981).

2. J. Larsson, P. A. Heimann, A. M. Lindenberg, P. J. Schuck, P. H. Bucksbaum, R. W. Lee, H. A. Padmore, J. S. Wark, R. W. Falcone, "Ultrafast structural changes measured by time-resolved X-ray diffraction", Appl. Phys. A $\underline{66}$, 587-591 (1998).

3. C. Rose-Petruck, R. Jimenez, T. Guo, A. Cavalleri, C. W. Siders, F. Raksi, J. A. Squier, B. C. Walker, K. R. Wilson, C. P. J. Barty, "Picosecond-milliangstrom lattice dynamics measured by ultrafast X-ray diffraction", Nature 398, 310-312 (1999).

4. C. W. Siders, A. Cavalleri, K. Sokolowski-Tinten, C. Toth, T. Guo, M. Kammler, M. Horn von Hoegen, K. R. Wilson, D. von der Linde, C. P. J. Barty, "Detection of nonthermal melting by ultrafast X-ray diffraction", Science 286, 1340-1342 (1999).

5. C. Rischel, A. Rousse, I. Uschmann, P.-A. Albouy, J.-P. Geindre, P. Audebert, J.-C. Gauthier, E. Forster, J.-L. Martin, A. Antonetti, "Femtosecond time-resolved X-ray diffraction from laser-heated organic films", Nature 390, 490-492 (1997).

6. J. S. Wark, R. R. Whitlock, A. A. Hauer, J. E. Swain, P. J. Solone, "Subnanosecond x-ray diffraction from laser-shocked crystals", Phys. Rev. B 40, 5705-5714 (1989).

7. D. Kalantar, E. A. Chandler, J. D. Colvin, R. Lee, B. A. Remington, S. V. Weber, L. G. Wiley, B. H. Failor, A. Hauer, J. S. Wark, M. Meyers, G. Ravichandran, "Transient X-ray diffraction used to diagnose shock compressed solids on the Nova laser ", Lawrence Livermore National Laboratory, UCRL-JC-127927-ABS, 1997.

8. R. W. Schoenlein, W. P. Leemans, A. H. Chin, P. Volfbeyn, T. E. Glover, P. Balling, M. Zolotorev, K.-J. Kim, S. Chattopadhyay, C. V. Shank, "Femtosecond X-ray pulses at 0.4 A generated by $90^{\circ}$ Thomson scattering: A tool for probing the structural dynamics of materials", Science 274, 236-238 (1996).

9. W. P. Leemans, R. W. Schoenlein, P. Volfbeyn, A. H. Chin, T. E. Glover, P. Balling, M. Zolotorev, K.-J. Kim, S. Chattopadhyay, C. V. Shank, "Interaction of relativistic electrons with ultrashort laser pulses: Generation of femtosecond x-rays and microprobing of electron beams", IEEE J. Quant. Elec. 33, 1925-1933 (1997).

10. V.P. Yanovsky, T. Ditmire, G. Hays, T. Cowan, G. LeSage, M. D. Perry, "Multip-terawatt laser-linac facility", Conference on Lasers and Electron Optics, 410, (1999), Washington, DC, OSA Technical Digest.

11. T. Ditmire, M. D. Perry, "High intensity physics with a table-top 20 TW laser system ", Lawrence Livermore National Laboratory, UCRL-ID-133293, 1999.

12. E. Esarey, S. K. Ride, P. Sprangle, "Nonlinear Thomson scattering of intense laser pulses from beams and plasmas", Phys. Rev. E 48, 3003-3021 (1993).

13. F. V. Hartemann, H. A. Baldis, A. K. Kerman, A. Le Foll, N. C. Luhmann Jr, B. Rupp, "Three-dimensional theory of emittance in Compton scattering and X-ray protein crystallography", Phys. Rev. E $\underline{64},(2001)$. 


\title{
Replacing Ti:sapphire regenerative amplifiers with an optical parametric chirped pulse amplifier
}

\author{
I. Jovanovic \\ B. J. Comaskey \\ C. A. Ebbers \\ R. A. Bonner \\ D. M. Pennington
}

\section{INTRODUCTION}

Optical parametric chirped pulse amplification (OPCPA) ${ }^{1}$ offers several advantages when compared to regenerative chirped pulse amplification using Ti:sapphire ${ }^{2}$ or Cr:LiSAF. These advantages include low cumulative nonlinear phase (Bintegral), greater wavelength flexibility, and high gain achieved without the use of gated electro-optic modulators and multipass amplification. High-energy OPCPA systems pumped by large aperture, low repetition rate glass laser systems have produced up to $0.5 \mathrm{~J}$ of amplified stretched pulses, subsequently recompressed to $300 \mathrm{fs}^{3}$ However, OPCPA using commercially available $\mathrm{Q}$-switched pump lasers have, to date, only produced $600 \mu \mathrm{J}$ of amplified signal ${ }^{4}$ with a pump-tosignal conversion efficiency near $0.3 \%$. This low efficiency limits the attractiveness of OPCPA to facilities with high energy, shorter pulse pump lasers. We report chirped pulse amplification of broadband $1054 \mathrm{~nm}$ pulses to $31 \mathrm{~mJ}$ by single passing only $40 \mathrm{~mm}$ of gain material pumped by a commercial Q-switched laser, with a pump to signal conversion efficiency of $6 \%$.

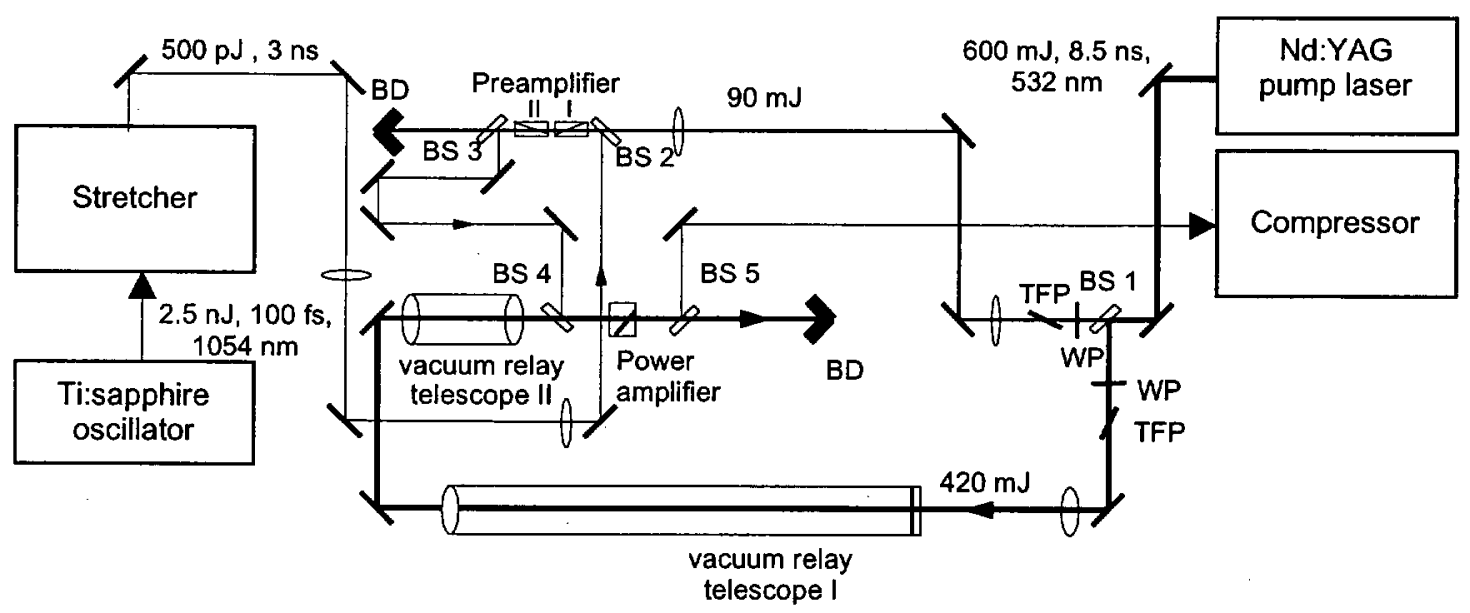

Fig. 1. Experimental setup. BS-beamsplitter, TFP-thin film polarizer, $W P$-waveplate, $B D$-beam dump.

\section{DESCRIPTION}

Our system is presented in Fig. 1. The optical parametric amplifier consists of three $\beta$-barium borate (BBO) crystals. The size of the first two crystals is $4 \times 4 \times 15 \mathrm{~mm}^{3}$, while the third crystal is $10 \times 10 \times 10 \mathrm{~mm}^{3}$, permitting scaling to large incident pump pulse energy by increasing the pump beam diameter. The crystals are cut at $22.8^{\circ}$ for type $\mathrm{I}$ angular phase matching. The first two crystals are configured as a preamplifier, with no idler separation between the crystals. $90 \mathrm{~mJ}$ is split from the pump pulse to pump the preamplifier, while $420 \mathrm{~mJ}$ is used to pump the power amplifier. The peak intensity of the pump beam in the preamplifier is $450 \mathrm{MW} / \mathrm{cm}^{2}$. The seed beam is introduced into the crystal at $1^{\circ}$ external angle with respect to the pump beam, perpendicular to the crystal principal plane. The output from the first BBO crystal was $1.8 \mu \mathrm{J}$, (Gain=3700), and from the second BBO crystal we obtained $1.5 \mathrm{~mJ}$ (Gain $=830)$. The amplified signal and the idler beam are spatially separated after propagating $30 \mathrm{~cm}$ out of the preamplifier.

The pump beam diameter in the power amplifier is $3.5 \mathrm{~mm}$, with peak intensity of $430 \mathrm{MW} / \mathrm{cm}^{2}$, and the expected small signal gain is 160 . A saturated gain of 20 was obtained from the power amplifier BBO crystal, which amplifies the signal beam up to $31 \mathrm{~mJ}$ with incident $1.5 \mathrm{~mJ}$ from the preamplifier. The input stretched pulse is $3 \mathrm{~ns}$ in length, interacting with the $8.5 \mathrm{~ns}$ (FWHM) pump pulse. Maximum pump to signal conversion efficiency in the power amplifier crystal is achieved when the preamplifier output is $1.5 \mathrm{~mJ}$, and equals $25 \%$ to the signal beam in the temporal window defined by the seed FWHM pulsewidth. The preamplifier signal output is controlled via the incident pump energy to the preamplifier. The measured bandwidth of our output beam is $16.5 \mathrm{~nm}$ FWHM, consistent with the absence of bandwidth narrowing due to intrinsic broad bandwidth of a type I difference frequency mixing process and saturation. Fig. 2 shows the measured spectra 
for stretched seed, amplified signal from the preamplifier and the power amplifier. In our configuration we saturate the OPCPA in order to obtain large extracted energy, at the expense of the recompressed pulse contrast level, which remains limited by the hard clip of the spectrum near its FWHM, which occurs in the pulse stretcher. The amplified signal spectrum is modulated by strong saturation in the power amplifier and resembles a top-hat shape near the point of maximum conversion efficiency.

A single-grating compressor is used to compress the amplified signal pulses. Stretched $500 \mathrm{pJ}$ seed pulses were compressed to $280 \mathrm{fs}$ (Fig. 3a), limited by the spherical and chromatic aberrations occurring in the pulse stretcher, which contains a lens-based refractive telescope The intensity autocorrelation of the recompressed pulse at an energy of $31 \mathrm{~mJ}$ is shown in Fig. 3b. The recompressed amplified pulse duration is measured to be $310 \mathrm{fs}, 10 \%$ longer compared to the best achieved seed recompression before amplification in OPA. Recompressed pulse pedestal is increased after amplification due to strong spectral modulation in the power amplifier. Our calculations show negligible self-phase modulation (0.04 rad) due to short beam path through the gain medium, which is a significant advantage over regenerative multipass systems.

\section{SUMMARY}

In summary, we have demonstrated the most efficient optical parametric chirped pulse amplifier to date pumped by a commercial Q-switched pump laser. The wavelength and pulse energy level obtained from our system is ideal for seeding high energy Nd:glass amplifiers of high peak power lasers. A gain of $6 \times 10^{7}$ was obtained with broad bandwidth without the use of electro-optic modulators or multipass amplification. We expect the focused intensity from our system to be on the order of $10^{16} \mathrm{~W} / \mathrm{cm}^{2}$. Although our system did not exploit the full bandwidth capabilities of OPA, we demonstrated high gain, conversion efficiency and good amplified pulse beam quality in OPCPA, which gives a viable cause for an all OPA-based kilojoule level ultrashort pulse source. With a suitable high energy pump laser and current compression grating technology, powers in excess of $10^{16} \mathrm{~W}$ are feasible using OPCPA ${ }^{5}$.

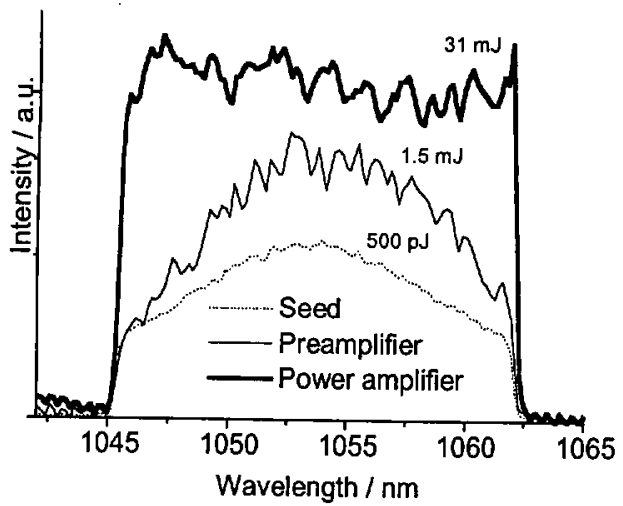

Fig. 2. Seed and amplified signal spectra from preamplifier and power amplifier
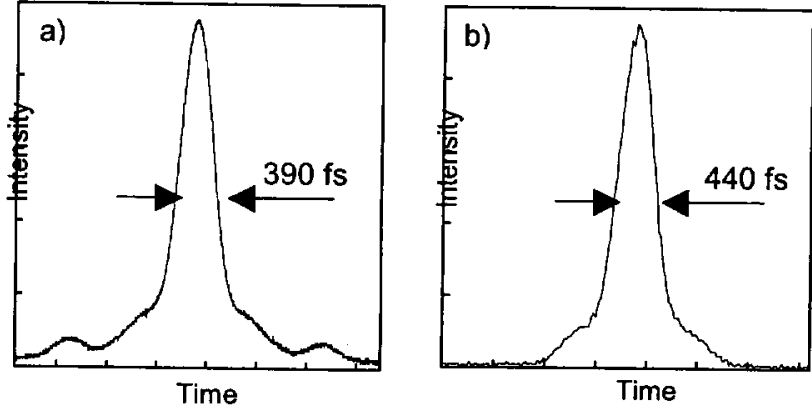

Fig. 3. Recompressed pulse intensity autocorrelation for a) seed pulses, and b) amplified pulses. Indicated is FWHM of the autocorrelation trace. Deconvolved pulse width is a) $280 \mathrm{fs}$, b) $310 \mathrm{fs}$.

\section{ACKNOWLEDGMENTS}

The authors would like to thank Mark Hermann and Chris Barty of Lawrence Livermore National Laboratory for their helpful critiques, comments, and suggestions.

This work is performed under the auspices of the U.S. Department of Energy by Lawrence Livermore National Laboratory under Contract W-7405-Eng-48.

\section{REFERENCES}

1. A. Dubeitis, G. Jonu_auskas, and. A. Piskarskas, Opt. Commun. 88, 437 (1992).

2. K. Yamakawa and C. P. J. Barty, IEEE J. Sel. Topics Quant. Electronics 6, 658 (2000).

3. I. N. Ross, J. L. Collier, P. Matousek, C. N. Danson, D. Neely, R. M. Allott, D. A. Pepler, C. Hernandez- Gomez, and K. Osvay, Appl. Opt. 39, 2422-2427 (2000).

4. S. K. Zhang, M. Fujita, H. Yoshida, R. Kodama, H. Fujita, M. Nakatsuka, Y. Izawa, and C. Yamanaka, Proc. SPIE 3886, 588-595 (1999).

5. I. N. Ross, P. Matousek, M. Towrie, A .J. Langley, J. L. Collier, Opt. Commun. 144, 125-133 (1997). 


\title{
Highly efficient tabletop optical parametric chirped pulse amplifier at $1 \mu \mathrm{m}$
}

\author{
I. Jovanovic ${ }^{* a, b}$ \\ B. J. Comaskey ${ }^{a}$ \\ C. A. Ebbers ${ }^{a}$ \\ R. A. Bonner ${ }^{a}$ \\ Edward C. Morse ${ }^{b}$ \\ ${ }^{a}$ National Ignition Facility Programs, Lawrence Livermore National Laboratory \\ ${ }^{b}$ Department of Nuclear Engineering, University of California at Berkeley
}

\begin{abstract}
Optical parametric chirped pulse amplification (OPCPA) is a scalable technology for ultrashort pulse amplification. Its major advantages include design simplicity, broad bandwidth, tunability, low B-integral, high contrast, and high beam quality. OPCPA is suitable both for scaling to high peak power as well as high average power.

We describe the amplification of stretched $100 \mathrm{fs}$ oscillator pulses in a three-stage OPCPA system pumped by a commercial, single-longitudinal-mode, Q-switched Nd:YAG laser. The stretched pulses were centered around $1054 \mathrm{~nm}$ with a FWHM bandwidth of $16.5 \mathrm{~nm}$ and had an energy of $0.5 \mathrm{~nJ}$. Using our OPCPA system, we obtained an amplified pulse energy of up to $31 \mathrm{~mJ}$ at a $10 \mathrm{~Hz}$ repetition rate. The overall conversion efficiency from pump to signal is $6 \%$, which is the highest efficiency obtained with a commercial tabletop pump laser to date. The overall conversion efficiency is limited due to the finite temporal overlap of the seed $(3 \mathrm{~ns})$ with respect to the duration of the pump $(8.5 \mathrm{~ns})$. Within the temporal window of the seed pulse the pump to signal conversion efficiency exceeds $20 \%$. Recompression of the amplified signal was demonstrated to $310 \mathrm{fs}$, limited by the aberrations initially present in the low energy seed imparted by the pulse stretcher. The maximum gain in our OPCPA system is $6 \times 10^{7}$, obtained through single passing of $40 \mathrm{~mm}$ of beta-barium borate. We present data on the beam quality obtained from our system $\left(\mathrm{M}^{2}=1.1\right)$.

This relatively simple system replaces a significantly more complex Ti:sapphire regenerative amplifier-based CPA system used in the front end of a high energy short pulse laser. Future improvement will include obtaining shorter amplified pulses and higher average power.
\end{abstract}

\section{INTRODUCTION}

Generation and amplification of ultrashort (femtosecond) laser pulses is important for a myriad of scientific and commercial applications. Scientific applications of high peak power short-pulse lasers include high-harmonic and x-ray generation, generation and acceleration of electron and proton beams, and fast ignition for inertial confinement fusion. On the commercial side, high average power short-pulse laser materials processing ${ }^{1}$ and biomedical applications such as laser surgery ${ }^{2}$ are becoming more present as the femtosecond sources become cheaper, more robust and reliable. Chirped pulse amplification (CPA) ${ }^{3}$ is the state-of-the-art technology that allows laser amplification of ultrashort pulses to high pulse energies, while avoiding damage in the laser amplifiers induced by high intensity associated with ultrashort pulses. Ti:sapphire is an ubiquitous laser gain material used for ultrashort pulse amplification, which is the result of its extremely broad spectral bandwidth and favorable mechanical properties. To obtain energetic femtosecond pulses at $1 \mu \mathrm{m}$, hybrid systems have been used previously, such as a combination of a Ti:sapphire front end and a high-energy Nd:glass laser. ${ }^{4}$ By producing most of the laser gain in the broadband Ti:sapphire front end, sufficient bandwidth exists in Nd:glass to produce high-energy pulses as short as several hundred femtoseconds. One problem with the $1 \mu \mathrm{m}$ front end based on Ti:sapphire is the relatively large number of passes necessary to produce sufficient gain, since the gain of Ti:sapphire is relatively small near $1 \mu \mathrm{m}$. As an example, the number of passes in a $1 \mathrm{~mJ}$ Ti:sapphire regenerative amplifier is often greater than $100 .{ }^{5}$ As a result, relatively large dispersion and B-integral occurs in the system. Regenerative laser cavity produces prepulses, which are spaced at an integer multiple of the cavity round-trip time prior to the main pulse.

*Corresponding author; e-mail: jovanovic1@1lnl.gov 
Prepulses are amplified in the subsequent amplifiers, and they experience amplification which is often greater than the amplification of the main pulse. The resulting prepulses can become energetic enough to perturb the target and produce undesirable experimental conditions.

An alternative convenient approach exists to obtain broad bandwidth amplification at $1 \mu \mathrm{m}$ and avoid complicated regenerative amplification schemes. Optical parametric amplifiers (OPAs) ${ }^{6}$ can be used in CPA as an alternative to laser amplifiers. The technique was demonstrated in $1992,{ }^{7}$ and termed parametric chirped pulse amplification (PCPA) ${ }^{7}$ and, more recently, optical parametric chirped pulse amplification (OPCPA). ${ }^{8}$ As opposed to laser amplification, OPCPA is an instantaneous nonlinear process, in which the energy is transferred from the high-frequency pump to lower frequency signal and idler waves. The signal wave is used in subsequent amplification in a laser system, if desired, while the idler wave at the difference frequency is discarded.

The first demonstration of OPCPA featured modest stretching ratios $(\sim 18)$ and $\mu \mathrm{J}$-level amplified pulse energies. ${ }^{7}$ Subsequently, arguments were made for scaling OPCPA to high peak power by using large-aperture KDP crystals. ${ }^{8}$ More recent experimental development produced TW-level pulses using OPCPA pumped by a large glass laser. ${ }^{9}$ While a high conversion efficiency of $20 \%$ in an OPCPA system based on a quasi-phase-matched OPA pumped by a fiber laser has been reported, ${ }^{10}$ experiments that use commercial tabletop Q-switched Nd:YAG lasers for pumping OPAs were relatively inefficient, with maximum reported energies of $0.6 \mathrm{~mJ}$ and conversion efficiencies of $0.6 \%{ }^{11,12}$ This was a result of both the pump laser characteristics and the particular OPCPA design. Here we report, to our knowledge, the most efficient $1 \mu \mathrm{m}$ OPCPA system pumped directly by a simple Q-switched Nd:YAG pump laser, which is normally used to pump Ti:sapphire. Our OPA exhibited efficiency of $6 \%$, which is a ten-fold improvement over previous results. In the subsequent chapters, we will describe the advantages of using OPCPA compared to regenerative CPA. We describe our numerical model for OPCPA and system design, followed by the results of our experiments and conclusion.

\section{CHARACTERISTICS OF OPCPA}

OPCPA has numerous advantages compared to conventional regenerative amplification systems. Most of those advantages are related to the shortness of amplification path and the single-pass architecture. Design simplicity is the inherent characteristic of OPCPA. Some of important characteristics of OPA design include precise timing between signal and pump, and relay imaging of the desired pump profile onto OPA crystals. Relay imaging of the near-field pump profile is important because, in many cases, near field of the pump laser is a flat top beam, which facilitates transversely uniform amplification. Additionally, pump uniformity reduces the risk of crystal damage. Relay imaging often requires vacuum telescopes to prevent air breakdown in the beam focus. This can be sometimes avoided by using a large $f-$ number focusing, at the expense of the increasing physical size of the system.

One of the most important advantages of OPCPA is the elimination of electro-optic modulators. This includes the modulators inside the regenerative laser cavity, and the pulse slicer that is normally used after the pulse stretcher. It is unnecessary to select a single pulse from the oscillator pulse train, because only the pulse that is temporally overlapped with the pump will be amplified. Pulse contrast in such OPCPA system that does not employ pulse selection prior to amplification is essentially determined by the total signal gain in the system. Some parametric fluorescence can be also produced, which is temporally coincident with the amplified pulse. Since amplification cavities are not used, no cavityrelated prepulses exist in OPA such as in regenerative amplifiers.

The amplification length in OPA is typically very short - even when nanosecond pulses are used, typical length is on the order of a few $\mathrm{cm}$. Short amplification length results in negligible accumulated nonlinear phase (B-integral). Typical amplification bandwidth is broad, on the order of $100 \mathrm{~nm} \cdot \mathrm{cm}$, which allows amplification of ultrashort pulses. OPCPA is more flexible than laser CPA with respect to the selection of center wavelength for amplification. Broad bandwidth is naturally achieved in a degenerate or a nearly degenerate OPA that operates in collinear geometry. Broad bandwidth around other center wavelengths can be also achieved in nondegenerate OPCPA using a noncollinear geometry. Additional flexibility of center wavelength can be achieved by using quasi-phase matching and alternative pump wavelengths.

Good beam quality can be obtained from OPA, which is a characteristic of a process which has relatively narrow angular tolerance. In effect, OPA acts like a spatial filter and can produce very good beam quality. For high average power applications, it is important that OPA does not exhibit significant intrinsic heat load. The absence of quantum defect allows all the energy that enters the nonlinear crystal to leave it in the form of optical field, except for minimal material absorption. This positions OPCPA as a technology with a potential to produce ultrashort pulses with average power in excess of $1 \mathrm{~kW}$. 
The most problematic feature with OPCPA is the performance limit when tabletop commercial pump lasers are used for pumping OPCPA. In the first approximation, the conversion efficiency in OPCPA is determined by the temporal overlap of signal and pump. Since the pump pulse width in simple tabletop pump lasers is often relatively long compared to the stretched signal pulse width, relatively poor conversion efficiency results.

Relatively high beam quality is required from the pump laser in OPCPA that uses critical angular phase matching due to narrow angular tolerance. ${ }^{13}$ The desired transverse and temporal profile of the OPCPA pump laser is a top-hat. A uniform pump beam produces uniform amplification in space and in time (spectrum) of the chirped signal beam in the small-signal regime. When depletion of the pump beam occurs, it is also desirable that the spatial and temporal profile of the signal is a top-hat, which maximizes efficiency. This can be approximately achieved by using an OPA preamplifier in which some back-conversion occurs. Alternatively, gaussian temporal profile can also be used, in a combination with a double-hump-shaped seed, which can produce a gaussian beam with high conversion efficiency.

OPCPA has to operate in the regime of high pump depletion to achieve high stability, ${ }^{14}$ which is not an inherent characteristic of a nonlinear process in the small-signal regime.

\section{NUMERICAL MODEL}

We developed a numerical model of OPCPA and used it to optimize the performance of our preamplifier and the power amplifier. We base our numerical model on the system of coupled differential equations that govern difference frequency generation:

$$
\begin{aligned}
& \frac{d A_{1}}{d z}=i \frac{2 \omega_{1}}{n_{1} c} d_{e f f} A_{2}^{*} A_{3} \exp (i \Delta k z), \\
& \frac{d A_{2}}{d z}=i \frac{2 \omega_{2}}{n_{2} c} d_{e f f} A_{1}^{*} A_{3} \exp (i \Delta k z), \\
& \frac{d A_{3}}{d z}=i \frac{2 \omega_{3}}{n_{3} c} d_{e f f} A_{1} A_{2} \exp (-i \Delta k z) .
\end{aligned}
$$

We denote by $A_{1}, A_{2}$, and $A_{3}$ the amplitude of the electric field of the signal, idler, and pump, respectively; $d_{e f f}$ is the effective nonlinearity, and $\Delta k$ is the wave vector mismatch. Eqs. (1)-(3) describe the propagation of traveling monochromatic waves with negligible group velocity dispersion (GVD). This approximation is allowed, since we are modeling nanosecond pulses. Small dephasing introduced by GVD is negligible over the length of nonlinear crystal. We do not include diffraction explicitly, assuming that the beam quality of the pump and seed is relatively good, so that it allows the great majority of the angular spectrum within the angular acceptance of OPA. In nanosecond OPAs, beam focusing is relatively weak and the resulting dephasing is small. Similarly, apart from depletion effects, pump profile can be assumed constant along the amplification length.

An important feature of our experiments is the spatial and temporal nonuniformity of pump and signal pulses. This implies that the coupled differential equations Eqs. (1)-(3) cannot be used directly, but have to be solved on a spatiotemporal grid that captures the characteristics of pump and seed pulses. In addition, birefringent walk-off and optional noncollinearity reduce the effective amplification length and introduce a more complicated spatial coupling of pump, signal, and idler. This is very important when the beam diameter is comparable to total transverse walk-off over the crystal length.

Intensity distribution at a point $(x, y, t)$ is related to the amplitude of electric field:

$$
I_{i}(x, y, t)=2 n_{i} \sqrt{\frac{\varepsilon_{0}}{\mu_{0}}}\left|A_{i}(x, y, t)\right|^{2}, \quad i=1,2,3,
$$

which can be normalized as following:

$$
\int_{-\infty}^{\infty} \int_{-\infty}^{\infty} \int_{-\infty}^{\infty} I_{i}(x, y, t) d x d y d t=E_{i}, \quad i=1,2,3,
$$

where $E$ is the integrated pulse energy. The effect of beam walk-off and noncollinearity is modeled as follows:

$$
\begin{aligned}
& x_{1}^{\prime}=x_{1}+\Omega_{1 x} z, y_{1}^{\prime}=y_{1}+\Omega_{1 y} z, \\
& x_{2}{ }^{\prime}=x_{2}+\Omega_{2 x^{2}}, y_{2}{ }^{\prime}=y_{2}+\Omega_{2 y} z,
\end{aligned}
$$




$$
x_{3}{ }^{\prime}=x_{3}+\rho z,
$$

where $\rho$ is the walk-off angle, $\left(\Omega_{x}, \Omega_{y}\right)$ are the noncollinear angles, and $\left(x^{\prime}(z), y^{\prime}(z)\right)$ are the coordinates corrected for walk-off and noncollinearity at the axial distance $z$. Wave vector mismatch can be modeled for a point in time $t$ in collinear geometry as

$$
\Delta k_{z}(t) \approx 2 \pi\left(\frac{n_{3}}{\lambda_{3}}-\frac{n_{1}(t)}{\lambda_{1}(t)}-\frac{n_{2}(t)}{\lambda_{2}(t)}\right)
$$

Using those definitions, the system of differential equations Eqs. (1)-(3) can be written as

$$
\begin{aligned}
& \frac{d A_{1}\left(x_{1}{ }^{\prime}, y_{1}{ }^{\prime}, t\right)}{d z}=i \frac{2 \omega_{1}(t)}{n_{1}(t) c} d_{e f f} A_{2}^{*}\left(x_{2}{ }^{\prime}, y_{2}{ }^{\prime}, t\right) A_{3}\left(x_{3}{ }^{\prime}, y, t\right) \exp (i \Delta k(t) z), \\
& \frac{d A_{2}\left(x_{2}{ }^{\prime}, y_{2}{ }^{\prime}, t\right)}{d z}=i \frac{2 \omega_{2}(t)}{n_{2}(t) k} d_{e f f} A_{1}^{*}\left(x_{1}{ }^{\prime}, y_{1}{ }^{\prime}, t\right) A_{3}\left(x_{3}{ }^{\prime}, y, t\right) \exp (i \Delta k(t) z), \\
& \frac{d A_{3}\left(x_{3}{ }^{\prime}, y, t\right)}{d z}=i \frac{2 \omega_{3}(t)}{n_{3}(t) c} d_{e f f} A_{1}\left(x_{1}{ }^{\prime}, y_{1}{ }^{\prime}, t\right) A_{2}\left(x_{2}{ }^{\prime}, y_{2}{ }^{\prime}, t\right) \exp (-i \Delta k(t) z) .
\end{aligned}
$$

In the next step, electric field discretization is performed using $(D X, D Y, D T)$ points in a spatial and temporal window size $(W X, W Y, W T)$, with the following normalization condition:

$$
2 n_{i} \sqrt{\frac{\varepsilon_{0}}{\mu_{0}}} \frac{W X}{D X} \frac{W Y}{D Y} \frac{W T}{D T} \sum_{j=1}^{D X} \sum_{k=1}^{D Y} \sum_{l=1}^{D T}\left|A_{i}(j, k, l)\right|^{2}=E_{i}, \quad i=1,2,3 .
$$

We solve system (9) using numerical integration to arrive with the expected energy, transverse beam profile and temporal/spectral profile of the amplified pulse.

\section{SYSTEM DESIGN AND RESULTS}

We used our numerical model to optimize the design of our OPCPA system. Initially, we measured the spatio-temporal evolution of the pump pulse. Our pump laser was a Spectra-Physics GCR 270-10 Q-switched tabletop pump laser, operating in a single longitudinal mode. Operation of the pump laser in single longitudinal mode is important, because the temporal modulation of pump pulse is imprinted on the amplified pulse spectrum. Additionally, since OPCPA crystals are often driven close to their damage threshold, strong temporal modulation can lead to instantaneous intensity beyond the material damage threshold. The pulse evolution measurement was performed using a scanning pinhole and a fast silicon diode in the image plane of the second harmonic of the pump. The numerical fit to the data in radial and temporal dimensions is shown in Fig. 1.

The most prominent feature of the characterized pump pulse is that the pulse build-up starts preferentially in the center of the laser cavity. At the back of the pulse, radially more distant parts of the pulse achieve appreciable intensity, while the intensity in the center drops due to the depletion of the laser gain medium. In effect, the pulse exhibits different transverse spatial profile at different points in time. In order to model the performance of OPCPA as an instantaneous process, it is important to include this particular pulse shape in the numerical model.

We present our OPCPA system design in Fig. 2. We used a mode-locked Ti:sapphire oscillator to generate the seed pulses. The oscillator was a Spectra-Physics Tsunami, operating at a center wavelength of $1054 \mathrm{~nm}$, with $16.5 \mathrm{~nm}$ FWHM bandwidth, producing 100-fs pulses. In this experiment, a pulse selector was used to select a single oscillator pulse at a repetition rate of $10 \mathrm{~Hz}$. While there is no fundamental reason to select a single pulse in an OPCPA system because the selection is performed by OPA, we still used a pulse slicer in order to obtain more reliable low-background measurement of the amplified pulse spectrum and profile. The pulse is introduced into the stretcher, which is based on a single grating and a plano-convex lens. A stripe-mirror design of the stretcher grating allows the pulse to be expanded to $3 \mathrm{~ns}$ in a relatively compact setup. The plano-convex lens introduces chromatic and spherical aberrations, eventually limiting the recompressed pulse width to several hundred fs.

The OPA is a quasi-two-stage amplifier, consisting of a high-gain preamplifier and a power amplifier. A novel design is introduced in the high-gain preamplifier, with two closely spaced beta-barium borate (BBO) crystals arranged in a walkoff compensating scheme. Independent angular tuning is realized for both crystals. The small air gap $(\sim 2 \mathrm{~mm})$ between the crystals provides for several advantages of this design. First, small dephasing in air allows high gain. Only single relay imaging of the pump beam is necessary for both crystals, and the pump beam is reused after the first crystal, where it is essentially undepleted. Finally, perfect timing of pump and seed can be achieved in both crystals by timing the pump 
and the seed in the first crystal only. The idler is propagated from the first into the second crystal, since the required bandwidth does not necessitate idler separation after propagating through the first crystal. Both preamplifier crystals were $15 \mathrm{~mm}$ long, and cut at $22.8^{\circ}$ to facilitate type I angular phase matching. All the crystals were cut with a $2^{\circ}$ wedge on the output surface to reduce the effect of parasitic oscillation.

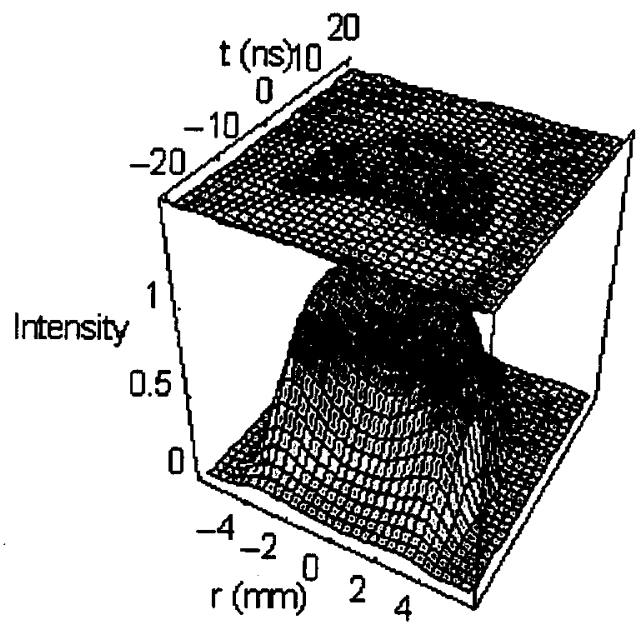

Figure 1. Spatio-temporal evolution of the pump laser. Shown is the numerical fit to the data taken on our pump laser.

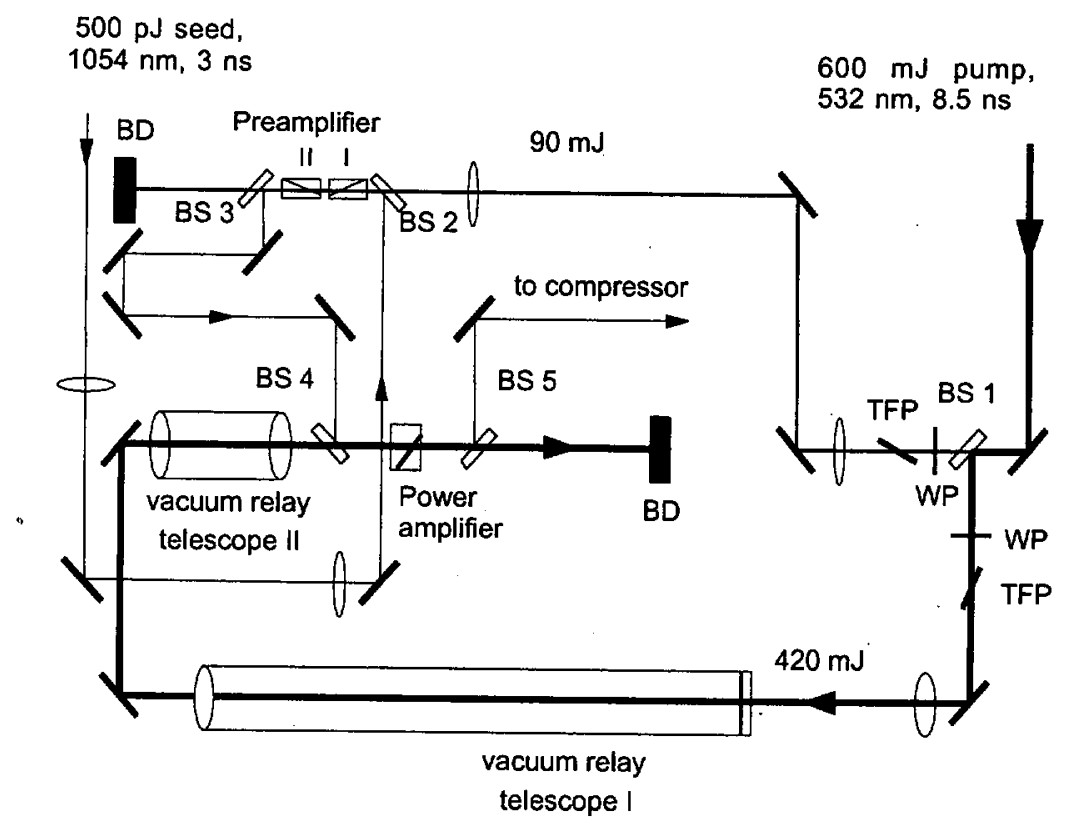

Figure 2. OPCPA setup. BS-beamsplitter, TFP-thin film polarizer, WP-waveplate, BD-beam dump

$500-\mathrm{pJ}$ stretched seed pulses are introduced into the preamplifier by reflection from a dichroic beamsplitter, at a $0.5^{\circ}$ external noncollinear angle with respect to the pump beam. The preamplifier is pumped by $85 \mathrm{~mJ}$ of pump energy, producing a peak intensity of $450 \mathrm{MW} / \mathrm{cm}^{2}$. The intensity we used allowed us to run our system at an appreciable repetition rate of $10 \mathrm{~Hz}$, with a minimal risk of crystal damage. The obtained maximum amplified pulse energy from the preamplifier was $1.5 \mathrm{~mJ}$, for a gain of $3 \times 10^{6}$. The idler is angularly separated and discarded after amplification in the 
preamplifier, while the signal is appropriately delayed and introduced into the final amplifier crystal. While the aperture of the preamplifier crystals is $4 \mathrm{~mm}^{2}$, the power amplifier is a $10 \mathrm{~mm}$-long BBO crystal of sufficient aperture $\left(10 \mathrm{~mm}^{2}\right)$ to handle high pulse energy available from our pump laser. The power amplifier was pumped by $420 \mathrm{~mJ}$ of energy from the pump laser, with the maximum intensity of $420 \mathrm{MW} / \mathrm{cm}^{2}$. The maximum amplified signal energy was $31 \mathrm{~mJ}$, which is $20 \%$ of the calculated pump energy in the temporal window defined by the pump pulse. With the overall efficiency of $6 \%$, this system represents the most efficient OPCPA system pumped by a tabletop Q-switched pump laser. We measured the output from the power amplifier and compared the results with our numerical model described above. With several different energies used to seed the power amplifier from the preamplifier, a good agreement was obtained between the calculations and the experiment (Fig. 3).

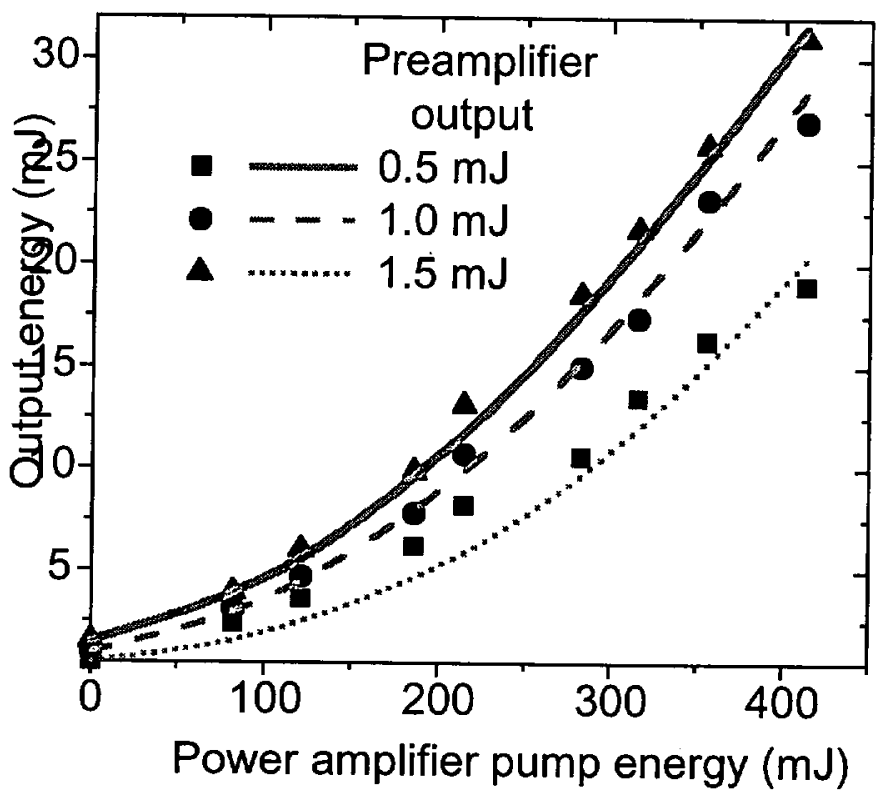

Figure 3. Amplified signal energy in the power amplifier for several inputs from the preamplifier. Indicated by lines are the results obtained from our numerical model.

Seed and amplified pulse spectra were measured, and the results are shown in Fig 4. The hard-clip points in the spectrum are the result of spectral slipping on the stretcher lens. After amplification in preamplifier, which does not operate near saturation, spectrum is slightly narrowed at its FWHM. The clip points in the spectrum remain the same, however, indicating that this spectral narrowing is a temporal CPA effect. After amplification in the saturated power amplifier, the spectrum is modified and resembles a top-hat shape. This is the result of saturated conversion and the onset of backconversion in the pulse temporal center, while the pulse wings are still converting with relatively high efficiency. This hard spectral shape eventually limits the recompressed pulse contrast. To verify the depletion of the pump beam in the power amplifier, we scattered the residual pump and monitored the instantaneous power on a fast silicon diode. As shown in Fig. 5, when we maximized our amplified pulse energy from the system by adjusting the seed-pump delay, the point of maximum pump depletion did not occur at the point in time when the pump pulse power is maximum. This can be understood by considering the spatio-temporal evolution of the pump pulse. Using the pulse evolution data in Fig. 1, and by calculating the peak power of the central $70 \%$ of the transverse pump size, we were able to determine that the central part of the pulse peaks 2-3 ns earlier compared to the pump pulse integrated across the transverse profile. The result of this calculation is also shown in Fig. 5. Since the beam center is more important for amplification in our system, this effect is consistent with expectations.

We measured the beam quality from our system, and by optimizing the beam overlap and minimizing the noncollinear angle in the power amplifier, we were able to obtain beam quality which is close to diffraction limit $\left(\mathrm{M}^{2}=1.1-1.2\right)$. In Fig. 6 we show the measured beam profiles in the near field and in the far field. 


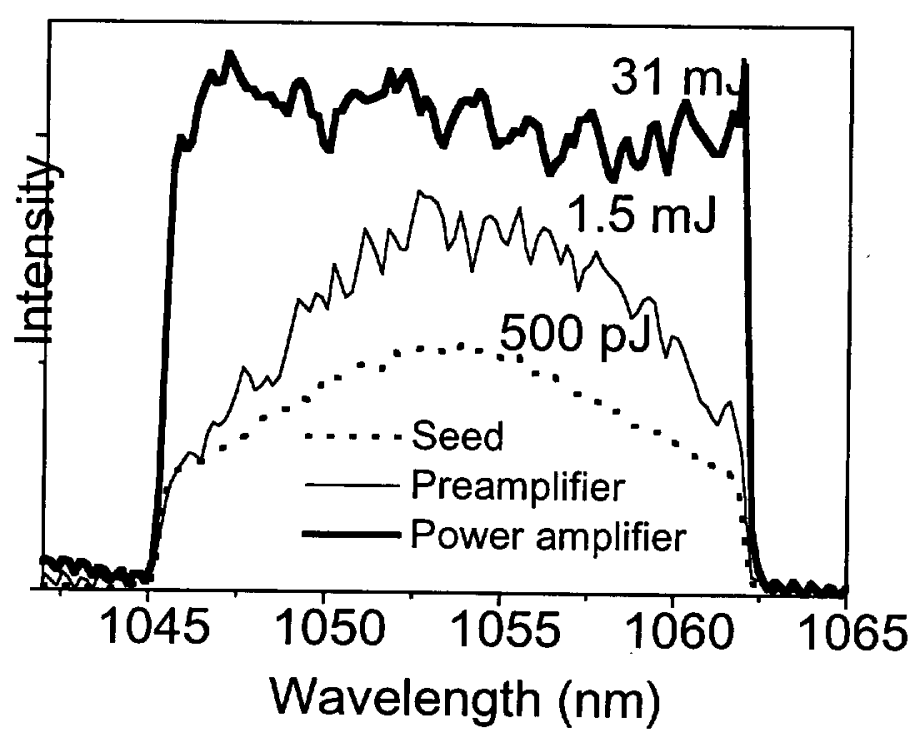

Figure 4. Seed and amplified signal spectra in $O P C P A$

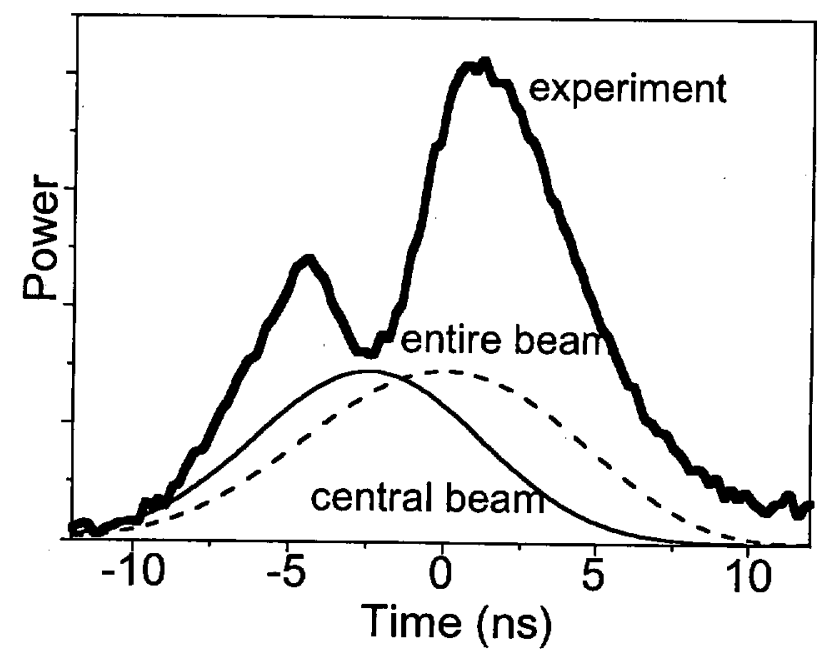

Figure 5. Maximum energy extraction occurs at the temporal peak of the central $70 \%$ of the pump pulse

We recompressed the amplified pulses in a single-grating compressor, with $50 \%$ efficiency. The obtained 15 -mJ pulses were characterized using intensity autocorrelation. We initially recompressed the seed pulses prior to amplification to 280 fs (Fig. 7 (a)). After amplification, the recompressed pulse width was 310 fs (Fig. 7 (b)). This small inconsistency may be the result of spectral modification in the amplification process, and a possible small uncertainty between the two autocorrelation devices used in the measurement (a scanning autocorrelator was used in Fig. 7 (a), while a single-shot autocorrelator was used in Fig. 7 (b)). As mentioned previously, we attribute the recompression results which are 3 times worse than the transform limit to uncompensated aberrations that arise in the pulse stretcher. Since the exact nature and impact of aberrations if unknown, a conservative gaussian deconvolution factor was used when calculating the pulse width from the measured autocorrelation traces. 

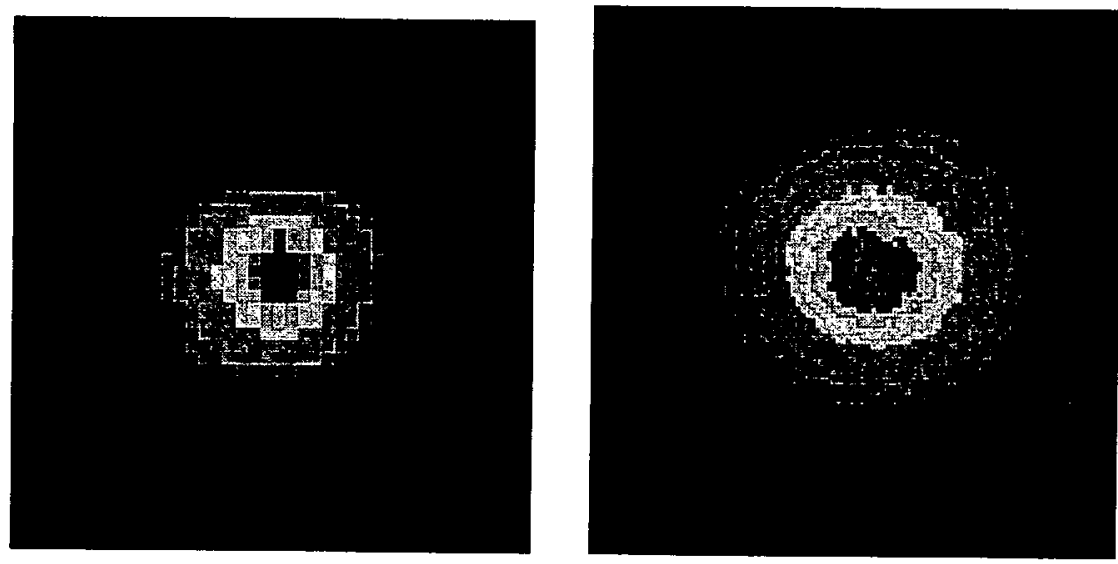

Figure 6. Amplified pulse at full energy, in (a) near field and (b) far-field

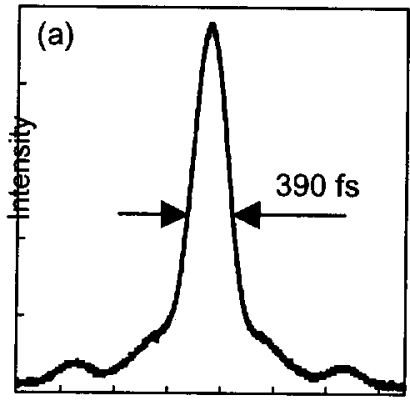

Time

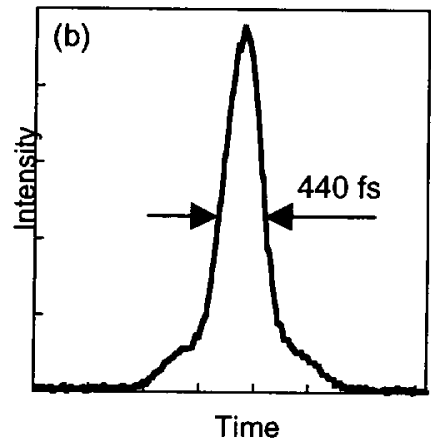

Time

Figure 7. Recompressed pulse intensity autocorrelation, (a) before amplification and (b) after amplification. Deconvolved pulse width is (a) $280 \mathrm{fs}$, and (b) $310 \mathrm{fs}$.

\section{CONCLUSION}

In conclusion, we demonstrated, to our knowledge, the most efficient OPCPA system to date pumped by a tabletop, commercial, Q-switched pump laser. In a single pass through only $40 \mathrm{~mm}$ of gain medium, a gain of $6 \times 10^{7}$ was realized. The conversion efficiency of $6 \%$ is limited by the spatio-temporal evolution of the pump pulse and the mismatch in the pulse duration between the signal and the pump pulse. This indicates that the future development of shorter-pulse nanosecond pump lasers suitable for OPCPA is desirable to improve the efficiency of the technique.

While our system did not demonstrate the transform-limited pulses, the obtained system is nevertheless applicable as a front end for a large glass-based petawatt-scale laser system, or as a stand-alone system usable for such applications as materials processing. OPCPA proved as a valuable technique with sufficient potential to substitute for regenerative amplification in Ti:sapphire at $1 \mu \mathrm{m}$, and allowed such simplifications as the elimination of electro-optic switching. Further developments of high average power pump lasers suitable for pumping OPCPA may lead to future record-setting average power in ultrashort pulses.

\section{ACKNOWLEDGEMENTS}

The authors wish to acknowledge generous support of Deanna M. Pennington and Hector Baldis and useful discussions with Paul S. Banks and Michael D. Perry. This work was performed under the auspices of the U. S. Department of Energy by the University of California, Lawrence Livermore National Laboratory under Contract No. W-7405-Eng-48. 


\section{REFERENCES}

1. B. C. Stuart, M. D. Feit, S. Herman, A. M. Rubenchik, B. W. Shore, and M. D. Perry, "Optical ablation by highpower short-pulse lasers," J. Opt. Soc. Am. B 13, pp. 459-468, 1996.

2. J. Neev, L. B. Da Silva, M. D. Feit, M. D. Perry, A. M. Rubenchik, B. C. Stuart. "Ultrashort pulse lasers for hard tissue ablation," IEEE Journal of Selected Topics in Quantum Electronics 2, pp. 790-800, 1996.

3. D. Strickland and G. Mourou, "Compression of amplified chirped optical pulses," Opt. Commun. 56, pp. 219221, 1985.

4. M. D. Perry, D. Pennington, B. C. Stuart, G. Tiethohl, J. A. Britten, C. Brown, S. Herman, B. Golick, M. Kartz, J. Miller, H. T. Powell, M. Vergino, and V. Yanovsky, "Petawatt laser pulses," Opt. Lett. 24, pp. 160-162, 1999.

5. B. C. Stuart, S. Herman, and M. D. Perry, "Chirped-pulse amplification in Ti:sapphire beyond $1 \mu \mathrm{m}$," IEEE J. Quantum Electron. 31, pp. 528-538, 1995.

6. R. A. Baumgartner and R. L. Byer, “Optical Parametric Amplification,” IEEE J. Quantum Electron. 15, pp. 432444, 1979.

7. A. Dubietis, G. Jonusauskas, and A. Piskarskas, "Powerful femtosecond pulse generation by chirped and stretched pulse parametric amplification in BBO crystal," Opt. Commun. 88, pp.437-440, 1992.

8. I. N. Ross, P. Matousek, M. Towrie, A. J. Langley, and J. L. Collier, "The prospects for ultrashort pulse duration and ultrahigh intensity using optical parametric chirped pulse amplifiers," Opt. Commun. 144, pp. 125-133, 1997.

9. I. N. Ross, J. L. Collier, P. Matousek, C. N. Danson, D. Neely, R. M. Allott, D. A. Pepler, C. HernandezGomez, and K. Osvay, "Generation of terawatt pulses by use of optical parametric chirped pulse amplification." Appl. Opt. 39, pp. 2422-2427, 2000.

10. A. Galvanauskas, A. Hariharan, D. Harter, M. A. Arbore, and M. M. Fejer, "High-energy femtosecond pulse amplification in a quasi-phase-matched parametric amplifier," Opt. Lett. 23, pp. 210-212, 1998.

11. J. Collier, C. Hernandez-Gomez, I. N. Ross, P. Matousek, C. N. Danson, and J. Walczak, "Evaluation of an ultrabroadband high-gain amplification technique for chirped pulse amplification facilities," Appl. Opt., 38, pp. 7486-7493, 1999.

12. S. K. Zhang, M. Fujita, H. Yoshida, R. Kodama, H. Fujita, M. Nakatsuka, Y. Izawa, and C. Yamanaka, "Study on High Gain Broadband Optical Parametric Chirped Pulse Amplification," Proc. SPIE, K. Mima, G. L. Kulcinski, and W. Hogan, editors, 3886, pp. 588-595, 2000.

13. I. Jovanovic, B. J. Comaskey, and D. M. Pennington, "Angular effects and beam quality in optical parametric amplification," J. Appl. Phys. 90, pp. 4328-4337, 2001.

14. S. K. Zhang, M. Fujita, M. Yamanaka, M. Nakatsuka, Y. Izawa, C. Yamanaka, "Study of the stability of optical parametric amplification," Opt. Commun. 184, pp. 451-455, 2000. 


\title{
Nondegenerate optical parametric chirped pulse amplification
}

\author{
I. Jovanovic ${ }^{1,2}$ \\ B. C. Stuart ${ }^{1}$ \\ C. A. Ebbers ${ }^{1}$ \\ M.R. Hermann ${ }^{I}$ \\ E. C. Morse ${ }^{2}$ \\ 'Lawrence Livermore National Laboratory, Livermore, California 94550 \\ ${ }^{2}$ Department of Nuclear Engineering, University of California at Berkeley, Berkeley, California 94720
}

\begin{abstract}
We present the first recompression of amplified pulses in a highly nondegenerate optical parametric chirped pulse amplifier. 60 -fs recompressed pulse width and up to $2 \mathrm{~mJ}$ pulses were obtained in our experiments.

\section{INTRODUCTION}

Generation and amplification of ultrashort laser pulses has been at the forefront of laser research in the recent period, driven primarily by newly accessible physics phenomena and commercial applications such as materials processing. The interest in optical parametric chirped pulse amplification (OPCPA) [1] for ultrashort pulse amplification has been motivated by its favorable properties. Large single-pass gain achievable in a $\sim \mathrm{cm}$ length of nonlinear material pumped by nanosecond pump laser eliminates the need for regenerative amplification. Broad bandwidth capabilities and scalability make OPCPA a possible amplification technology for future multi-PW sources [2]. Absence of thermal load should allow short pulse amplification up to $\mathrm{kW}$-level average power. While the ultrashort amplification capabilities of optical parametric amplifiers (OPAs) have been demonstrated down to $5 \mathrm{fs}$, the compression of ultrashort ( $<100 \mathrm{fs}$ ) pulses in thick OPCPA crystals remains largely unexplored. Here, we present the first pulse compression in highly nondegenerate OPCPA and obtain the shortest pulses produced in OPCPA to date.
\end{abstract}

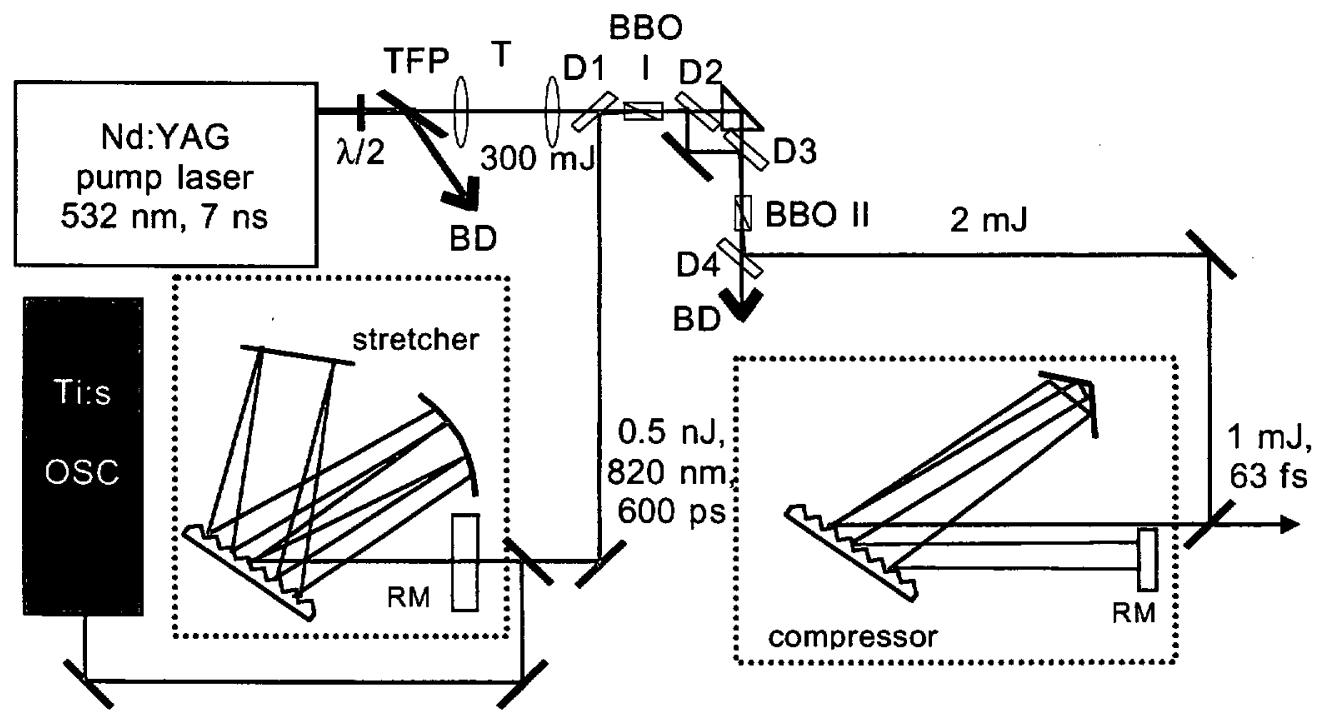

Fig. 1. Experimental setup. TFP-thin film polarizer, T-telescope, D-dichroic. N/2-waveplate,TFP-thin film polarizer, $B D$-beam dump, RM-roof mirror.

\section{DESCRIPTION}

The design of our OPCPA system is presented in Fig. 1. A mode-locked oscillator, which produces 20 -fs pulses centered at $820 \mathrm{~nm}$, is used as a seed source. An all-reflective stretcher is used to stretch the seed pulses to $600 \mathrm{ps,}$ with a spectral cut-off width in excess of $100 \mathrm{~nm}$. The OPA consists of two antireflection-coated beta-barium borate 
(BBO) crystals. The crystals are cut at $23.8^{\circ}$ to facilitate type I phase matching at an external noncollinear angle of $3.7^{\circ}$. The noncollinear angle is optimized numerically to maximize the gain bandwidth. The length of each crystal is $15 \mathrm{~mm}$, and they have a wedged output surface to eliminate parasitic oscillation. The pump beam is relay imaged between the two crystals and the beam diameter is adjusted to $3 \mathrm{~mm}$, for a peak intensity near $450 \mathrm{MW} / \mathrm{cm}^{2}$. We obtained a maximum gain of $4 \times 10^{6}$ from the OPA when the noncollinear plane was chosen to be perpendicular to the principal plane of the crystal. The result were pulses with energies of up to $2 \mathrm{~mJ}$ amplified in a single pass through only $30 \mathrm{~mm}$ of gain material. The measured seed and amplified signal spectra are shown in Fig 2 . We observe a shift of the center wavelength to $830 \mathrm{~nm}$, which is consistent with the gain bandwidth in nondegenerate BBO OPA, which is centered at longer wavelengths (near $850 \mathrm{~nm})$. The small bandwidth narrowing $(<2 \mathrm{~nm})$ at the FWHM observed when the OPA operates far below saturation $(0.5 \mathrm{~mJ})$ can be attributed to this spectral shift. At the point near saturation ( $2 \mathrm{~mJ}$ ), the spectrum is modified further and the amplified FWHM is increased to $35 \mathrm{~nm}$. Spectral broadening is the result of different rate of nonlinear conversion for the spectral components of different initial intensity. We recompressed our pulses in a single-grating compressor, and the autocorrelation trace is shown in Fig. 3. The measured FWHM autocorrelation of the recompressed pulse is $104 \mathrm{fs}$, which is nearly 2 times longer than the FWHM of the calculated autocorrelation of the transform-limited pulse with the measured spectrum (Fig. 2). With the inclusion of the spectral phase in the system, the calculated FWHM of the autocorrelation is $108 \mathrm{fs}$, which is within our experimental error, indicating that we produced 60 -fs pulses.

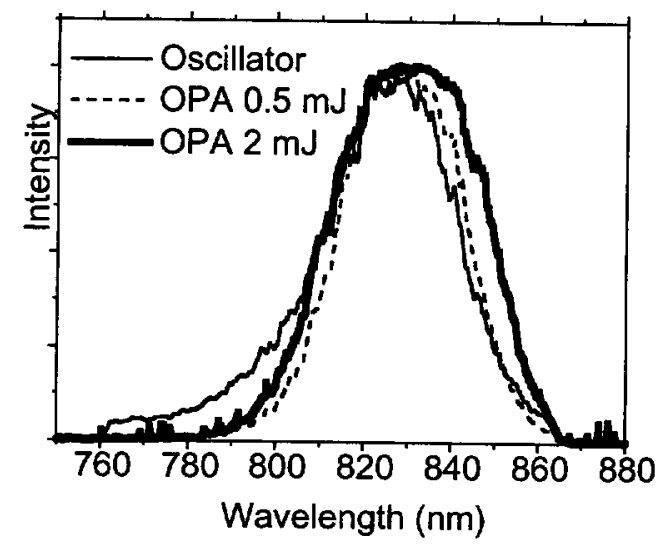

Fig. 2. Seed and amplified signal spectra from the nondegenerate OPCPA system

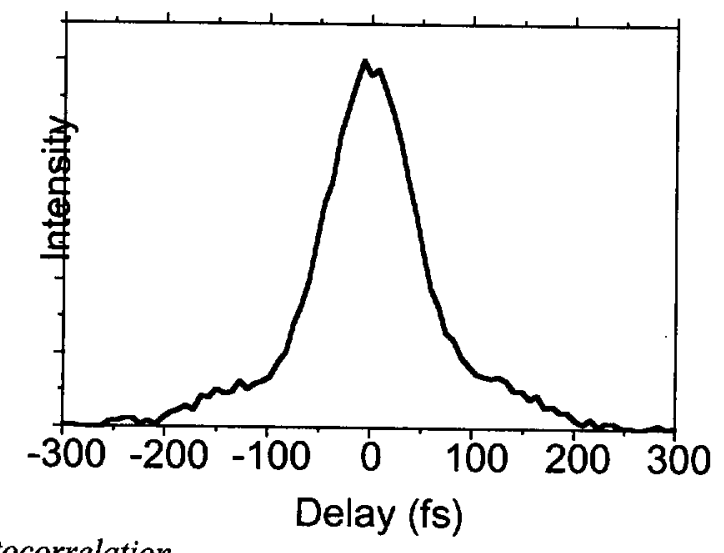

Fig.3. Recompressed pulse autocorrelation

\section{SUMMARY}

In summary, we report for the first time the compression of pulses amplified in nondegenerate OPCPA. The reported 60 -fs pulses are the shortest pulses reported to date produced in an OPCPA system. A careful compensation of spectral phase in our system should enable 30-fs transform-limited pulses.

This work was performed under the auspices of the U. S. Department of Energy by Lawrence Livermore National Laboratory under Contract No. W-7405-Eng-48. 


\section{REFERENCES}

1. A. Dubietis, G. Jonusauskas, and A. Piskarskas, "Powerful femtosecond pulse generation by chirped and stretched pulse parametric amplification in BBO crystal," Opt. Commun. 88, 437-440 (1992).

2. I. N. Ross, P. Matousek, M. Towrie, A. J. Langley, J. L. Collier, "The prospects for ultrashort pulse duration and ultrahigh intensity using optical parametric chirped pulse amplifiers," Opt. Commun. 144, 125 (1997). 


\section{Solid-State Heat-Capacity Laser AND TESTS Resarch ACtivity Highlights}

\section{A 10-kW Solid-State Heat- Capacity Laser System Installed at HELSTF, White Sands Missile Range}

Under the support of the U.S. Army's Space and Missile Defense Command, and in collaboration with industrial partners (Raytheon, General Atomics, Northrup/Grumman PolyScientific, PEI, Armstrong Laser Technology, and others), LS\&T is developing high-averagepower (100-kW-class) heat-capacity laser technology for applications in tactical short-range air defense missions. The ultimate vision is an electrically powered, solid-state weapon that can be deployed on a hybrid electric vehicle.

To establish a solid technical basis for the heat-capacity laser operation, and to support development of an adaptive resonator, we have built a $10-\mathrm{kW}$ prototype (nine-disk $\mathrm{Nd}$ :glass laser pumped by flashlamps). During the summer of 2001, this laser (see photo below) was delivered to the Army's High Energy Laser Systems Test Facility (HELSTF) at White

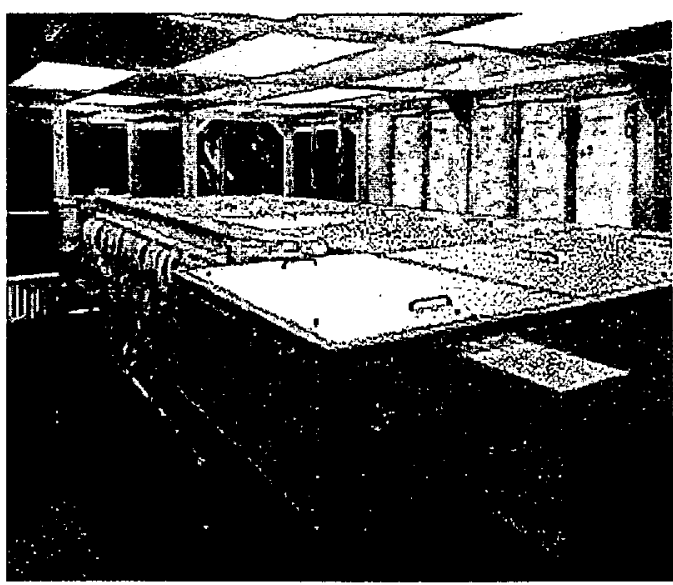

The 10-kW laser system installed at HELSTF to support material interaction testing.

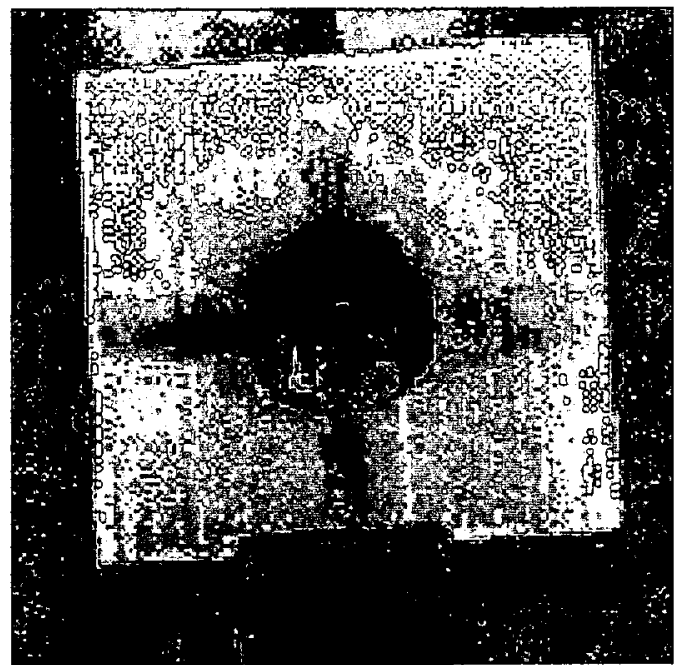

A 1-in.-thick aluminum target after a 2-sec laser burst produced a hole through it.

Sands Missile Range to support material interaction testing.

On August 31, 2001, our milestone of 10$\mathrm{kW}$ average power for $10 \mathrm{sec}$ was exceeded when we obtained an average energy/pulse of $640 \mathrm{~J}$. With the $20-\mathrm{Hz}$ repetition rate, this resulted in an average power approaching 13 $\mathrm{kW}$ during the 10-sec burst.

On September 28, 2001, a ribbon-cutting ceremony attended by a large number of media reporters as well as high-ranking Army officials was held to commemorate the delivery of the laser.

During the ribbon-cutting ceremo-ny, the laser was fired at a 1-in.-thick aluminum target. The photo at top of column shows the target at the end of a 5-sec burst. The hole produced by the focused laser beam penetrated the 1-in. thickness in 2 sec.

The laser will remain at White Sands where it will be used for laser/target interaction experiments until early summer of 2002. At that 


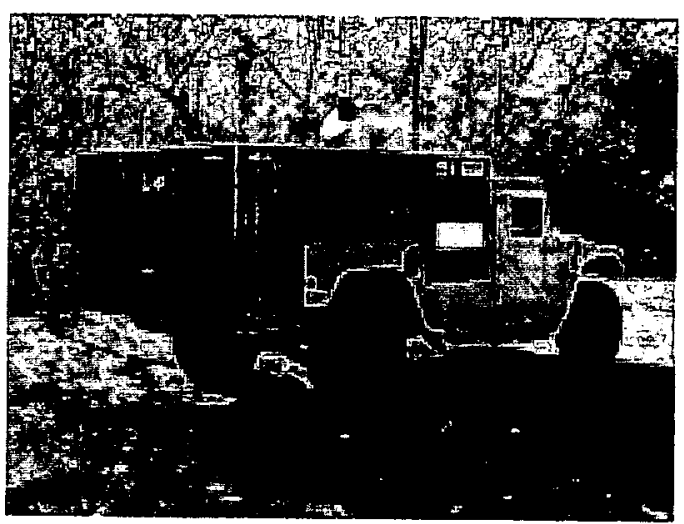

An actual 1:1 scale model of a 100-kW laser system installed on a hybrid electric HMMWV.

time, the laser will be brought back to Livermore so that a new deformable mirror may be installed.

Currently, the laser is operating with a stable resonator which is ideally suited to the target interaction experiments. A beam quality of approximately $3.5 \times$ the diffraction limit has been demonstrated with an unstable resonator with no deformable mirror correction. With the new deformable mirror and an unstable resonator, we expect to achieve the desired goal of $2 \times$ the diffraction limit over the entire 10 -sec run time. The beam footprint of the $10-\mathrm{kW}$ laser is roughly square with a $6-\times 6.5-\mathrm{mm}$ spot on the target.

Future plans call for a $100-\mathrm{kW}-$ class, diodepumped, solid-state laser utilizing neodymiumdoped gadolinium gallium garnet (Nd:GGG) crystalline laser media for better thermal characteristics and operating efficiency. With the support of LS\&T Advanced Lasers and Component, we have recently completed the fabrication and testing of a $42-\mathrm{kW}$ laser-diode array for pumping of Nd:GGG slabs. We will demonstrate a small-scale Nd:GGG heat-capacity laser at $200 \mathrm{~Hz}$ in late 2002 and replicate as closely as possible features that would be found in a fieldable unit (see photo above). For example, prime power for the laser will be provided by a bank of $\mathrm{Li}$-ion batteries.

We plan to complete three-slab heat-capacity laser fabrication and testing in 2003 . The scheduled completion of the $100-\mathrm{kW}$ system could be as early as 2005 , depending on continuing funding support.

\section{2-kW Diode Array Delivered to the HELSTF Program}

Under the support of the U.S. Army's Space and Missile Defense Command, we have recently completed the fabrication of a $42-\mathrm{kW}$ peak power laser-diode array for pumping of high-average-power solid-state heat -capacity laser for applications in tactical short-range air defense missions. Figure 1 shows the laserdiode module which is constructed from 280 edge-emitting laser-diodes mounted on a backplane packaged by 28 closely packed Silicon Monotlithic Microchannels tiles ( SiMMs) arranged in $4 \times 7$ configuration. This pump module is designed to deliver $42 \mathrm{~kW}$ of $808 \mathrm{~nm}$ radiation when operated at $200 \mathrm{~Hz}$ or higher duty cycles.

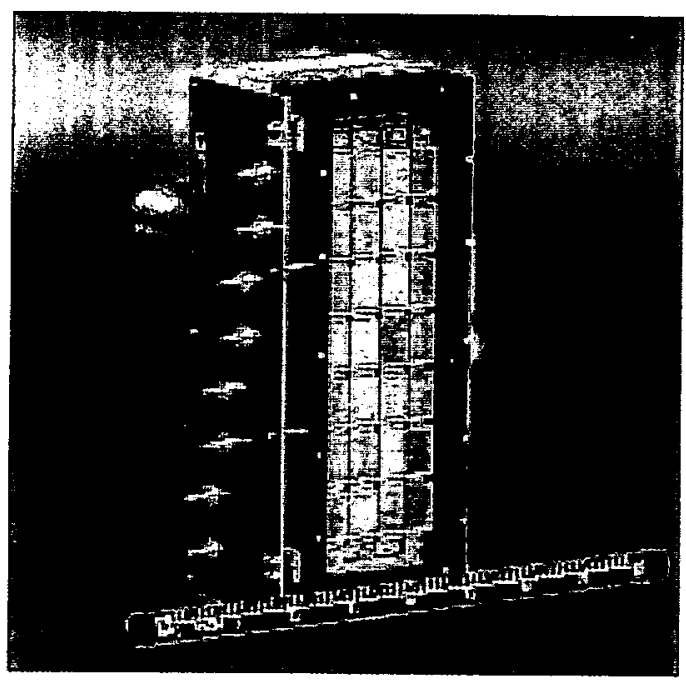

Figure 1, $100 \mathrm{~kW}$-class diode array module developed for pumping of high-average - power solid-state lasers.

The production of high-average-power laserdiode arrays on the scale demonstrated here has relied on the development of a silicon based diode packaging technology that has been actively pursued at LLNL for the last 15 years. The silicon package which serve to cool the laser diode bars are produced by photolithography and etching techniques used in the microelectronic industry. Using this technology, we were able to manufacture thousands of miniscule $30-\mu \mathrm{m}$-wide channels in silicon substrates. Water flow through these microchannels 
aggressively cools the laser diode bars, which are mounted on the silicon at a location less than $200 \mu \mathrm{m}$ from the channels. By mounting 10 diode bars onto a single heatsink, a 10-bar package (referenced as a tile and shown in the photograph in Fig. 2) can be easily assembled to serve as an unit cell from which larger twodimensional diode arrays can be built up through tiling. Noteworthy of this design is that the brightness (i.e., W/ $\mathrm{cm}^{2}$-str) of the array is extremely high, by virtue of the compact tiling of the heatsinks, and the precision placement of 10-element microlens arrays on each tile.

Over the years, we have developed several types of diode packaging technologies for highpower applications. Special considerations were given to both the ease of fabrication and power scalability. In the current SiMMs design, we were able to preserve the same aggressive heat removal capability that characterized our original rack and stack silicon microchannel cooled package. This requirement was met by incorporating microchannels into the silicon directly below the location of the attached laser diode bars.

The SiMMs package has a very tight thermal circuit, with only 177 microns of silicon

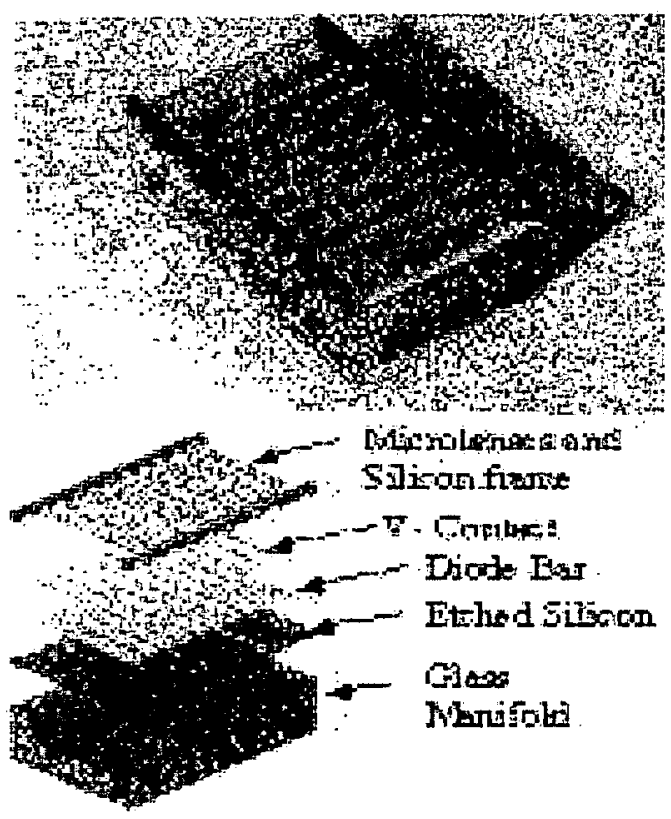

Figure 2. The SiMMs package. Top: Photograph of an individual SiMMs package. Bottom: Assembly drawing showing the various SiMMs package components in an exploded view. The V-grooves on the front surface of the silicon enable us to precisely position the laser diode bars and microlenses with micron precision. separating the heat generating laser diode bars and the microchannel fins that define the cooling channels. AS shown in Figure 2, each SiMMs package holds 10 individual $1 \mathrm{~cm}$ long laser diode bars in a single monolithically cooled silicon tile. Individual $1 \mathrm{~cm}$ diode bars produce $150 \mathrm{~W}$ of output radiation leading to an effective irradiance of $1 \mathrm{~kW} / \mathrm{cm} 2$ for the extended 2-dimensional diode arrays that are built up with individual tiles. Presently these arrays are operated at this output level at a $10 \%$ duty factor, but the aggressive thermal capability of the microchannel cooling approach allows substantially higher duty factor operation when it is required in future systems.

In order to fully utilize the light emitted from laser diode bars (particularly along the fast axis which has a 30 degree divergence angle), we have also designed microlenses to collect radiation along the fast axis. A single step microlens-mounting technique was developed to eliminate the production steps necessary to individually attach microlenses to each diode bar, dramatically reducing package cost. To achieve good optical performance, microlenses must be placed with a positional tolerance of a few microns relative to the diode bar emitter facets and each individual diode bars within a tile must also be positioned relative to one another with at least this same few micron accuracy.

Using a V-groove technology on the front surface of the package accomplishes precision placement of the laser-diode bars. V-grooves are generated using the same etching technology that is used to fabricate the microchannels in silicon and serve as pads to which the laser diode bars are attached and precisely registered. Because the V-grooves are defined lithographically, output facets of individual bars can be located with micron precision relative to one another over the entire SiMMs tile. Single-step microlens mounting is accomplished using precision frames fabricated in the form of silicon runners. Lenses are preloaded and glued into these silicon runners forming a ladder-like structure consisting of 10 lenses as shown in Figure 2. These microlenses have effectively reduced the beam divergence of the array to $<1$ degree.

Using diode bars procured from the Coherent Semiconductor Group we successfully achieved $1.5 \mathrm{~kW}$ of output power from a single tile (10-bar SiMMs package) with wall plug 
efficiencies approaching 50\%. We plan to optimize the optical performance of the $42 \mathrm{~kW}$ array module and demonstrate pumping of Nd:GGG slab laser in future experiments. The SiMMs pack- age represents a breakthrough in high power diodearray packaging technology enabling us to scale the output of $2 \mathrm{D}$ diode arrays to $100 \mathrm{~kW}$ or larger with extremely high brightness. 


\title{
Material Removal by a Heat Capacity Laser ${ }^{*}$
}

\author{
C. D. Boley \\ C. B. Dane \\ E. A. Stappaerts
}

\begin{abstract}
This report describes the modeling of material removal by a solid state heat capacity laser. Two hydrodynamic models of laser-materials interactions were employed to explain data collected with the testbed laser built during FY98. One of these models, in which material is removed by vaporization, produced interesting trends but poor agreement with experiment. Liquid ejection was inferred to play an important role. The second model, which handles some liquid motion effects, gave much better agreement with experiment. Detailed results are presented. The virtues and shortcomings of the models are also discussed.
\end{abstract}

\section{INTRODUCTION}

In collaboration with industrial partners, LLNL is developing solid state laser of high average power and high pulse energy for DoD defensive applications. An initial accomplishment was to build a flashlamp-pumped Nd:glass laser capable of 10-s bursts at an average power of about $1.4 \mathrm{~kW}$. This three-slab testbed was completed in 1998. Next to be built was a flashlamp-pumped Nd:glass laser capable of 10-s bursts at an average power of $10 \mathrm{~kW}$. In 2001 it was delivered to the High Energy Laser System Test Facility (HELSTF) at White Sands Missile Range.

The three-slab testbed laser was used to perform demonstrations of material removal from coupon targets of steel, aluminum, and a graphite composite. The main diagnostics were the energy per pulse, the temporal pulse shape, the shape of the hole, and the number of pulses necessary to penetrate the coupons. Here we present simulations of these experiments, for the two metal coupons. Two models were employed: a 1D model, which describes thermal effects in the condensed matter and hydrodynamic effects in the ablated vapor, and a 2D model which describes the hydrodynamics of the entire solid-liquidvapor system. It was assumed that the effects of the first pulse are representative of those from later pulses. The first model was found to be inadequate to explain the observed removal, while the second model gave results reasonably consistent with experiment.

In the following sections, we describe the experiment, the models and their predictions, and our conclusions and directions for further work.

\section{SUMMARY OF EXPERIMENTAL RESULTS}

For the demonstration shots ${ }^{1}$, efficient extraction from the 3-slab amplifier was achieved via a 4-pass configuration. The output pulse, containing about $80 \mathrm{~J}$, was focused to a $3 \times 3 \mathrm{~mm}^{2}$ spot on the coupon, giving a fluence of about $900 \mathrm{~J} / \mathrm{cm}^{2}$. Figure 1 shows the calculated waveform ${ }^{2}$, which is close to what was observed. The pulse, of length about $350 \mu \mathrm{s}$, consisted of about 20 spikes (relaxation oscillations). The width of the spikes increased from a few $\mu$ s, early in the pulse, to about 20 $\mu \mathrm{s}$, near the end. The peak irradiance on target decreased from about $36 \mathrm{MW} / \mathrm{cm}^{2}$ for first spike to $1 \mathrm{MW} / \mathrm{cm}^{2}$ for the last.

The resulting holes have the rough shape of the beam and are quite sharp. An estimate of the material removed per pulse can be obtained simply by dividing the thickness of the coupons ( $2.3 \mathrm{~mm}$ for both steel ${ }^{3}$ and aluminum) by the number of pulses (13 for steel, 8 for aluminum). This gives about $180 \mu \mathrm{m}$ and $290 \mu \mathrm{m}$, respectively, per pulse. If there is a significant temperature rise from pulse to pulse, the material removal would increase with pulse count. Our calculations in Section 4 indicate, however, that the temperature rise is very small in these experiments. During the final pulse, removal should increase because material is ejected from the opposite face as well as the near face. The quoted estimates might represent an average of the removal per pulse.

\section{MODELING VIA THALES}

THALES is a l-dimensional hydrodynamics and vaporization code, originally developed at LLNL to model drilling by copper vapor lasers, in the intensity range $10^{8}-10^{11} \mathrm{~W} / \mathrm{cm}^{2}$. It has also been used to model ablation in NIF beam dumps.

\footnotetext{
* This work was performed at LLNL in partnership with Raytheon Electronic Systems, under the support of the U.S. Army Space and Missile Defense Command.
} 


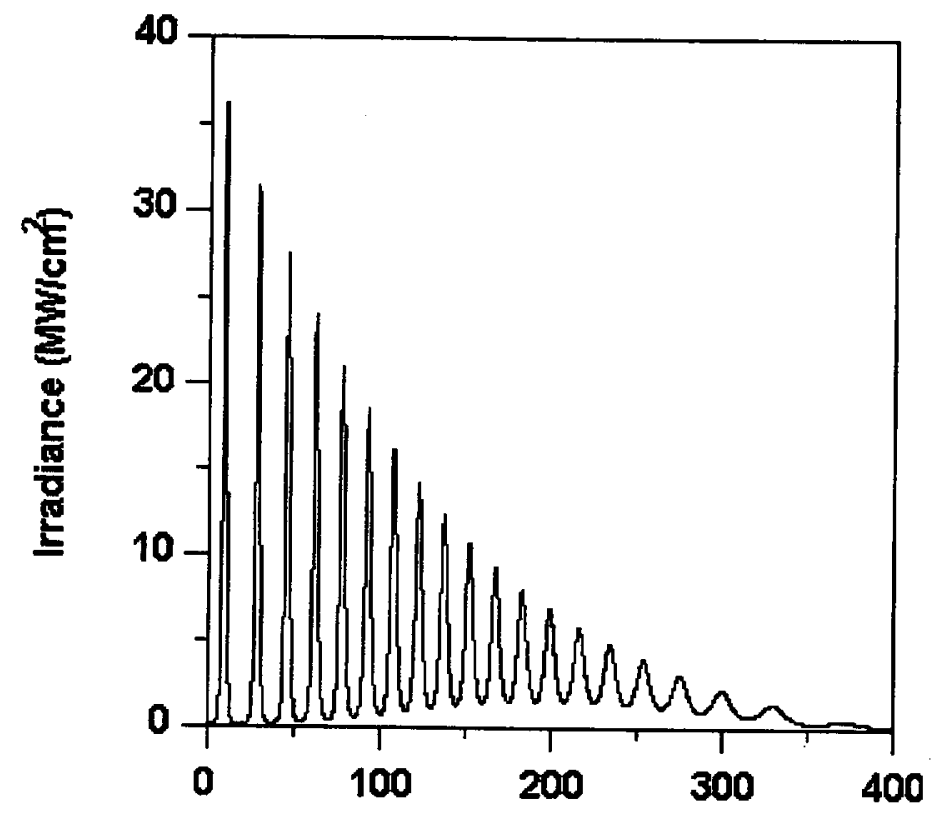

Fig. 1. Pulse irradiance at beam center for coupon tests.

Time (microsec)

The geometry of the model is illustrated in Fig. 2. From left to right in the figure, the model describes the solid, the moving melt line, the liquid, the moving ablation surface, a thin region of molecular flow (the Knudsen layer), a region of hydrodynamically expanding vapor, the vapor/air contact surface, a region of compressed air, the shock front, and finally, the ambient air. In the solid and liquid, the model describes thermal conduction, using available data ${ }^{6}$. The melt line moves in accordance with the Stefan condition (speed proportional to the discontinuity in the thermal flux). Within the Knudsen layer $^{7,8}$, the molecular velocity distribution function changes from a one-sided Maxwellian with reflux (at the surface of the liquid) to a full local Maxwellian (a few mean free paths within the vapor). At the beginning of the layer, the pressure of emitted atoms equals the vapor pressure at the local temperature. Thus the material removal mechanism is vaporization.

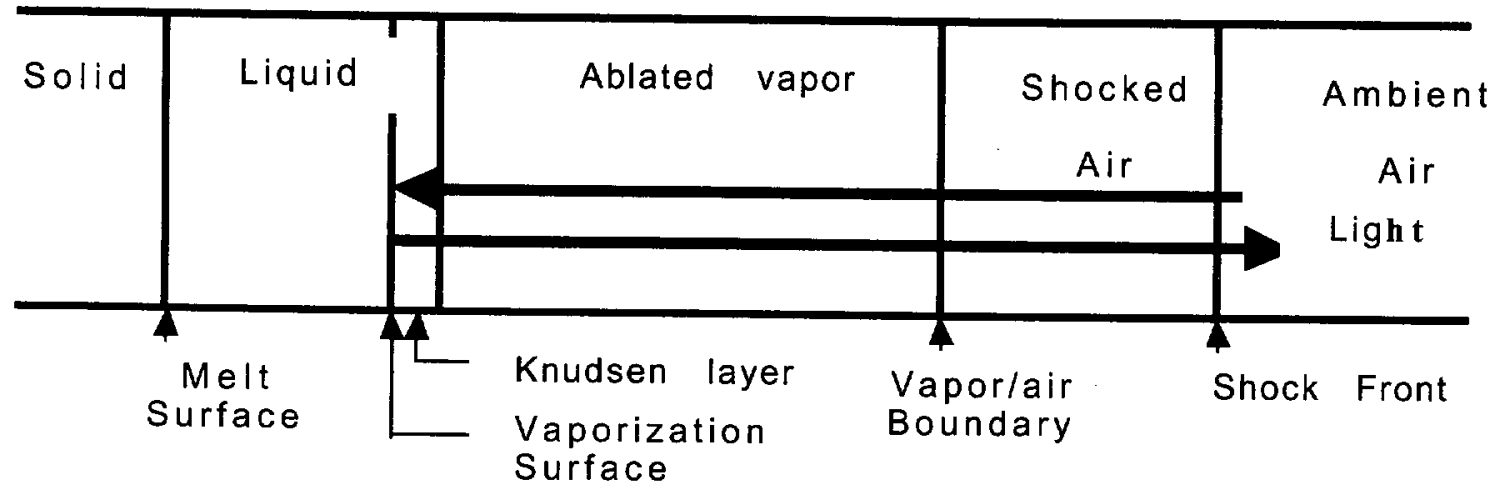

Fig. 2. Geometry of experiment according to THALES model (not to scale).

In the vapor and air, the 1-dimensional hydrodynamic equations are solved. The incident and reflected light rays are propagated, with absorption in the vapor occurring via photoionization and inverse bremsstrahlung. The compressed air is generally cool and dense, with negligible absorption. We employ the absorption formulation of Zel'dovich and Raizer', in which the vapor atoms are treated as hydrogen-like with empirical ionization potentials. This model is also used to set up a Saha equation of state in the vapor. The vapor/air boundary is treated as a tangential discontinuity (continuous velocities and pressures) 10. Since the model is one-dimensional, it cannot account for the Rayleigh-Taylor instability at the vapor/air surface, but it estimates the typical growth rate, with wavelength equal to the beam diameter (or, for a noncircular beam, the equivalent beam diameter containing the same energy). The shock front satisfies the Rankine-Hugoniot relations. Some further details of the THALES model are given in Appendix A. 
At the ablation surface, light is absorbed according the temperature-dependent absorption coefficient. This is taken from a simple MHD model, fitted to the available absorption data. Details are given in Appendix B.

Before applying the code, we can estimate the maximum value of the removal by vaporization, assuming that all the absorbed energy goes into melting and vaporization. This requires that the vapor remain transparent, as will hold if the vapor temperature rises no more than a few thousand degrees, depending on the material, and that comparatively little energy is transported into the material. The thickness removed is approximately

$$
\Delta z \approx \frac{\alpha F}{\rho\left[C_{s}\left(T_{m}-T_{0}\right)+H_{m}+C_{l}\left(T_{v}^{0}-T_{m}\right)+H_{v}^{0}\right]}
$$

where $\alpha$ is the absorptivity of the material, $F$ is the incident fluence, $C_{s}$ and $C_{l}$ are typical specific heats per unit mass of the solid and liquid, respectively, and $H_{m}$ and $H_{v}$ are the heats of melting and vaporization per mass. The vaporization temperature increases with pressure, while the heat of vaporization decreases with temperature. For the moment, we simply use the values at the boiling point (atmospheric pressure), appending a superscript. In THALES itself, the best available temperature-dependent material properties are used. The energies in the denominator above are dominated by the heat of vaporization. While the absorptivity is a poorly known parameter, as discussed in the appendix, here we estimate it as 0.4. Then the estimated removal depths for steel and aluminum turn out to be about $55 \mu \mathrm{m}$ and $95 \mu \mathrm{m}$, respectively. The approximate ratio of these depths follows from the facts that the heat of vaporization of aluminum is about 1.7 times that of steel while the density of aluminum is about $1 / 3$ that of steel. These depths are only about $30 \%$ of the respective experimental values, indicating that vaporization alone is unlikely to be sufficient. Hence we anticipate that THALES simulations will not tell the full story.

As noted, this estimate for the removal depends on the existence of a transparent vapor. If the vapor is heated sufficiently to become absorbing, energy can be transferred to the material by thermal conduction in the resulting plasma, and a different regime of operation results ${ }^{4,5}$.

We show in Fig. 3 the calculated temperature at the liquid edge (just before the Knudsen layer) during the simulation of the steel coupon. The temperature displays the same pattern of spikes as the pulse. The maximum temperature, reached at the peak of the first few spikes, is almost $5000 \mathrm{C}$. Near the end of the pulse, the temperature at the top of a spike has decreased to about $3300 \mathrm{C}$. The spatially averaged temperature in the vapor (weighted by the density) increases to about $2700 \mathrm{C}$ and drops to about $600 \mathrm{C}$ after $1000 \mu \mathrm{s}$. The density-averaged temperature in the compressed air generally remains below 1000 C. In this regime, both the vapor and the air are transparent. The same holds true for the aluminum coupon.

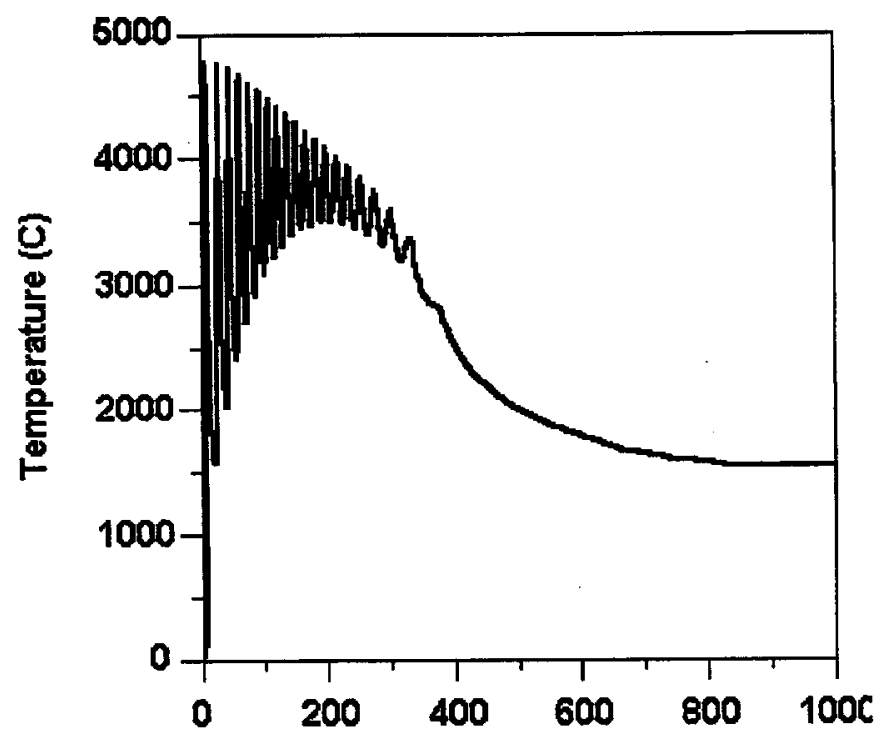

Time (microsec)

Fig. 3. THALES predictions for liquid edge temperature of steel coupon. 
Figure 4 gives the calculated phase separation versus time for the steel and aluminum coupons. In each case, the shock front moves steadily at somewhat less then $1 \mathrm{~km} / \mathrm{s}$. The metal/air interface tends to decelerate after the pulse, and in the case of aluminum it is eventually pushed back by the shocked air. The Rayleigh-Taylor instability (which is not in the model) has a growth time of $\sim 30 \mu \mathrm{s}$ on the spatial scale of the beam diameter. Hence the division between metal vapor and air may beam, as is the air. The lawe our regime, however, since the vapor remains essentially neutral and is transparent to the case, a melt layer opens up during the applicatione dependence of the material phases in the coupons themselves. In each and $3 \mathrm{~ms}$ for aluminum). The maximum melt greater for aluminum because its thermal diffusivity is about $52 \mu \mathrm{m}$ for steel and $195 \mu \mathrm{m}$ for aluminum. The width is temperature range.

As shown in Fig. 4, the calculated removal depths for steel and aluminum are $43 \mu \mathrm{m}$ and $75 \mu \mathrm{m}$, respectively. These depths are less than the maximum values estimated earlier ( $55 \mu \mathrm{m}$ and $95 \mu \mathrm{m}$, respectively), as would be expected. Each represents about $25 \%$ of the average observed removal. Thus the model confirms that vaporization alone does not account for the that the sums of the removal depths appears to be an important effect. This appears plausible when we note, heuristically, respectively) are closer to the observed removal.
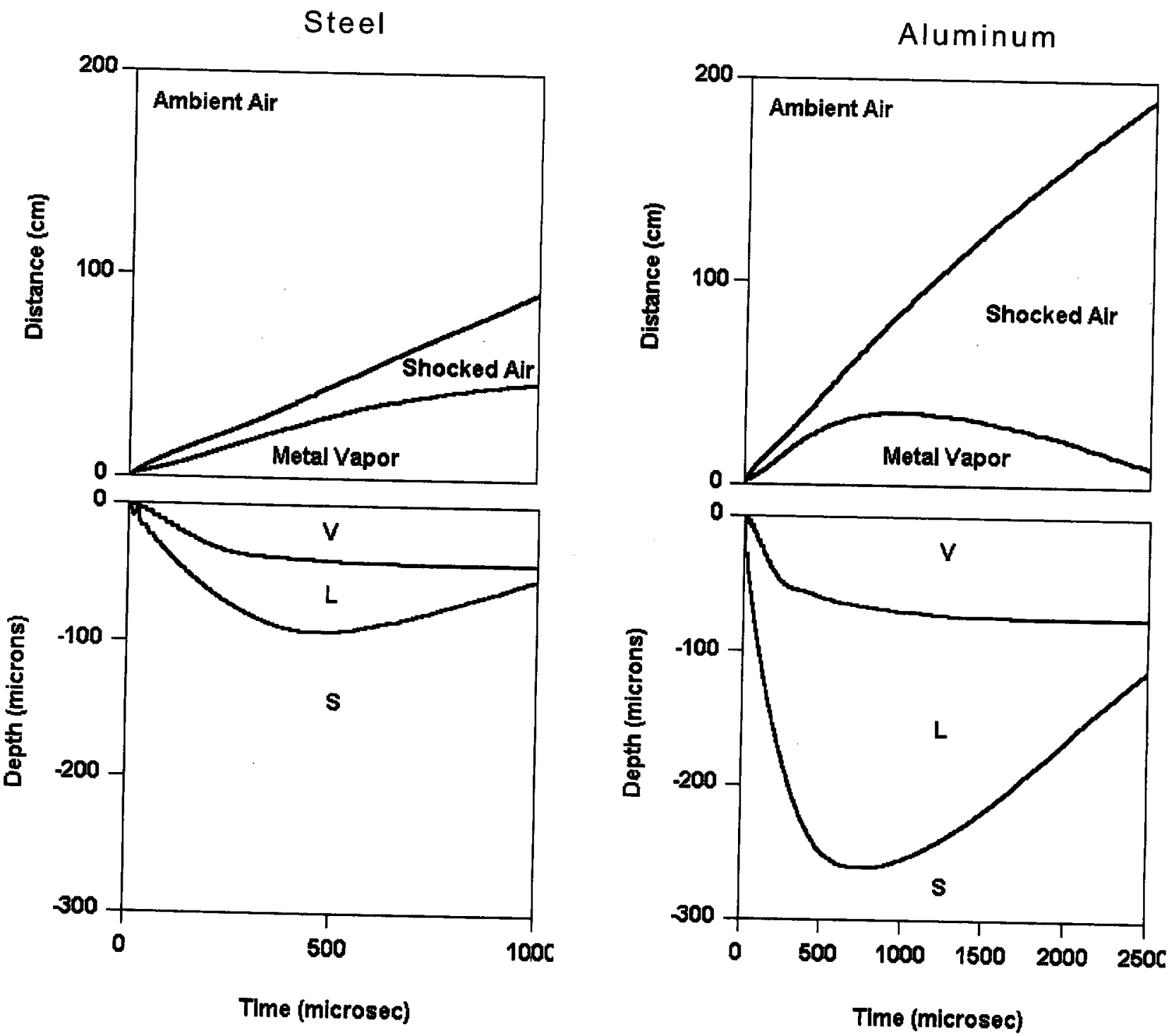

Fig. 4. Phase separation versus time for steel and aluminum coupons, according to THALES calculations. Upper plots: metal/air surface and shock front. Lower plots: regions of solid (S), liquid (L), and vapor below original surface $(V)$. 


\section{MODELING VIA CALE}

CALE $^{\prime \prime}$ is a 2-dimensional hydrodynamics code developed at LLNL. It simulates fluid flow on a mesh which can be set as Lagrangian, Eulerian, or arbitrarily between the two. This treatment is termed ALE, or arbitrary Lagrangian-Eulerian; the C in the name of the code refers to the $\mathrm{C}$ programming language. The code also simulates elastic effects in the solid. It uses equation of state data (pressure and internal energy as functions of density and temperature) and elastic data (such as the elastic moduli and yield strength) provided by the user ${ }^{2}$. CALE has a laser absorption model in which a ray deposits a specified fraction of its energy at the critical surface, where the plasma frequency equals the light frequency $\left(n_{e} \sim 10^{21} \mathrm{~cm}^{-3}\right.$ for $1 \mu \mathrm{m}$ light). This fraction was taken as 0.4 for steel and 0.5 for aluminum, for reasons noted later. Our reason for using the code was that it offers a unified treatment of solid, liquid, and gas phases. In principle, the equation of state provides the energetics associated with transitions among these phases.

In CALE, we employed cylindrical $(r-z)$ symmetry, with the coupon modeled as a large disk centered on the $z$-axis with a 2$\mathrm{mm}$ thickness in the $\mathrm{z}$ direction. Atmospheric air was present on the side of the coupon facing the laser. The other side of the coupon provided a distant boundary condition, since it received negligible energy during the pulse. The laser beam, modeled as azimuthally symmetric, was centered on the $z$-axis and extended radially to $\mathrm{r} \sim 0.17 \mathrm{~cm}$ (following simply from the energy and fluence). Following rough experimental guidance, the beam profile was taken as Gaussian, with the irradiance at the beam edge equal to half the central irradiance. We were primarily interested in phenomena occurring no more than a few hundred microns below the surface and a few $\mathrm{mm}$ perpendicular to the beam (i.e. the beam size). However, we had to allow for the expanding vapor, which extended a few $\mathrm{cm}$ (primarily in $z$ ) after the pulse duration. Therefore, fine resolution was required within the coupon, in order to measure material removal, but resolution requirements were less stringent within the air and expanding vapor. Within the coupon, the grid was chosen as Eulerian (fixed), with zones gradually expanding in size away from the area affected by the laser. The smallest resolution in $z$ (parallel to the beam) was $16 \mu \mathrm{m}$, while that in $r$ was about $113 \mu \mathrm{m}$. The latter size was chosen so that the beam extended 15 zones radially. Within the air, the grid was allowed to follow the Lagrangian/Eulerian prescription of the code, and so it expanded as vapor was ejected. The original widths in $z$ of successive zones increased from $16 \mu \mathrm{m}$ (near the coupon surface) to a few $\mathrm{cm}$.

Figure 5 shows the material grid, the expanding vapor, and a selection of laser rays, early in time (15.5 $\mu$ s), in the simulation of steel. Note that a few rays have found a critical surface somewhat removed (about 300 microns) from the edge of the metal. This is an anomaly of the ionization model, which is taken from Thomas-Fermi theory. The model overestimates the ionization at modest temperatures. Later in the run, the rays penetrate more uniformly.

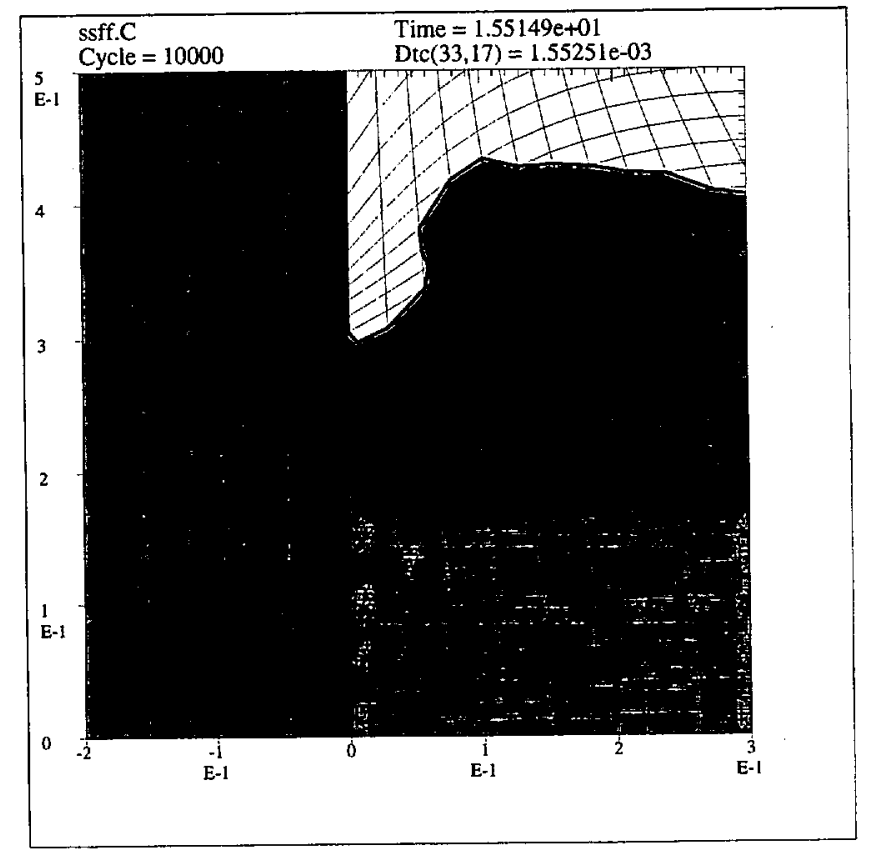

Fig. 5. Expanding vapor and sample rays at an early time $(15.5 \mu \mathrm{s})$ of the CALE simulation of steel. The vertical and horizontal directions correspond to $r$ and $z$, respectively. Dimensions are in $\mathrm{cm}$. The beam, entering from the right, has a radius of about $0.17 \mathrm{~cm}$. 
Figure 6 shows snapshots of the expanding steel vapor at three times, equal to one-third, two-thirds, and the full pulse length. The dark region corresponds to zones which are predominantly metal. The expanding vapor is diverted vertically
from the beam (in the $r$ direction) by according to the ALE algorithm. To avoid artificial zones in the problem continue for several $\mathrm{cm}$ beyond effects at the boundary of the expanding vapor and air, the actual condensation, with a typical density of about $10^{-3} \mathrm{~g} / \mathrm{cm}^{3}$.
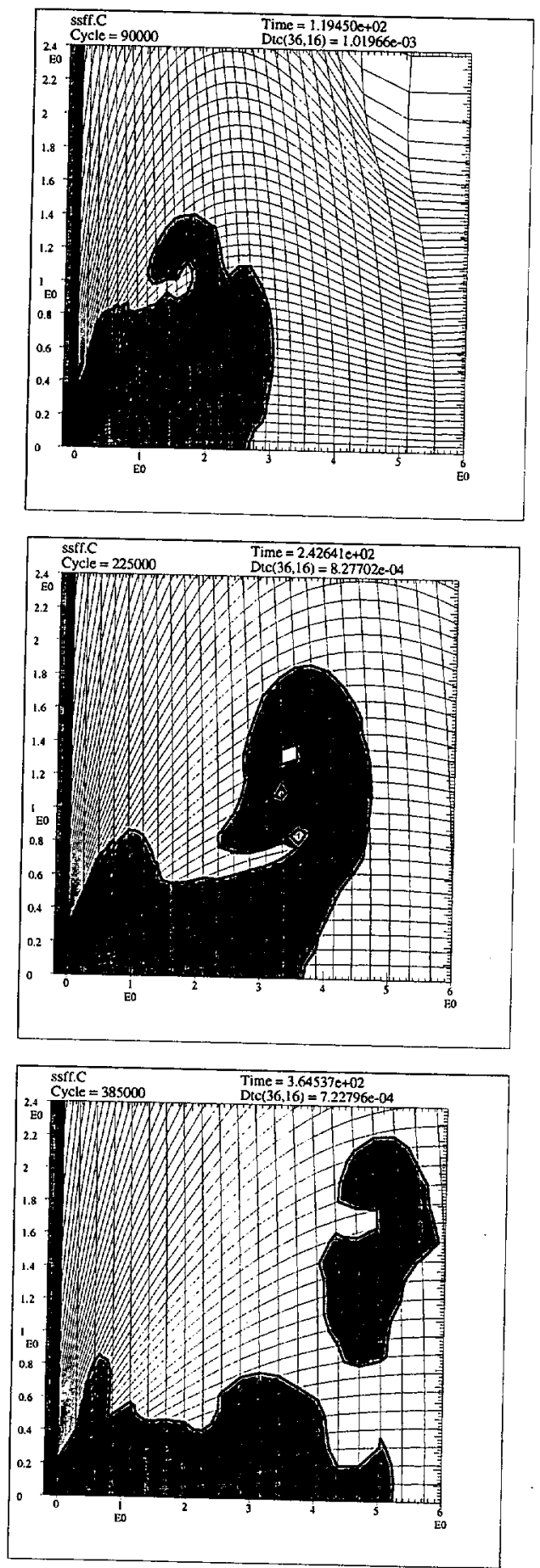

Fig. 6. Expanding steel vapor, at times corresponding to approximately $1 / 32 / 3$, and full pulse length $(119,243$, and 365 $\mu s)$, according to CALE. The three pictures have the same scale, with the horizontal scale (z) 2.5 times greater than the
vertical scale $(r)$. 
Turning to the detailed effect of the laser on the material, we show in Fig. 7 the density distribution within the steel coupon at the same three times as above. This depicts graphically the formation of a hole having the radius of the beam and a maximum central depth of about $230 \mu \mathrm{m}$. Near the edge of the hole, the density decreases sharply, dropping by an order of magnitude across a single zone (of resolution $16 \mu \mathrm{m}$ ). This indicates that the liquid layer is very thin. The minimum vapor density in the hole is about $10^{-4}$ solid density.

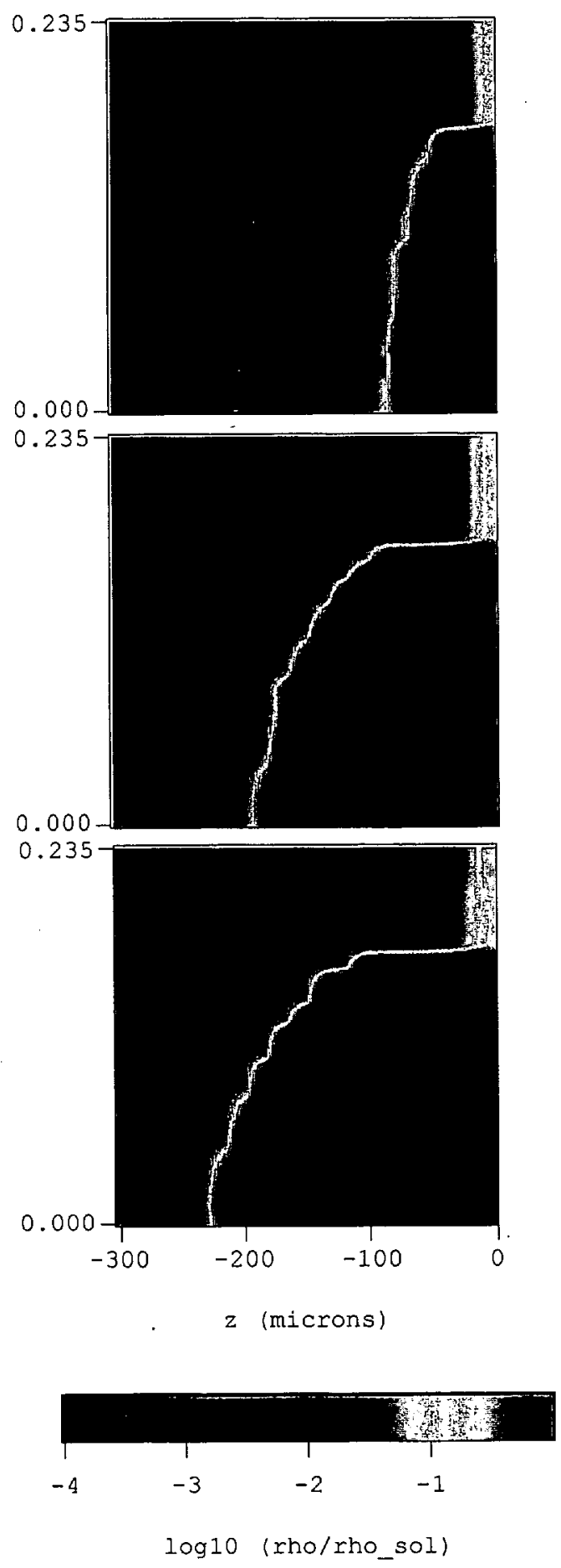

Fig. 7. Density distribution in the steel coupon, as calculated by CALE, at the same times as in Fig. 6. The color scale gives the logarithm of the density normalized by the normal solid density. The beam enters from the right. 
The temperature distribution within the hole in the steel coupon is shown in Fig. 8. During the pulse, the temperature reaches a maximum of about $4000 \mathrm{C}$ near the edge of the hole and decreases near the entrance. Fig. 9 shows the temperature at the hole entrance and beam center, as a function of time. It oscillates around about $3000 \mathrm{C}$. Not all details of the structure are shown, since the time resolution in this plot is fairly coarse (a few $\mu \mathrm{s})$.

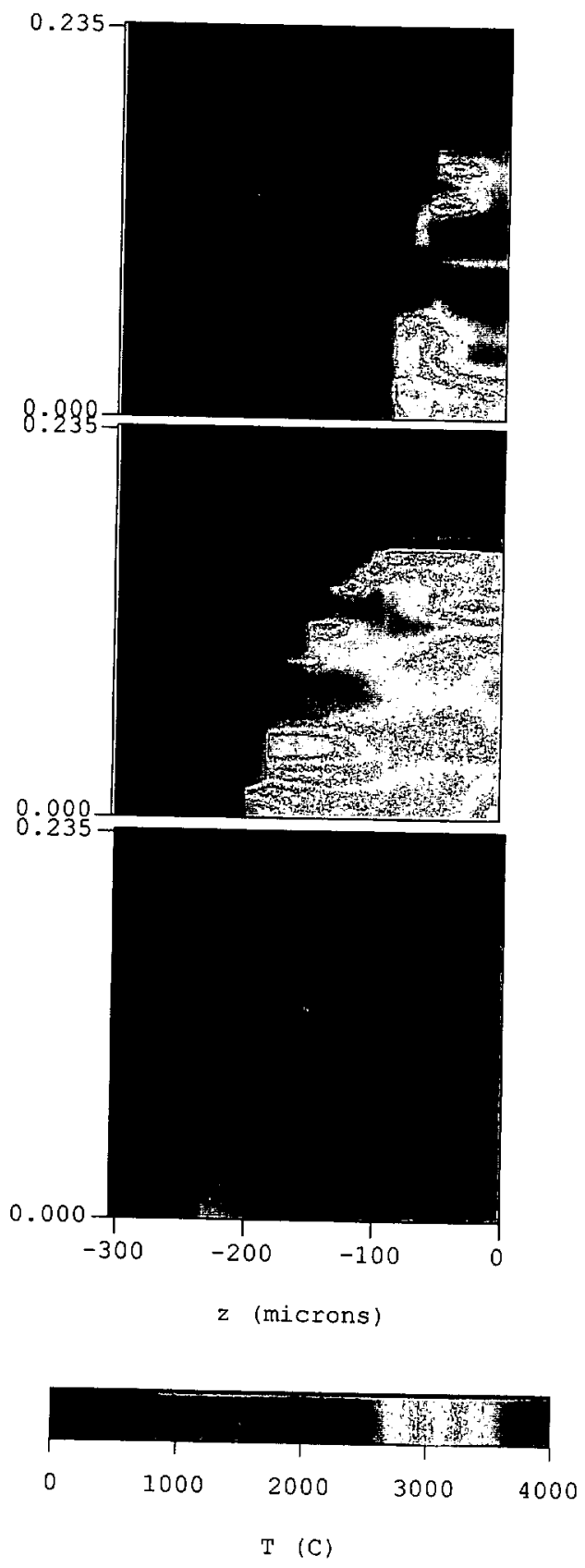

Fig. 8. Temperature distributions in the steel coupon at the same times as in Fig. 8, in the CALE calculation.

The left-hand plot in Figure 10 shows the average hole depth versus time during the pulse. It is interesting to note that both steel and aluminum show the same staircase pattern. This pattern correlates with the beam energy delivered prior to the particular time. At the end of the pulse, the hole depths are 178 microns and 230 microns for steel and aluminum, respectively. In steel, the depth is essentially the same as the observed average removal depth (180 microns), while in aluminum it is almost $80 \%$ of the observed depth. Here we have taken the edge of the hole to be defined as the point at which the density is half of the solid density. In the case of steel, for example, the point at which the density is $1 / 5$ solid density is 


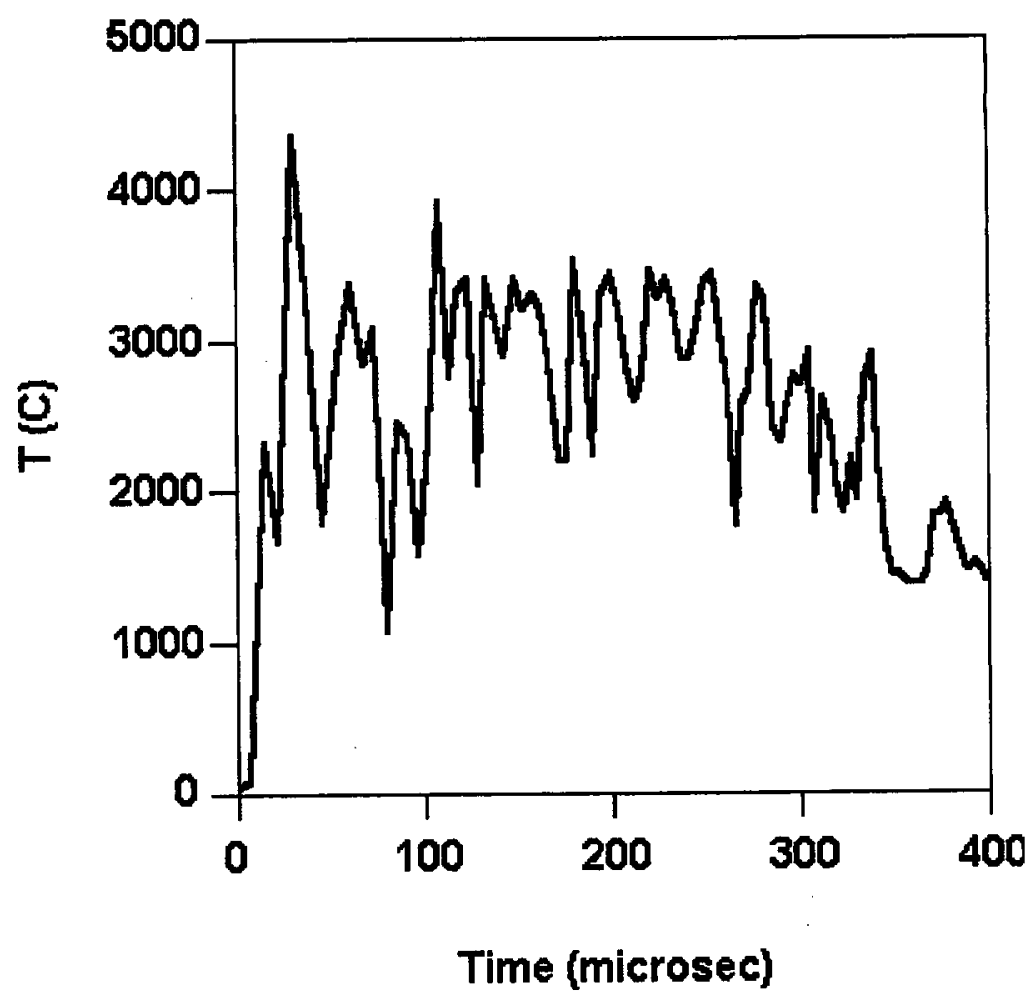

Fig. 9. Vapor temperature at the hole entrance (beam center), for the steel coupon, in the CALE calculation. The points are chosen every few $\mu$ s.
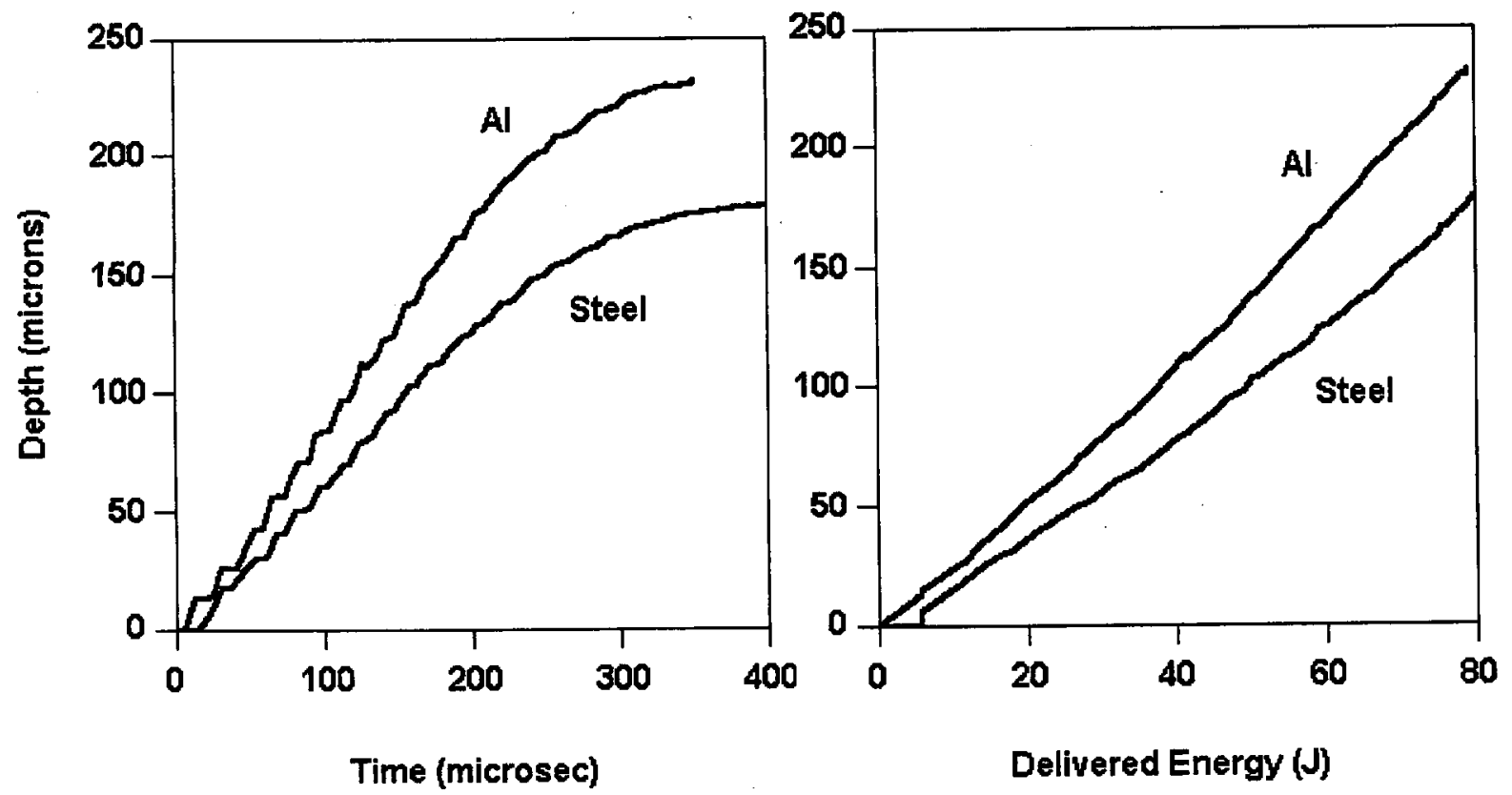

Fig. 10. Average hole depth versus time (left) and versus delivered beam energy (right), as calculated with CALE.

The dependence of the instantaneous hole depth on delivered pulse energy is shown in the right-hand plot of Fig. 10. Here the time has been eliminated and the depth is plotted directly as a function of delivered beam energy. The hole depth increases linearly with energy, with a threshold of a few joules in the case of steel. This plot should not be confused with a plot of removal depth versus total pulse energy. The latter is fixed throughout the runs. 
Figure 11 shows the rough phase separation in the coupon at about $2 / 3$ of the pulse length. Since the phases are not sharply defined in CALE, we somewhat arbitrarily denote the liquid as the region having a density between 0.4 and 0.9 solid density. This region is not well resolved in the hole, as it is generally no more than a single zone thick (16 $\mu \mathrm{m}$ in this case). Within the "liquid", the velocity field is directed almost entirely outward (antiparallel to the beam), showing no appreciable envisaged in liquid ejection scenarios.

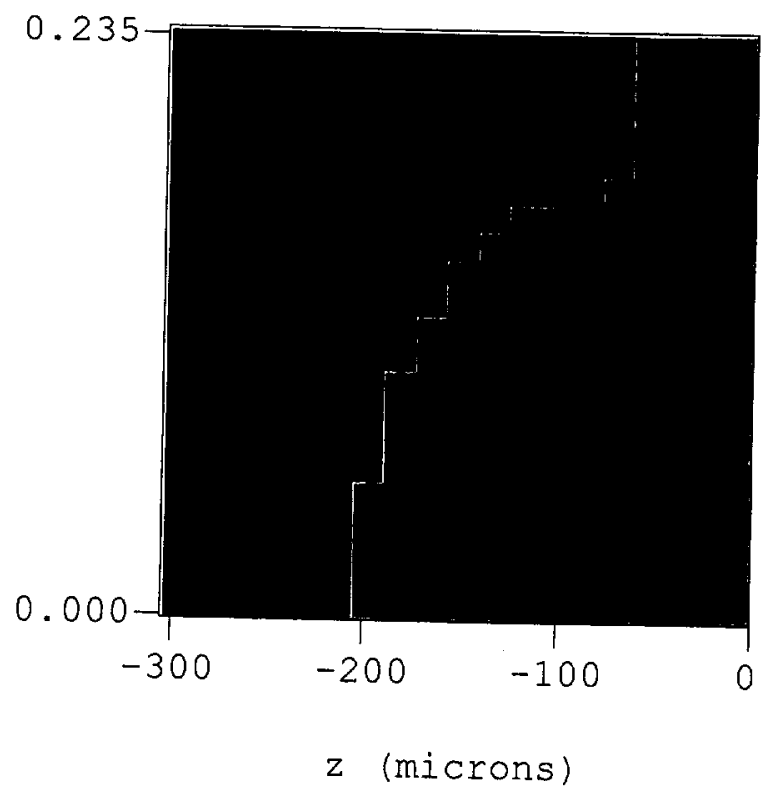

Fig. 11. Schematic phase separation in the steel coupon at $234 \mu$ s. The green area indicates zones with density lying between 0.4 and 0.9 solid density; the blue and red denote regions of higher and lower densities, respectively.

Having simulated the first pulse, we now turn to succeeding pulses, which arrive every $100 \mathrm{~ms}$. We did this only in the case of steel, but the conclusions are expected to carry over to the case of aluminum. Between pulses, the residual heat in the foil code we employed conduction and radiation. We modeled this process, in two dimensions, via the code ANSYS. In this in the THALES runs. The raterial properties (thermal conductivity, specific heat, and latent heat of melting) as were used the thermal diffusivity increases as the liquid cools. Thete thermal relaxation. A mechanism supporting this is the fact that centerline, was only a few degrees above room temp. The maximum final temperature, at the edge of the hole along the beam thermal diffusivity.

We proceeded to use CALE to simulate the second pulse on steel, using the thermalized hole of the first pulse as the initial $2 / 3$, and full pulse times characteristics during the run were similar to those of the first pulse. The growth of the hole, at $1 / 3$, third pulse gave similar is shown in Fig. 12. The average hole depth grew by 154 microns, to a total of 332 microns. The summarized in Fig. 13. The final density the hole depth by 156 microns to a total of 488 microns. These results are trend of the first thr. 13. The final density profiles of the first three pulses are shown together in Fig. 14. Extrapolating the practice, the effects of pulses, we see that the steel coupon width of $2.3 \mathrm{~mm}$ would be attained during the 15 th pulse. In side. Experimentally, as noted, 13 pulses were required

The CALE results are impressively close to experiment. However, there remain a few outstanding issues. First, the material absorptivity in the code is required to be a single constant, independent of temperature. Compared with the sophistication of the rest of the code, this is a rather crude assumption. The metallic absorptivity typically increases with temperature, as noted in Appendix B. Hence we adopted the strategy of making runs for various choices of the absorptivity $\alpha$ and comparing the resulting best choice was $\alpha \sim 0.4$, as position and time) with that associated with the given absorptivity. For steel, the aluminum, for which we simply used $\alpha=0.5$. 

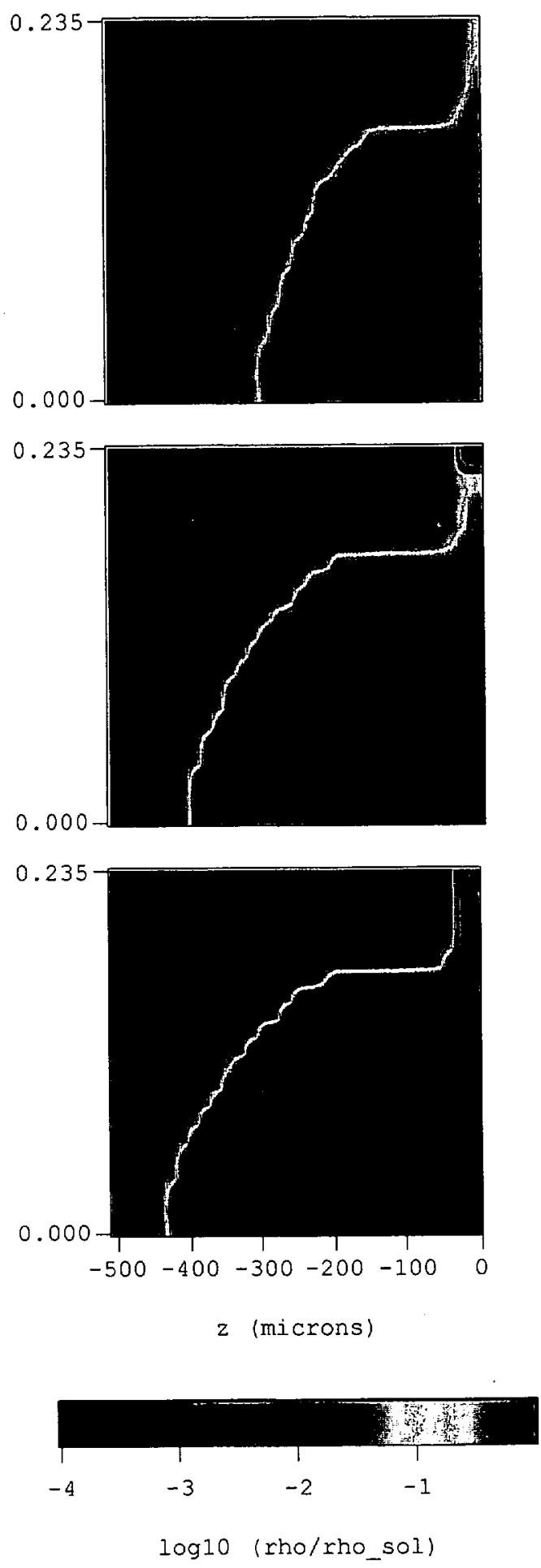

Fig. 12. Density distribution in the steel coupon during the second pulse, as calculated by CALE, at times of approximately $1 / 3,2 / 3$, and full pulse length $(122,245$, and $366 \mu \mathrm{s})$. The color scale gives the logarithm of the density normalized by the normal solid density. 


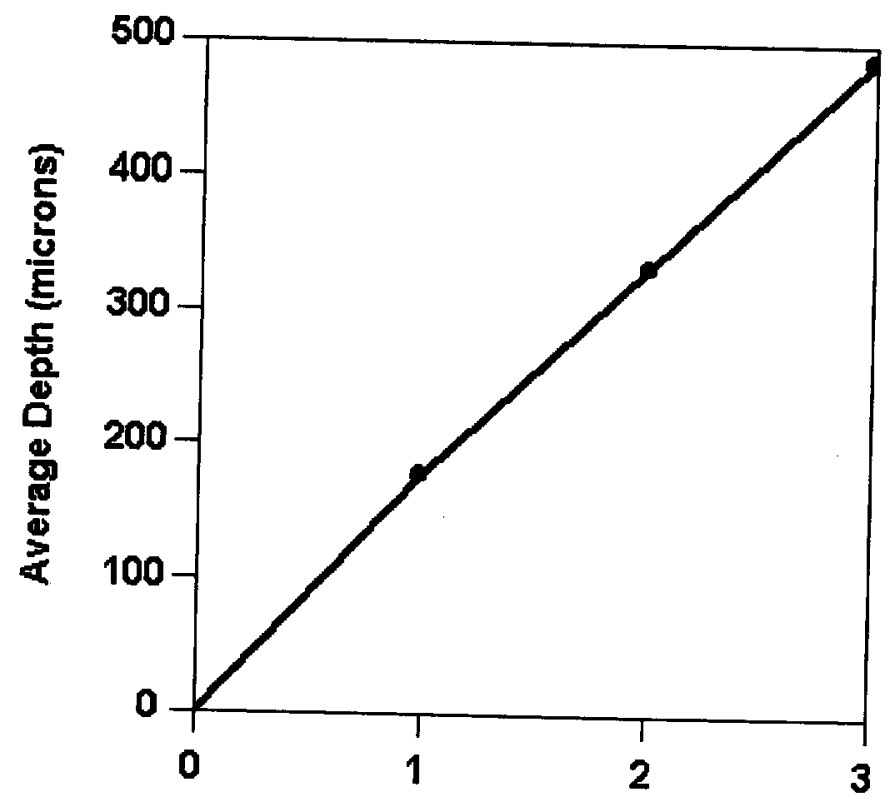

Pulse \#

Fig. 13. Removal versus pulse number, on the steel coupon.

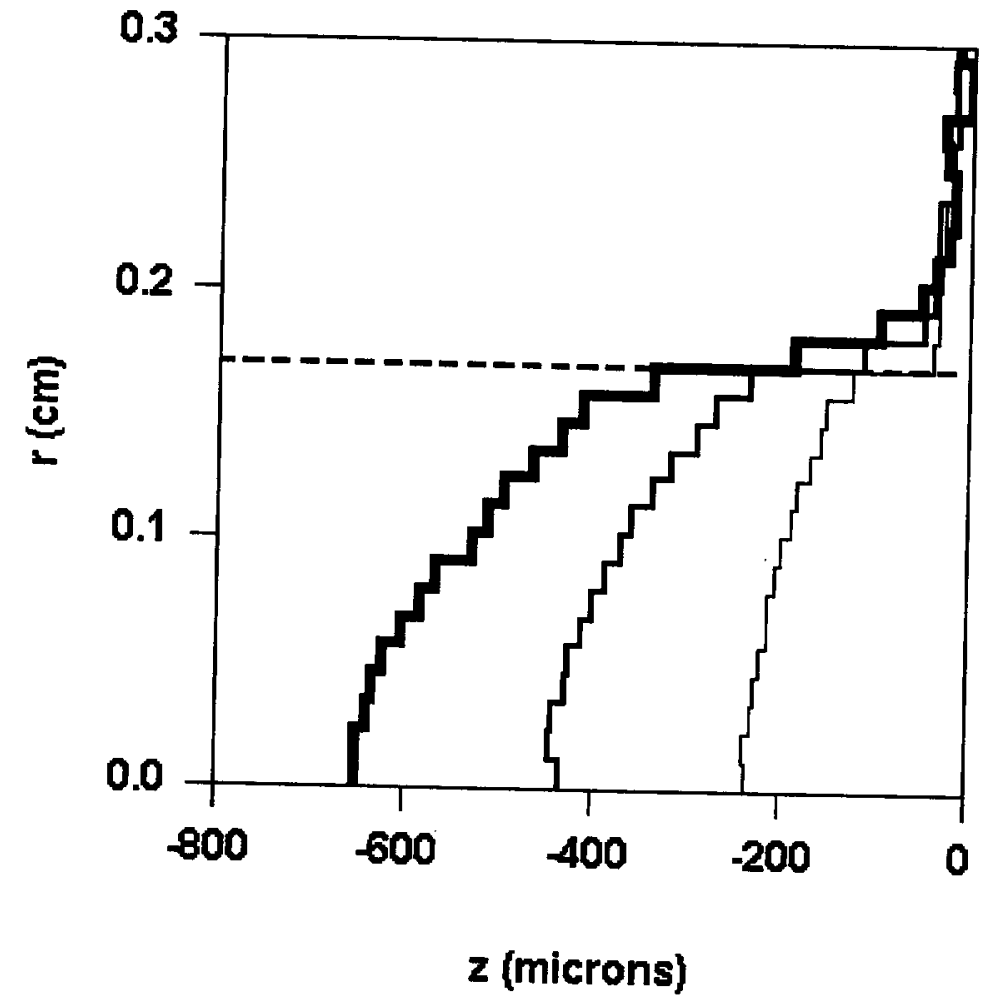

Fig. 14. Final density profiles of the first three pulses on the steel coupon, according to CALE. 


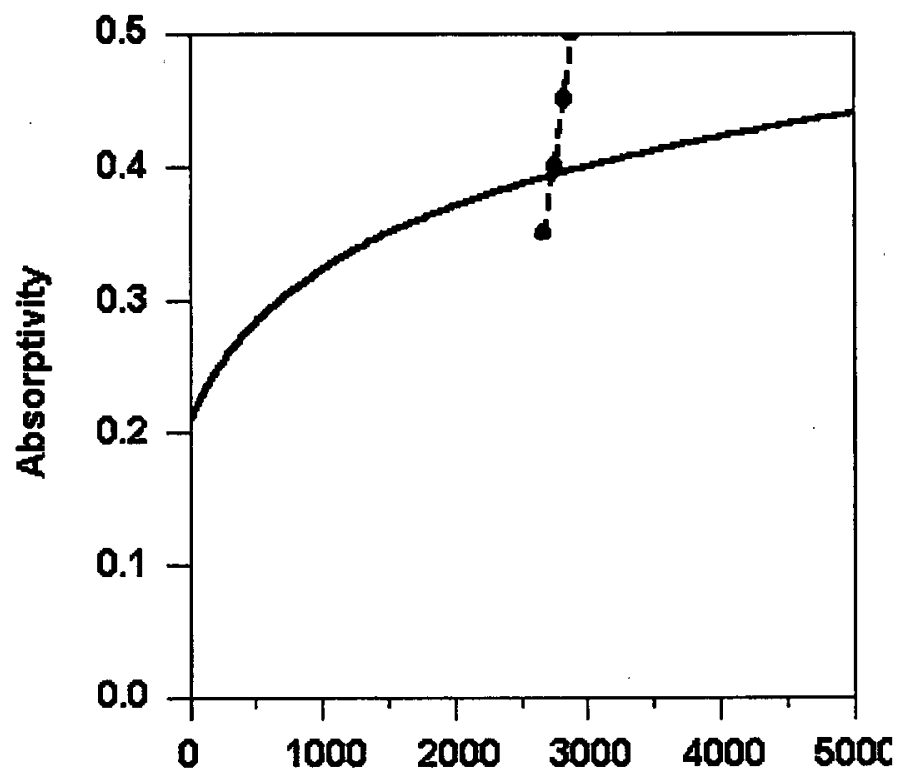

$\mathrm{T}(\mathrm{C})$

Fig. 15. Solid line: model of steel absorptivity for I- $\mu$ m light (solid line). The dotted line shows average edge temperatures, as calculated by $C A L E$, for four assumed values of the absorptivity. The curve for absorptivity by aluminum is similar to that for steel.

Second, the role of the liquid requires further study. The liquid layer (defined roughly in terms of density) is thin and poorly resolved, and it shows no sign of radial motion in response to pressure gradients. One explanation might be an insufficiently fine mesh. Another possible explanation is that, while CALE has equation-of-state information regarding the phases and transition energies, it treats the liquid as inviscid. For liquid iron near the melting temperature, the shear viscosity is about 0.06 poise $^{13}$ (accurate to about $20 \%$ ), corresponding to a kinematic viscosity of $8 \times 10^{-3} \mathrm{~cm}^{2} / \mathrm{s}$. The kinematic viscosity is about the same for aluminum, and it is also comparable to that of water at standard conditions. Estimating the Reynolds number based on a liquid width of $10 \mu \mathrm{m}$ and a speed of $10^{3} \mathrm{~cm} / \mathrm{s}$, we have $\operatorname{Re} \sim 100$. This will increase above the melting point, since the liquid viscosity decreases exponentially with temperature (for $\mathrm{Fe}$, by a factor of two at 300 deg above melting), but it seems unlikely that Re would be sufficiently high to trigger hydrodynamic instabilities. This reasoning led us to justify an inviscid treatment. It might be the case, nevertheless, that viscosity plays a discernible role in the liquid dynamics.

Surface tension is also neglected by CALE. In its absence, we would expect that (1) the dynamics of the melt surface would be inaccurate, (2) bubbles within the melt would not be properly described, and (3) condensation in the vapor would be impossible. The first two effects might prove significant, but the third would not affect material removal, as long as the condensation did not significantly absorb the beam. As we noted earlier, the CALE results show no condensation in the vapor.

A final issue, not particular to CALE itself, is that the equations of state employed here ${ }^{12}$ are sparse in the thermodynamic regime of interest. We have adjusted a few points to avoid anomalies of interpolation. However, it would be desirable to have complete, reliable tables for our regime.

\section{CONCLUSIONS}

We have described simulations of metal coupon tests by the 3-slab heat capacity testbed laser at LLNL. Two models were discussed -- a 1D model (THALES) simulating removal by vaporization, and a 2D model (CALE) simulating removal by the unified hydrodynamics of the solid/liquid/vapor system ${ }^{14}$. The first model was found to be insufficient to explain the observed removal. The second model, which was considerably more complicated, gave results fairly consistent with experiment. It is reasonable to believe that more refined treatments will also involve a multidimensional hydrodynamic code. Such treatments should include a more realistic absorption model and, ideally, effects of viscosity and surface tension. 


\section{APPENDIX A. SOME DETAILS OF THALES} This appendix gives some further details regarding the THALES model ${ }^{4}$. In the condensed matter, the model employs the
$1 \mathrm{D}$ thermal conduction equation

$$
\rho C \frac{\partial T}{\partial t}=\frac{\partial}{\partial z} \lambda \frac{\partial T}{\partial z},
$$

with $\lambda$ the thermal conductivity. The melt surface moves according to the difference of heat fluxes at the boundary, $\rho H_{m} \&_{m}=-[\lambda \partial T / \partial z]_{S}^{L}$, where $H_{m}$ is the latent heat and the $z$ coordinate increases from the solid to the liquid (to the right in Fig. 2). In the vapor, the code solves the $1 \mathrm{D}$ hydrodynamic equations for the mass density $\rho$, the $z$ -
component of the velocity field $u$, and the temperature $T$, which are

$$
\begin{aligned}
& \frac{\partial \rho}{\partial t}+\frac{\partial}{\partial z}(\rho u)=0, \\
& \rho\left(\frac{\partial}{\partial t}+u \frac{\partial}{\partial z}\right) u+\frac{\partial p}{\partial z}=0, \\
& \rho C_{v}\left(\frac{\partial}{\partial t}+u \frac{\partial}{\partial z}\right) T+T\left(\frac{\partial p}{\partial T}\right)_{\rho} \frac{\partial u}{\partial z}=\frac{\partial}{\partial z} \lambda \frac{\partial T}{\partial z}+\kappa\left[I^{(+)}-I^{(-)}\right],
\end{aligned}
$$

with $C_{\nu}$ the specific heat per unit mass at constant volume, and $\kappa$ the optical absorption coefficient. In this problem, the vapor is too cool to radiate appreciably. For sufficiently high temperatures ( $\mathrm{T} \sim 1 \mathrm{eV}$ ), a surface blackbody loss term is
included in the energy equation.

The incident laser intensity satisfies

$$
\left(\frac{\partial}{\partial t}-c \frac{\partial}{\partial z}\right) I^{(+)}=-c \kappa I^{(+)},
$$

and a similar equation with opposite spatial derivative holds for the reflected intensity. Absorption in the vapor, as embodied in the function $K(\rho, T)$, is treated in detail in THALES. However, this is unimportant in the problem at hand since the relatively low irradiance allows the vapor to remain transparent.

In the region of compressed air, the hydrodynamic equations again apply. Since the dissociation and ionization energies are high, the thermodynamics of air is handled simply via an effective $\gamma=1.4$. At the shock front, the fluid velocity and density are related to the overpressure $p^{\prime}=p / p_{0}-1$ via the Rankine-Hugoniot relations

$$
\begin{aligned}
& \left.u=\frac{c_{0} p^{\prime}\left[1+(\gamma+1) \frac{p^{\prime}}{\gamma}\right]^{-1 / 2}}{2 \gamma}\right]^{\prime}, \\
& \rho=\rho_{0}\left[1+(\gamma+1) \frac{p^{\prime}}{2 \gamma}\right]\left[1+(\gamma-1) \frac{p^{\prime}}{2 \gamma}\right]^{-1},
\end{aligned}
$$

with the subscript $o$ referring to ambient air. The speed of the shock front is

$$
\frac{\&}{s}=c_{0}\left[1+(\gamma+1) \frac{p^{\prime}}{2 \gamma}\right]^{1 / 4} .
$$


The overall numerical approach in THALES is to convert the equations of the model into a system of ordinary differential equations in time by suitably differencing the spatial terms, and then to advance the ODEs via a variant of the LSODE solver package $^{15}$. To handle the moving melt, vaporization, contact, and shock surfaces, we introduce dimensionless coordinates in each phase. Thus in the vapor we take $\xi=\left(z-z_{v}\right) /\left(z_{c}-z_{v}\right)$, with $z_{v}$ and $z_{c}$ the coordinates of the vaporization surface and contact surface, respectively. When the time and space derivatives are transformed to these coordinates, the moving boundaries are eliminated in favor of a nonlinear convective term. The equations are differenced in space with an Eulerian grid in $\xi$. The method of differencing employs the pseudocharacteristic method of lines ${ }^{16}$. Finally, to stabilize possible large gradients, artificial viscosity terms ${ }^{17}$ are added to the velocity and temperature equations.

\section{APPENDIX B. ABSORPTION MODEL}

At the surface of the condensed matter, light is absorbed according to the empirical absorption coefficient. This generally increases with frequency and temperature, but the available data ${ }^{18}$ are scattered and limited to low temperatures, where surface preparation is important. Hence a primitive temperature-dependent model was employed. For perpendicular incidence, the absorption coefficient has the form

$$
\alpha=1-\left|\frac{n-1}{n+1}\right|^{2},
$$

with $n$ the complex index of refraction. In MHD with a single electron collision frequency $\mathrm{v}$, this is given by

$$
n^{2}(\omega)=1-\frac{\Omega_{p}^{2}}{\omega^{2}}(1+i v / \omega)^{-1}
$$

where $\omega$ is the light frequency and $\Omega_{p}$ is the plasma frequency. The electron density is taken as the condensed number density, with the appropriate number of valence electrons. The collision frequency was assumed to increase as $T^{1 / 2}$, corresponding to a reaction rate with a constant cross section. The coefficient was chosen so that the absorptivity matched the measured absorptivity at the highest available temperature and at a frequency near that of 1- $\mu \mathrm{m}$ light. The resulting absorptivity for iron, as a function of temperature (Fig. 15), increases from slightly more than 0.2 at room temperature to slightly less than 0.5 at $5000 \mathrm{C}$. For aluminum, extrapolation of the very scattered data gives an unrealistically low value at a few thousand degrees. Hence the fit was adjusted to give about the same absorptivity as steel. While these choices appear reasonable, the absorption model should certainly be improved. Most helpful would be data at a temperature of a few thousand degrees.

\section{ACKNOWLEDGMENTS}

We would like to thank P.A. K. Amala, R. A. Managan, and P. A. Pincosy for guidance on CALE, and L. B. Hagler for guidance on ANSYS. We are also indebted to G. F. Albrecht, the late H. T. Powell, and A. M. Rubenchik for discussions.

This work was performed under the auspices of the U.S. Department of Energy by Lawrence Livermore National Laboratory under Contract No. W-7405-ENG-48.

\section{REFERENCES}

1. The coupon tests were performed by C. B. Dane and S. N. Fochs at LLNL.

2. We are indebted to M. D. Rotter (LLNL) for providing the calculated waveform.

3. In the demonstrations, cold rolled steel (about $98 \% \mathrm{Fe}$ ) was used. In the THALES simulation, we model this as pure iron. In the CALE simulations, the equation of state and material properties correspond to stainless steel. The differences are not expected to be important.

4. C. D. Boley and J. T. Early, "Computational Model of Drilling with High Radiance Pulsed Lasers," Proc. Intern. Conf. on Applications of Lasers and Electro-Optics, Orlando, FL, Vol. 79, p. 499 (1994).

5. M. A. Norton, C. D. Boley, J. E. Murray, K. Sinz, and K. Neeb, "Long-Lifetime, Low-Contamination Metal Beam Dumps for NIF Spatial Filters," Third Inter. Conf. on Solid State Lasers for Application to Inertial Confinement Fusion, SPIE Vol. 3492, pp. 901-911 (1999).

6. Y. S. Touloukian et al., Thermophysical Properties of Matter, Vol. I, Thermal Conductivity - Metallic Elements and Alloys, IFI/Plenum, New York, 1970; C. Y. Ho, R. W. Powell, and P. E. Liley, "Thermal Conductivity of the 
Elements," J. Phys. Chem. Ref. Data, Vol. 3, Suppl. 1 (1974); R. Hultgren et al., "Selected Values of the Thermodynamic Properties of the Elements," American Society for Metals, 1973.

7. S. I. Anisimov, "Vaporization of Metal Absorbing Laser Radiation," Sov. Phys. JETP 27, 182-183 (1968).

8. C. J. Knight, "Theoretical Modeling of Rapid Surface Vaporization with Back Pressure," AIAA J. 17, $519-523$ (1979).

9. Ya. B. Zel'dovich and Yu. P. Raizer, Physics of Shock Waves and High-Temperature Hydrodynamic Phenomena, Academic Press, 1966.

10. L. D. Landau and E. M. Lifshitz, Fluid Mechanics, Pergamon Press, 1959.

11. The primary developer of CALE is R. E. Tipton, LLNL.

12. The equation of state and elastic properties were taken from tables available at LLNL.

13. T. Iida and R. I. L. Guthrie, The Physical Properties of Liquid Metals, Clarendon Press, Oxford, 1988.

14. It should be pointed out that THALES can be run much more speedily than CALE. A typical THALES execution time on a UNIX workstation is 20 minutes, while a CALE run, or actually a combination of several runs, on a workstation having four times greater clock rate consumes 35-45 hours.

15. A. C. Hindmarsh, "ODEPACK, a Systematized Collection of ODE Solvers," in Scientific Computing, R. S. Stepleman et al. (eds.), North-Holland, Amsterdam, 1983 (Vol. I of IMACS Transactions on Scientific Computing), pp. 55-64.

16. M. B. Carver, "Pseudo Characteristic Method of Lines Solution of the Conservation Equations," J. Comp. Phys. 35, 57-76 (1980); Y.-Z. Wang and S. H. Johnson, "Pseudocharacteristic Method of Lines Simulation of Single- and TwoPhase One-Dimensional Flow Transients," Proc. IMACS 11th World Congress, Vol. 2, pp. 201-204, Oslo, 1985.

17. P. J. Roache, Computational Fluid Dynamics, Hermosa Publishers, Albuquerque, 1972.

18. Y. S. Touloukian and D. P. DeWitt, Thermophysical Properties of Matter, Vol. 7, Thermal Radiative Properties Metallic Elements and Alloys, IFU/Plenum, New York, 1970. 


\section{Special Project: Laser Material Processing Resarch ACtivity Highlights}

\section{Laser Peening Increases the Corrosion Resistance of Metal}

In a joint research effort with the Yucca Mountain Project (YMP), we are evaluating laser peening (Figure 1) as a technique to

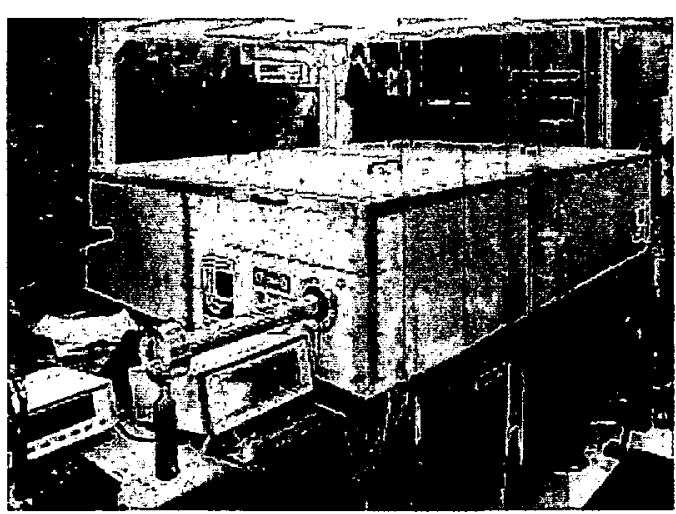

Figure 1. LLNL s LasershotSM Peening System.

improve the corrosion resistance and service lifetime of metal canisters designed for final disposal of high-level radioactive waste, dismantled reactors, and retired weapon components. Nuclear waste around the country will be stored underground at Yucca Mountain in welded canisters of Alloy 22. These canisters are required to last 10,000 years without leakage. However, the process of welding the end caps on these canisters can cause tensile stress that allows defects to grow into cracks and accelerate corrosion. Previous work (see August 2000 LS\&T Program Update) demonstrated that laser peening can transform tensile stress into compressive stress deep into the material and prevent the growth of such cracks. In recent tests, we found that laser peening not only stops crack propagation in welds, it also retards the overall corrosion rate of metal.
By optimizing the process parameters such as laser pulse duration ( 10 to $30 \mathrm{~ns}$ ), fluence intensity ( 50 to $300 \mathrm{~J} / \mathrm{cm}^{2}$ ) and number of treatment pulses, we were able to induce compressive stress deep into the metal. Figure 2 shows the residual stresses induced by laser peening on titanium measured at various depth levels. Compressive residual stress extending to depth of several millimeters is achieved with the laser pulses at various fluence intensities.

We have conducted a number of stress-corrosion cracking (SCC) and surface corrosion experiments on 304 and 316 stainless-steel

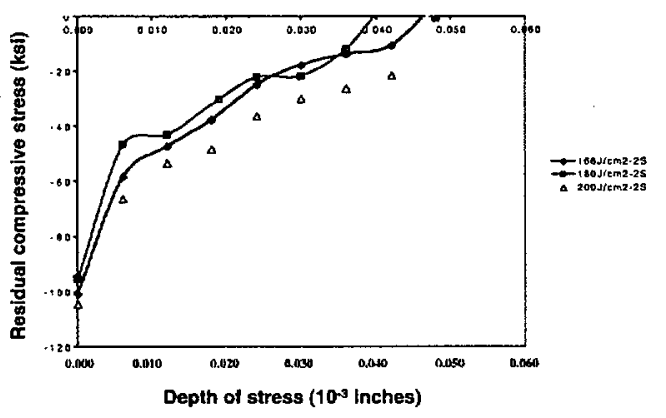

Figure 2. Laser peening induces compressive stress on titanium.

weld. Figure 3 shows results from 304 stainless specimens bathed in $40 \% \mathrm{MgCl}_{2}$ solution at $160^{\circ} \mathrm{C}$, which accelerates cracking and corrosion. On the unpeened weld, cracks perpendicular to the weld developed within 24 hours, while no observable cracks were detected on the peened weld even after weeks of exposure.

We have also assembled a high-tempera-ture reactor to perform SCC experiments on largesize (foot-scale) test samples. Two foot-scale stainless-steel welds were tested. The first 


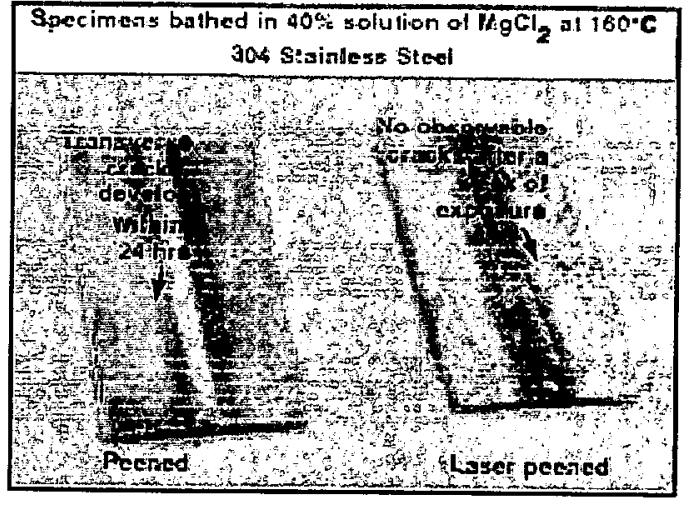

Figure 3. Laser-peened 304 stainless-steel weld on the right showed no cracking even after a week in $160 ; \mathrm{C} \mathrm{MgCl} 2$ solution.

experiment was on a seam-weld made by two pieces of $12-\times 5.5-\times 0.5$-in. 316 stainless steel. One side of the welds was laser-peened and the other side of the same weld was not. Two bottomless Erlenmeyer flasks were used to boil the $\mathrm{MgCl}_{2}$ solution at $156^{\circ} \mathrm{C}$. The unpeened weld area showed cracks and corrosion within 5 days, while the laser-peened weld area showed no signs of cracking or corrosion even after 10 days of exposure. This result is consistent with the electrochemical data obtained by French researchers earlier [P. Peyre et al., Materials Science and Engineering, A280, 294-302, (2000)]. Laser peening appears to increase the free corrosion potential and reduce the passive current densities of metal.

The second experiment was conducted on a similar size of 316 stainless steel weld. Laser peening was performed on a selected area of the welds. A foot-scale bottomless Erlenmeyer flask was used to hold the $\mathrm{MgCl}_{2}$ solution over both the peened and unpeened areas. After 5 days bathed in the boiling $\mathrm{MgCl}_{2}$ solution, cracks developed on the surface at the unpeened area while the laser-peened area showed no observable cracks. Cracks originating from the unpeened area appeared to propagate, bypass, and cease at the peened zone (see Figure 4).

Our experiments demonstrated that laser peening can significantly improve the resistance of metal to stress-corrosion cracking and surface corrosion. We are working closely with industry to commercialize this technology. Besides nuclear waste disposal, the U.S. industry could apply this technology to improve reactor safety and reliability and to extend the

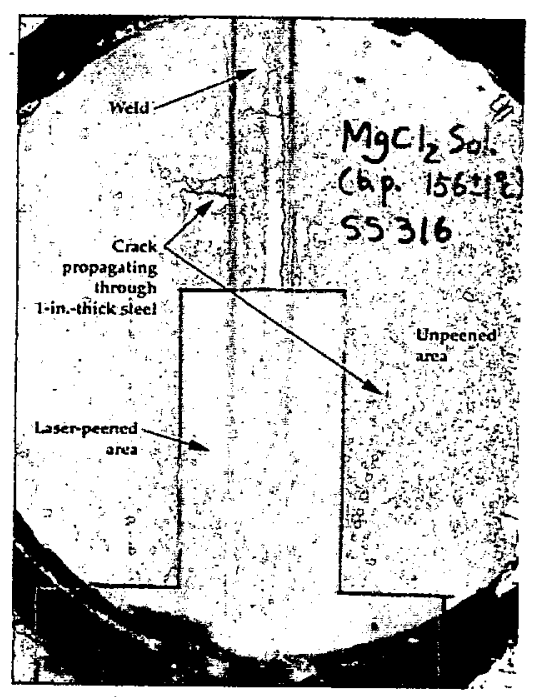

Figure 4. Laser-peened area of a welded 316 stainless steel showed no observable cracks even after 5 days in $156 ; \mathrm{C} \mathrm{MgCl} 2$ solution.

operational life of reactor components (internals, tubes, bolts, and pins) so that boilingwater reactors and pressurized water reactors will have a higher service life and lower operating cost.

\section{Joint Research Develops Laser Peenforming A New Precision Metal-Forming Technology}

In a collaborative effort with Metal Improvement Co. Inc, of Paramus, N.J., we are evaluating the effectiveness of laser peenforming to precisely form metal panels for aerospace and defense applications. Using a kilowatt-class pulsed Nd:glass laser, we are able to selectively place residual compressive stress into the surfaces of thick metal panels to form them into precise and complex shapes without yielding the metal and leaving both surfaces in crack-resistant compressive stress state.

Similar to the laser peening process originally developed for increasing fatigue and corrosion resistance of metals, the peenforming technique, recently developed by LS\&T, uses a high-energy and high-intensity solid-state laser to impress a deep level of residual stress into selected surfaces of the metal. The strain associated with the compressive residual stress causes the treated surface to elongate, effectively curving the metal within the peened area. 
By optimizing the process parameters such as laser fluence $\left(-100 \mathrm{~J} / \mathrm{cm}^{2}\right)$, intensity (5 to $10 \mathrm{GW} / \mathrm{cm}^{2}$ ), and number and location of treatment pulses, we are able to precisely form a broad range of aluminum alloys to contours and shapes that could not be adequately made using conventional methods.

Figure 5 shows three aerospace-grade 2024 T-3 aluminum alloy plates 1 in., $3 / 4$ in., and $5 / 8$ in. thick formed by the laser peenforming technique. We were able to form them to precise curvatures and bend radius by adjusting the laser peening parameters and peening location. We have also formed a 5/8-in.-thick aluminum plate (Figure 6) into a precise saddle shape with 150-in. radius of curvature concave and convex on alternating surfaces.

Forming is critically important to the fabrication of structural wing skins for large com- works well but is limited to only 7000 series aluminum to which age creep forming can be applied. The process is not precise, and parts have to be repeatedly treated and checked in a forming jig until the fit meets specification. Using laser peenforming, one could potentially make the 40-meter outer skin of an airplane wing fit precisely onto its frame with just one or two treatments.

The laser peenforming process will dramatically change aircraft manufacturing in terms of cost and efficiency as well as the introduction of a new capability to the design and forming of metal components. The ability to form large structural members using lighter-weight panels and fewer joints can significantly reduce aircraft weight and increase payload and fuel efficiency. We are working closely with industry to commercialize this technology.

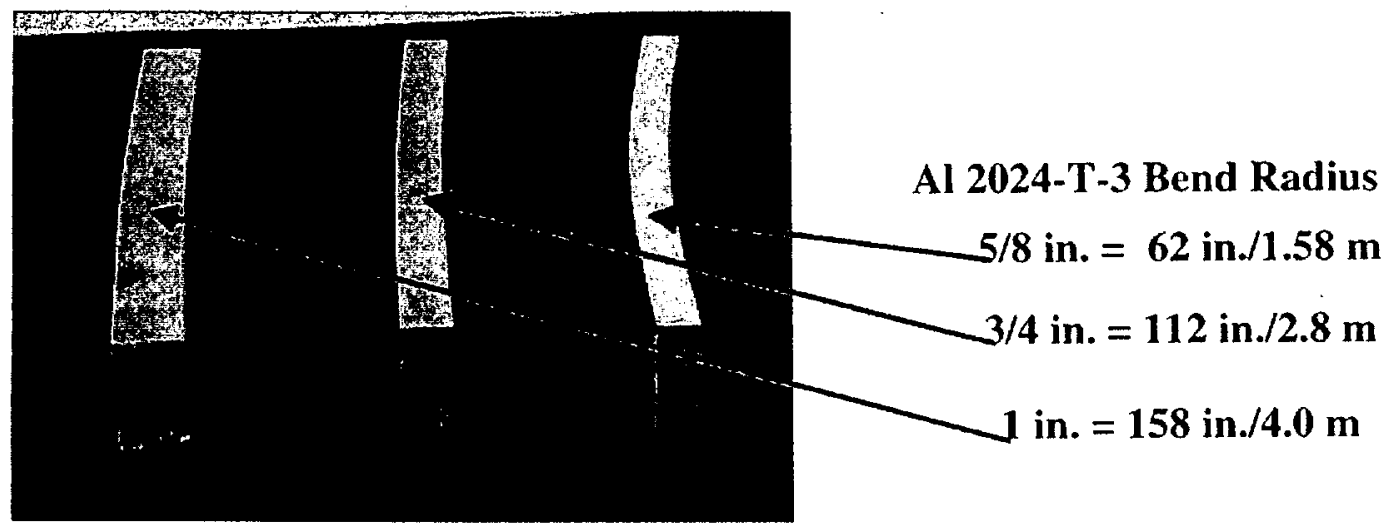

Figure5. Thick aluminum metal sections have been formed by laser peenforming without yielding the metal and leaving both surfaces in a crack-resistant compressive stress state.

mercial and military aircraft. Many

components on aircraft such as wing skins, elevator and rudder panels, and winglets need to be formed to precise complex curvatures both to meet aerodynamic requirements and to fit precisely on the airframe for stress-free fastening. These structural components cannot be bent using conventional hydraulic or other force forming techniques because bending causes undesirable yielding of metal. Metal yielding causes loss of mechanical strength and always leaves surfaces in tension, thus susceptible to fatigue fracture and corrosion cracking. In the past, the aerospace industry has used an iterative forming process (repeated mechanical shot peening and thermal age creeping) to form and shape metal panels. This iterative process

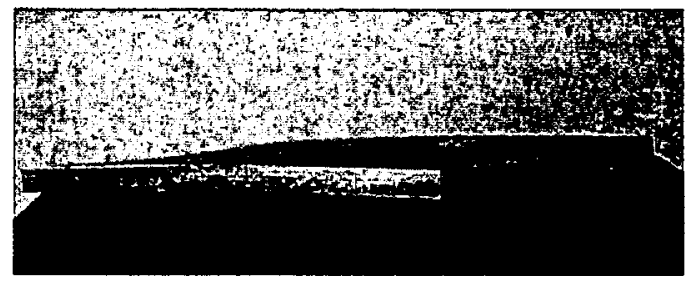

Figure 6. A 5/8-in.-thick 2024 T-3 aluminum plate formed to a saddle shape by laser peenforming. Laser peenforming can form metal panels with complex curvatures. 


\section{DISCLAIMER}

This document was prepared as an account of work sponsored by an agency of the United States Government. Neither the United States Government nor the University of California nor any of their employees, makes any warranty, express or implied, or assumes any legal liability or responsibility for the accuracy, completeness, or usefulness of any information, apparatus, product, or process disclosed, or represents that its use would not infringe privately owned rights. Reference herein to any specific commercial product, process, or service by trade name, trademark, manufacturer, or otherwise, does not necessarily constitute or imply its endorsement, recommendation, or favoring by the United States Government or the University of California. The views and opinions of authors expressed herein do not necessarily state or reflect those of the United States Government or the University of California, and shall not be used for advertising or product endorsement purposes.

This work was performed under the auspices of the U.S. Department of Energy by University of California Lawrence Livermore National Laboratory under Contract W-7405-ENG-48.

This report has been reproduced directly from the best available copy.

Available to DOE and DOE contractors from the Office of Scientific and Technical Information

P.O. Box 62, Oak Ridge, TN, 37831

Prices available from (615) 576-8401, FTS 626-8401

Available to the public from the National Technical Information Service, U.S. Department of Commerce, 5285 Port Royal Rd., Springfield, VA 22161

May 2002

UCRL-ID-134972-01

Scientific Editor: Hao-Lin Chen

Publication Editor: Cindy Cassady

For further information please contact:

Dr. Lloyd A. Hackel: hackel1@llnl.gov, (925) 422-9009

Dr. Hao-Lin Chen: chen4@llnl.gov, (925) 422-6198 


\title{
The Effects of Process Variations on Residual Stress Induced by Laser Peening
}

\author{
Jon E. Rankin ${ }^{1}$ \\ Michael R. Hill ${ }^{1}$ \\ Hao-Lin Chen ${ }^{2}$ \\ Lloyd A. Hackel ${ }^{2}$ \\ ${ }^{1}$ Mechanical and Aeronautical Engineering Department, \\ University of Califomia, Davis, CA 95616 \\ ${ }^{2}$ Laser Science and Technology Program, \\ Lawrence Livermore National Laboratory, Livermore, CA 94550
}

\begin{abstract}
Measurements of the distribution of residual stress with depth from the surface in laser peened coupons were made in a highstrength aluminum alloy. Residual stresses were measured using slitting (also known as the crack compliance method). Measurements were made on several coupons to: compare laser peening (LP) and shot peening residual stresses; ascertain the influence of several LP parameters on the residual stress imposed; determine whether tensile residual stress existed outside the peened area; assess the variation of residual stress with in-plane position relative to the layout of the laser spots used for peening; and, determine the importance of a uniform spatial distribution of laser energy within the laser spot. Residual stress $0.1 \mathrm{~mm}$ from the surface due to LP and due to shot peening were comparable and the depth of the compressive stress for LP was far greater than for shot peening. Variations of most LP parameters did not significantly alter residual stress at shallow depths. Residual stresses adjacent to the peened area were found to be compressive. Decreased levels of surface residual stress were found when laser spots had a non-uniform distribution of laser intensity.
\end{abstract}

\section{INTRODUCTION}

Laser peening is an emerging surface treatment, capable of imparting compressive surface residual stress and improving the resistance of components to fatigue failure. While the general mechanical concept is similar to conventional shot peening, laser peening (LP) offers certain advantages. First, LP leaves a more desirable surface than does shot peening (SP) [1,2]. This improved surface condition may result in improved resistance to fatigue crack initiation. Second, while LP and SP create residual stresses of similar magnitude, the compressive stresses extend far deeper from the surface for LP [3], thereby offering improved resistance to the growth of near-surface, macroscopic cracks. These two characteristics may therefore lead to significant improvements in fatigue life of treated components. This work presents measurements of the distribution (or, "profile") of LP-induced residual stress with distance from the surface under a variety of processing conditions, for a high strength aluminum alloy used in aerospace structure (7049 T73). The term "measure" is used throughout this paper; technically, however, released strain is measured while residual stress is determined via an inverse elastic analysis.

Potential improvements in fatigue life make LP an attractive process, but its adoption will likely depend on verifying its performance relative to current surface treatments. Because SP is the most common surface residual stress treatment for aerospace structure, effects of LP are often compared to effects of SP. Considering that the beneficial effects of either type of peening on fatigue life are primarily due to the near-surface residual stress, the first objective of this paper is to compare the residual stress profile produced by LP to that produced by SP in 7049 T73 aluminum.

The residual stress profile due to LP is influenced by several process variables. One basic process parameter is the energy density, or "fluence", of the laser pulse. A second important parameter is the size of the laser spot. A third important parameter is the number layers of peening applied to a component and, for multi-layer peening, a fourth important parameter is the spatial offset, or "overlap", from one peening layer to another. The influences of these process variables on the resulting residual stress profiles produced by LP are not wholly understood. Therefore, the second objective of this paper is to assess the variation of the LP-induced residual stress profile with changes in laser energy, laser spot size, and layer overlap. Since residual stresses must be in equilibrium, tensile residual stress must exist somewhere in a part containing compressive residual stress. The third objective is to examine the in-plane variation of residual stress adjacent to a laser peened area to determine whether tensile residual stresses are present. Since the physical scale of LP is far larger than that of SP (LP spots are a factor 10 to 1.00 larger than SP impacts), the fourth objective of this paper is to determine the in-plane variation of residual stresses the LP area. To assess the effect of non-uniform laser energy on the residual stress profile, the final objective is to examine LP residual stresses when one corner of laser spot was systematically degraded. 


\section{METHODS}

The objectives described above were addressed by measuring residual stress in a series of coupons peened in various ways. Residual stresses were measured by relaxation, using the slitting method (also called the compliance method or the crack compliance method).

Slitting. The general procedure for slitting is to gradually extend a slit into the specimen surface and measure near-slit strain as a function of slit depth. Strain due to slitting was measured using a metallic foil gage placed near the slit (Figure 1) and the slits were cut using wire electrical discharge machining (EDM). Strain versus depth data were then input to an inverse elastic analysis to compute the variation of pre-slit residual stress with depth from the surface (i.e., the stress profile). The inverse elastic analysis assumed a finite slit in a semi-infinite half-space, as described by Prime and Finnie [4]. The analysis accounted for the finite slit width $w$, gage length $L_{g}$, and gage position $s$ (Figure 1). Further details on the slitting method are omitted for brevity, but have been summarized by Prime [5].

Stress profiles for each measurement were preliminarily calculated for polynomial orders of $0^{\text {th }}$ through $7^{\text {th }}$ order. The polynomial order for the stress expansion was selected for each set of measured strain data by considering the root mean square of the error between measured strain and the fitted strain. Low-order polynomial series generally exhibit high strain fit error, and increasing order decreases error only for a limited number of terms. Including excessively high order terms results in a system with poor numerical conditioning, which magnifies the effect of experimental error on the calculated residual stress. Order selection and error estimation were recently discussed by Hill and Lin [6].

Careful attention was paid to several aspects of the experiments. Strain gages had $L_{g}=0.79 \mathrm{~mm}$ and gage locations were at least 2 cut depths from coupon edges, along the slit. A waterproofing system consisted of a layer of acrylic covered by a layer of paraffin wax and protected the gages from water used for EDM. The gage positions $s$ were nominally $1.8 \mathrm{~mm}$, which allowed room for the coatings. Rankin et al [7] described further experiment details.

Measurement Sites. Seven coupons were used to address the five objectives described above. These coupons were cut from a single 7049 T73 aluminum forging. A commercial provider performed shot peening to AMS-S-13165, using 0.48$0.71 \mathrm{~mm}$ cast steel shot, $200 \%$ coverage, and $0.010 \mathrm{~A}-0.014 \mathrm{~A}$ Almen intensity. LP was performed on a novel Nd:glass, flash lamp pumped laser. The laser system is capable of an average power of $600 \mathrm{~W}$, pulse width of 10 to $100 \mathrm{~ns}$, pulse energy of up to $100 \mathrm{~J}$ and a repetition rate of up to $6 \mathrm{~Hz}$. Details on a similar laser system were previously reported by Dane et al [8] and a discussion of its use for LP was reported by Hammersley et al [9]. The LP parameters used for each of the seven coupons are listed in Table 1 (which also has a listing of the measurement sites on these coupons, described below). Initial measurements of residual stress were made on treated rectangular surfaces with planar dimensions $9.5 \times 50 \mathrm{~mm}$ and with coupon thickness of $25 \mathrm{~mm}$ (coupons 5ST and 6LT). All other coupons were $9.5 \mathrm{~mm}$ thick and had planar dimensions at least $25 \times 38 \mathrm{~mm}$.

Table 1: Experimental coupons and measurement sites

\begin{tabular}{|c|c|c|c|c|c|c|c|}
\hline Coupon & Site(s) & $\begin{array}{c}\text { Fluence } \\
{\left[\mathrm{J} / \mathrm{cm}^{2}\right]}\end{array}$ & \multicolumn{2}{c|}{$\begin{array}{c}\text { Pulse width } \\
1[\mathrm{~ns}]\end{array}$} & $\begin{array}{c}\text { Size } \\
{[\mathrm{ns}]}\end{array}$ & $\begin{array}{c}\text { Overlap } \\
{[\% \text { size }]}\end{array}$ & Purpose or Effect \\
\hline \hline 5ST & $0-\mathrm{S}$ & -- & -- & -- & -- & -- & SP residual stress \\
\hline 6LT & $0-\mathrm{L}$ & 60 & 18 & 18 & 5 & 10 & Compare LP to SP \\
\hline \multirow{2}{*}{$4 \mathrm{~A}$} & 1 & 60 & 17 & 12 & 5 & 10 & Shortened second pulse \\
\cline { 2 - 9 } & 2 & 45 & 17 & 12 & 5 & 10 & Reduced laser energy \\
\hline 4AT & 3 & 60 & 17 & 12 & 3.2 & 10 & Reduced spot size \\
\hline 6AT & $4-6$ & 45 & 17 & 12 & 5 & 50 & Adjacent to edge of LP area \\
\hline 6A & $7 \mathrm{a}-9 \mathrm{a}$ & 45 & 17 & 12 & 5 & 50 & In-field variations \\
\hline 7AT & $10 \mathrm{a}-13 \mathrm{a}$ & 45 & 17 & 12 & 5 & 50 & Conrer-attenuated laser spots \\
\hline
\end{tabular}

Residual stress profiles were measured at fifteen sites on the seven coupons. A schematic representation of all measurement sites within the laser peening fields is shown in Figure 2. Note that the figure shows the sites relative to the laser spot layouts employed in peening and does not indicate the proximity of the sites to one another. A double ellipse denotes each site, where the center indicates the measurement location and the major axis lies along the slit direction.

The collection of coupons and measurement sites (Table 1 and Figure 2) was capable of addressing the five objectives of the present work. Results at sites 0-S and 0-L allow a comparison between SP and LP residual stress profiles. Measurements at sites 1,2, and 3 compare the effects of laser pulse fluence (laser energy per spot area) and laser spot size on the residual stress profiles. Comparison of results at site $0-\mathrm{L}$ and site 1 shows the effect of a smaller second-layer pulse width (18 ns for site $0-\mathrm{L}$ and $12 \mathrm{~ns}$ for site 1 ; the $1 \mathrm{~ns}$ difference of the first pulse was assumed to be insignificant). Comparison of results for site 2 with those of site 7a shows the effect of layer overlap. Results at sites 4, 5, and 6 show the in-plane variation of the residual stress profile adjacent to the laser peened area. Sites $7 \mathrm{a}, 8 \mathrm{a}, 9 \mathrm{a}$, and $11 \mathrm{a}$ investigate the in-plane variations of the 
residual stress profile within the peened area. Peening on coupon 7AT was performed with one corner of the laser spot intentionally attenuated (masked) as shown in Figure 2(d); comparison of the results at sites 10a and 11a with those at 12a and 13a shows the effect of spatially uniform laser energy.

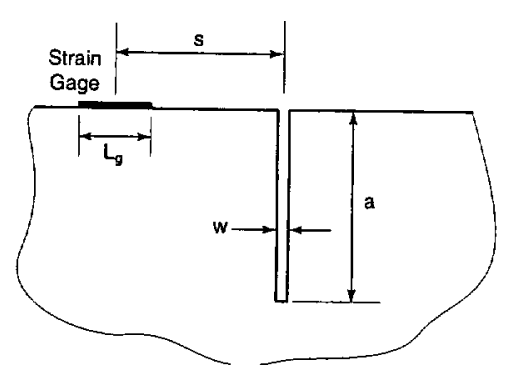

Figure 1: Strain gage installation schematic

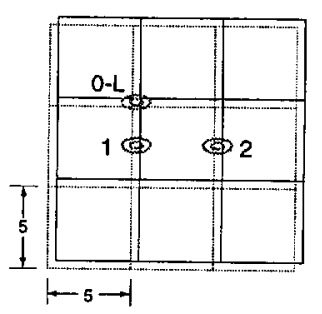

(a)

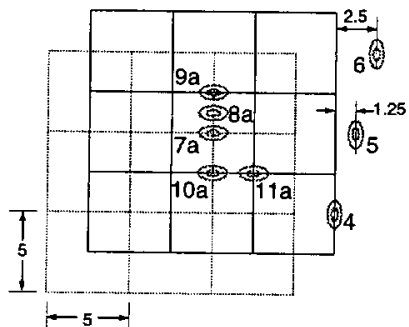

(c)

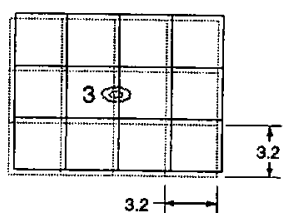

(b)

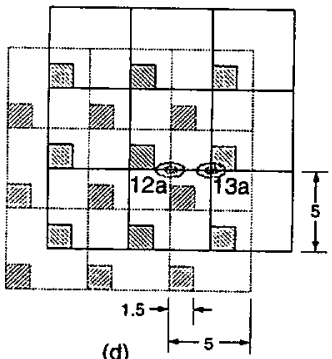

(d)

$$
\begin{array}{r}
- \text { Layer } 1 \\
- \text { Layer } 2 \\
\hline
\end{array}
$$

Figure 2: All measurement sites relative to peening fields: (a) $10 \%$ overlap and $5 \times 5 \mathrm{~mm}$ spot, (b) $10 \%$ overlap and $3.2 \times 3.2 \mathrm{~mm}$ spot, (c) $50 \%$ overlap and $5 \times 5 \mathrm{~mm}$ spot, (d) $50 \%$ overlap and $5 \times 5 \mathrm{~mm}$ spot with a $1.5 \times 1.5 \mathrm{~mm}$ corner attenuation as indicated with cross hatching

\section{RESULTS}

Stress profiles for SP and for variations in LP process parameters show interesting trends (Figure 3). Results at sites 0-S and 0 -L indicate that compressive residual stress extended far deeper into the surface for LP than for SP (Figure 3(a)), but that SP generated a larger compressive residual stress close to the surface. Decreasing the pulse width of the second-layer from 18 to $12 \mathrm{~ns}$ increased the near-surface residual stress to a similar magnitude as produced by SP (compare Figure 3(a) and (b)). Reducing the laser fluence from 60 to $45 \mathrm{~J} / \mathrm{cm}^{2}$ resulted in residual stress that was of similar magnitude near the surface, but that decreased more rapidly with distance from the surface (Figure 3(b)). Laser spot sizes of 3.2 and $5.0 \mathrm{~mm}$ produced very similar residual stress profiles (Figure 3(c)). Coupons treated with 10\% and 50\% layer overlap had similar levels of residual stress close to the surface, but the residual stress decreased more rapidly with depth for $10 \%$ overlap (Figure 3(d)). In general, residual stress at the surface can be as high with LP as for SP, while the depth of the compressive residual stress is far greater for LP than for SP.

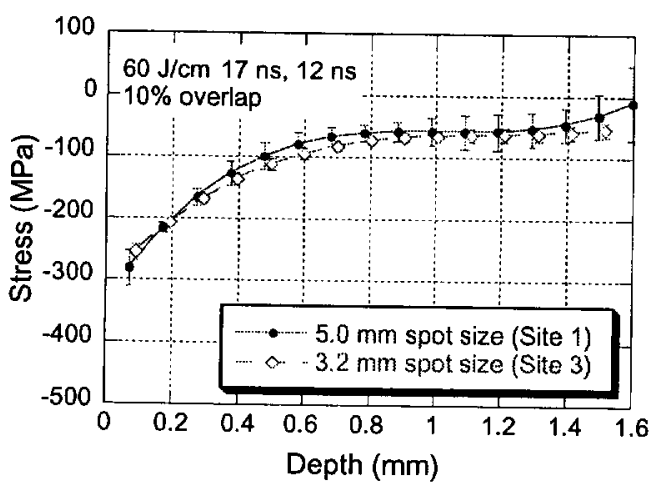

(c)

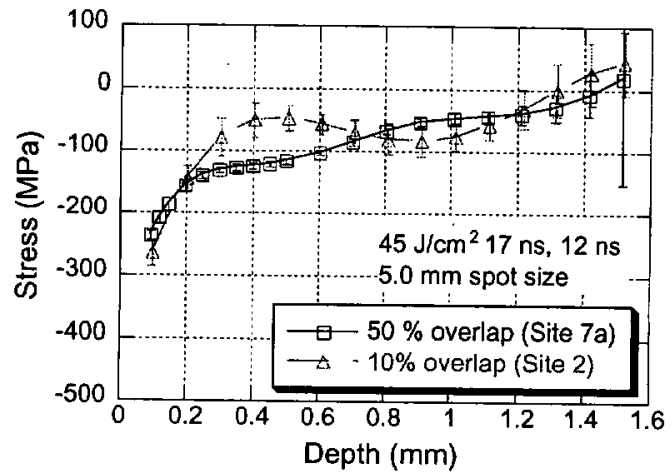

(d)

Figure 3: Comparison of peening processes: (a) SP vs. LP (b) effect of energy density, (c) effect of spot size, (d) effect of
layer overlap. 
Stress adjacent to the peened area was significantly lower than that within the peened area (Figure 4 (a)). Both near and below the surface, the residual stress magnitude decreased with distance from the peened area. No tensile residual stresses were found outside the peened area.

Variations of the residual stress profile within the peened area were limited to the near-surface region (Figure 4(b)). The highest surface residual stress was found at the edge of a layer-two spot (site 9a), but because results are not available for all sites at shallow depths, it is not possible to conclude which location had the highest or lowest surface stress. In the region between 0.10 and $0.20 \mathrm{~mm}$, the largest stresses occurred at the center of a layer-two spot (site $7 \mathrm{a}$ ) with the difference between sites being as much as $100 \mathrm{MPa}$. Below $0.60 \mathrm{~mm}$, all locations had similar stress profiles.

The effects of corner attenuation were also limited to the near-surface region (Figure 4 (c)). At the edge of a layer-two spot (sites $10 \mathrm{a}$ and $12 \mathrm{a}$ ), residual stresses at depths less than $0.30 \mathrm{~mm}$ were significantly altered by corner attenuation, with the attenuated site having much lower stress close to the surface. At the center of a layer-one spot (sites 11a and 13a), nearsurface residual stresses were unaffected by corner attenuation.

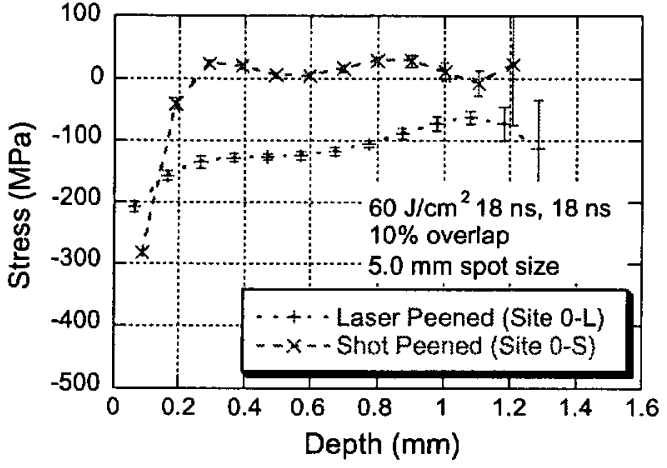

(a)

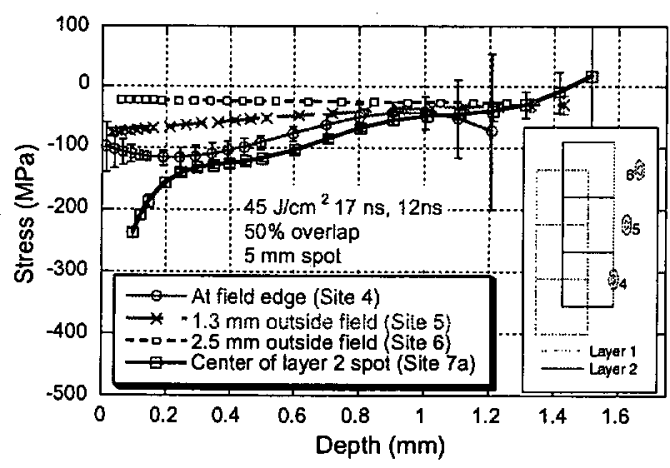

(a)

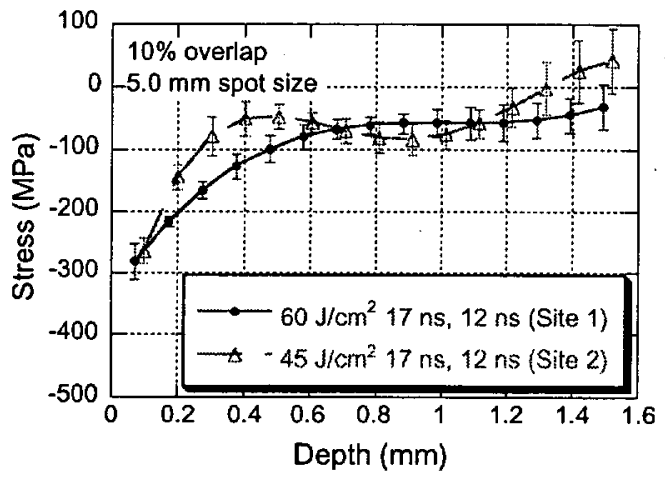

(b)

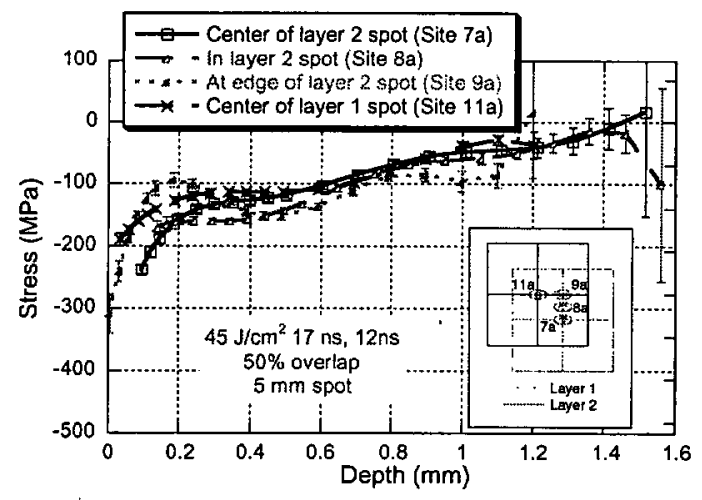

(b)

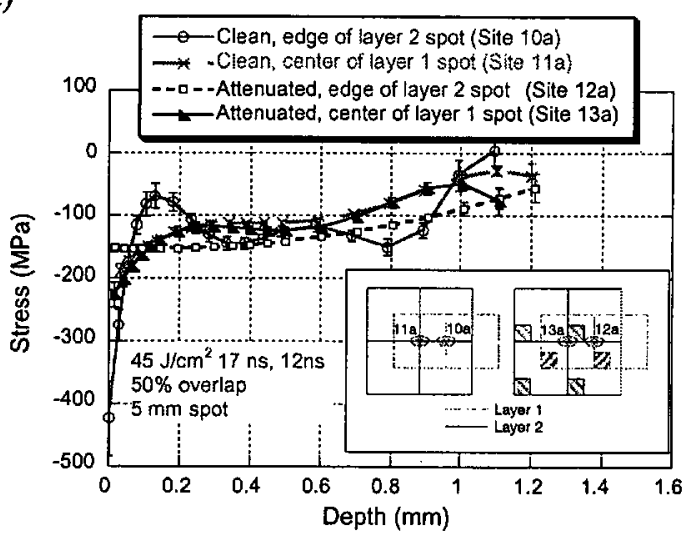

(c)

Figure 4: (a) Residual stress adjacent to a laser peened area, (b) in-plane variations of the residual stress profile, and (c) residual stress with and without corner attenuation 


\section{DISCUSSION}

Several of the key findings mentioned above may have important implications for potential LP applications. Compared with SP, compressive surface residual stresses due to LP were of similar magnitude near the surface and extended far deeper into the surface of the component (Figure 3(a)). The greater depth of compressive residual stress for LP has previously been found to enhance fatigue performance to a greater degree than occurs with SP [3,9-11].

Near-surface residual stresses due to LP were significantly affected by only a single parameter. Residual stress at depths less than $0.20 \mathrm{~mm}$ were significantly increased when the pulse width for the second-layer laser was decreased from 18 to $12 \mathrm{~ns}$ (compare results at site $0-\mathrm{L}$ and site 1 in Figure 3 ). Other parameter variations produced little change in near-surface stress (Figure 3), indicating that near-surface stress was robust to variations of fluence, spot size, and layer overlap.

Residual stresses at depths between 0.20 and $0.60 \mathrm{~mm}$ were significantly affected by both laser fluence and layer overlap. Higher compressive residual stresses were found in this depth range with increased fluence and with increased layer overlap. The amount of the residual stress increase for both parameters was largest near a depth of $0.40 \mathrm{~mm}$ and was approximately $75 \mathrm{MPa}$ in each case.

The fact that surface tensile residual stress was not found adjacent to the laser peened area near the surface suggests that LP should not have detrimental effects on fatigue performance. However, the results here may not be representative of what occurs in other geometries. Further, it may be that surface tensile residual stress exists further from the peened area than examined here.

The results show that in-plane variations of residual stress did exist within the laser peened area (Figure 4 (b)), which suggests that fatigue cracks may initiate and grow at preferred locations (i.e., at areas of lower magnitude stress). It would therefore be of value for LP fatigue test programs to incorporate fractographic evaluation to determine whether in-plane variations of residual stress lead to preferred sites for crack initiation and growth.

Since one location in the intentionally attenuated field had a significantly reduced residual stress (compare site $10 \mathrm{a}$ with site 12a, Figure 4 (c)), applications of LP should be careful to ensure a uniform spatial distribution of laser energy in the laser spots used for peening.

\section{ACKNOWLEDGMENTS}

UC Davis received financial support from Metal Improvement Company Inc. and LLNL received financial support from the National Center for Manufacturing Science (USA).

\section{REFERENCES}

[1] P. Peyre, et al., Mater. Sci. and Eng. A Vol. 280 (2000), p. 294.

[2] P.K. Sharp and G. Clark: The Effect of Peening on the Fatigue Life of 7050 Aluminum Alloy (Defence Science \& Technology Organisation, Victoria, Australia 2001).

[3] P. Peyre, et al., Mater. Sci. and Eng. A Vol. 210 (1996), p. 102.

[4] M.B. Prime and I. Finnie, J. Eng. Mater. and Tech. Vol. 118 (1996), p. 410.

[5] M.B. Prime, Appl. Mech. Rev. Vol. 52 (1999), p. 75.

[6] M.R. Hill, W.-Y. Lin, J. Eng. Mater. and Tech. (in press).

[7] J.E. Rankin, et al., sumbitted to J. Eng. Mater. and Tech.

[8] C.B. Dane, et al., J. Quant. Elect. (IEEE) Vol. 31 (1995), p. 148.

[9] G. Hammersley, et al., Opt. and Lasers in Eng. Vol. 34 (2000), p. 327.

[10] P. Peyre, et al., Surf. Eng. Vol. 11 (1995), p. 47.

[11] A.H. Clauer, in Surface Performance of Titanium (TMS, Warrendale, PA 1996), p. 217. 


\section{University of California}

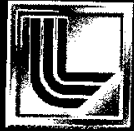

Lawrence Livermore National Laboratory

Laser Programs

PO Box 808 • Livermore, CA • 94551

Visit our NIF Programs Website at: http://www./ln/.gov/nif/

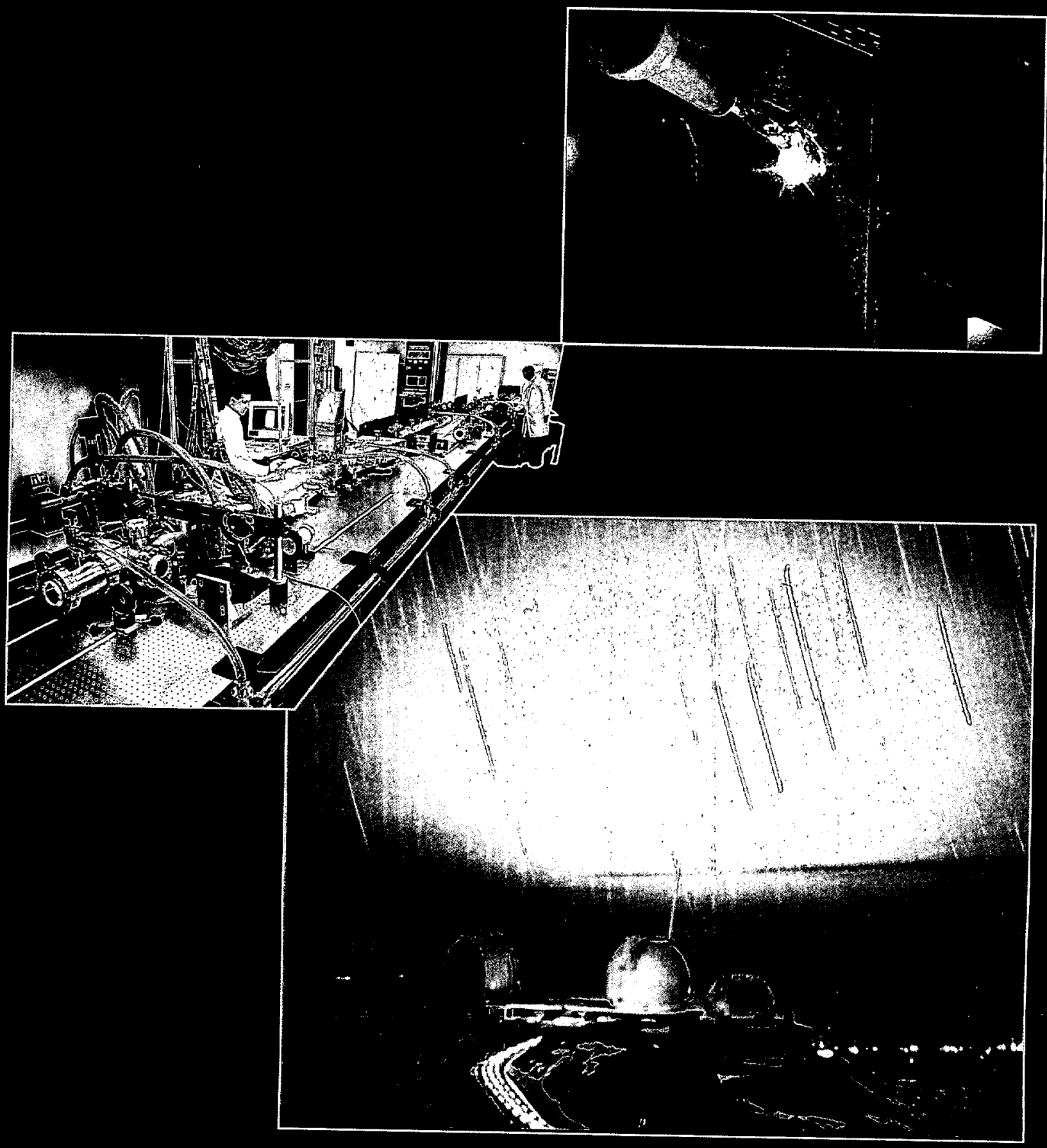

